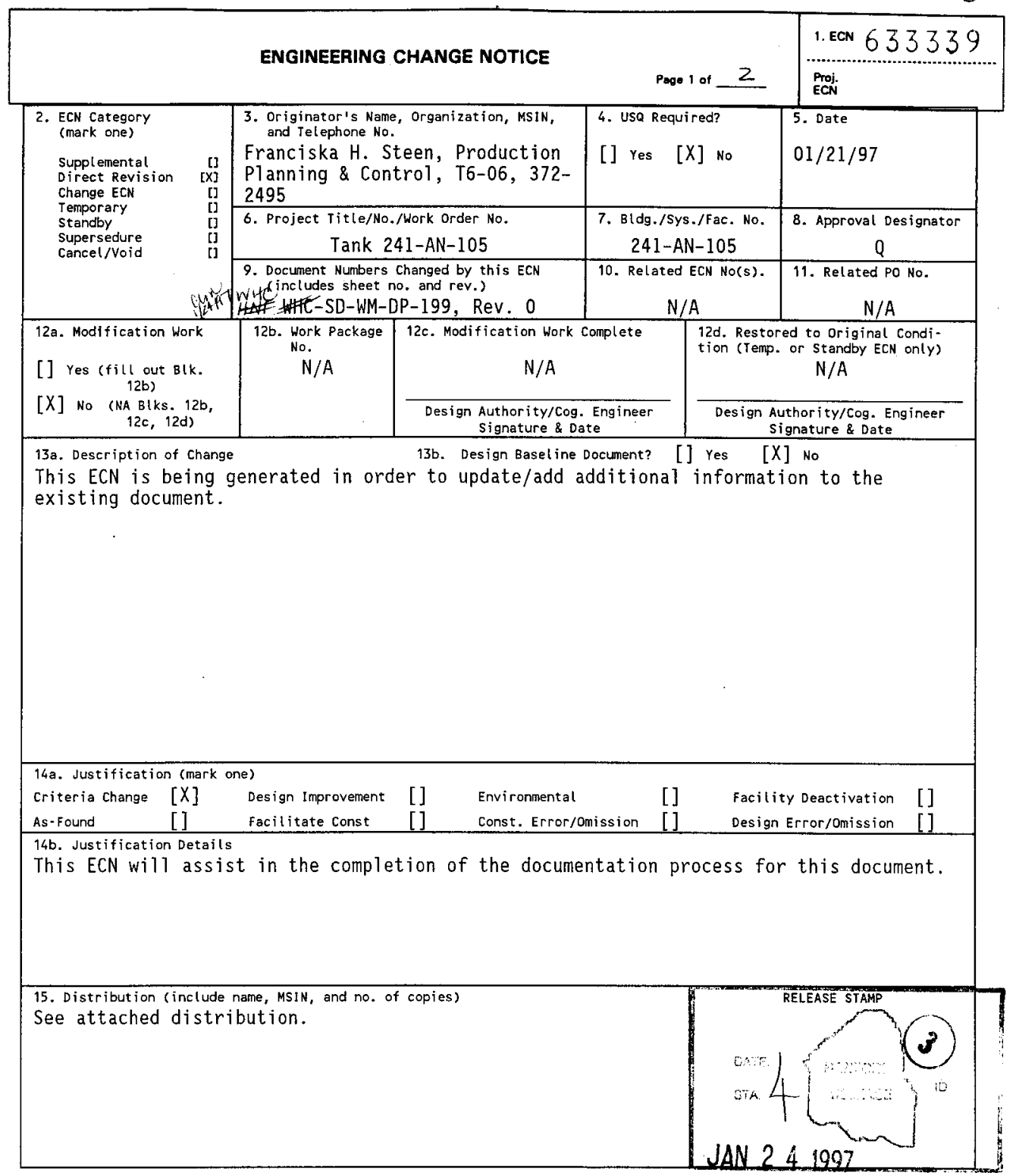

A-7900-013-2 (05/96) GEF095 


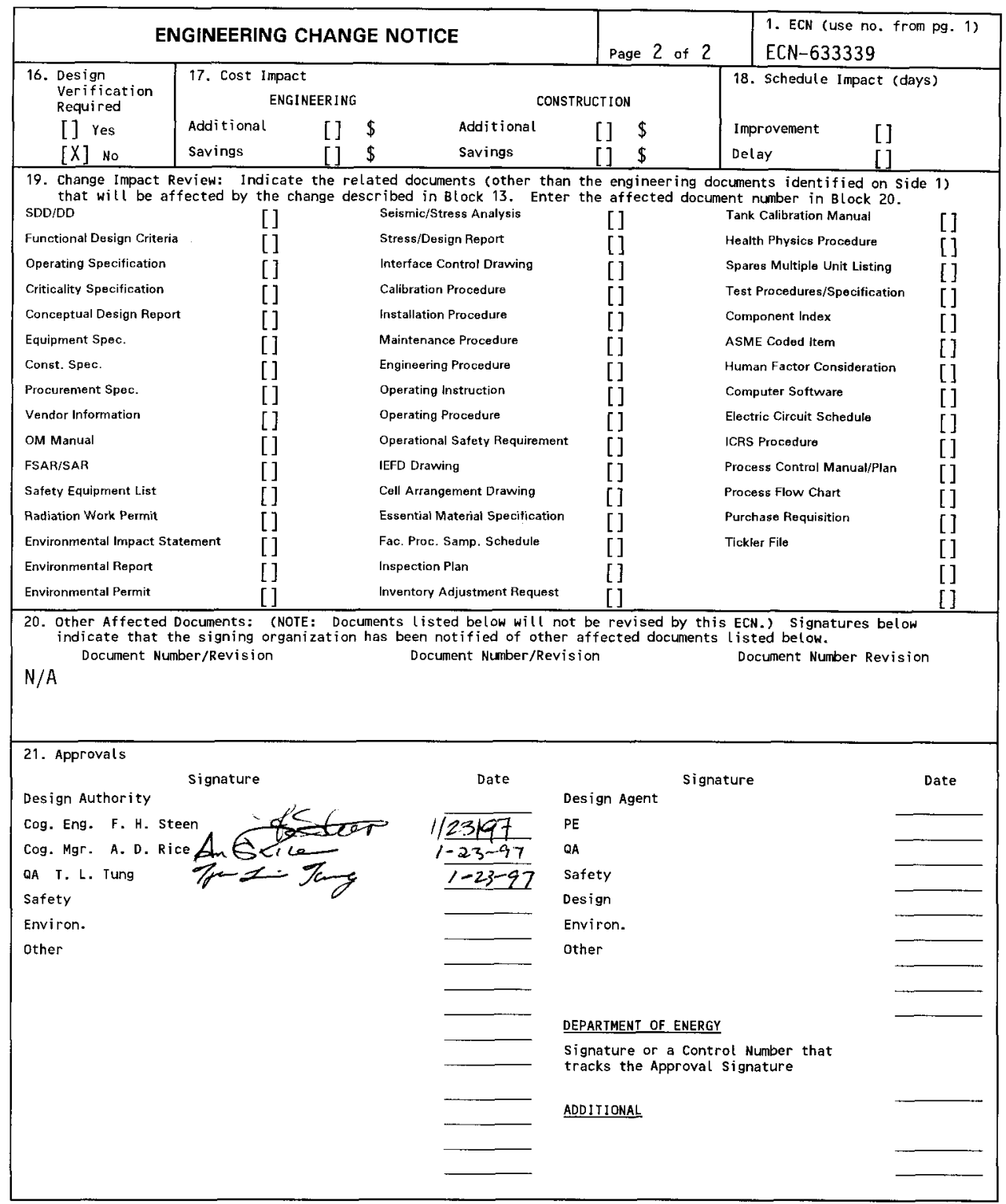




\title{
Tank 241-AN-105, Cores 152 and 153 Analytical Results for the Final Report
}

\author{
Franciska H. Steen \\ Rust Federal Services of Hanford, Inc., Richland, WA 99352 \\ U.S. Department of Energy Contract DE-AC06-87RL10930
}

$\begin{array}{lll}\text { EDT/ECN: } & \text { ECN-633339 } & \text { UC: } 2070 \\ \text { Org Code: } & 7576 \mathrm{~B} & \text { Charge Code: MD378 } \\ \text { B\&R Code: } & \text { EW } 3120074 & \text { Total Pages: } 2496\end{array}$

Key Words: Tank 241-AN-105, Tank AN-105, AN-105, AN Farm, Core 152, Core 153, Analytical Results, Final Report

Abstract: $N / A$

TRADEMARK DISCLAIMER. Reference herein to any specific commercial product, process, or service by trade name, trademark, manufacturer, or otherwise, does not necessarily constitute or imply its endorsement, recommendation, or favoring by the United States Government or any agency thereof or its contractors or subcontractors.

Printed in the United States of America. To obtain copies of this document, contact: WHC/BCS Document Control Services, P.O. Box 1970, Mailstop H6-08, Richland WA 99352, Phone (509) 372-2420; Fax (509) 376-4989.
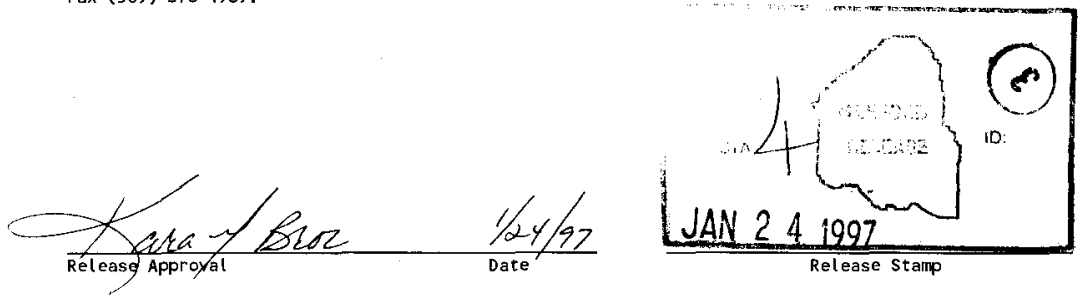

Approved for Public Release 
RECORD OF REVISION

(1) Document Number

HNF-SD-WM-DP-199

(2) Title

Tank 241-AN-105, Cores 152 and 153 Analytical Results for the Final Report CHANGE CONTROL RECORD

\begin{tabular}{|c|c|c|c|c|c|}
\hline \multirow{2}{*}{ (3) } & \multirow{2}{*}{ Revision } & \multirow{2}{*}{ (4) } & \multirow{2}{*}{ Description of Change - Replace, Add, and Delete Pages } & \multicolumn{2}{|c|}{ Authorized for Release } \\
\hline & & & & (5) Cog. Engr. & (6) Cog. Mgr. \\
\hline & 0 & & $\begin{array}{l}\text { Initially released } 09 / 20 / 96 \text { on EDT- } \\
617495\end{array}$ & F.H. Steen & A.D. Rice \\
\hline & & & & $600,1 / 23197$. & \\
\hline & 1 RS & & Incorporate per ECN-633339 & E.H. Steen & A.D. Rice \\
\hline & & & 1 - 1 & $\tan \times 1 / 23 / 97$ & An $<>$ - $-2,-9$ \\
\hline & & & & & \\
\hline & & & & & \\
\hline & & & & & \\
\hline & & & & & \\
\hline & & & & & \\
\hline & & & & & \\
\hline & & & & & \\
\hline & & & & & \\
\hline & & & & & \\
\hline & & & & & \\
\hline & & & & & \\
\hline & & & & & \\
\hline & & & & & \\
\hline & & & & & \\
\hline & & & & & \\
\hline & & & & & \\
\hline & & & & & \\
\hline & & & & & \\
\hline & & & & & \\
\hline & & & & & \\
\hline & & & & & \\
\hline & & & & & \\
\hline & & & & & \\
\hline & & & & & \\
\hline & & & & & \\
\hline & & & & & \\
\hline & & & & & \\
\hline & & & & & \\
\hline & & & & & \\
\hline & & & & & \\
\hline
\end{tabular}


HNF

WHC-SD-WM-DP-199, REV. 1

xims $V_{24}(4)$

ANALYTICAL SERVICES

TANK 241-AN-105, CORES 152 AND 153 ANALYTICAL RESULTS FOR THE FINAL REPORT

Project Coordinator: FRANCISKA H. STEEN

Prepared for the U.S. Department of Energy

Office of Envirommental Restoration and Waste Management

by

222-S Laboratory

Rust Federal Services of Hanford Inc.

P.0. Box 700

Richland, Washington 
HNF-SD-WM-DP-199, REV. 1

WHC-SD-WM-DP-199, REV. 1

THIS PAGE WAS INTENTIONALLY LEFT BLANK 


\section{HNF-SD-WM-DP-199, REV. 1}

WHC-SD-WM-DP-199, REV , 1

TABLE OF CONTENTS

Narrative . . . . . . . . . . . . . . . . . . . . 1

AN-105 Sample Breakdown (Attachment 1) . . . . . . . . . . 13

Sample Data Summary . . . . . . . . . . . . . . . . . . . . . 56

Photographs . . . . . . . . . . . . . . . . . . . . . 164

Sample Handling . . . . . . . . . . . . . . . . . . . . 203

Extrusion Workl ist \#9966 (3481) . . . . . . . . . . . . . . 216

Extrusion Worklist \# 9967 (3482) . . . . . . . . . . . . . . 217

Extrustion Workl ist \# 9968 (3483) . . . . . . . . . . . . 218

Extrusion Worklist \# 9969 (3491) .............. . 219

Extrusion Worklist \# $9970(3492)$. . . . . . . . . . . . . 220

Extrusion Worklist \# 9971 (3493) . . . . . . . . . . . . . . 221

Extrusion Norklist \# 9972 (3494) . . . . . . . . . . . . . . 222

Extrusion Work1 ist \# $9973(3495)$. . . . . . . . . . . . . . 223

Extrusion Workl ist \# 10012 (3502) . . . . . . . . . . . . . . . . 224

Extrusion Worklist \#10014 (3503) . . . . . . . . . . . . . 225

Extrusion Worklist \# $10015(3504)$. . . . . . . . . . . . . . . 226

Extrusion Worklist \#10110 (3505) . . . . . . . . . . . . . . 227

Extrusion Workl ist \# 10181 (3512) . . . . . . . . . . . . . 228

Extrusion Worklist \#10182 (3513) . . . . . . . . . . . . 229

Extrusion Workl ist \# $10183(3514)$. . . . . . . . . . . . . 230

Extrusion Workl ist \# 10388 (3588) ................ 231

Extrusion Workl ist $\# 10389(3589)$. . . . . . . . . . . . . . 232

Extrusion Worklist \# 10390 (3590) . . . . . . . . . . . . 233

Extrusion Workl ist \#10611 (3630) ............... 234

Extrusion Workl ist \# $10613(3637)$. . . . . . . . . . . . . . . 235

Extrusion Workl ist \# 10614 (3638) . . . . . . . . . . . . . . . . 236

Extrusion Workl ist \# 10615 (3639) ................. 237

Extrusion Work1 ist \# 10652 (3663) ................ . 238

Extrusion Workl ist \#10653 (3664) ................ 239

Extrusion Workl ist \#10654 (3665) . . . . . . . . . . . . . 240

Extrusion Work1 ist \# $10655(3666)$. . . . . . . . . . . . . . . 241

Extrusion Work1 ist \# 11399 (4154) . . . . . . . . . . . . . 242

Extrusion Workl ist \# 11400 (4155) ................ 243

Extrusion Work1 ist \# 11401 (4156) ................. 244

Extrusion Workl ist \# 11693 (4324) . . . . . . . . . . . . . . . . 245

Extrusion Workl ist \# $11702(4323)$. . . . . . . . . . . . . . 246

Extrusion Work1 ist \# 11780 (4326) ................. 247

Extrusion Workl ist \#11781 (4327) . . . . . . . . . . . . . . 248

Extrusion Workl ist \#11782 (4328) ............... . 249

Extrusion Work 1 ist \# $11932(4384)$. . . . . . . . . . . . . . 250 


\section{HNF-SD-WM-DP-199, REV. 1}

WHC-SD-WM-DP-199, REV. 1

TABLE OF CONTENTS (Continued)

Extrusion Work1 ist \# 11934 (4385) . . . . . . . . . . . . 251

Extrusion Work1 ist \# 11936 (4386) . . . . . . . . . . . . . 252

Sample Preparations . . . . . . . . . . . . . . . . . . 253

Fusion Digestion Workl ist \# $11117(3827,3893,3894,3895)$. . . 255

Fusion Digestion Workl ist \# $11146(3889,3890,3896,3897)$. . . 257

Fusion Digestion Workl ist \# $11147(3891,3892)$. . . . . . . . . 259

Fusion Digestion Workl ist \# $12107(4314,4317,4337)$. . . . . . . 260

Fusion Digestion Worklist \# $12108(4441,4442,4443)$. . . . . . . 261

Fusion Digestion Workl ist \# $12109(3984,3985,3986)$. . . . . . . 262

Fusion Digestion Worklist \# $12383(4256,4261)$. . . . . . . . . . 263

Water Digestion Worklist \# $11150(3907,3915)$. . . . . . . . . 264

Water Digestion Workl ist \#11151 $(3829,3908)$. . . . . . . . . . . 265

Water Digestion Workl ist \#11152 $(3909,3910)$. . . . . . . . . . . 266

Water Digestion Workl ist \# $11153(3911,3912)$. . . . . . . . . . . 267

Water Digestion Workl ist \# $11154(3913,3914)$. . . . . . . . . . . 268

Water Digestion Workl ist \# $12025(3990,3991)$. . . . . . . . . . . 269

Water Digestion Workl ist \# 12026 (3992) . . . . . . . . . . . . . 270

Water Digestion Workl ist \# $12113(4316,4339)$. . . . . . . . . . 271

Water Digestion Work1 ist \# $12114(4319,4447)$. . . . . . . . . . . 272

Water Digestion Workl ist \# $12115(4448,4449) . . . . . . . . . . .273$

Water Digestion Workl ist \# 12253 (593) . . . . . . . . . . . . . 274

Water Digestion Workl ist \# $12385(4258,4263) . . . . . . . . . . .275$

Water Digestion Workl ist \# $13659(5325,5326$,$) . . . . . . . . . 276$

Acid Digestion Worklist \# $11148(3906,3898,3828,3899,3900,3901) 277$

Acid Digestion Worklist \# $11149(3902,3903,3904,3905)$. . . . 279

Acid Digestion Workl ist \# $12110(4315,4318,4338,4444)$. . . . . 281

Acid Digestion Workl ist \# $12111(3988,4445,4446) \ldots . . . . . . .283$

Acid Digestion Workl ist \# $12112(3987,3989)$. . . . . . . . . . 285

Acid Digestion Workl ist \# 12252 (592) . . . . . . . . . . . . . . 286

Acid Digestion Worklist \# 12264 (4144) . . . . . . . . . . . . . 287

Acid Digestion Worklist \# $12384(4257,4262)$. . . . . . . . . . . 288

AN-105 Bulk Density Worksheets . . . . . . . . . . . . . . . . 289

Inorganic Analyses . . . . . . . . . . . . . . . . . . . . . 304

Differential scanning Calorimetry (DSC)

DSC Workl ist \# 12274 (4039) . . . . . . . . . . . . . . . 306

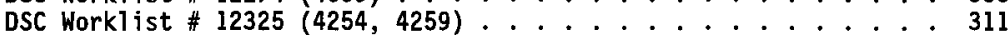

Thermogravimetric Analyes (TGA)

TGA Worklist \# 12278 (4039) . . . . . . . . . . . . . . . . 317

TGA Work 1 ist \# $12334(4254,4259)$. . . . . . . . . . . . . . 324 


\title{
HNF-SD-WM-DP-199, REV. 1
}

\author{
WHC-SD-WM-DP-199, REV. 1
}

TABLE OF CONTENTS (Continued).

\begin{abstract}
IC Worklist \# $11955(3913,3914)$. . . . . . . . . . . . . . 794
IC Worklist \# $12409(4308,4309)$. . . . . . . . . . . . . . . . . 806

IC Workl ist \# $12410(4333,4419)$. . . . . . . . . . . . . . . . . . 817

IC Workl ist \# $12411(4420,4421)$. . . . . . . . . . . . . . . . . . . 828

IC Worklist \# $12412(4433,4434)$. . . . . . . . . . . . . . . . 841

IC Worklist \# $12420(4039)$. . . . . . . . . . . . . . . . . 852

IC Worklist \# $12473(3744)$. . . . . . . . . . . . . . . 861

IC Workl ist \# $12475(3990)$. . . . . . . . . . . . . . . . . . 872

IC Workl ist \# $12476(3992)$

IC Work1 ist \# $12478(4339,4316)$. . . . . . . . . . . . . . . . . 896

IC Work l ist \# $12479(4319,4447)$. . . . . . . . . . . . . . . . 908

IC Work list \# $12502(4309,4333)$. . . . . . . . . . . . . . . . . 920

IC Work list \# $12503(4419,4420)$. . . . . . . . . . . . . . . . . 930

IC Workl ist \# $12504(4421,4433)$. . . . . . . . . . . . . . 940

IC Workl ist \# 12505 (4434) . . . . . . . . . . . . . . . . . . . . 952

IC Workl ist \# $12506(3990,3991)$. . . . . . . . . . . . . . . . 960

IC Workl ist \# $12508(4339,4316)$. . . . . . . . . . . . . . . 972

IC Work list \# $12509(4319,4447)$. . . . . . . . . . . . . . . . 983

IC Worklist \# $12683(3747,3739)$. . . . . . . . . . . . . . . . . 996

IC Workl ist \# $12832(4258,4263)$. . . . . . . . . . . . . . . . 1007

IC Workl ist \# $13020(4258,4263)$. . . . . . . . . . . . . . . 1020

IC Worklist \# $13023(3744)$. . . . . . . . . . . . . . . . . 1031

IC Workl ist \# $13202(3991)$. . . . . . . . . . . . . . . . . 1046

IC Workl ist \# $13311(3744)$. . . . . . . . . . . . . . . . . . 1056

IC Workl ist \# $13601(4420,4421)$. . . . . . . . . . . . . . . . 1068

IC Workl ist \# $13814(4448,4449)$. . . . . . . . . . . . . . . 1078

IC Worklist \# $14081(3909,3910)$. . . . . . . . . . . . . . . . 1090

IC Workl ist \# $14082(4308,4831)$. . . . . . . . . . . . . . . . 1102

IC Work list \# $14520(5325,5326)$. . . . . . . . . . . . . . . . 1112

IC Workl ist \# $14704(5325,5326)$. . . . . . . . . . . . . . . . 1123

IC Workl ist \# $15170(3913,3914,3992)$. . . . . . . . . . . . . . 1135

IC Workl ist \# $15198(3911,3912)$. . . . . . . . . . . . . . . . 1152

IC Workl ist \# $15476(4258,4263)$. . . . . . . . . . . . . . . . . 1162

IC Worklist \# 15852 (4419) . . . . . . . . . . . . . . . . . 1174

Inductively Coupled Plasma Spectroscopy Analyses (ICP)

ICP Work1 ist \# 10858 (3631) . . . . . . . . . . . . . . . . 1181

ICP Work 1 ist \# $11595(3732,3739,3740,3741,3742)$. . . . . . . . 1188

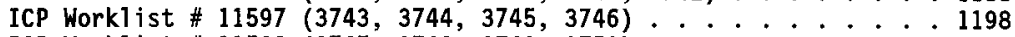

ICP Work l ist \# $11598(3747,3748,3749,3750)$. . . . . . . . . . . 1207

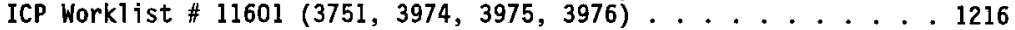

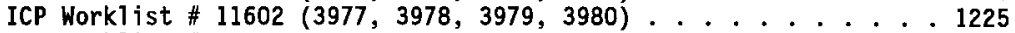

ICP Work 1 ist \# $11976(3828,3898,3899,3900,3901,3906)$. . . . . 1234

ICP Work 1 ist \# $12085(3902,3903,3904,3905)$. . . . . . . . . . . 1243

ICP Work1 ist \# $12098(3827,3893,3894,3895)$. . . . . . . . . . . 1252

ICP Work1 ist \# $12131(3889,3890,3896,3897)$. . . . . . . . . 1261
\end{abstract}




\section{HNF-SD-WM-DP-199, REV. 1}

WHC-SD-WM-DP-199, REV. 1

TABLE OF CONTENTS (Continued)

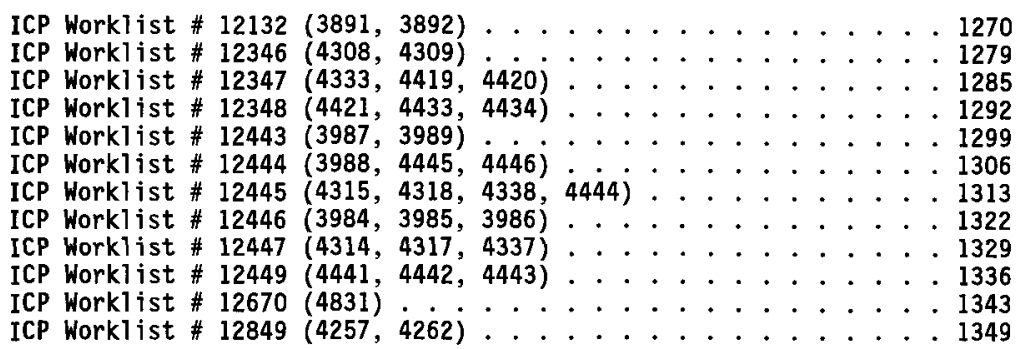

Total Inorganic Carbon/Total Organic Carbon Analyses (TICTOC)

TICTOC Worklist \# 12388 (4049) . . . . . . . . . . . . . 1356 TICTOC Worklist \# $12402(4254,4259)$. . . . . . . . . . . . 1376

Uranium Analyses (U)

U Worklist \# $12764(4256,4261)$. . . . . . . . . . . . . . . . . . 1406

U Workl ist \# $14454(4003)$. . . . . . . . . . . . . . . . . . 1419

U Work1 ist \# $14576(5546)$. . . . . . . . . . . . . . . . . . . 1428

Radiochemical Analyses ................. . 1437

Tritium Analyses (H3)

H3 Workl ist \# $12793(4258)$. . . . . . . . . . . . . . . 1439

H3 Work1 ist \# 14427 (4003) . . . . . . . . . . . . . . . . . 1446

H3 Work1 ist \# $14765(4831)$. . . . . . . . . . . . . . . . 1453

H3 Workl ist \# 15289 (4263) . . . . . . . . . . . . . . . . . . 1460

Total Alpha Analyses (AT)

AT Work1 ist \# $11366(3743,3744,3745,3746,3747) \ldots . . . . . .1466$

AT Workl ist \# $11612(3975,3976,3977,3978,3979,3980)$. . . . . 1482

AT Worki ist \# $11862(3893,3827)$. . . . . . . . . . . . 1500

AT Workl ist \# $11866(3889,3890)$. . . . . . . . . . . . . . 1509

AT Workl ist \# $11977(3732,3739,3740,3741,3742) . . . . . . . . .1518$

AT Work list \# $12123(3891,3892) \ldots . . . . . . . . . . . . .1534$

AT Workl ist \# $12275(4039,4419,4420,4433,4434)$. . . . . . . . 1543

AT Workl ist \# $12280(4308,4309,4333) \ldots . . . . . . . . . . .1563$

AT Workl ist \# $12461(3985)$. . . . . . . . . . . . . . . . . . . . 1575

AT Workl ist \# $12463(4317,4337)$. . . . . . . . . . . . . 1585

AT Workl ist \# $12465(4442,4443)$. . . . . . . . . . . . . . . . . 1595

AT Workl ist \# 12602 (3984) . . . . . . . . . . . . . . . . . 1604

AT Work1 ist \# 12603 (4421) ..................... . . 1611

AT Workl ist \# $12953(3748,3749,3750,3751) \ldots . . . . . . . .1618$ 


\section{HNF-SD-WM-DP-199, REV. 1}

WHC-SD-WM-DP-199, REV. 1

TABLE OF CONTENTS (Continued)

Total Alpha/Total Beta Analyses (AT/TB)

AT/TB Workl ist \# 11611 (3974) . . . . . . . . . . . . . . 1631

AT/TB Workl ist \# $12752(4256,4261) \ldots \ldots . . . . . . . . .1643$

Total Beta Analyses (TB)

TB Worklist \# $12309(4049) \ldots \ldots . \ldots . . \ldots 1661$

Gamma Energy Analyses (GEA)

GEA Workl ist \# 12312 (4049) . . . . . . . . . . . . . . 1668

GEA Workl ist \# $12759(4256,4261) \ldots \ldots . . \ldots \ldots 1687$

GEA Worklist \#14451 (4003) ................ . . 1714

Iodine-129 Analyses (I-129)

I-129 Workl ist \# $12313(4049) \ldots . . . . . . . . . . . . .1733$

I-129 Workl ist \# $12795(4258,4263) \ldots . . . . . . . . . .1752$

I-129 Work1 ist \# $14553(4003) \ldots \ldots . . . . . . . . . .1778$

Strontium Analyses (Sr-90)

Sr-90 Work1 ist \# $12516(4049) \ldots \ldots$. . . . . . . . . . . 1796

Sr-90 Workl ist \# $13052(4256,4261) \ldots . . . . . . . . . . .1804$

Sr-90 Worklist \# 15407 (4003) . . . . . . . . . . . . 1814

Technetium-99 Analyses (Tc-99

Tc-99 Workl ist \# $12763(4256,4261) \ldots . . . . . . . . . . .1820$

Tc-99 Workl ist \# $13001(4049,414) \ldots . . . . . . . . .1830$

Tc-99 Work1 ist \# 14447 (4003) . . . . . . . . . . . . 1839

Neptunium-237 Analyses (Np-237)

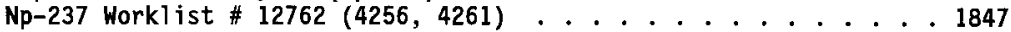

Np-237 Workl ist \# 12315 (4049) . . . . . . . . . . . . . . . 1857

Np-237 Worklist \# 14429 (4003) . . . . . . . . . . . 1864

Americium-241 Analyses (Am-241)

Am-241 Workl ist \#12311 (4049) ................. . 1871

Am-241 Workl ist \# $13071(4256,4261)$. . . . . . . . . . . . . . 1890

Am-241 Workl ist \#14426 (4003) . . . . . . . . . . . . . . . 1918

Plutonium Analyses (Pu)

Pu Workl ist \#12316 (4049) . . . . . . . . . . . . . . 1937

Pu Workl ist \# $12761(4256,4261)$. . . . . . . . . . . . . . . 1957

Pu Workl ist \# $14431(4003)$. . . . . . . . . . . . . . . . 1985

Part II Tank 241-AN-105, Cores 152 and 153, Analytical Results for the 45. Day Report

Narrative . . . . . . . . . . . . . . . . . . . . 2-1 


\section{HNF-SD-WM-DP-199, REV. 1}

WHC-SD-WM-DP-199, REV. 1

TABLE OF CONTENTS (Continued)

AN-105 Sample Breakdown (Attachment 1) . . . . . . . . . 2-12

Sample Data Summary . . . . . . . . . . . . . . . . . 2-51

Chain of Custody Forms . . . . . . . . . . . . . . . . 2-90

Inorganic Analyses . . . . . . . . . . . . . . . . . 2-138

Differential Scanning Calorimetry (DSC)

DSC Workl ist \# $11288(3732,3739)$. . . . . . . . . . . . . 2-141

DSC Worklist \# $11289(3740,3741)$. . . . . . . . . . . . . . . . 2-147

DSC Workl ist \# $11290(3742,3743)$. . . . . . . . . . . . . . . . 2-153

DSC Worklist \# $11291(3744,3745)$. . . . . . . . . . . . . . . 2-159

DSC Worklist \# $11292(3746,3747)$. . . . . . . . . . . . . . 2-165

DSC Workl ist \# $11293(3748,3749)$. . . . . . . . . . . . . . . . 2-171

DSC Workl ist \# $11294(3750,3751)$. . . . . . . . . . . . . 2-176

DSC Workl ist \# $11308(3731)$. . . . . . . . . . . . . . . . . . 2-182

DSC Workl ist \# $11499(3792,3793)$. . . . . . . . . . . . . 2-186

DSC Worklist \# $11500(3794,3795)$. . . . . . . . . . . . . . . 2-192

DSC Work list \# $11501(3796,3797)$. . . . . . . . . . . . . 2-198

DSC Workl ist \# $11502(3798,3816)$. . . . . . . . . . . . . . . 2-204

DSC Worklist \# $11503(3817)$.................. . . 2-210

DSC Work1 ist \# $11658(3974,3975)$. . . . . . . . . . . . . . . 2-214

DSC Workl ist \# $11659(3976,3977)$. . . . . . . . . . . . . . 2-220

DSC Workl ist \# $11660(3978,3979,3980)$. . . . . . . . . . . . . 2-226

DSC Workl ist \# $11663(3981,3982,3983)$. . . . . . . . . . . . 2-235

DSC Workl ist \# $12274(4039,4308)$. . . . . . . . . . . . 2-243

DSC Workl ist \# $12327(4310,4311)$. . . . . . . . . . . . . 2-249

DSC Work1 ist \# $12328(4335,4435)$. . . . . . . . . . . . 2-255

DSC Workl ist \# $12329(4436,4437)$. . . . . . . . . . . . . 2-261

DSC Work list \# $12330(4309,4333)$. . . . . . . . . . . . . . 2-267

DSC Work 1 ist \# $12331(4419,4420)$. . . . . . . . . . . . 2-274

DSC Work 1 ist \# $12332(4421,4433)$. . . . . . . . . . . . . . . 2-280

DSC Work1 ist \# 12333 (4434) . . . . . . . . . . . . . . . . . . 2-287

DSC Work1 ist \# $12356(3731,4254,3792,3793,3794,3795,3796$,

3797) ..................... 2-291

DSC Work1 ist \# $12357\left(3798,3816^{\circ}, 3817^{\circ}, 42 \dot{\circ}^{\circ}, 3981^{\circ}, 3982^{\circ}, 39 \dot{8}^{\circ}, 4310^{\circ}\right.$

4311) . . . . . . . . . . . . 2-294

DSC Work1 ist \# $12358(4335,4435,4436,4437,4433,4434) . . .02-297$

DSC Worklist \# 12359 (3732, 4039, 3739, 3740,3741, 3742, 3743, 3744

3745) ................. 2-299

DSC Work1 ist \# $12360(3746,3747,3748,3749,3750,3751,3974,3975$

$3976,3977) \cdot 0^{\circ} \cdot 0^{\circ} \cdot 0^{\circ} \cdot{ }^{\circ} \cdot j^{\circ} \cdot 2-302$

DSC Work1 ist \# $12361(3978,3979,3980,4308,4309,4333,4419,4420$

4421)................ 2-... 205 


\section{HNF \\ TABLE OF CONTENTS (Continued)}

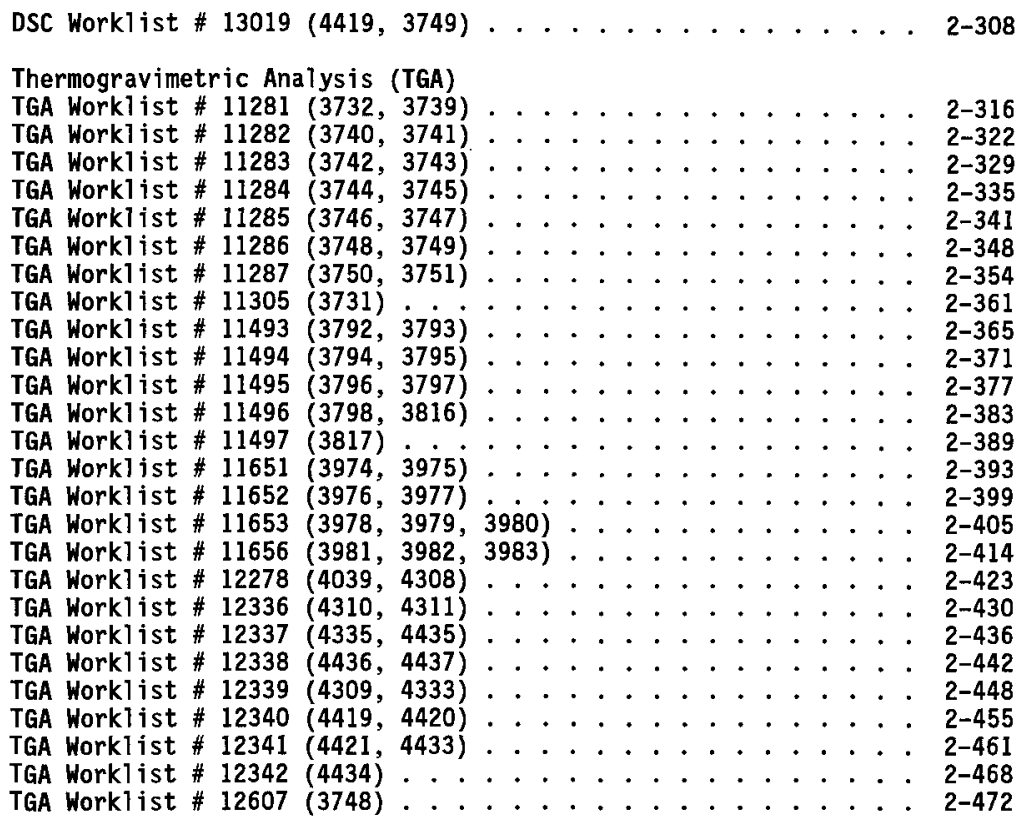

This Document consists of two sections.

Part I consists of pages 1 through 2003, plus pages $i i, 2,14,57,165,204$, $254,290,305,950,951$ and 1438 were intentionally left blank.

Part II consists of pages 2-1 through 2-475, and pages 2-2, 2-12.1, 2-52, 291, 2-139 and 2-140 were intentionally left blank.

TRADEMARKS :

Perkin-Elmer is a Registered Trademark of Research and Manufacturing Company, Inc. Mettler is a Registered Trademark of Mettler Electronics. 
HNF-SD-WM-DP-199, REV. 1

WHC-SD-WM-DP-199, REV: I

NARRAT IVE

1 
HNF-SD-WM-DP-199, REV. 1

WHC-SD-WM-DP-199, REV. 1 -

THIS PAGE WAS INTENTIONALLY LEFT BLANK

2 
HNF-SD-WM-DP-199, REV. 1

WHC-SD-WM-DP-199, REV. 1

222-S ANALYTICAL SERVICES

TANK 241-AN-105, CORES 152 AND 153, ANALYTICAL RESULTS FOR THE FINAL REPORT

This document is the analytical laboratory report for tank 241-AN-105 push mode core segments collected between June 10,1996 and June 28, 1996. The segments were subsampled and analyzed in accordance with the Tank 241-AN-105 Push Mode Core Sampling and Analysis Plan (TSAP) (Steen, 1997), the Safety Screening Data Quality Objective (DQO) (Dukelow, et al,, 1995) and the Flammable Gas Data Quality Objective (DQO) (Benar, 1995). The analytical results are included in the data summary table (Table 1).

\section{Sample Handling}

Attachment 1 is a cross reference to relate the tank farm identification numbers to the 222-S Laboratory LABCORE/LIMS sample numbers. These diagrams show the make-up of the core composites and secondary analyses requested. These diagrams and any changes made supersede the diagrams presented in Rev. 0 of this document. Sample handling and appearance information for the subsegments may be found in Rev. 0 of this document.

Two solid and one liquid core composite were made from core 152 . The core composite worksheets are included in this document.

Selected segments from core $152(15,17,19$ and 21$)$ and core $153(4,16,18$ and 20$)$ were sampled using the Retained Gas Sampler (RGS) and extruded by the Process Chemistry and Statistical Analysis Group. Core 152 segment 14 was originally identified as a RGS sample. However since the integrity of the sample was compromised due to a leaking sampler seal it was extruded as a non-RGS sample.

\section{Field Blank}

A field blank was provided to the 222-S laboratory with core 153 . It underwent the same analysis as the drainable liquid as instructed by the TSAP (Steen, 1997). 


\section{HNF-SD-WM-DP-199, REV. 1}

\section{WHC-SD-WM-DP-199, REV. 1}

\section{Analytical Results Summary}

The data summary report (Table 1) compiles the analytical results that satisfy all applicable DQOs.

\section{Inorganic Analyses}

\section{Differential Scanning Calorimetry (DSC)}

The DSC analyses were performed on the core composite subsamples. The analyses were performed in duplicate on direct subsamples. The exothermic energy based on dry weight of subsample was calculated for all subsamples. The average of the TGA results for each subsample was used in the dry weight correction for that subsample. The results for all DSC analyses and the raw data for the core composite subsamples are included in this report.

High RPDs ( $>20 \%$ ) were reported for the solid core composite subsamples. The RPDs can be attributed to the heterogenous nature of the samples and no reruns were requested. The standard recoveries for this analysis were within the required limits.

\section{Thermogravimetric Analysis (TGA)}

The TGA analyses were performed on the core composite subsamples. The analyses were performed in duplicate on direct subsamples. Results were determined by summing the weight loss steps which occurred below $220^{\circ} \mathrm{C}$; weight loss steps above this were not used to determine the result. The results for all TGA analyses and the raw data for the core composite subsamples are included in this report. The standard recoveries and RPDs for this analysis were within the required limits.

\section{Sp.G.-Specific Gravity/Bulk Density}

Bulk density analyses were performed on direct solid subsamples. The results and raw data for all subsamples are reported in this revision. Specific gravity analyses were performed on the liquid subsamples. The results are provided in Table 1. The raw data are included in this revision. There are no exceptions to the quality control parameters stated in the TSAP for these samples.

\section{Inductively Coupled Plasma Spectrophotometry (ICP)}

The liquid subsamples were prepared for analysis by an acid adjustment of the direct subsample. This is indicated by a " $D$ " in the aliquot class (A\#) column in Table 1. Solid subsamples were prepared for analysis by performing both an acid digest as indicated by an " $\mathrm{A}$ " and a fusion as indicated by an " $F$ ". 


\section{HNF-SD-WM-DP-199, REV. 1 WHC-SD-WM-DP-199, REV. 1}

Only the $\mathrm{QC}$ for requested analytes $(\mathrm{Li}, \mathrm{Na}, \mathrm{Al}, \mathrm{Cr}, \mathrm{Ca}, \mathrm{Fe}, \mathrm{K}, \mathrm{U}, \mathrm{Zn}, \mathrm{Zr}, \mathrm{Ba}, \mathrm{Si}, \mathrm{B}, \mathrm{Bi}, \mathrm{Mn}, \mathrm{Ni})$ were reviewed for this report. Other "opportunistic" analyte results are included in Table 1. These analytes do not have customer defined $Q C$ parameters and are not discussed. High RPDs $(>20 \%)$ were reported for several analytes $(\mathrm{Li}, \mathrm{Al}, \mathrm{Cr}, \mathrm{Ca}, \mathrm{Fe}, \mathrm{Zn}, \mathrm{Zr}, \mathrm{Si}, \mathrm{B}, \mathrm{Mn}, \mathrm{Ni}$ ) and were the result of sample inhomogeneity. Reruns were not requested. Table 2 shows a comparison of the result of the sample with that of the serial dilution for those required analytes that had a spike recovery outside of the $75 \%$ to $125 \%$ range as set by the TSAP and the Quality Assurance Plan for the 222-S Laboratories (LabQAP). Spike recoveries outside of the required range were reported for $\mathrm{Al}, \mathrm{Ca}, \mathrm{Cr}, \mathrm{Fe}, \mathrm{Li}, \mathrm{K}, \mathrm{Si}, \mathrm{Na}$ and $\mathrm{Zr}$. Serial dilutions are performed by diluting a sample by five-fold. The results from the serial dilution may be found in the raw data and are signified by an " $L$ " at the end of the sample number. For acceptable performance the percent difference between the serial dilution and the undiluted results must be $s 10$ percent, one sample had a serial dilution greater than ten percent. This inaccuracy can be attributed to the low concentration of the analyte with respect to the detection limit. There did not appear to be any analytical anomalies or difficulties during the analyses of $\mathrm{Ba}$ or $\mathrm{U}$. 


\section{HNF-SD-WM-DP-199, REV. 1 \\ WHC-SD-WM-OP-199 REV. 1}

Table 2. ICP Serial DHlution Raw Results for Tank 2A1-AN-10S

\begin{tabular}{|c|c|c|c|c|}
\hline Sample ID & Analyto & $\begin{array}{l}\text { Semple Result Undilited } \\
(\mathrm{u} / \mathrm{mL} \text { ) }\end{array}$ & $\begin{array}{c}\text { Serial Difution Result } \\
\text { (ug/mL) }\end{array}$ & \% Difference \\
\hline S961003732 & $\mathbf{A}$ & 43830.00 & 44750.00 & 2.1 \\
\hline S96T003732 & $\mathrm{N}$ & 252600.00 & 259500.00 & 2.7 \\
\hline 5967003730 & $\mathrm{Na}$ & 230100.00 & 242800.00 & 1.6 \\
\hline S95T003730 & A & 40860.00 & 41100.00 & 0.8 \\
\hline 5967003740 & A & 40880.00 & 41250.00 & 0.9 \\
\hline S96T003740 & $\mathrm{Na}$ & 238800,00 & 243000.00 & 1.8 \\
\hline Sœ6T00374t & $\mathbf{A}$ & 40860.00 & 41190.00 & 0.8 \\
\hline 5907003744 & $\mathrm{Na}$ & 251000.00 & 255100.00 & 1.6 \\
\hline S०6T003742 & $\mathbf{K}$ & 6676.00 & 6400.00 & 4.1 \\
\hline \$967003742 & $\mathbf{A}$ & 42370.00 & 43690.00 & 3.1 \\
\hline S96T003742 & $\mathrm{Na}$ & 250900.00 & 260700.00 & 3.9 \\
\hline SE6T003743 & $\mathbf{K}$ & 7099.00 & 7127.00 & 0.4 \\
\hline S96T003743 & $\mathbf{A}$ & 45450.00 & 46130.00 & 1.5 \\
\hline S96T003743 & $\mathrm{Na}$ & 266500.00 & 271800.00 & 2.0 \\
\hline S90T003744 & $\mathrm{Na}$ & 213900.00 & 211500.00 & 1.1 \\
\hline S98T003744 & $\mathbf{A}$ & 35000.00 & 36040.00 & 3.0 \\
\hline S96T003744 & $\mathbf{K}$ & 5513.00 & 5658.00 & 2.6 \\
\hline S96T003745 & $\mathrm{Na}$ & 236000.00 & 236000.00 & 0.0 \\
\hline S\$8T003745 & $\mathbf{A}$ & 37500.00 & 37570.00 & 0.2 \\
\hline S96T003745 & $\mathbf{K}$ & 6056.00 & 5896.00 & 2.6 \\
\hline S96T003745 & $\bar{p}$ & 1240.00 & 1221.00 & 1.5 \\
\hline S967003746 & $\mathrm{Na}$ & 240400.00 & 245900.00 & 2.3 \\
\hline 5967003746 & $\mathrm{~A}$ & 41290.00 & 42210.00 & 2.2 \\
\hline S967003746 & $\mathrm{K}$ & 6192.00 & 6476.00 & 4.6 \\
\hline S967003747 & $\mathbf{A}$ & 41590.00 & 42400.00 & 1.8 \\
\hline S967003747 & $\mathrm{Na}$ & 243700.00 & 249900.00 & 2.5 \\
\hline 5867003748 & $\mathrm{Na}$ & 241000.00 & 245700.00 & 1.6 \\
\hline 5967003748 & $\mathbf{K}$ & 6491.00 & 5425.00 & 16.4 \\
\hline S967003740 & $\mathbf{A}$ & 41440.00 & 41730.00 & 0.7 \\
\hline 5\%3T003740 & $\mathrm{Na}$ & 240300.00 & 243200.00 & 1.2 \\
\hline $5 \% 6 T 003750$ & $\mathrm{No}$ & 237500.00 & 240700.00 & 1.3 \\
\hline S96T003750 & $\mathbf{A}$ & 40060.00 & 40320.00 & 0.6 \\
\hline S90T003750 & K & 6413.00 & 5795.00 & 9.6 \\
\hline S96T003751 & $k$ & 6072.00 & 6128.00 & 0.8 \\
\hline $5 \% 67003751$ & A & 38700.00 & 30420.00 & 1.9 \\
\hline 5967003751 & $\mathrm{Na}$ & 234100.00 & 241500.00 & 3.2 \\
\hline SBST003904 & $\mathrm{Na}$ & 1081.00 & 1116.00 & 3.2 \\
\hline 5967003975 & $\mathbf{A}$ & 35700.00 & 38480.00 & 7.8 \\
\hline S86T003975 & $\mathrm{Na}$ & 2339200.00 & 245100.00 & 2.5 \\
\hline 5967003976 & $\mathrm{Na}$ & 217700.00 & 223100.00 & 2.5 \\
\hline 5967003976 & $\overline{A l}$ & 37350.00 & 38020.00 & 1.8 \\
\hline S98T003976 & $\mathbf{K}$ & 5810.00 & 5708.00 & 1.8 \\
\hline s08T003977 & $\mathrm{Na}$ & 252000.00 & 257400.00 & 2.1 \\
\hline S96T003977 & A & 42260.00 & 43020.00 & 1.8 \\
\hline 5867003977 & $\mathbf{K}$ & 6682.00 & 6496.00 & 2.8 \\
\hline S85TC03078 & $\mathrm{K}$ & 6290.00 & 6246.00 & 0.7 \\
\hline S28T003078 & $\mathrm{Na}$ & 236900.00 & 238300.00 & 0.6 \\
\hline S96T003079 & $\mathrm{K}$ & 6291.00 & 6757.00 & 7.4 \\
\hline S98T003979 & A & 42930.00 & 43810.00 & 2.0 \\
\hline 596T003979 & $\mathrm{Na}$ & 252100.00 & 256500.00 & 1.7 \\
\hline S96T003980 & Al & 27420.00 & 27720.00 & 1.1 \\
\hline S96T003980 & $\mathrm{Na}$ & 223200.00 & 226200.00 & 1.3 \\
\hline S96T003988 & $\mathrm{Na}$ & 1013.00 & 1028.00 & 1.5 \\
\hline 5967004308 & $\mathrm{Na}$ & 258500.00 & 262800.00 & 1.7 \\
\hline S96T004309 & $\mathrm{K}$ & 6636.00 & 6603.00 & 0.5 \\
\hline S96T004309 & A & 43190.00 & 43050.00 & 0.3 \\
\hline S96T004300 & $\mathrm{Na}$ & 254400.00 & 254700.00 & 0.1 \\
\hline SOST004333 & $\mathbf{A}$ & 44310.00 & 44280.00 & 0.1 \\
\hline S96T004333 & $\mathrm{Na}$ & 265600.00 & 265000.00 & 0.2 \\
\hline S96T004419 & No & 262200.00 & 265300.00 & 1.2 \\
\hline 5967004419 & A & 44180.00 & 44190.00 & 0.0 \\
\hline SPOTO04420 & No & 246300.00 & 251800.00 & 2.2 \\
\hline $590 T 004420$ & $\mathbf{A}$ & 412900.00 & 12070.00 & 1.8 \\
\hline 500004420 & $\mathrm{~K}$ & 6638.00 & 6861.00 & 3.4 \\
\hline S9STOA4421 & $\mathrm{Na}$ & 254700.00 & 254100.00 & 0.2 \\
\hline SP6T004421 & $\mathbf{A}$ & 42550.00 & $\$ 2280.00$ & 0.6 \\
\hline 5961004421 & $\mathrm{~K}$ & 6433.00 & $67 \% 5.00$ & 5.6 \\
\hline $5 \% 6004433$ & $\mathrm{Na}$ & 269100.00 & 275000.00 & 2.2 \\
\hline S96T004434 & $\mathrm{Na}$ & 233100.00 & 243000.00 & 4.2 \\
\hline S96T004434 & $\mathbf{A}$ & 39400.00 & 37200.00 & 5.6 \\
\hline S98T004434 & $K$ & 6066.00 & 6211.00 & 2.4 \\
\hline S96T004831 & $\mathrm{Na}$ & 254400.00 & 260100.00 & 2.2 \\
\hline S96T004831 & K & 6493.00 & 6470.00 & 0.4 \\
\hline S96T004831 & A & 43390.00 & 44110.00 & 1.7 \\
\hline
\end{tabular}

\% Difference $=($ ABS $($ Sample - Serial) $) /$ Sample $\times 100$ 


\section{HNF-SD-WM-DP-199, REV. 1}

\section{WHC-SD-WM-DP-199, REV. 1}

\section{Ion Chromatography (IC)}

The IC analyses were performed on direct subsamples of liquid samples, as indicated by a blank in the A\# column in Table 1. The solid subsamples were prepared for analysis by performing a water digest. This is indicated with a "W" in the A\# column in Table 1.

High RPDs ( $>20 \%)$ were reported for several analytes and can be attributed to sample inhomogeneity. Spike recoveries outside of the required range (75\% to $125 \%)$ were reported for Acetate, $\mathrm{Cl}, \mathrm{Fl}, \mathrm{NO} 2$ and NO3. The chemist noted that the spike failures were due to matrix interferences from organic acids. Bromide was detected in 34 of the 52 subsamples submitted for analysis and was an indication of hydrostatice head fluid intrusion into the samples. The standard recoveries for this analysis were within the required limits.

\section{Total Inorganic/Organic Carbon Persulfate (TIC/TOC)}

TIC/TOC by persulfate/coulometry analyses were performed on the core composite samples. None of the results exceeded the TOC notification limit of $30,000 \mu \mathrm{gC} / \mathrm{g}$. Low concentrations of TIC and TOC were noted in some preparation blanks, however the levels of contamination are inconsequential when compared to the result of the sample and do not impact sample data quality. The standard recoveries, spike recoveries and RPDs for these analyses were within the required limits.

\section{OH}

The $\mathrm{OH}$ analyses were performed on direct liquid core composite subsamples and the field blank, as indicated by a blank in the A\# column in Table 1. The solid core composite subsamples were prepared for analysis by performing a water digest. This is indicated with a "W" in the A\# column in Table 1. The standard recoveries and RPDs for this analysis were within the required limits.

\section{Chromium VI (CrVI)}

The CrVI analyses were performed on direct liquid core composite subsamples and the field blank, as indicated by a blank in the A\# column in Table 1. The solid core composite subsamples were prepared for analysis by performing a water digest. This is indicated with a "W" in the A\# column in Table 1. The standard recoveries, spike recoveries and RPDs for this analysis were within the required limits. 


\section{WHC-SD-WM-DP-199, REV. 1}

\section{Radionuclide Analyses}

\section{Total Alpha (AT)}

Total alpha activity analyses were performed on direct liquid core composite subsamples and the field blank. The solid core composite subsamples were prepared for analysis by performing a fusion digest in duplicate. The fusion preparation is indicated with an " $\mathrm{F"}$ in the A\# column in Table 1. The results and the raw data for all subsamples are included in this revision. High RPDs $(>20 \%)$ were reported for the solid core composite subsamples and can be attributed to sample inhomogeneity. No reruns were requested due to the low alpha activity in the samples. The standard recoveries and spike recoveries for this analysis were within the required limits.

\section{Total Beta (TB)}

Total beta activity analyses were performed on direct liquid core composite subsamples and the field blank. The solid core composite subsamples were prepared for analysis by performing a fusion digest in duplicate. The fusion preparation is indicated with an " $F$ " in A\# column in Table 1. Low levels of beta activity were noted in some preparation blanks, however the levels of contamination are inconsequential when compared to the result of the sample and do not impact sample data quality. The standard recoveries, spike recoveries and RPDs for this analysis were within the required limits.

\section{Strontium $90\left({ }^{90} \mathrm{Sr}\right)$}

${ }^{90} \mathrm{Sr}$ analyses were performed on direct liquid core composite subsamples and the field blank. The solid core composite subsamples were prepared for analysis by performing a fusion digest in duplicate. The fusion preparation is indicated with an " $\mathrm{F}$ " in A\# column in Table 1. The standard recoveries and RPDs for this analysis were within the required limits.

\section{Gamma Energy Analysis (GEA)}

GEA analyses were performed on direct liquid core composite subsamples and the field blank. The solid core composite subsamples were prepared for analysis by performing a fusion digest in duplicate. The fusion preparation is indicated with an " $F$ " in A\# column in Table 1.

Actual detection limits for GEA analytes are not currently available. The latest GEA software does not currently report a minimum detectable activity (MDA). If an analyte is reported as "less than", the value reported is the detection limit.

Low levels of cesium activity were noted in some preparation blanks, however the levels of contamination are inconsequential when compared to the result of the sample and do not impact sample data quality. The standard recoveries and RPDs for this analysis were within the required limits. 


\section{HNF-SD-WM-DP-199, REV. 1}

\section{WHC-SD-WM-DP-199, REV. 1}

\section{Total Uranium (U)}

Uranium analyses were performed on direct liquid core composite subsamples and the field blank. The solid core composite subsamples were prepared for analysis by performing a fusion digest in duplicate. The fusion preparation is indicated with an "F" in A\# column in Table 1. The standard recoveries, spike recoveries and RPDs for this analysis were within the required limits.

\section{Plutonium $\left({ }^{239 / 240} \mathrm{Pu}\right)$}

${ }^{239 / 240} \mathrm{Pu}$ analyses were performed on direct liquid core composite subsamples and the field blank. The solid core composite subsamples were prepared for analysis by performing a fusion digest in duplicate. The fusion preparation is indicated with an " $F$ " in A\# column in Table 1. The standard recoveries and RPDs for this analysis were within the required limits.

\section{Iodine $\left({ }^{129} \mathrm{D}\right)$}

${ }^{129} \mathrm{I}$ analyses were performed on direct liquid core composite subsamples and the field blank, as indicated by a blank in the A\# column in Table 1. The solid core composite subsamples were prepared for analysis by performing a water digest. This is indicated with a "W" in the A\# column in Table 1. The standard recoveries and RPDs for this analysis were within the required limits.

\section{Technicium $\left.{ }^{99} \mathrm{Tc}\right)$}

${ }^{99} \mathrm{Tc}$ analyses were performed on direct liquid core composite subsamples and the field blank. The solid core composite subsamples were prepared for analysis by performing a fusion digest in duplicate. The fusion preparation is indicated with an "F" in A\# column in Table 1. The standard recoveries and RPDs for this analysis were within the required limits.

\section{Alpha Energy Analyses}

\section{Americium ( $\left.{ }^{241} \mathrm{Am}\right)$}

${ }^{241} \mathrm{Am}$ analyses were performed on direct liquid core composite subsamples and the field blank. The solid core composite subsamples were prepared for analysis by performing a fusion digest in duplicate. The fusion preparation is indicated with an " $F$ " in A\# column in Table 1. High RPDs were reported for the solid core composite samples. This can be attributed to the heterogenous nature of the samples. No rerun was requested due to the low americium activity in the samples. The standard recoveries for this analysis were within the required limits.

\section{Curium $\left({ }^{243 / 244} \mathrm{Cm}\right)$}

${ }^{243 / 244} \mathrm{Cm}$ analyses were performed on direct liquid core composite subsamples and the field blank. The solid core composite subsamples were prepared for analysis by performing a fusion digest in duplicate. The fusion preparation is indicated with an "F" in A\# column in Table 1. The RPDs for this analysis were within the required limits. 
HNF-SD-WM-DP-199, REV.

WHC-SD-WM-DP-199, REV. 1

\section{Procedures}

Table 3 lists the analytical procedures used for performing the sample analyses. Abbreviations for analyses are defined in the table notes.

\section{Table 3. Analytical Procedures}

\begin{tabular}{|c|c|c|c|}
\hline Analysis: & $\begin{array}{l}\text { Sample } \\
\text { Portion }\end{array}$ & Preparation & $\begin{array}{l}\text { Analysis } \\
\text { Procedure }\end{array}$ \\
\hline DSC & Solid/Liquid & $\mathrm{N} / \mathrm{A}$ & $\begin{array}{l}\text { LA-514-114 Rev. C-1 } \\
\text { LA-514-113 Rev. C-1 }\end{array}$ \\
\hline TGA & Solid/Liquid & N/A & $\begin{array}{l}\text { LA-514-114 Rev. C-1 } \\
\text { LA-560-112 Rev. B-1 }\end{array}$ \\
\hline Bulk Density & Solid & N/A & LO-160-103 Rev. B-0 \\
\hline Sp.G. & Liquid & N/A & LA-510-112 Rev. C-3 \\
\hline $\mathrm{U}$ & $\begin{array}{l}\text { Solid } \\
\text { Liquid }\end{array}$ & $\begin{array}{c}\text { LA-S49-141 Rev. F-0 } \\
\text { N/A }\end{array}$ & LA-925-009 Rev. A-1 \\
\hline IC & $\begin{array}{l}\text { Solid } \\
\text { Liquid }\end{array}$ & $\begin{array}{c}\text { LA-504-101 Rev. E-0 } \\
\text { N/A }\end{array}$ & LA-533-105 Rev. D-1 \\
\hline ICP & $\begin{array}{l}\text { Solid } \\
\text { Liquid }\end{array}$ & $\begin{array}{c}\text { LA-505-159 Rev. D-0 } \\
\text { LA-549-141 Rev. F-0 } \\
\text { N/A }\end{array}$ & $\begin{array}{l}\text { LA-505-151 Rev. D-3 } \\
\text { LA-505-161 Rev. B-1 }\end{array}$ \\
\hline $\mathrm{OH}-$ & $\begin{array}{l}\text { Solid } \\
\text { Liquid }\end{array}$ & $\begin{array}{c}\text { LA-504-101 Rev. E-0 } \\
\text { N/A }\end{array}$ & LA-211-102 Rev. C-0 \\
\hline
\end{tabular}


HNF-SD-WM-DP-199, REV. 1

WHC-SD-WM-DP-199, REV. 1

Table 3. Analytical Procedures

\begin{tabular}{|c|c|c|c|}
\hline TICTOC & Solid/Liquid & N/A & LA-342-100 Rev. D-0 \\
\hline $\mathrm{CR}(\mathrm{VI})$ & $\begin{array}{l}\text { Solid } \\
\text { Liquid }\end{array}$ & $\begin{array}{c}\text { LA-504-101 Rev. E-0 } \\
\text { N/A }\end{array}$ & LA-265-101 Rev. B-0 \\
\hline TOC & Liquid & N/A & LA-344-105 Rev. D-1 \\
\hline${ }^{3} \mathrm{H}$ & $\begin{array}{l}\text { Solid } \\
\text { Liquid }\end{array}$ & $\begin{array}{c}\text { LA-504-101 Rev. E-0 } \\
\text { N/A }\end{array}$ & LA-218-114 Rev. B-0 \\
\hline${ }^{129} \mathrm{I}$ & $\begin{array}{l}\text { Solid } \\
\text { Liquid }\end{array}$ & $\begin{array}{c}\text { LA-504-101 Rev. E-0 } \\
\text { N/A }\end{array}$ & LA-378-103 Rev. C-0 \\
\hline${ }^{\infty} \mathrm{Tc}$ & $\begin{array}{l}\text { Solid } \\
\text { Liquid }\end{array}$ & $\begin{array}{c}\text { LA-549-141 Rev. F-0 } \\
\text { N/A }\end{array}$ & LA-438-101 Rev. D-2 \\
\hline${ }^{27} \mathrm{~Np}$ & $\begin{array}{c}\text { Solid } \\
\text { Liquid }\end{array}$ & $\begin{array}{c}\text { LA-549-141 Rev. F-0 } \\
\text { N/A }\end{array}$ & LA-933-141 Rev. H-1 \\
\hline $\mathrm{AT} / \mathrm{TB}$ & $\begin{array}{l}\text { Solid } \\
\text { Liquid }\end{array}$ & $\begin{array}{c}\text { LA-549-101 Rev. F-0 } \\
\text { N/A }\end{array}$ & LA-508-101 Rev. D-2 \\
\hline GEA & $\begin{array}{l}\text { Solid } \\
\text { Liquid }\end{array}$ & $\begin{array}{c}\text { LA-549-101 Rev. F-0 } \\
\text { N/A }\end{array}$ & LA-548-121 Rev. E-0 \\
\hline${ }^{241} \mathrm{Am} /{ }^{244} \mathrm{Cm}$ & $\begin{array}{l}\text { Solid } \\
\text { Liquid }\end{array}$ & $\begin{array}{c}\text { LA-S49-101 Rev. F-0 } \\
\text { N/A }\end{array}$ & LA-953-103 Rev. B-0 \\
\hline${ }^{90} \mathrm{Sr}$ & $\begin{array}{c}\text { Solid } \\
\text { Liquid }\end{array}$ & $\begin{array}{c}\text { LA-549-141 Rev. F }-0 \\
\text { N/A }\end{array}$ & LA-220-101 Rev. D-1 \\
\hline${ }^{239} \mathrm{Pu}$ & $\begin{array}{c}\text { Solid } \\
\text { Liquid }\end{array}$ & $\begin{array}{c}\text { LA-549-101 Rev. F-0 } \\
\text { N/A }\end{array}$ & LA-943-128 Rev. B-0 \\
\hline
\end{tabular}




\section{HNF-SD-WM-DP-199, REV. 1}

\section{WHC-SD-WM-DP-199, REV. 1}

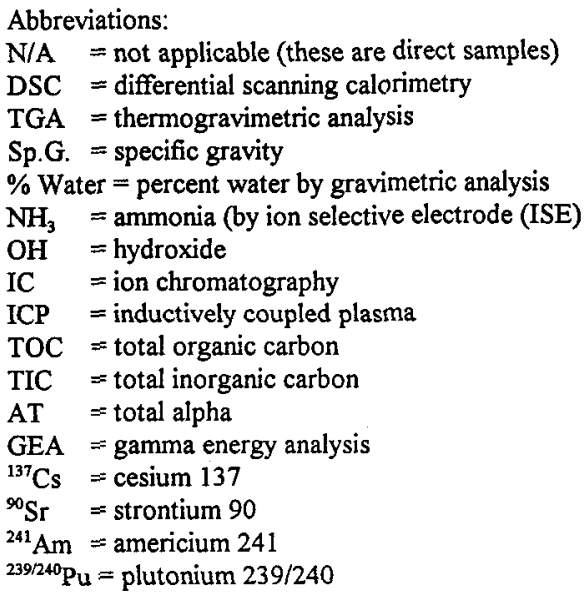

\section{References}

Steen, F. H., 1997, Tank 241-AN-105 Push Mode Core Sampling and Analysis Plan, WHC-SD-WM-TSAP-027, Rev. 1-A, Westinghouse Hanford Company, Richland, WA 99352.

Dukelow, G. T., J. W. Hunt, H. Babad, and J. E. Meacham, 1995, Tank Safety Screening Data Quality Objective, WHC-SD-WM-SP-004, Rev. 2, Westinghouse Hanford Company, Richland, WA 99352

Benar, C. J., 1995, Flammable Gas Data Quality Objective, WHC-SD-WM-DQO-004, Rev. 2, Westinghouse Hanford Company, Richland, WA 99352 


\section{HNF-SD-WM-DP-199, REV. 1}

WHC-SD-WM-DP-199, REV. I

ATTACHMENT 1

AN-105 SAMPLE BREAKDOWN 
HNF-SD-WM-DP-199, REV. 1

WHC-SD-WM-DP-199, REV. 1 
HNF-SD-WM-DP-199, REV. 1
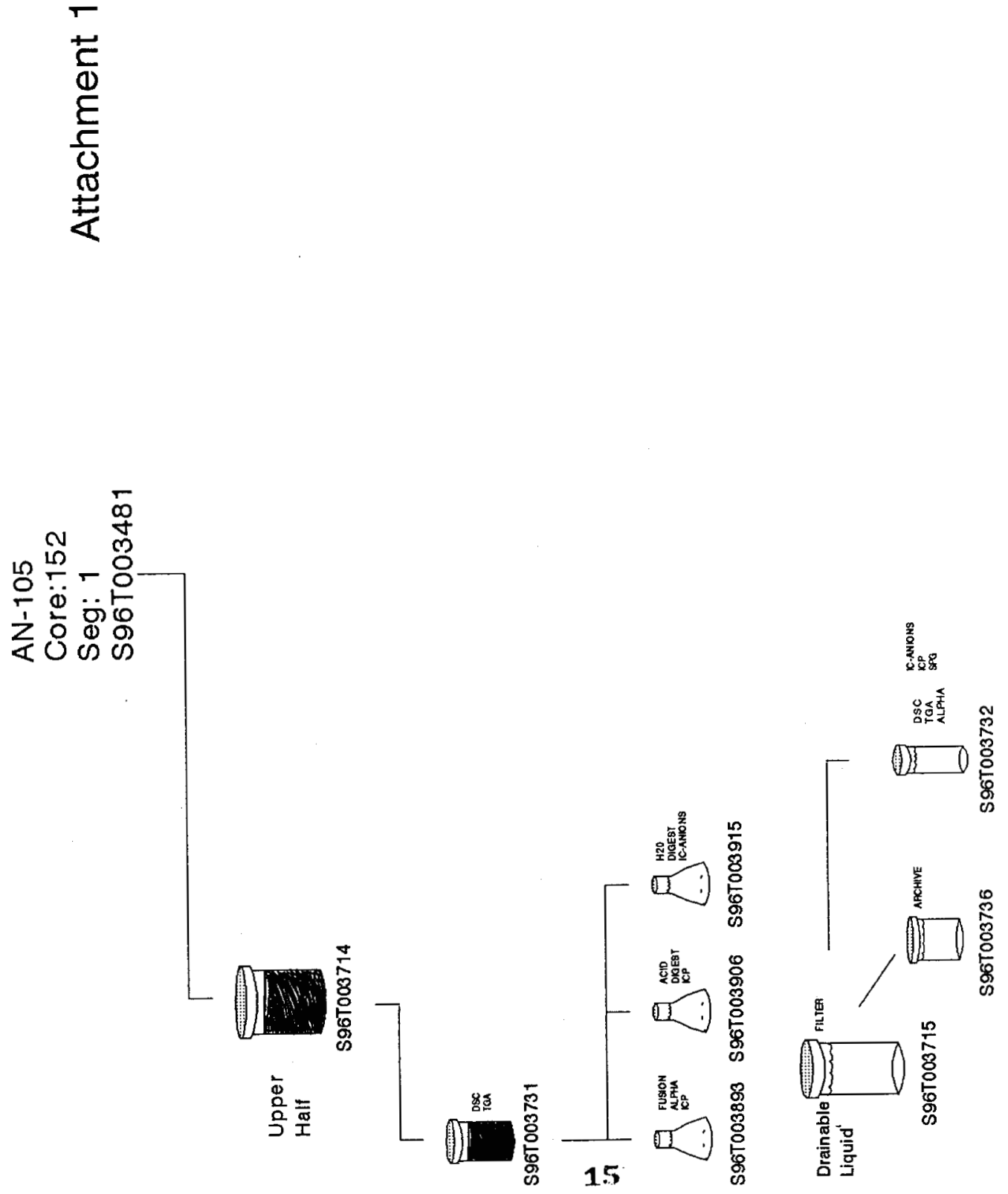
HNF-SD-WM-DP-199, REV. 1

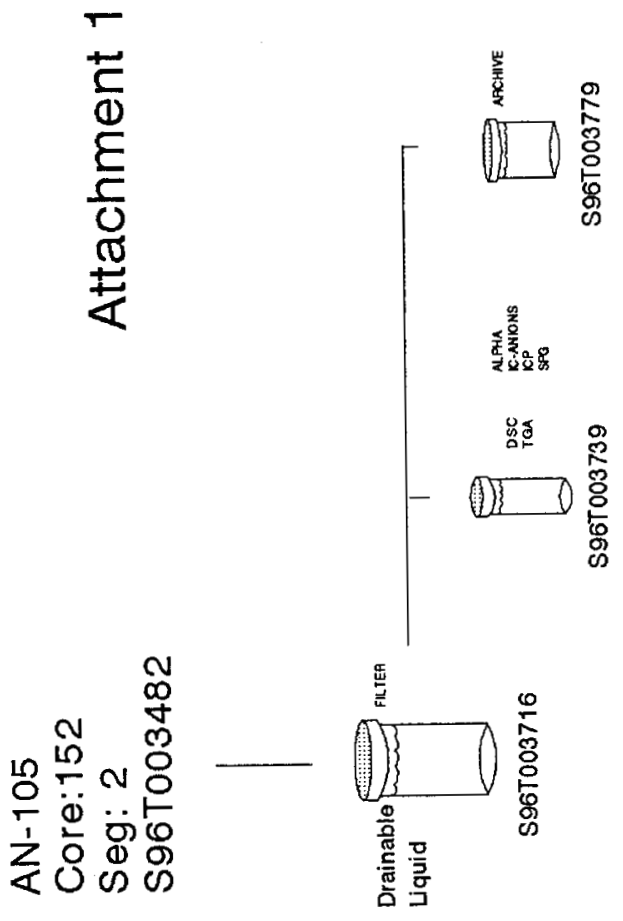


HNF-SD-WM-DP-199, REV. 1

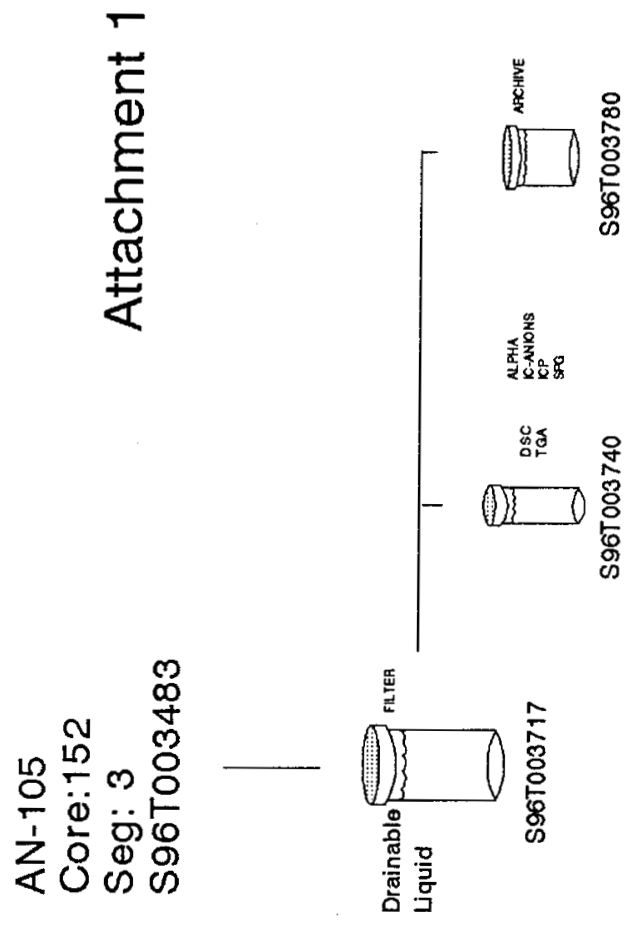


HNF-SD-WM-DP-199, REV. 1

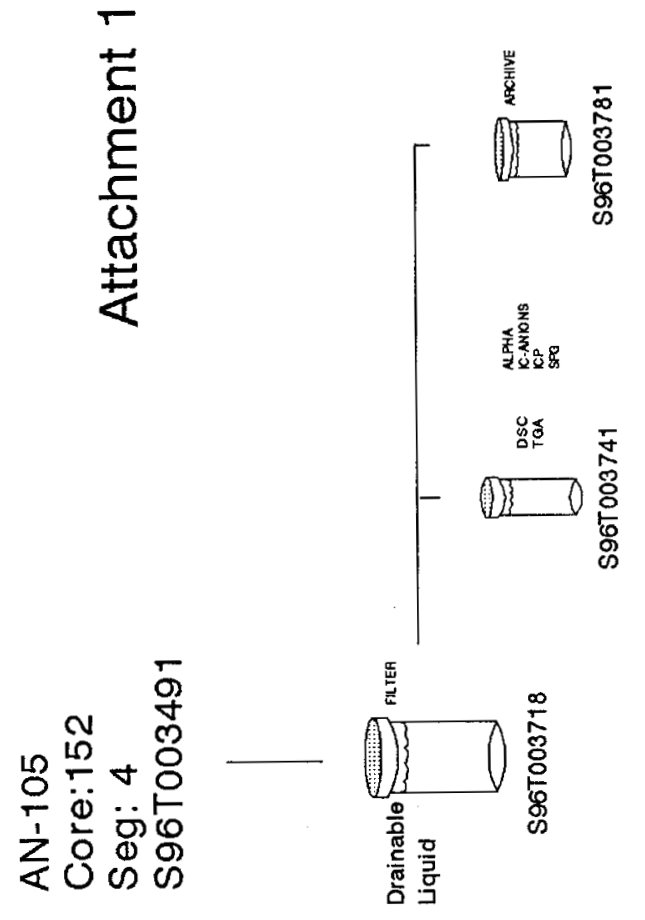




\section{HNF-SD-WM-DP-199, REV. 1}

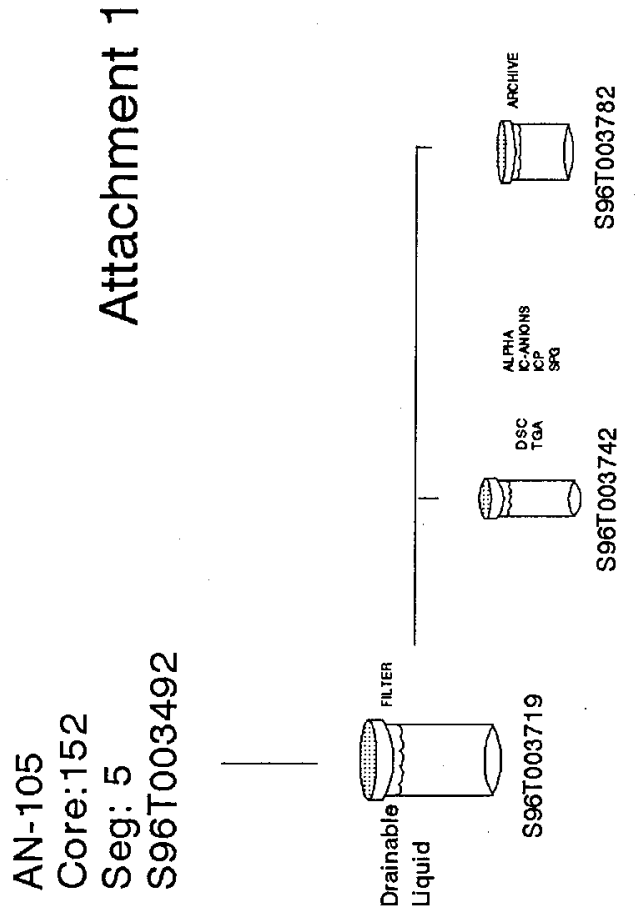




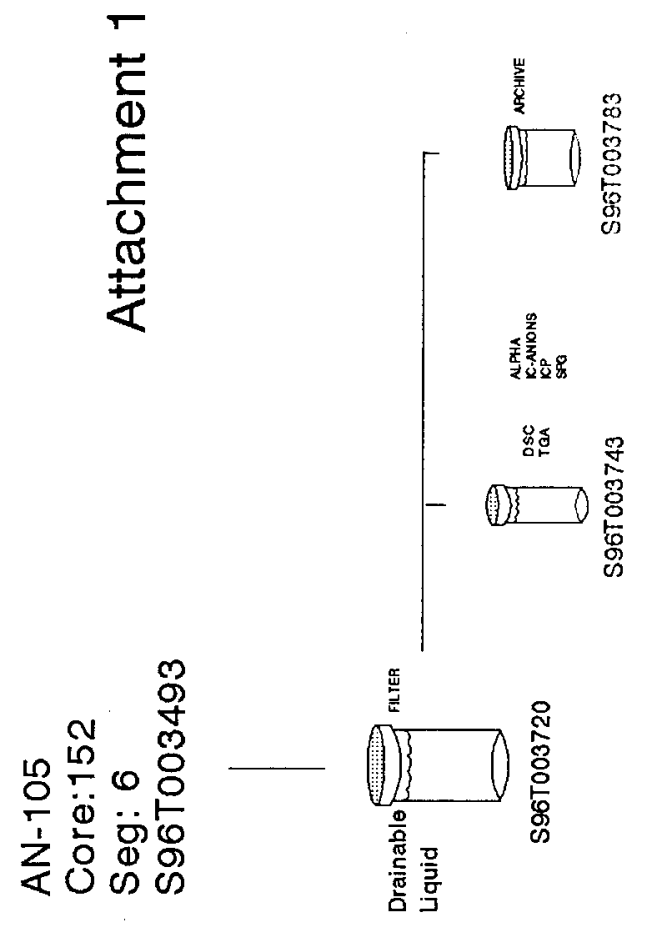


HNF-SD-WM:DP-199, REV. 1

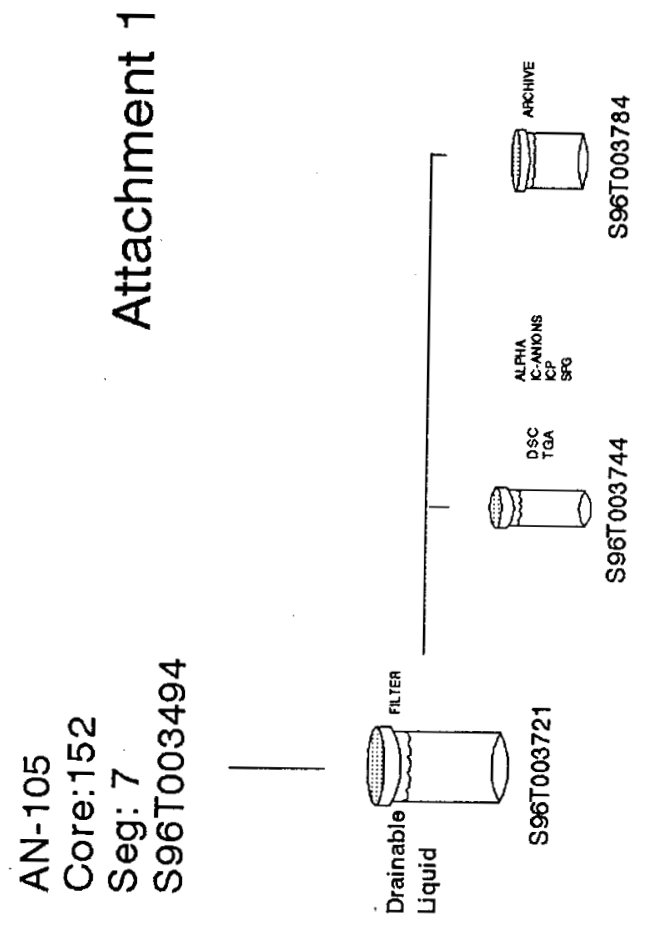


HNF-SD-WM:DP-199, REV. 1
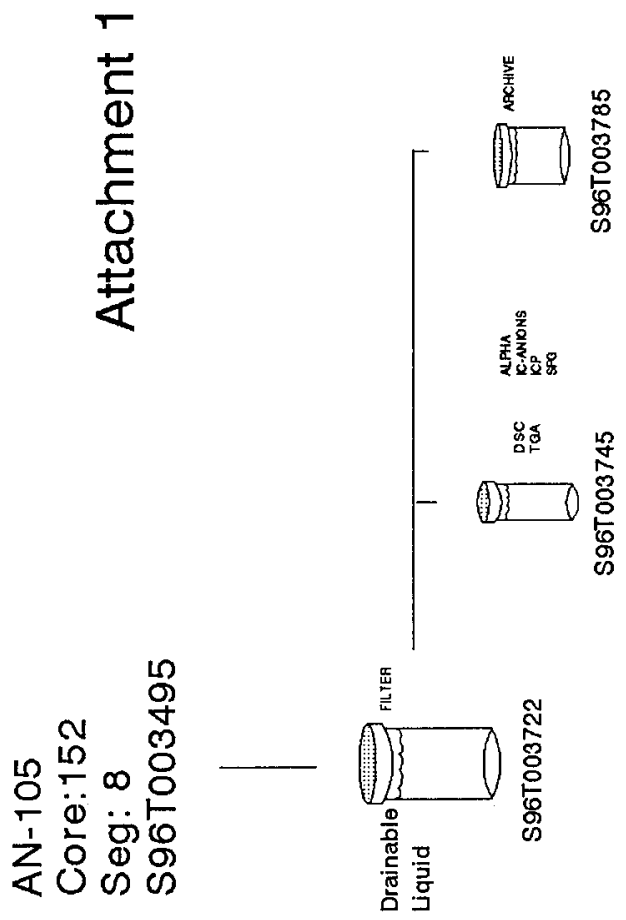
HNF-SD-WM-DP-199, REV. 1

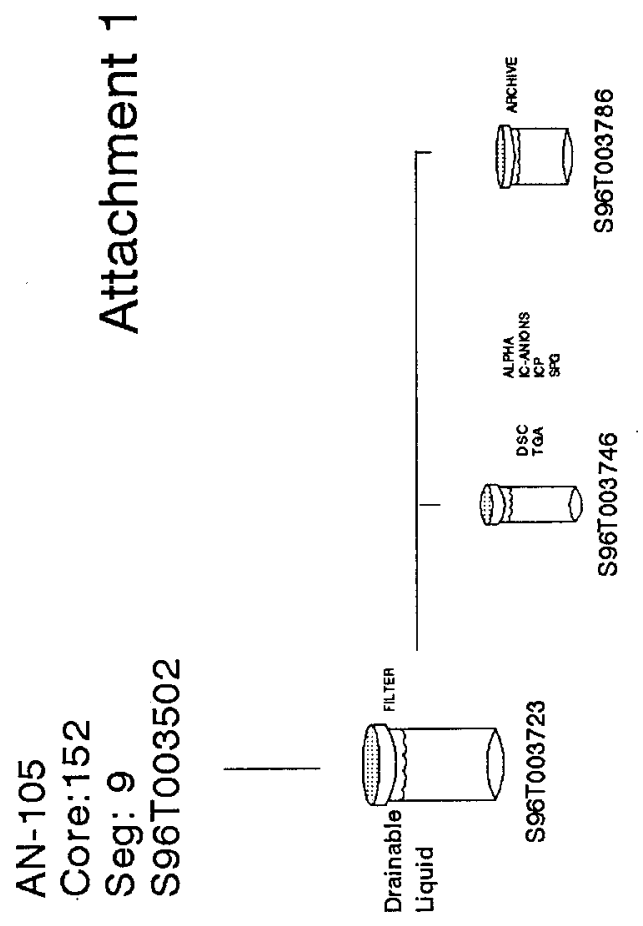


HNF-SD-WM-DP-199, REV. 1

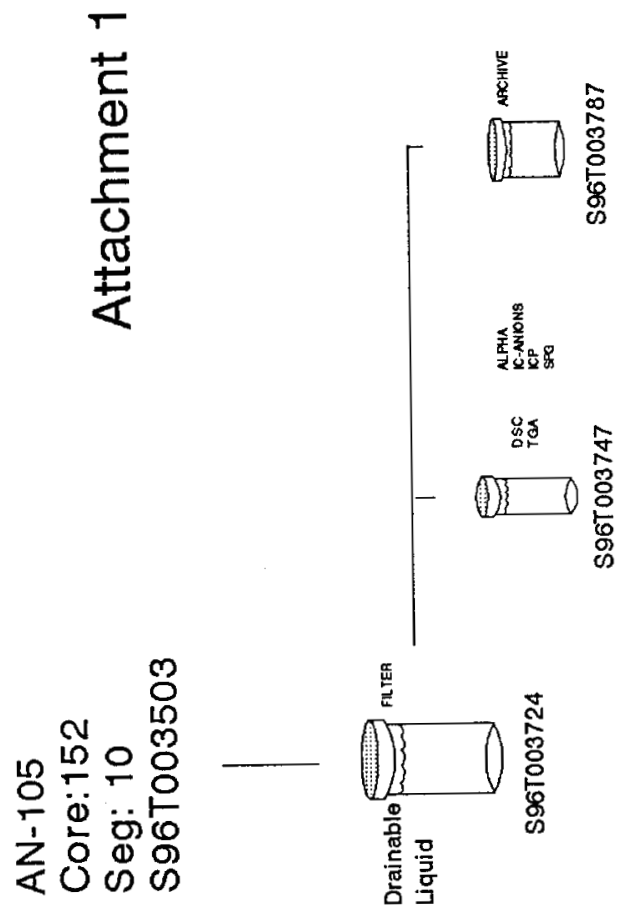


HNF-SD-WM-DP-199, REV. 1

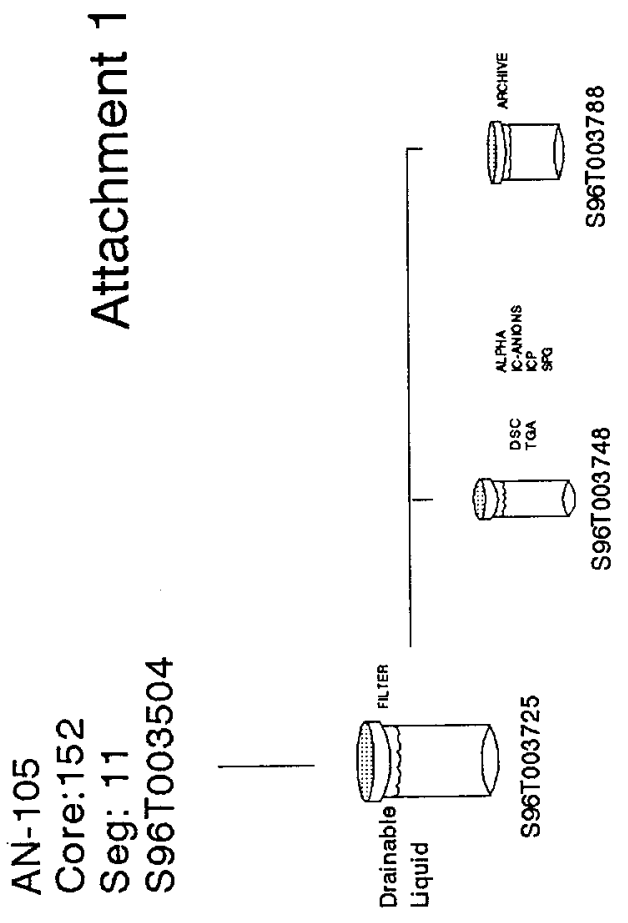


HNF-SD-WM-DP-199, REV. 1

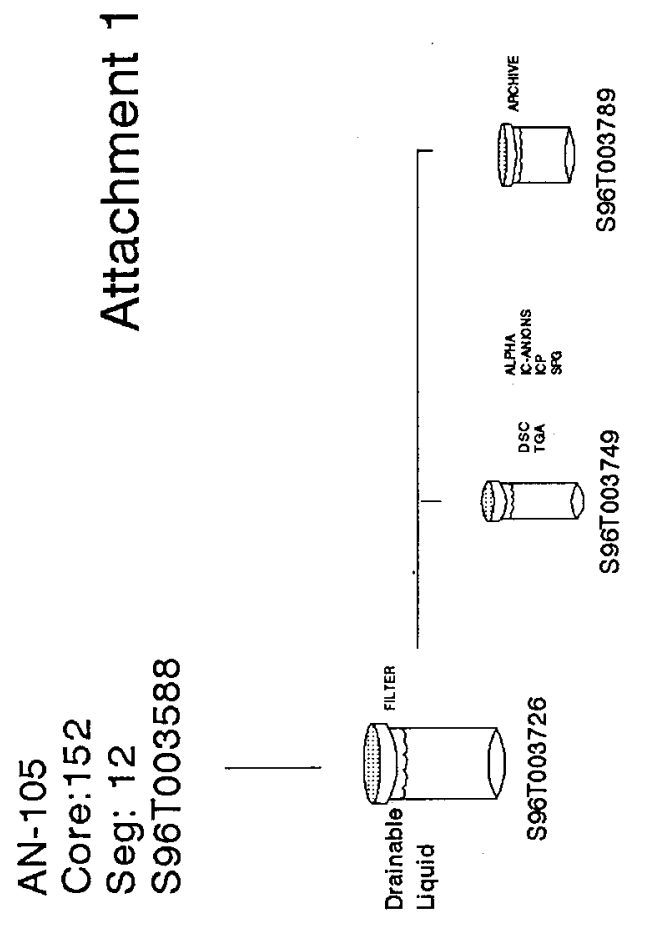


HNF-SD-WM-DP-199, REV. 1

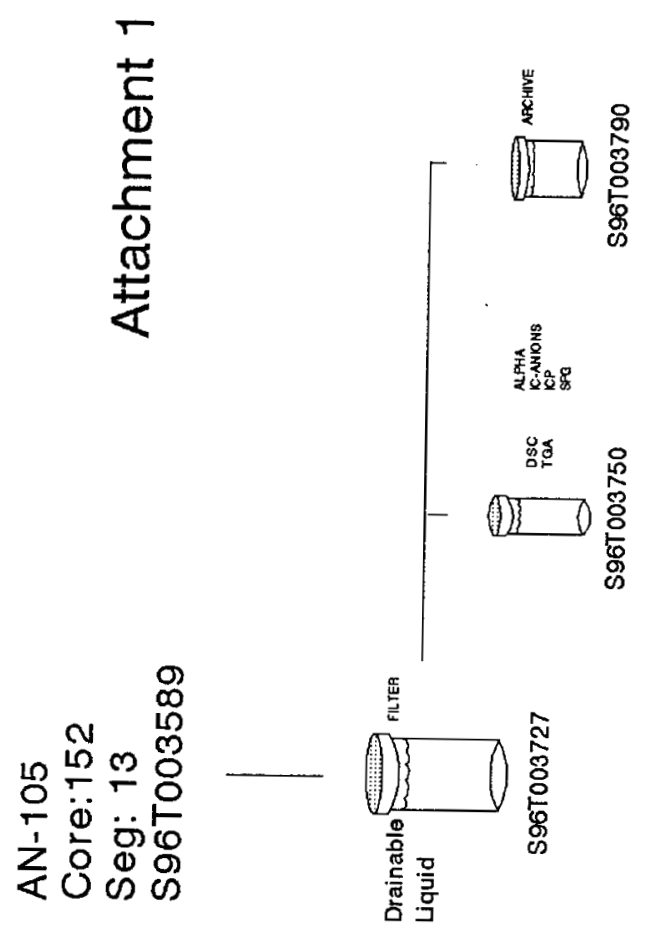




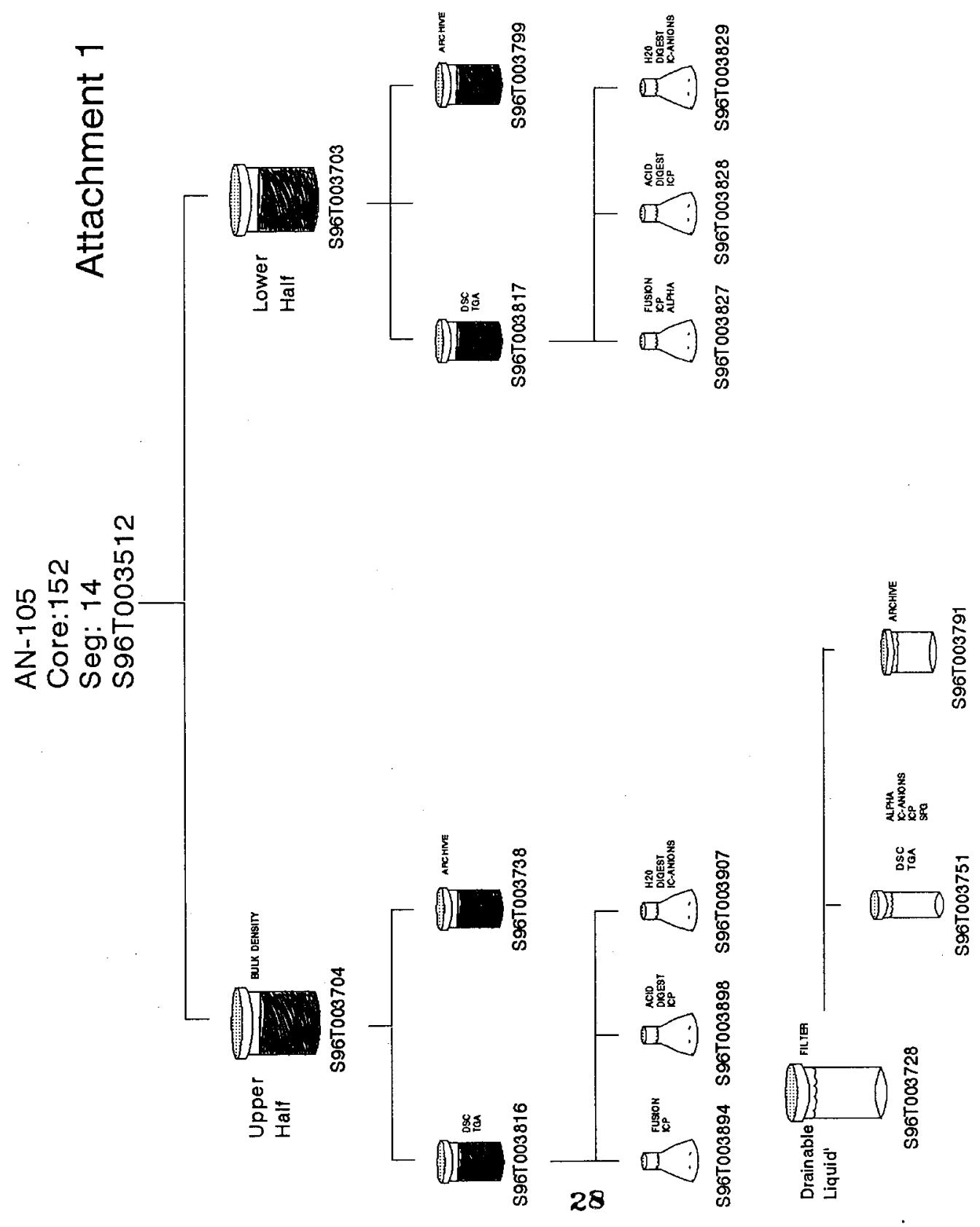


HNF-SD-WM-DP-199, REV. 1

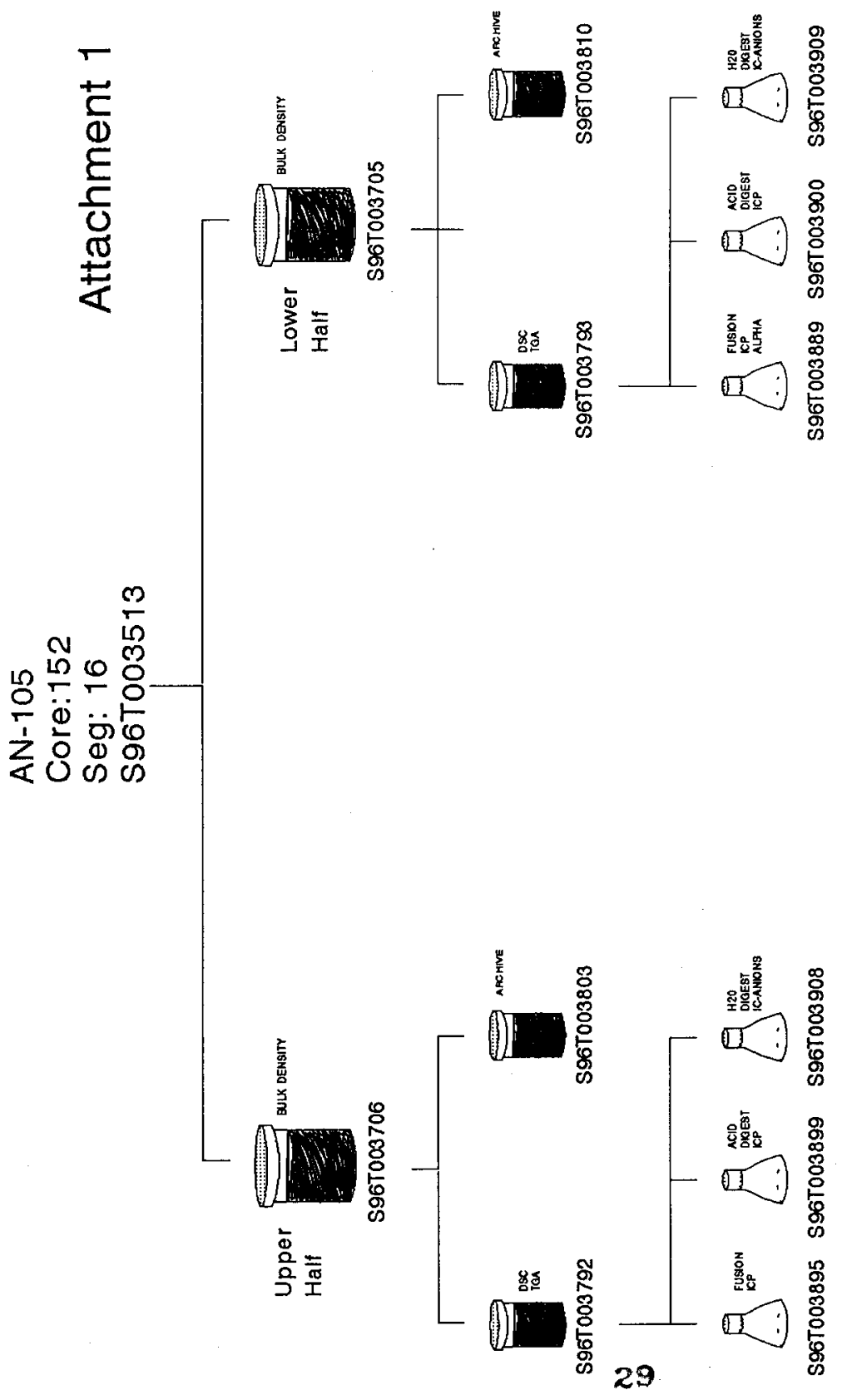


HNF-SD-WM-DP-199, REV. 1

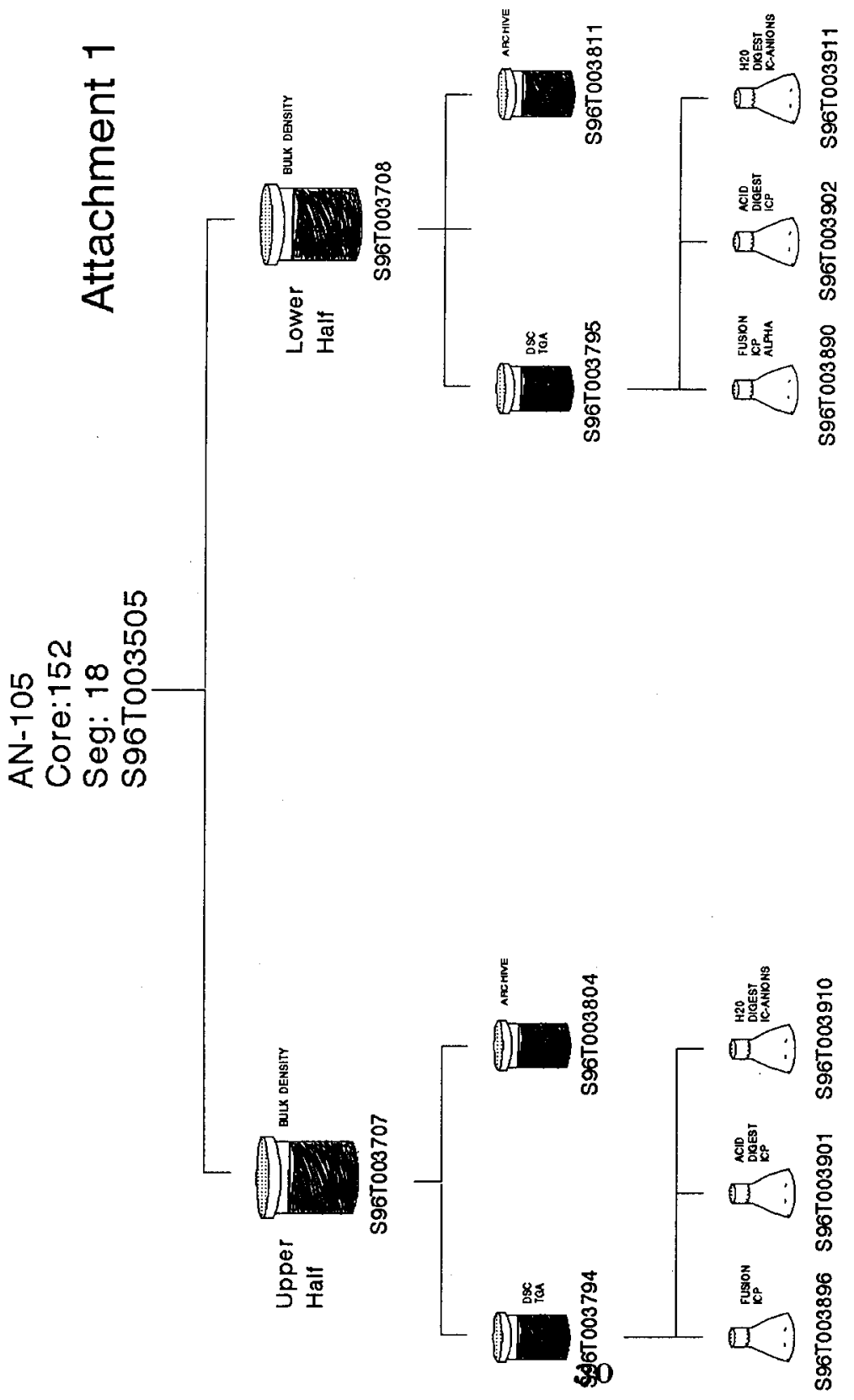




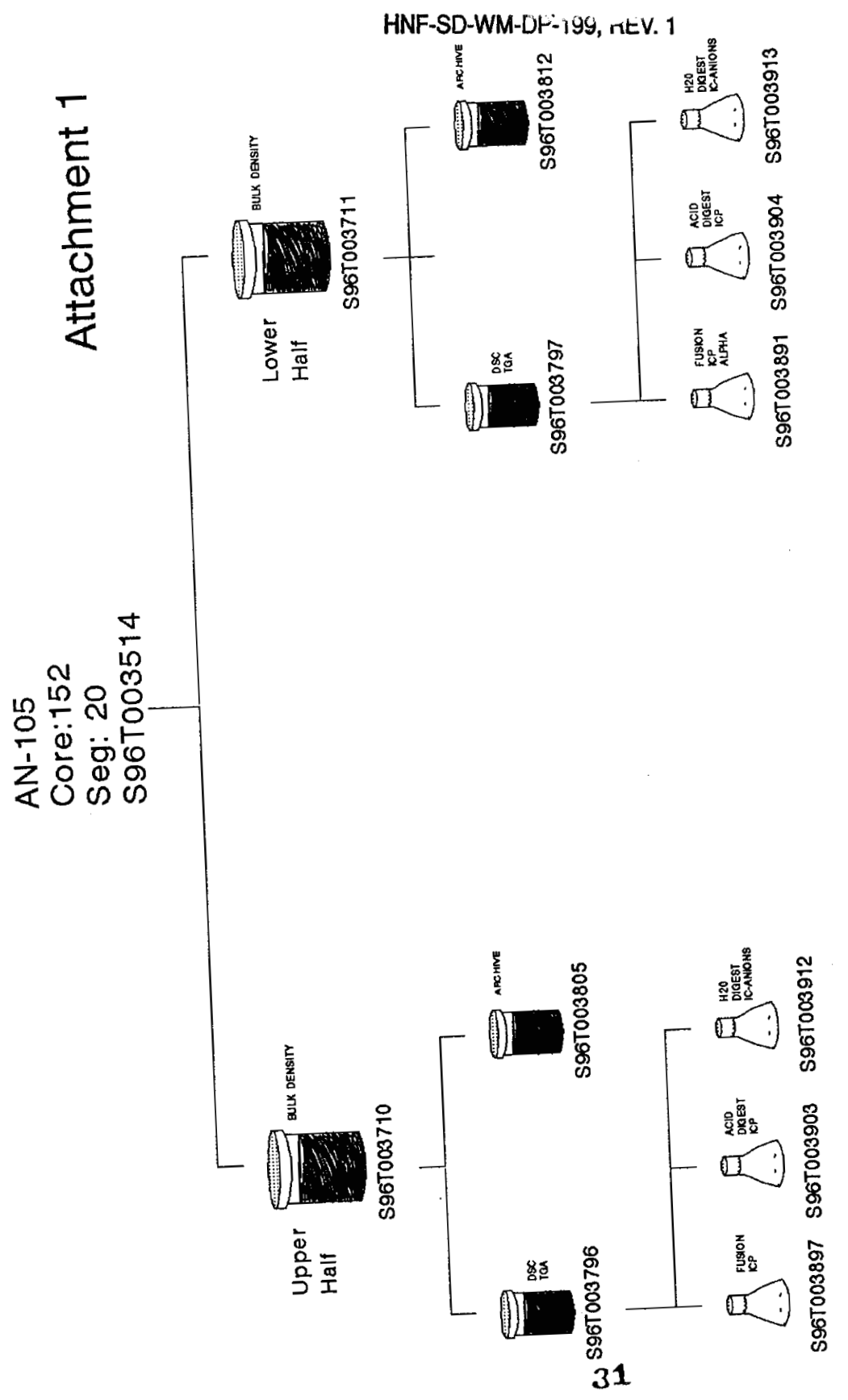


HNF-SD-WM-DP-199, REV. 1

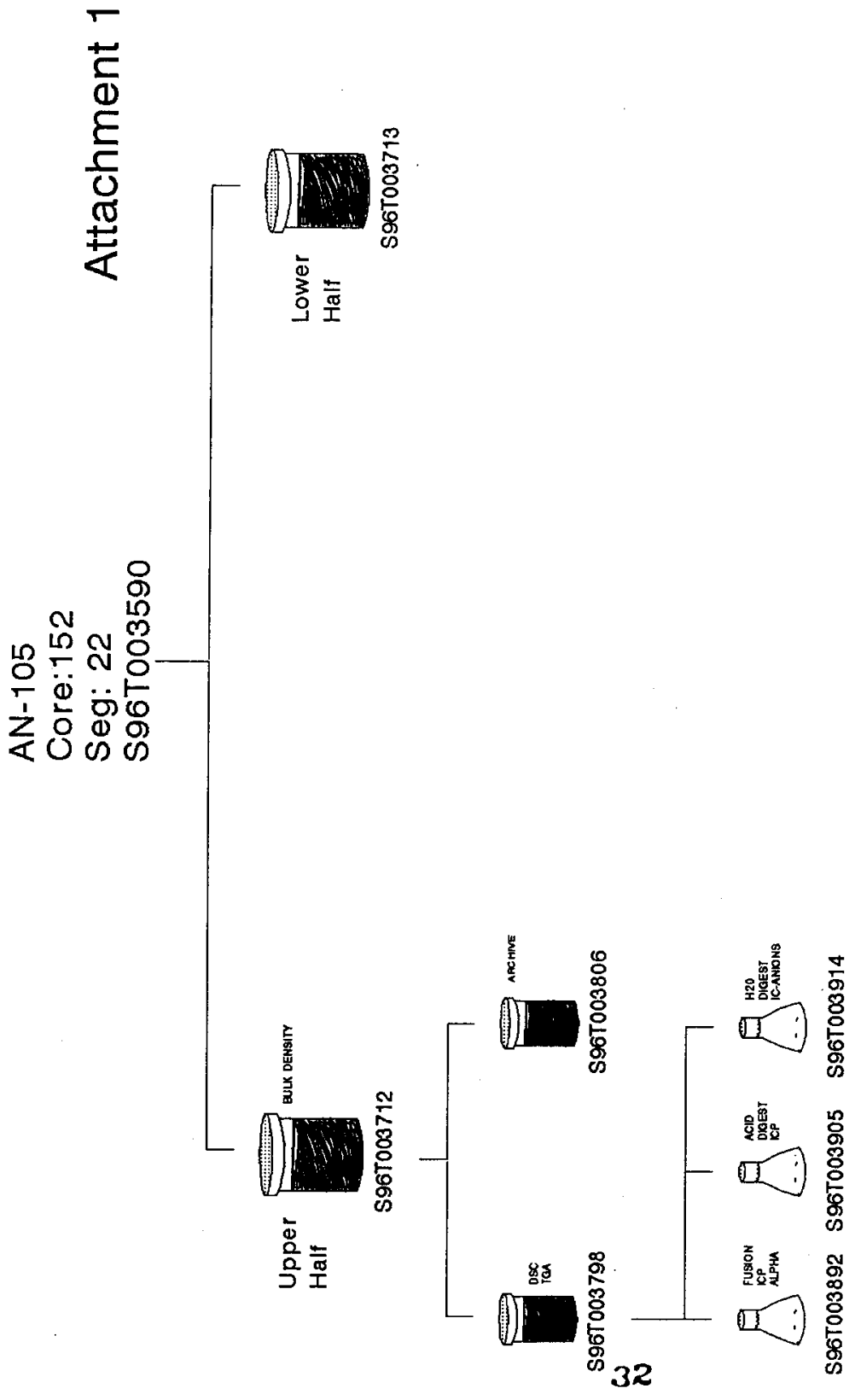


HNF-SD-WM-DP-199, REV. 1

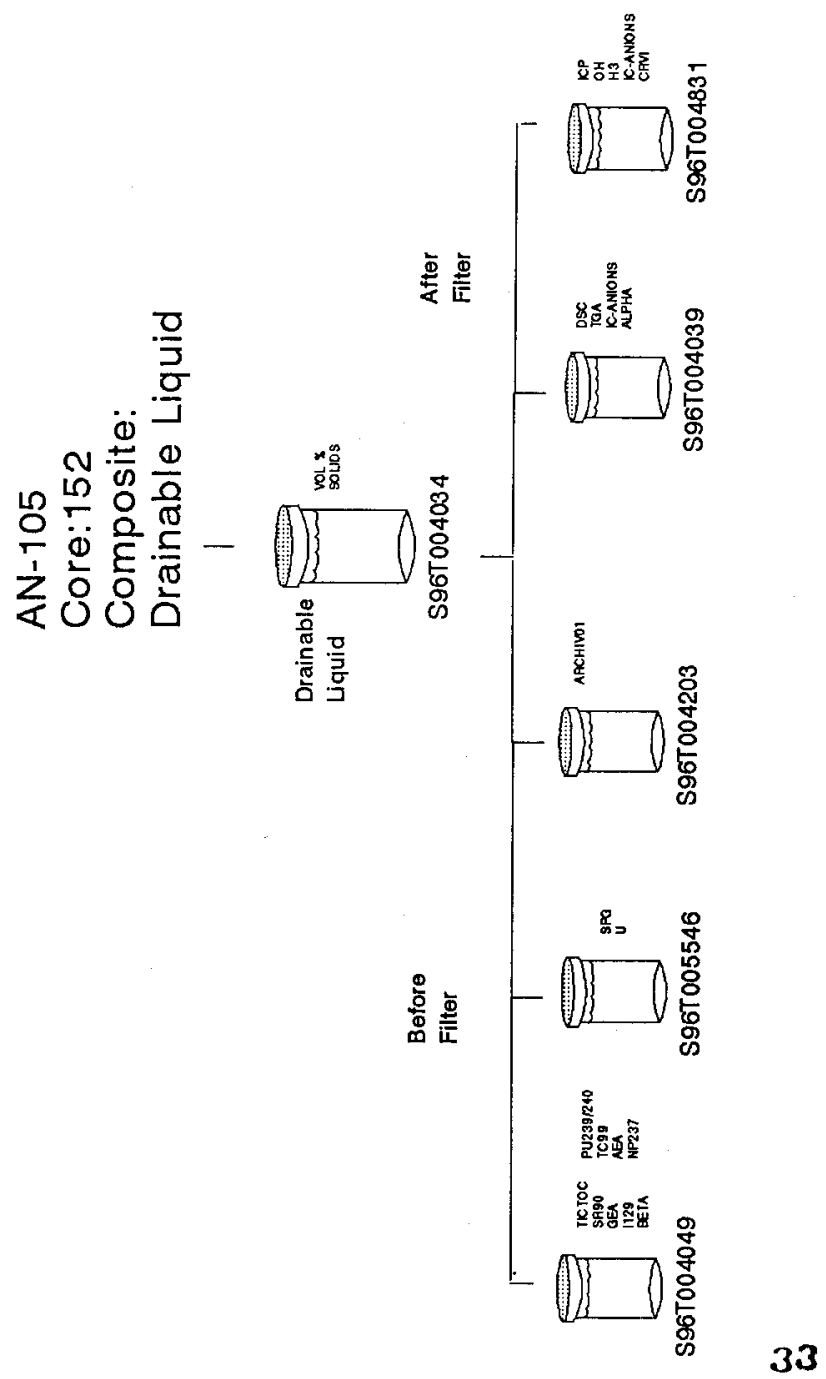




\section{HNF-SD-WM-DP-199, REV. 1}
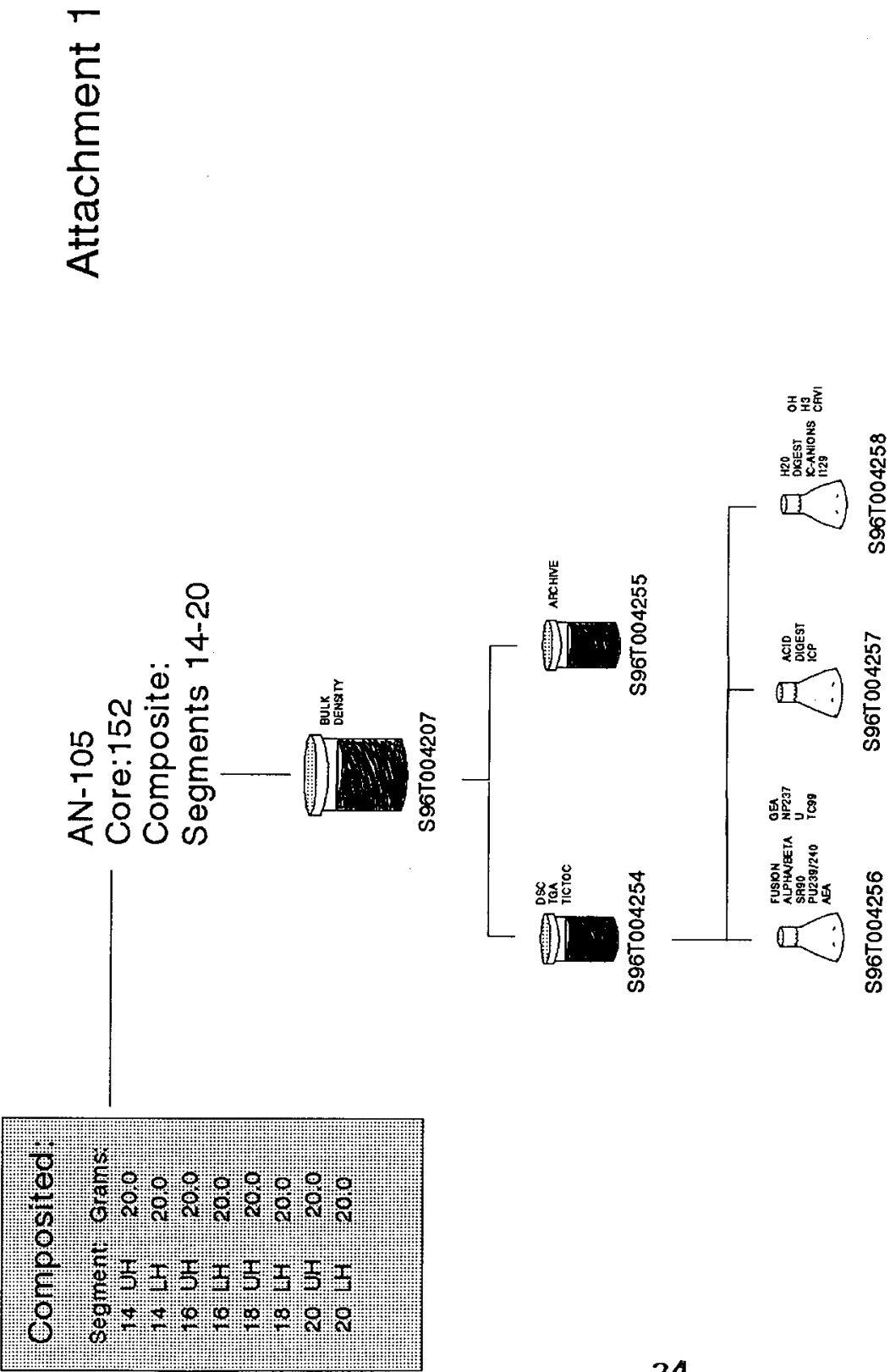

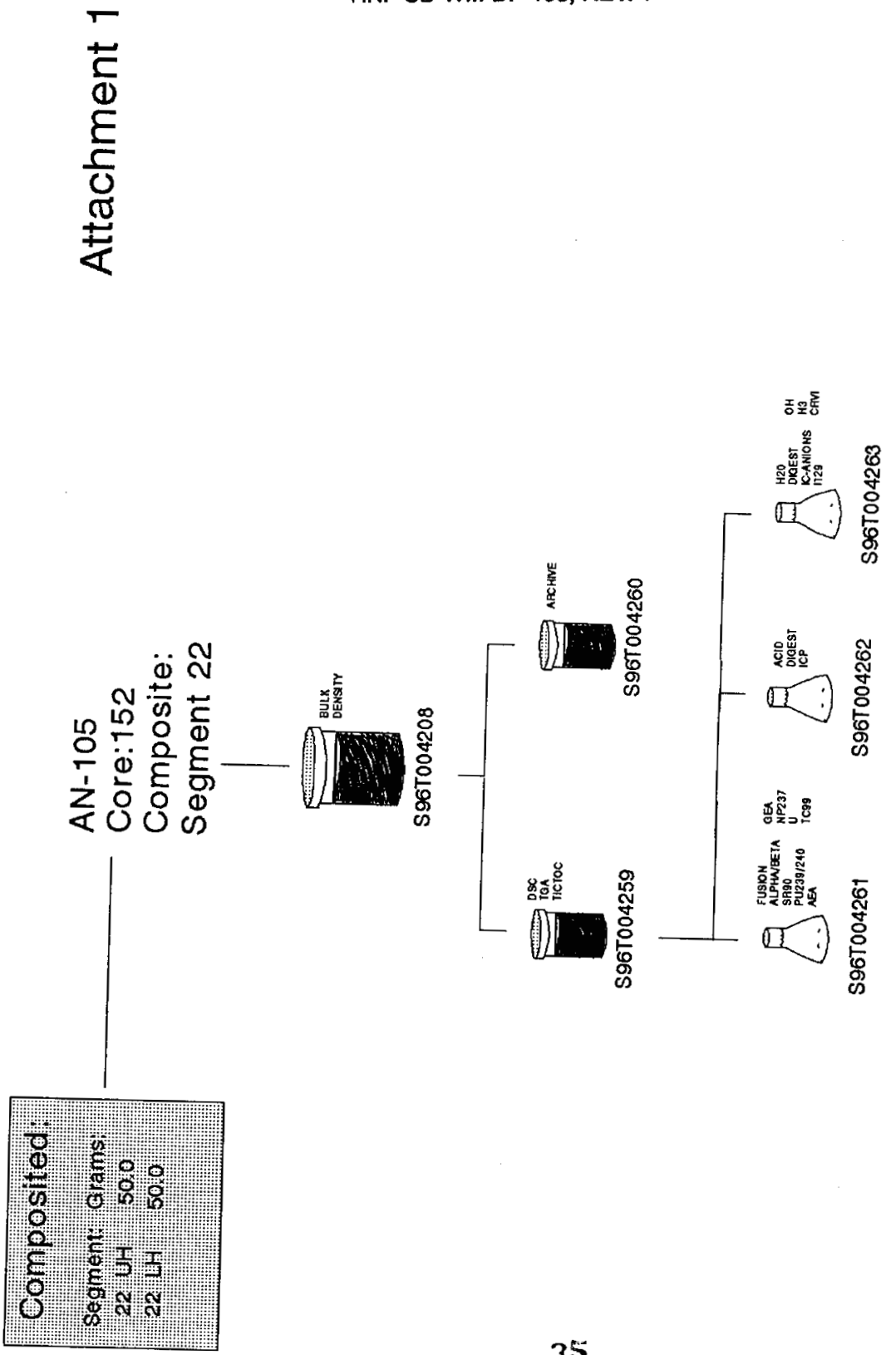
HNF-SD-WM-DP-199, REV. 1

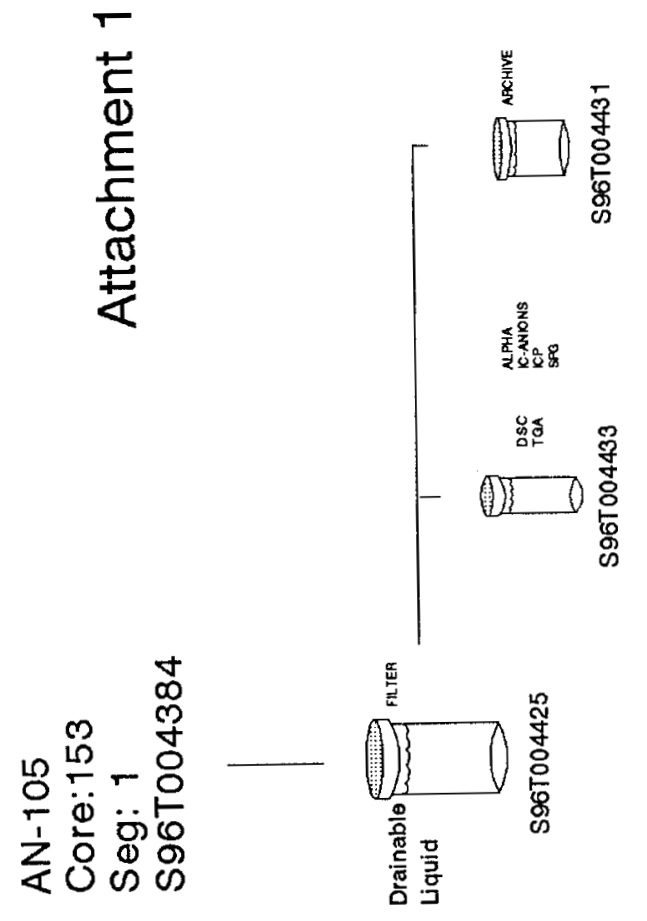


HNF-SD-WM-DP-199, REV. 1

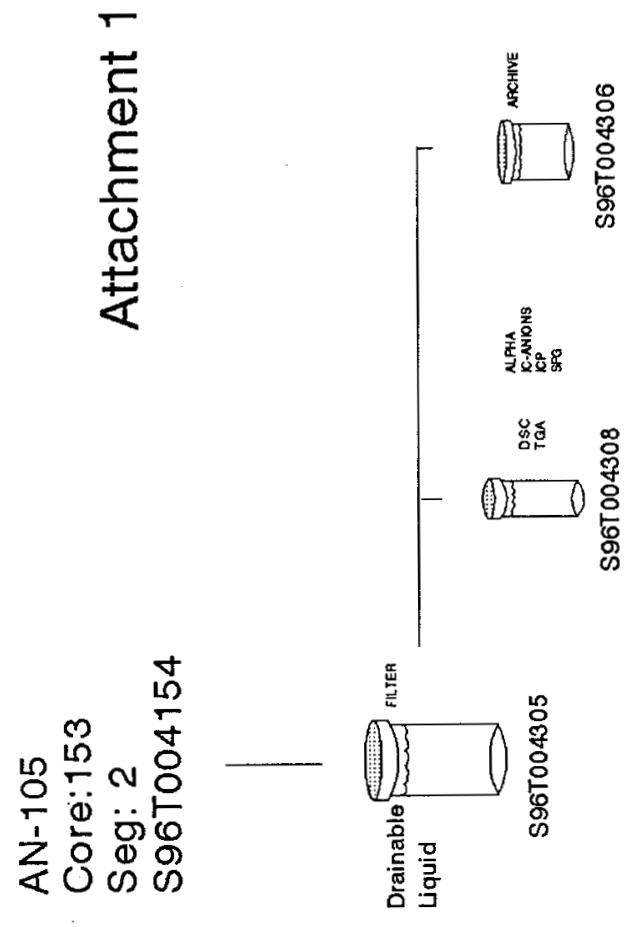


HNF-SD-WM-DP-199, REV. 1

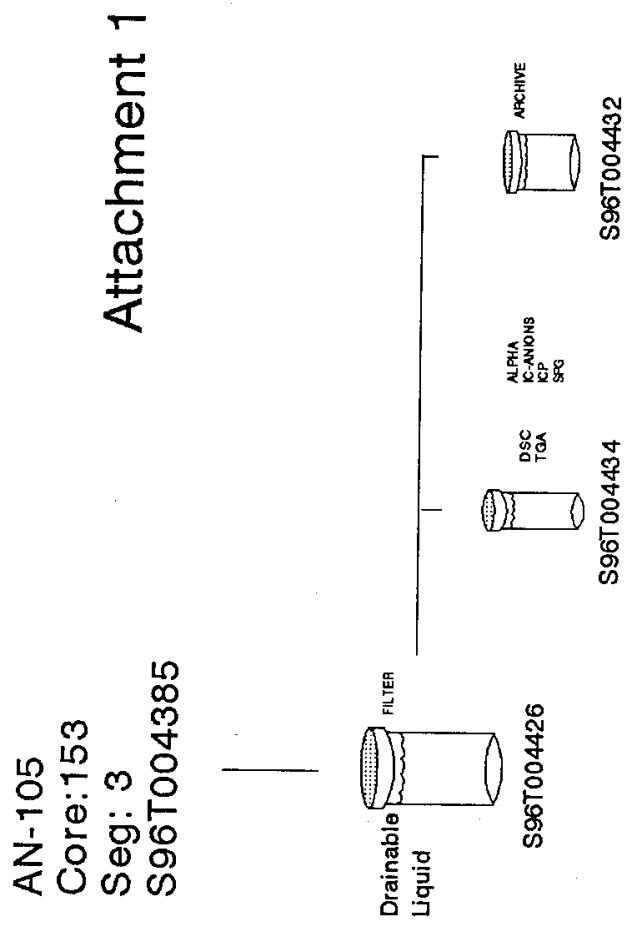



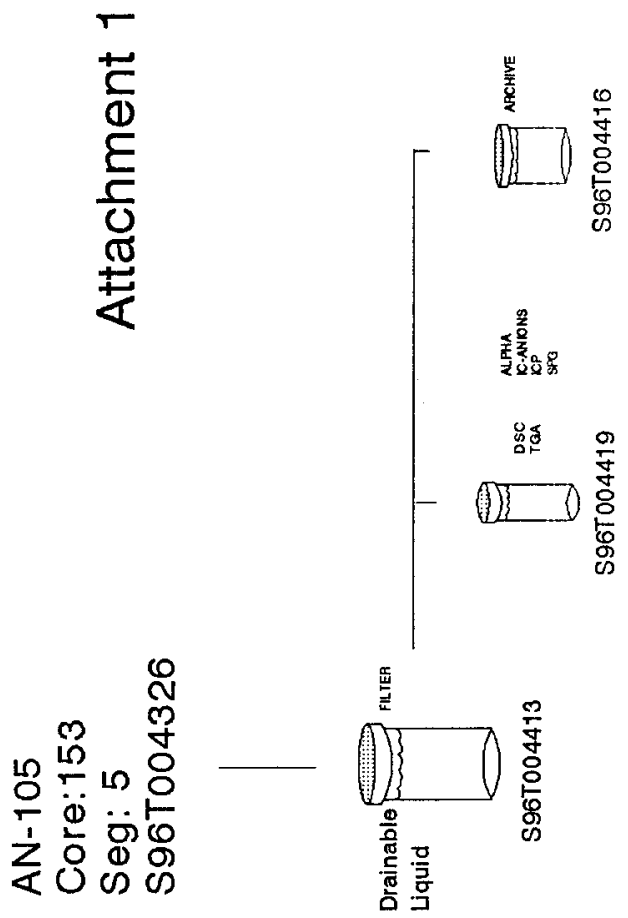


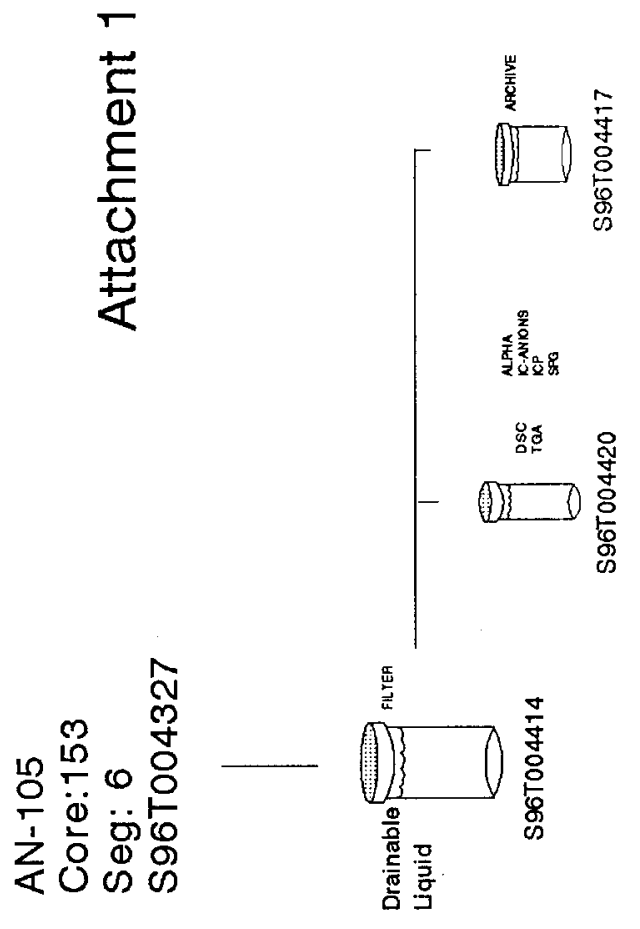


HNF-SD-WM-DP-199, REV. 1

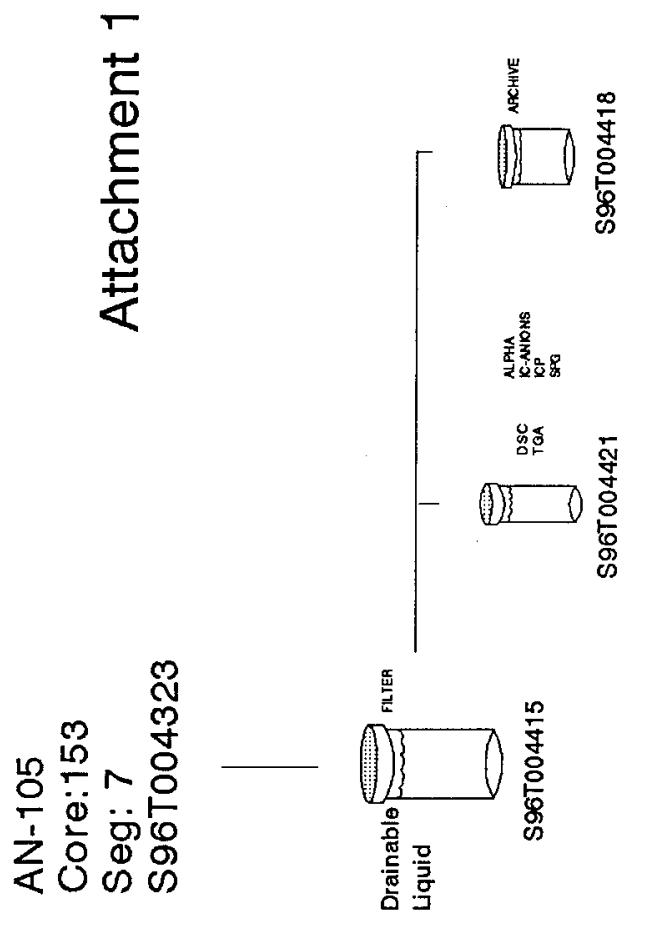


HNF-SD-WM-DP-199, REV. 1

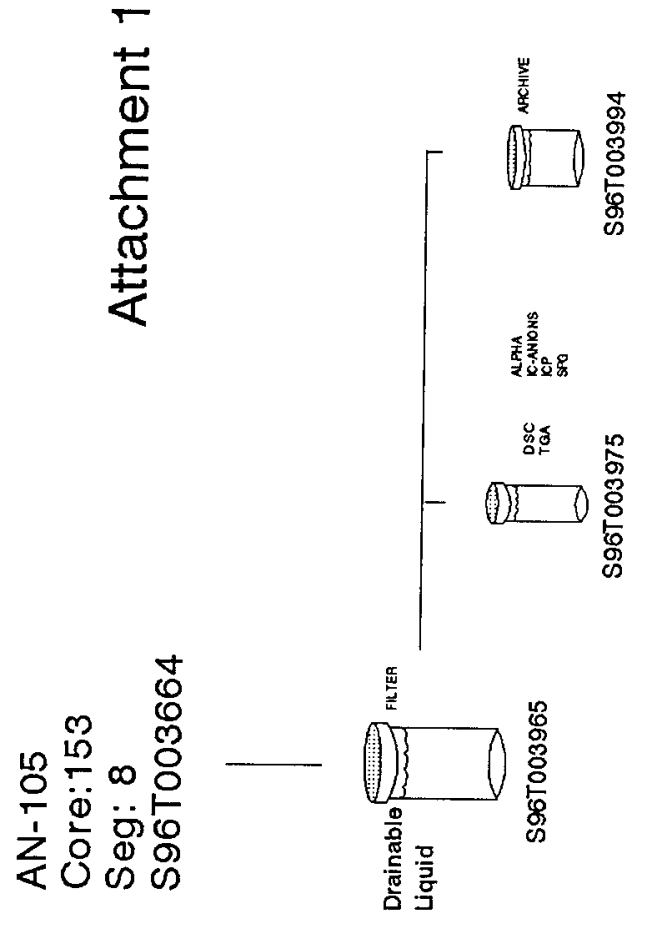


HNF-SD-WM-DP-199, REV. 1

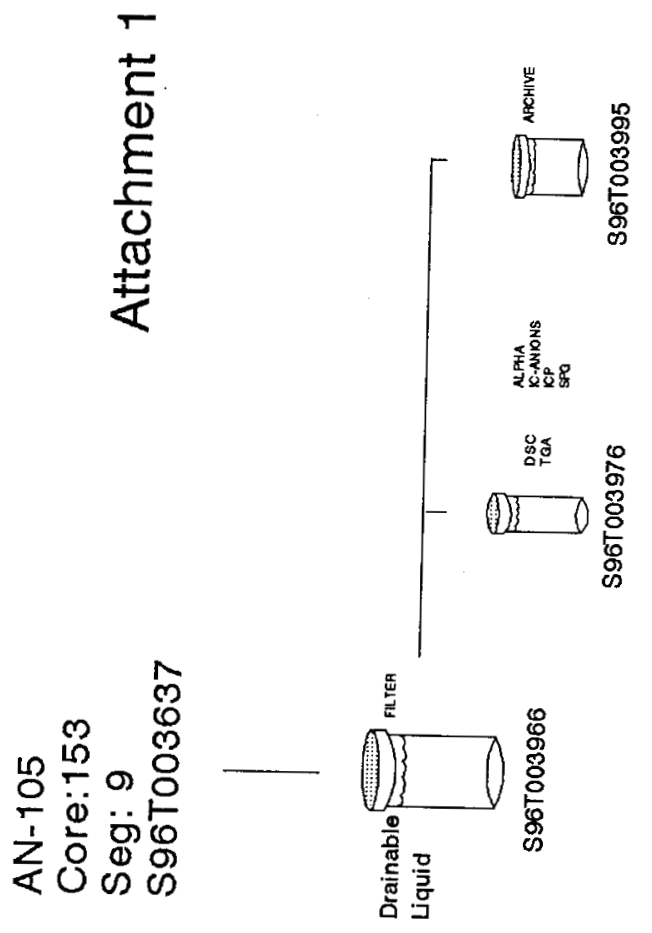


HNF-SD-WM-DP-199, REV. 1

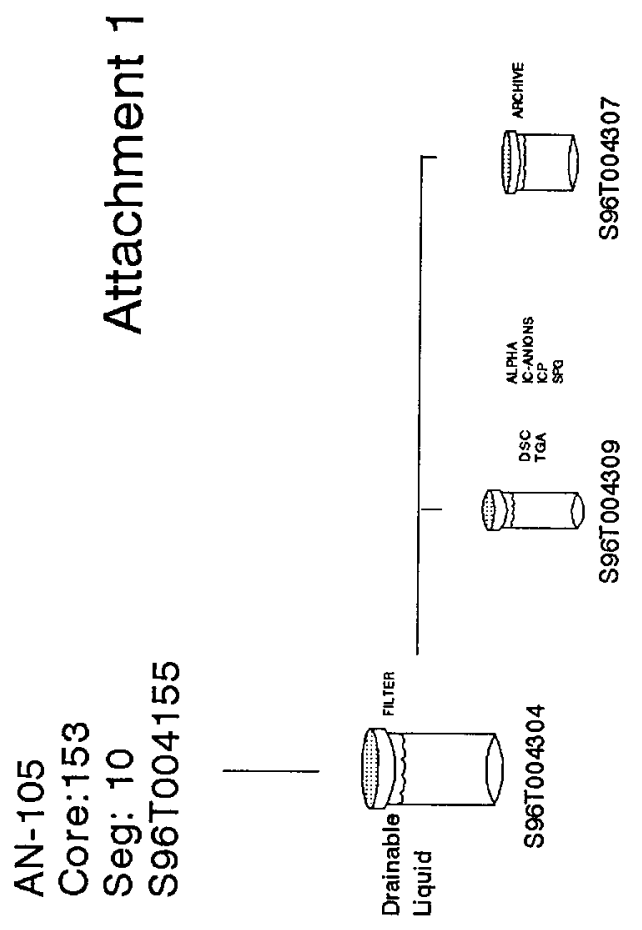


HNF-SD-WM-DP-199, REV. 1

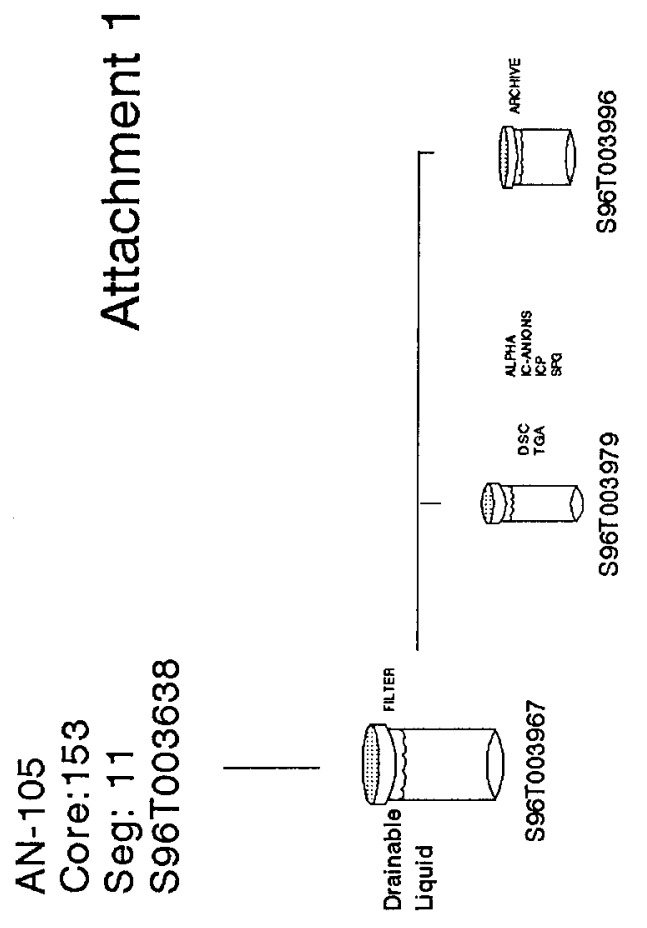


HNF-SD-WM-DP-199, REV. 1

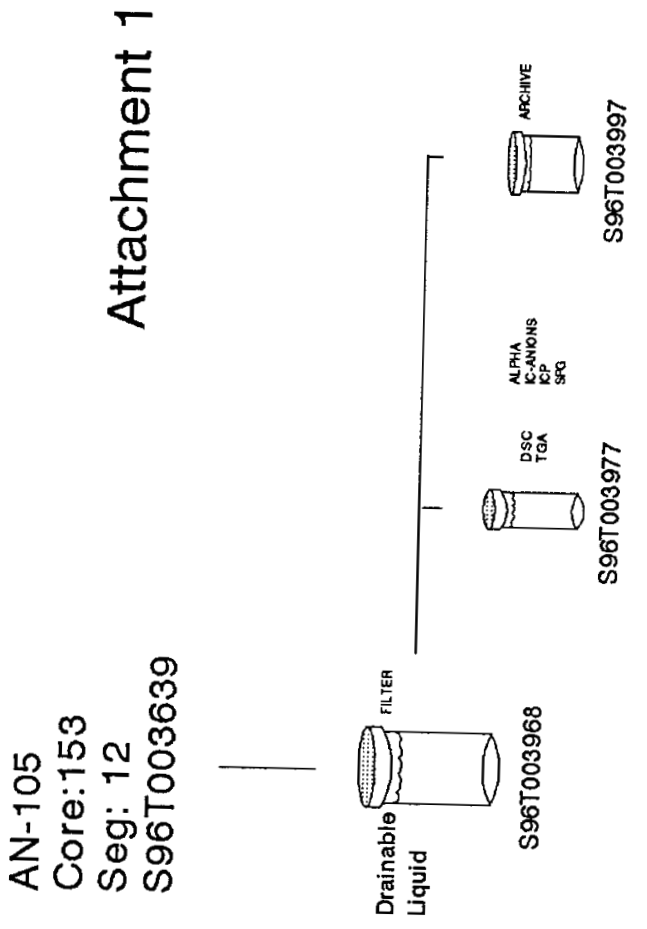


HNF-SD-WM-DP-199, REV. 1

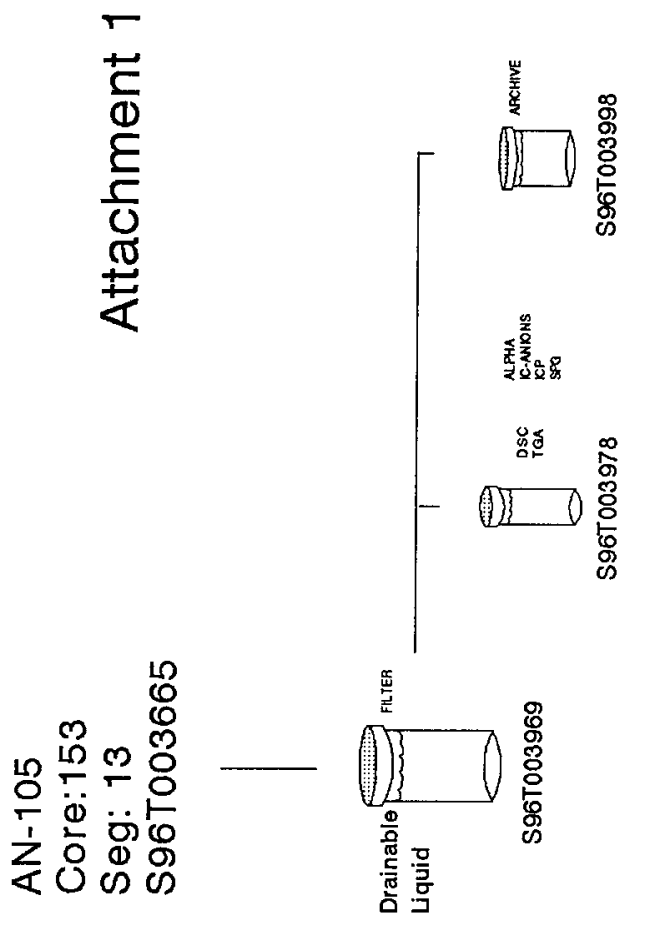


HNF-SD-WM-DP-199, REV. 1
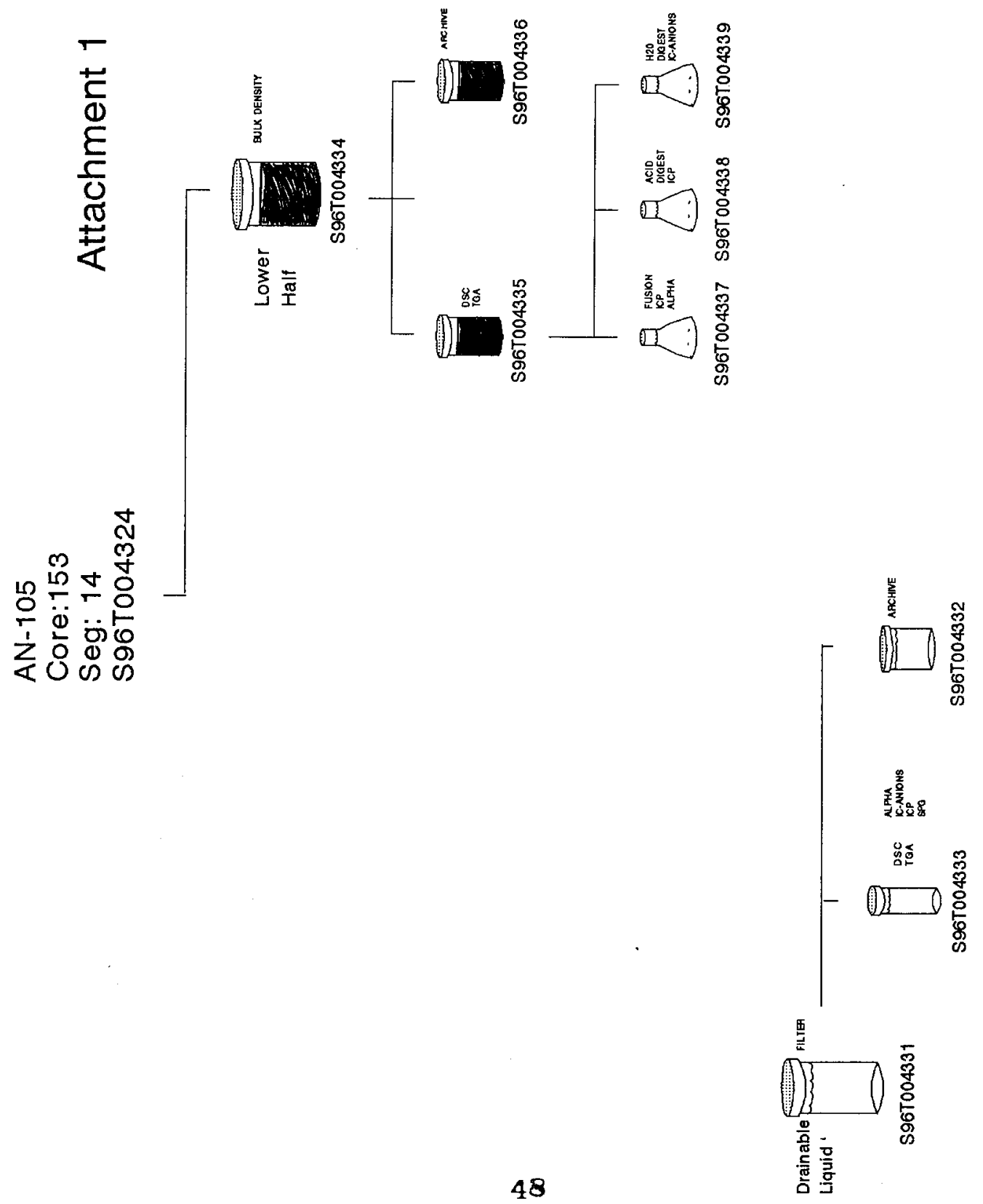
HNF-SD-WM-DP-199, REV. 1

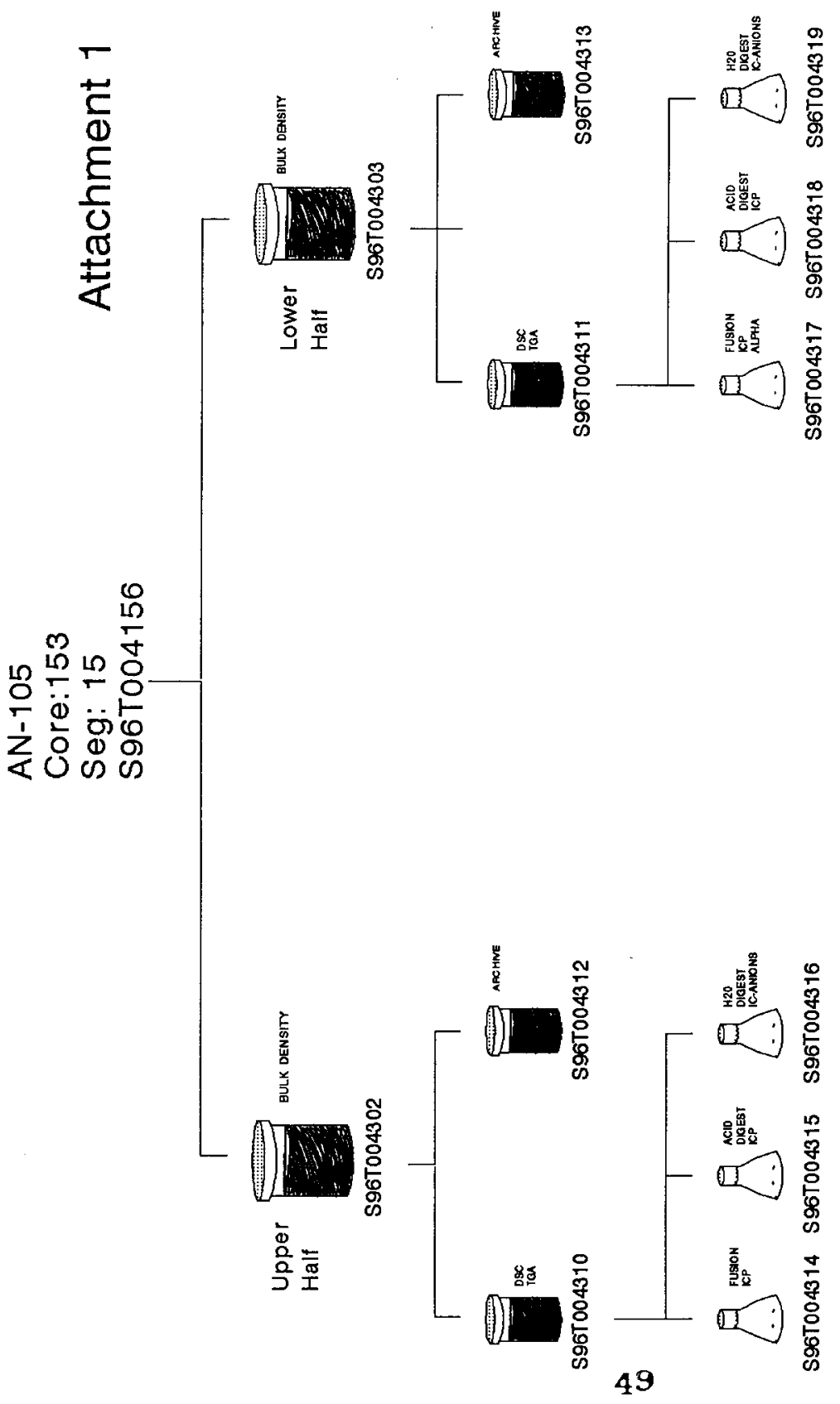


HNF-SD-WM-DP-199, REV. 1

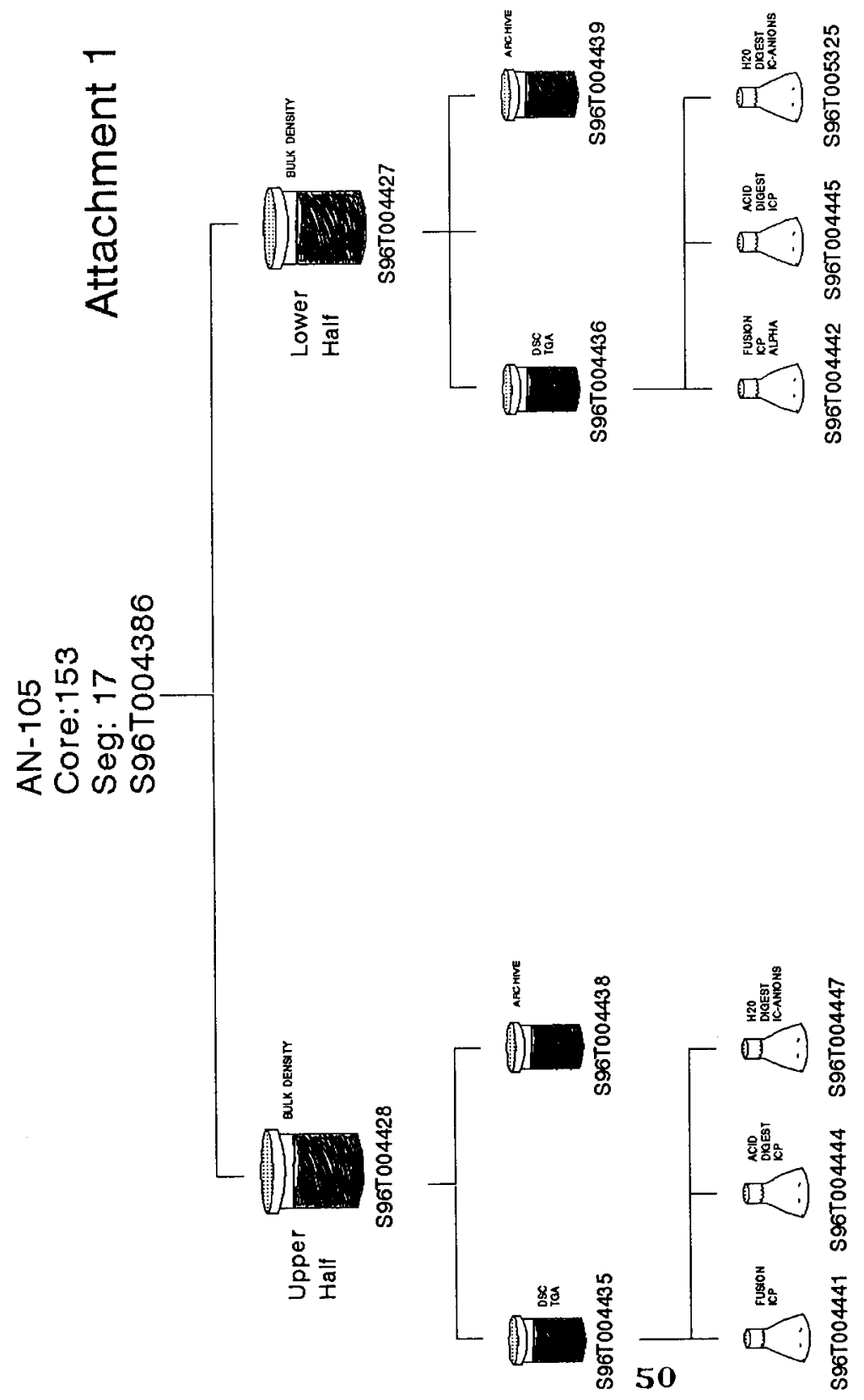


HNF-SD-WM-DPP-199, REV. 1

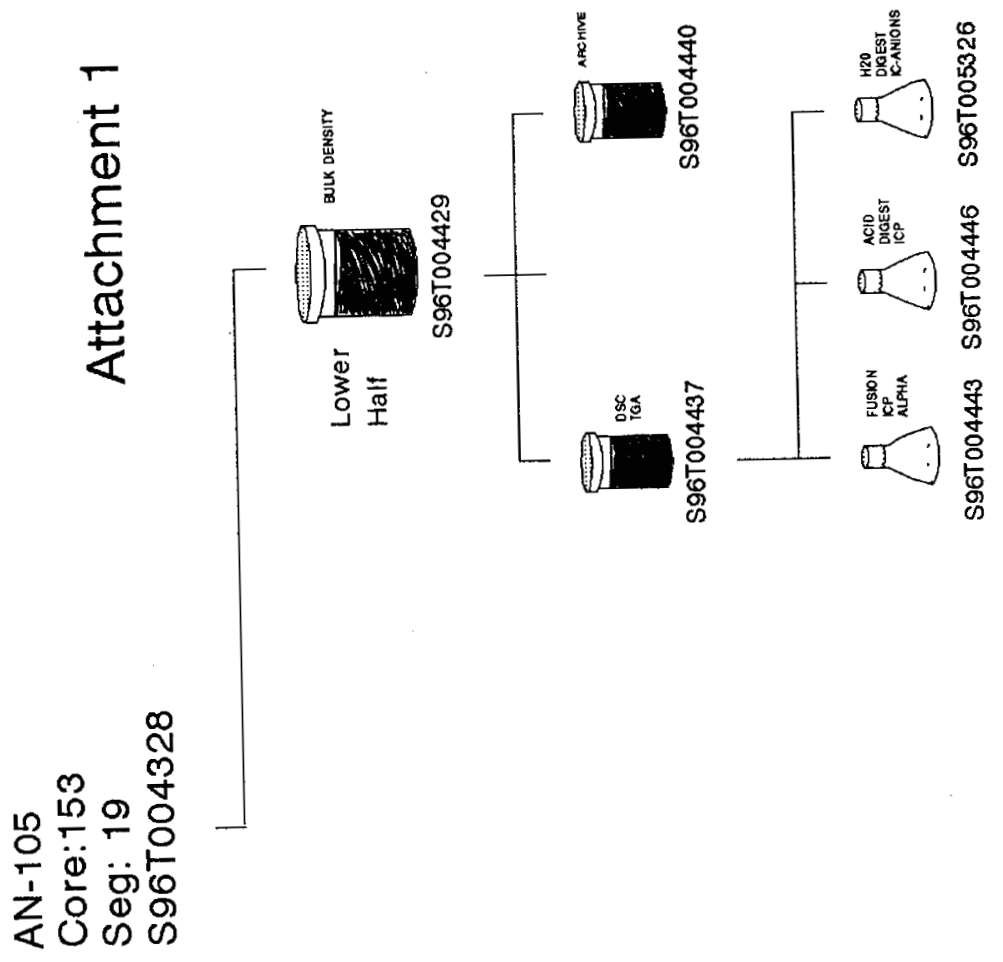

51 
HNF-SD-WM-DP-199, REV. 1
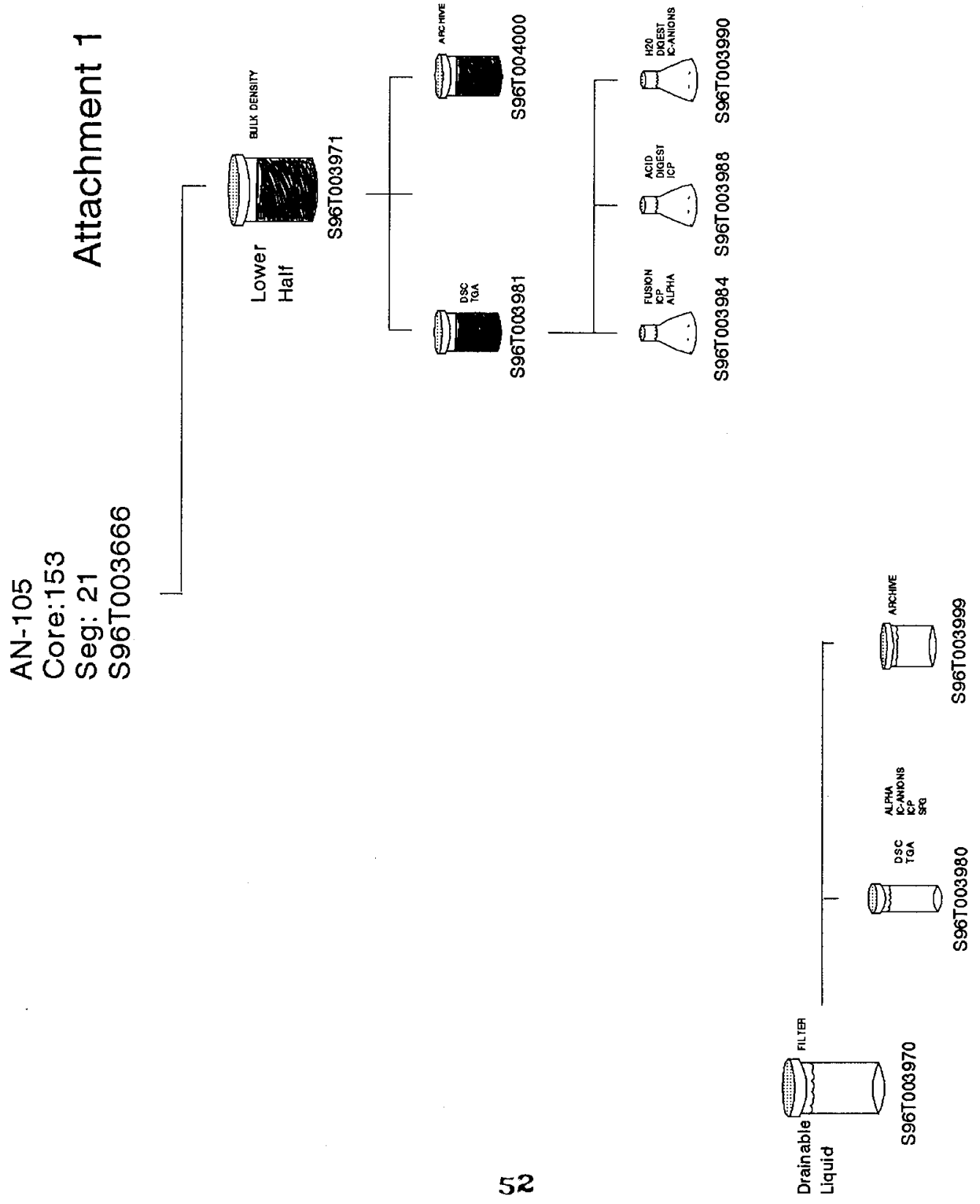
HNF-SD-WV-J--UE, - =v.

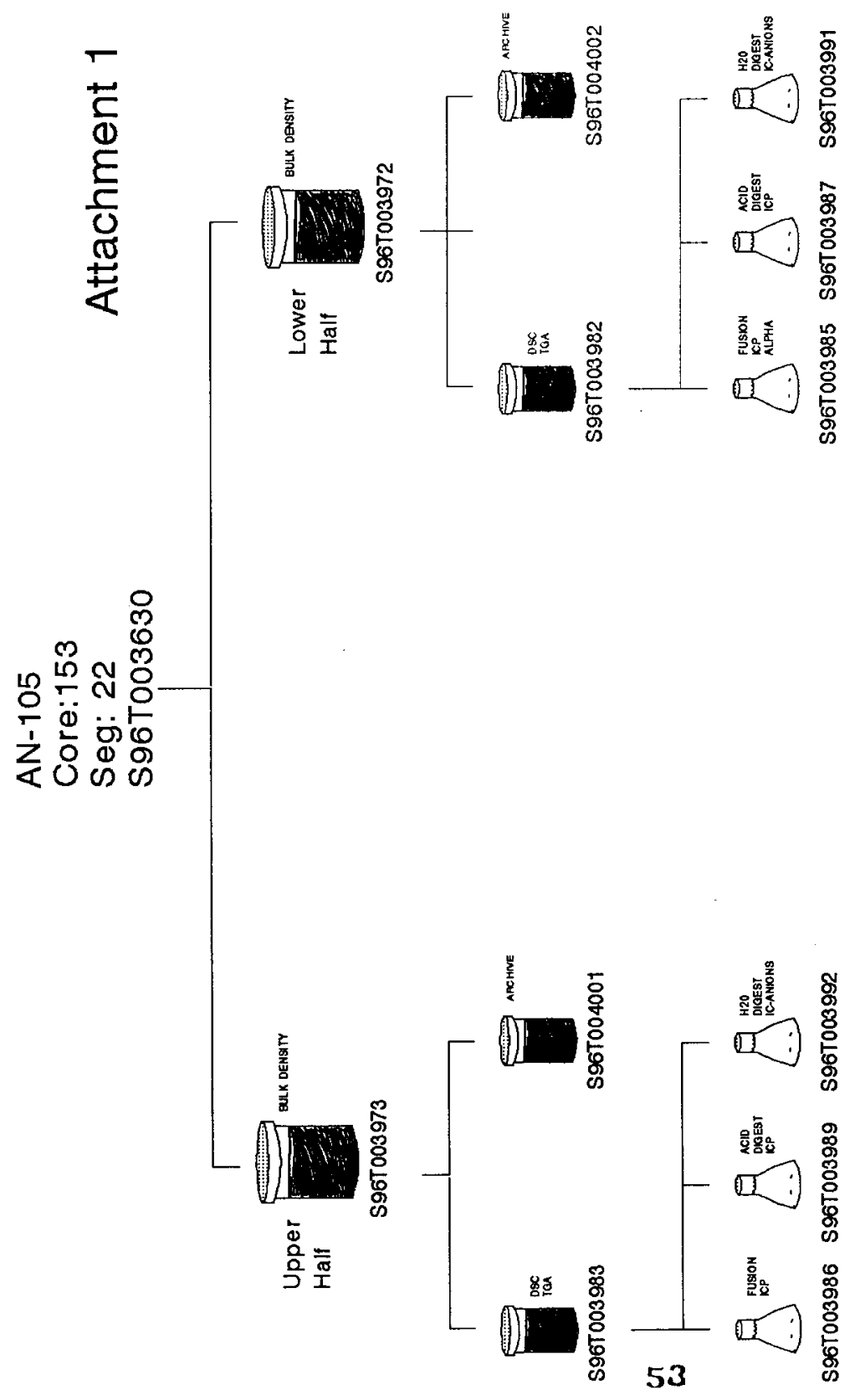


HNF-SD-WM-DP-199, REV. 1

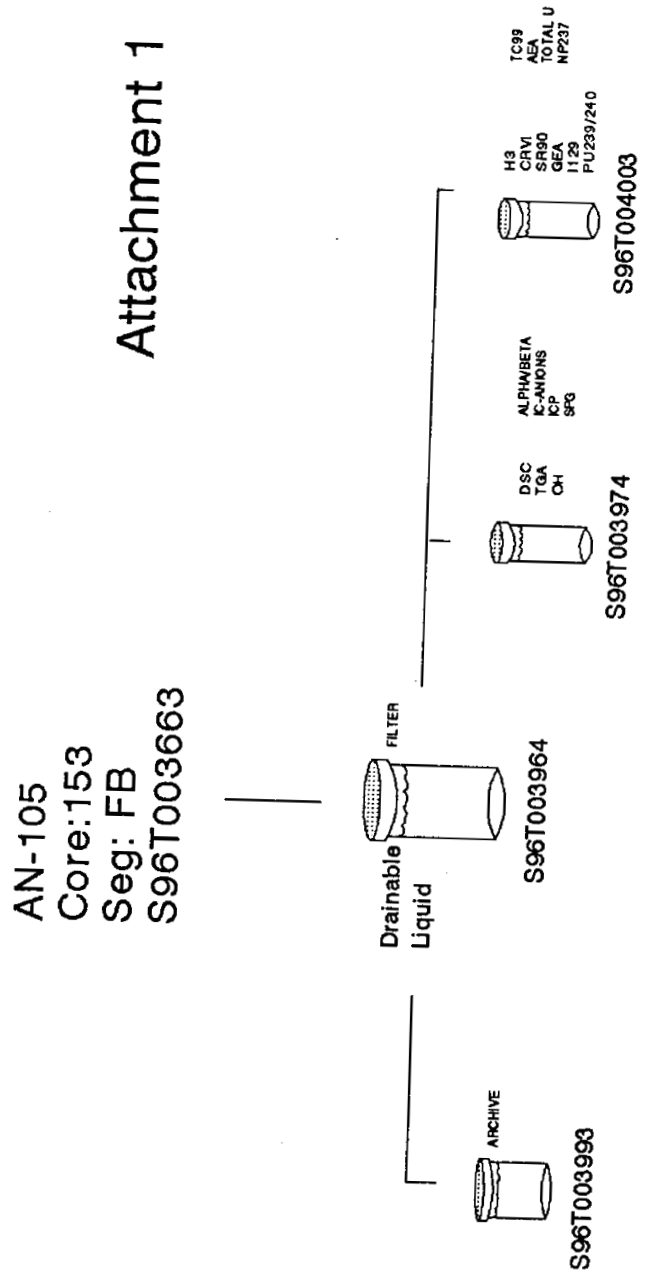


HNF-SD-WM-DP-199, REV. 1
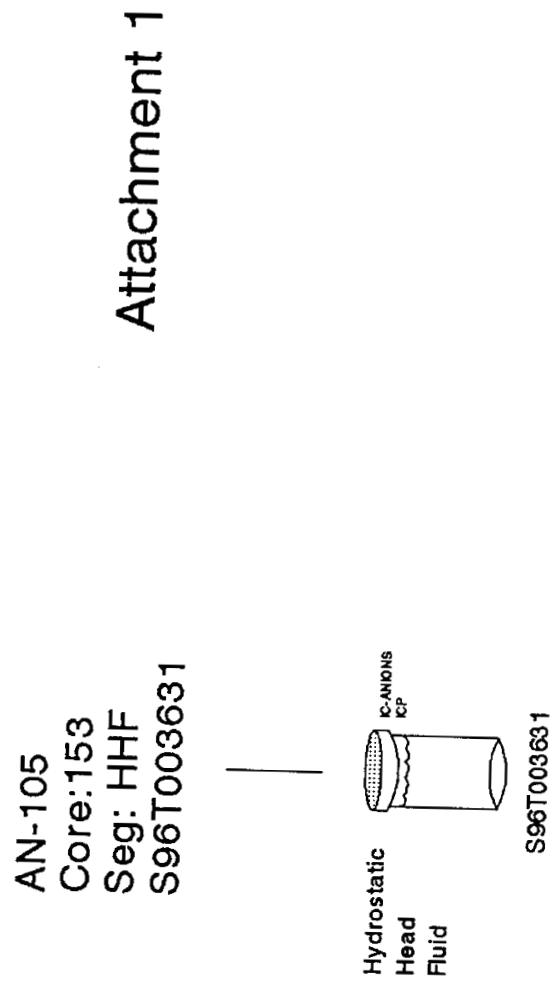


\section{HNF-SD-WM-DP-199, REV. 1}

WHC-SD-WM-DP-199, REV. I

SAMPLE DATA SUMMARY 


\section{HNF-SD-WM-DP-199, REV. 1}

WHC-SD-WM-DP-199, REV. 1

THIS PAGE WAS INTENTIONALLY LEFT BLANK 
x ब

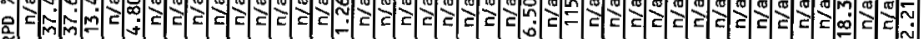

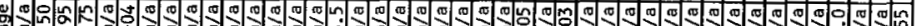

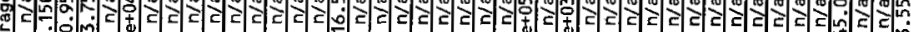

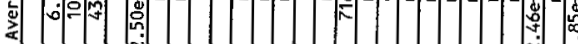

a 0 -

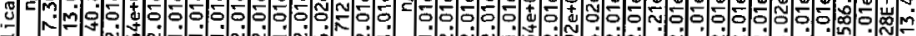

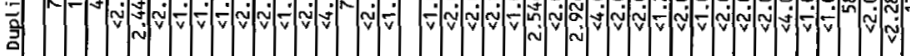

ثี

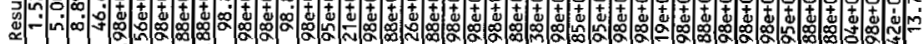

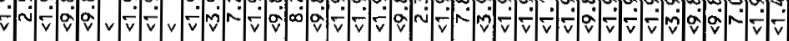

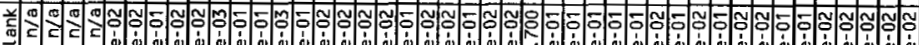

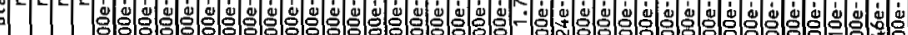

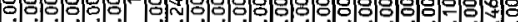

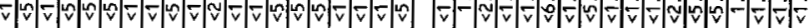

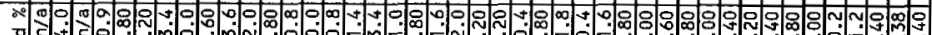
뭉 00 : 空

\section{1 잔}

의.

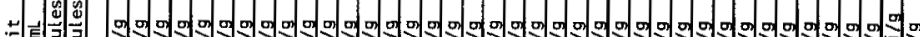

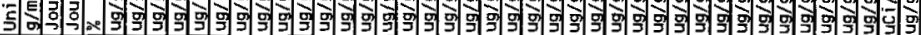

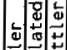

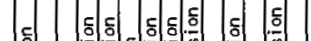

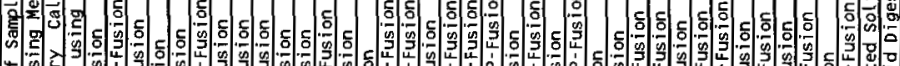

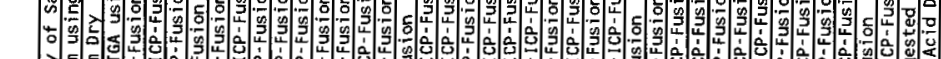

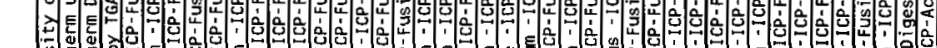
落

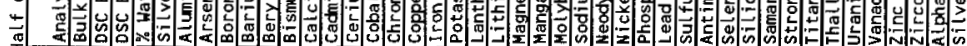

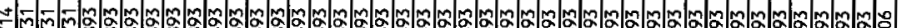
SCF 


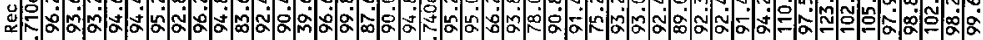
$\div \div$

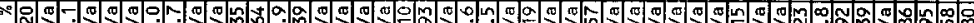

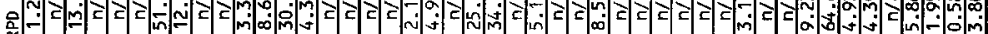

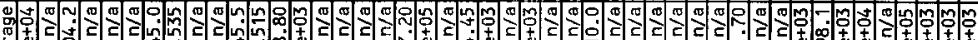

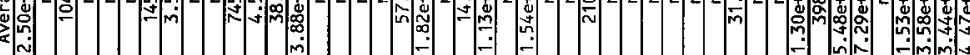

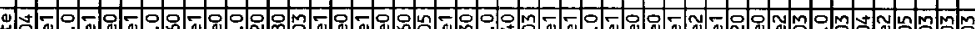

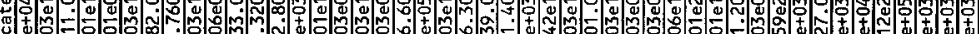

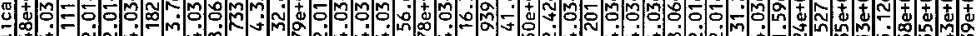

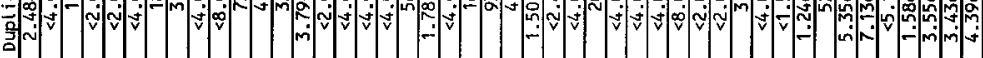

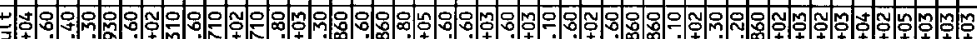

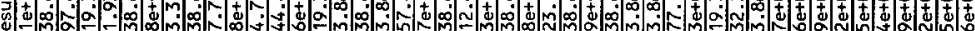

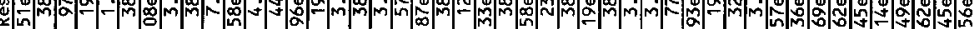

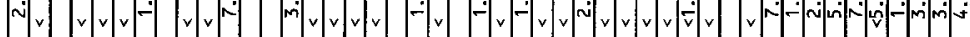

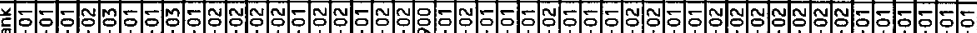

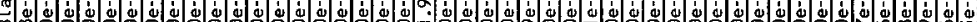
a

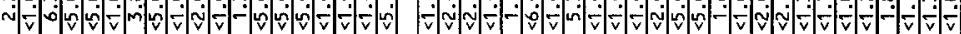

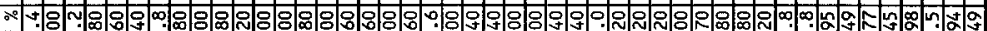

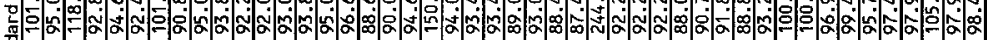
辡

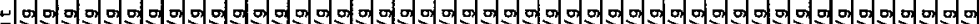

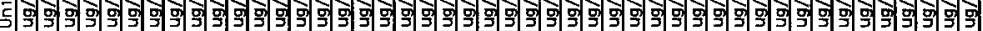

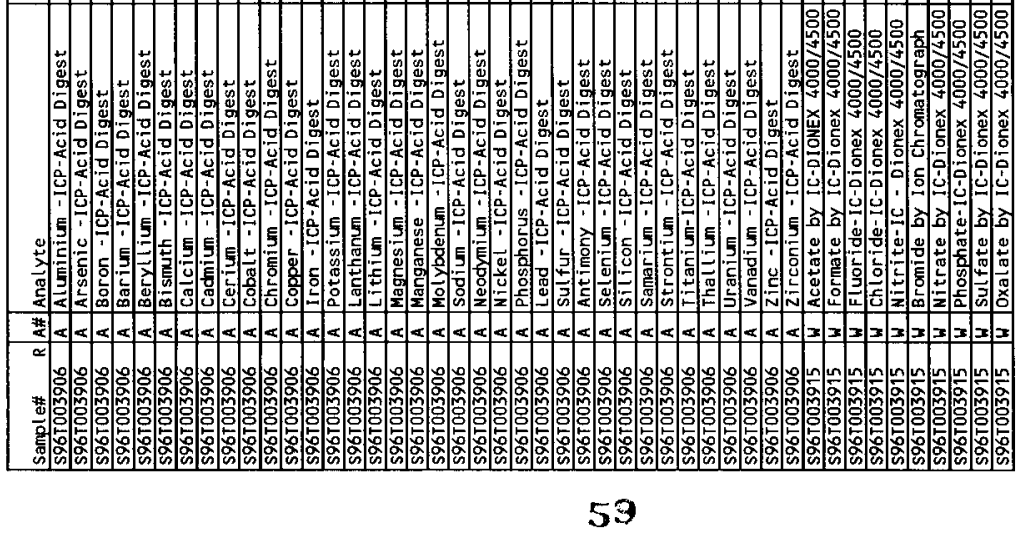




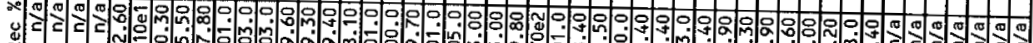
\& : a

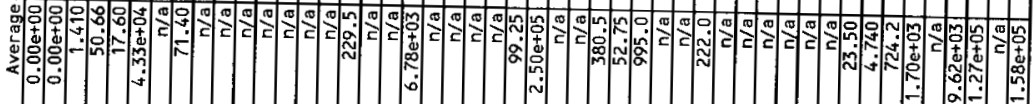

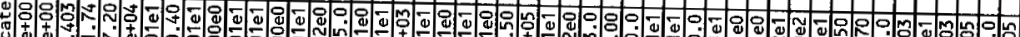
。ํ. -

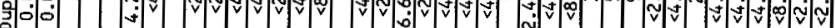

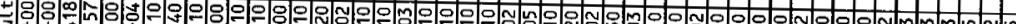
年

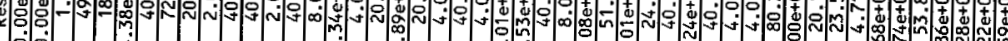

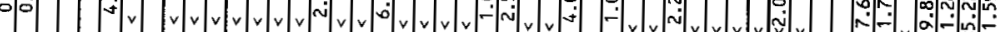

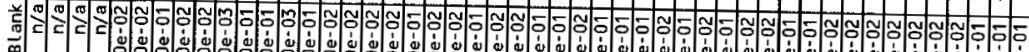
๓

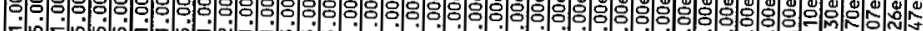

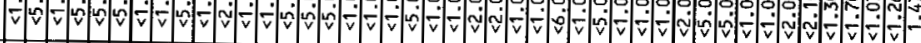
N

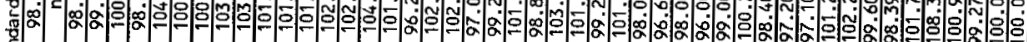
空

of 요

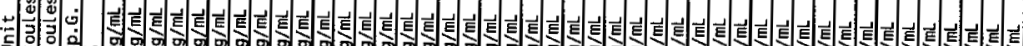

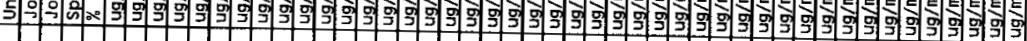

㻤

군

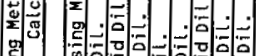

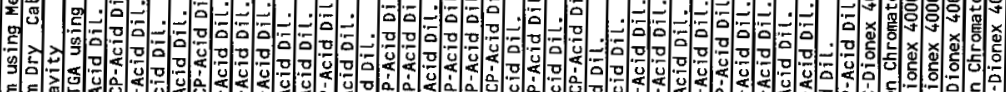

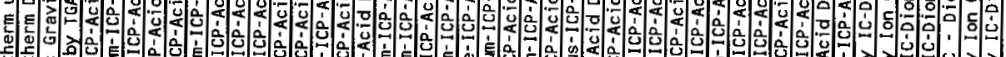

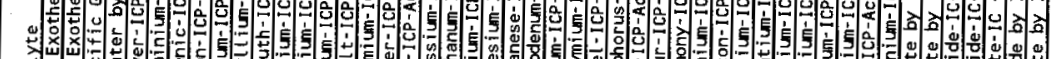

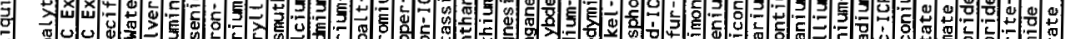

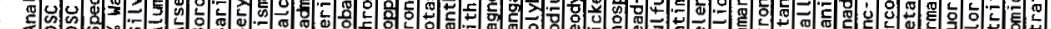
숭

m

$\stackrel{\ddot{g}}{\mathrm{~g}}$

$\ddot{q}$

$\hat{\alpha}$

$\div \dot{m}$

要总

N * 
HNF-SD-WM-DP-199, REV. 1

N WHC-SD-WM-DP-199, REV. 1

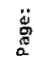

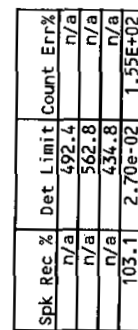

$\therefore$ :ण a $\infty$ 엥=

an
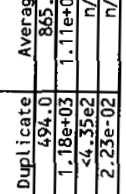

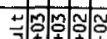

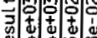

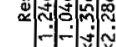

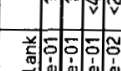

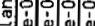

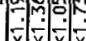

बतन

잉ㅎㅇ

,

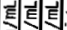

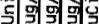

웅

路行行

홍으.

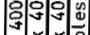

의의

의

일.

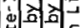

엔뭉

김 90

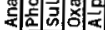

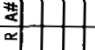

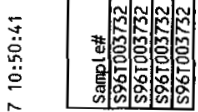

a

둥 
HNF-SD-WV-Y -499, $7 \cong V$.

민

\% :

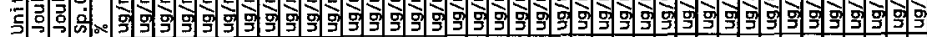

7$$
\div \div
$$

考泀

要

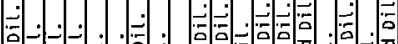

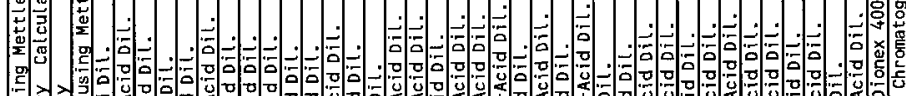
$\Rightarrow>0$ a

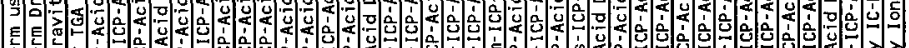

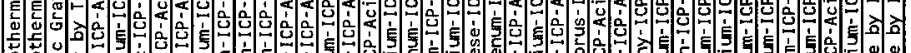

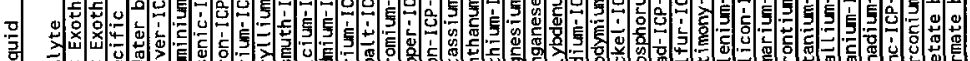

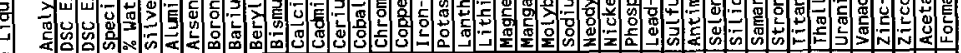

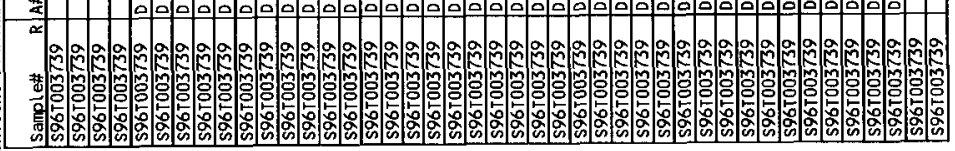


i

㟧 $-1-\infty-v b v-\ldots z z,-x=v$.

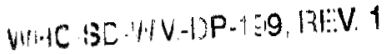

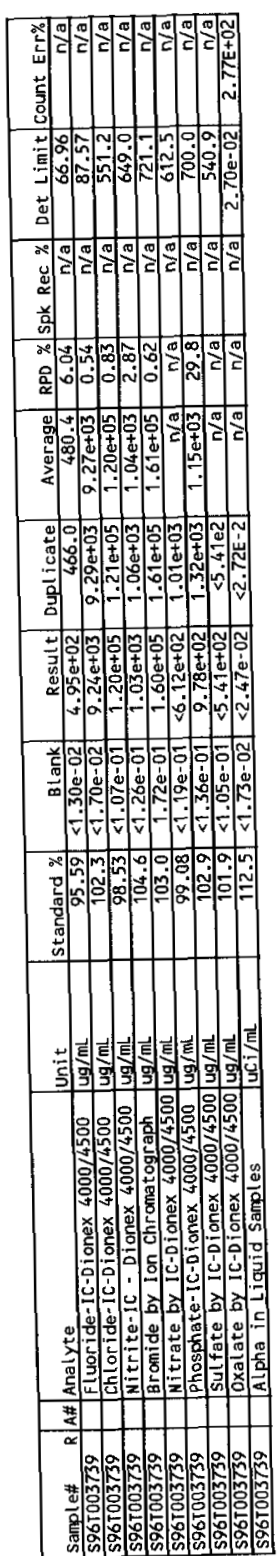

a

ํㅜㅇ 
HNF-SD-WM-DP-199, REV. 1

의

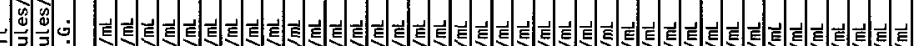

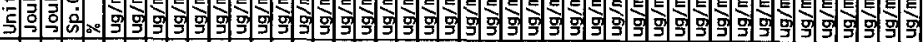

莺 产

$\frac{0}{3}$

产

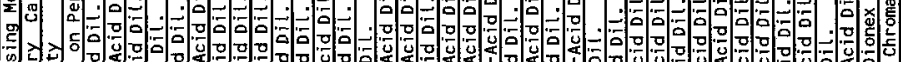

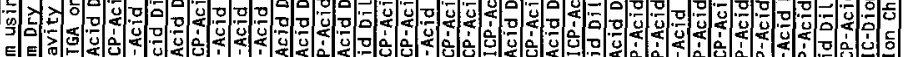
然 a

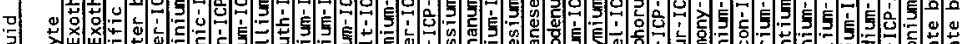

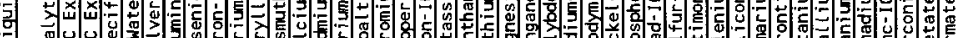

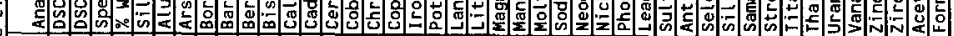

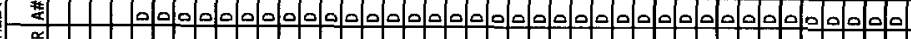
菩 


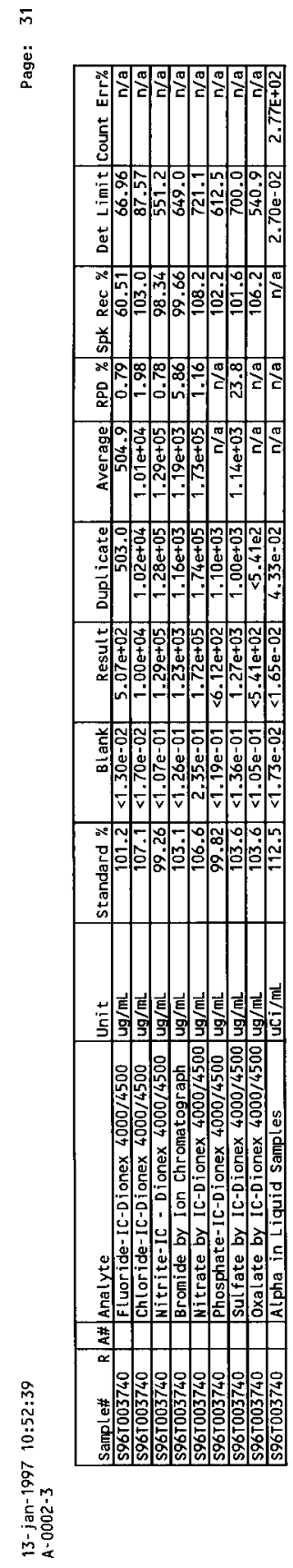

HNF-SD-WM-DP-199, REV. 1 WHC-SD-WM-DP-199, REV. 1 
$-1-3 J-v V v-+-2 z,-7=v$. 


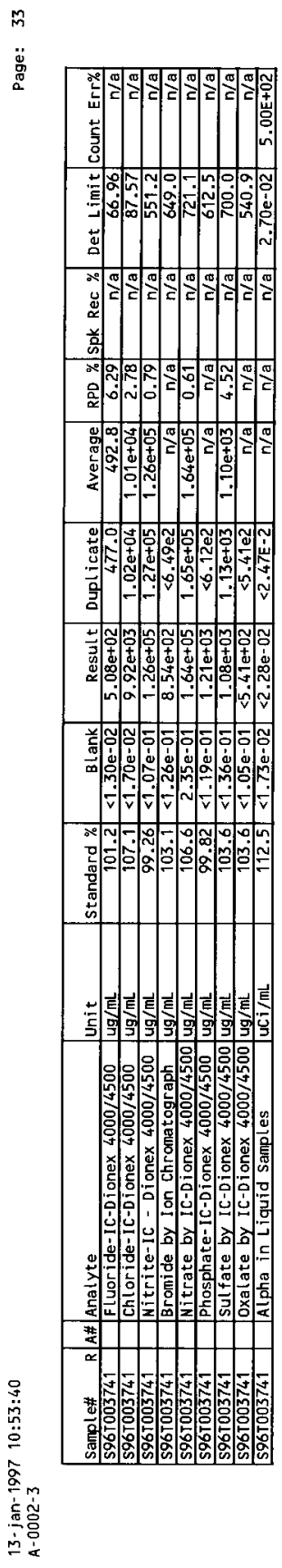

HNF-SD-WM-DP-199, REV. 1

WHC-SD-WM-DP-199, REV. 1 


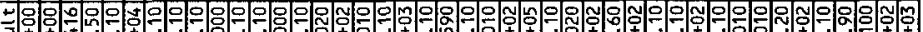

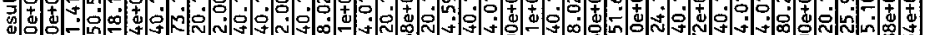
c

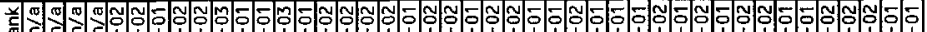

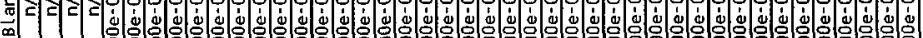
๓ (5)

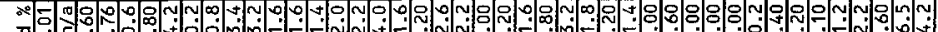

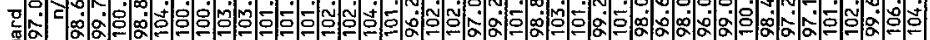
잉 कึ

?

요용

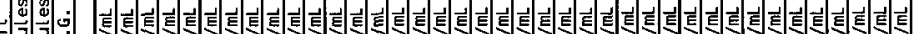

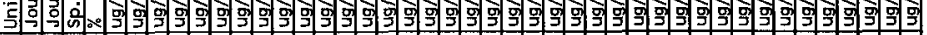

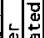

$\frac{0}{3}$

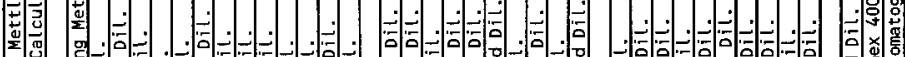
을

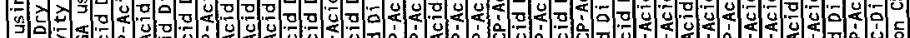

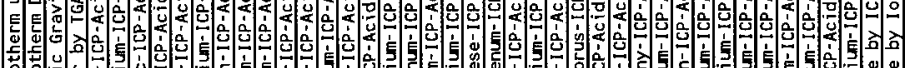

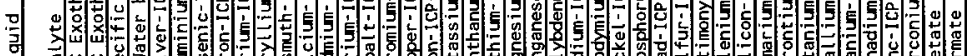

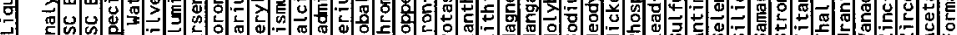

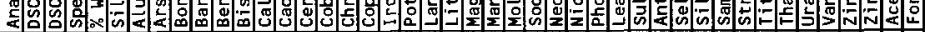

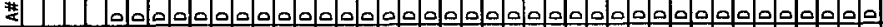




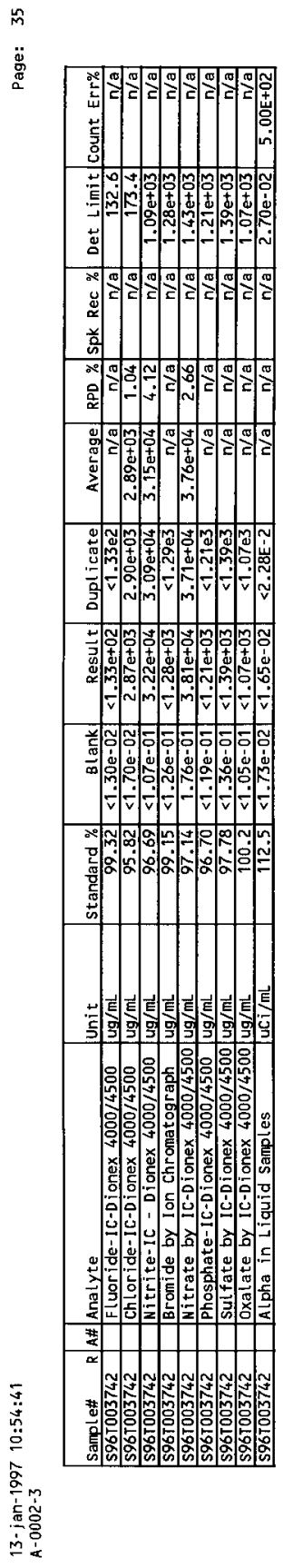

HNF-SD-WM-DP-199, REV. 1

WHC-SD-WM-DP-199, REV. 1

63 
츤

ㅇ․요

90

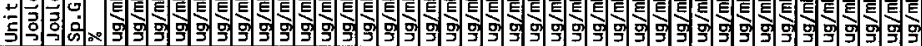

च

可 2

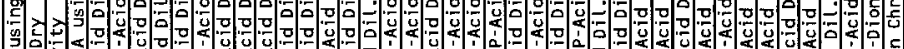
E

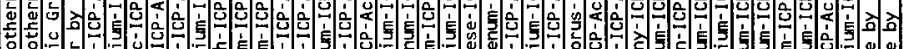

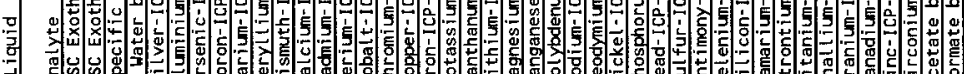

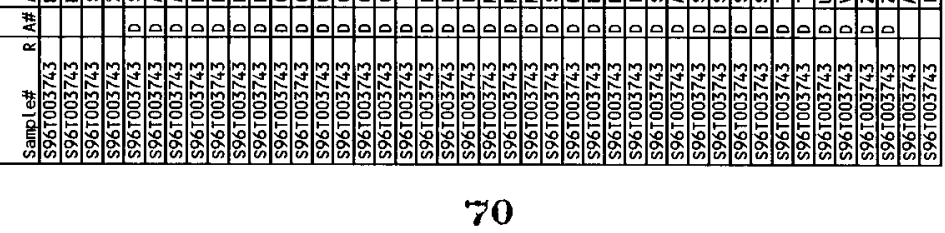




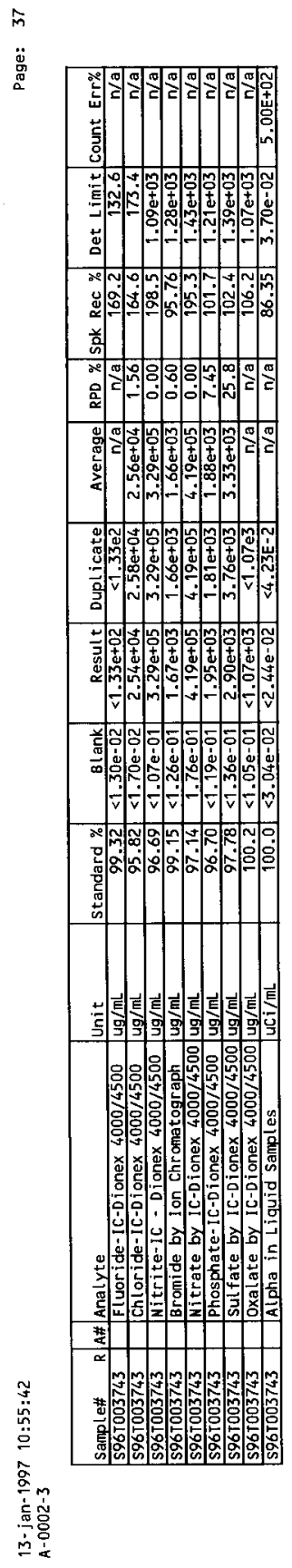

HNF-SD-WM-DP-199, REV. 1

WHC-SD-WM-DP-199, REV. 1 
HNF-SD-WM-DP-199, REV. 1

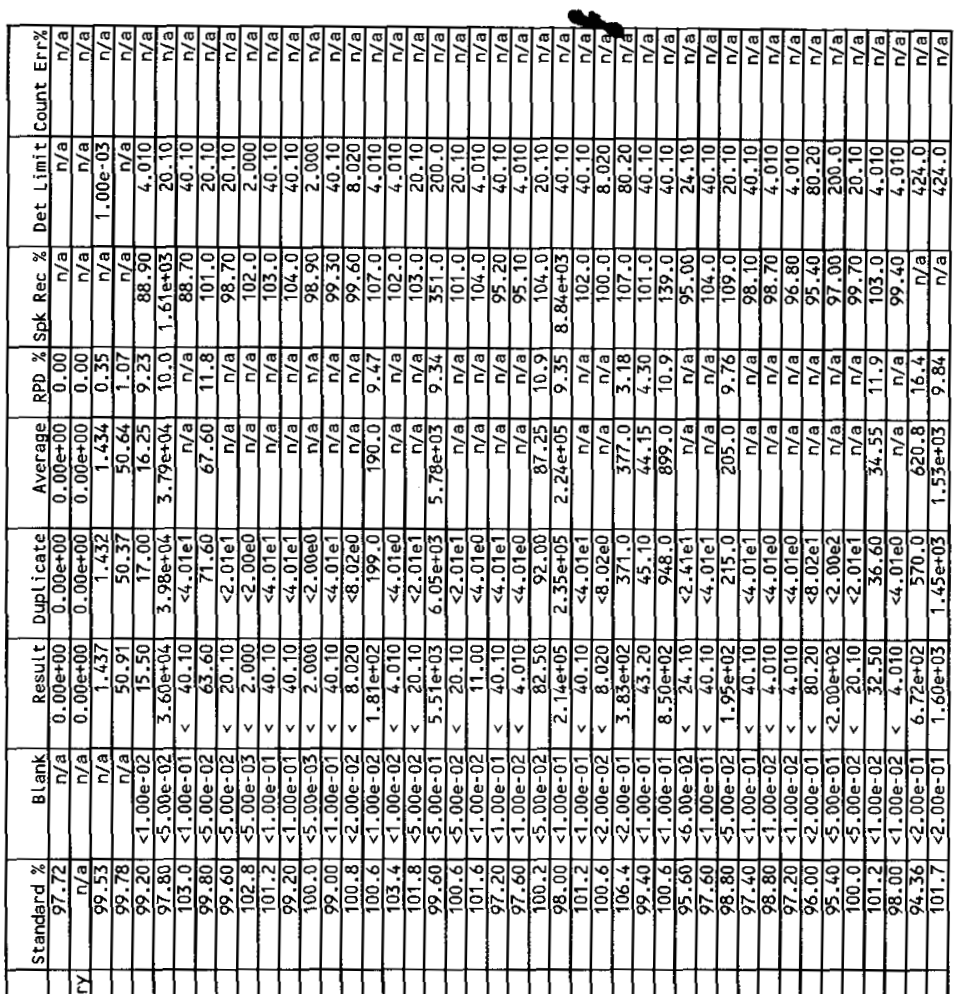

(a)

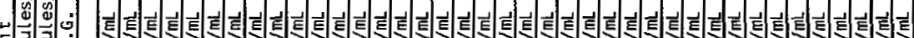

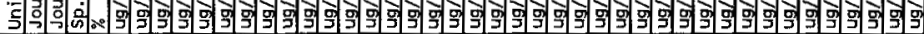

.

$\frac{3}{3}$

学 9

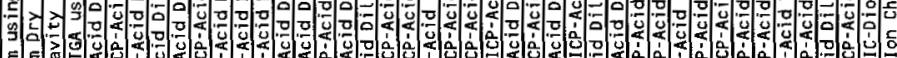
든 2.

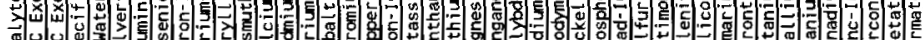

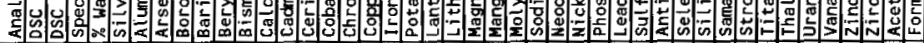

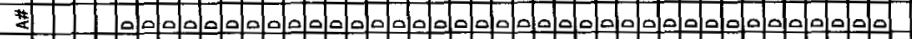


$\because v=-5,-v v-\ldots=, \cdots=v$.

$a$
$m$
0
0
0

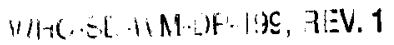

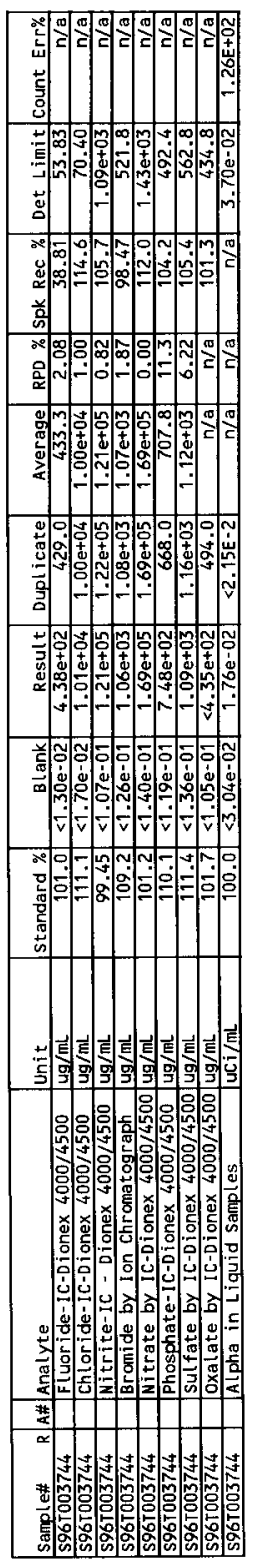

응

ํㅜㄹ 
.

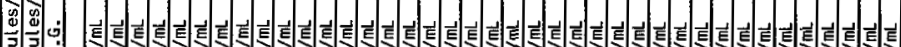

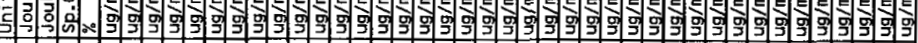

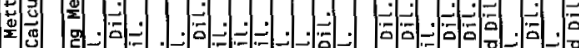
음

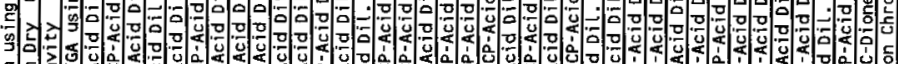
-

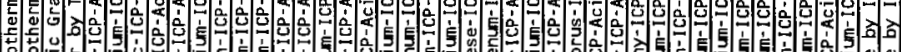

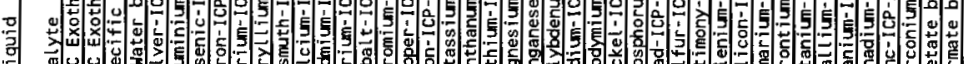

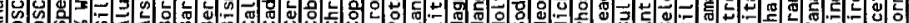
a 
WHC.SU-1/N IP 9 REV. 1
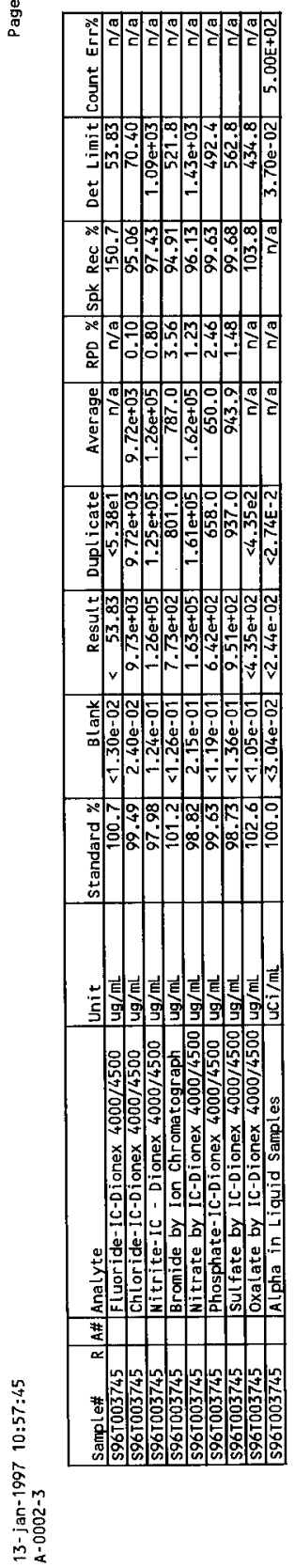


\section{HNF-SD-WM-DP-199, REV. 1}

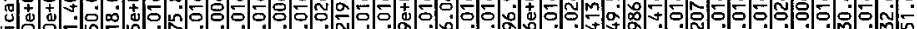

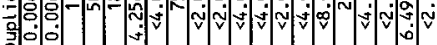

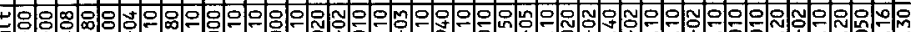

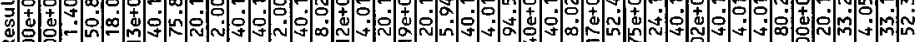

$\circ 0^{\circ}$

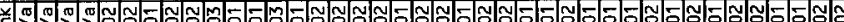

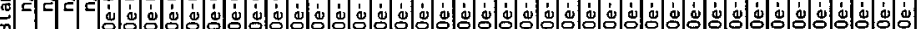

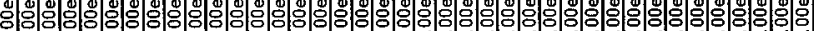

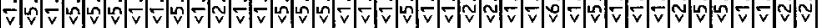

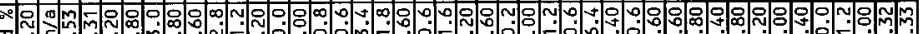
ta

홓

는

(1)

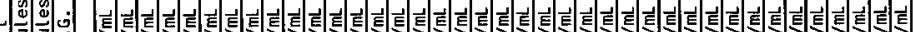

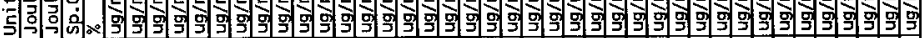

:

$\stackrel{\square}{a}$

形象

党

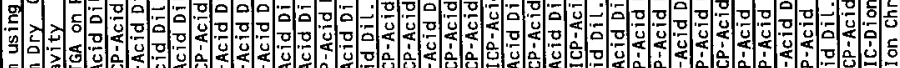

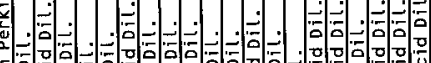

튼

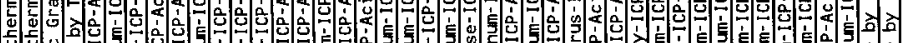

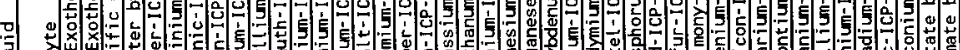

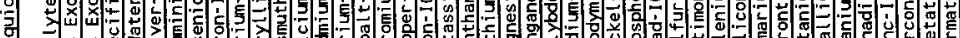

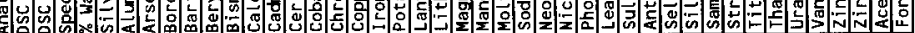

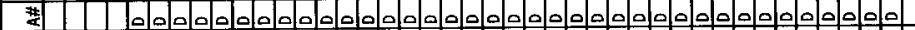

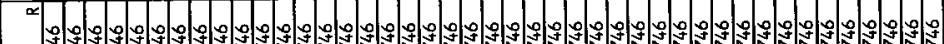

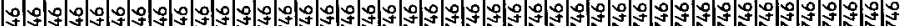

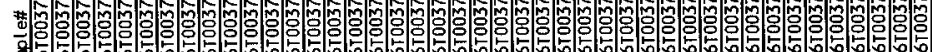




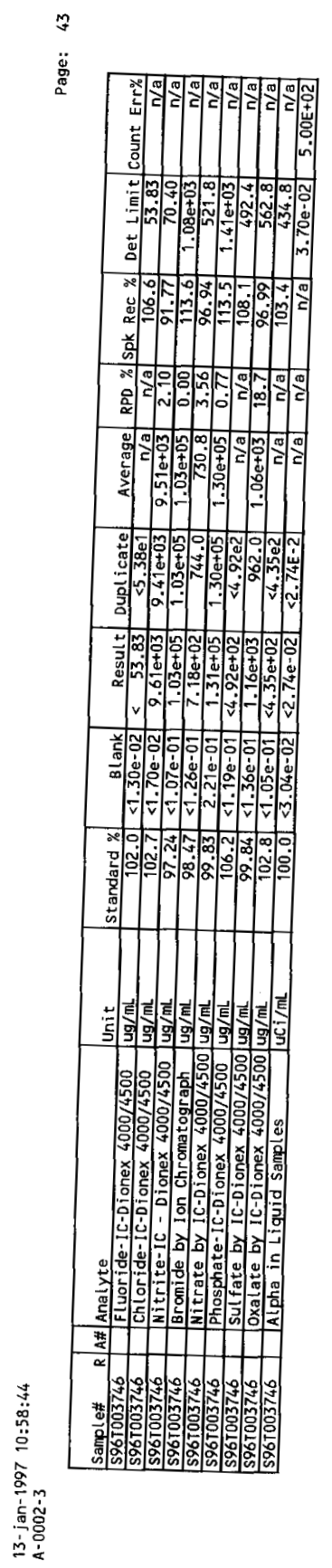

HNF-SD-WM-DP-199, REV. 1

WHC-SD-WM-DP-199, REV. 1 
HNF-SD-WM-DP-199, REV. 1

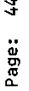

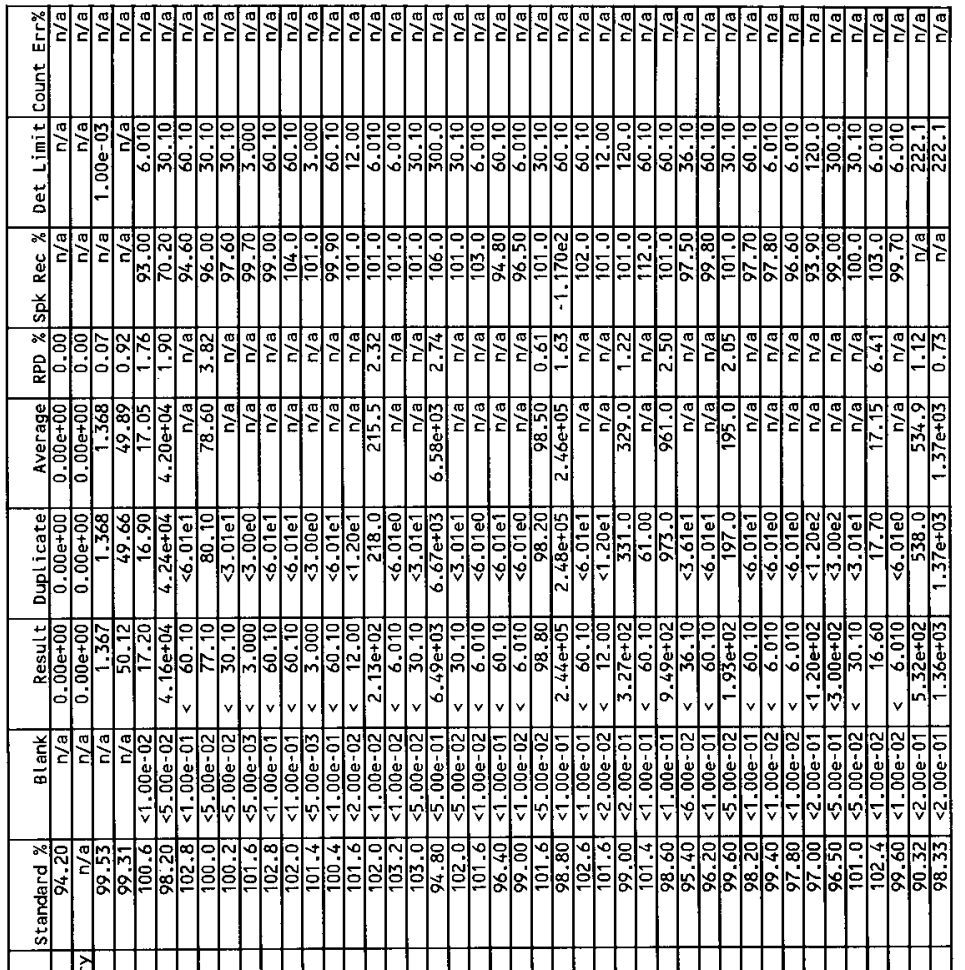

ㅇ․ 0

过

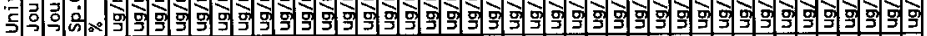

尊

㦴

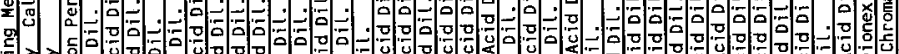
自 E

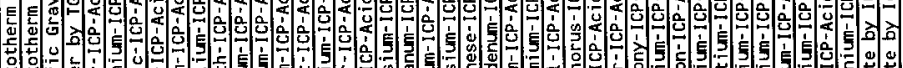

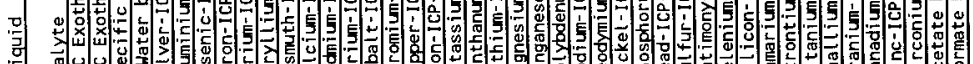

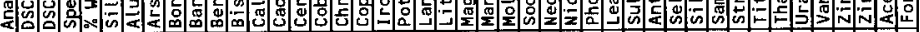

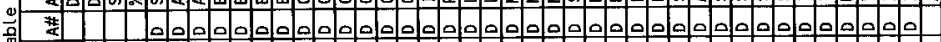
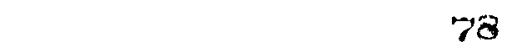
3

苛

HNF-SD-WM-DP-199, REV. 1

WHC-SD-WM-DP-199, REV. 1

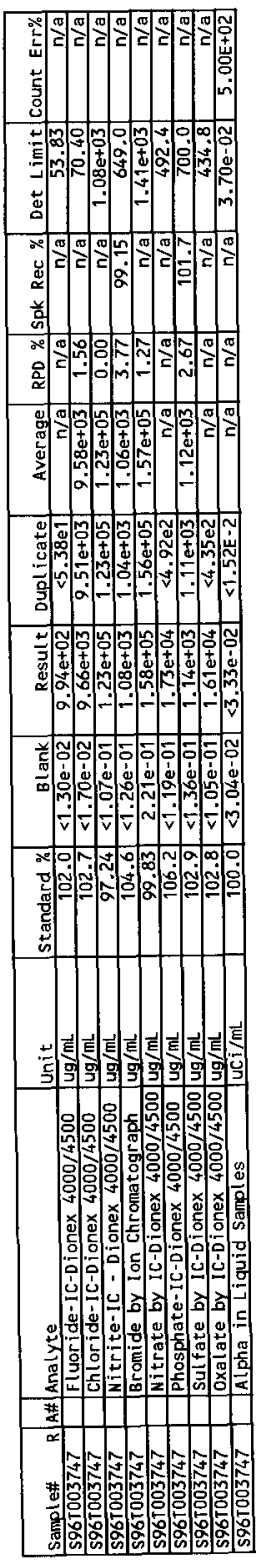

金

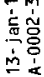




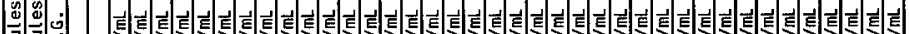

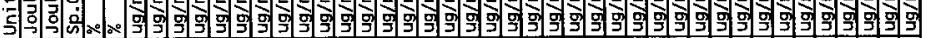

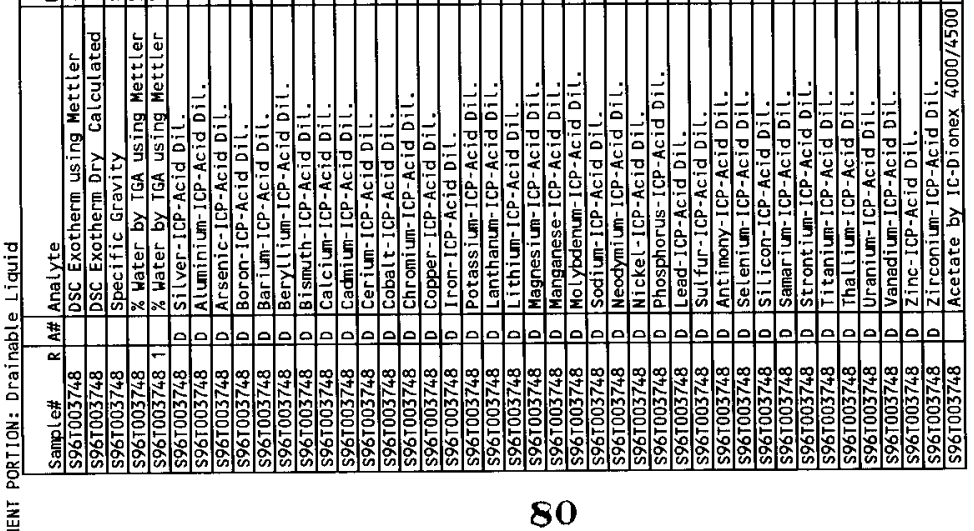




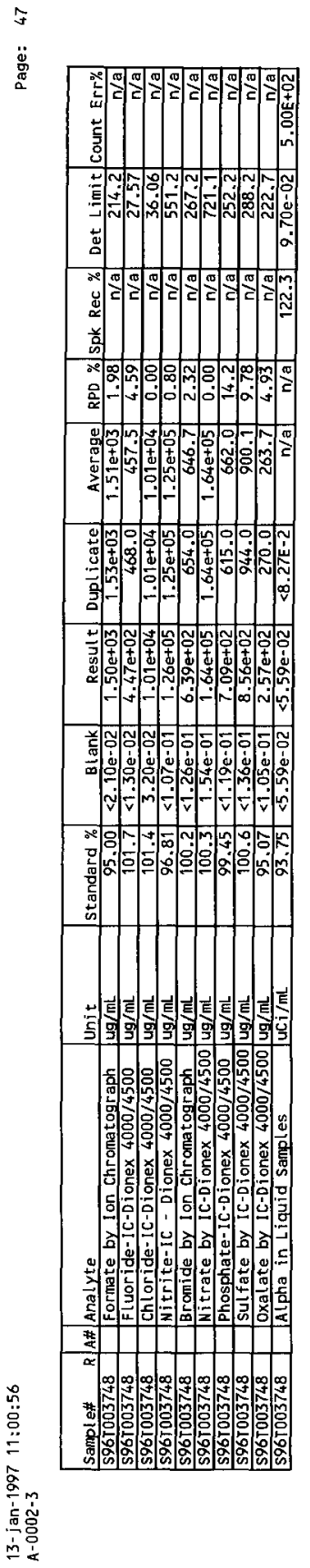

\section{HNF-SD-WM-DP-199, REV. 1 \\ WHC-SD-WM-DP-199, REV. 1}




\section{HNF-SD-WM-DP-199, REV. 1}

총

9.

.

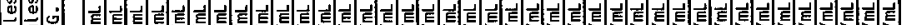

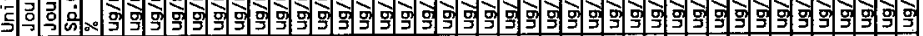

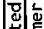

要

商

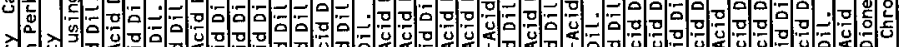

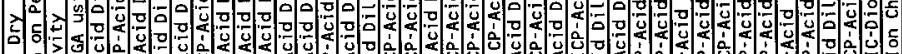

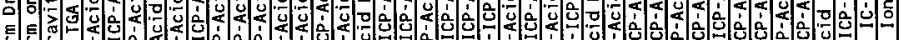

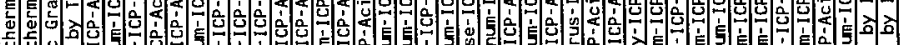

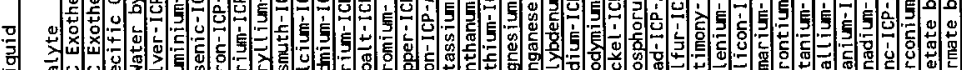

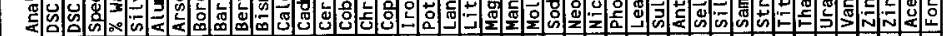

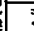

a a a a a a a a a a a a a a a a a a a a a a a

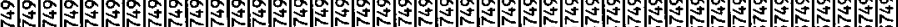


-TNF-SD-WV- $-99,7 E V$.

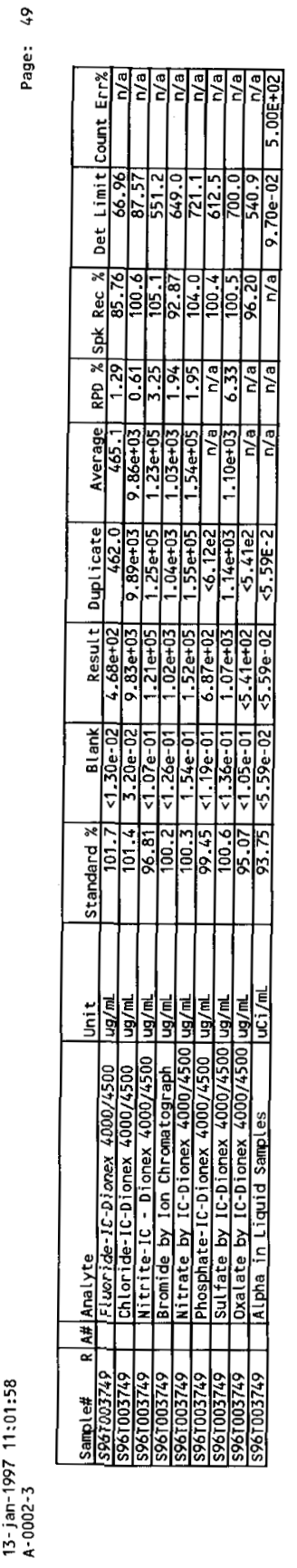


HNF-SD-WM-DP-199, REV. 1

$\stackrel{\circ}{\ddot{g}}$

WHC-SD-WM-DP-199, REV. 1

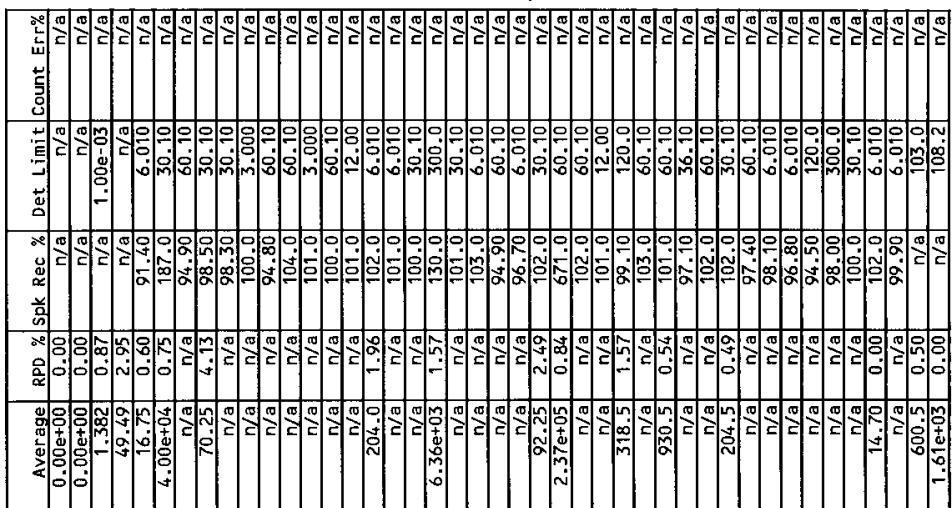

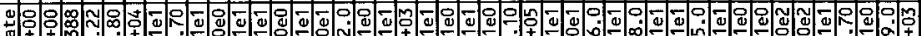
苟高?

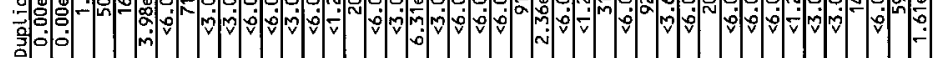

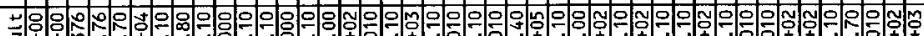

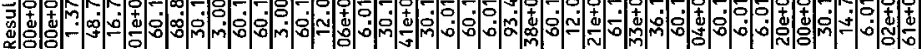

落

$\therefore \circ$

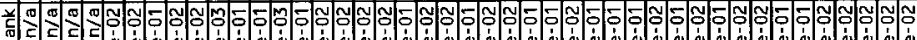

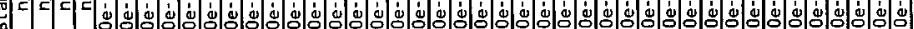

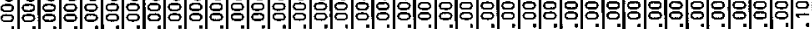

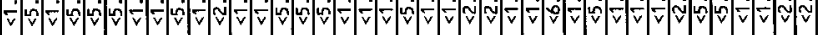

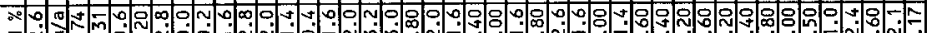

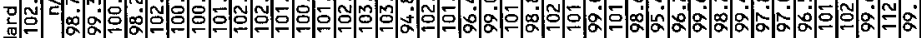
空 11

잉

送送

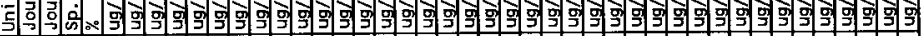
| 敬

@)

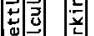

$\frac{m}{2}$

:

党

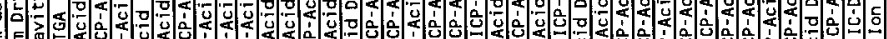

은

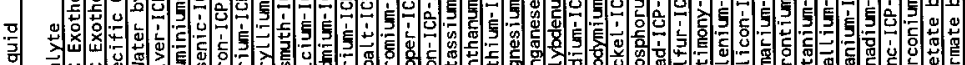

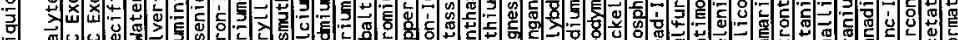

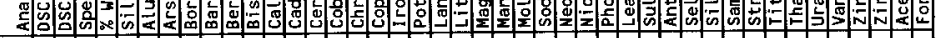

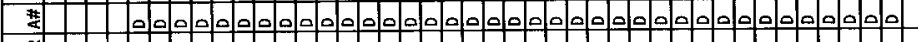

8

茴

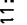

总

产

mín

票

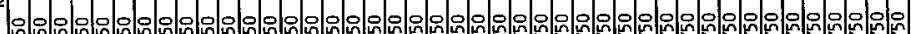

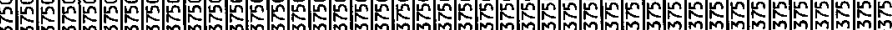

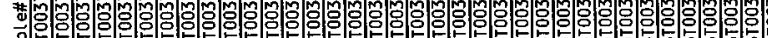

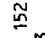




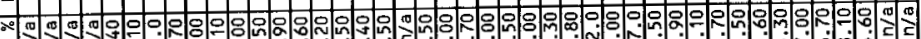
$y=2=20$ a

总 作

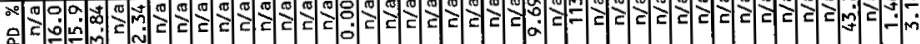

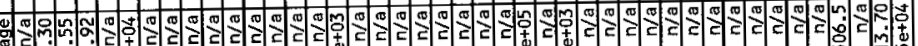

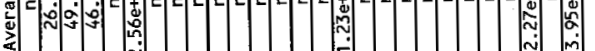

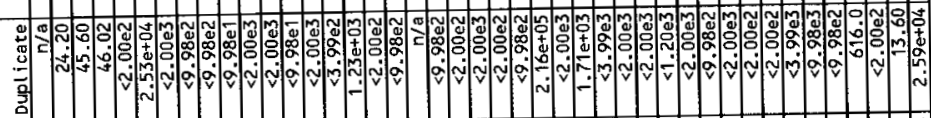

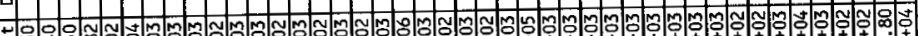

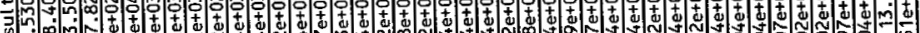

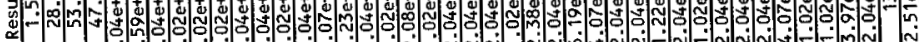

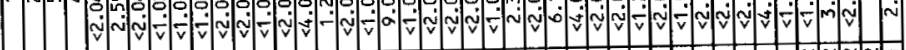

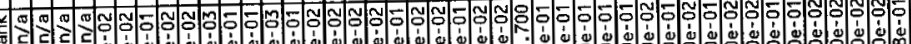

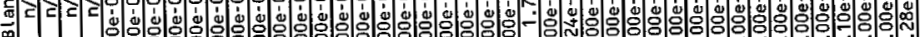
p c $\frac{\mathrm{n}}{\mathrm{c}}$ : $-1$

(a)

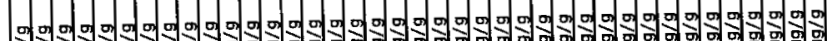

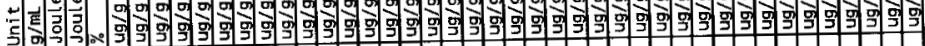




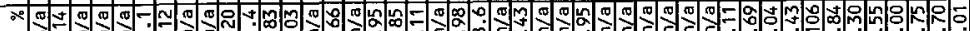

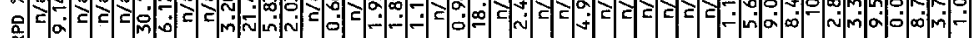

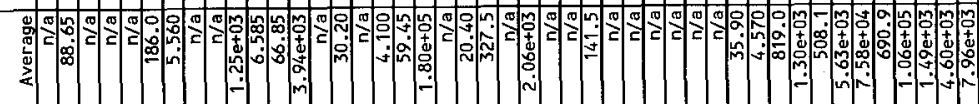

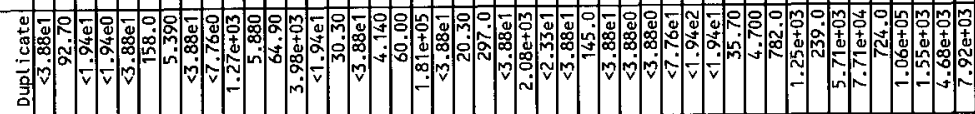

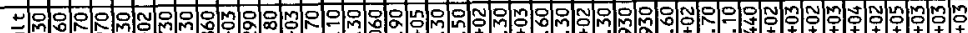
与.?

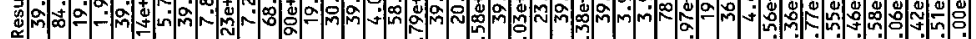

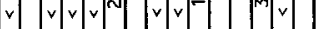

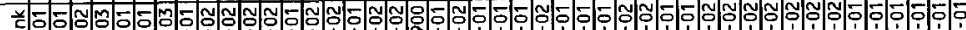

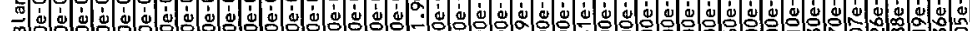
๓

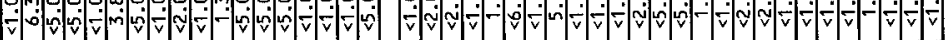
¿ co ํํำ

11

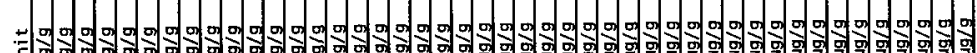

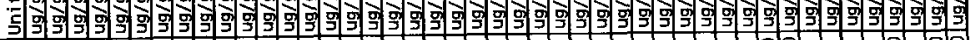

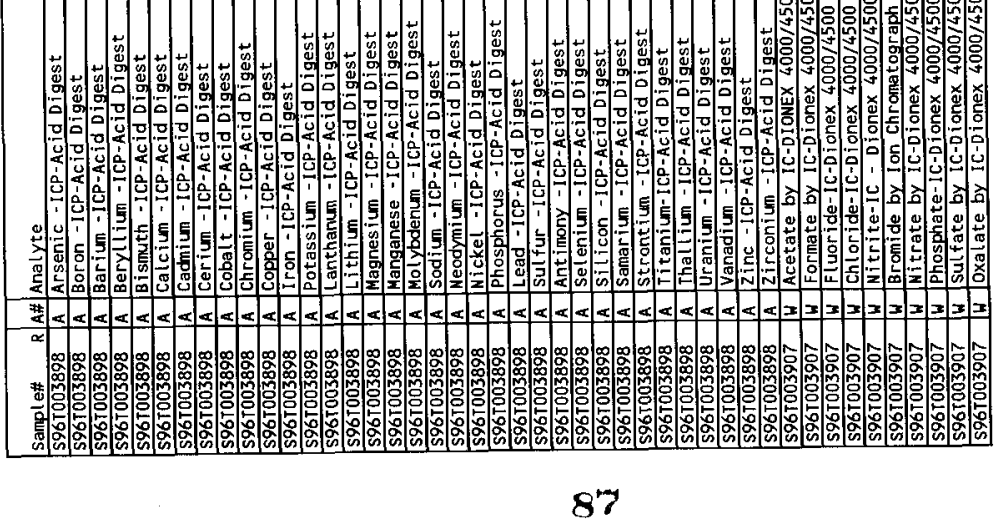




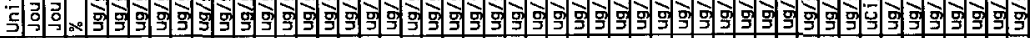

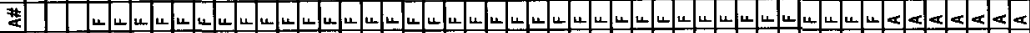




\section{HNF-SD-WM-DP-199, REV. 1}

แn

菏

WHC-SD-WM-DP-199, REV. 1

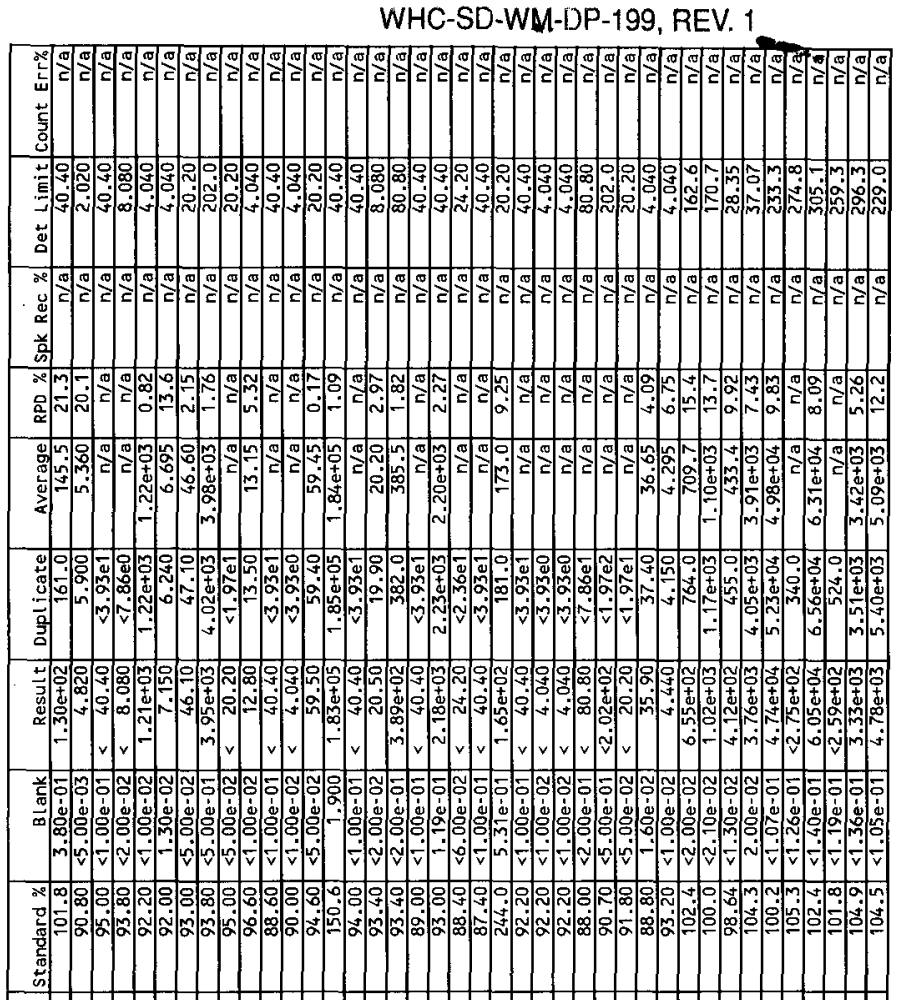

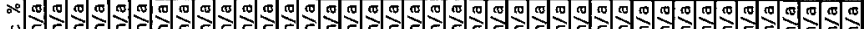

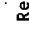

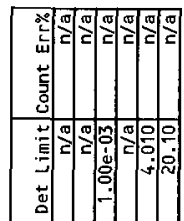

र०त बता तण

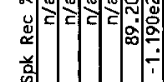

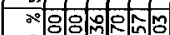

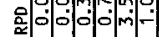

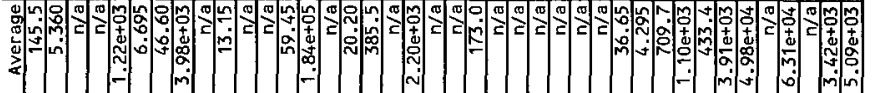

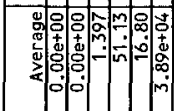

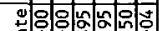

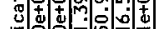

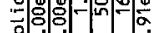

言.

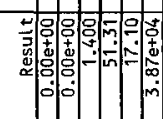

*ब可可

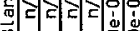

$\vec{\infty}$

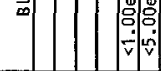

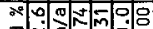

뎡의 뎡

용

虫

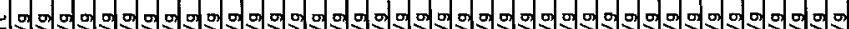

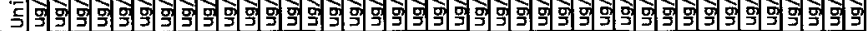

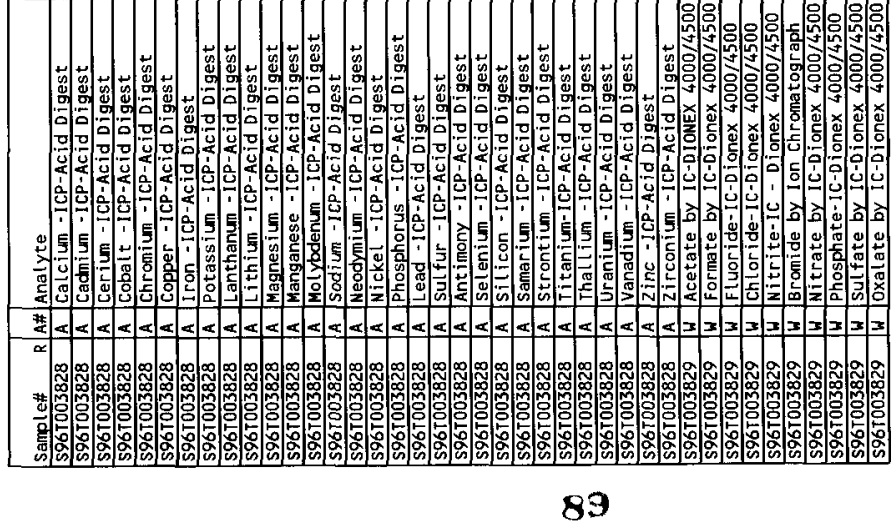

萢

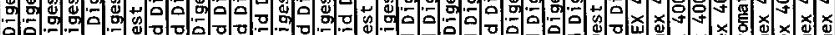

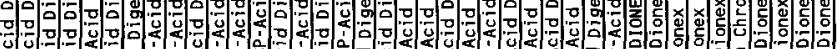

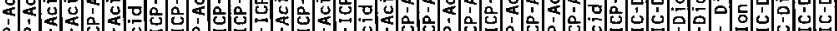

는

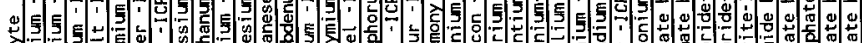

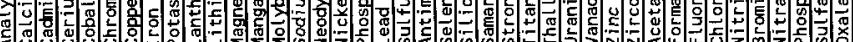

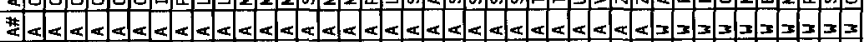




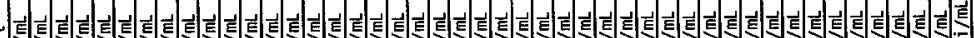

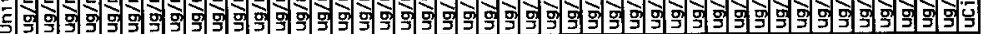

\begin{tabular}{|l}
\hline \\
\hline
\end{tabular}

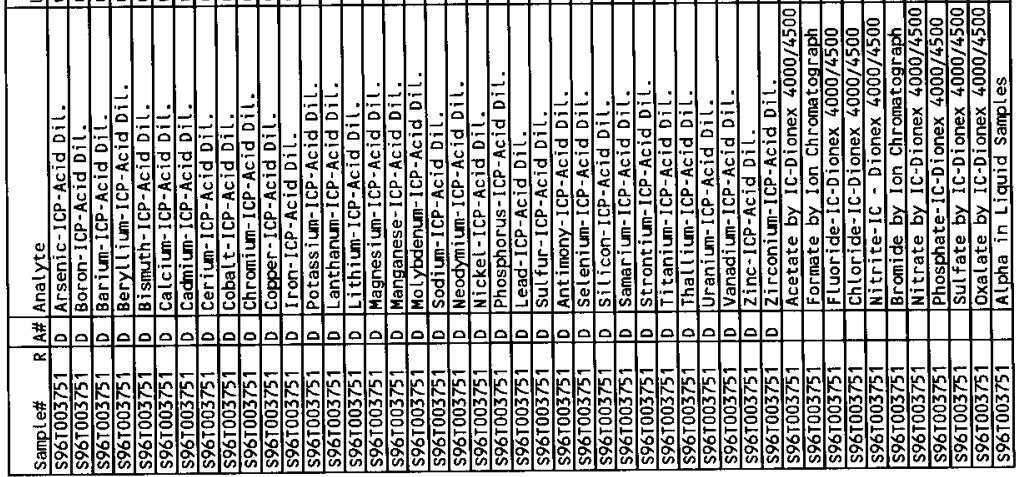
a 
HNF-SD-WM-DP-199, REV. 1

WHC-SD-WM-DP-199, REV. 1

$\underset{\ddot{g}}{\ddot{g}}$

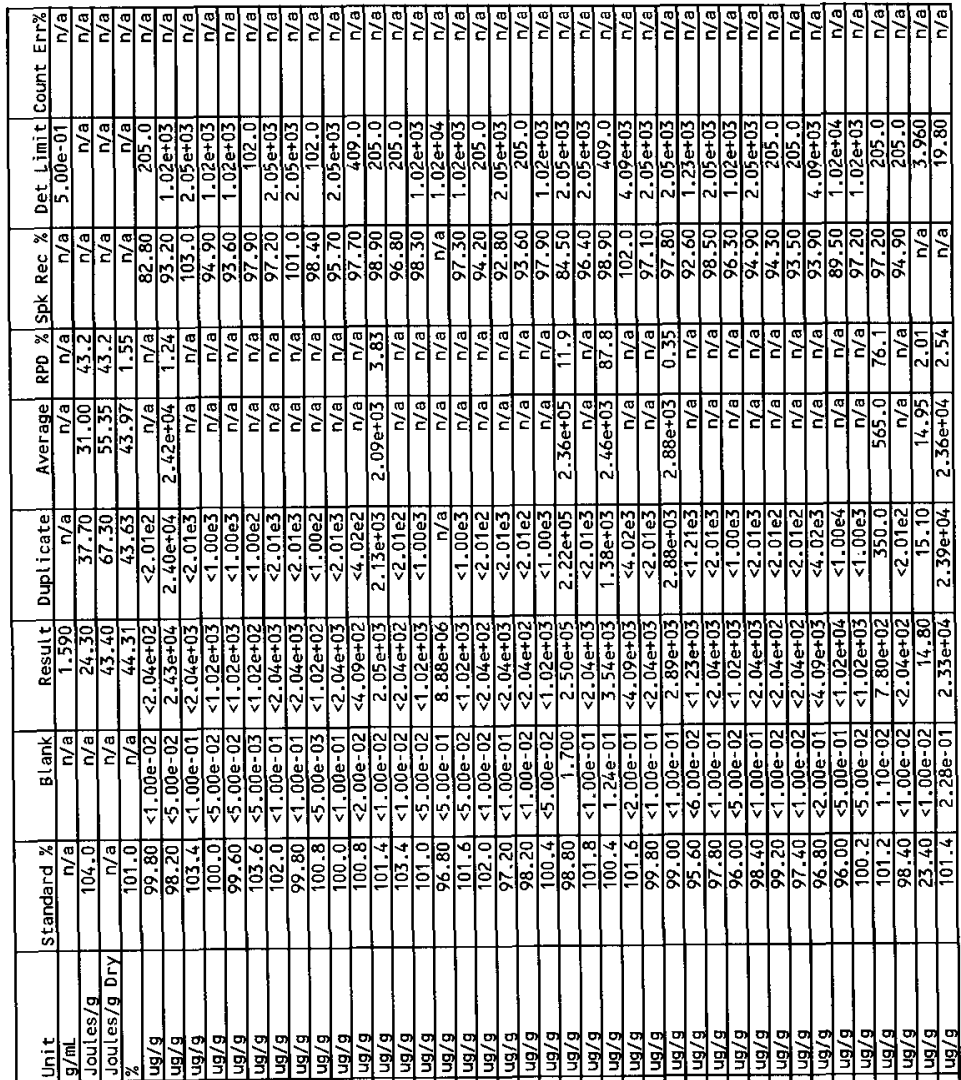

包

$=0$

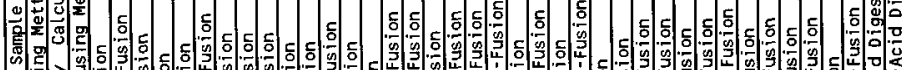
总

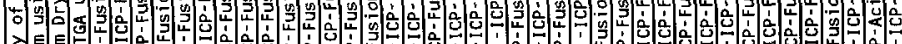

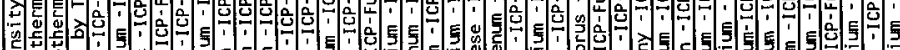

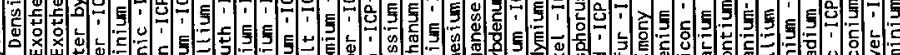

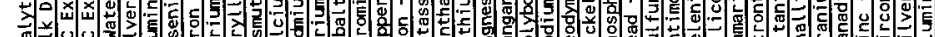

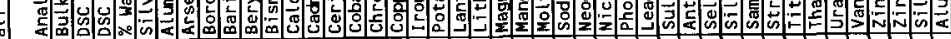

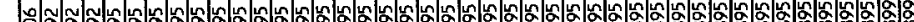
* 
HNF-SD-WM-DP-199, REV. 1

WHC-SD-WM-DP-199, REV. 1

$\underset{\ddot{d}}{\ddot{m}}$

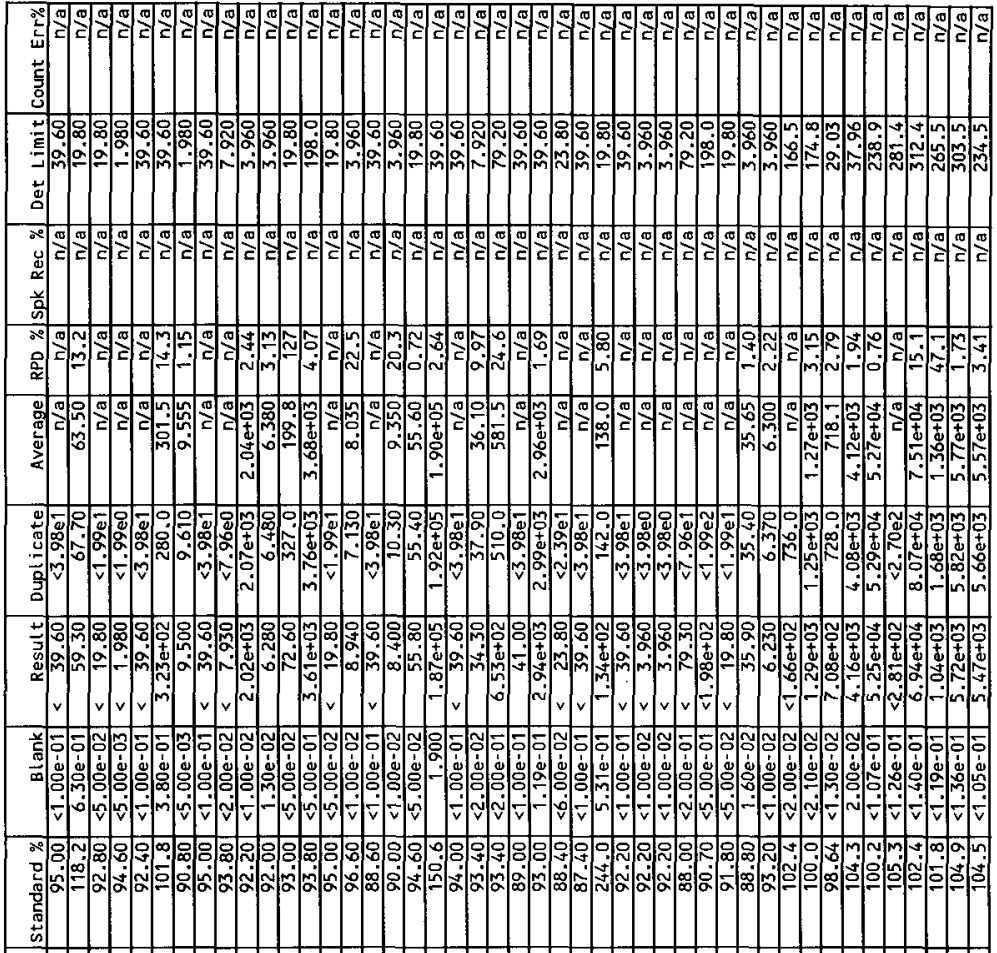

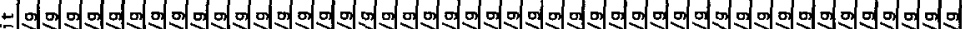

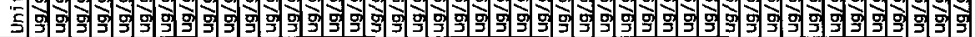

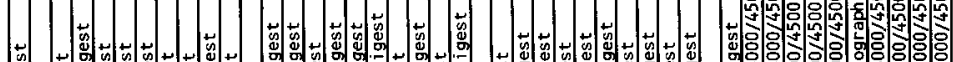

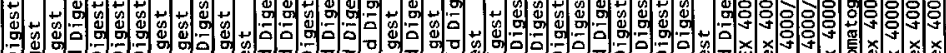
品

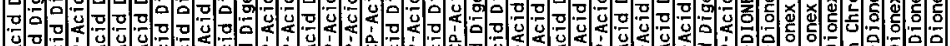

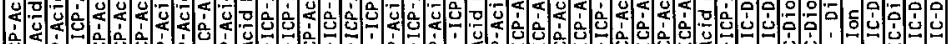

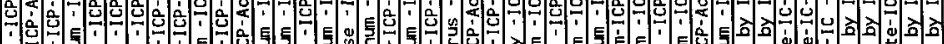

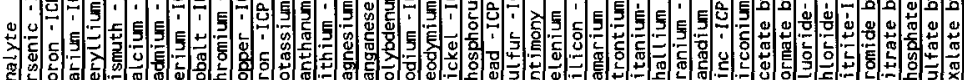

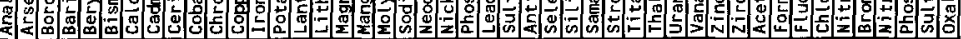

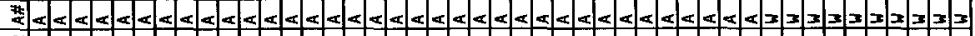




\section{HNF-SD-WM-DP-199, REV. 1}

츤

의 의

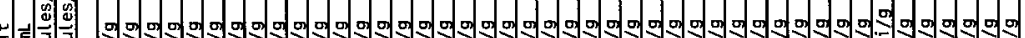

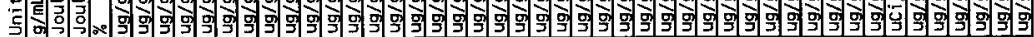
(a) 过

$\frac{1}{2}$

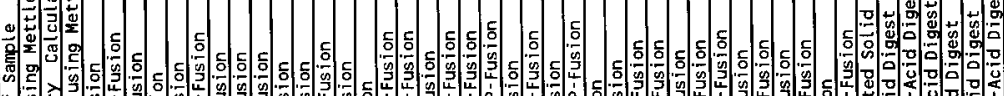
象

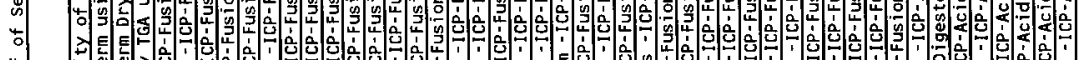

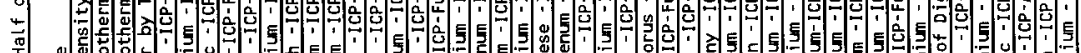
夏

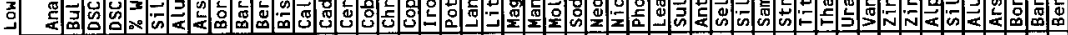


HNF-SD-VV - J-99, $P=V$.

WHC-SD-MMH.S-1S9, FEY 1

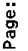

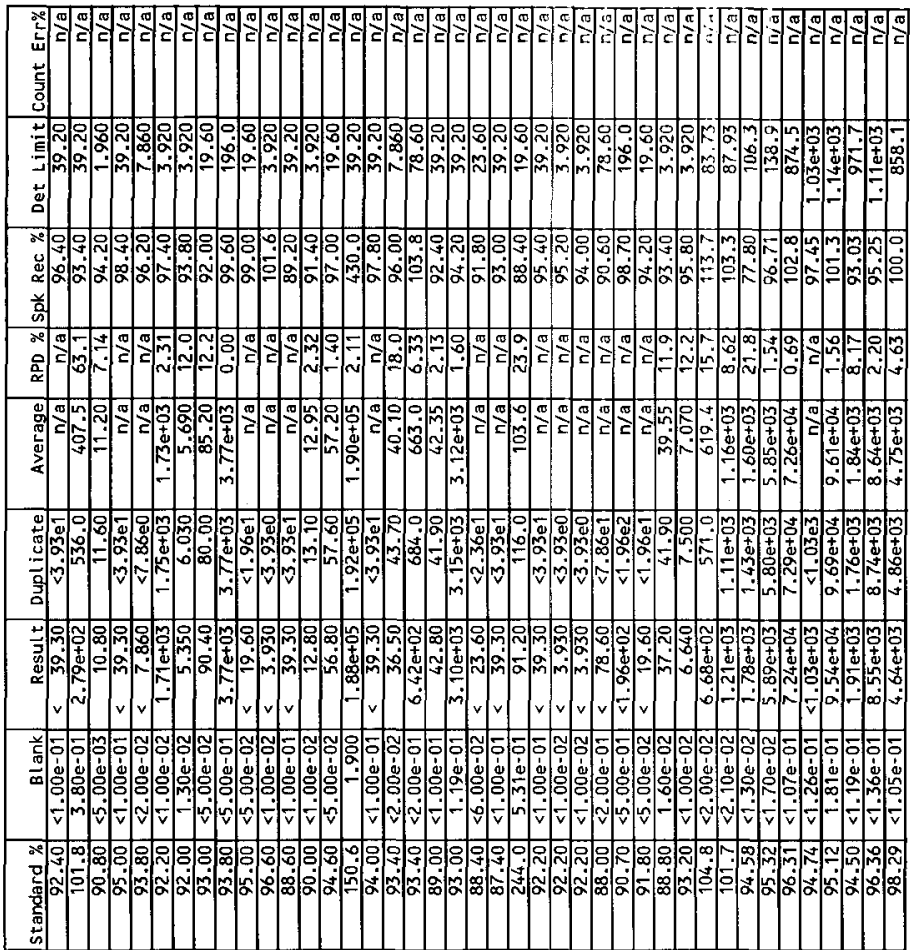

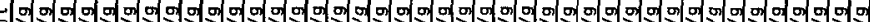

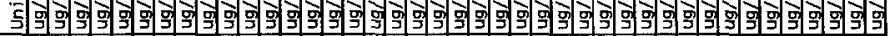

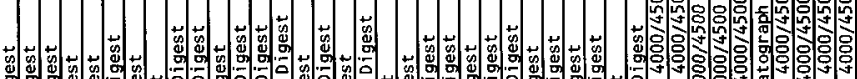

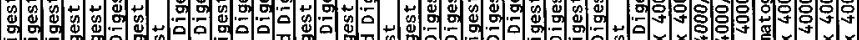
等

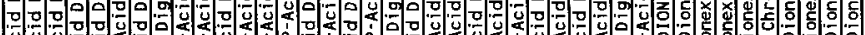

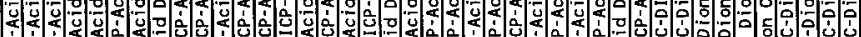
id

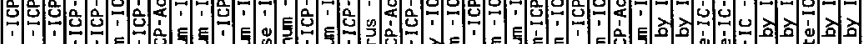

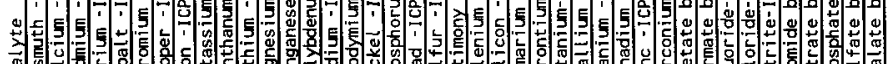

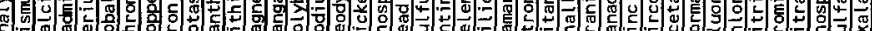

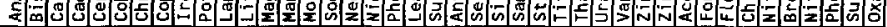

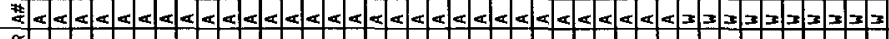
m 


\section{HNF-SD-WM-DP-199, REV. 1}

WHC-SD-WM-DP-199, REV. 1

$\underset{\ddot{a}}{\ddot{g}}$

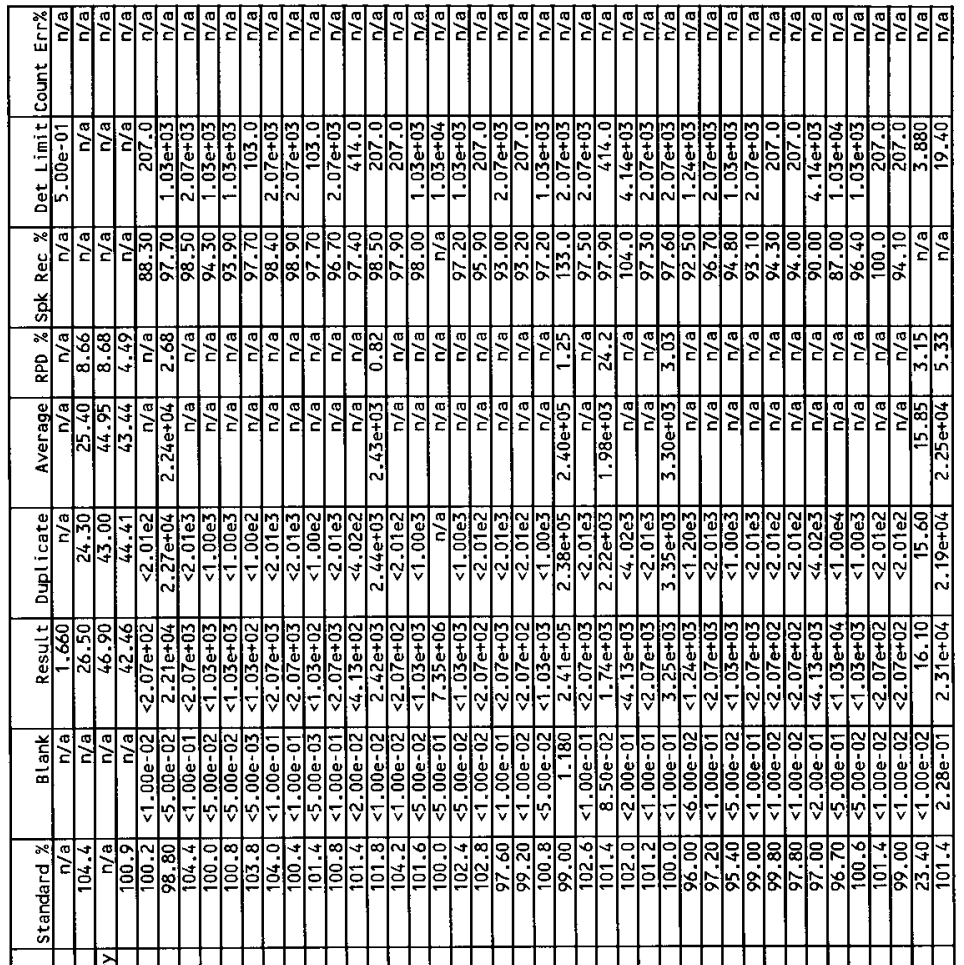

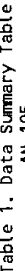

(2)

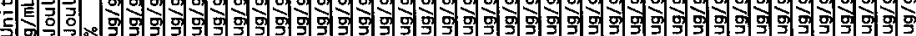

证育

.

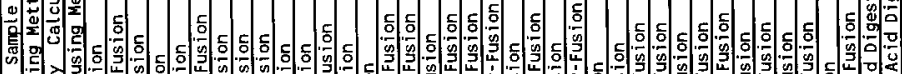

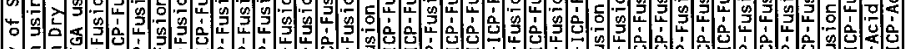

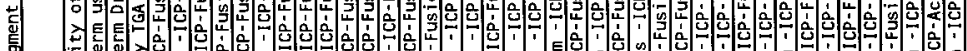

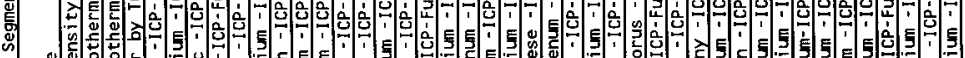

to

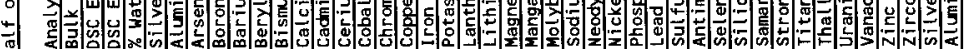

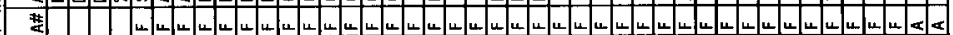

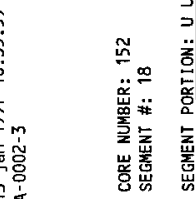

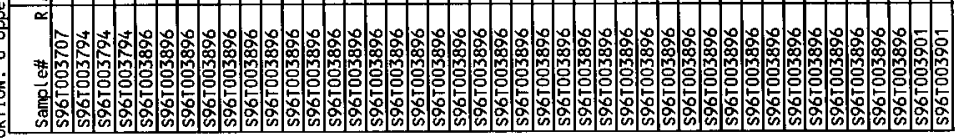




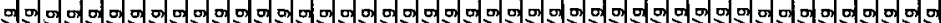

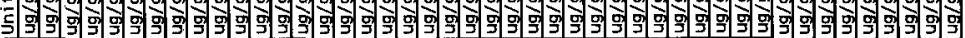

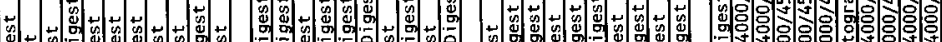
\% 0 a 뭉

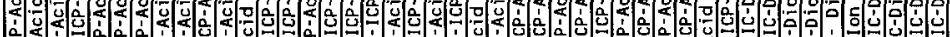
영

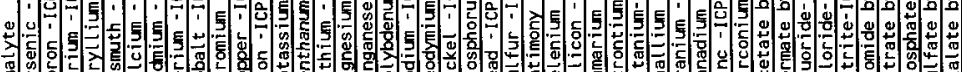

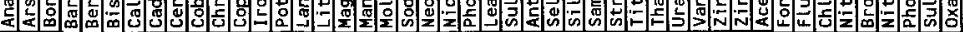

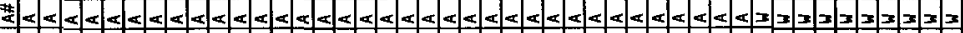




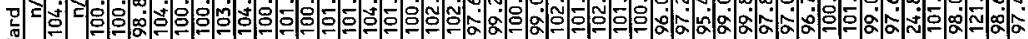
몸

is

웅

1 이의.

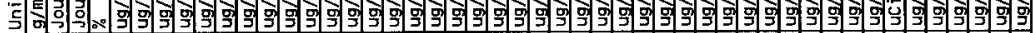

迹

4.

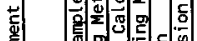

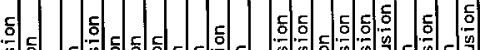

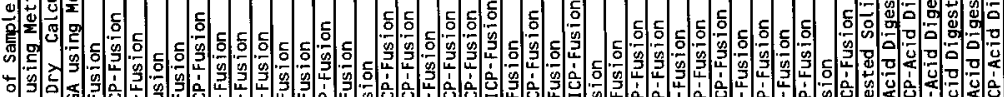

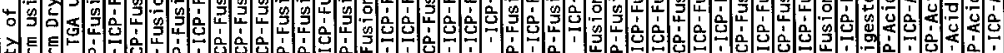

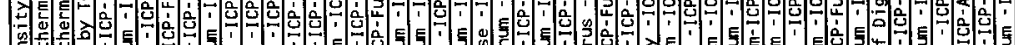

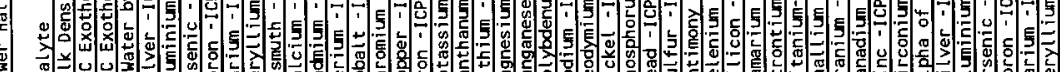

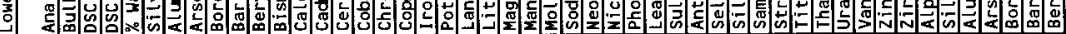

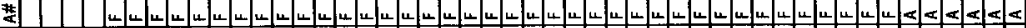

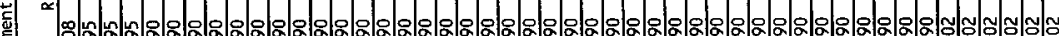

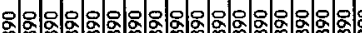




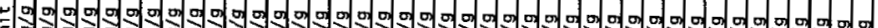

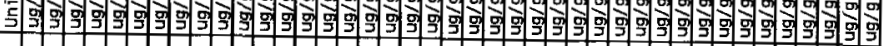

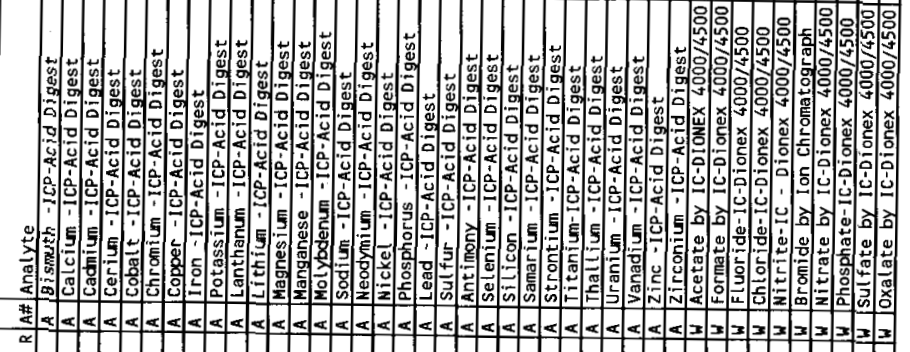




\section{HNF-SD-WM-DP-199, REV. 1}

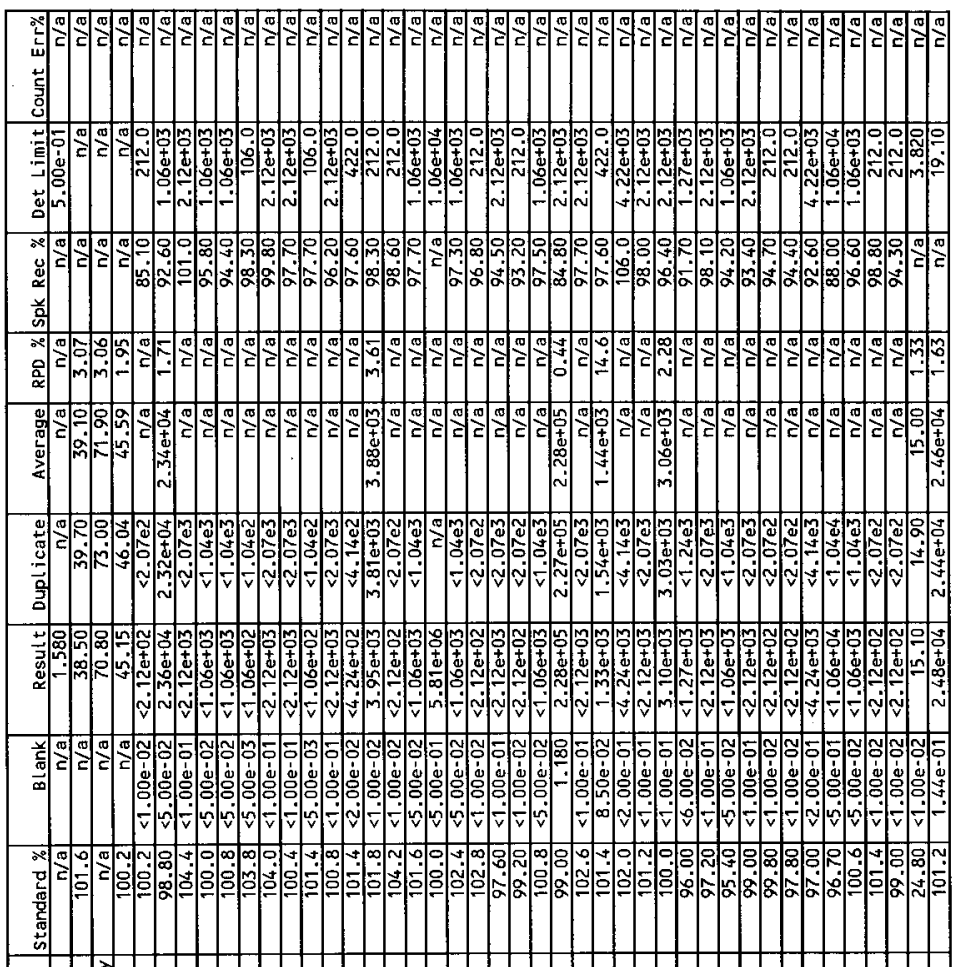

o. 0

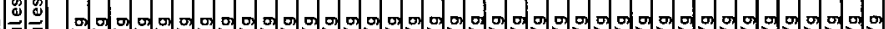

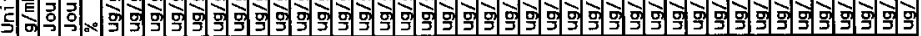

(5)

(.)

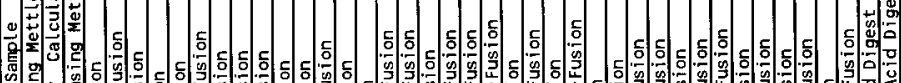

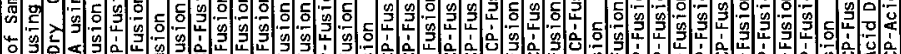
等

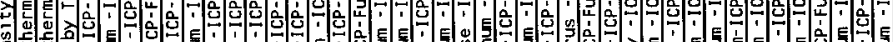

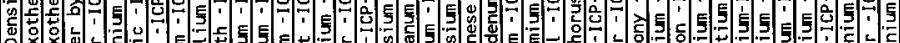
5

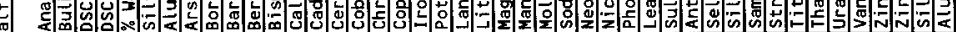

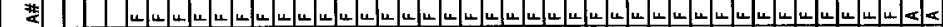

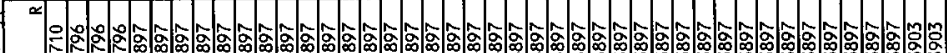
*

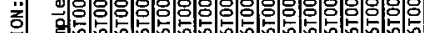

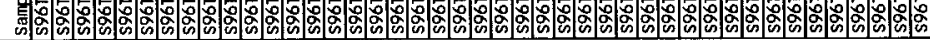




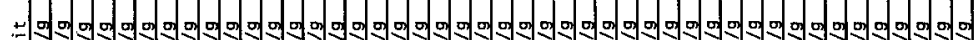

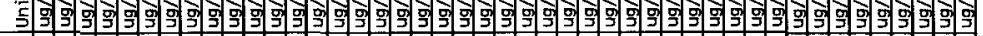

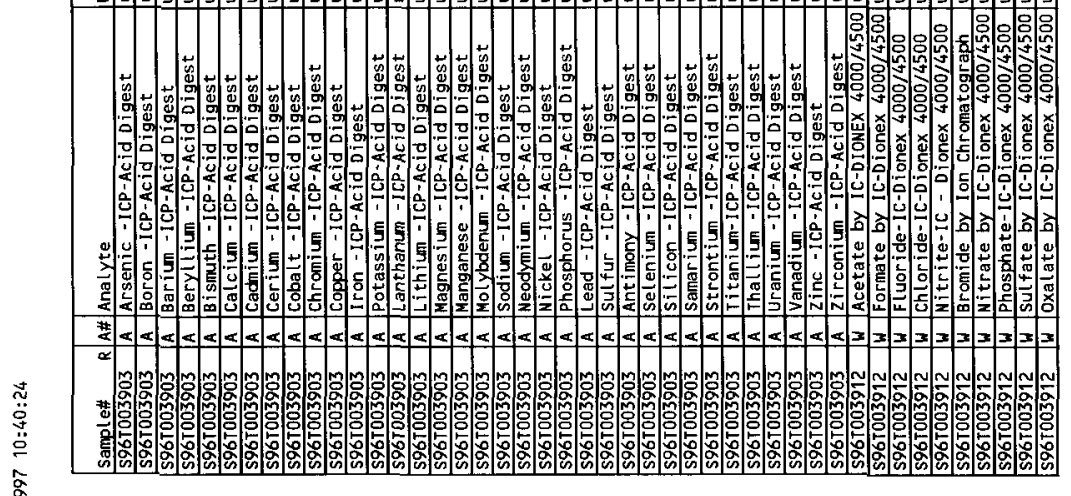
\& 
HNF-SD-WM-DP-499, PEV.

의 0

ติ

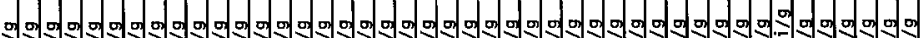

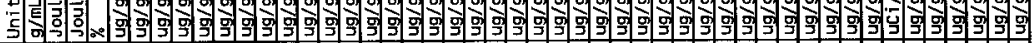

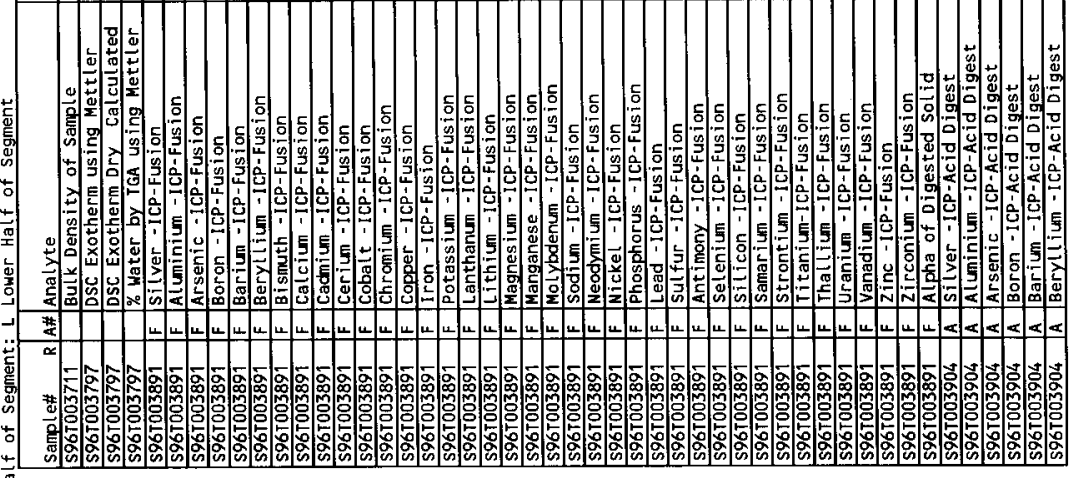

\&

完容 
HNF-SD-WM-DP-199, REV. 1

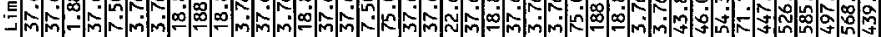
$\stackrel{\mathrm{g}}{\circ}$

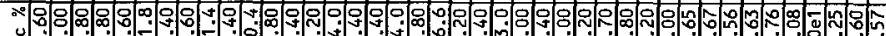

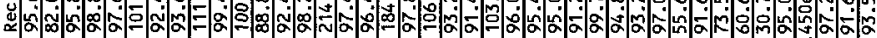
美 党

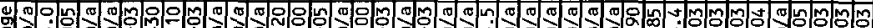

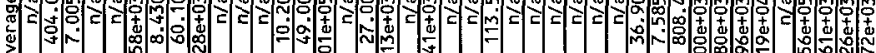

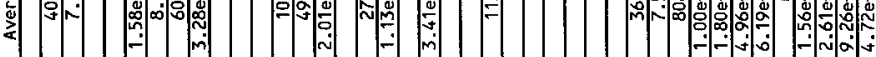

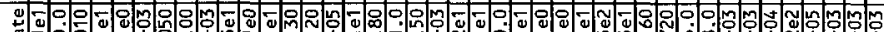

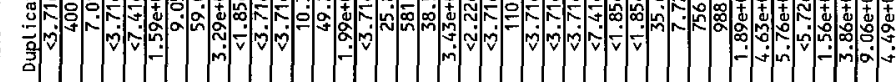

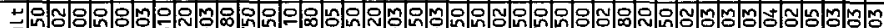

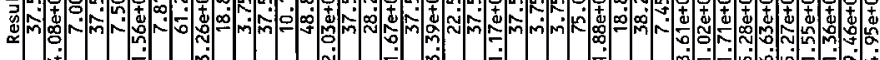

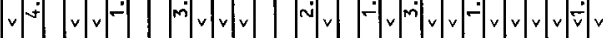

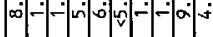

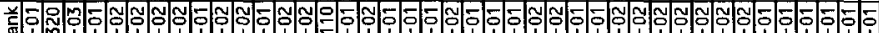

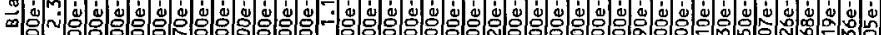

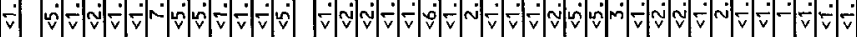

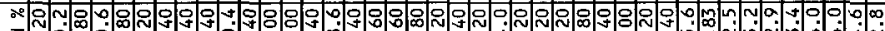

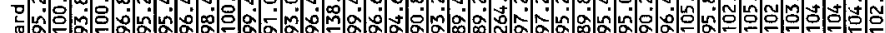
窎

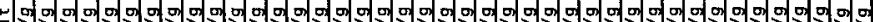

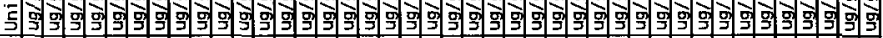

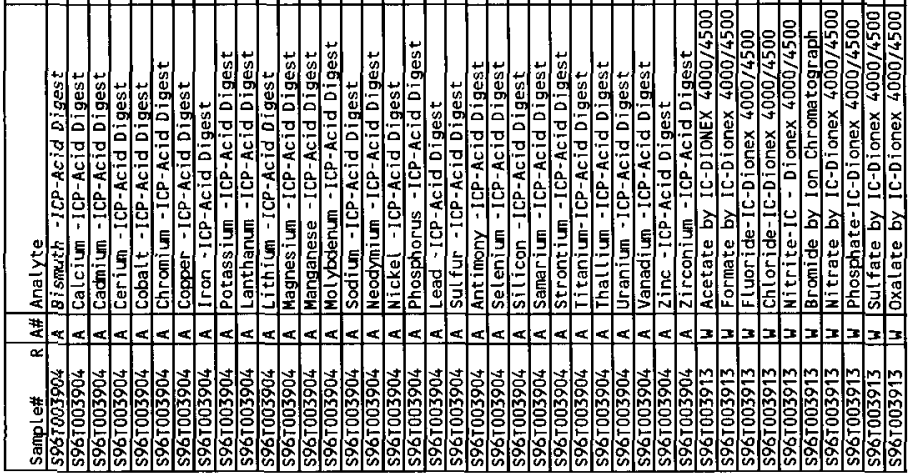

잉

동

웅 
ㅇ. ㅇ.?

웡

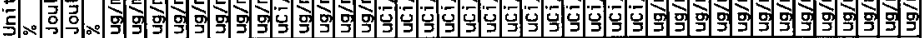
(

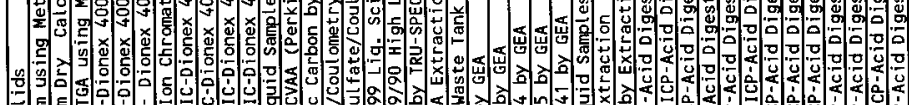

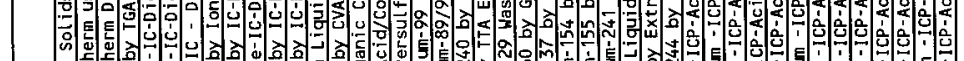

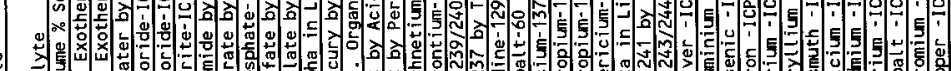

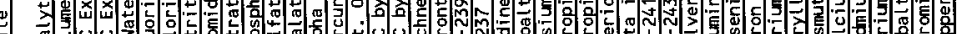

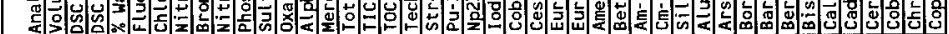
\#

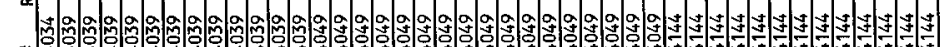

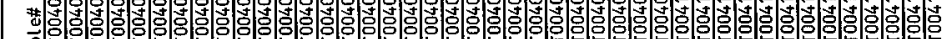

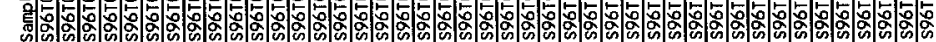
8 
HNF-SD-WM-DP-199, REV. 1

iv

$\underset{\mathrm{g}}{\mathrm{g}}$ WHC-SD-WM-DP-199, REV. 1

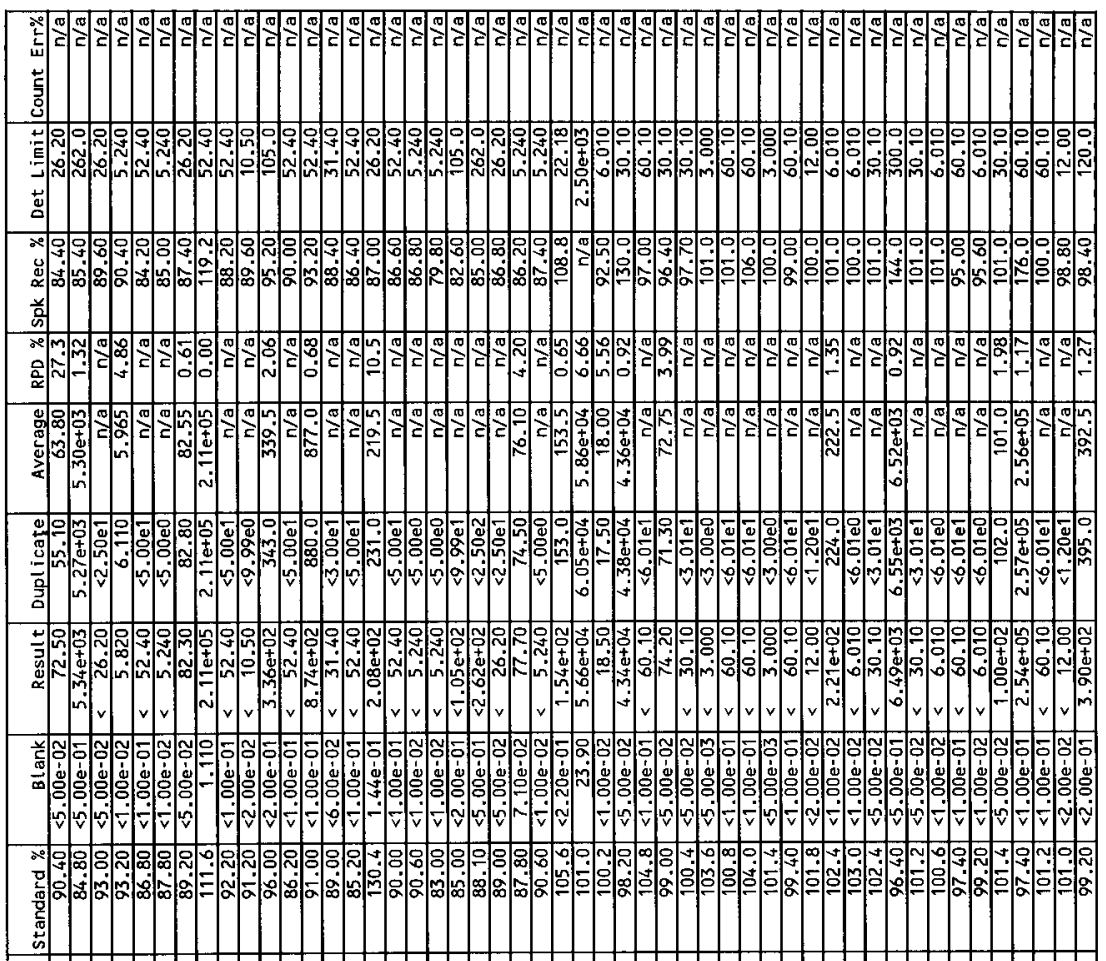

늘

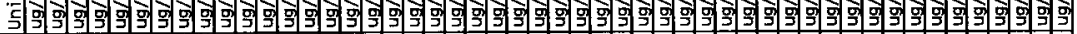

०.

갈

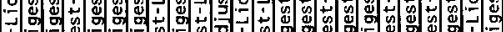

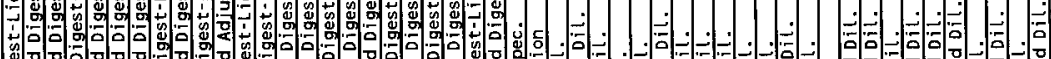

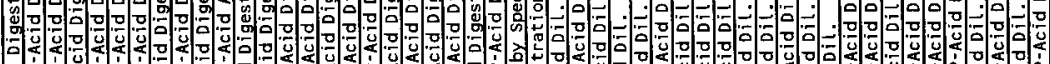

- 0

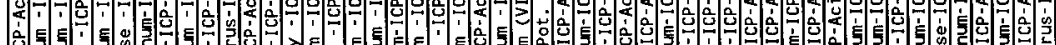

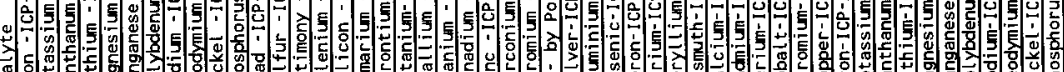

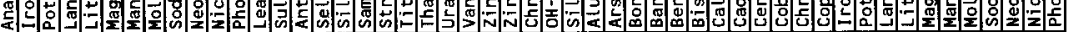
Z

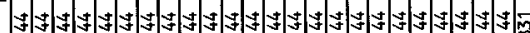




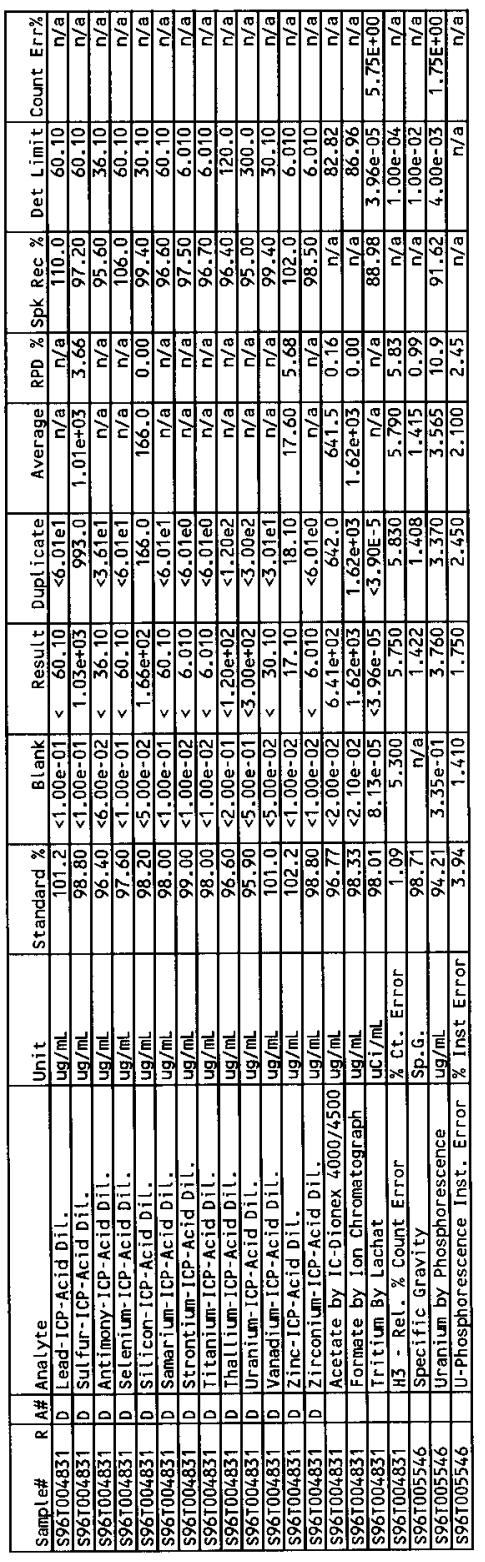

\%m

它望 
HNF-SD-WM-DP-199, REV. 1

WHC-SD-WM-DP-199, REV.

$\underset{\ddot{Z}}{\mathscr{Z}}$

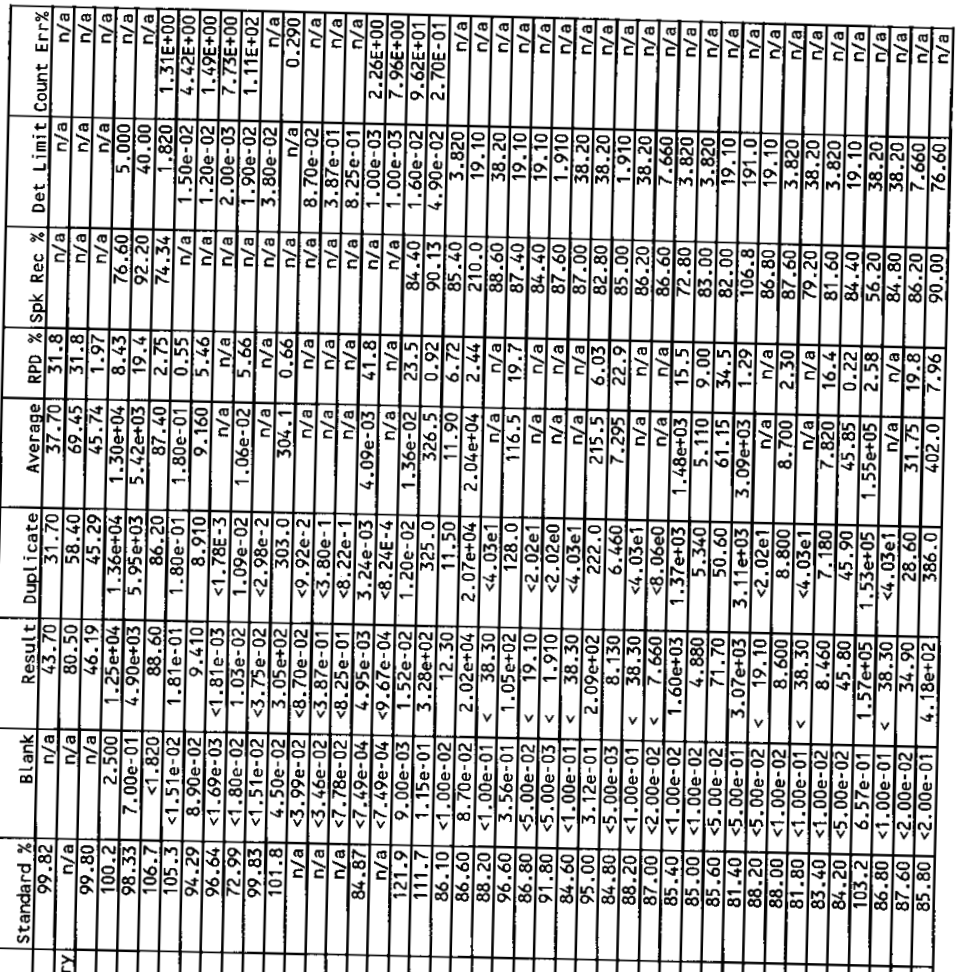

요

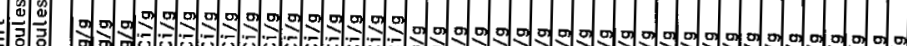

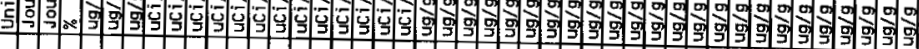

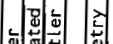

7 힌

这事

身

5 010

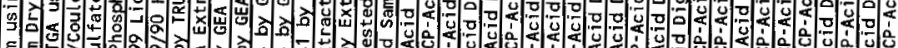

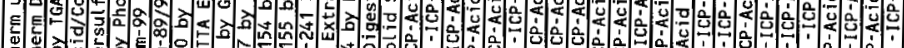

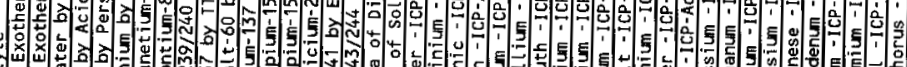

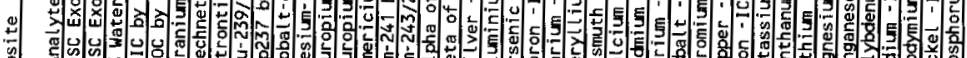

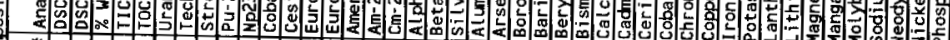

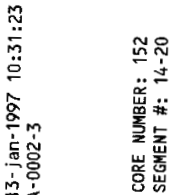

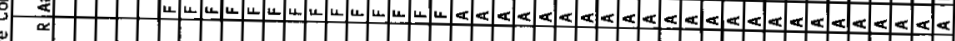

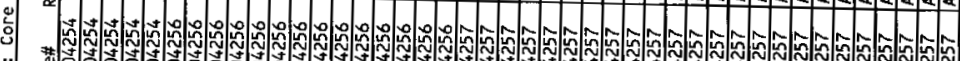

40

氙这迢

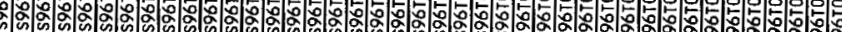

西




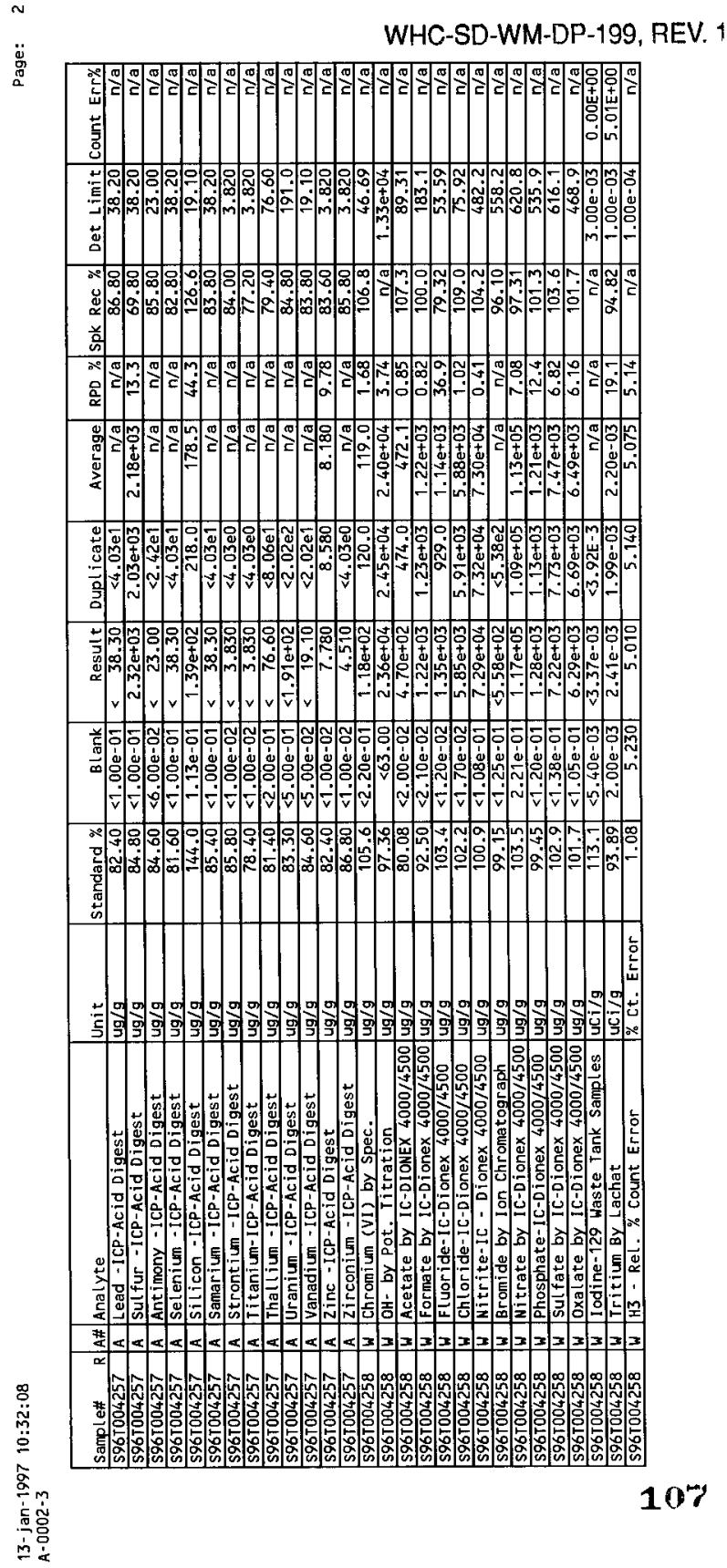


N
$\ddot{0}$
$\vdots$
0

\section{HNF-SD-WM-DP-199, REV. 1}

WHC-SD-WM-DP-199, REV. 1

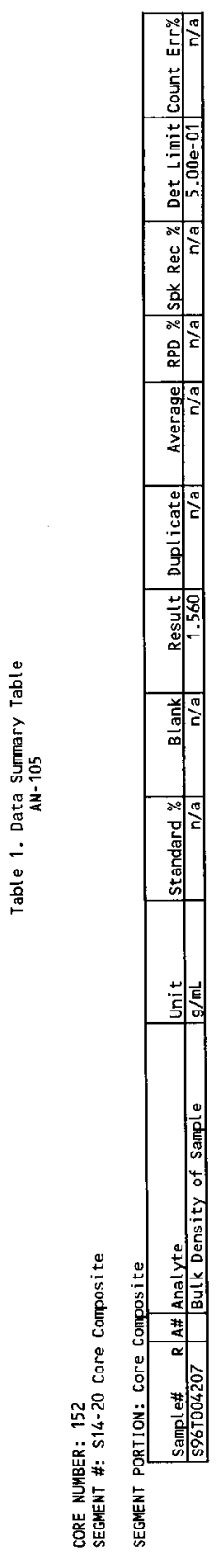




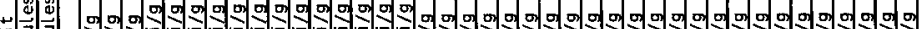

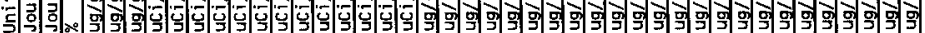

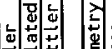

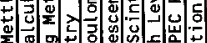

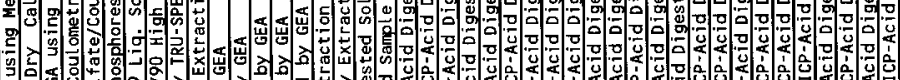

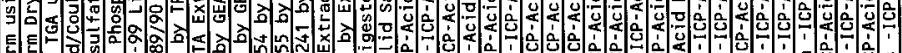

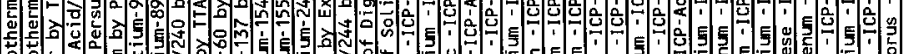

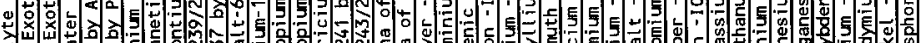
婪

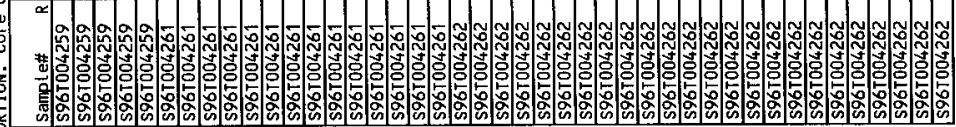




\section{HNF-SD-WM-DP-199, REV. 1}

WHC-SD-WM-DP-199, REV. 1

$\underset{j}{g}$

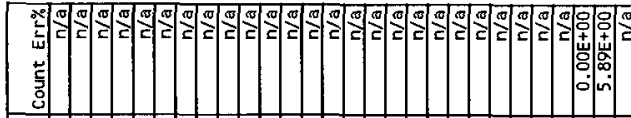

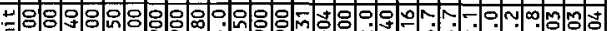

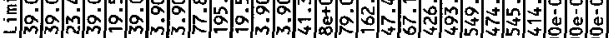
a

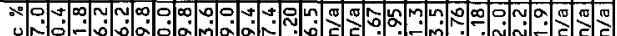
un $=0$

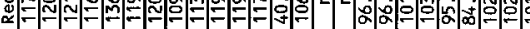
히

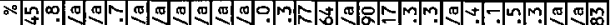

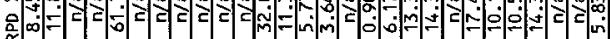

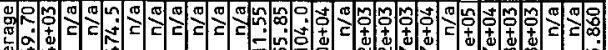

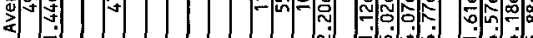

4 응 -

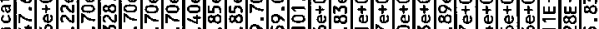

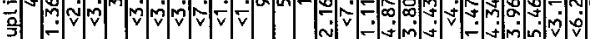

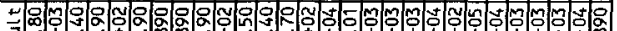

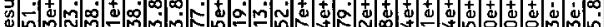

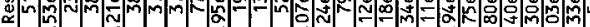

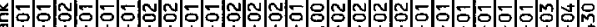

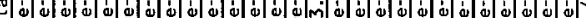

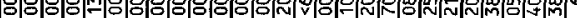

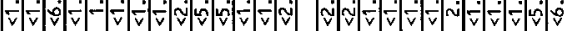

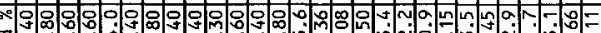
t क् s

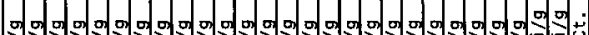

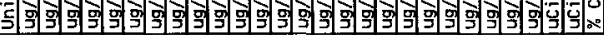

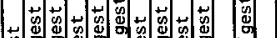

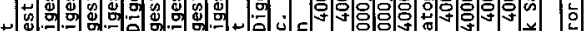

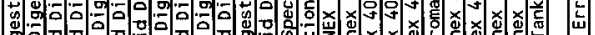

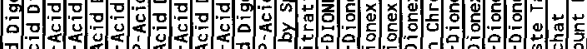
문

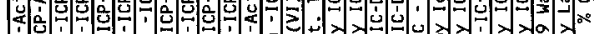
音

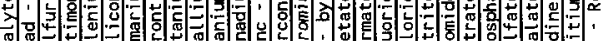

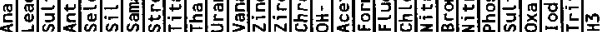

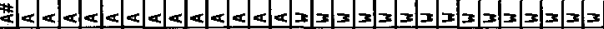

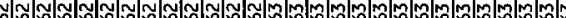

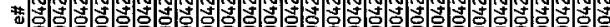

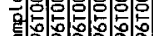

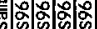

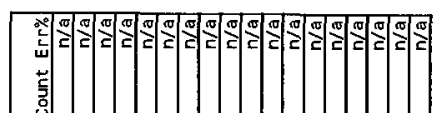

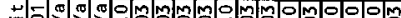

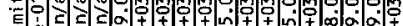

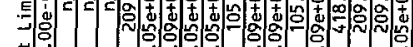
萡

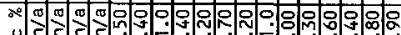

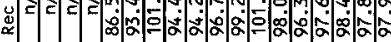
总

बतन

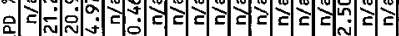

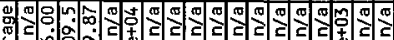

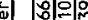
है

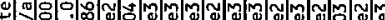

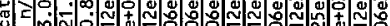
의 $N-0.0$ 을

- 000 ज క

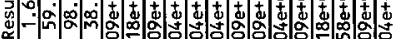

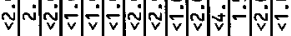

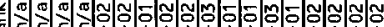

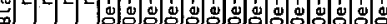

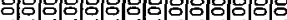

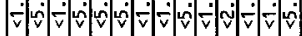

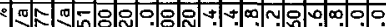
문 다영 蛋

음을

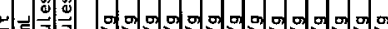

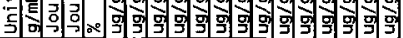

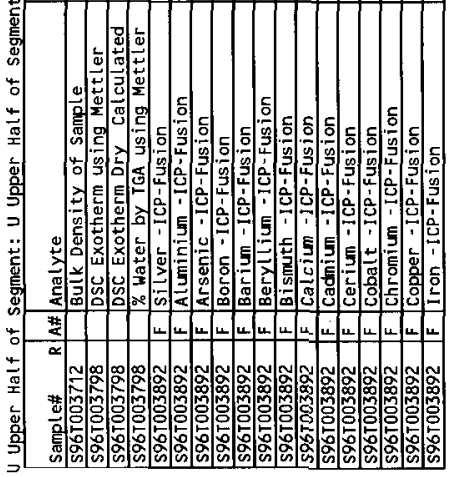

2 


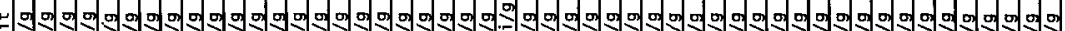

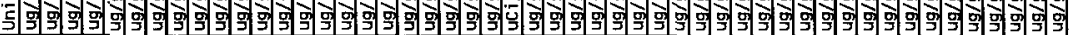

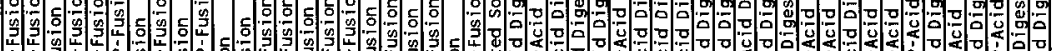
4.

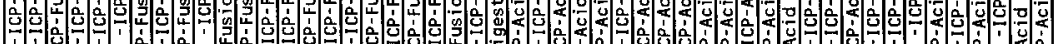
- 10 年

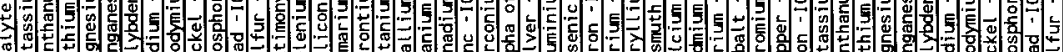

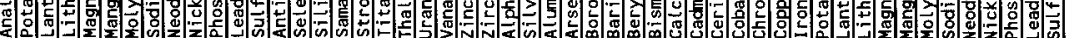

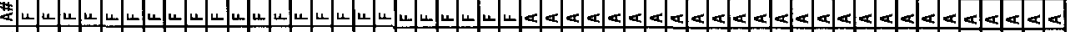
\%

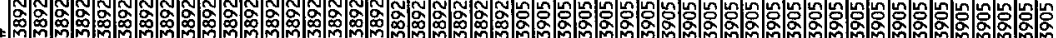
*

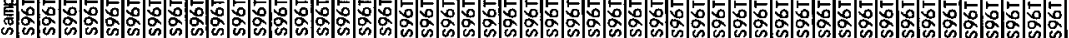
西 


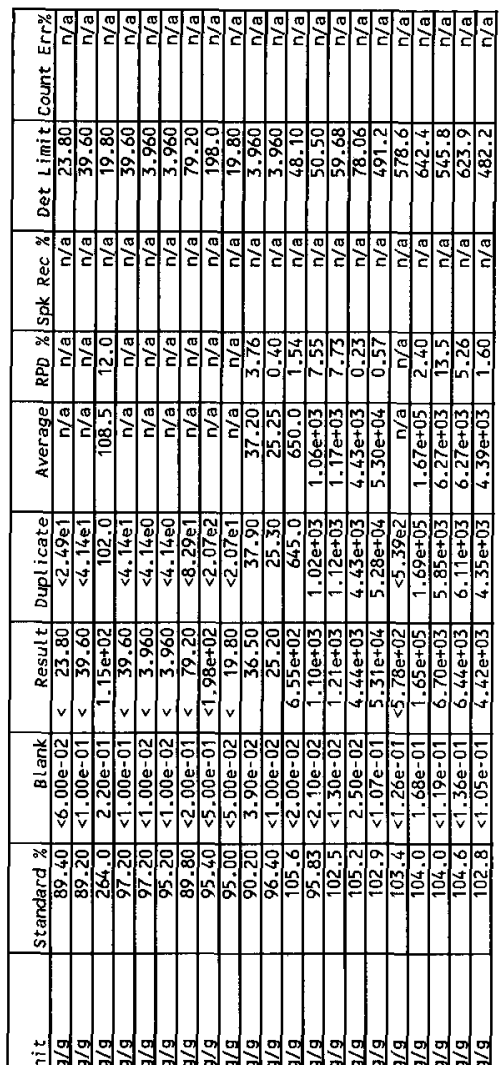

5)

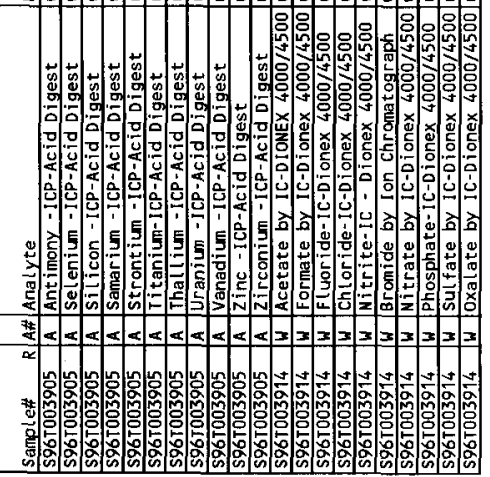


$a$
$\ddot{0}$
$\stackrel{0}{0}$ WHC-SO-WM-DP-199, REV. 1

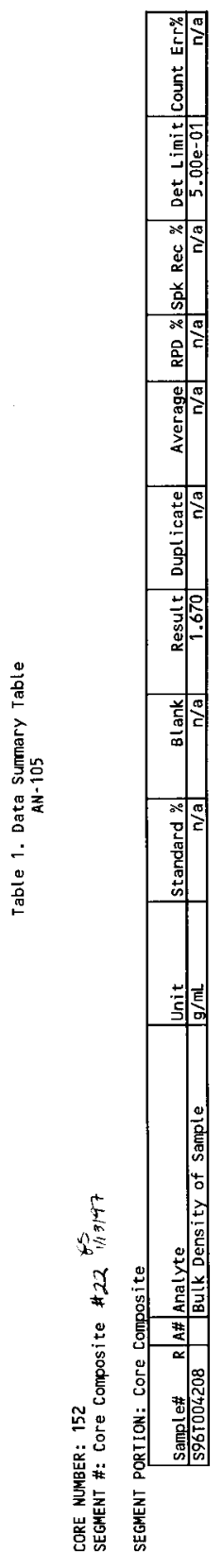




\section{HNF-SD-WM-DP-199, REV. 1}

品

WHC-SD-WM-DP-199, REV. 1

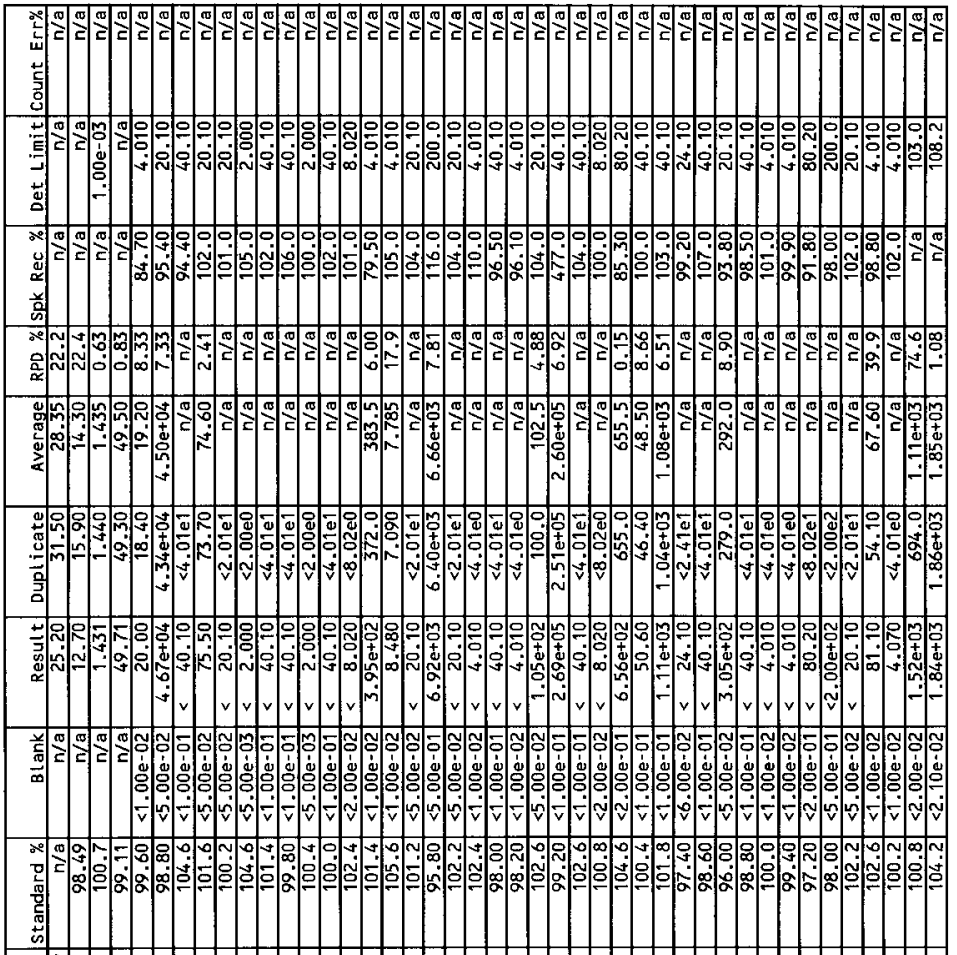

는

아요

氮总

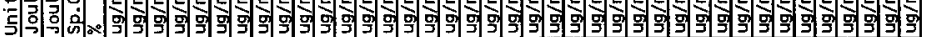

\section{-1 迹}

状

可

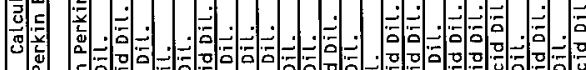

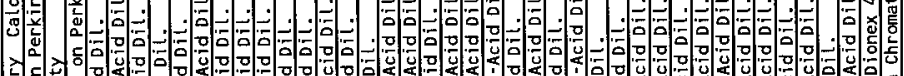

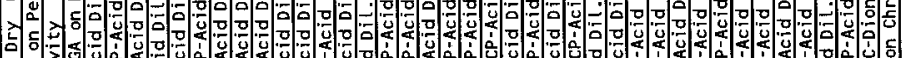

E $\varepsilon$ 焉

过至 0 닟 거 따

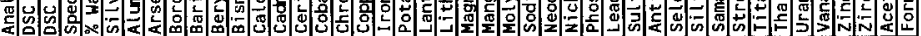

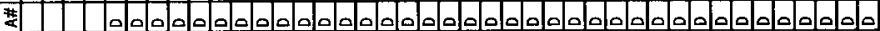

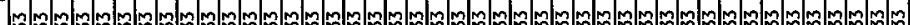
* 
$N$

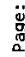

HNF-SD-WM-DP-199, REV. 1

WHC-SD-WM-DP-199, REV. 1

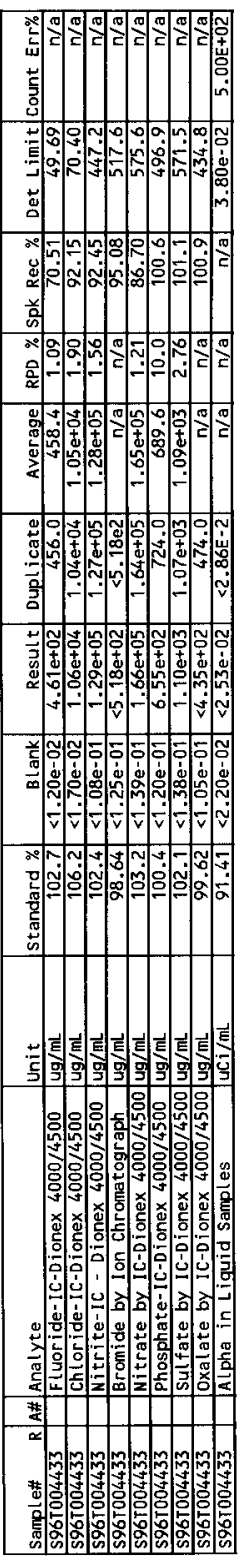

\&

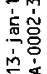




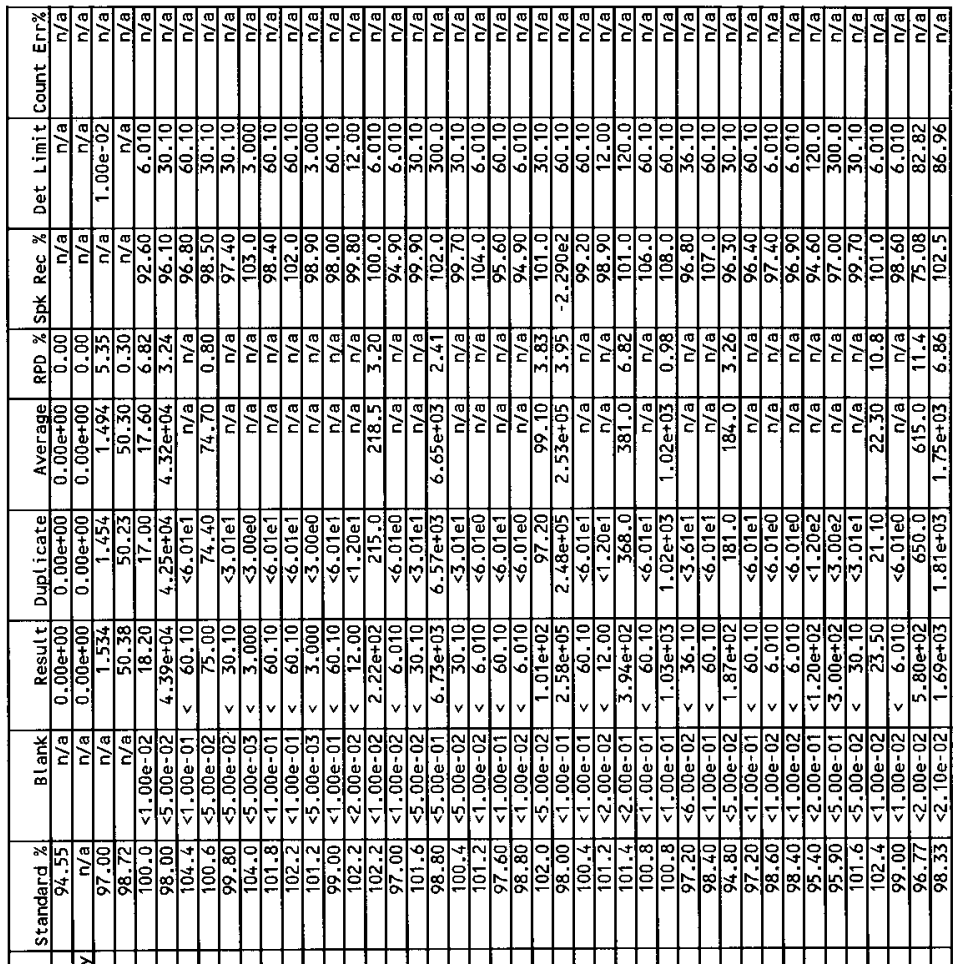

D.

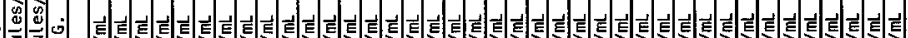

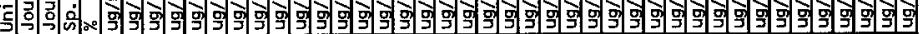

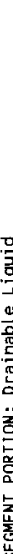$$
\text { 年) }
$$

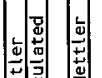

产 品

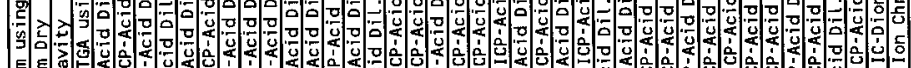

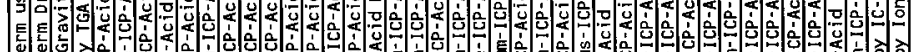

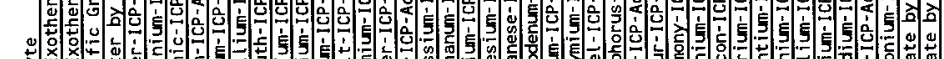

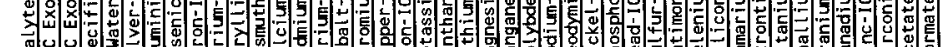

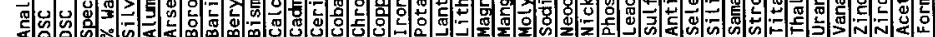

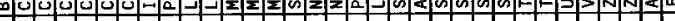

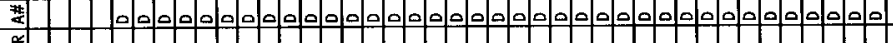

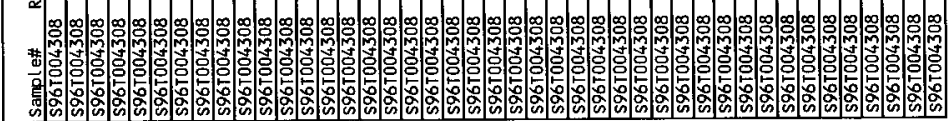




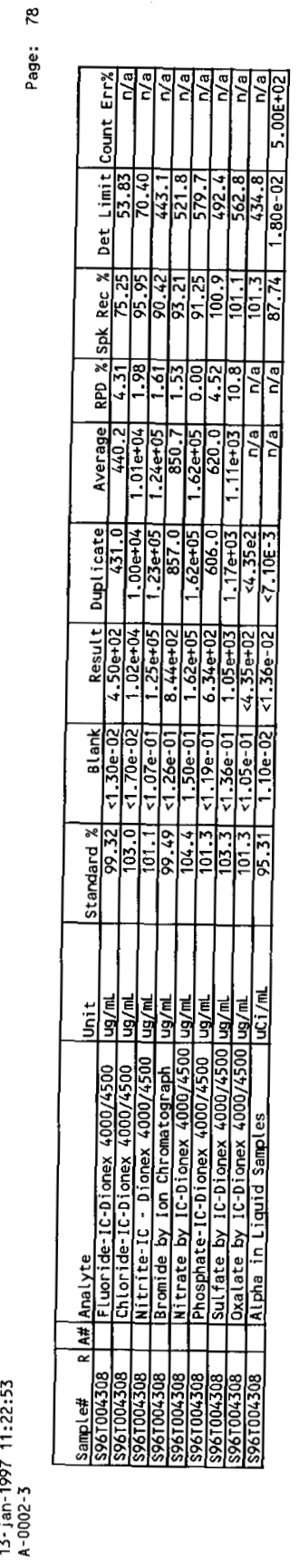

HNF-SD-WM-DP-199, REV. 1

WHC-SD-WM-DP-199, REV. 1 


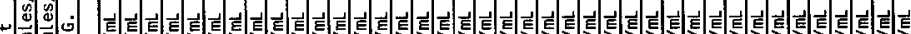

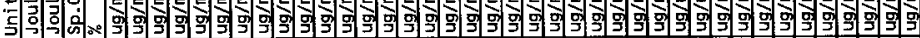




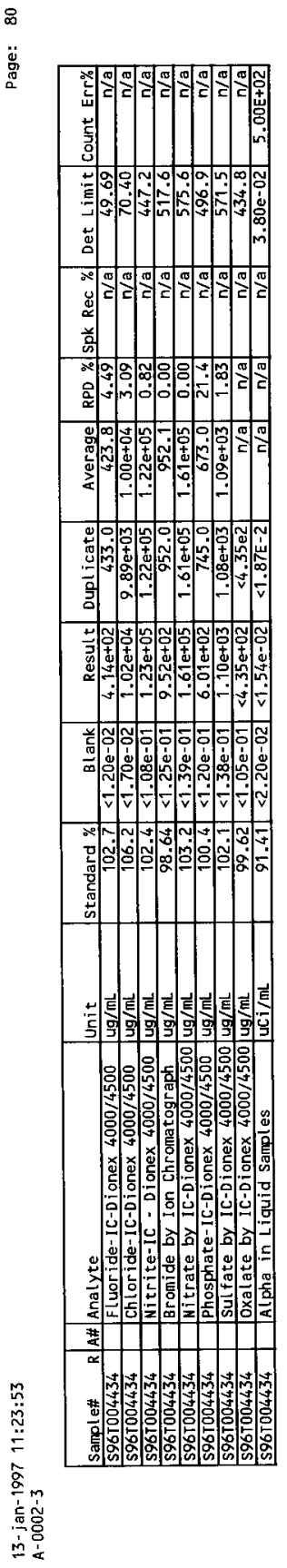

WHC-SD-WM-DP-199, REV. 1 


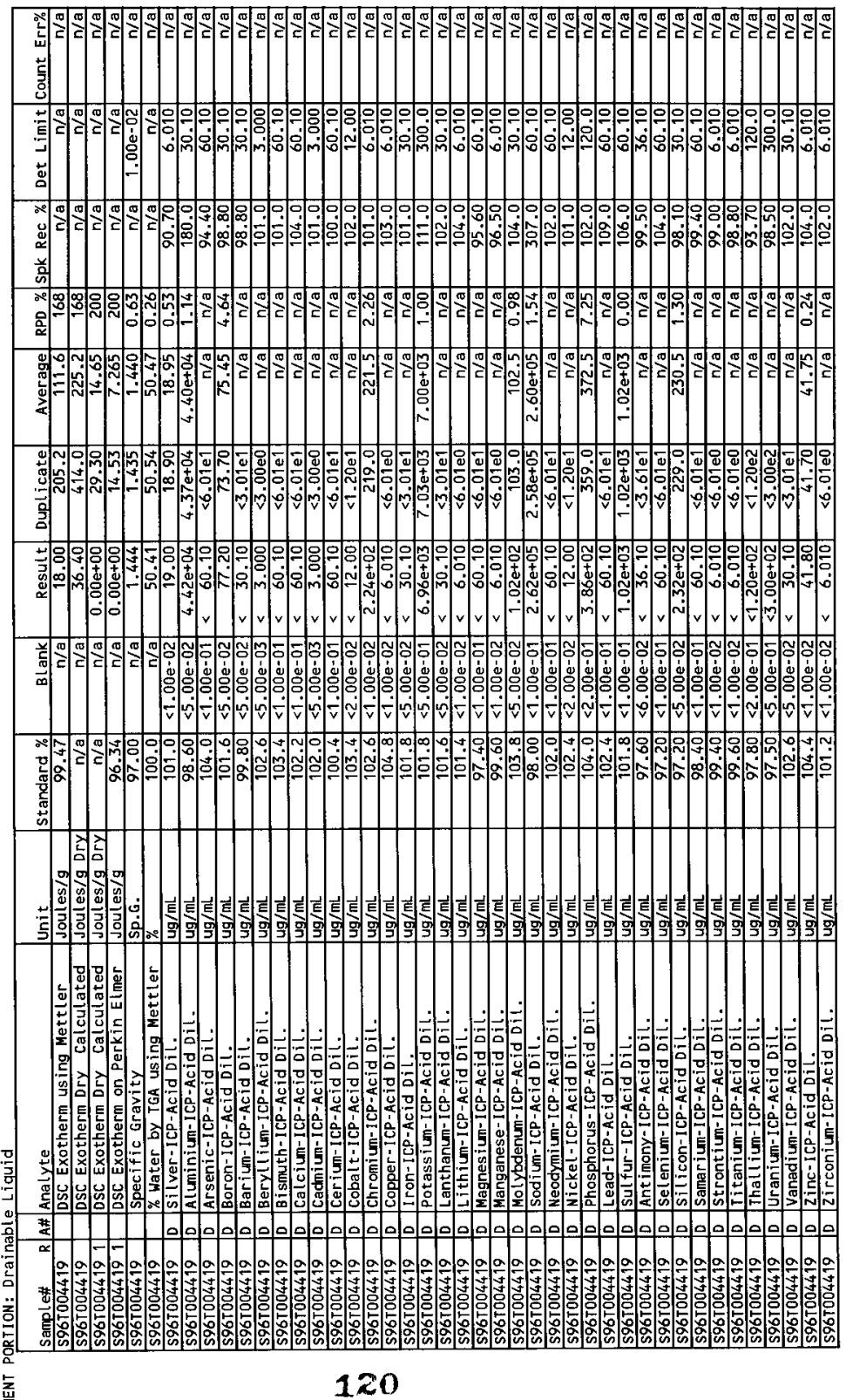



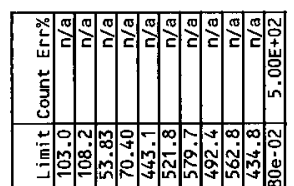

岁

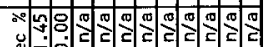
:

音

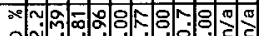

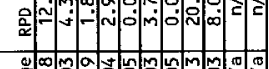

:

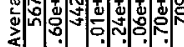

\&

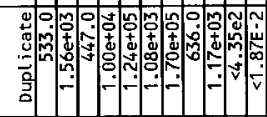

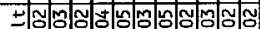

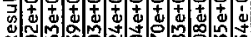

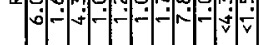

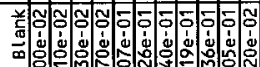

vi $\div-\therefore \therefore-\therefore$

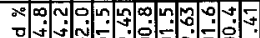

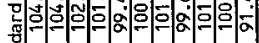

呁

$101+1+$

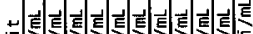

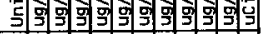
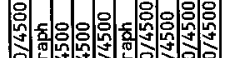

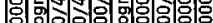

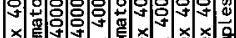

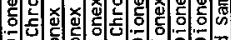

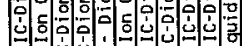

저의

40

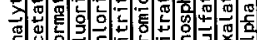

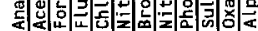

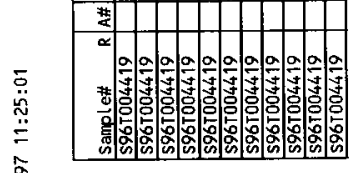



产空 
HNF-SD-WM-DP-199, REV. 1

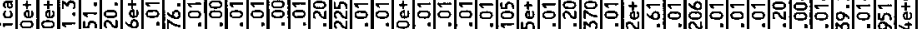

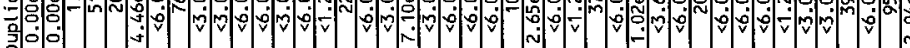

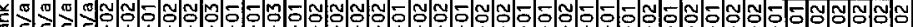

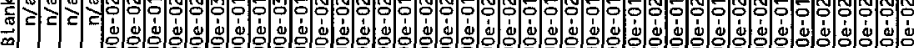

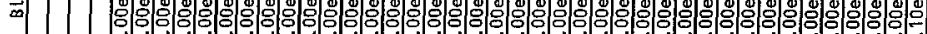

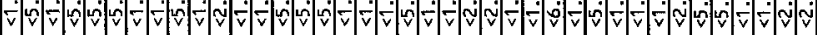

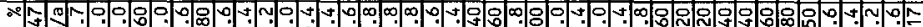
닐

댂.

\section{>}

on on

总包

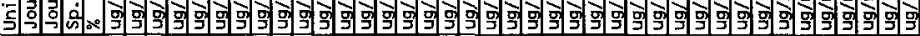

謧

至

$\frac{0}{2}$ 范

- $=:=0$

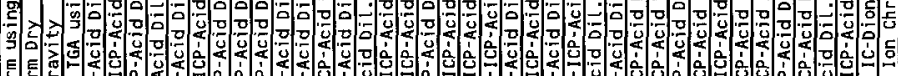

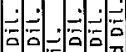

$=$

. . . 잉영

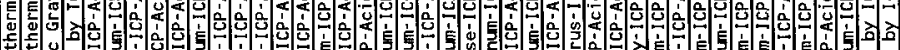

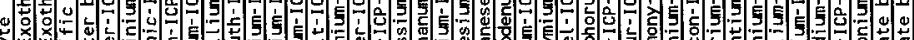

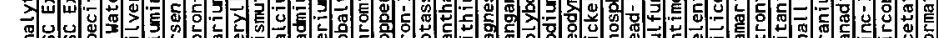

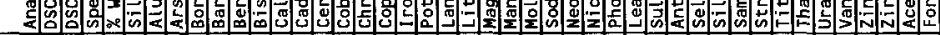

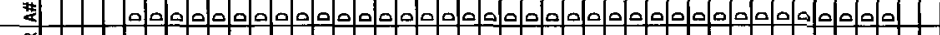

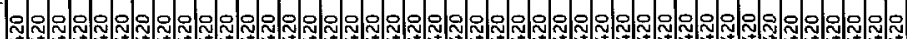

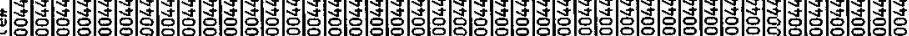
$\stackrel{m}{\stackrel{m}{n}}$ 


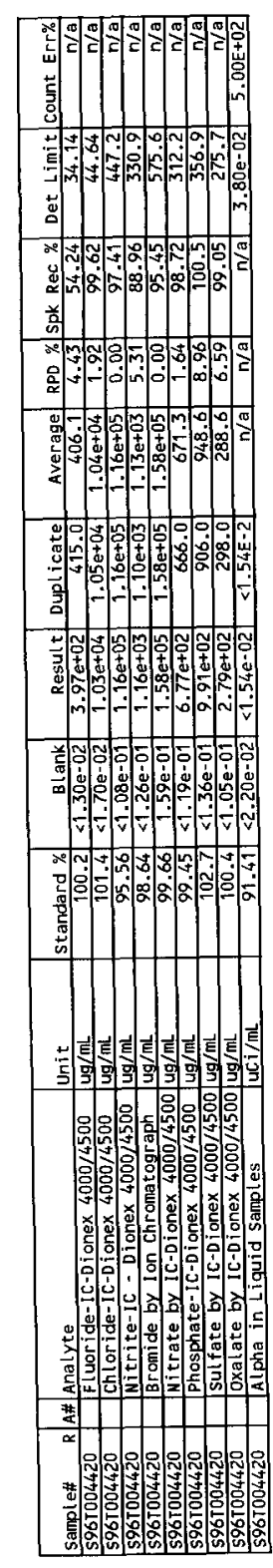

a

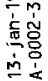


HNF-SD-WM-DP-199, REV. 1

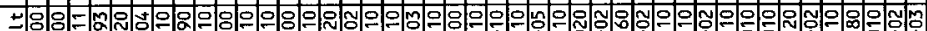

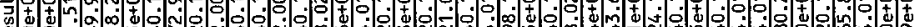
$\propto$ :

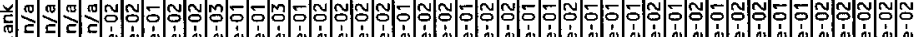

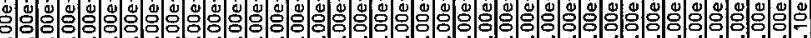

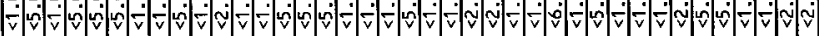

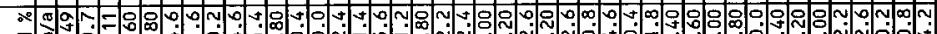

索

善

궁

(⿻)

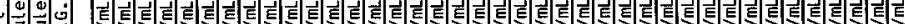

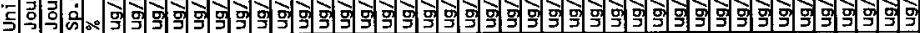

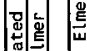 \\ 可}

䆑

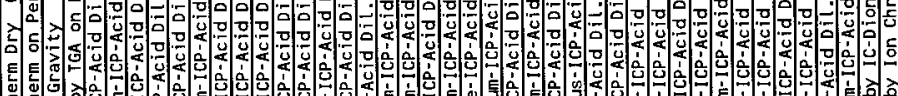

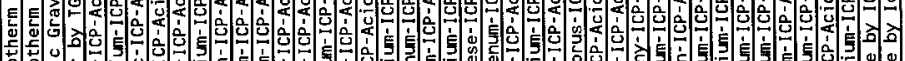

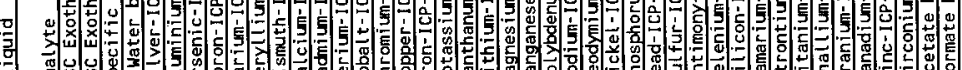

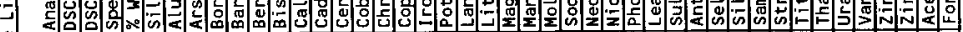




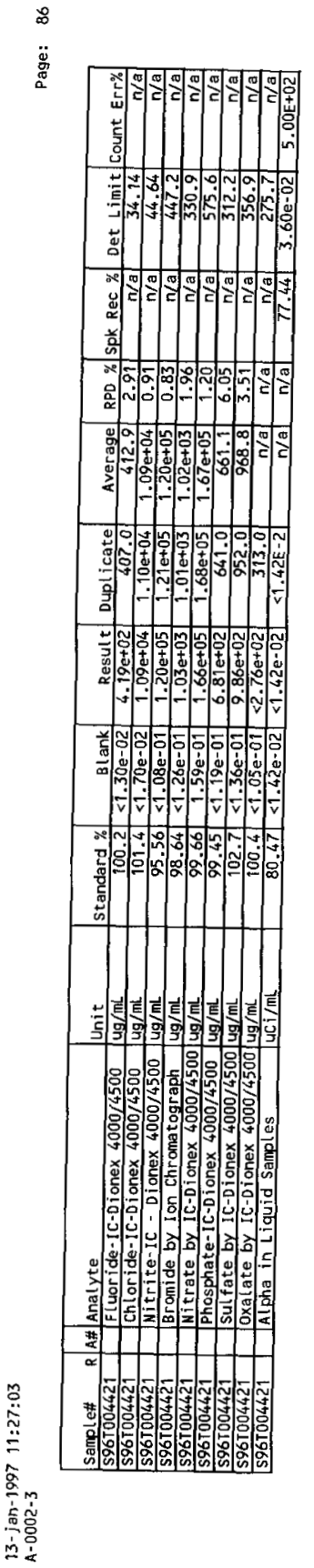

HNF-SD-WM-DP-199, REV. 1

WHC-SD-WM-DP-199, REV. 1 


\section{HNF-SD-WM-DP-199, REV. 1}

.0.

(2)

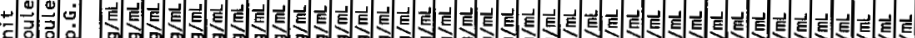

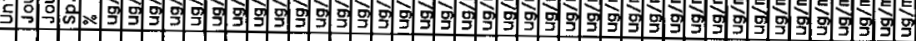

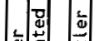

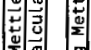

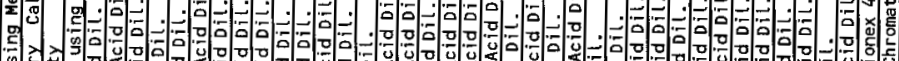

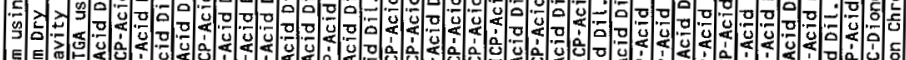
国 든

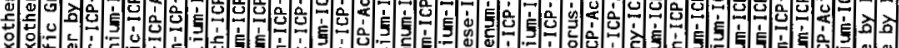

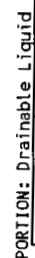

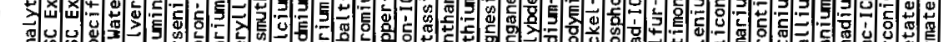

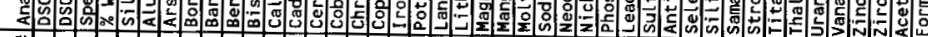

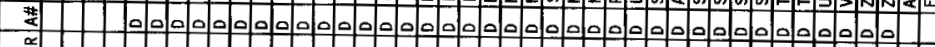




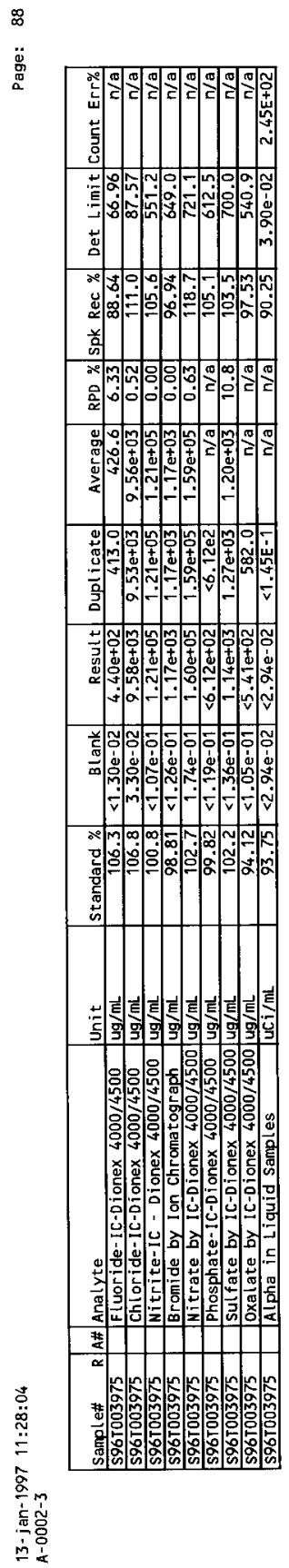

HNF-SD-WM-DP-199, REV. 1

WHC-SD-WM-DP-199, REV. 1 


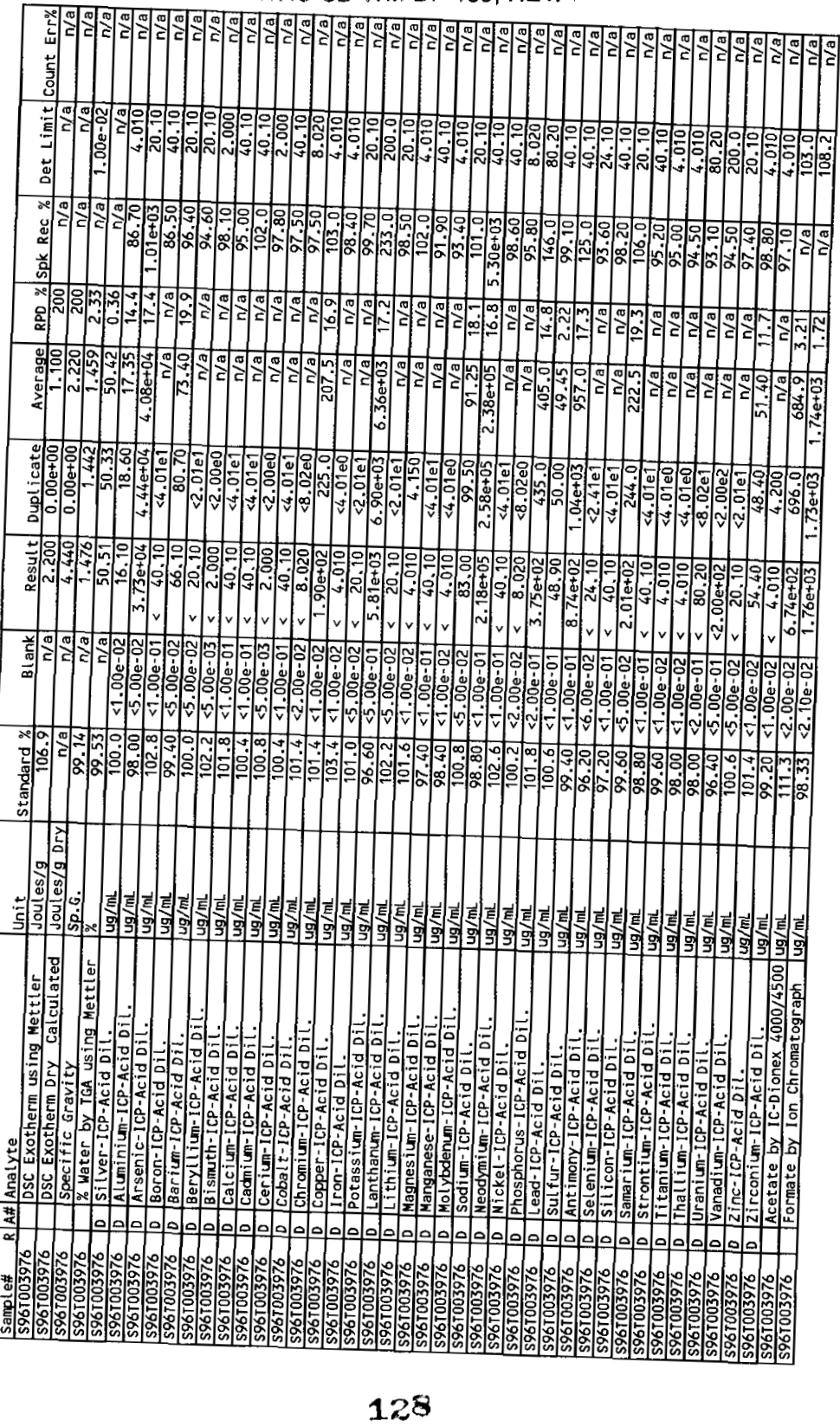




\section{HNF-SD-WM-DP-199, REV. 1}

WHC-SD-WM-DP-199, REV. 1
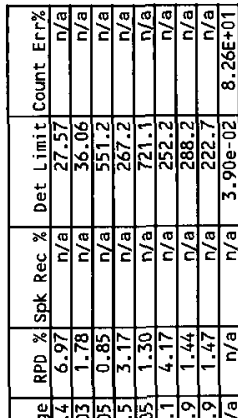

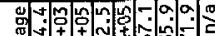
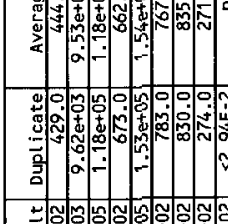

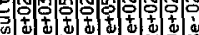
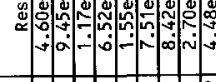

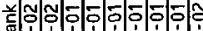

$=0$

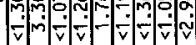

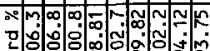

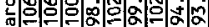

空
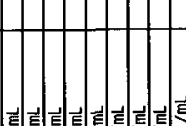

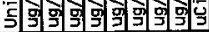

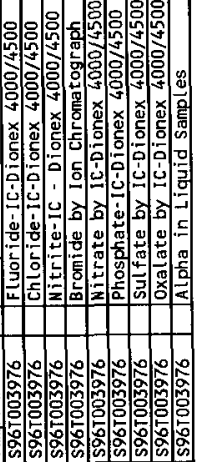

a

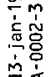




\section{HNF-SD-WM-DP-199, REV. 1 \\ WHC-SD-WM-DP-199, REV. 1}

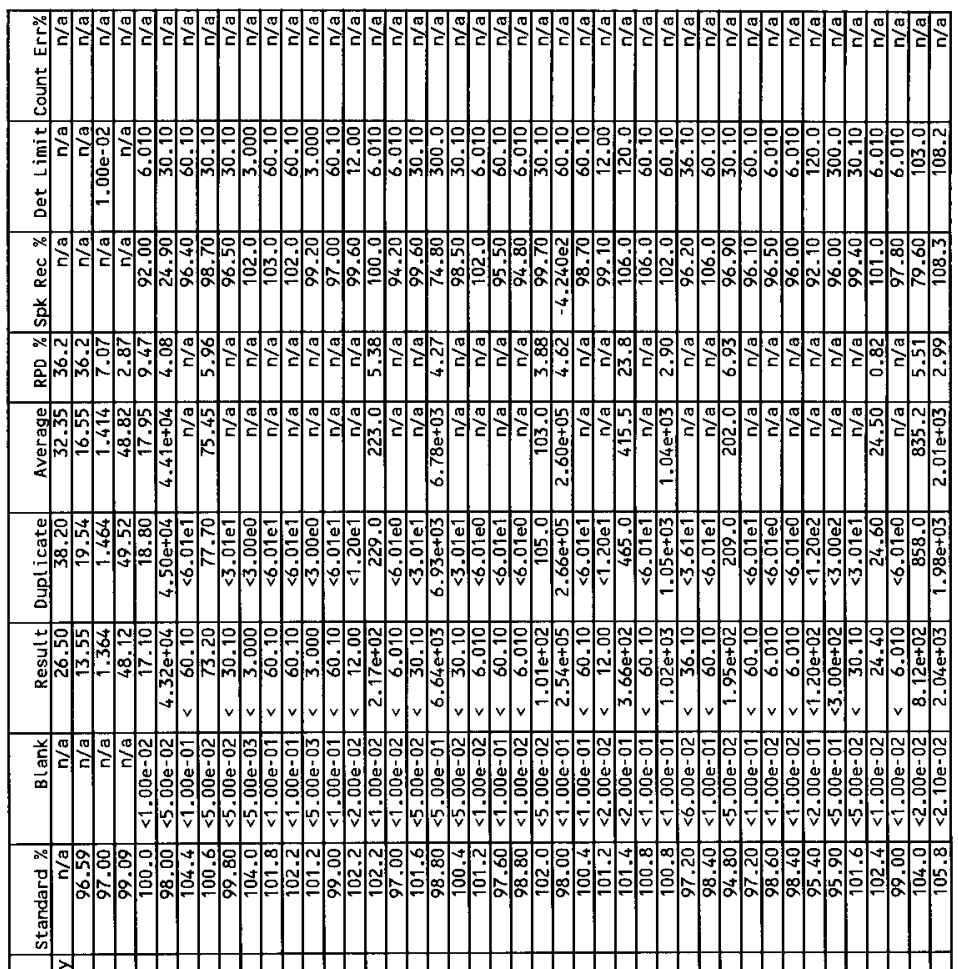

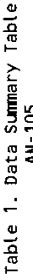

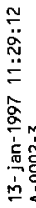

o. 0

की

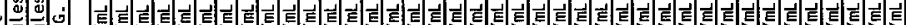

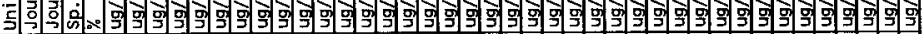

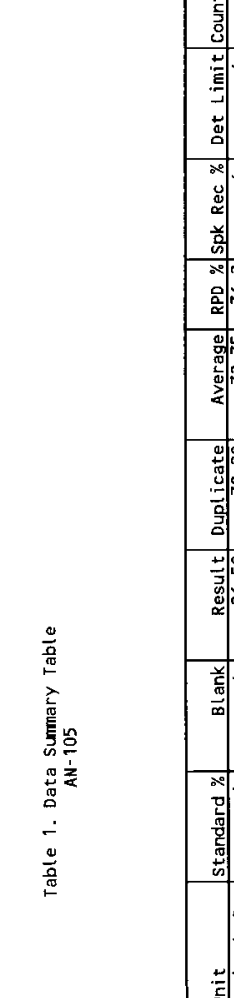

1.

要

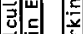

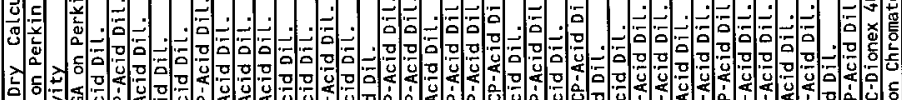

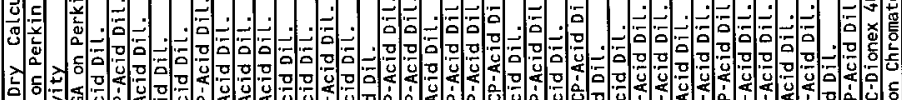

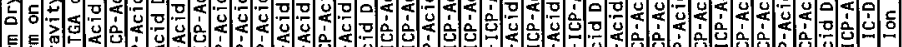

튼

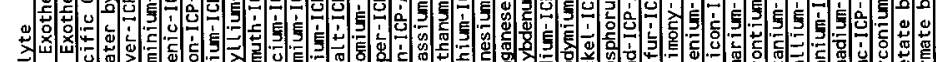

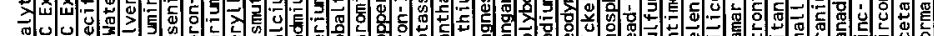

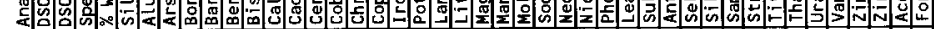

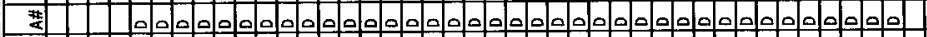

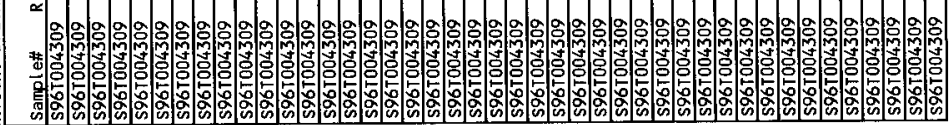

容 
$\approx$

o

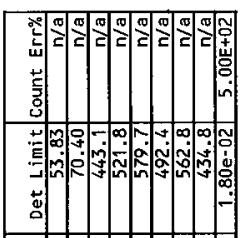

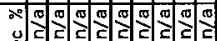

产

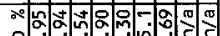

aेmm m m m $m=$

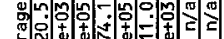

บे

$\therefore$

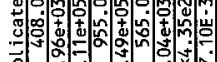

音

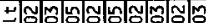

苟志士

m ma

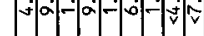

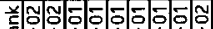

$=0$

$\therefore-\therefore \therefore \therefore$

anolomm $=$

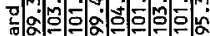

空

a

可司國

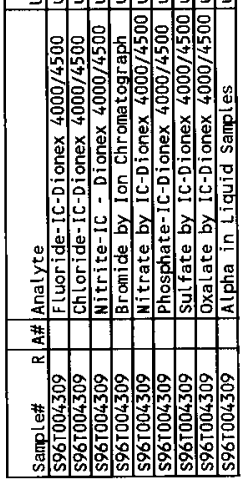



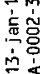

HNF-SD-WM-DP-199, REV. 1

WHC-SD-WM-DP-199, REV. 1 
HNF-SD-WM-DP-199, REV. 1

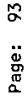

WHC-SD-WM-DP-199, REV. 1

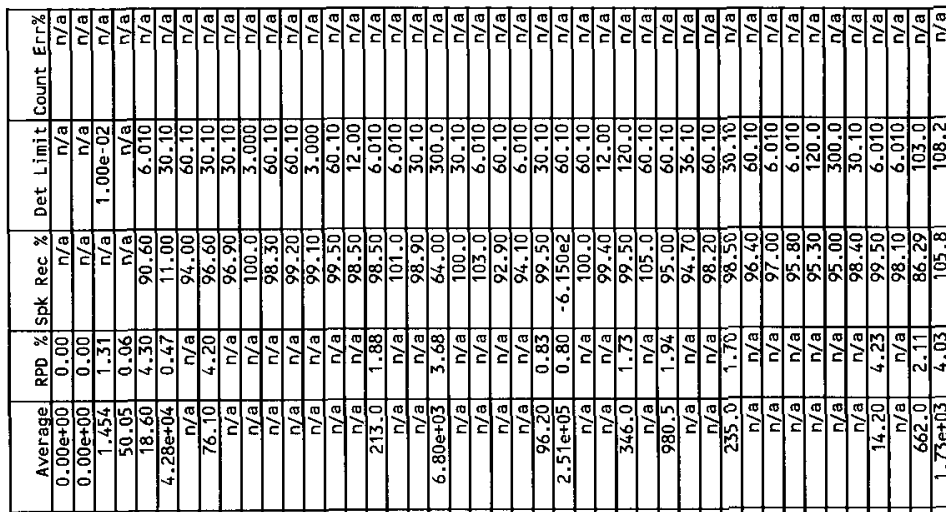

要

으을

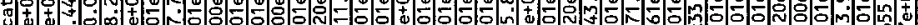

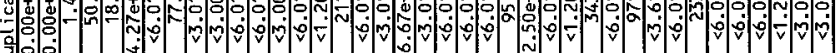

aेंo

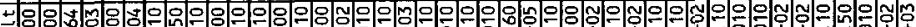

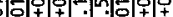

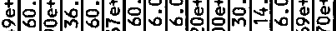

$\therefore$

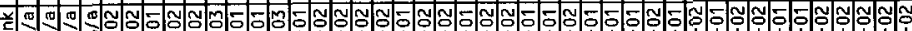

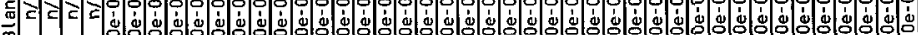
$\forall$ v

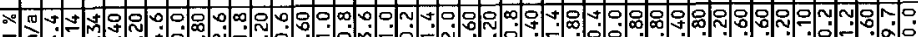

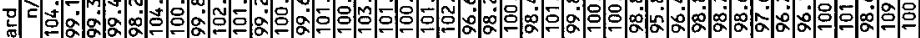
है⿴囗十)

요용

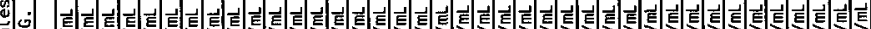

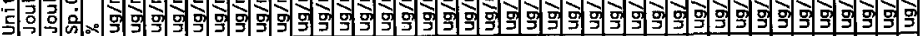

氙产

ज्ञ

可言

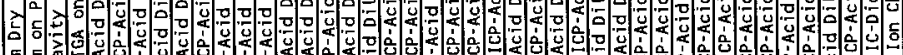

틀 E

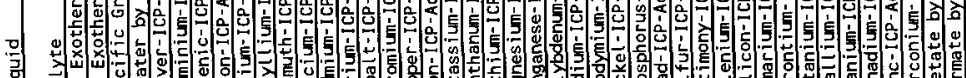

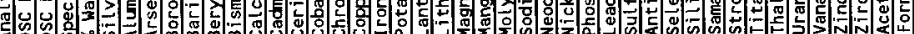

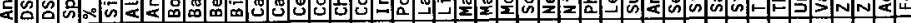

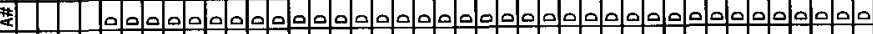

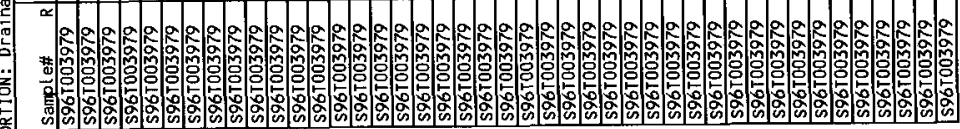




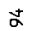

迥
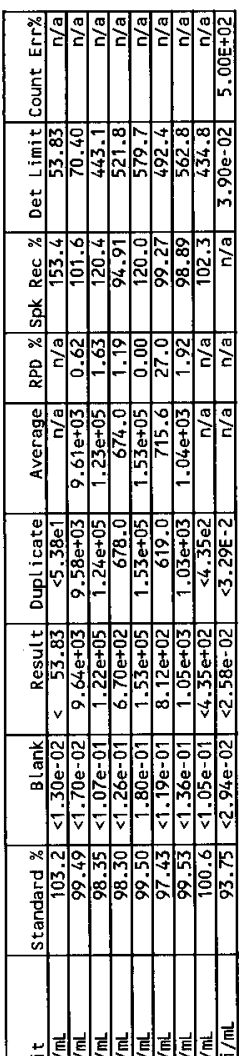

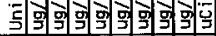

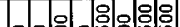

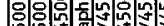

낭응형융

윙위

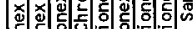

흐의의 의

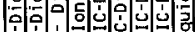

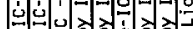

4 웡

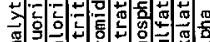

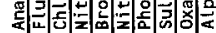

帮 000

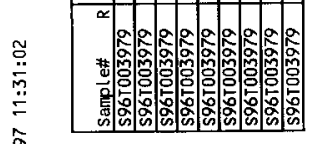

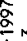

$\frac{2}{1}$

它号
HNF-SD-WM-DP-199, REV. 1

AHC,-SD-WM-DP-199, REV. 1 


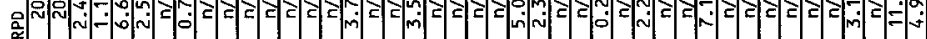

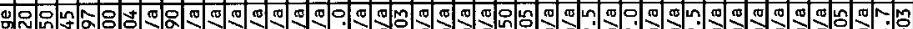
월 $\stackrel{0}{2}$

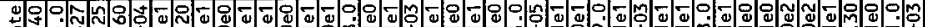

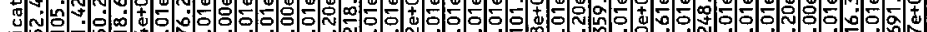

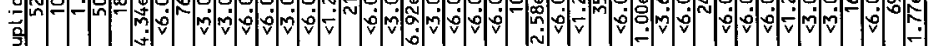
a

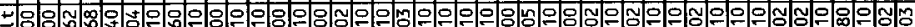
1

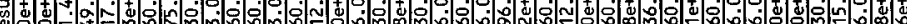

$\circ 0^{\circ}$

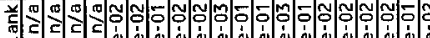

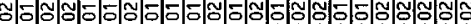
8 : 0 :

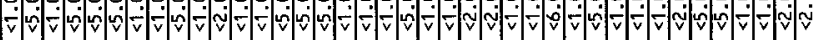

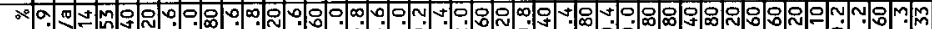
70 ํㅗㅇ (1)

की

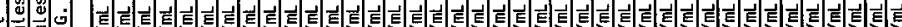

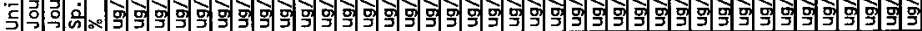

$=$

$\stackrel{\bar{m}}{=}$

$=$

$\hat{\alpha}$

Im

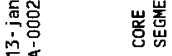

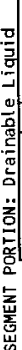

总

떤

힌

on.

$\therefore \quad \therefore \quad \therefore=0$

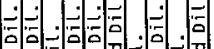

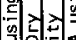

E

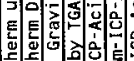

궁

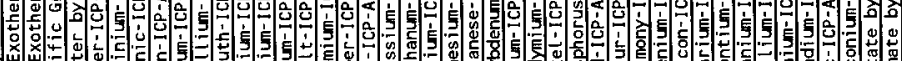

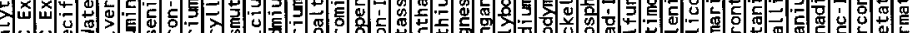

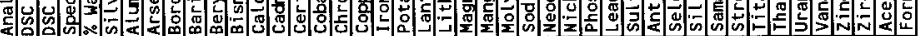

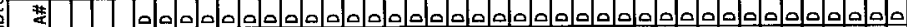

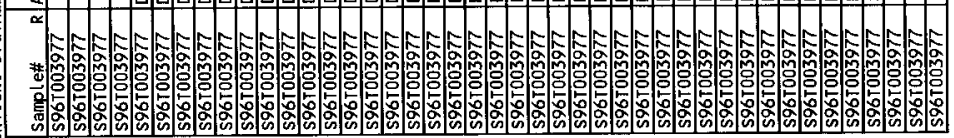




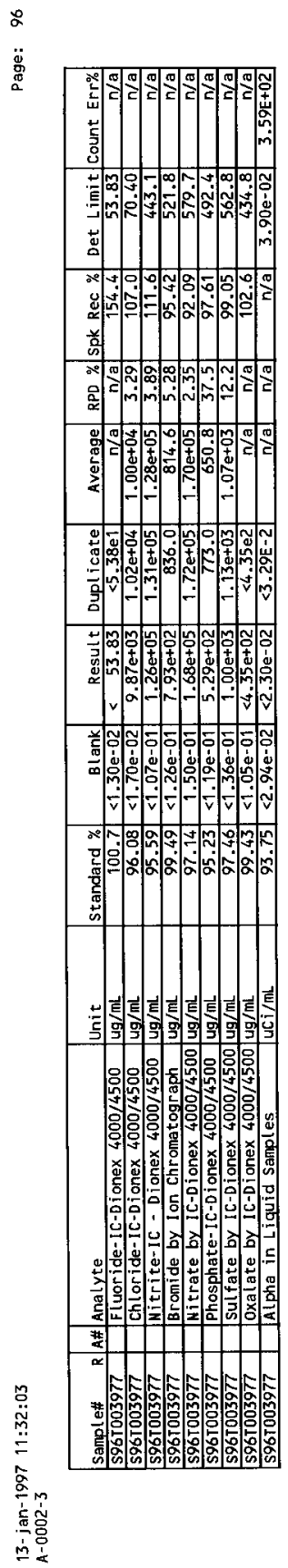

\author{
HNF-SD-WM-DP-199, REV. 1 \\ WHC-SD-WM-DP-199, REV. 1
}


HNF-SD-WM-JF-99, REV.

๙a WHC-SD-WM-DPB-199, REEV 1

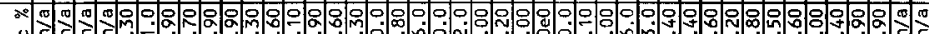

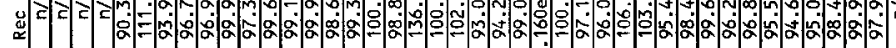
总

a

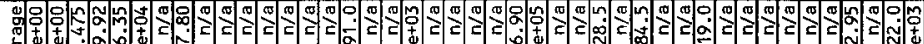

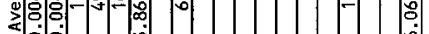

a.

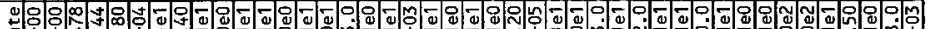

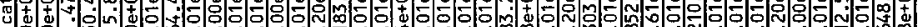
等

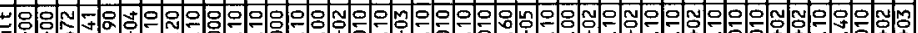
3 a s

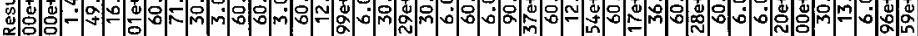
$\therefore \circ$

y0

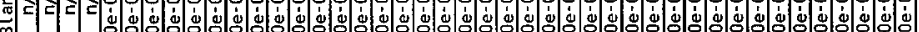

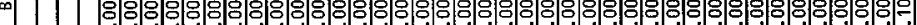

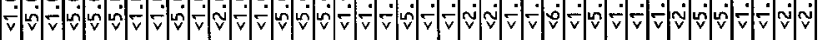

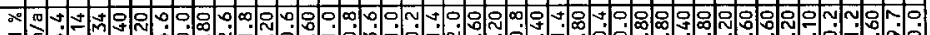

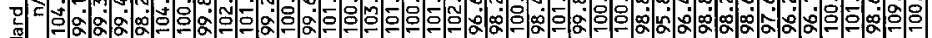
롱

줗

잉

1 过论

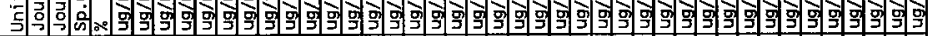

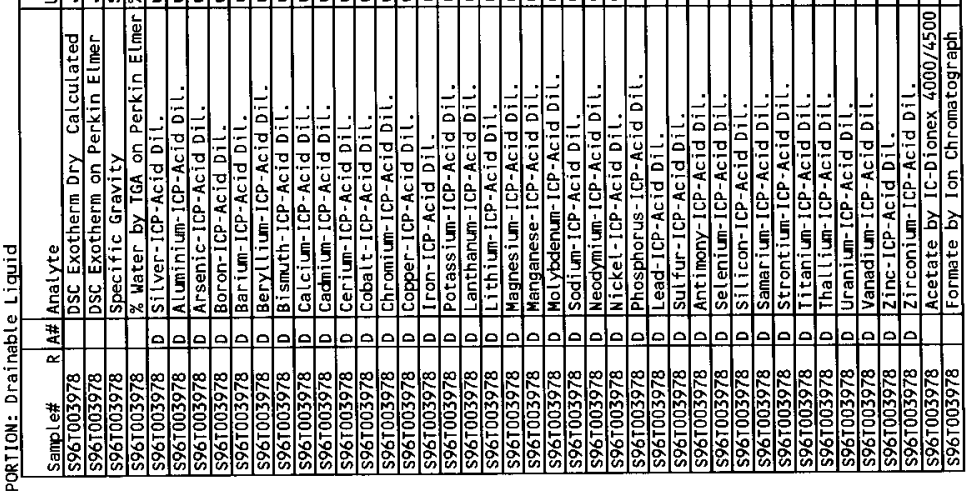




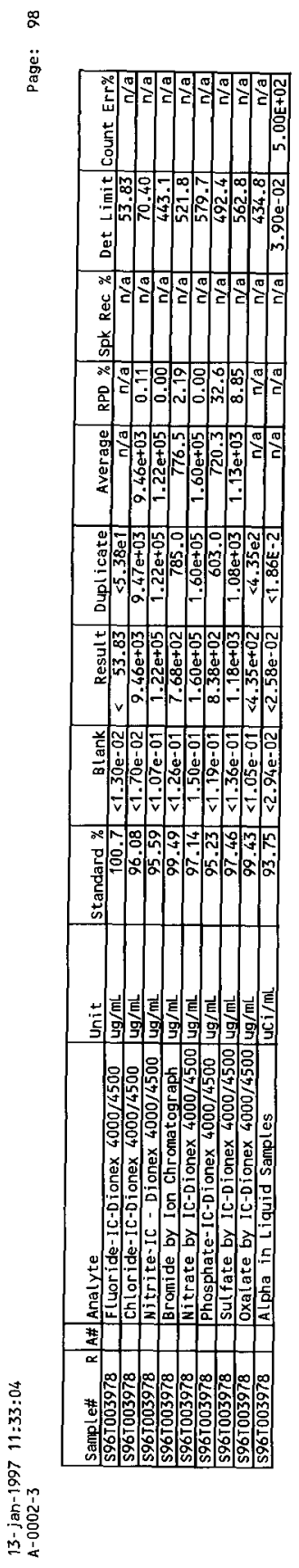

HNF-SD-WM-DP-199, REV. 1
WHC-SD-WM-DP-199, REV. 1 


\section{HNF-SD-WM-DP-199, REV. 1}

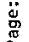

WHC-SD-WM-DP-199, REV. 1

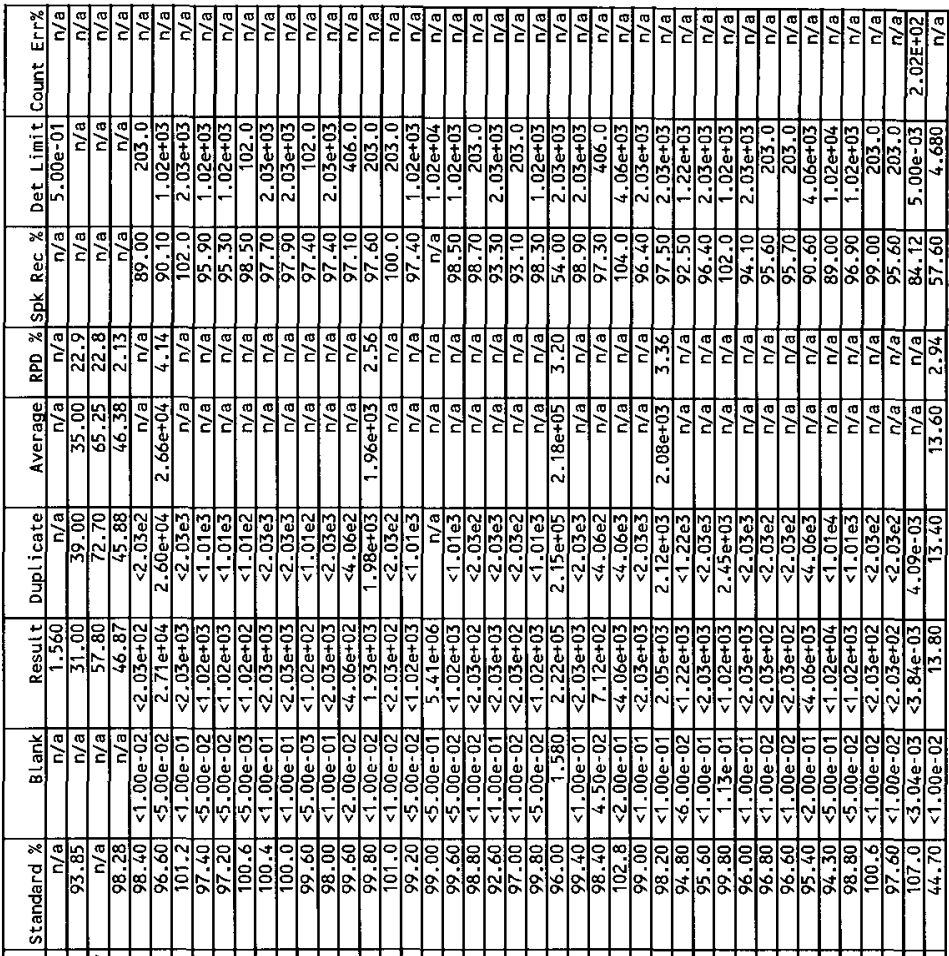

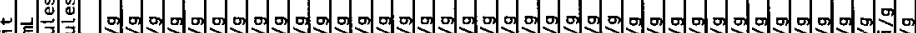

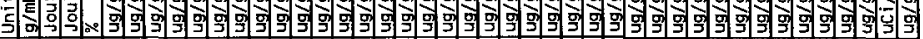

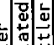

.

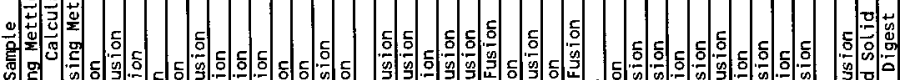

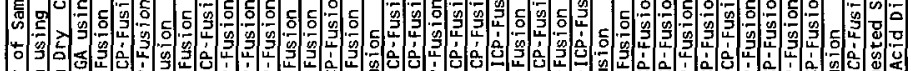
莗

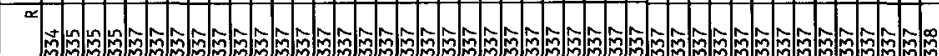




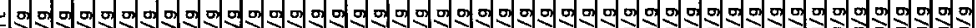

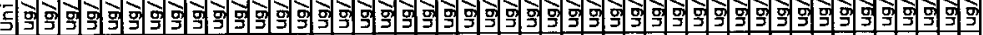

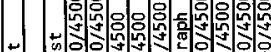

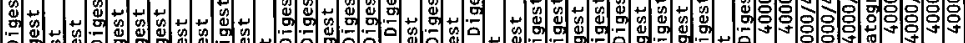

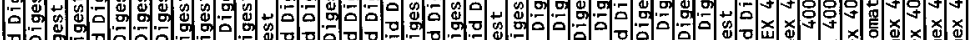

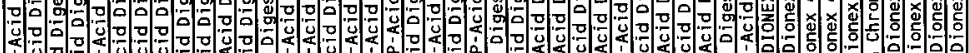

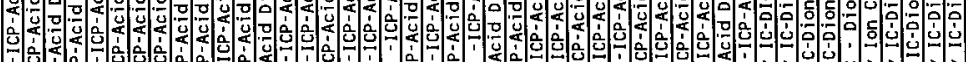

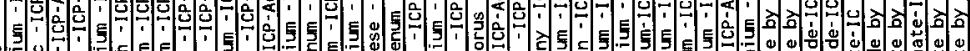

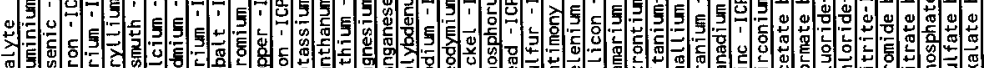

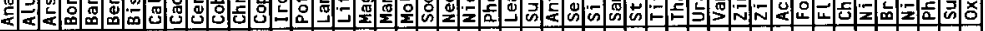

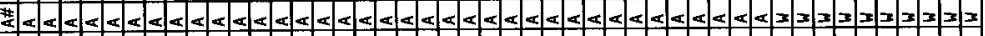

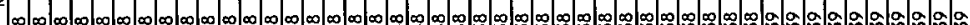
W

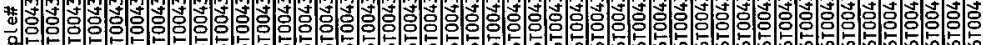




\section{HNF-SD-WM-DP-199, REV. 1}

$\overline{5}$

WHC-SD-WM-DP-199, REV. 1

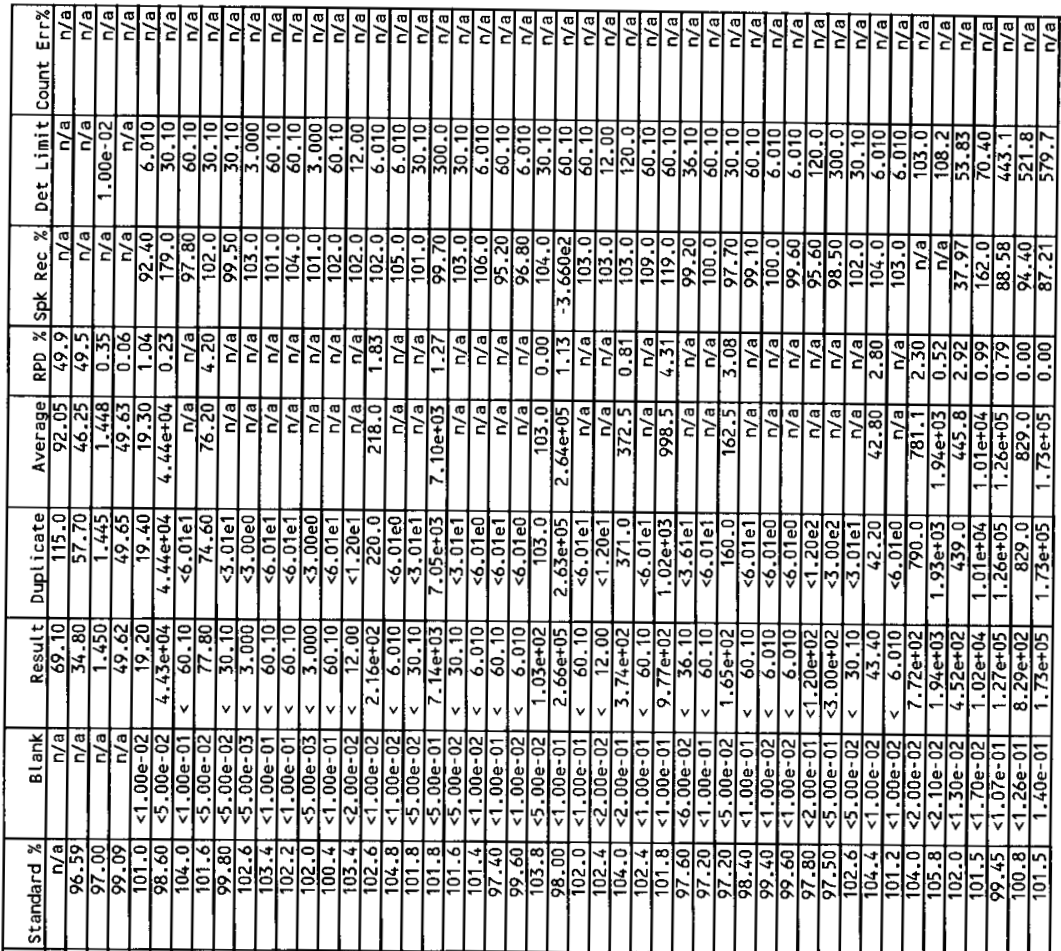

S.

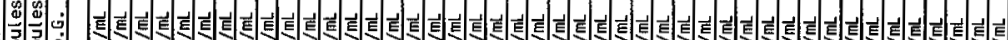

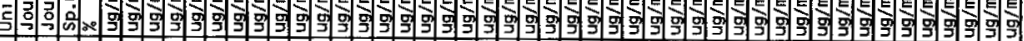

这魚

3

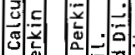

急竞

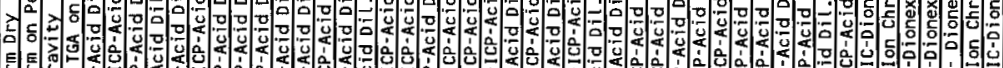

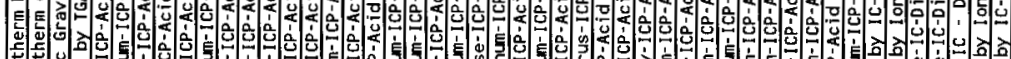

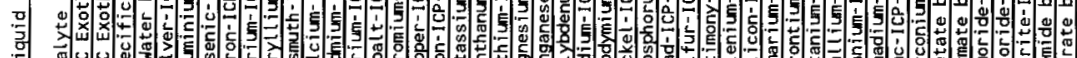

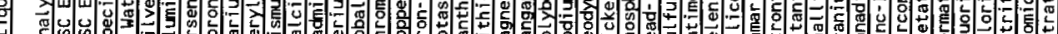

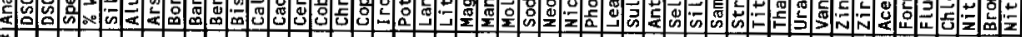

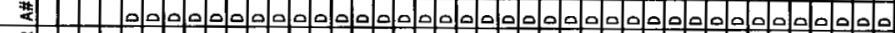


HNF-SD-WM-DP-199, REV. 1

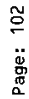

WHC-SD-WM-DP-199, REV. 1

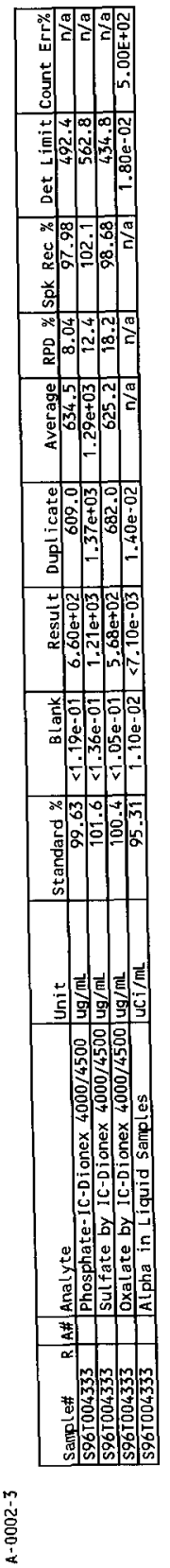




\section{HNF-SD-WM-DP-199, REV. 1}

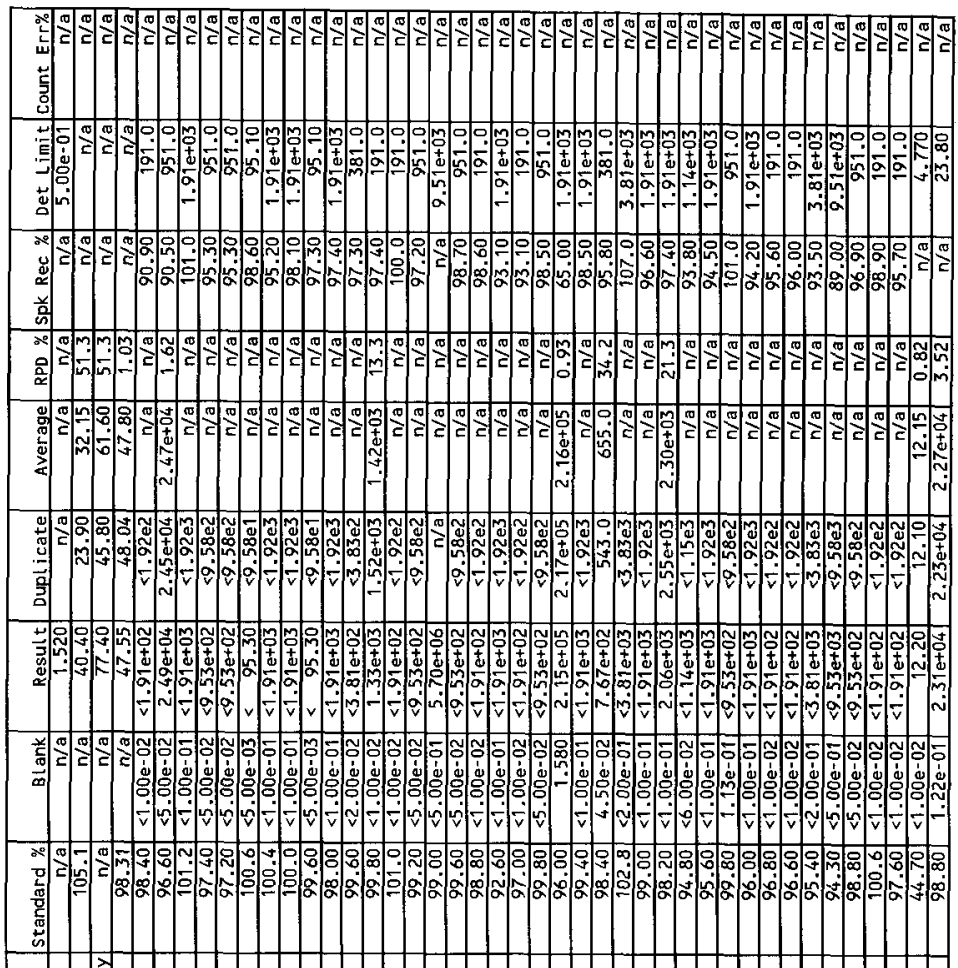

o. 0

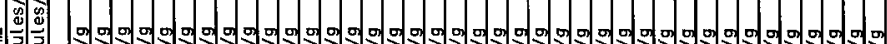

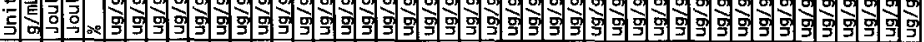
(2)

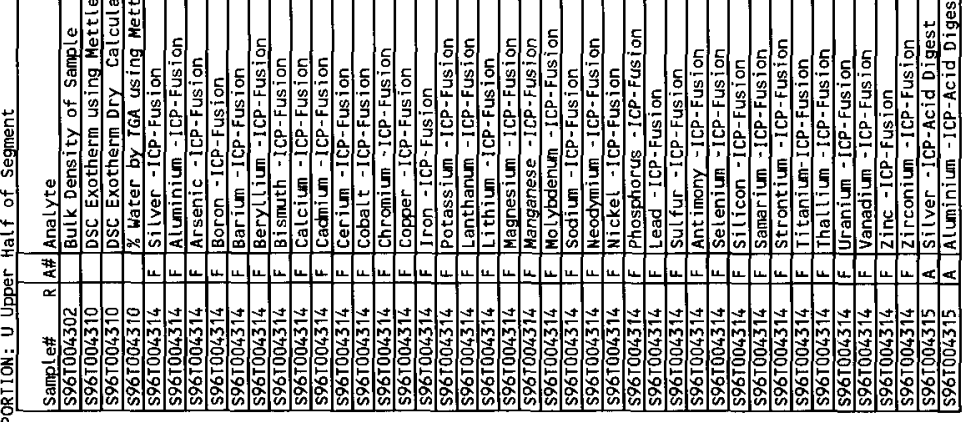

$\stackrel{2}{a}$

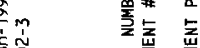




\section{HNF-SD-WM-DP-199, REV. 1}

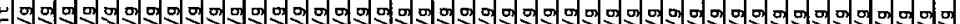
5)

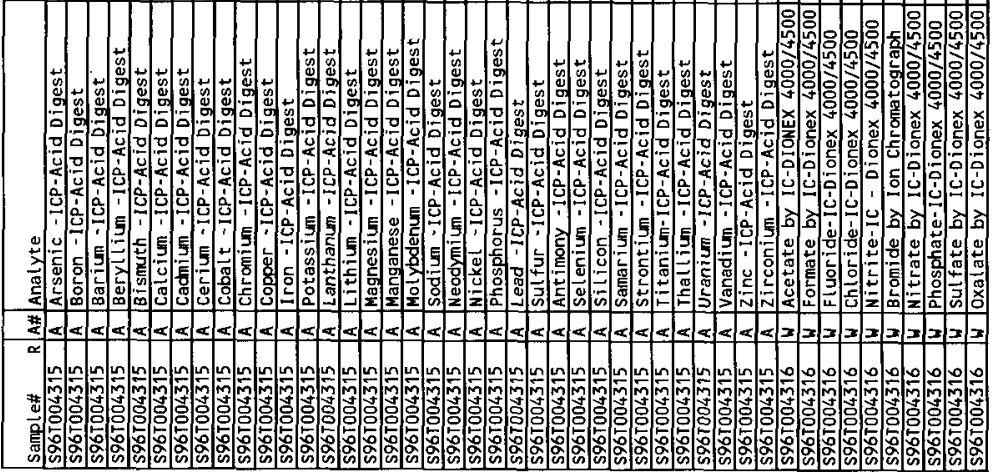
a 


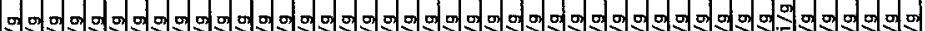

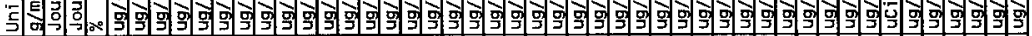

힣

过䊁

(.)

$\frac{10}{2}$

.

등

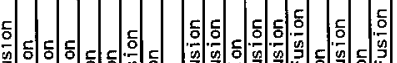

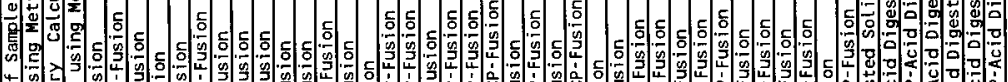

幽

䔽

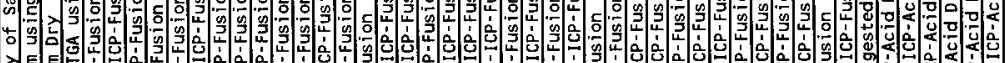

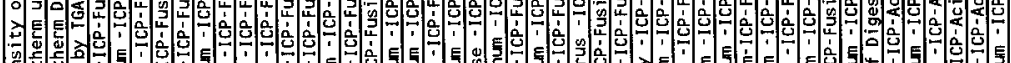

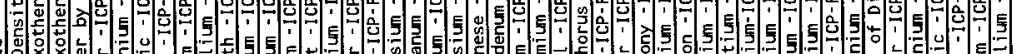

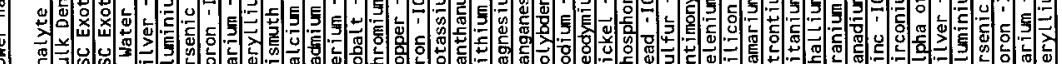

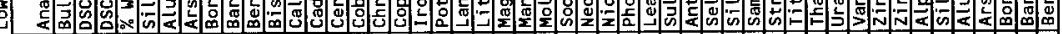

₹ $\infty$ 


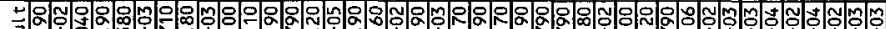

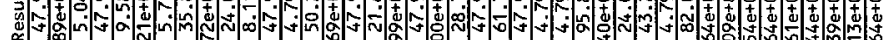

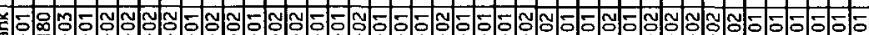

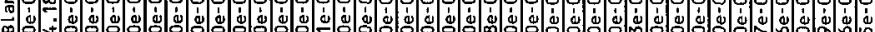
8

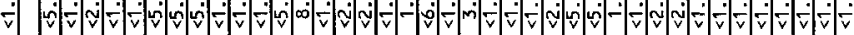

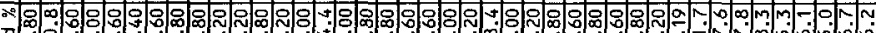
(a)

空

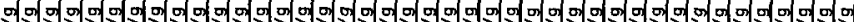

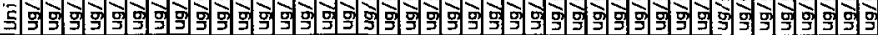

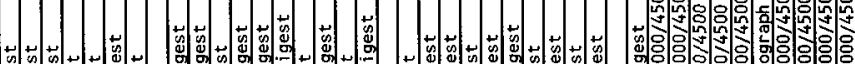

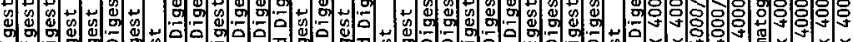

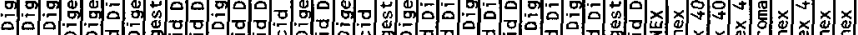
a t

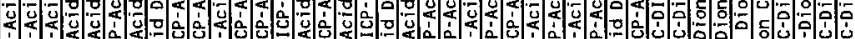

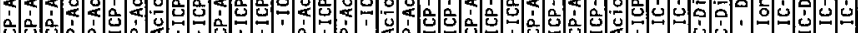

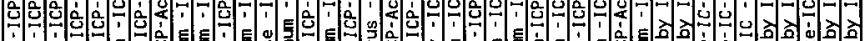

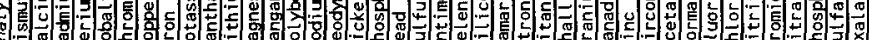
5.

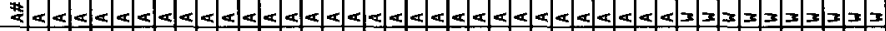




\section{HNF-SD-WM-DP-199, REV. 1}

(2)

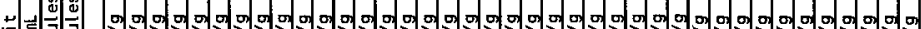

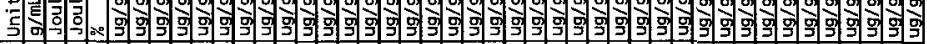

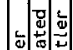

.

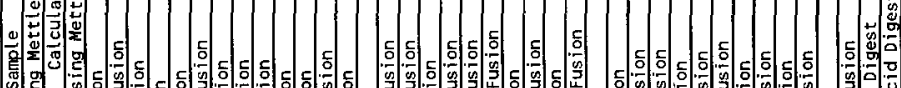

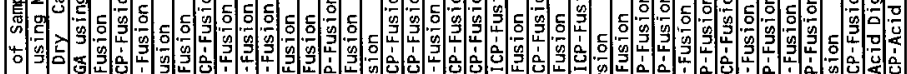
馗 
HNF-SD-WM-DP-199, REV. 1

趷

WHC-SD-WM-DP-199, REV. 1

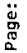

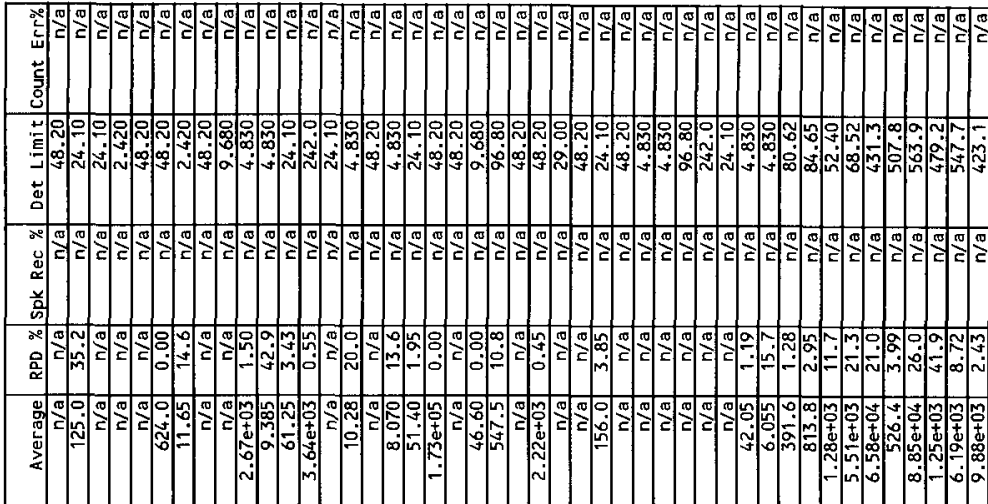

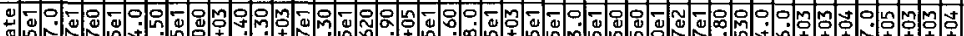

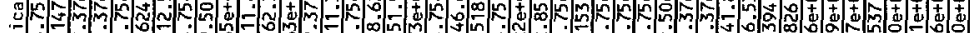

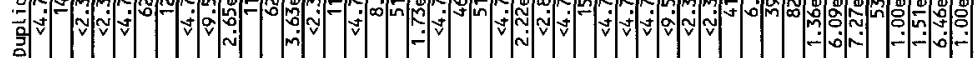

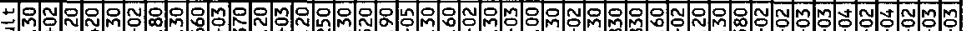
至

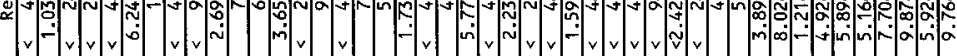

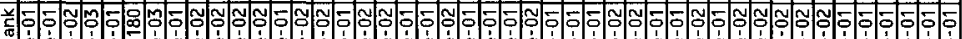
-

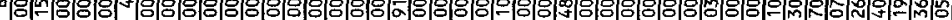

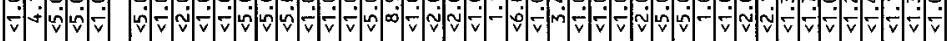

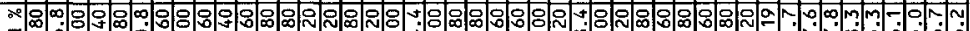

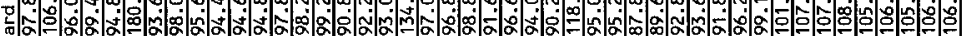
음 in

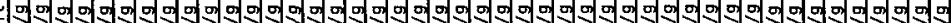

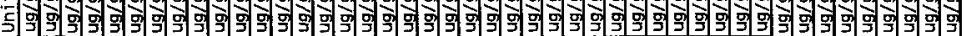

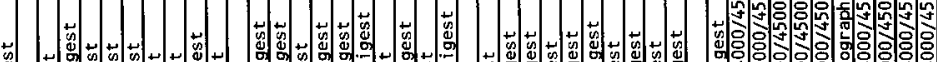
象 무의.

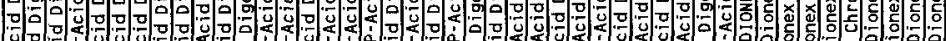

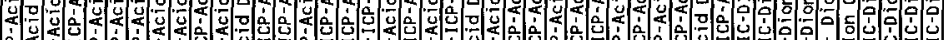

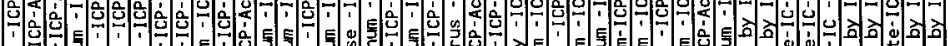

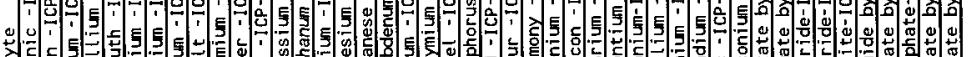

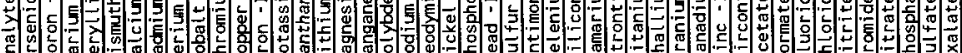

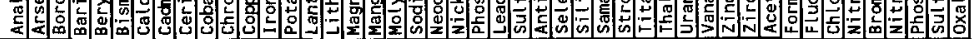

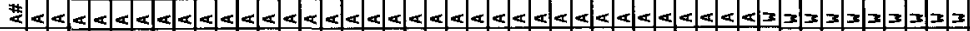
(a) 


\section{HNF-SD-WM-DP-199, REV. 1}

in

WHC-SD-WM-DP-199, REV. 1

若

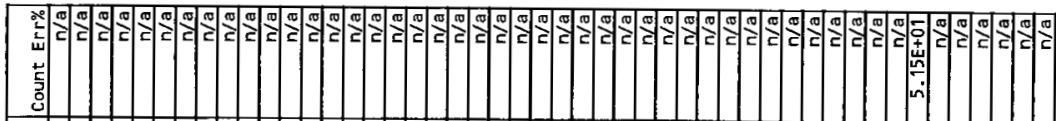

可

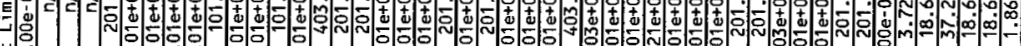
荡

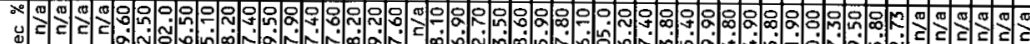

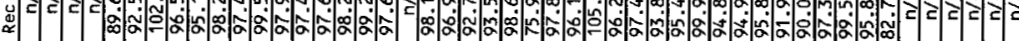
总

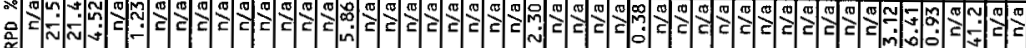

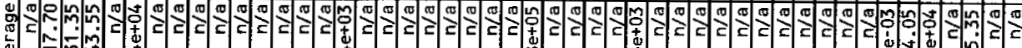
$2=5 m$ m

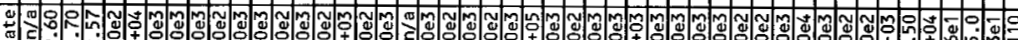

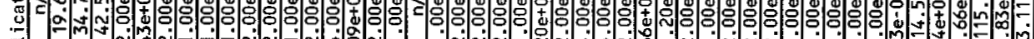
客

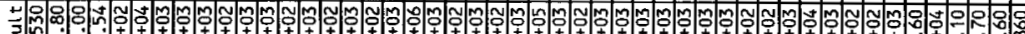

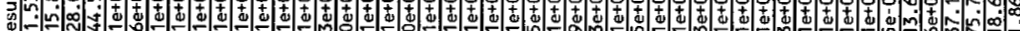

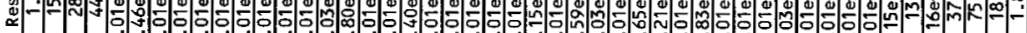

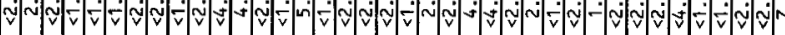

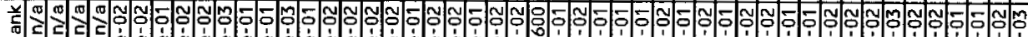
๑

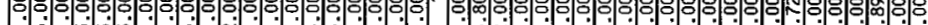

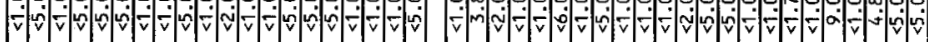

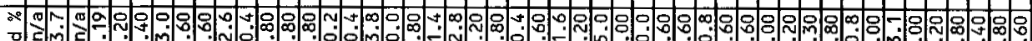
家 额

\section{त्रे}

의

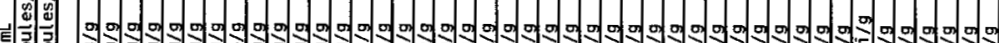

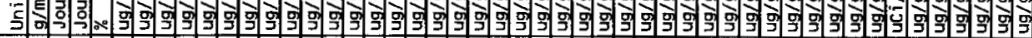

تี

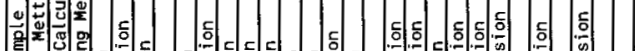

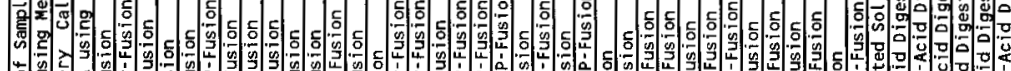

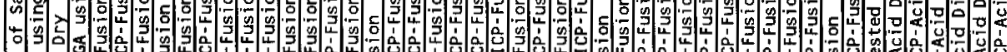

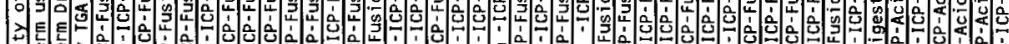

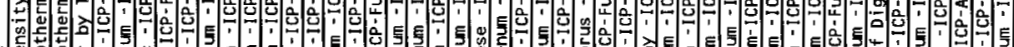

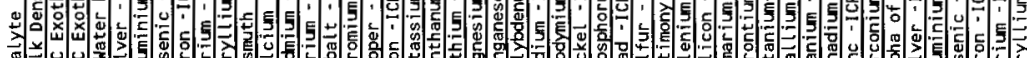

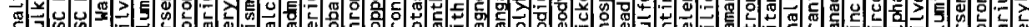

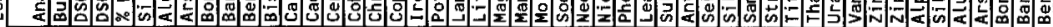

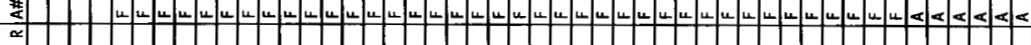


HNF-SD-WM-DP-199, REV. 1

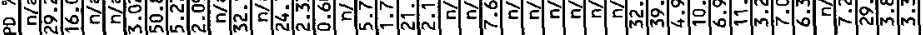

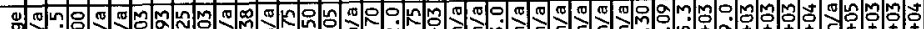

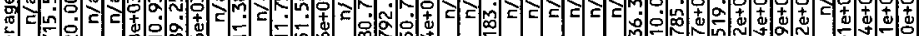

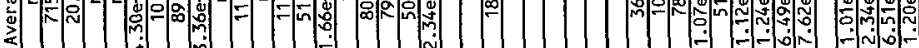

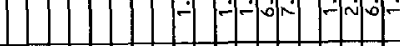

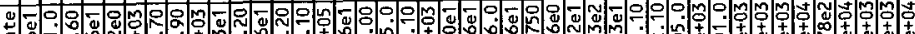

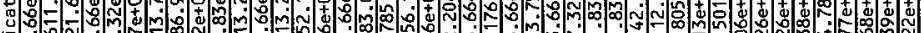
$=0$

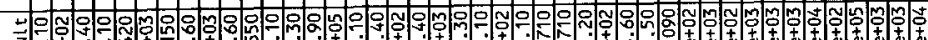

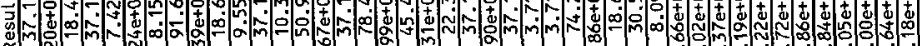

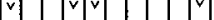

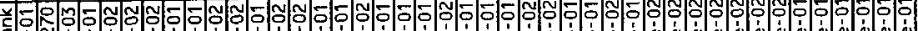

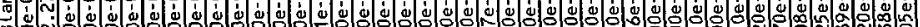
๓

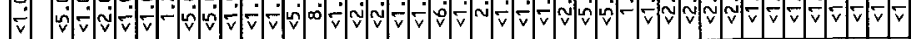

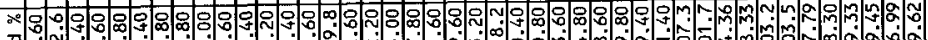
70

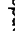
is

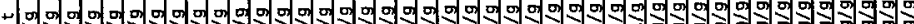

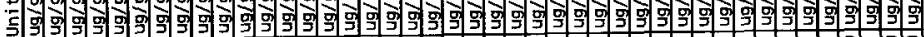

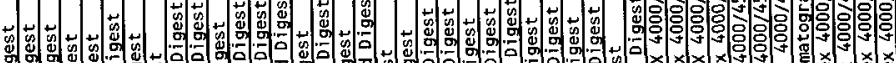

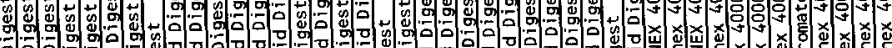

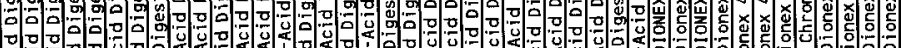

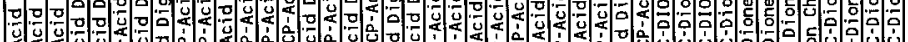

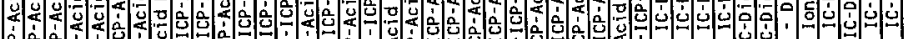

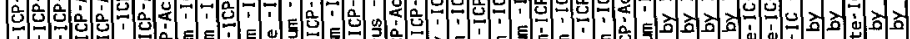

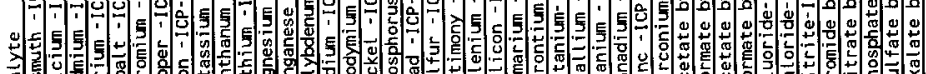
운

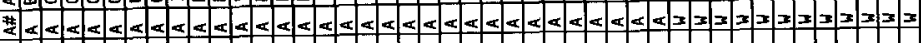

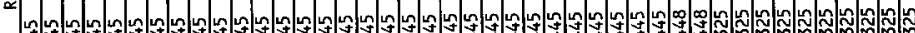




\section{HNF-SD-WM-DP-199, REV. 1}

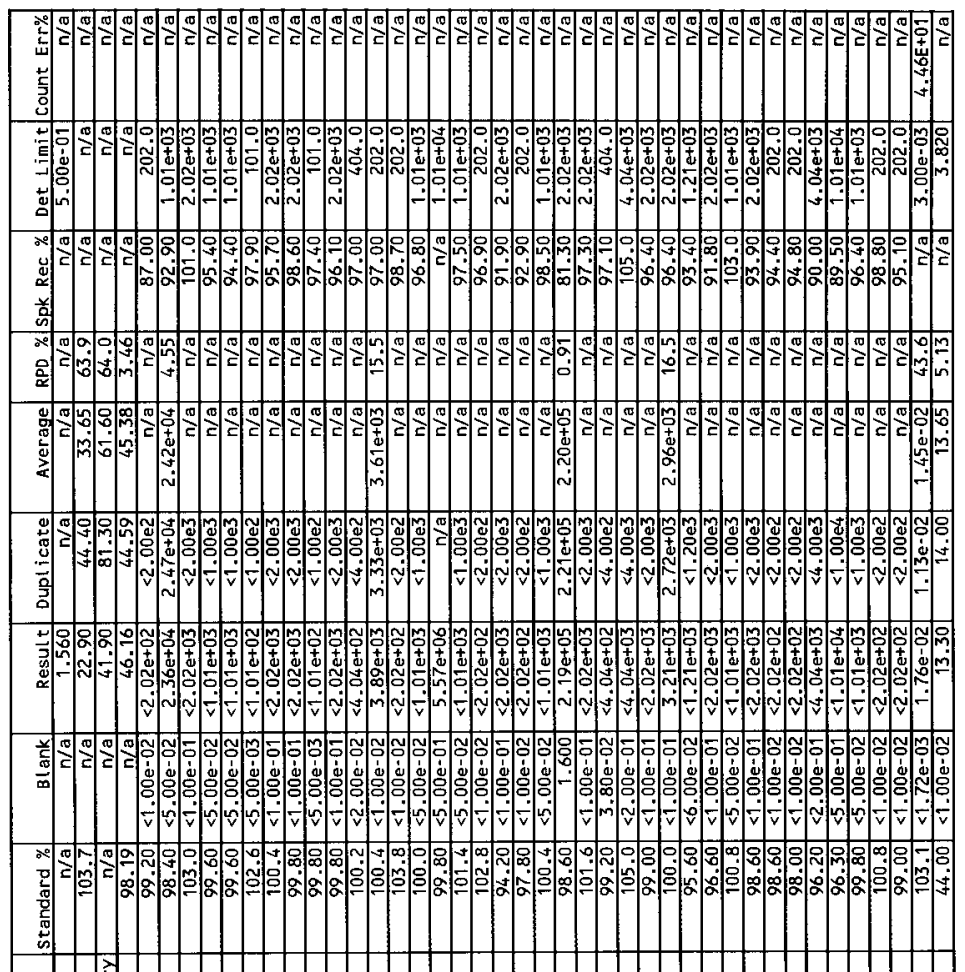

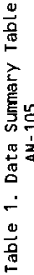

$140 \mathrm{O}$

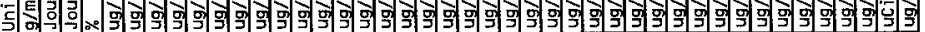

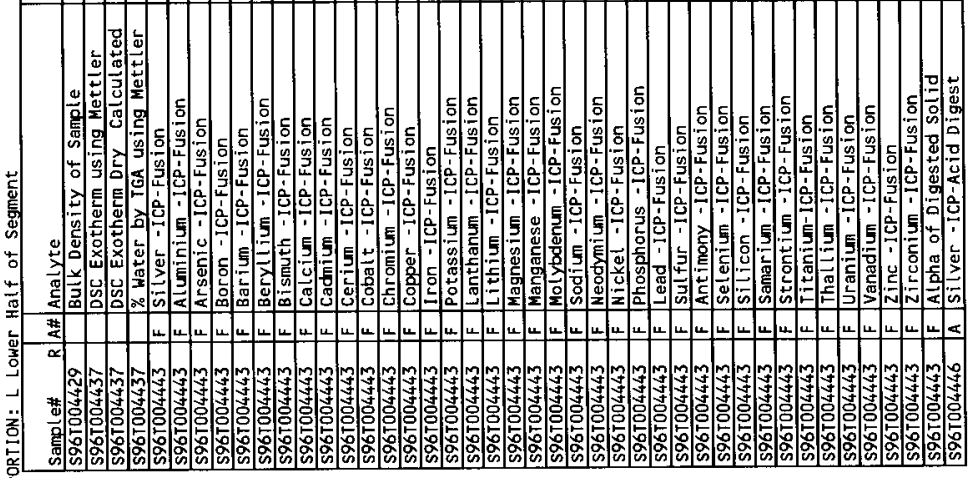


HNF-SD-WM-O - $99,-\because$.

WHC-SD-W/M-D $+1,12 E \mathrm{~V}, 1$

$\underset{8}{\$}$

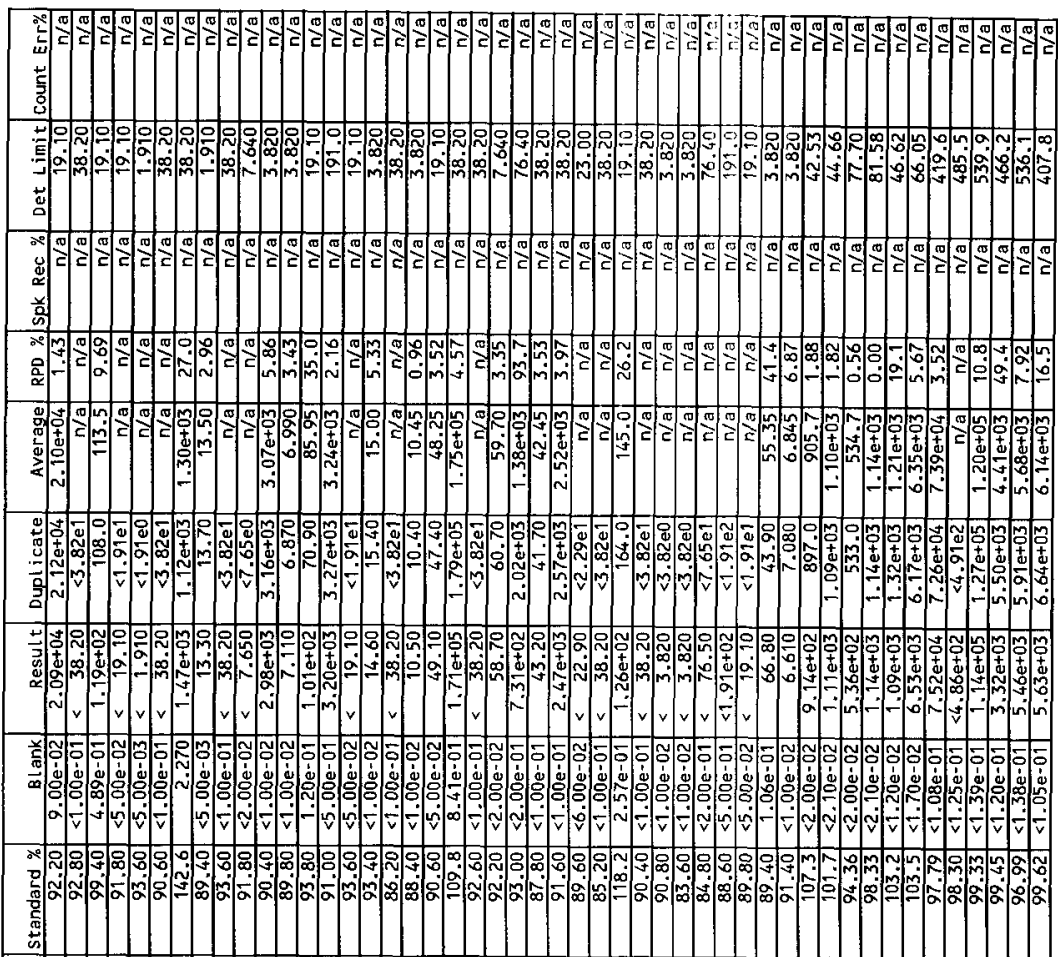

1090

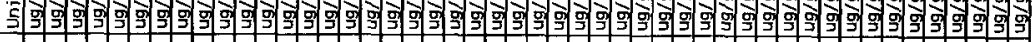

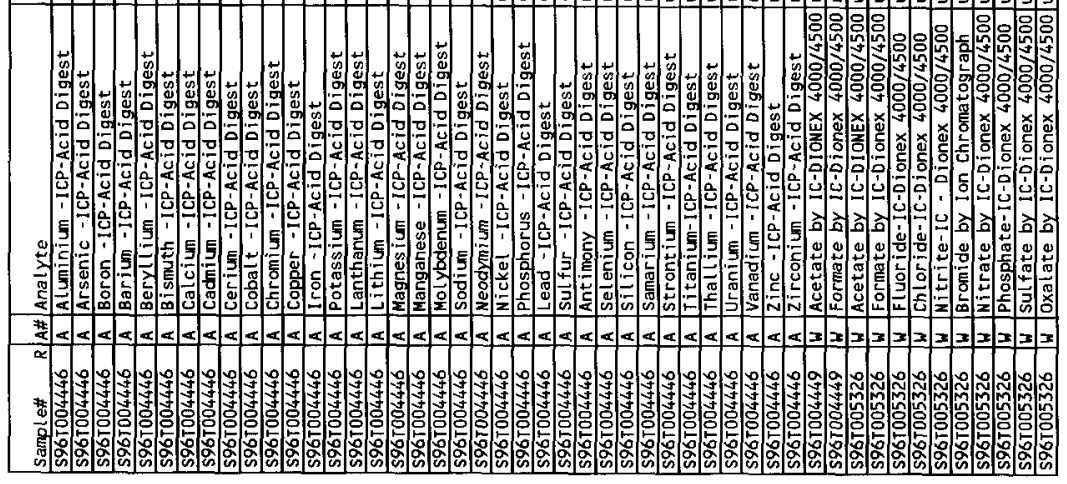


- 0 momm mom 自: 竞

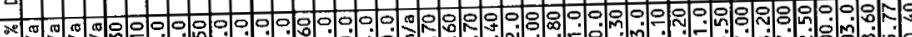
等

高

20m a

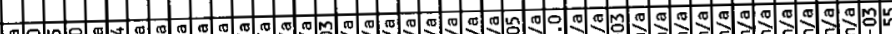

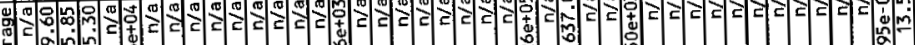
: $\frac{1}{4}+\infty$

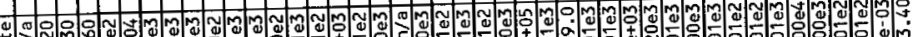
4 m

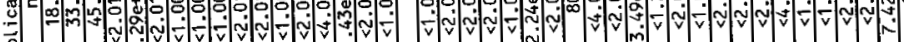
章

a

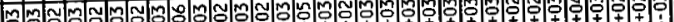

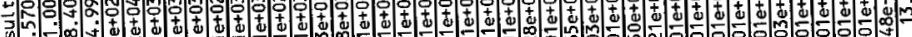
(-)

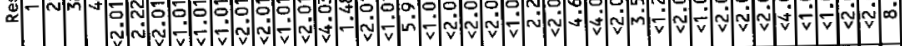
सबत्वत्व

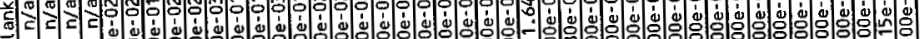
o ( D.

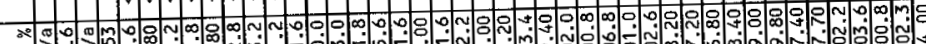
z c. is

ำ

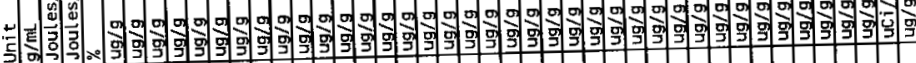

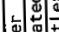

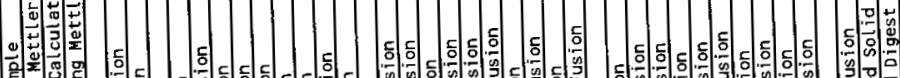

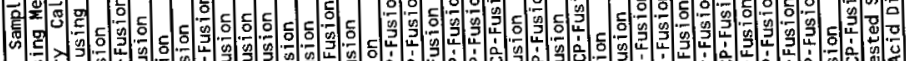

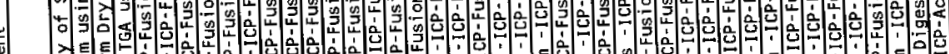

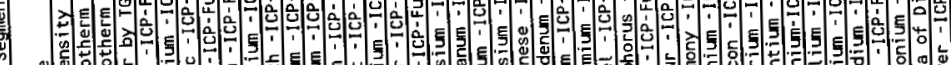

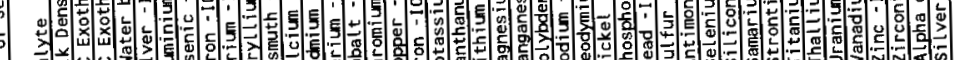

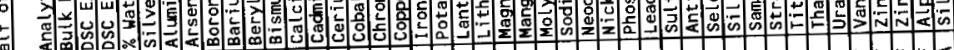

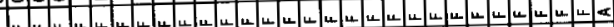
整

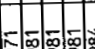<smiles>[Mg][AlH]</smiles>
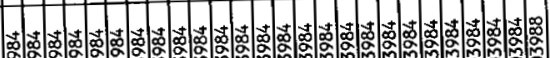


\section{HNF-SD-WM-DP-199, REV. 1}

$\underset{\ddot{0}}{\stackrel{0}{0}}$

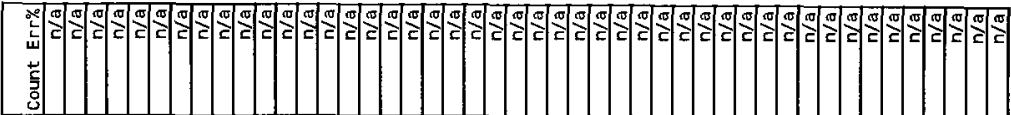

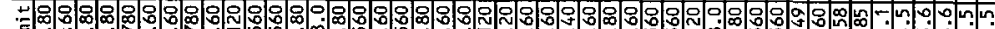

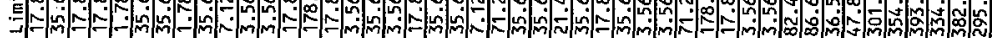
㟧

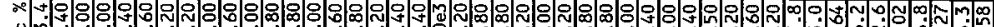

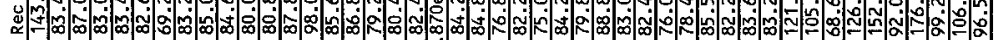
怠

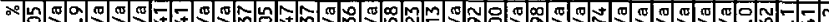

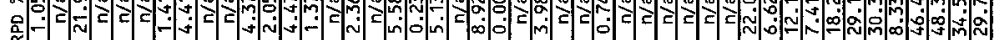

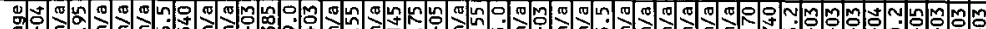

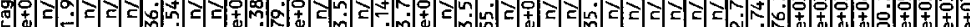
资高

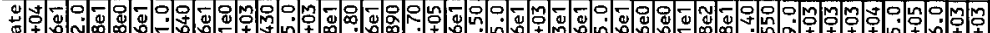

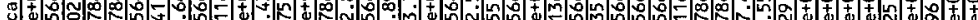

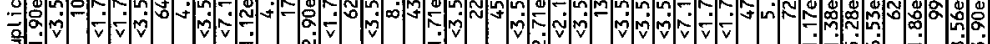
$-$

t40.0

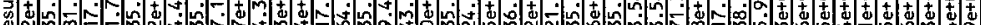

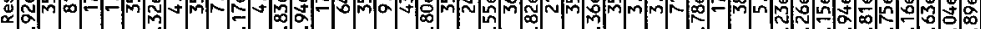

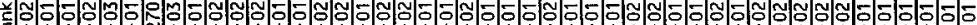

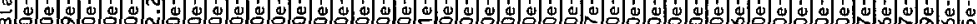
-

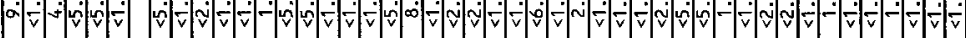

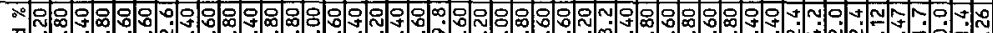

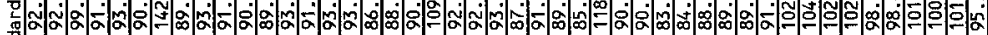
惫

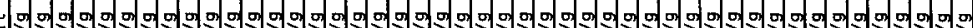

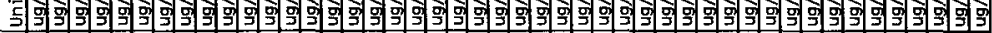

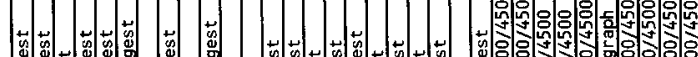

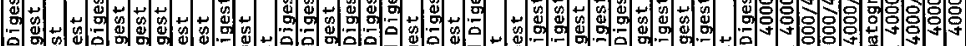
-

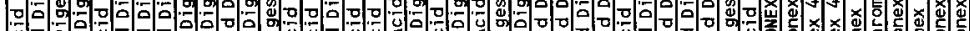

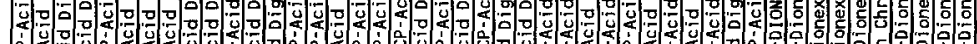

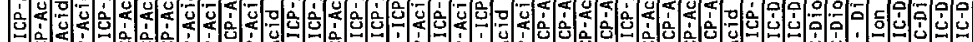
100

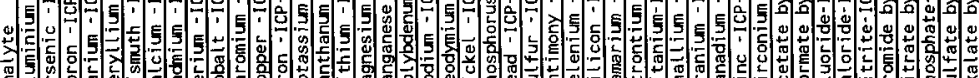
四

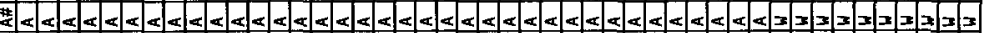




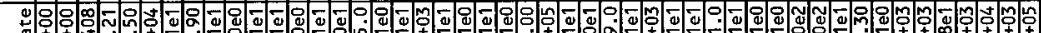

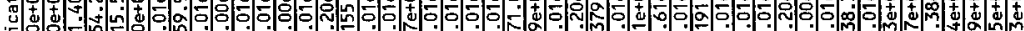

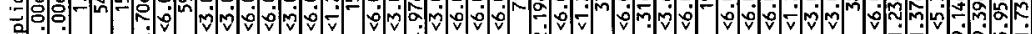
可.0

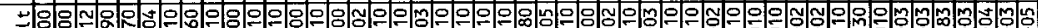

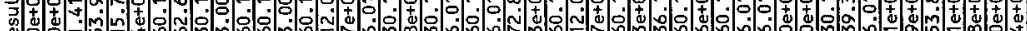
$\circ 0^{\circ}$

Ni

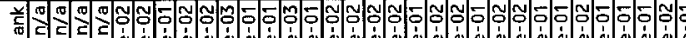

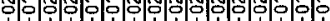
$\infty$

60

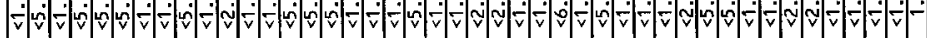

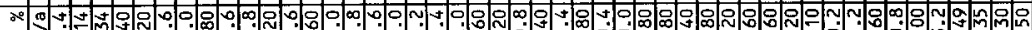

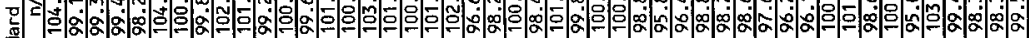

갛

9

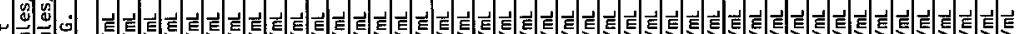

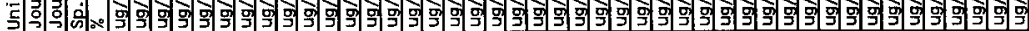

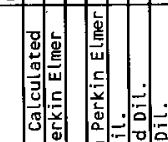

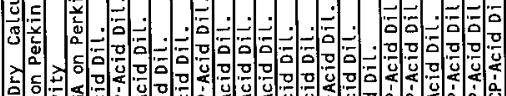

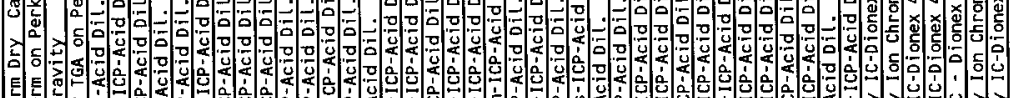

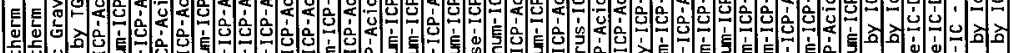

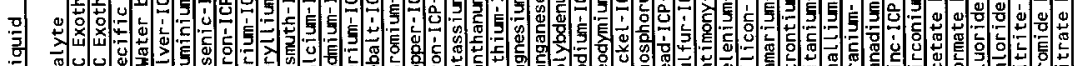

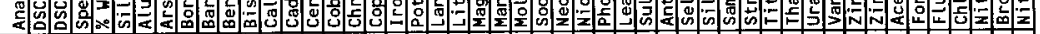

$\frac{a}{\ddot{q}}$

$\dddot{\because}$

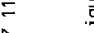

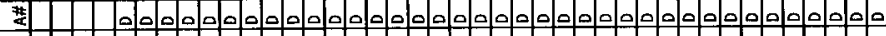

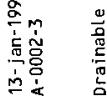


HNF-SD-WM-DP-199, REV. 1

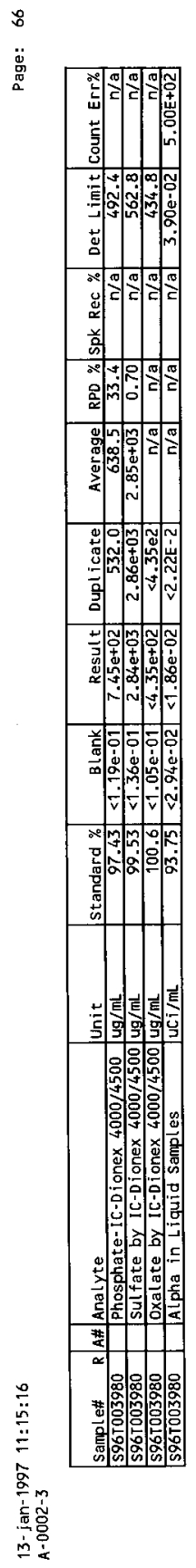

WHC-SD-WM-DP-199, REV. $?$ 
HNF-SD-WM-DP-199, REV. 1

-

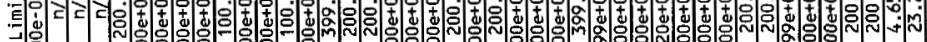

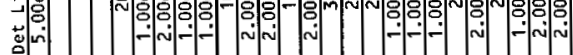

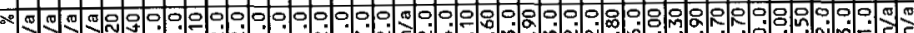

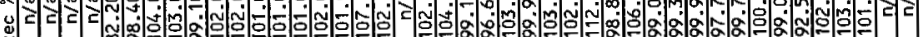
: 善

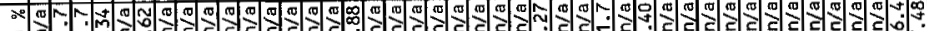
있 됭

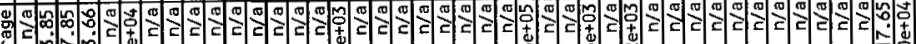

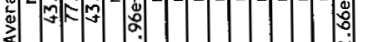

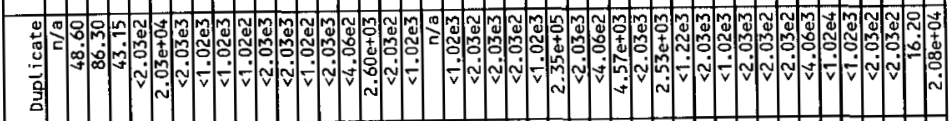

Hol

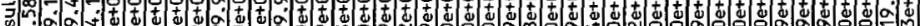
:

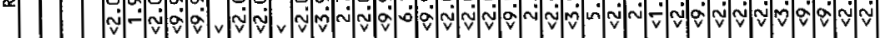

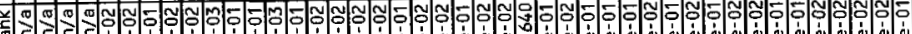

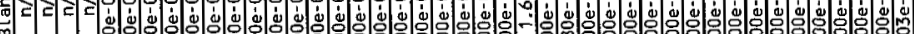

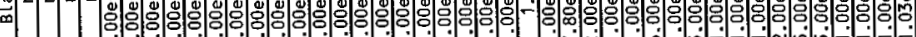

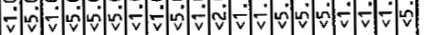

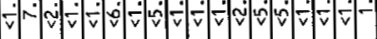

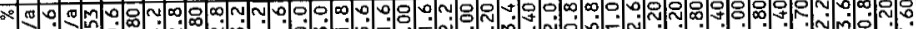
달 숭

ํํㅇ

$+$

요요

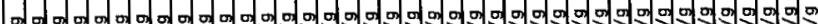

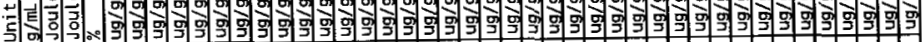

这这

(3)

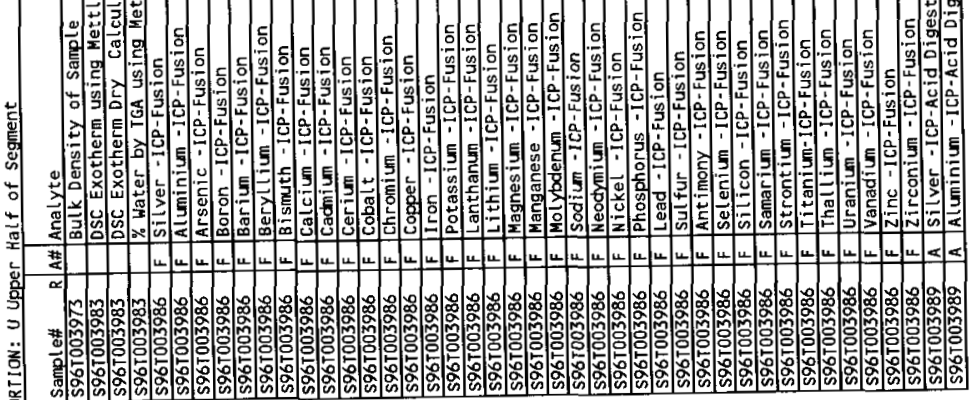




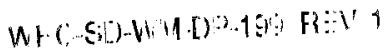

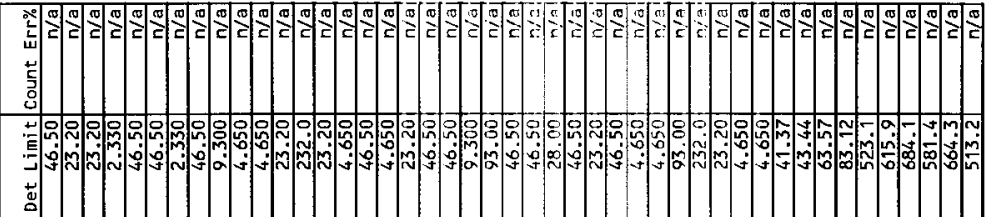

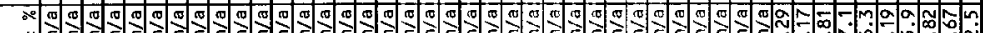

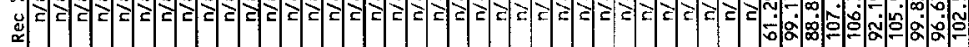

离 0
0

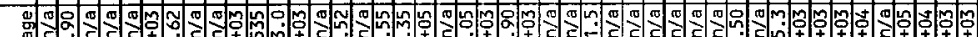

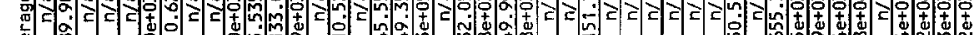

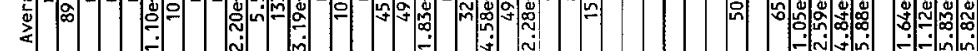

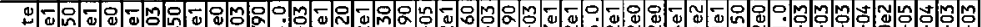

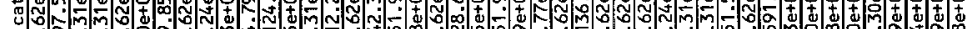

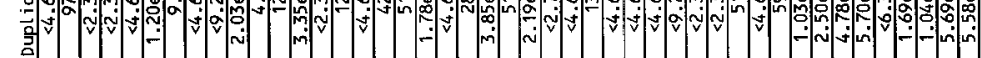

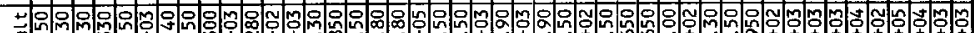

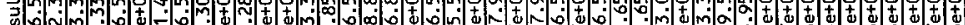

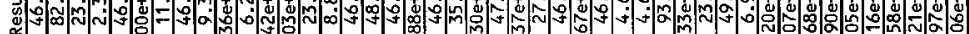

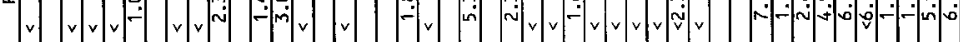

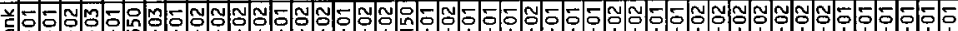
-

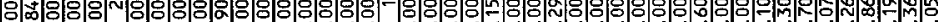

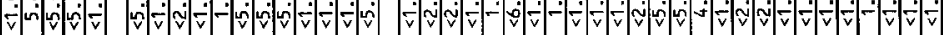

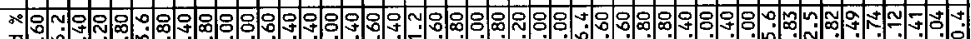
索 :

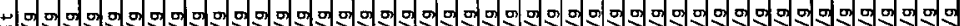

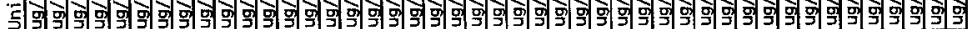

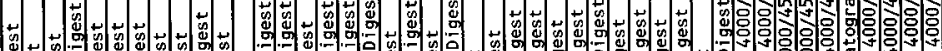

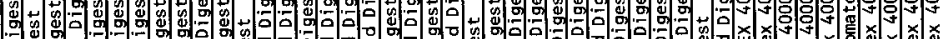

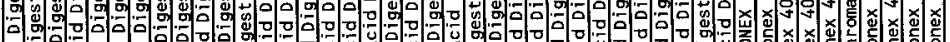
a 4.

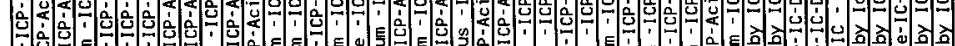
。

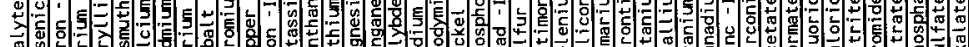

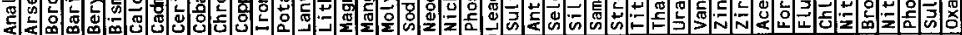

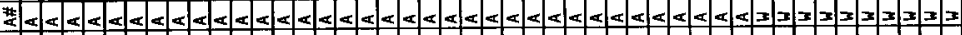

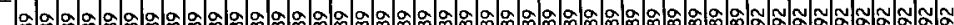

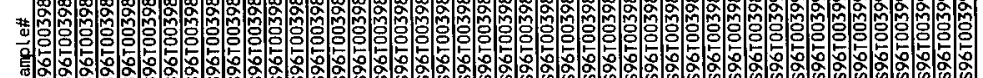

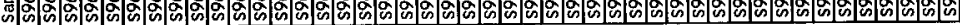


of 0

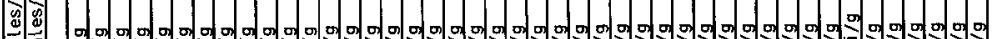

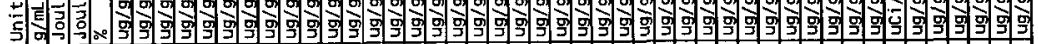

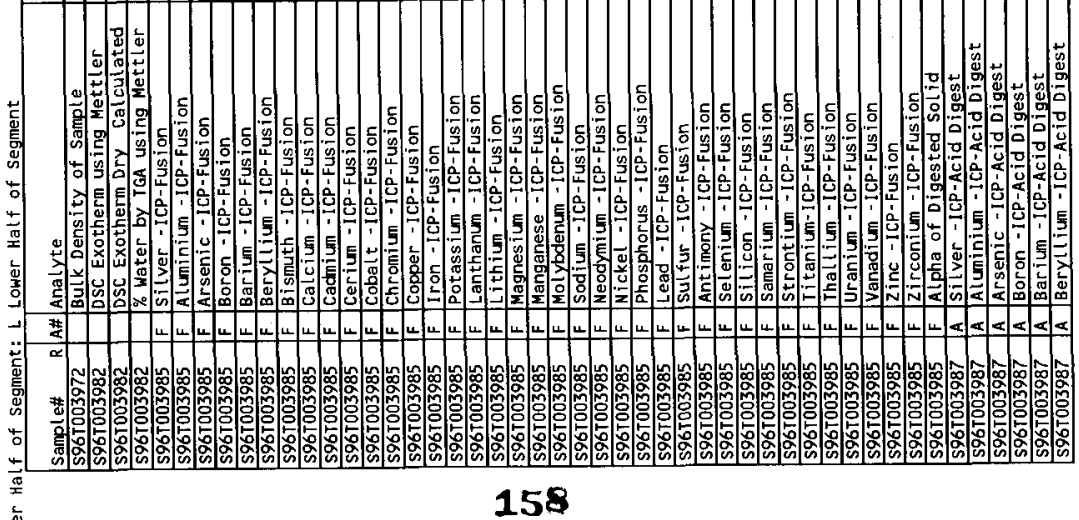


HNF-SD-WM-DP-199, REV. 1

R

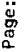

WHC-SD-WM-DP-199, REV. 1

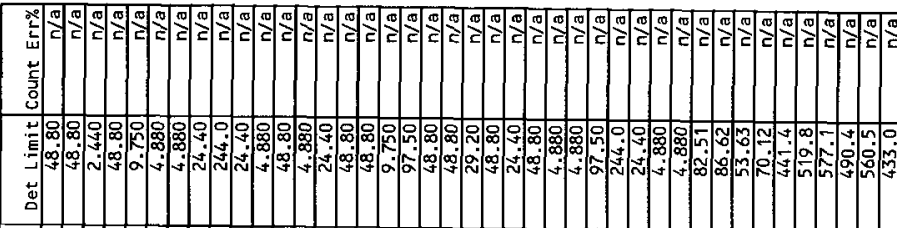

r

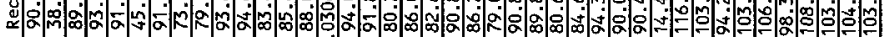
商

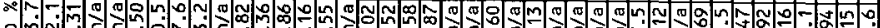
a

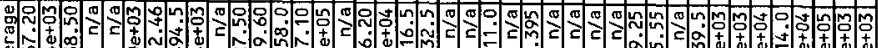

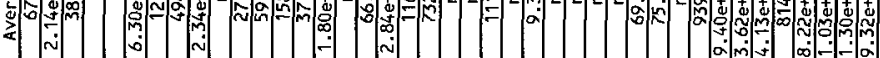

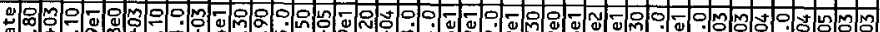

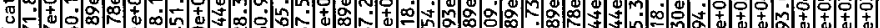

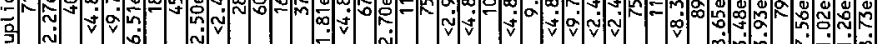

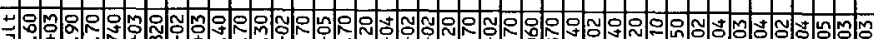

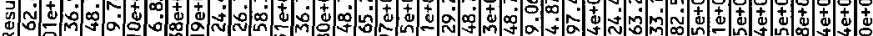

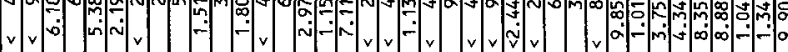

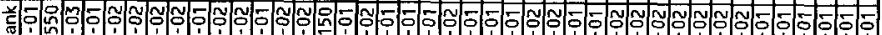

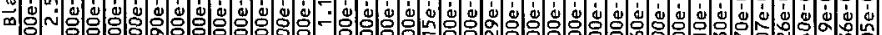

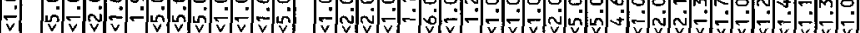

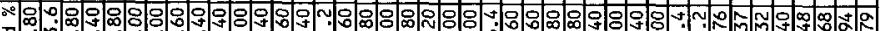

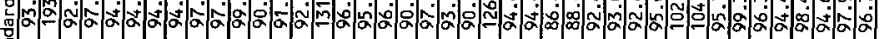
in

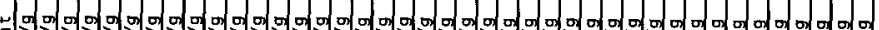

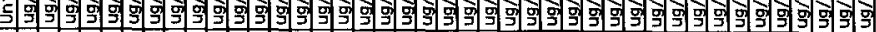

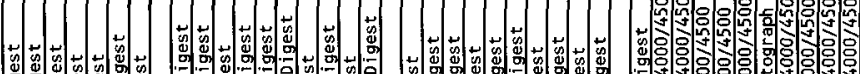

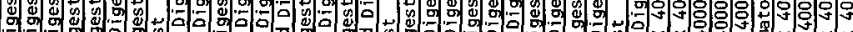

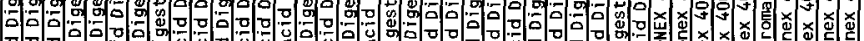

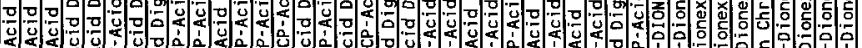

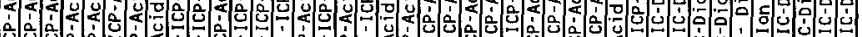

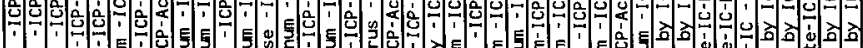

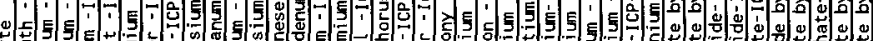

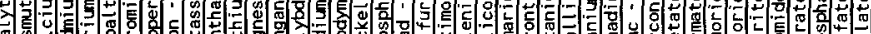

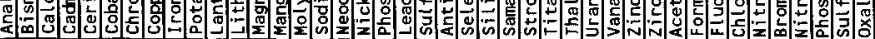

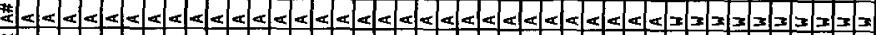

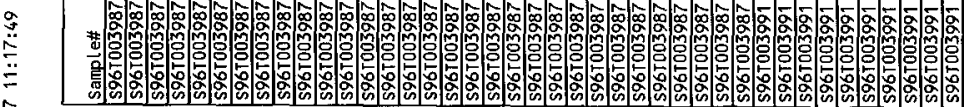
$\approx$

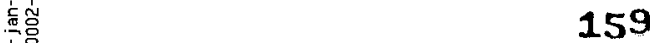

$m$ 

a

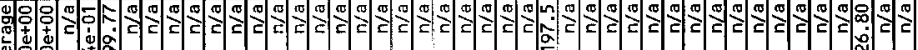

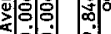

a. 2

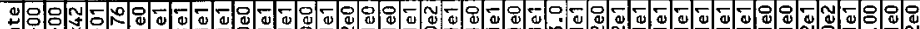
ฐ

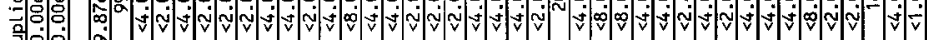

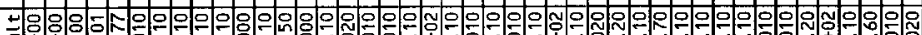

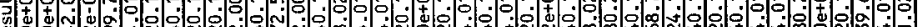
a

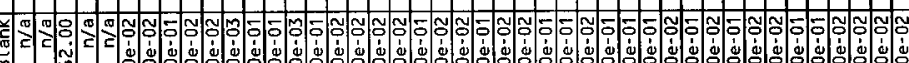

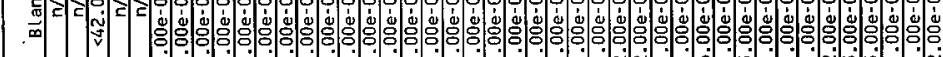

홀

에

क⿺辶大 등혀영

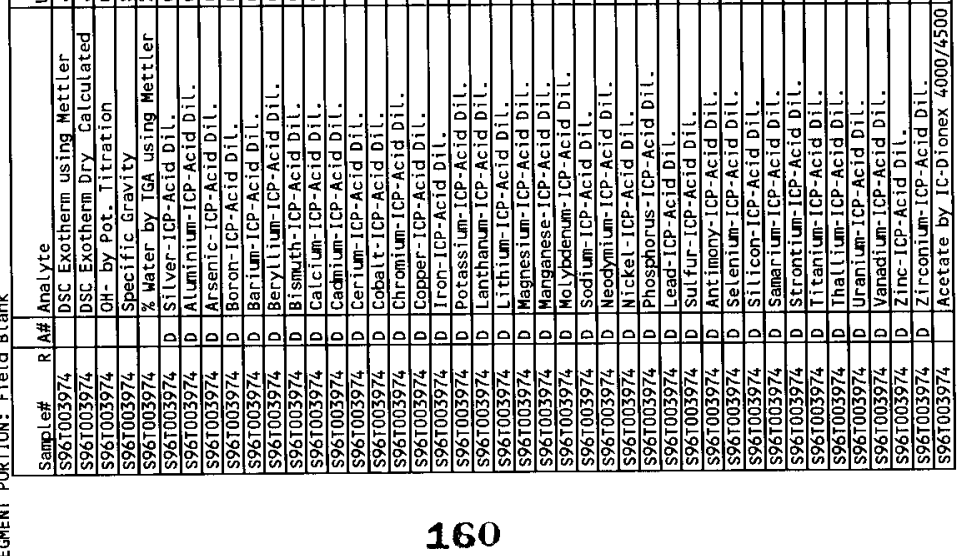


HNF-SD-WM-DP-199, REV. 1

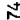

(3)

WHC-SD-WM-DP-199, REV. 1

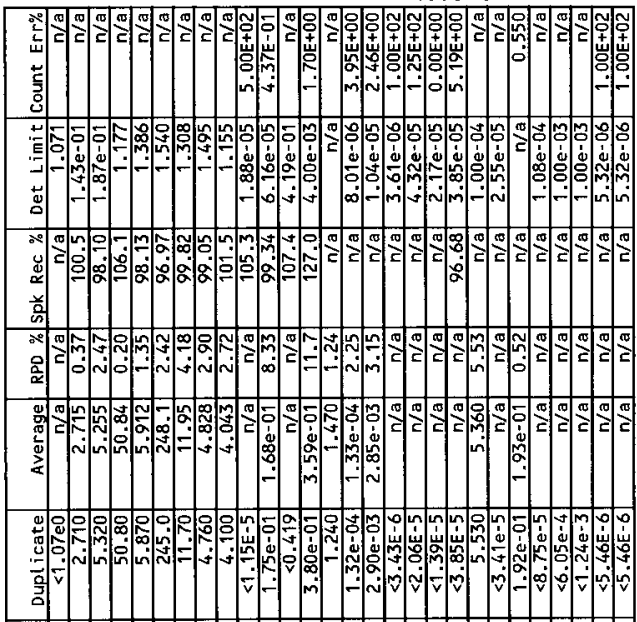

E- 0 -

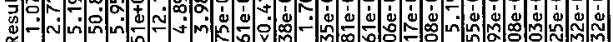

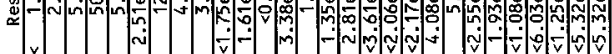
弟 중- - -

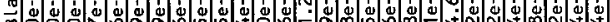

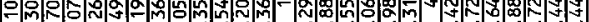

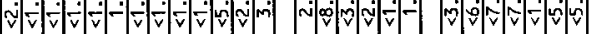
0
$\frac{m}{2}$
0

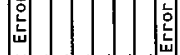

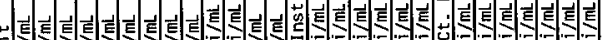

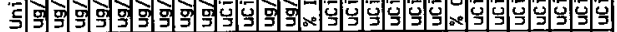

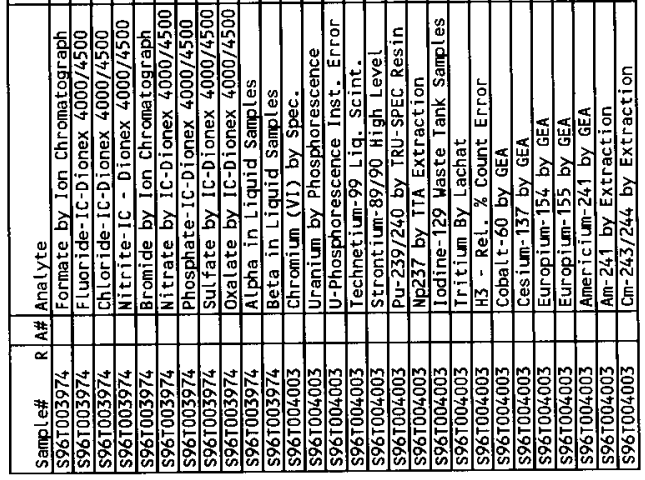

a

동 
HNF-SD-WM-DP-199, REV. 1

WHC-SD-WM-DP-199, REV. 1

$\ddot{a}$

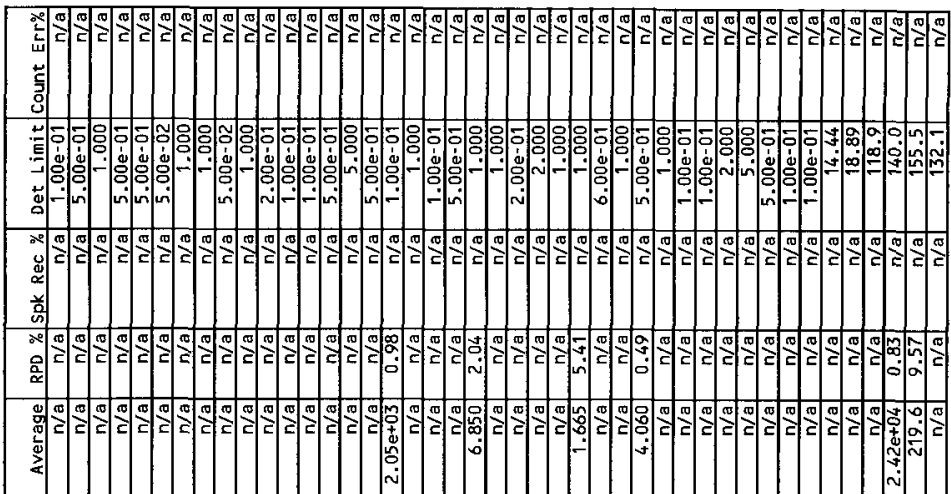

y-

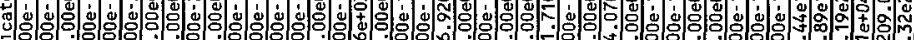

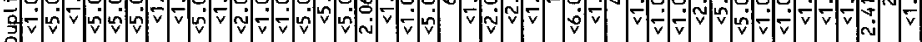

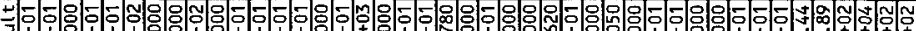

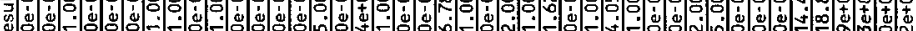
Q

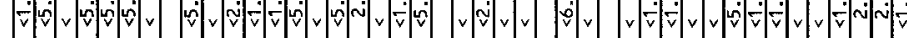
政0

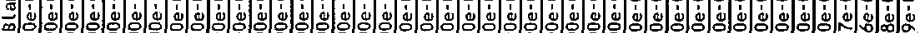

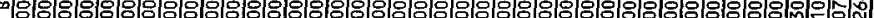

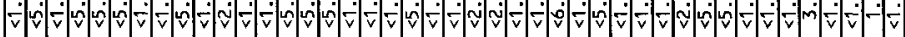

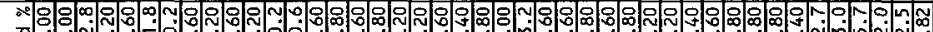
7.

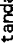

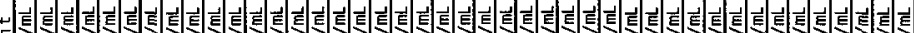

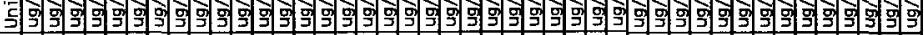

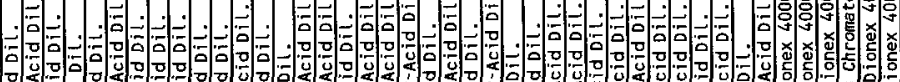
a

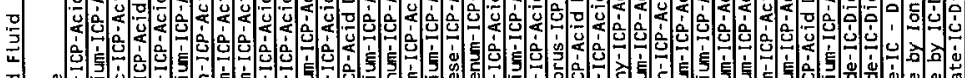

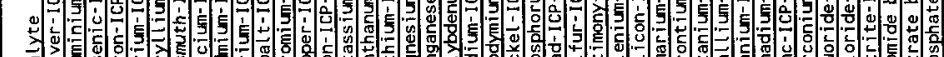

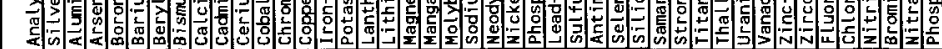
年

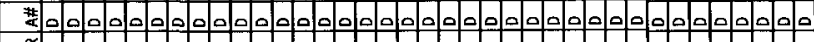

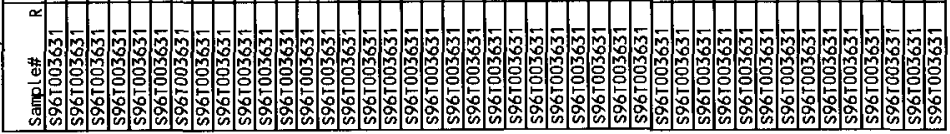


HNF-SD-WM-DP-199, REV. 1

$N$

茄

WHC-SD-WM-DP-199, REV. *

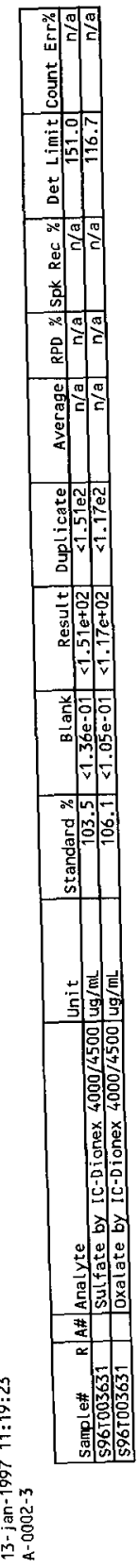




\section{HNF-SD-WM-DP-199, REV. 1}

WHC-SD-WM-DP-199, REV. 1

\section{PHOTOGRAPHS}




\section{HNF-SD-WM-DP-199, REV. 1}

WHC-SD-WM-DP-199, REV . 1

\section{THIS PAGE WAS INTENTIONALLY LEFT BLANK}

\subsection{5}




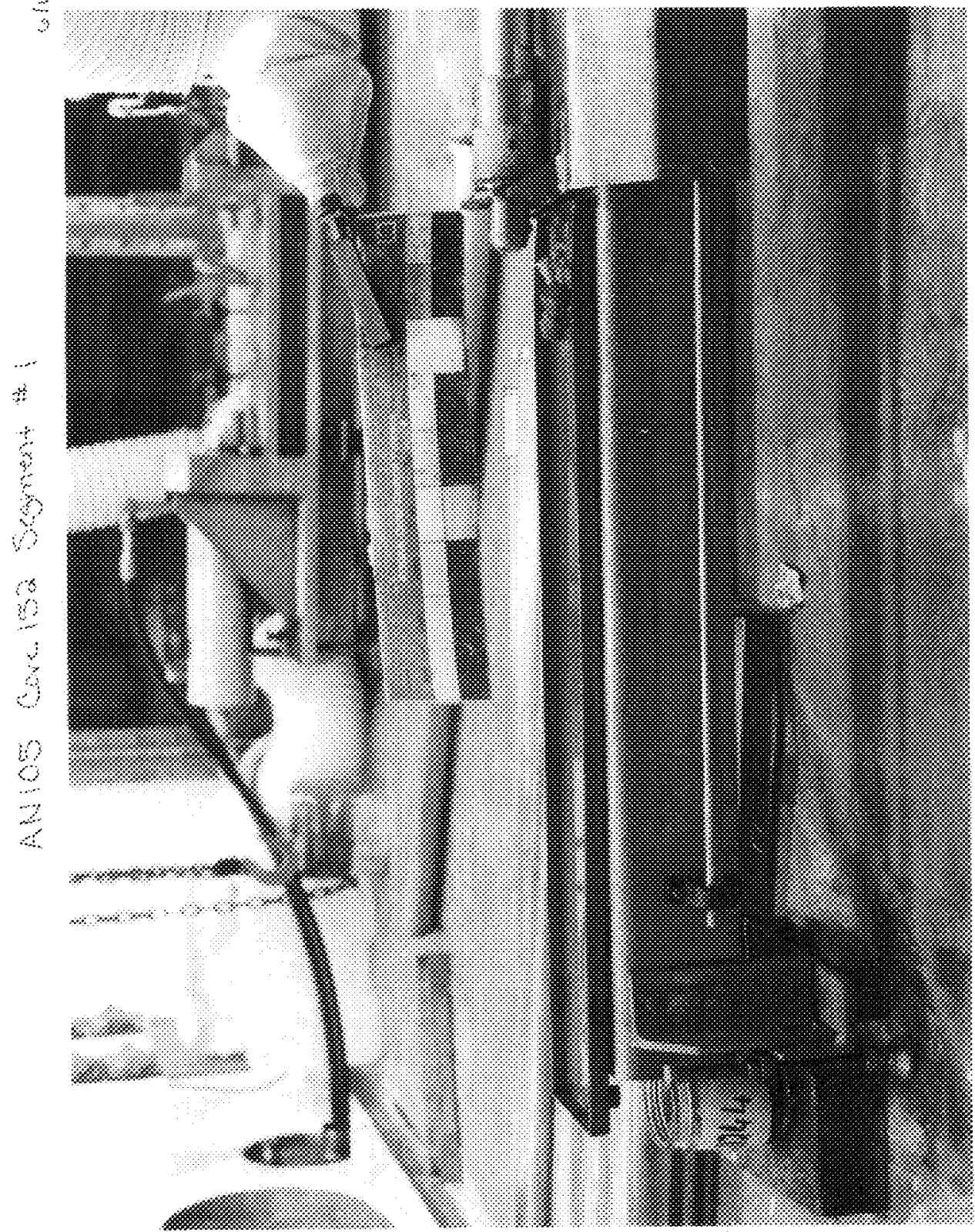




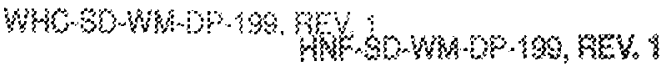

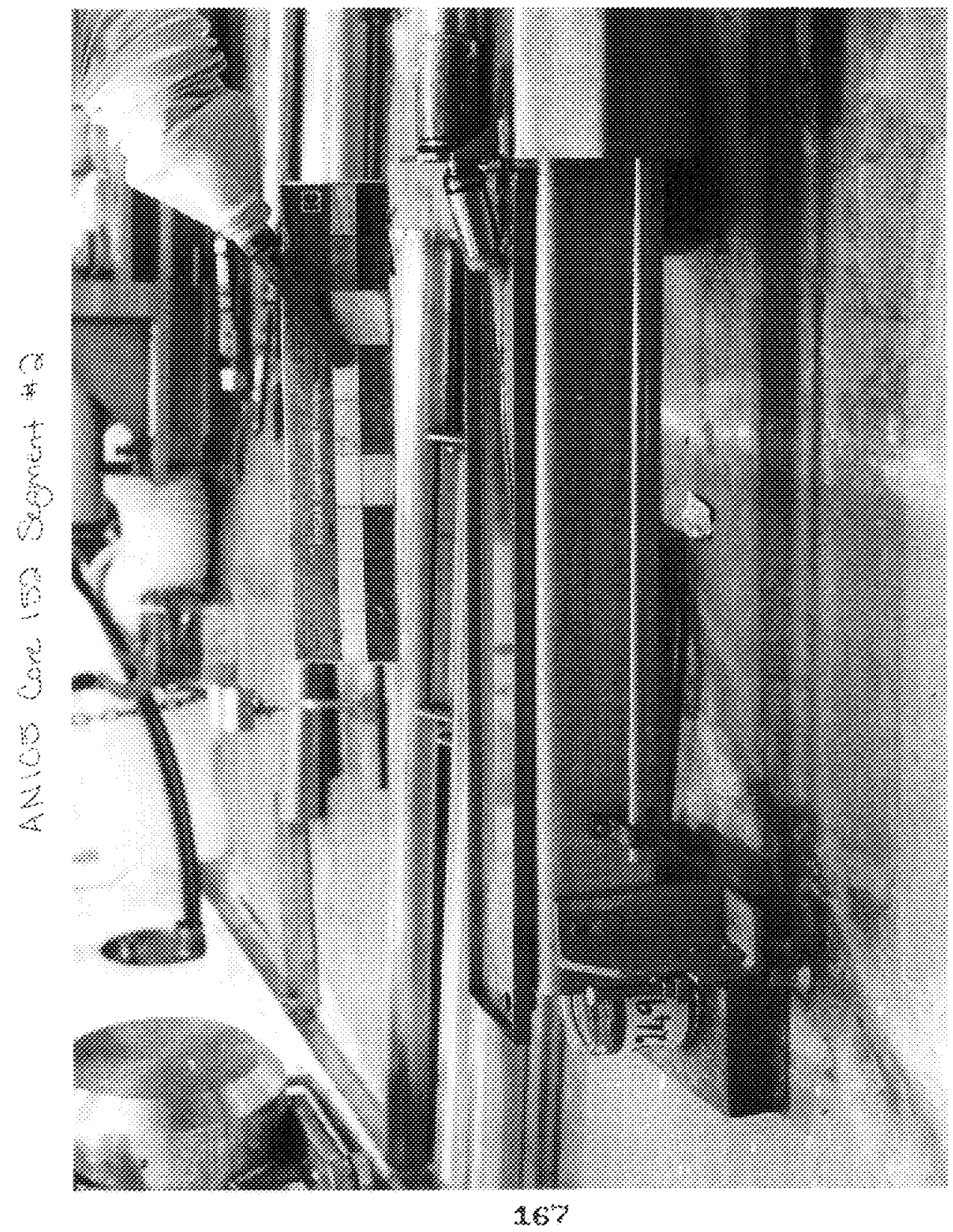



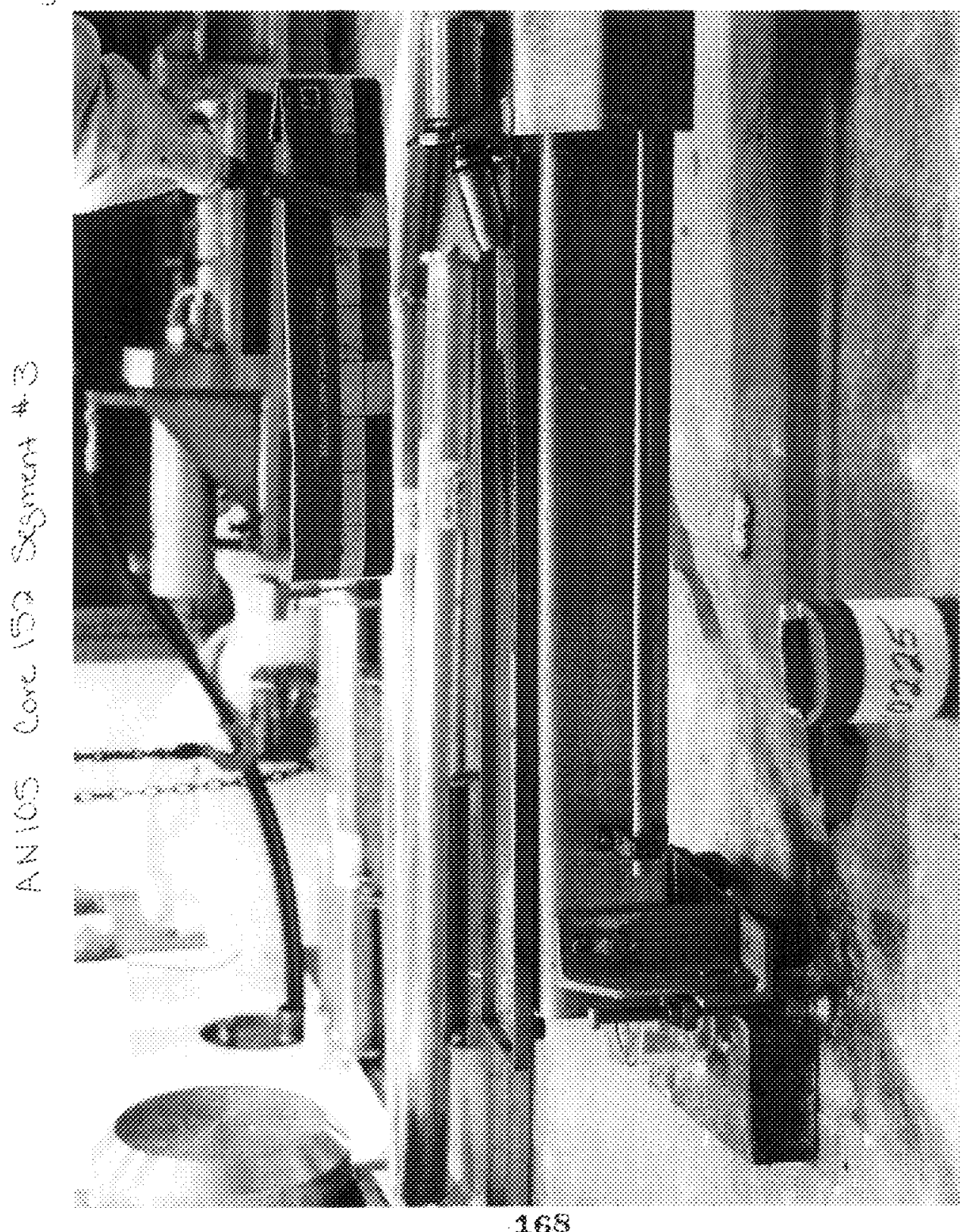


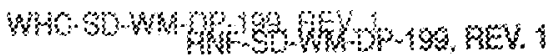

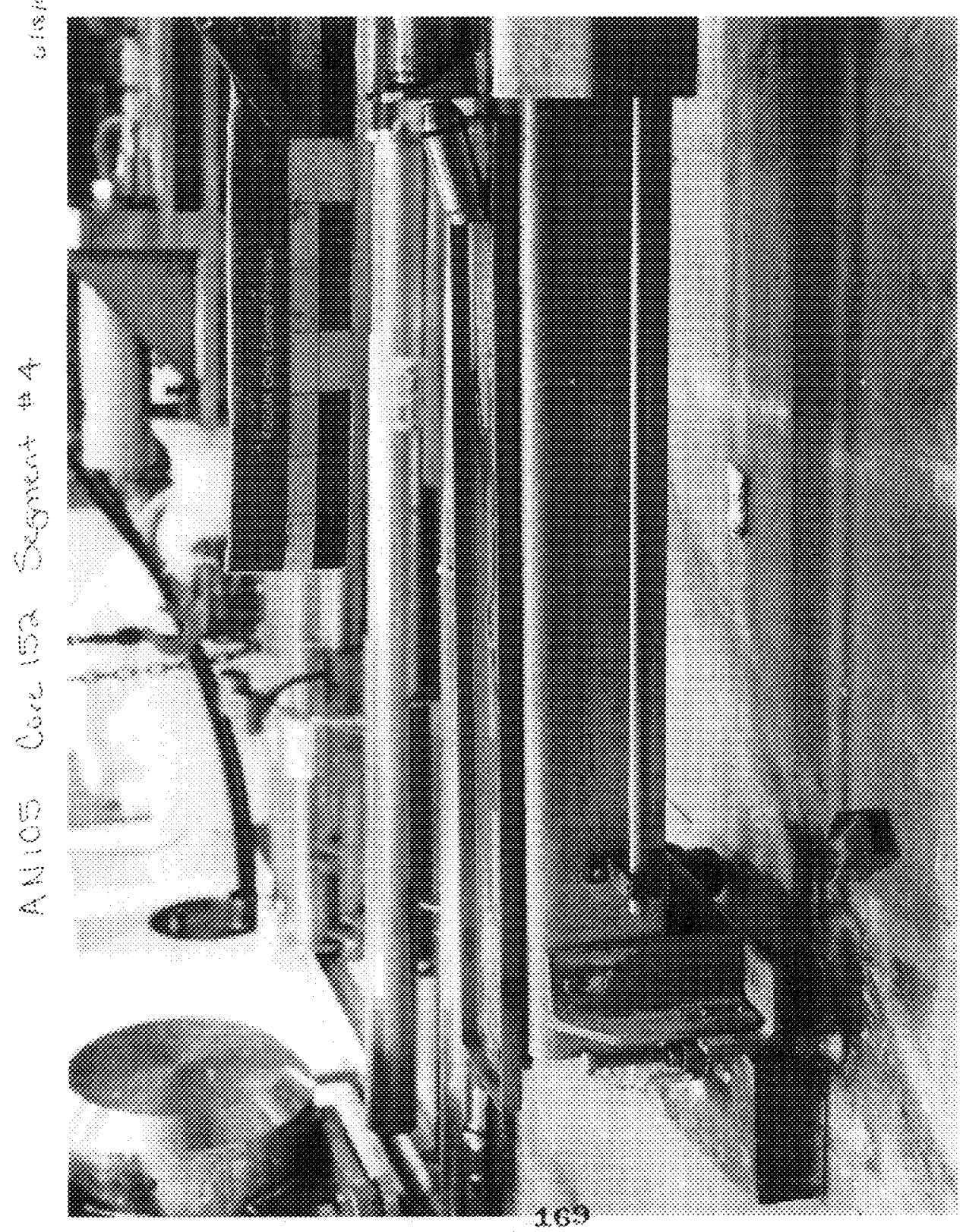




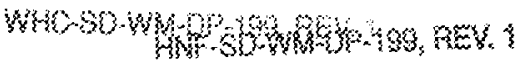

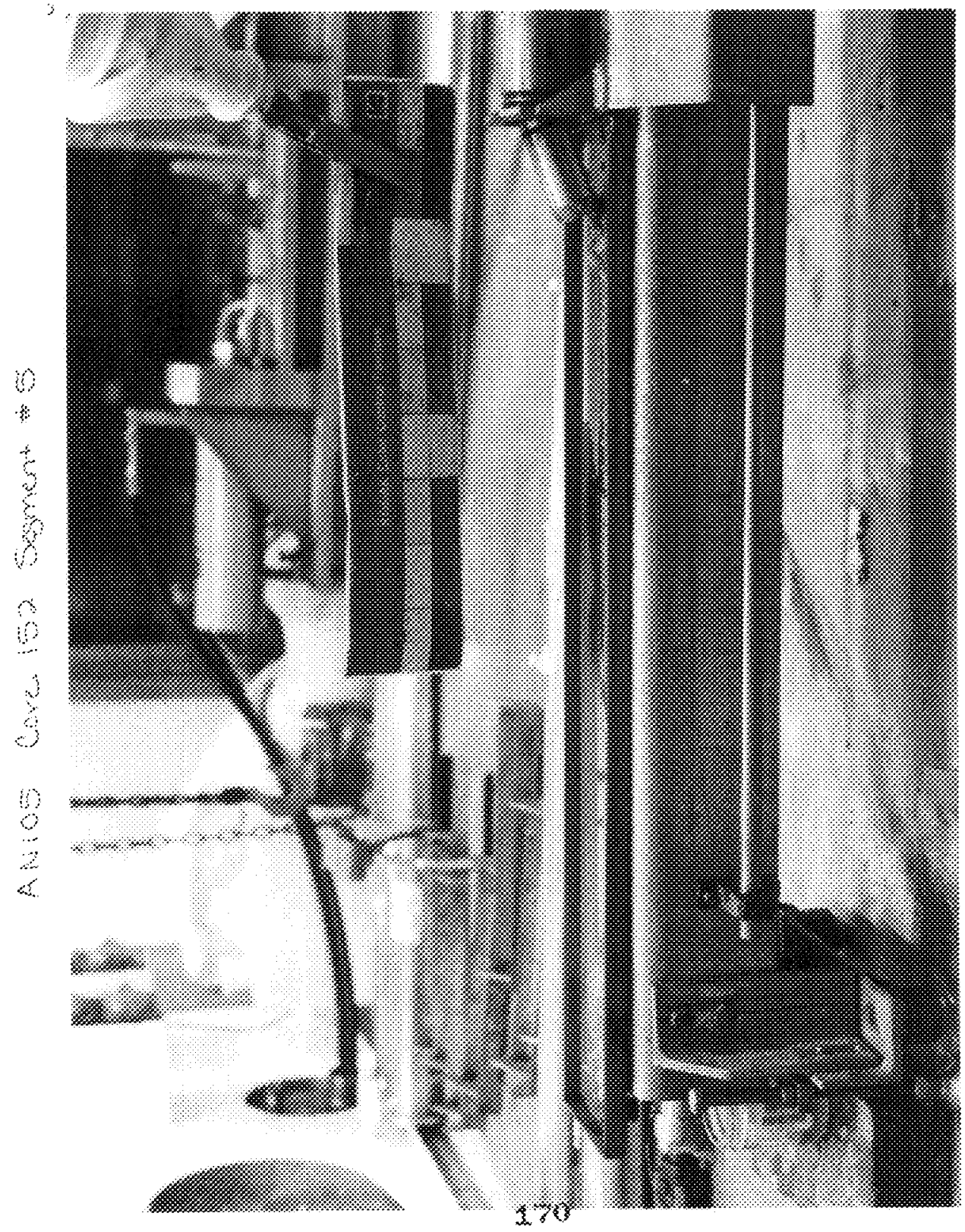




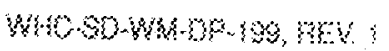

$\because$

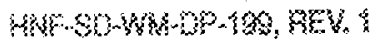

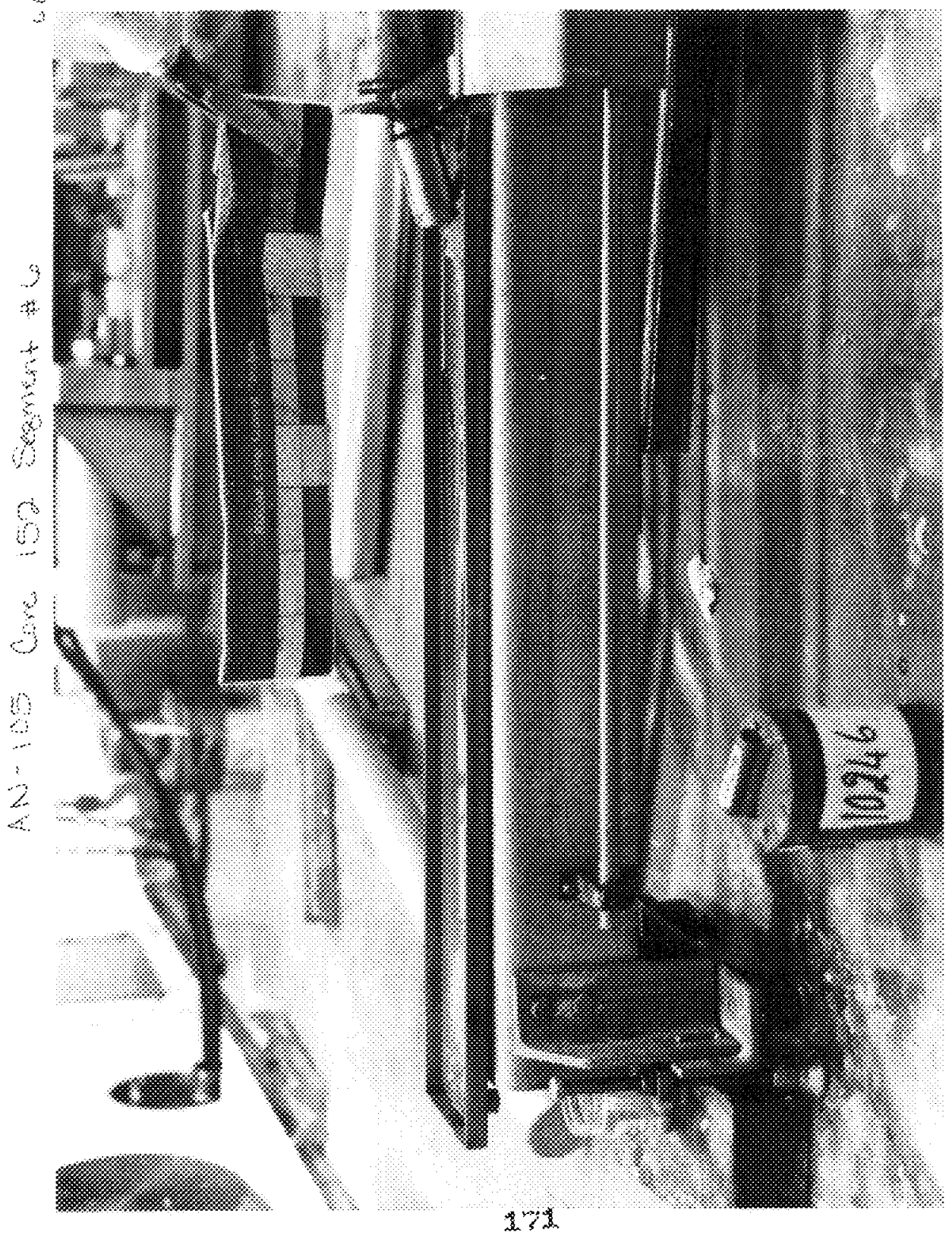


Wh-SWH 19,5

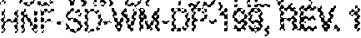

3

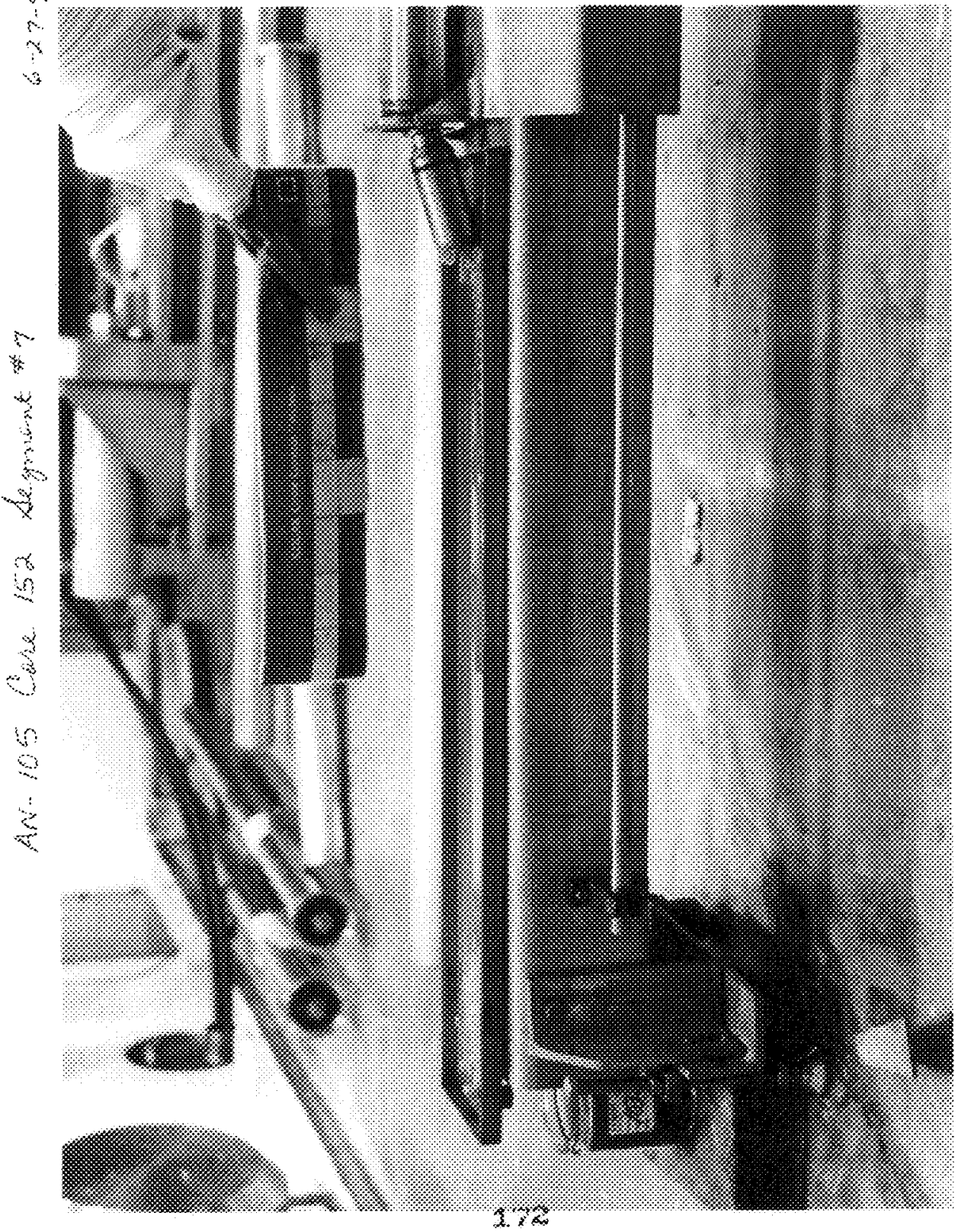


whe-

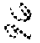

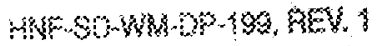

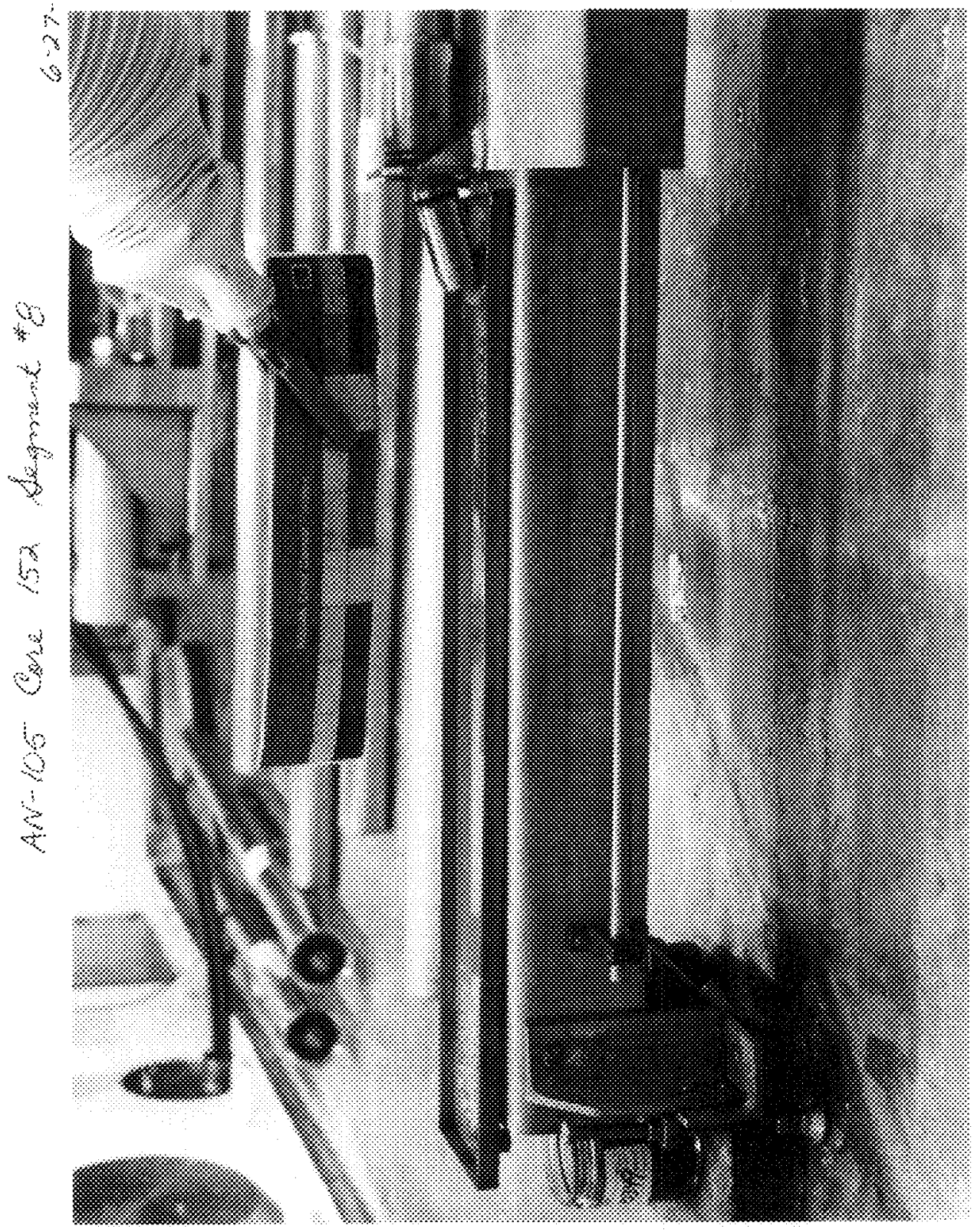


WHOSWMO 399 MEV

$\infty$ HWSO

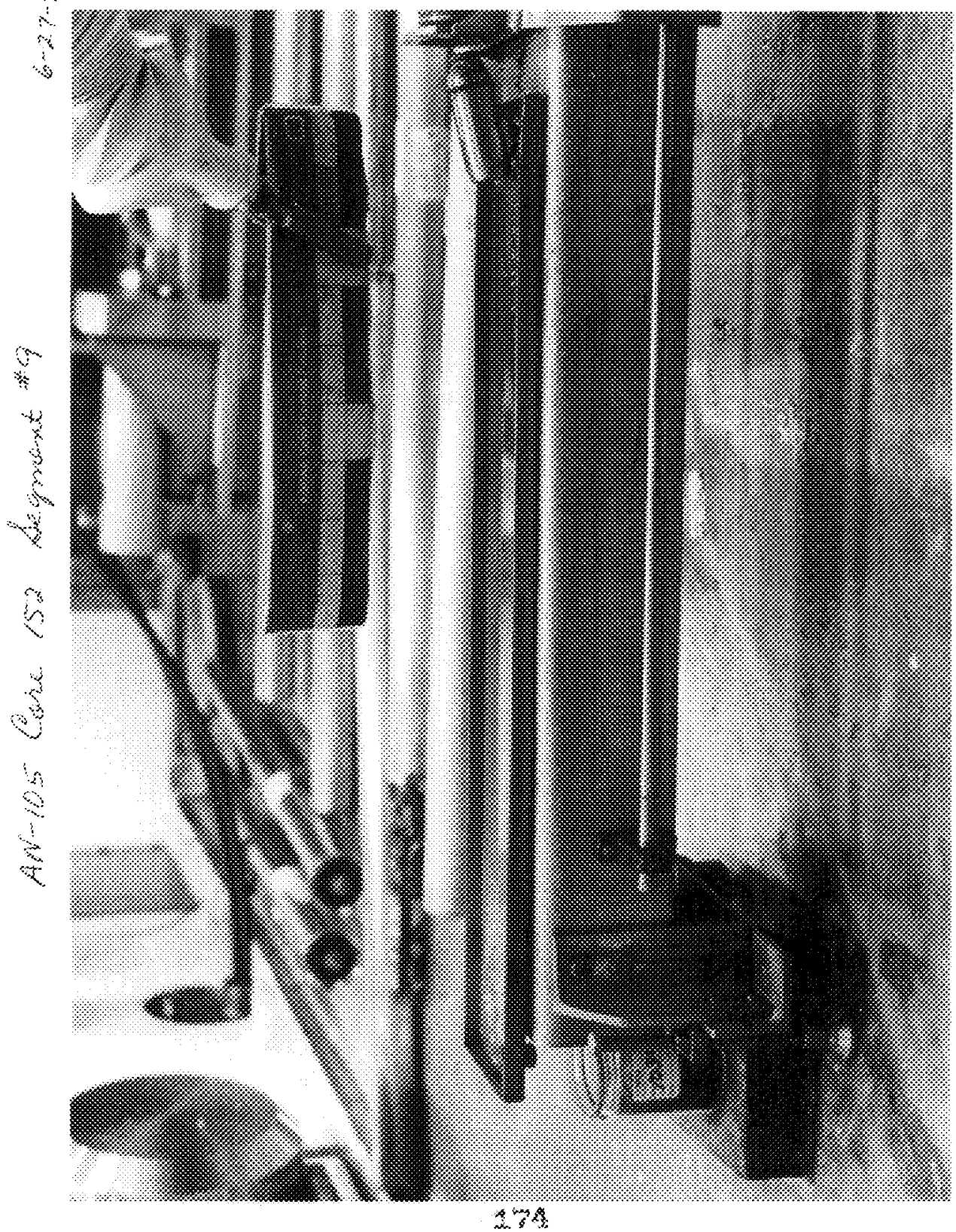


Wh-60\%

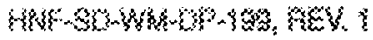

s

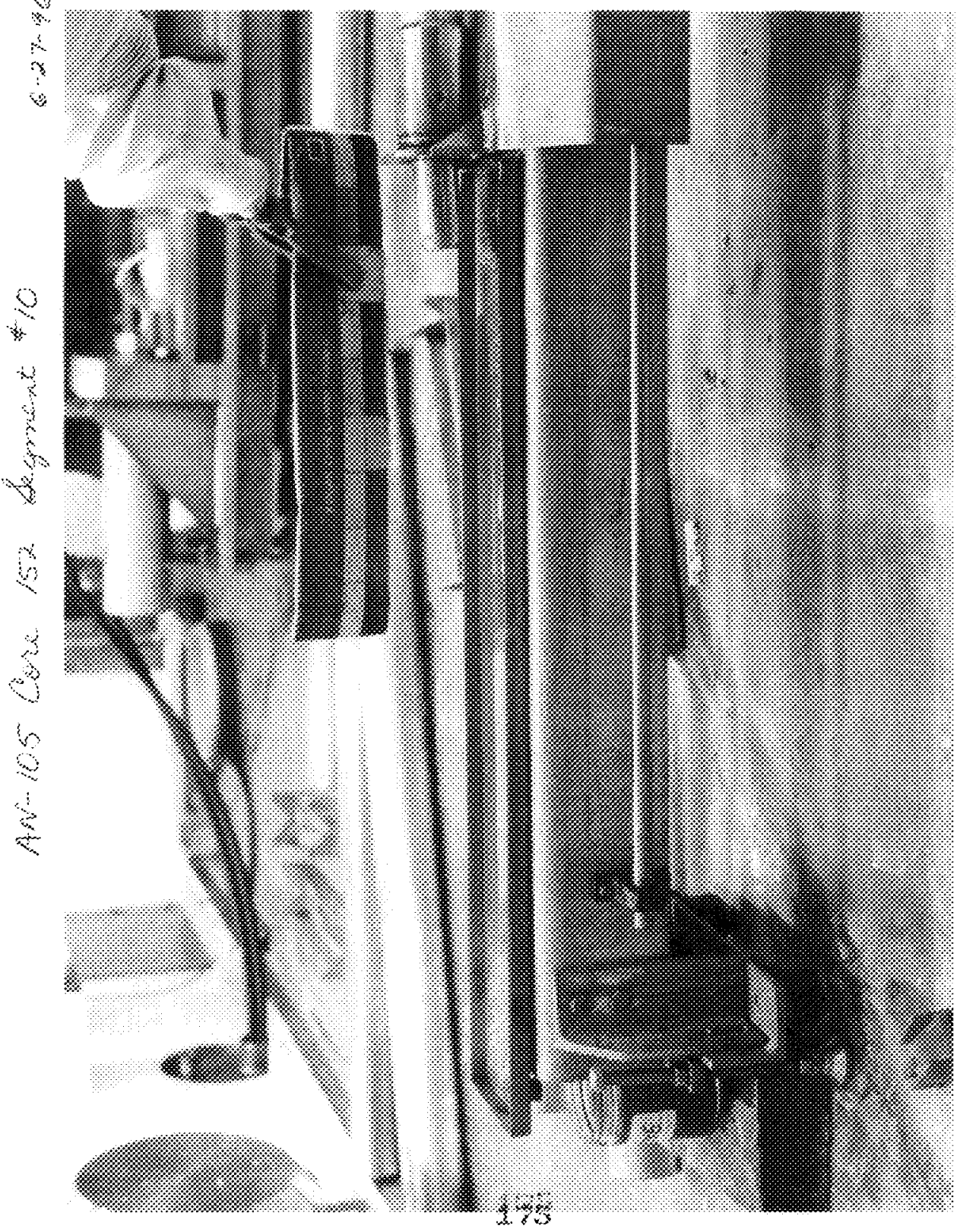


WHOSOWh

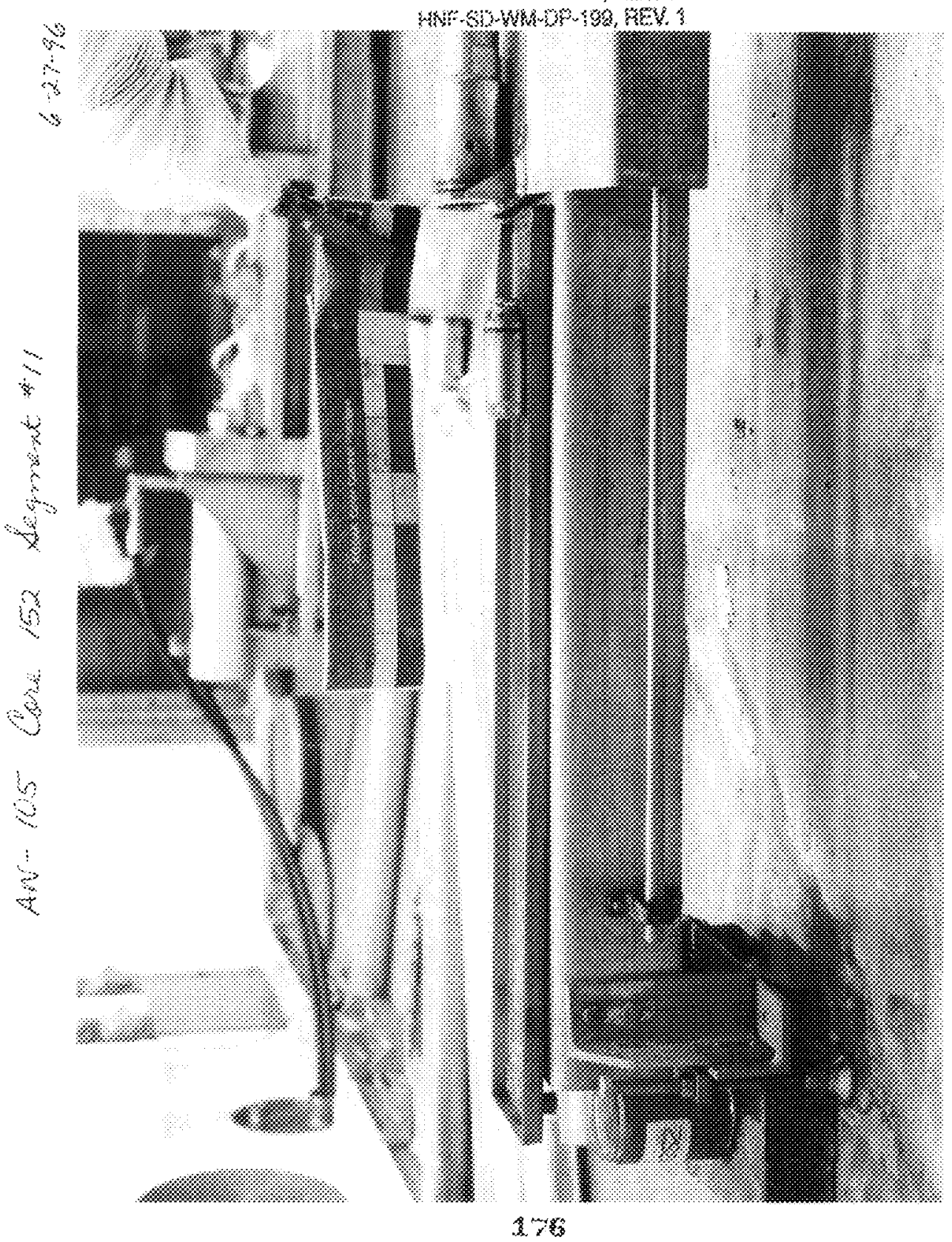




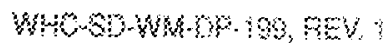

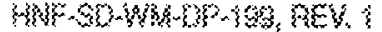

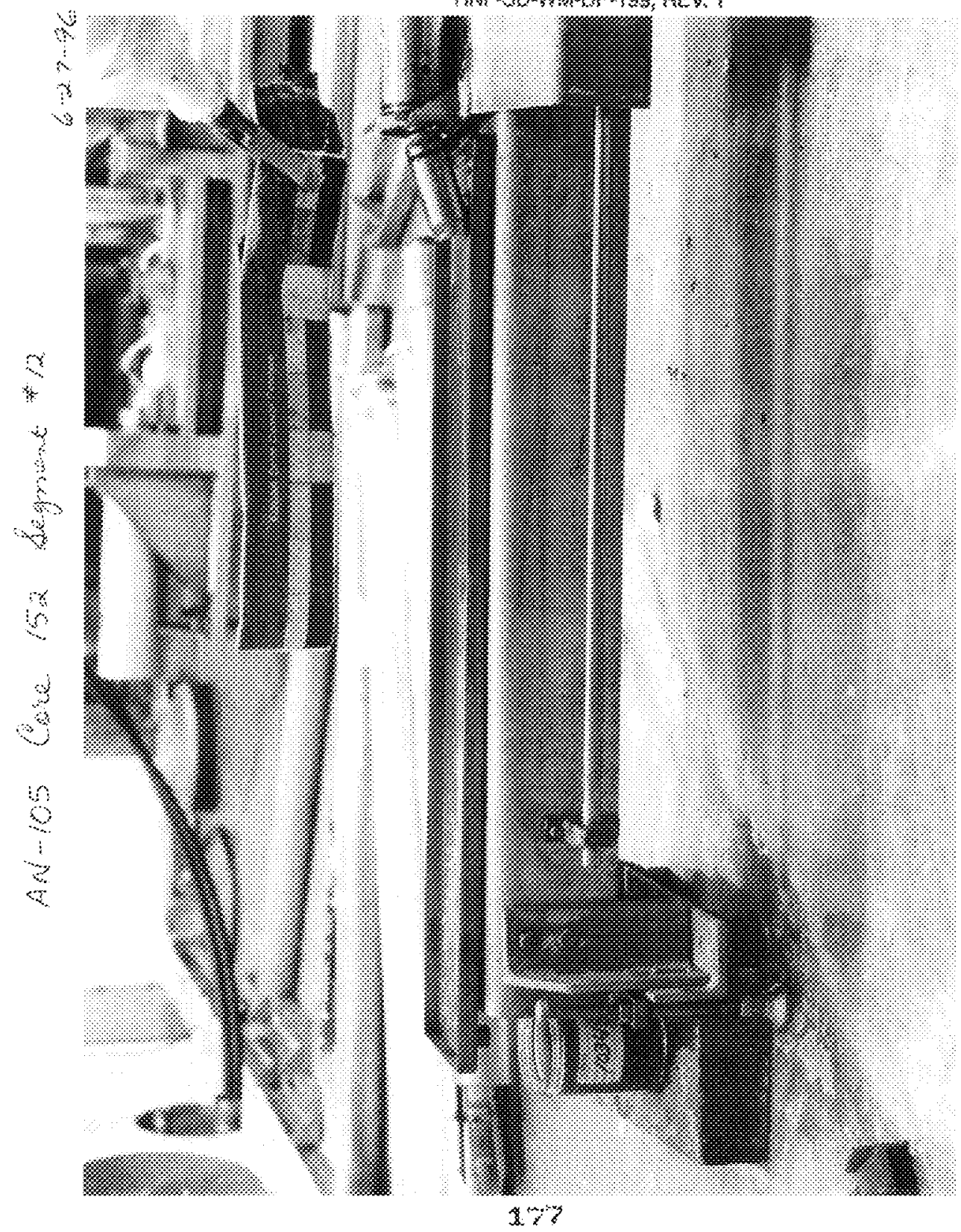




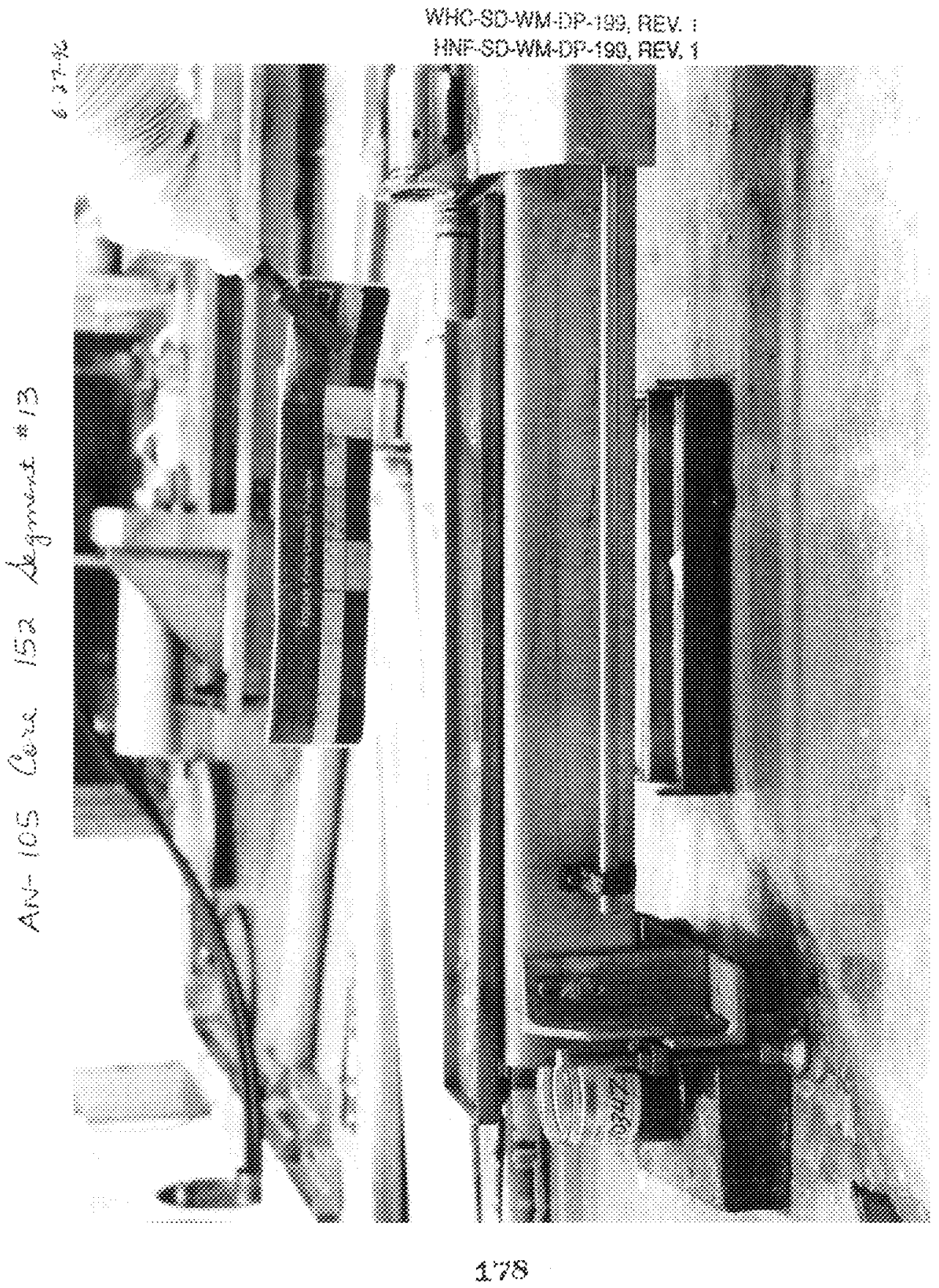


WH,

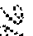

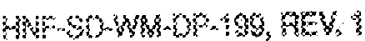

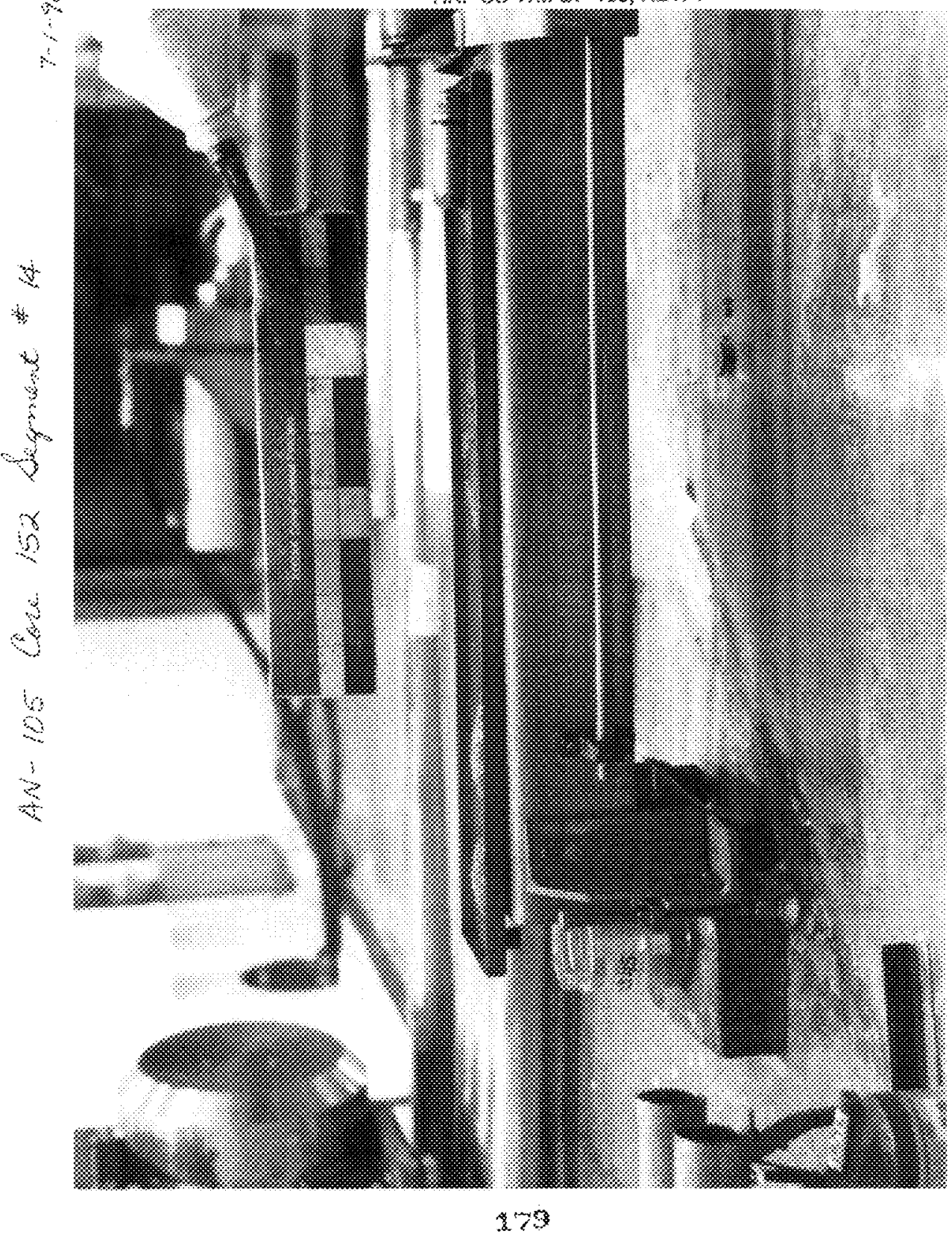


W40,

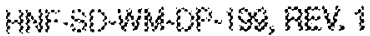

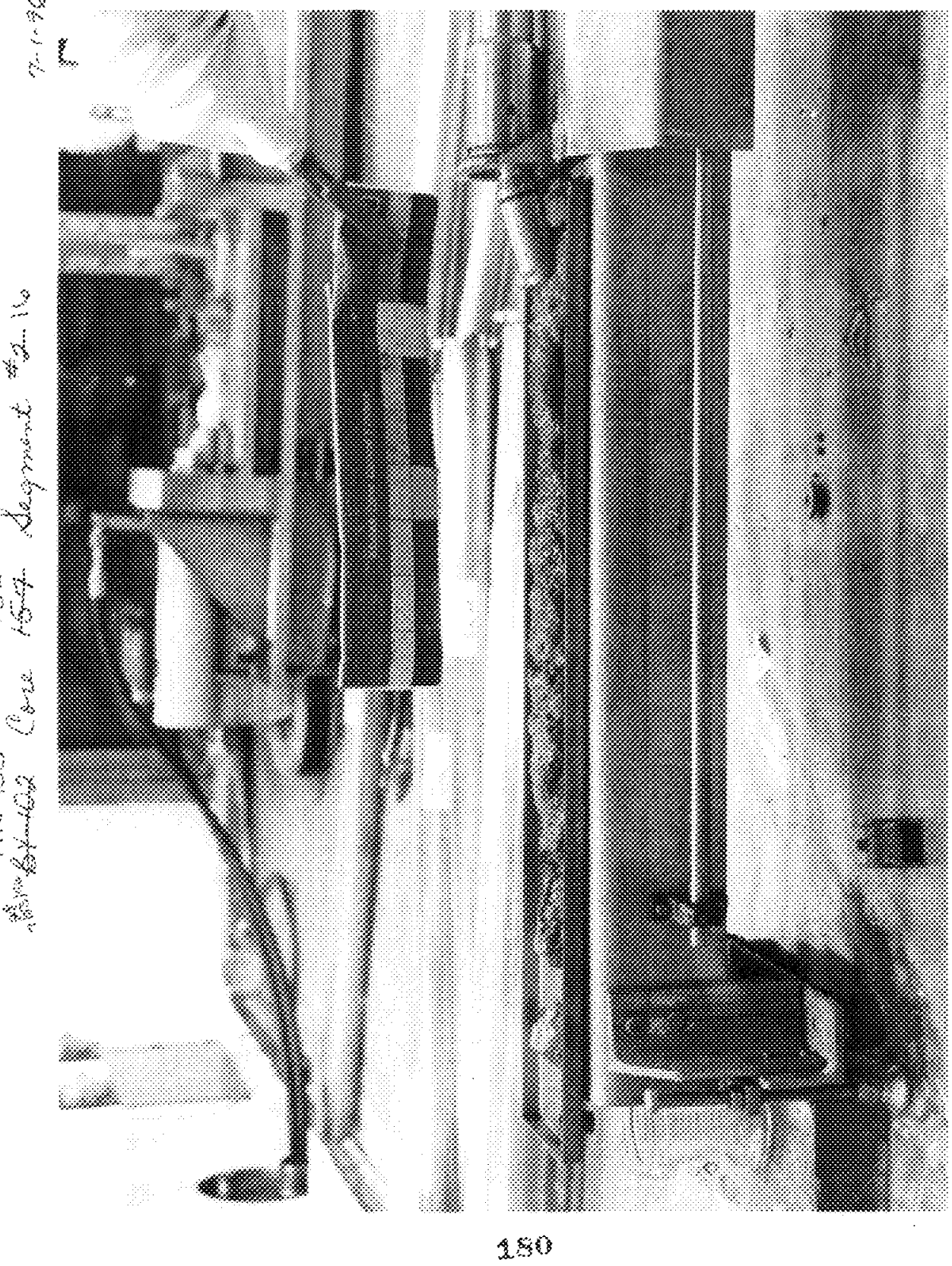




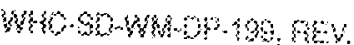
औरF 50 -

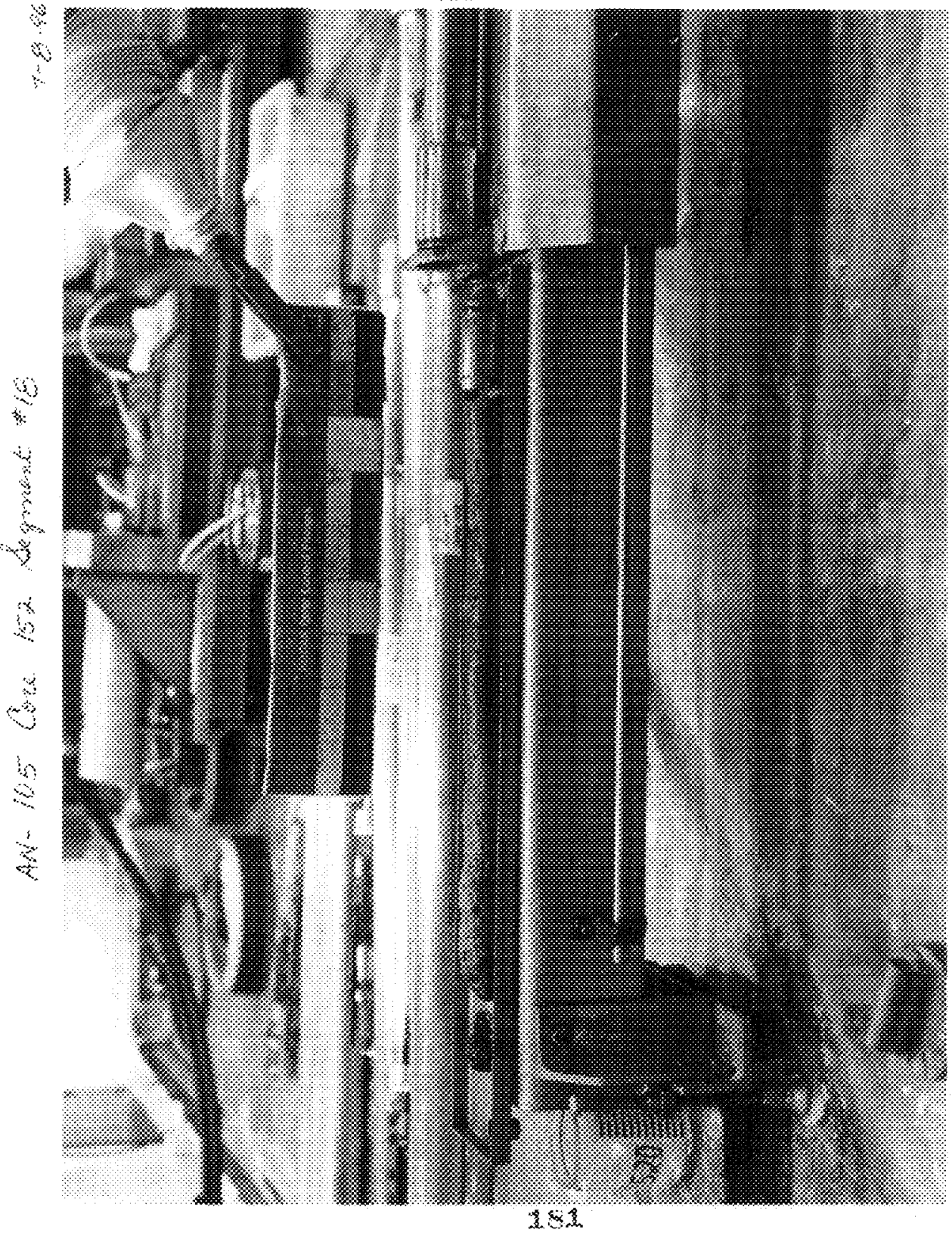




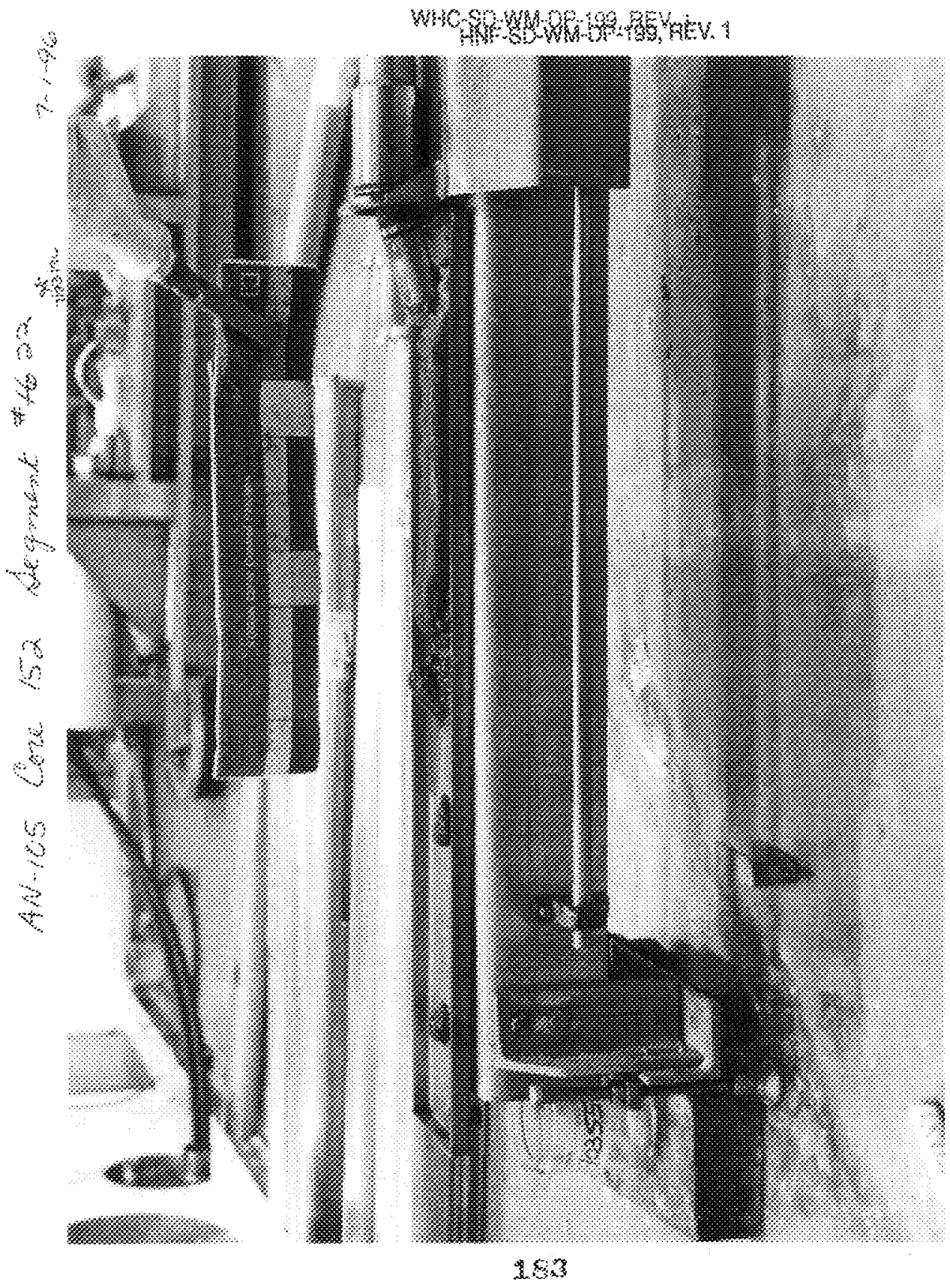




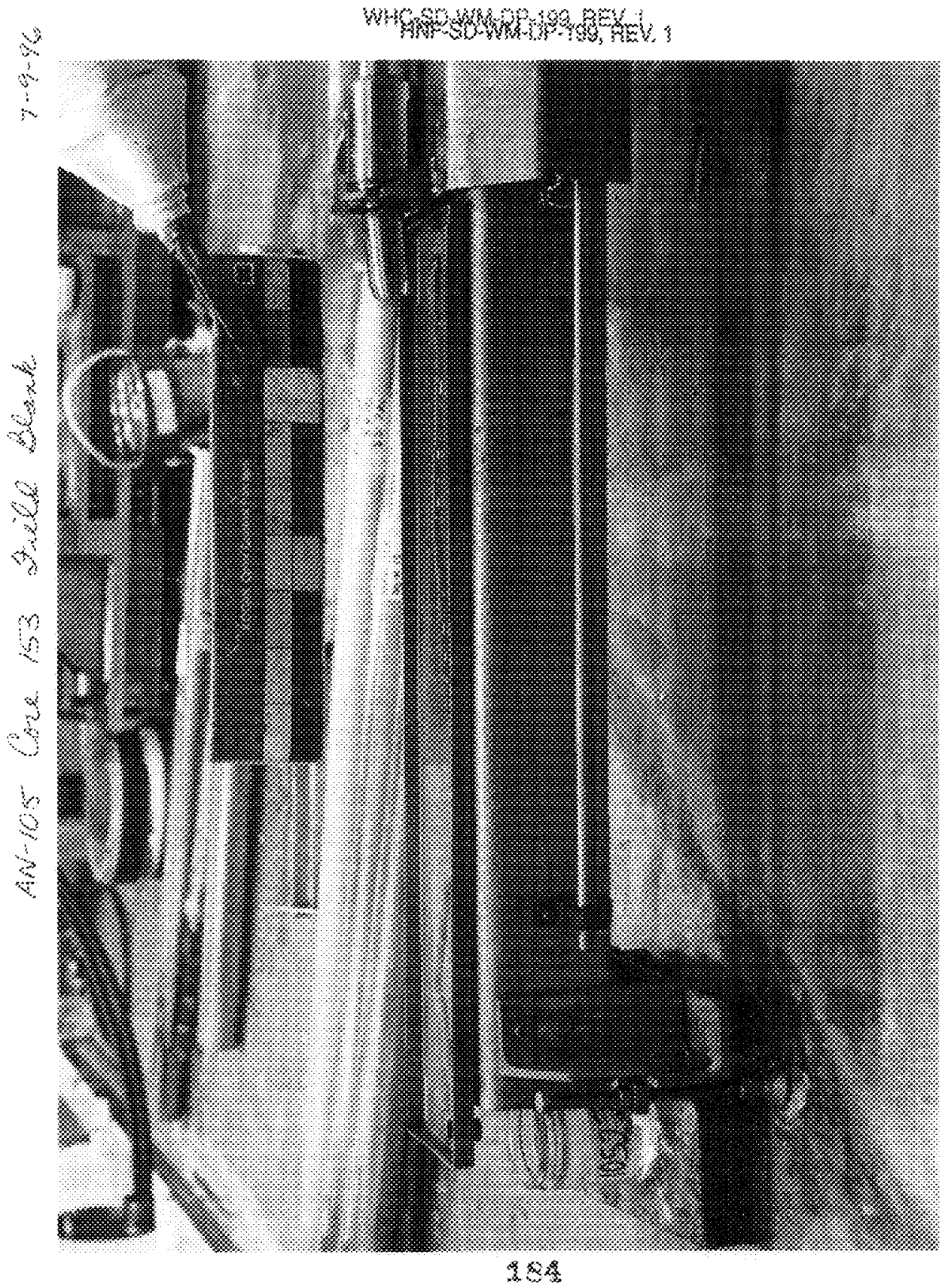




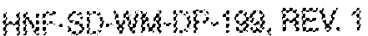

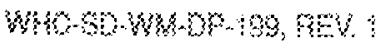

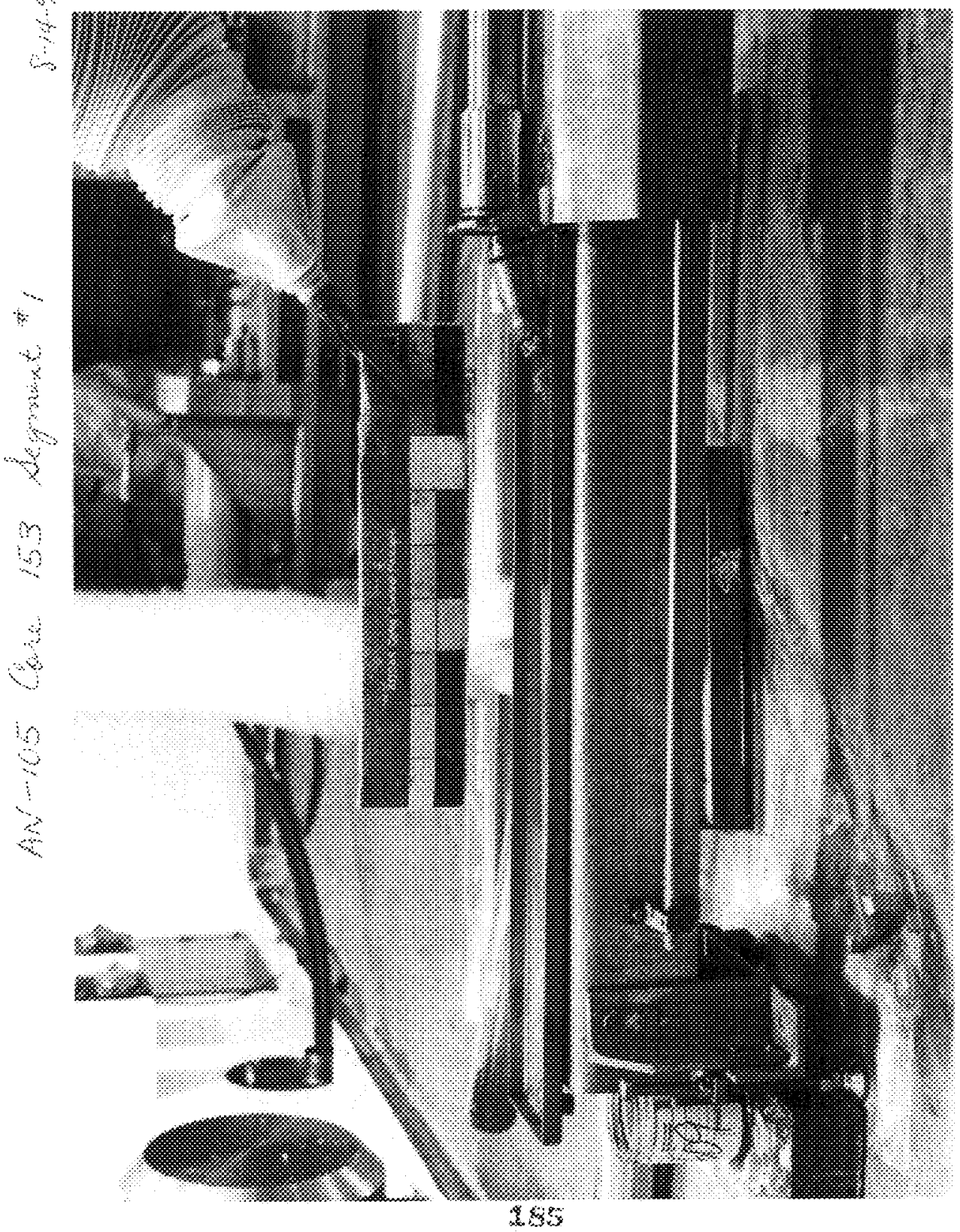


ME SOWM-OH

Wh, Su Whop-39, $9 \mathrm{QH}$

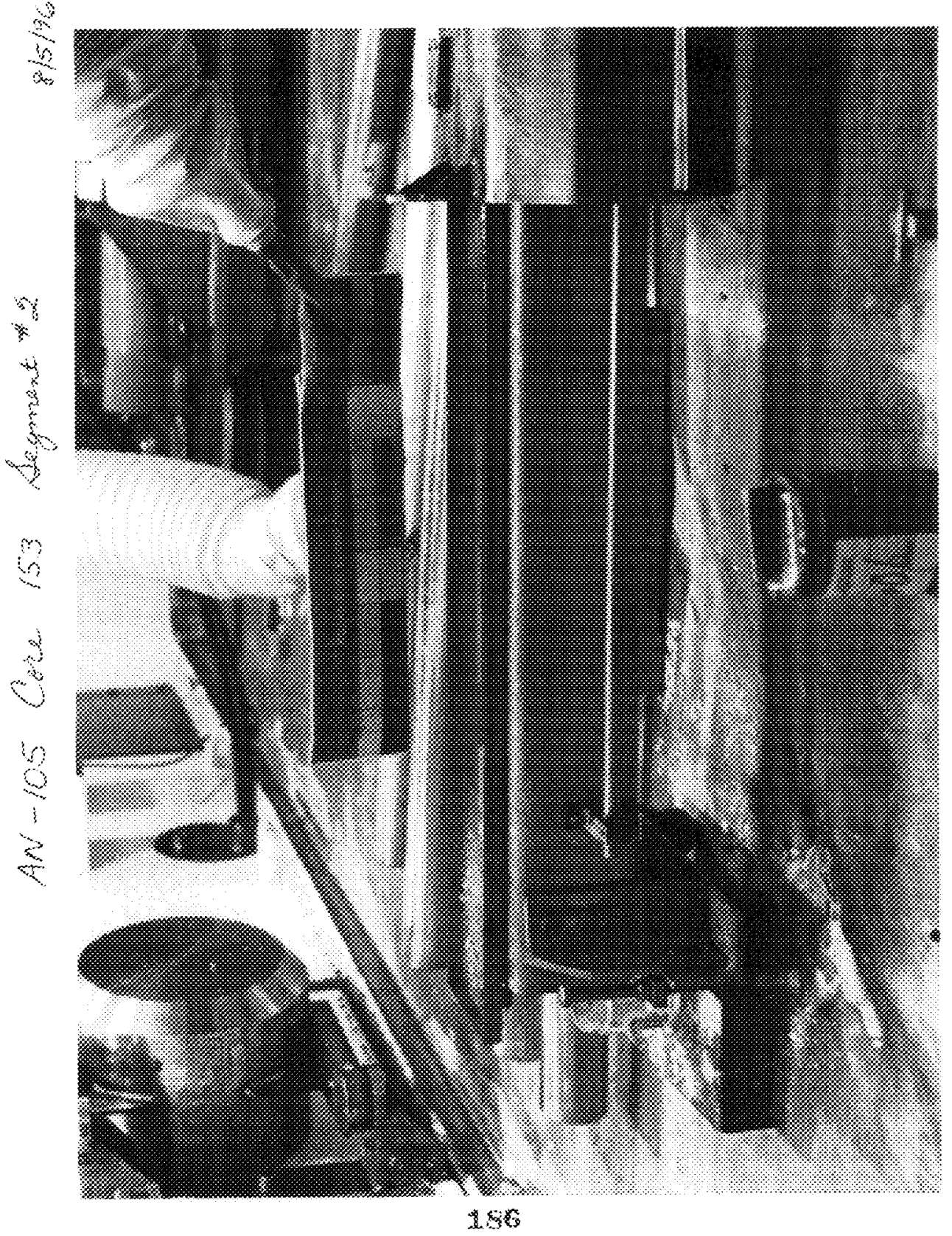




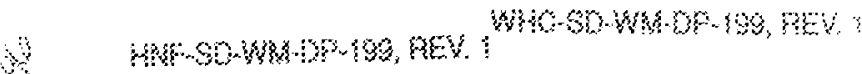

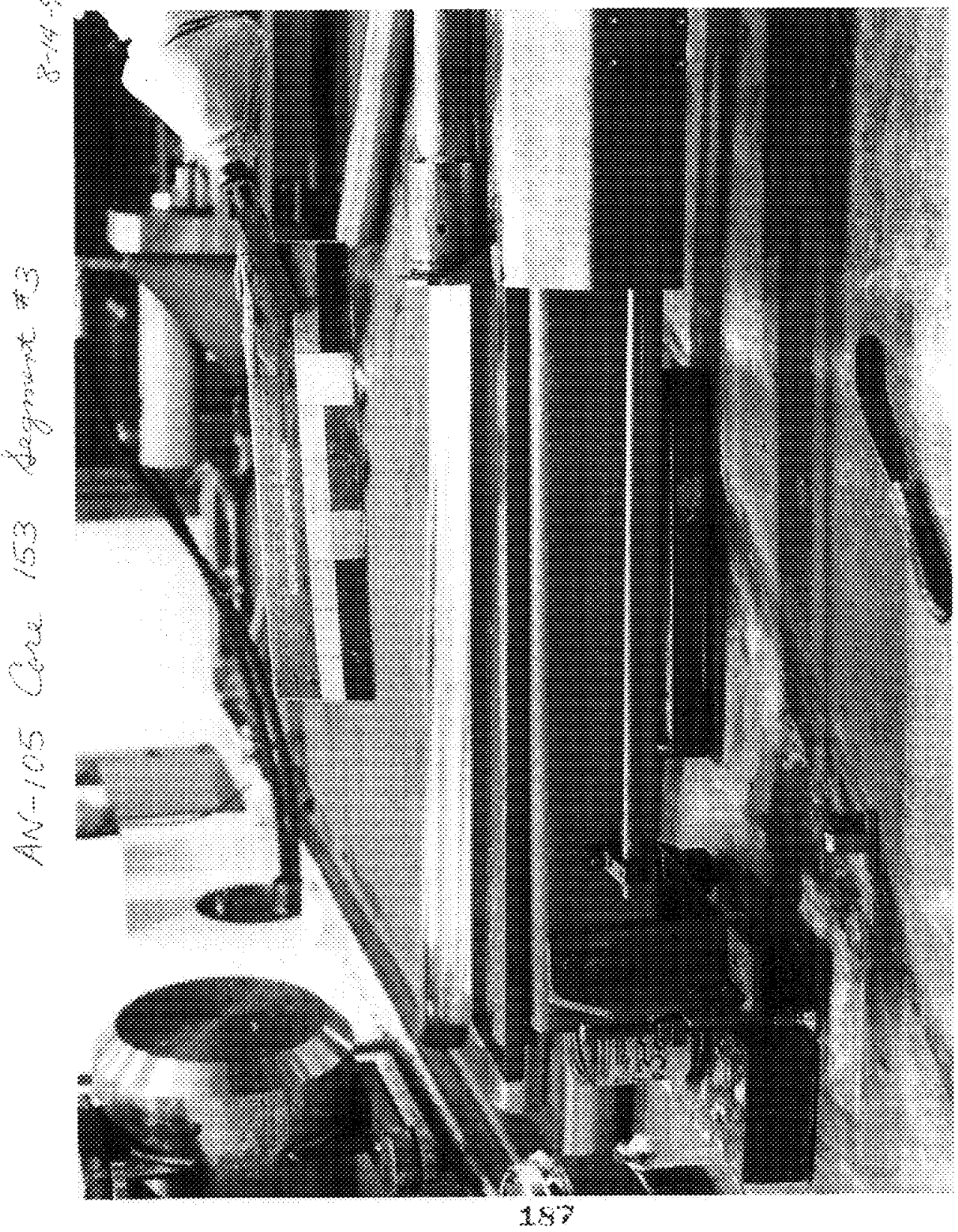




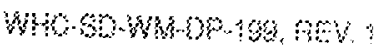

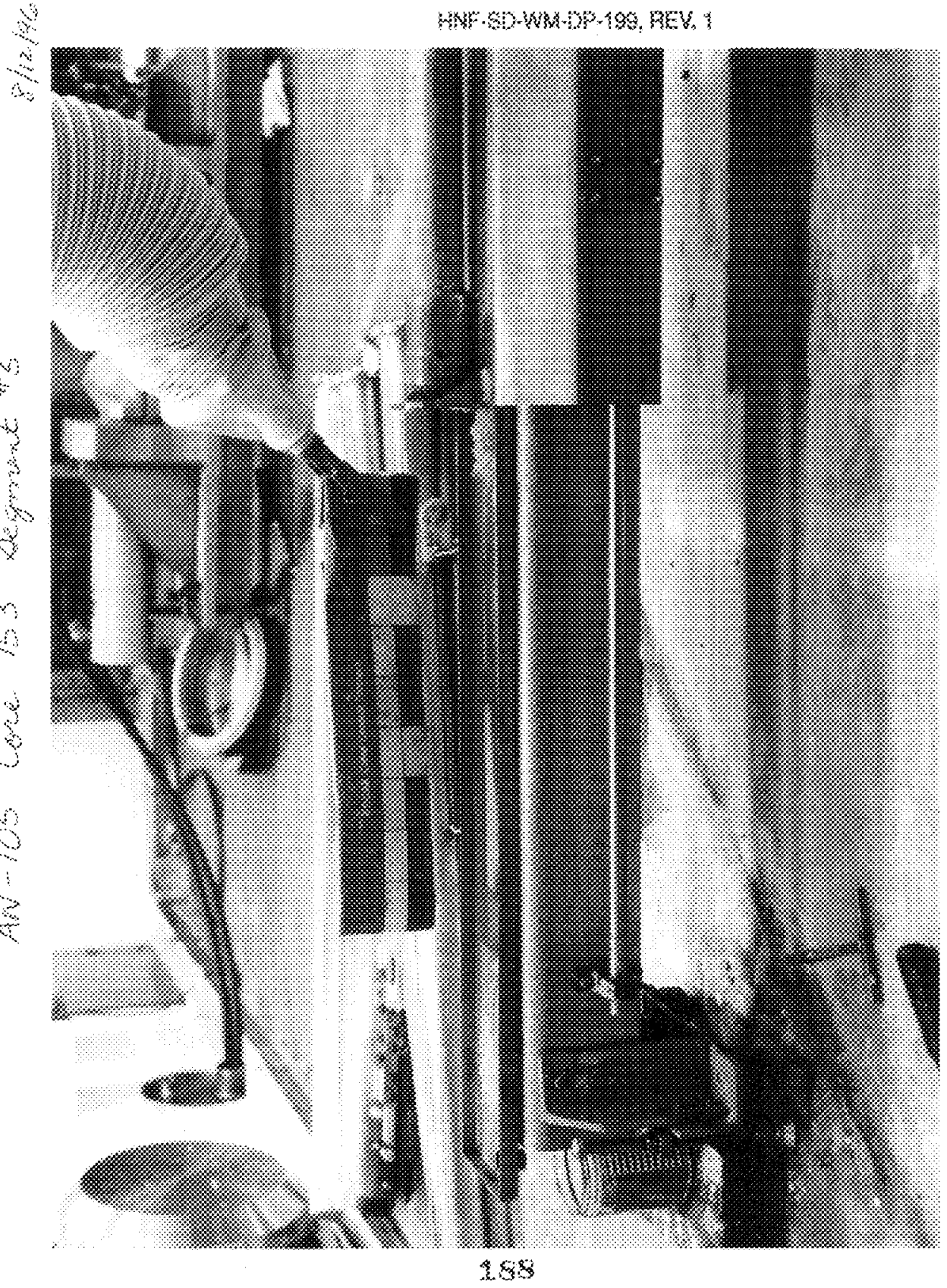




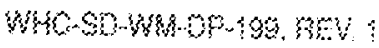

8

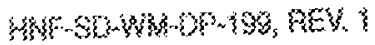
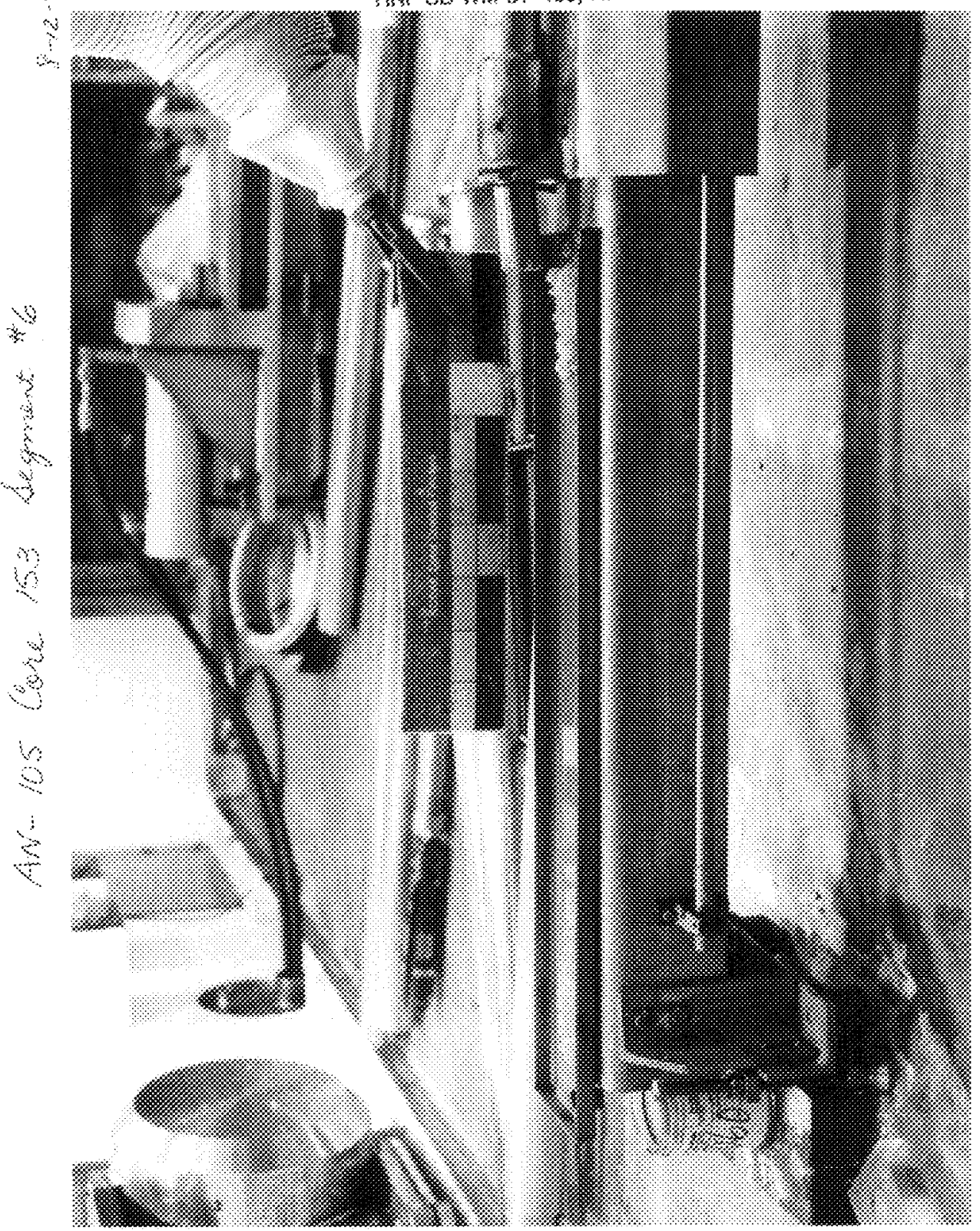


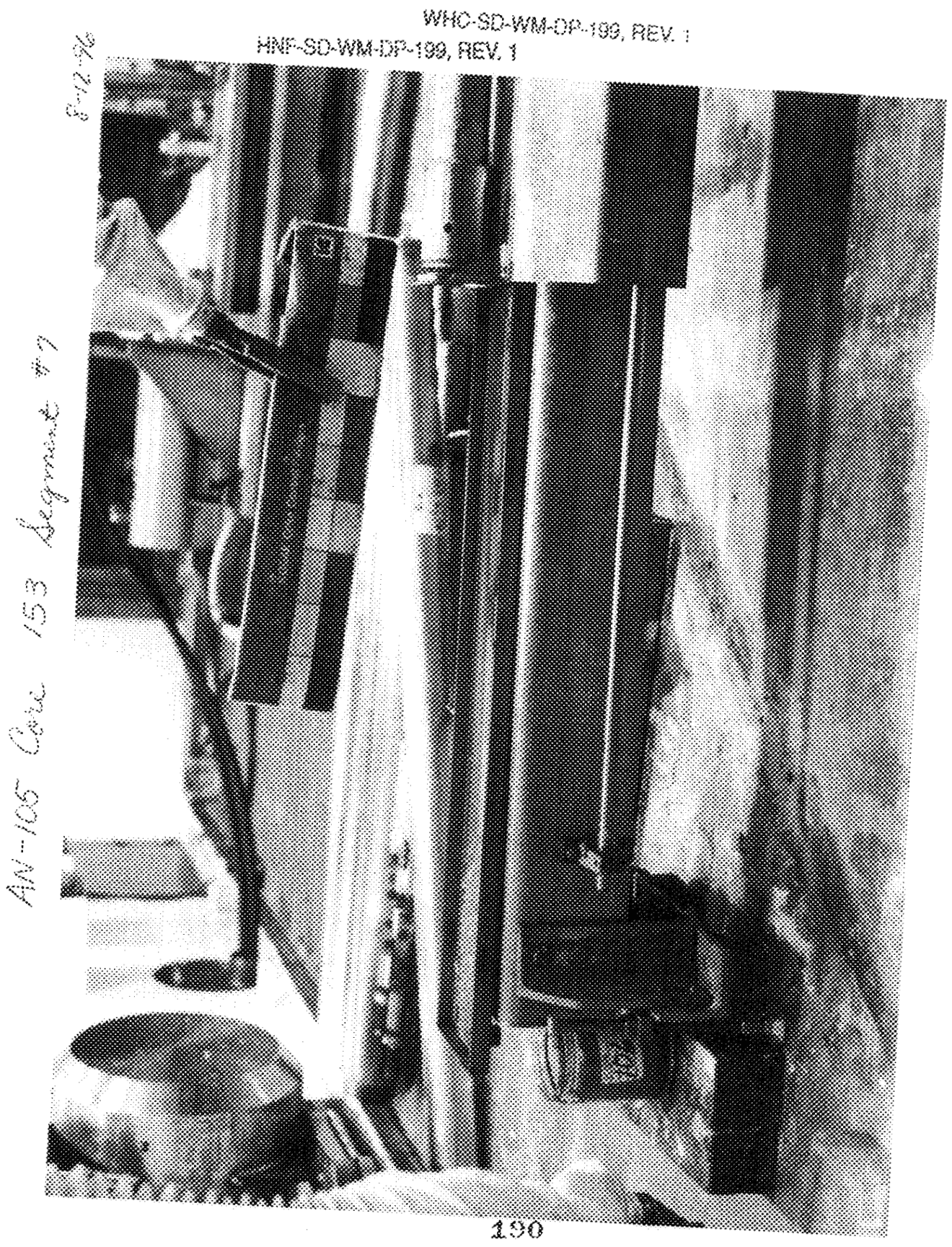


who-

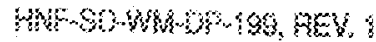

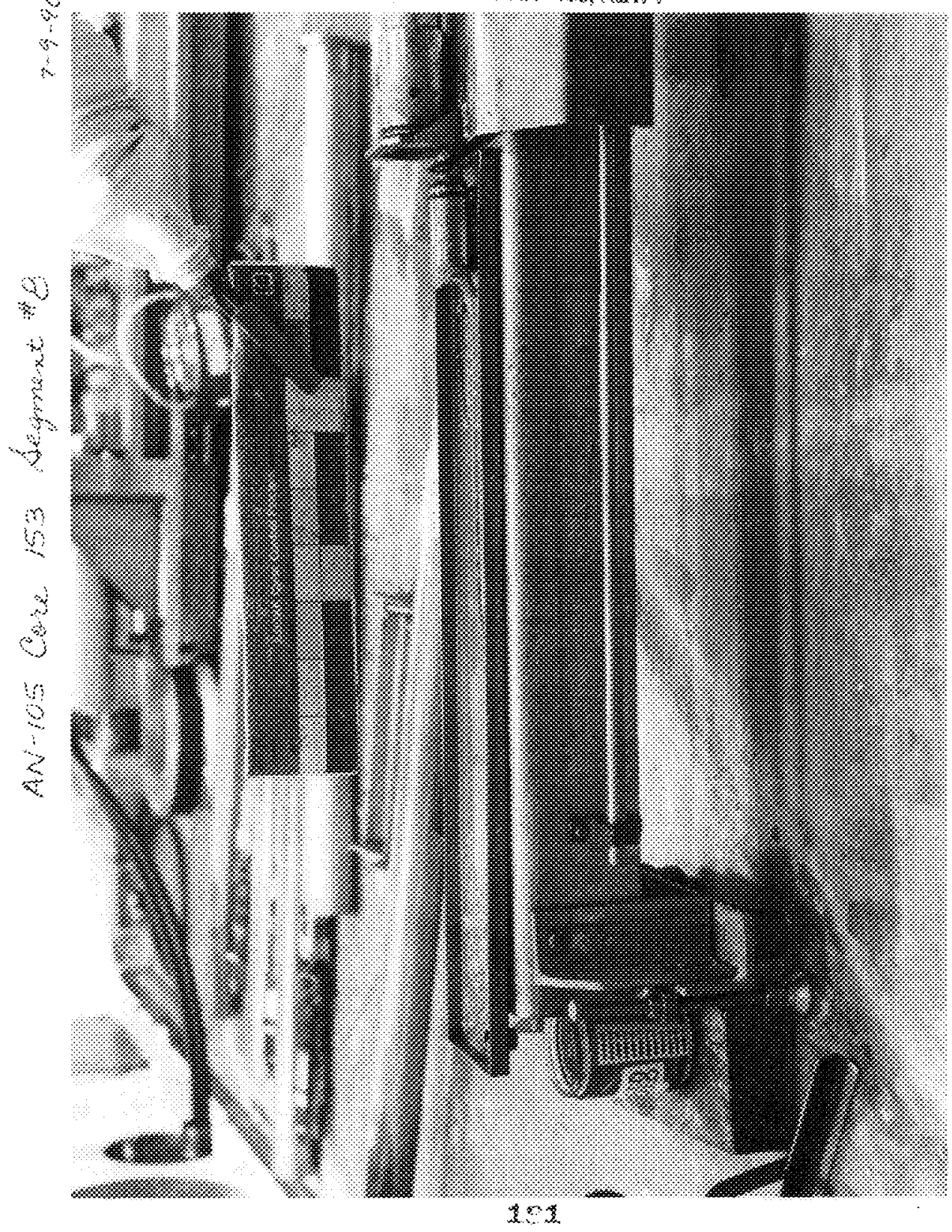


\&
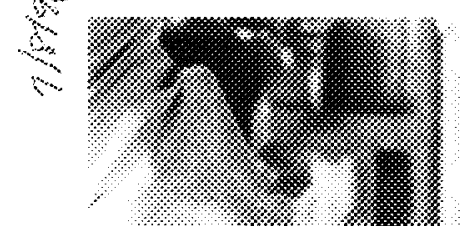

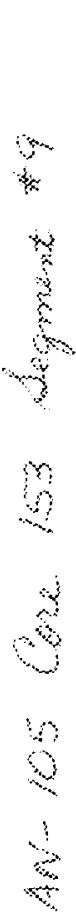
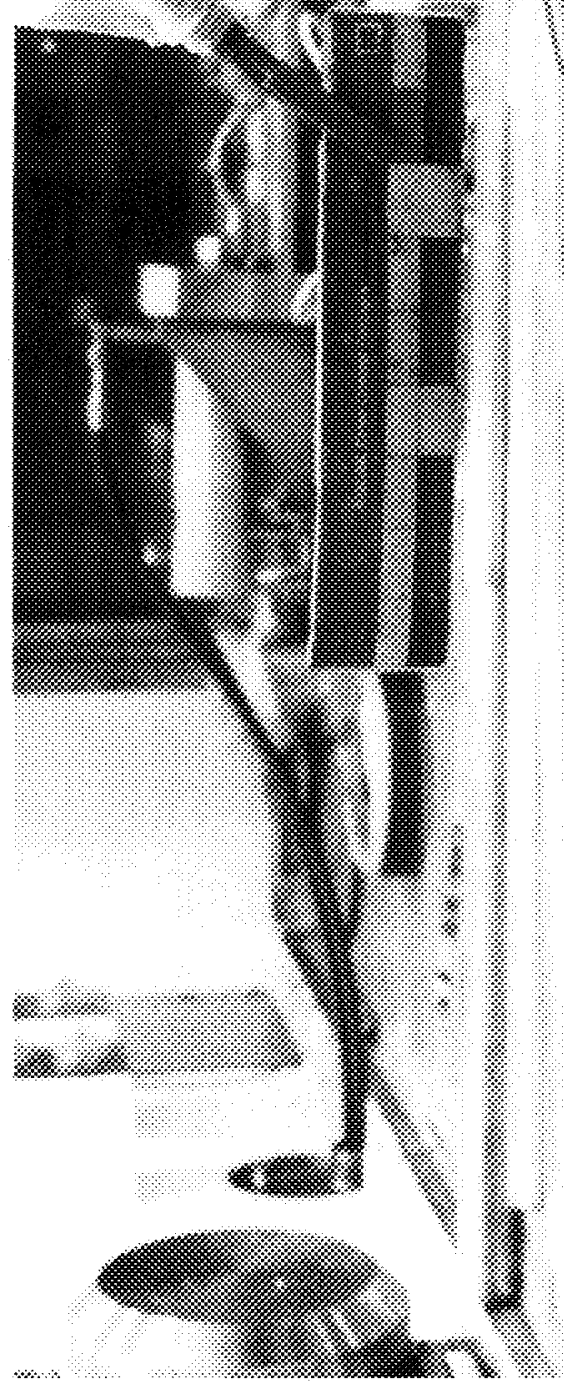

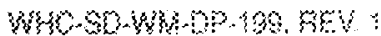

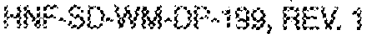
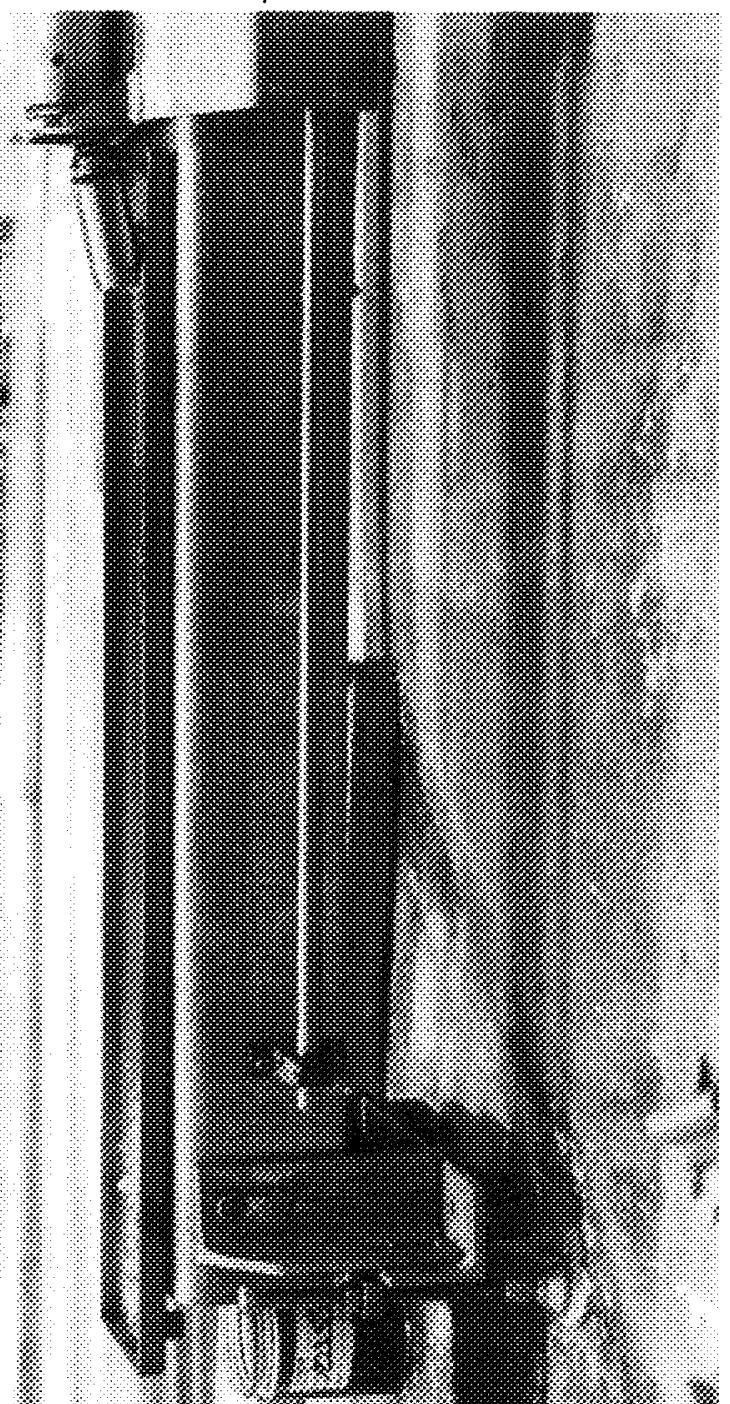

\%

\% $\%$ $\%$

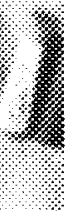


MHO

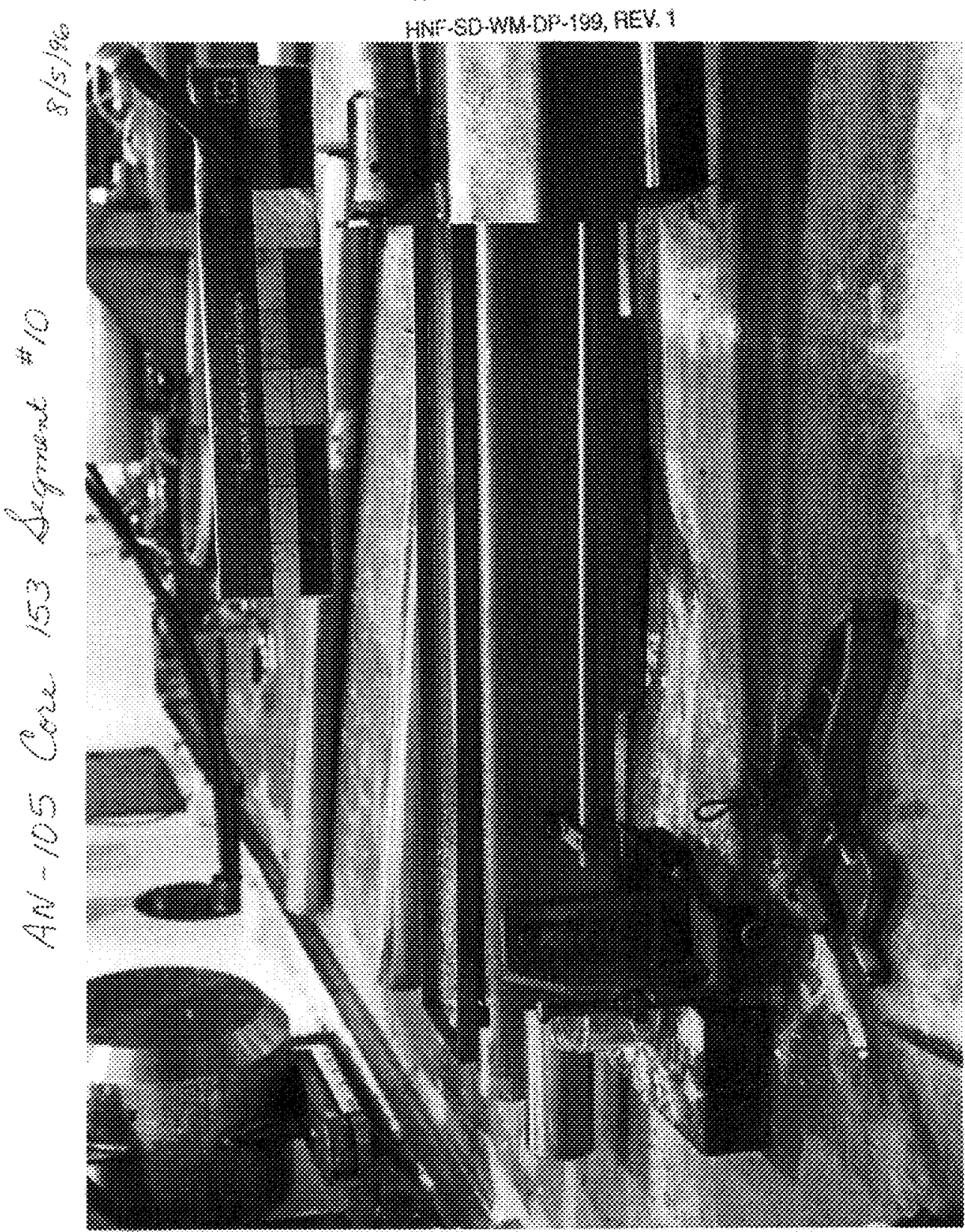


Who $\$$ Wh

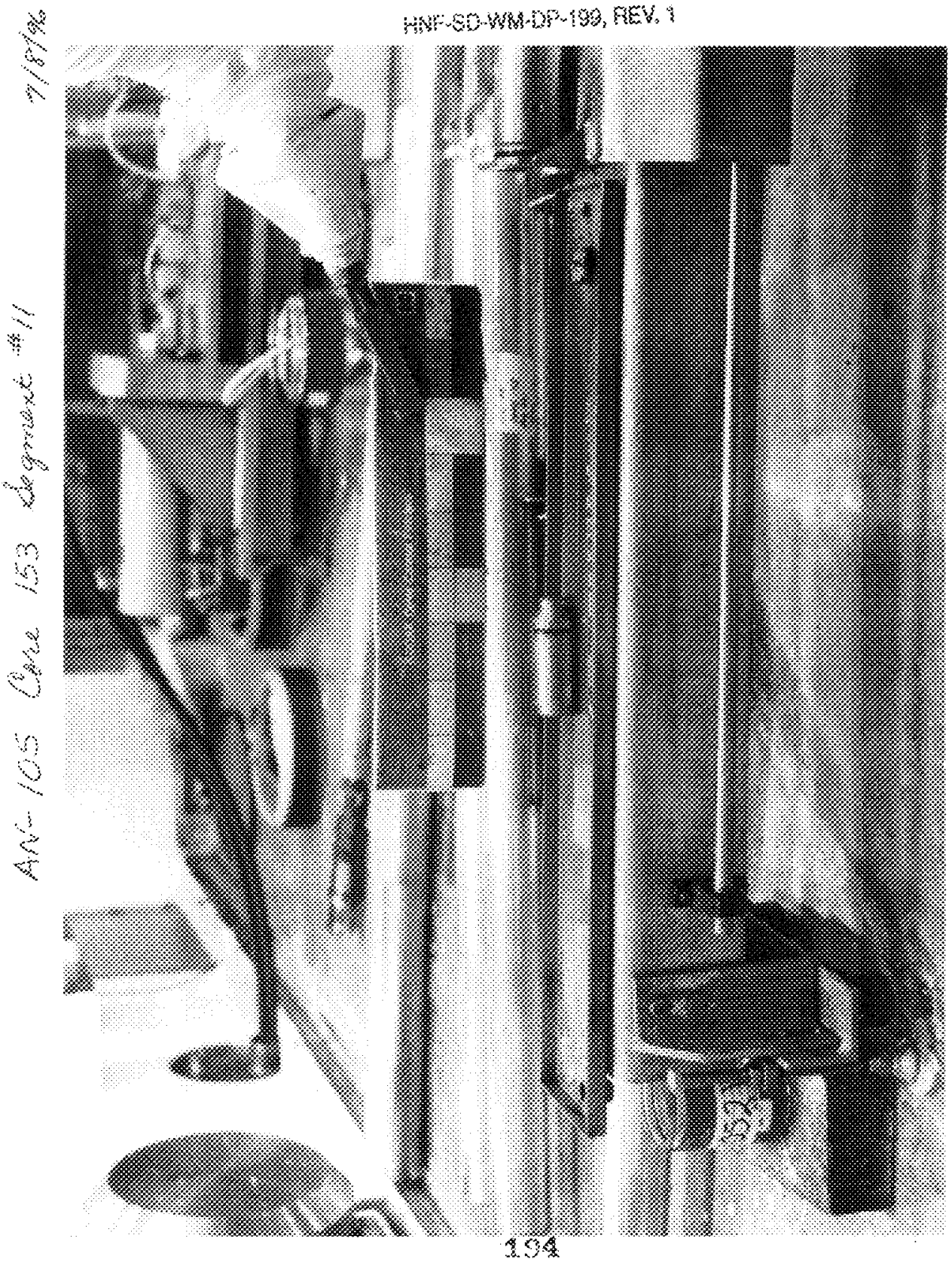




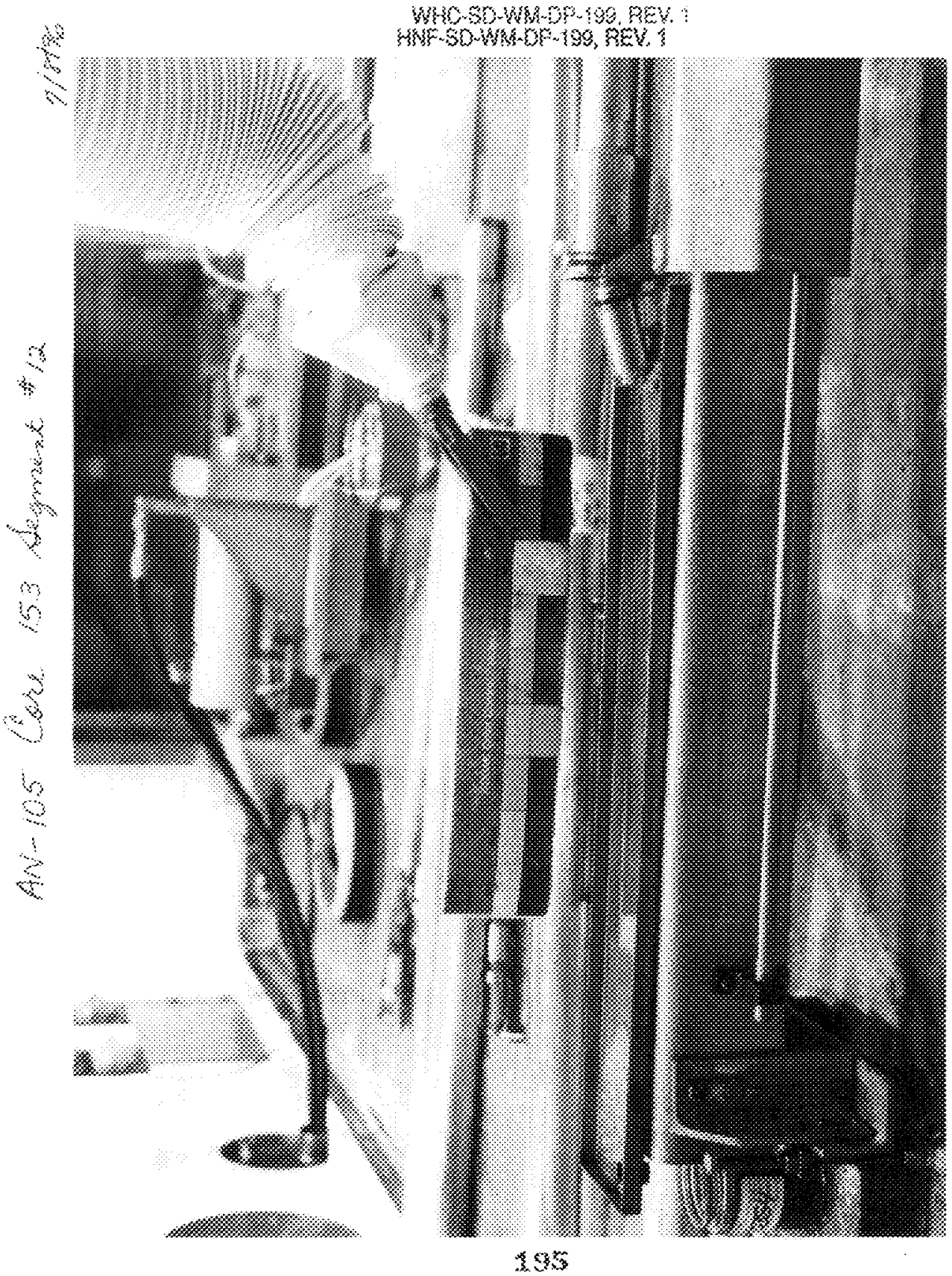




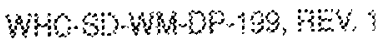

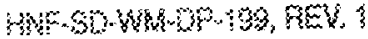

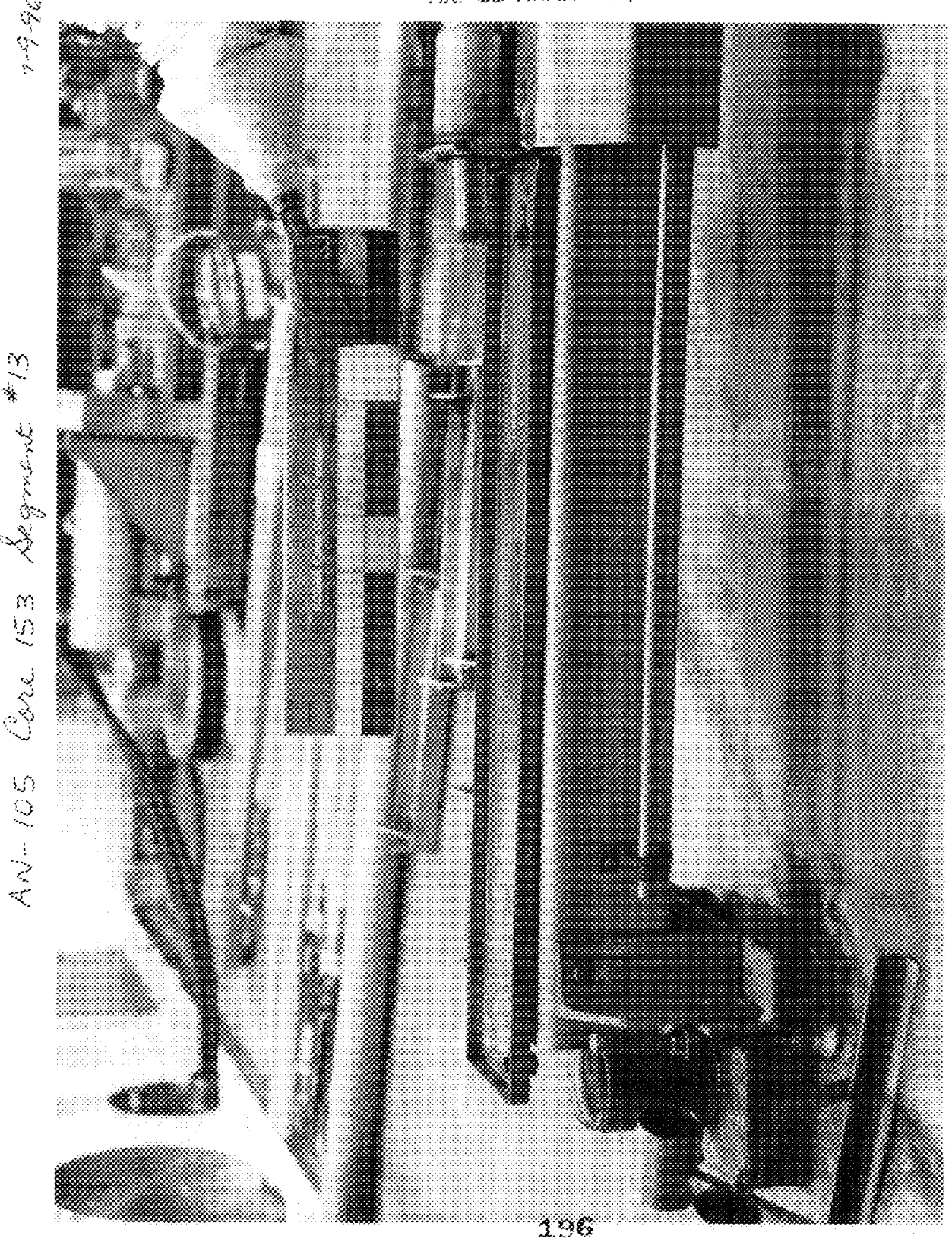




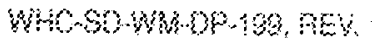

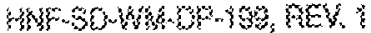

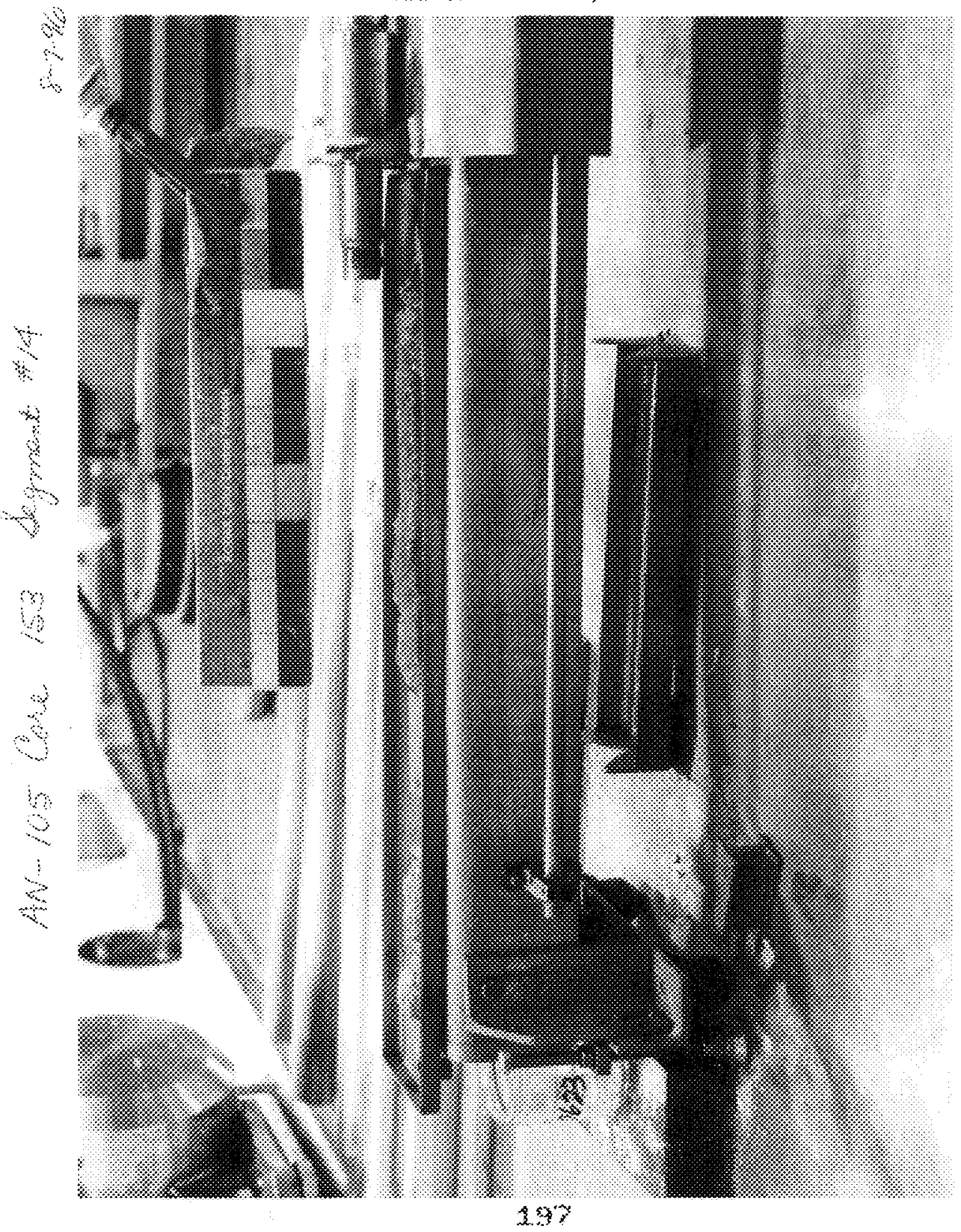




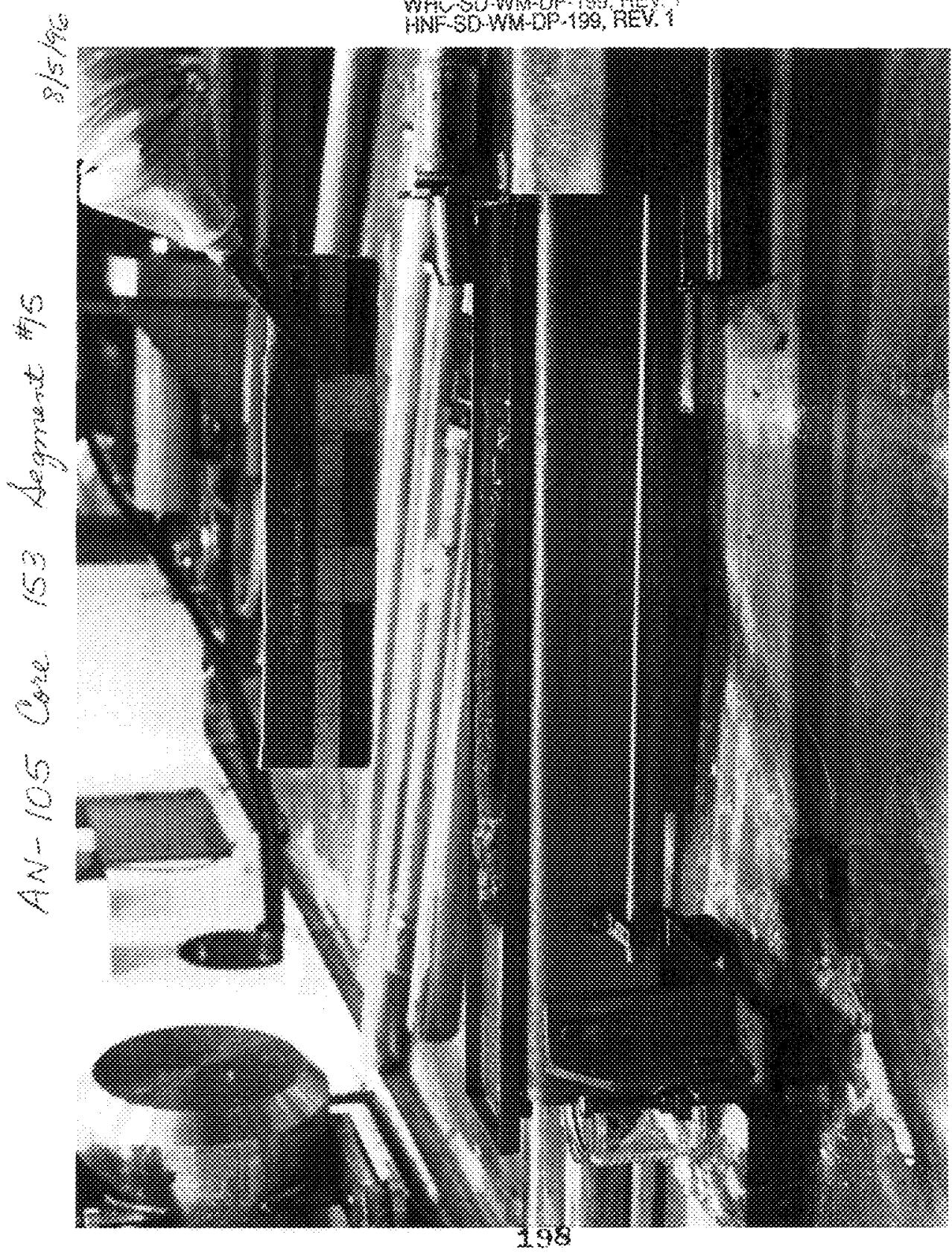


WH.90\%,

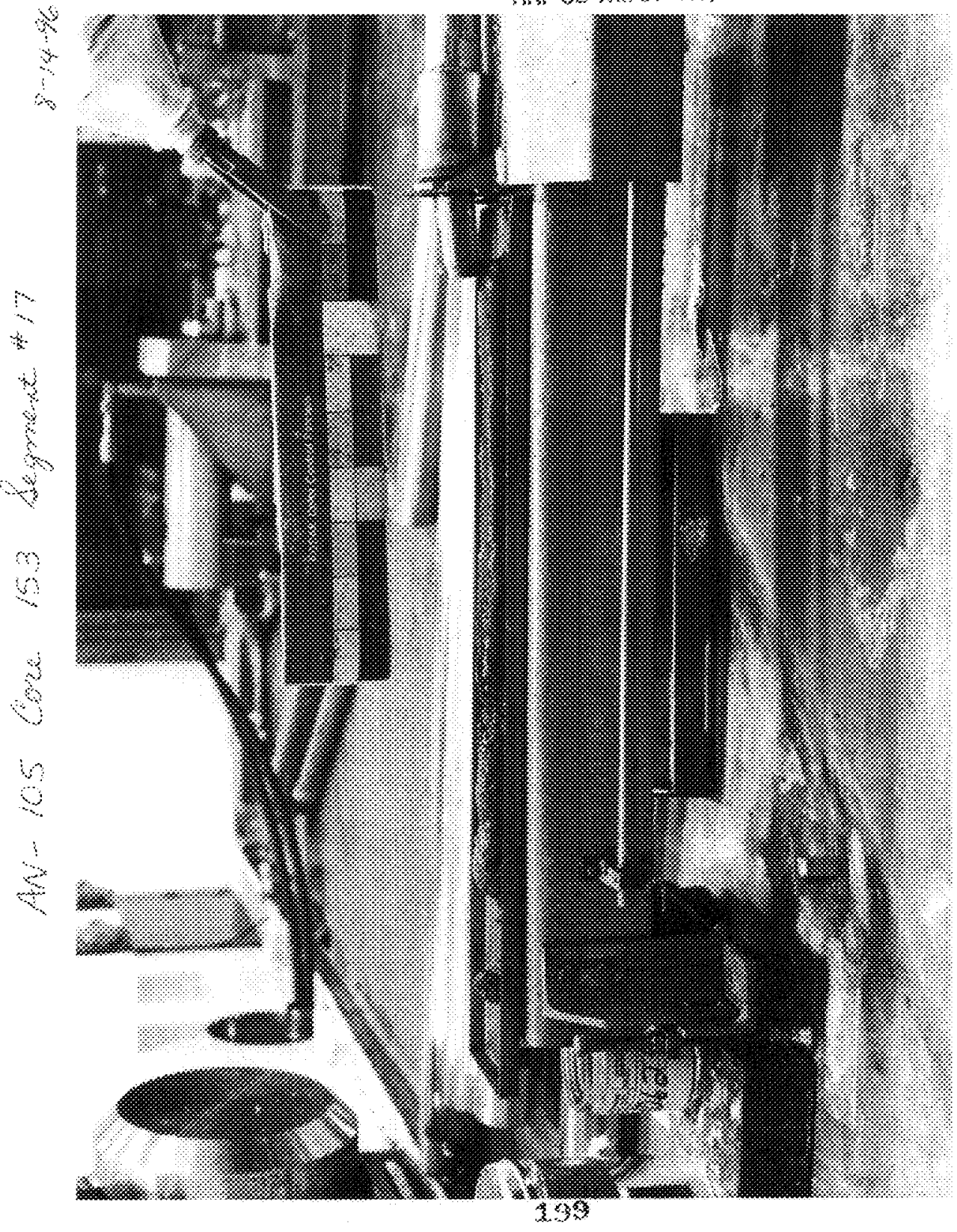


\$HCS0 Wh-Yo -190,

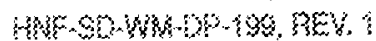

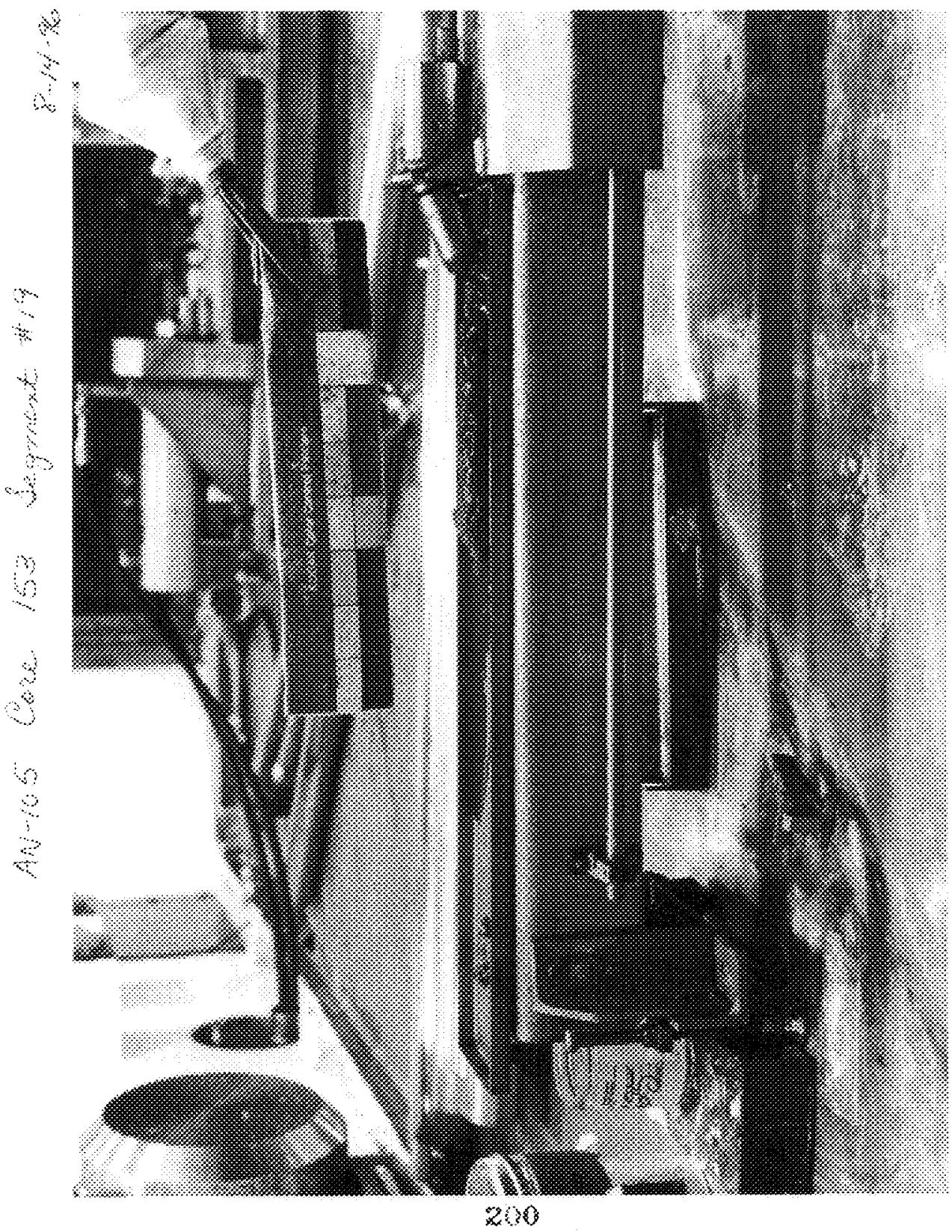




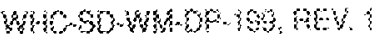

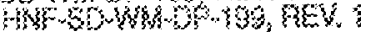

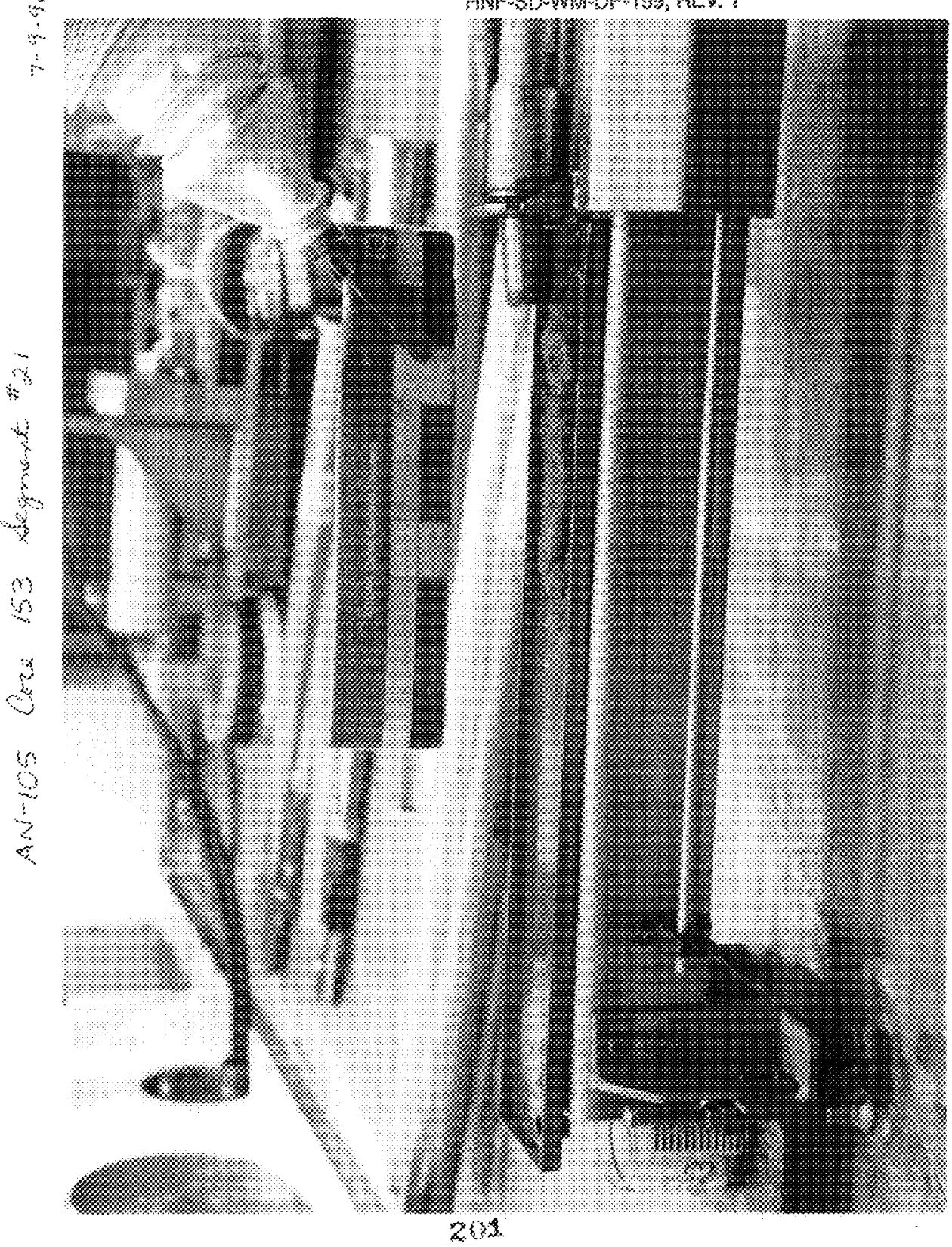




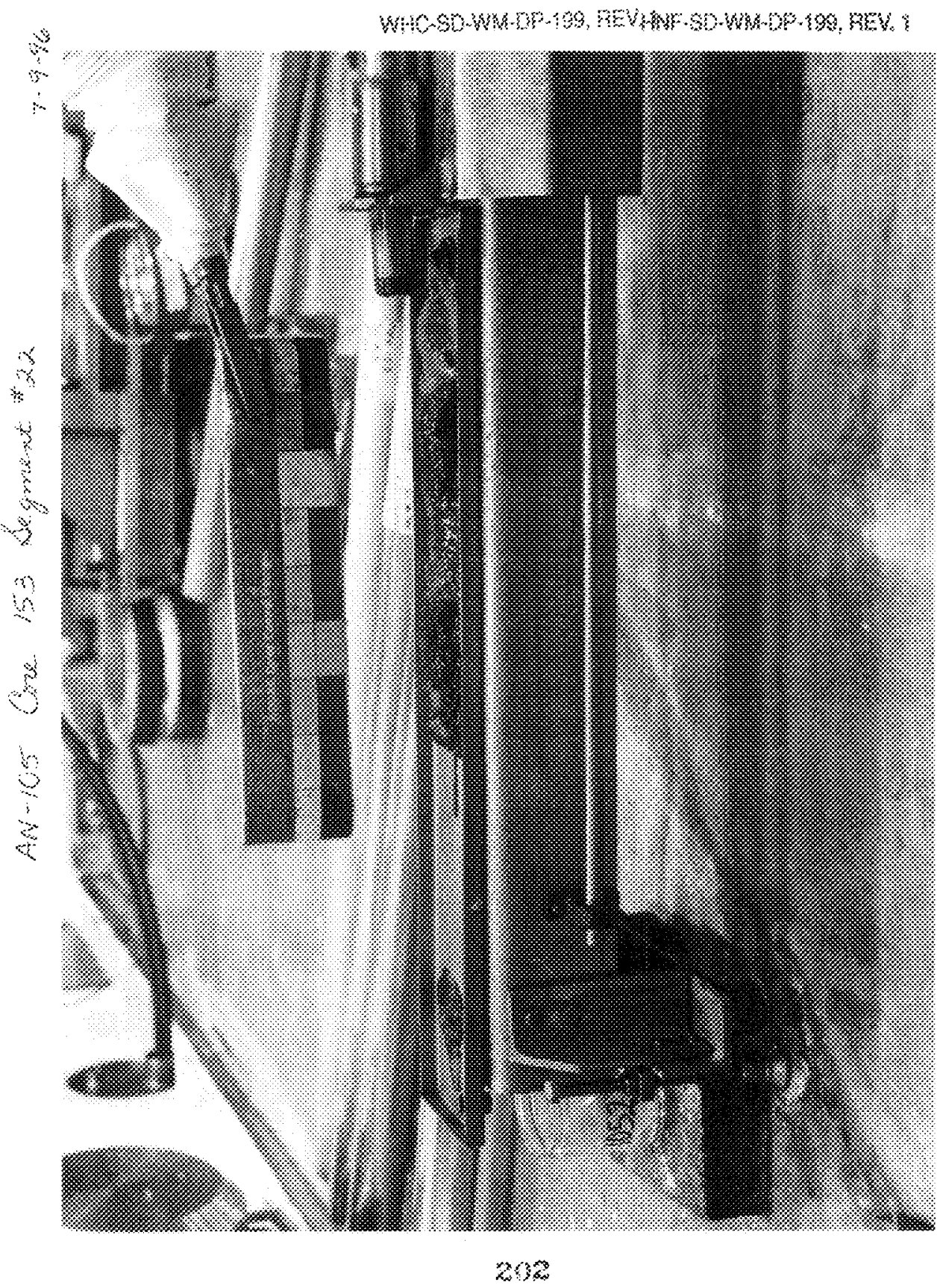




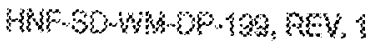

WHS- $35-4 M-3 P-199$, KEY.

SABEL KAXOLIRG 


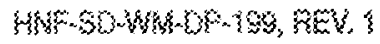

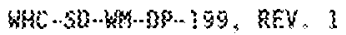

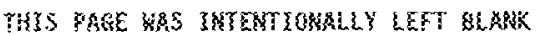





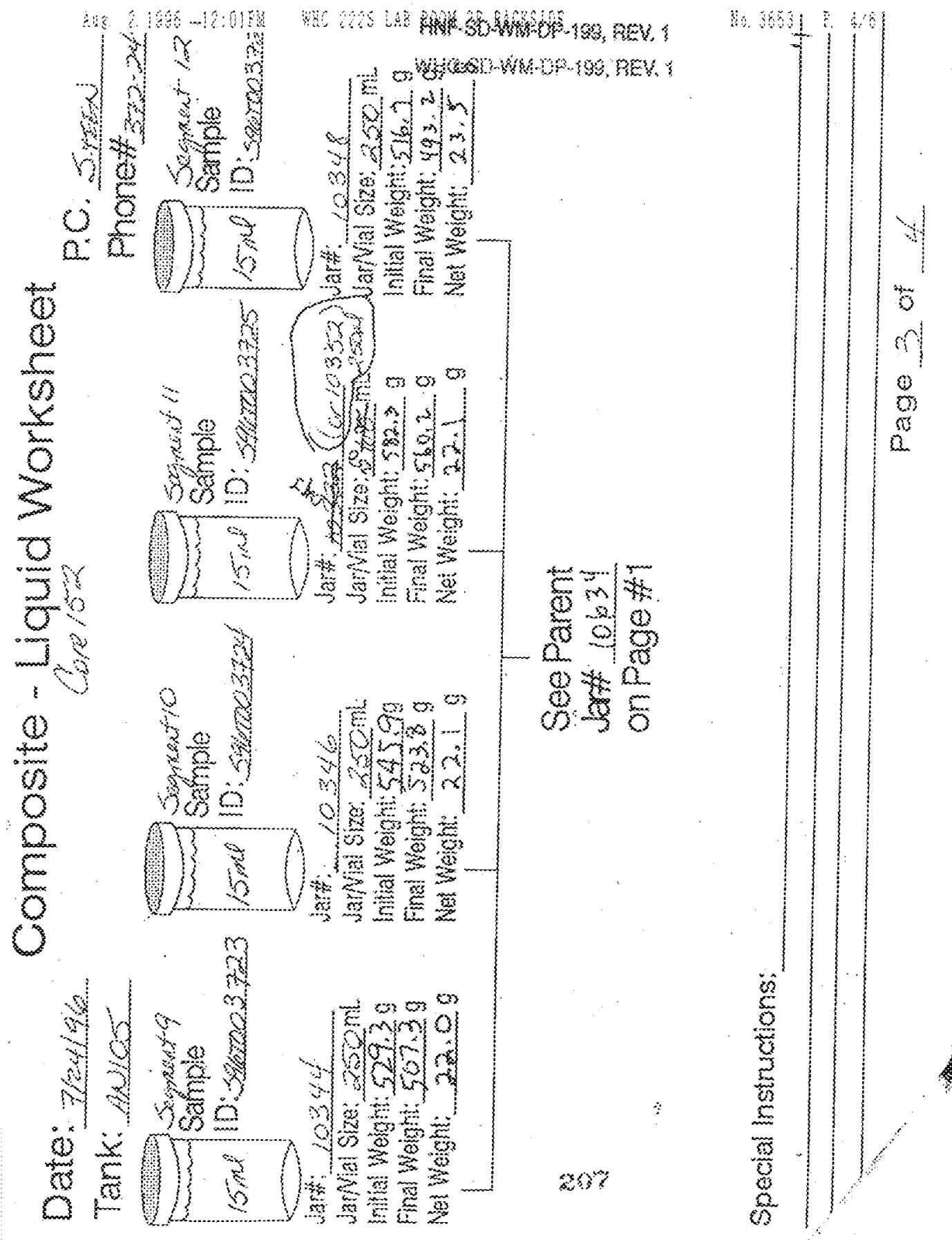




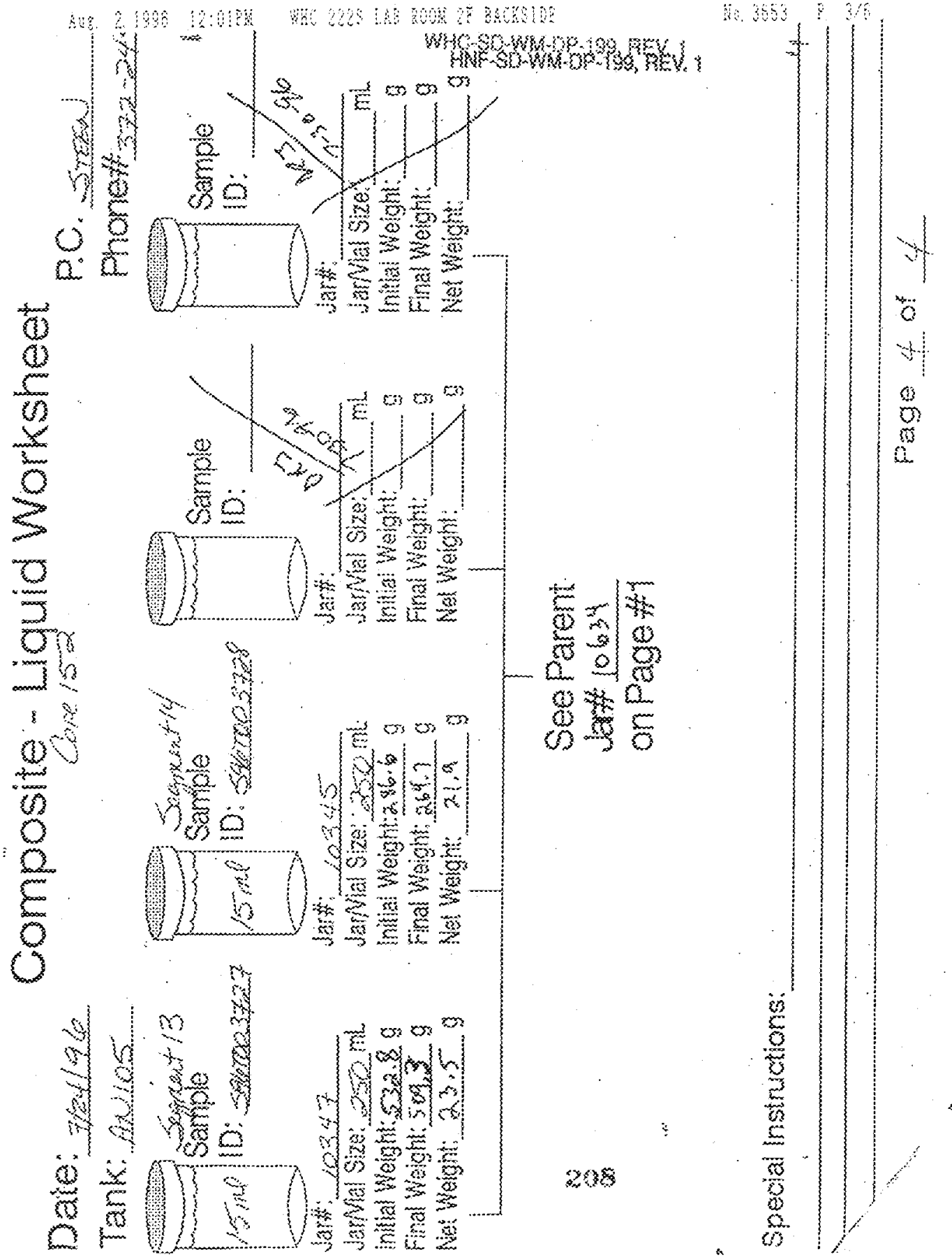




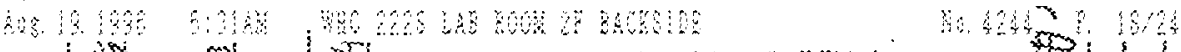

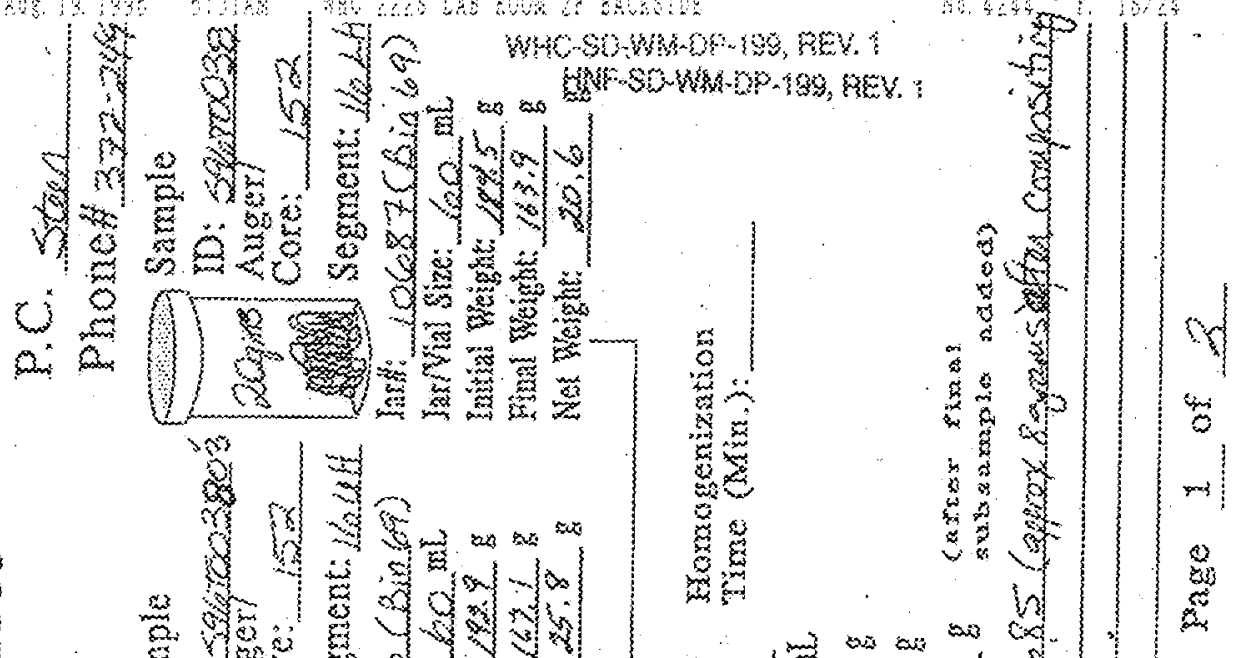




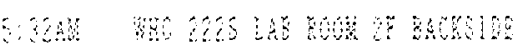

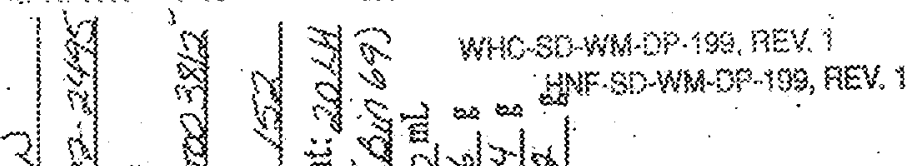

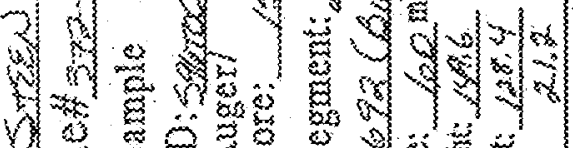

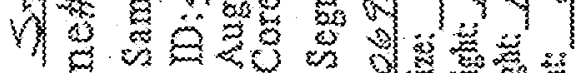

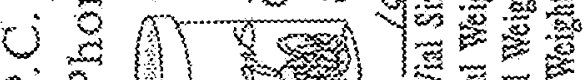

ais

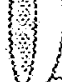

iv

ond

13

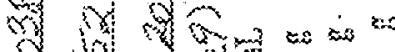

$Q$ रु $\cdots$ हो

जo

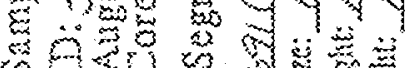

\%

(2)

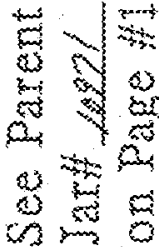

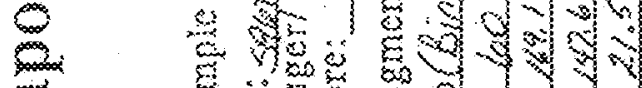

कod $\quad$ का

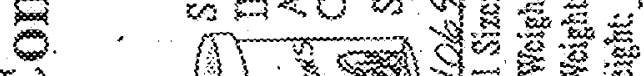

3

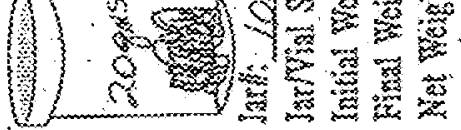

$>=$

8

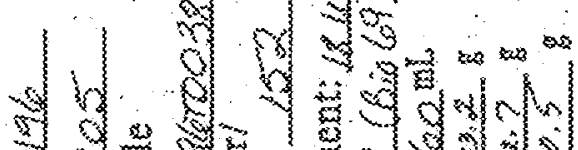

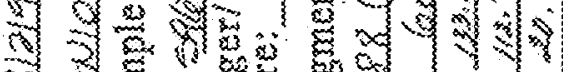

$x$ रो

1)

in

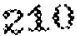

S

2

8

की 


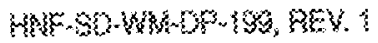

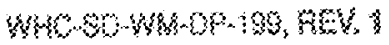

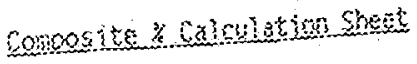

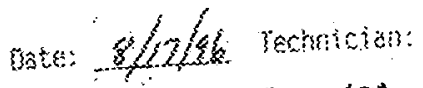

1) sement 50 \%

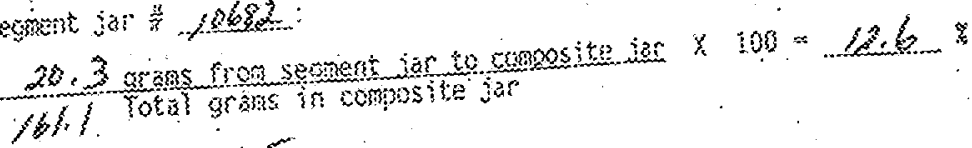

(2) Sespent jur 2068

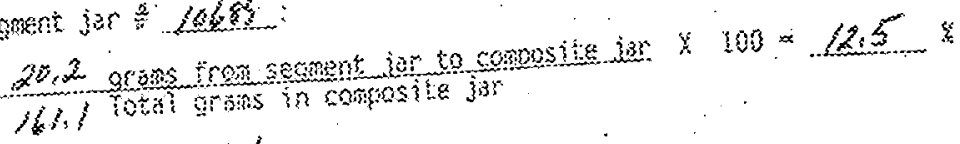

3) Segnet jor

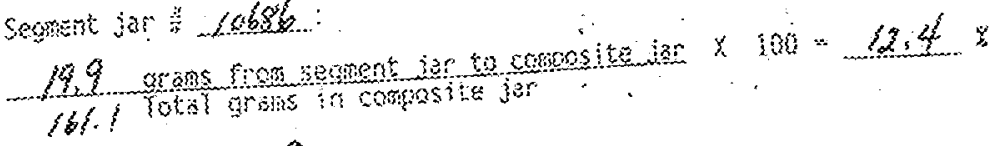

4) Segmen: jar

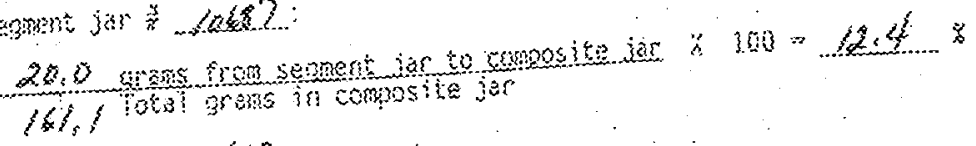

$5)$ seprit jor .068 :

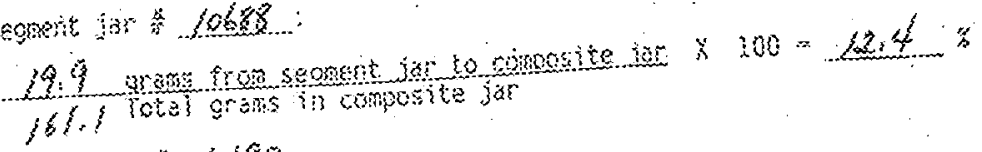

s) Geome jor is lobis.

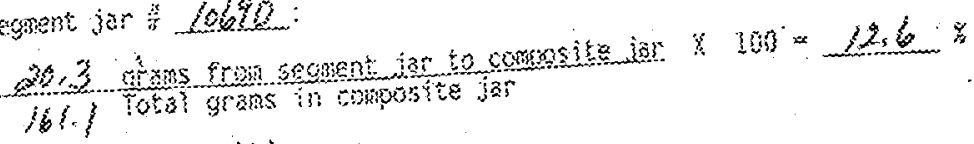

7) segent for 206 is:

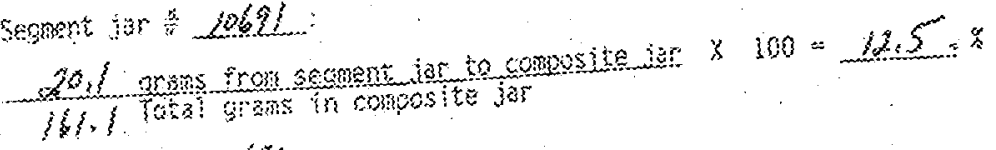

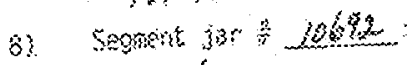

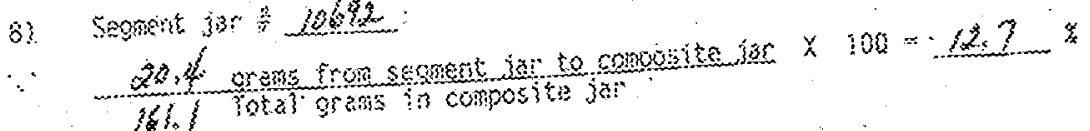

$9)$

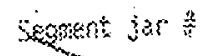

(1) Segent jor

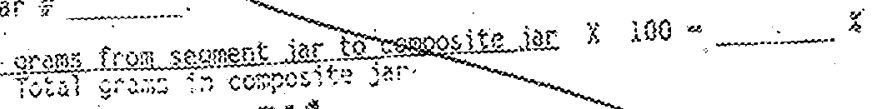
2,3

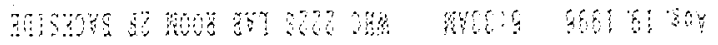




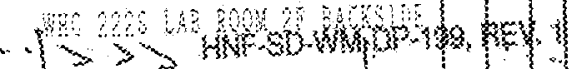

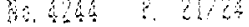

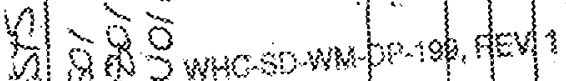

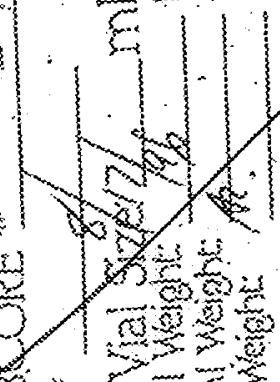

rod

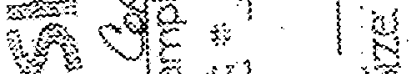

s.

2.

inar

(2)

rand

Exis

6

3

is

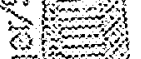

का

急

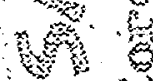

$B^{2}$

:

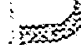

致

4 bs

ar an

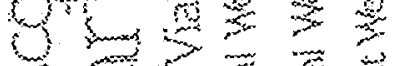

सि

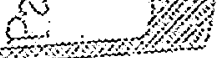

(

3

3

a)

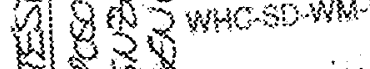
7 .

$\rightarrow$ W

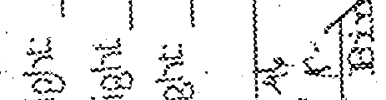

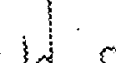

का

㲅

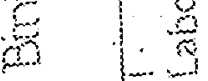

3 \%

$\frac{i}{5}$

$\therefore$ कर

in

को

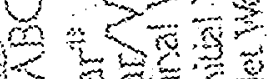

$3 \cos \sin$

(3)

2. 350

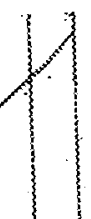




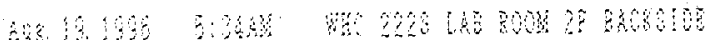

40424

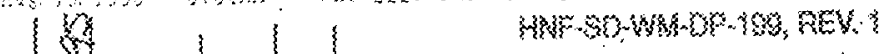
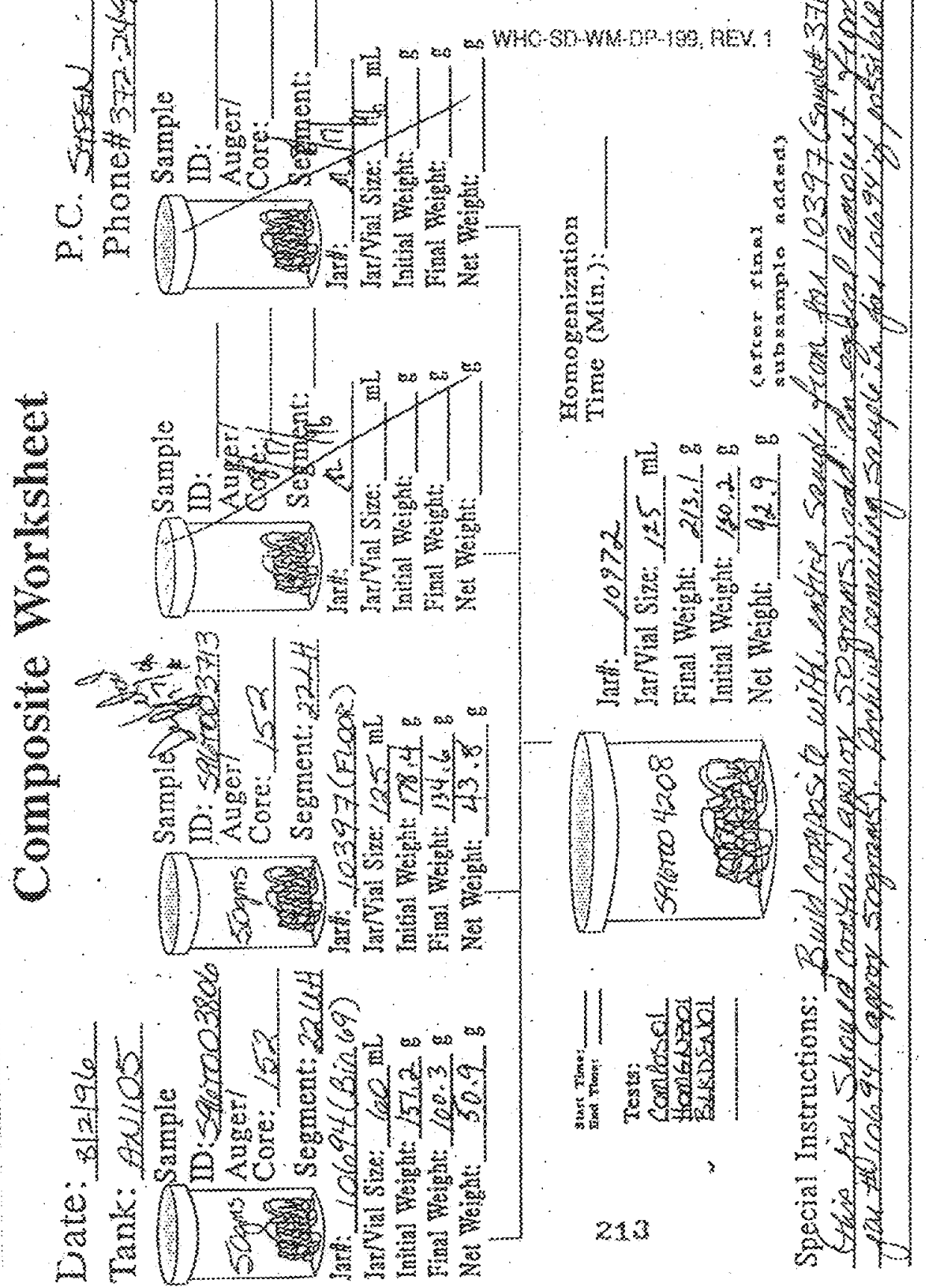


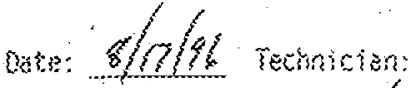

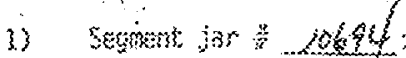

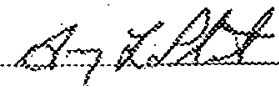

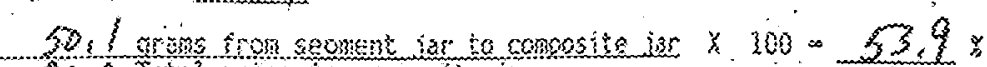
Q2. 4 jotal groms in composite jar

2) 3egers ja: 30377

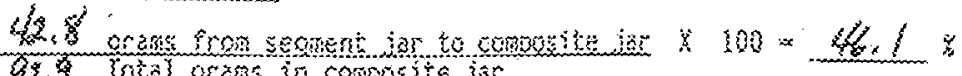

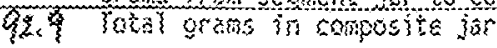

3) S\&uneti jor h

4) Segmonor

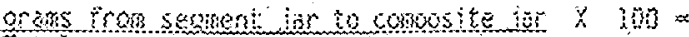
Texil orias in composite jor

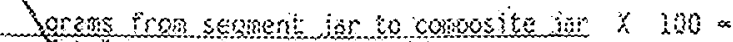
Gotal or ams in romposite jur

5) Senhent jar

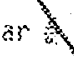

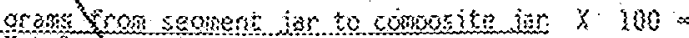
Total theins in compesite jur

$6)$ Segment $53 y^{\circ}$

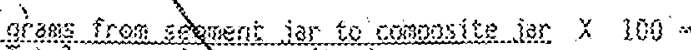
Total grans - in romosite jer

7) Segment jar

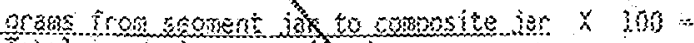
$: \%$ Total oraws in composibe iar

8) Sequent jer ju

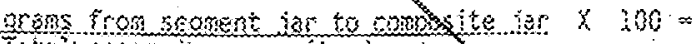
r Total grams in compost te jer

3) SEghent tai :

3hes from seoment iar to conos ira iar Totx grais in componte jar

30) Segrent 3 a : *
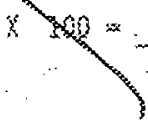

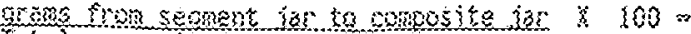

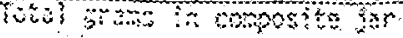




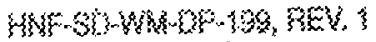

(a)

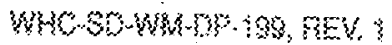
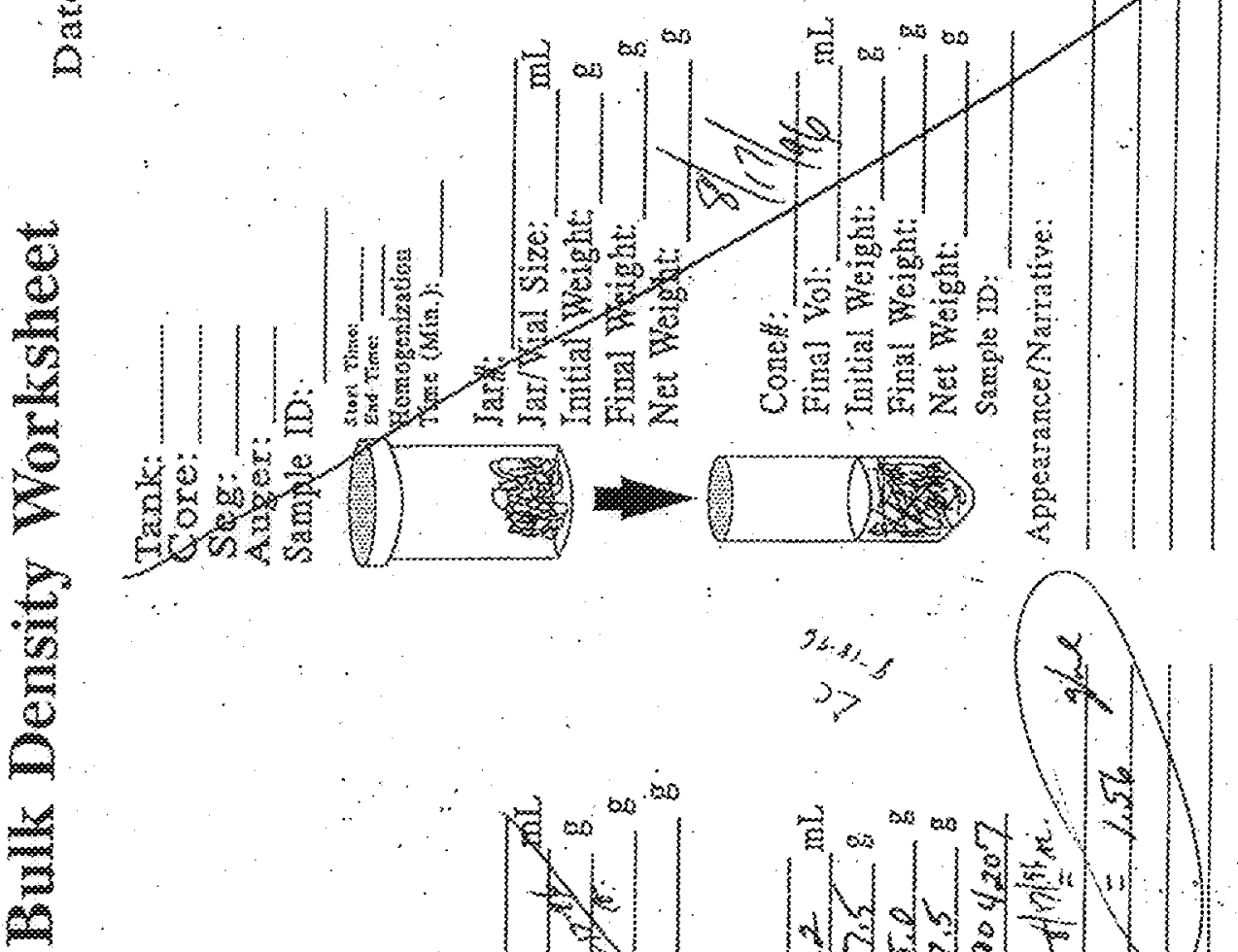
Anskrste

Zrasterstrent: BAOKY)

3 sosk $\ldots$........

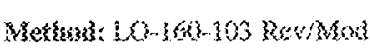

4

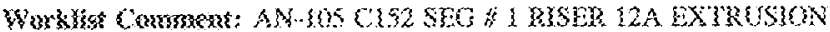

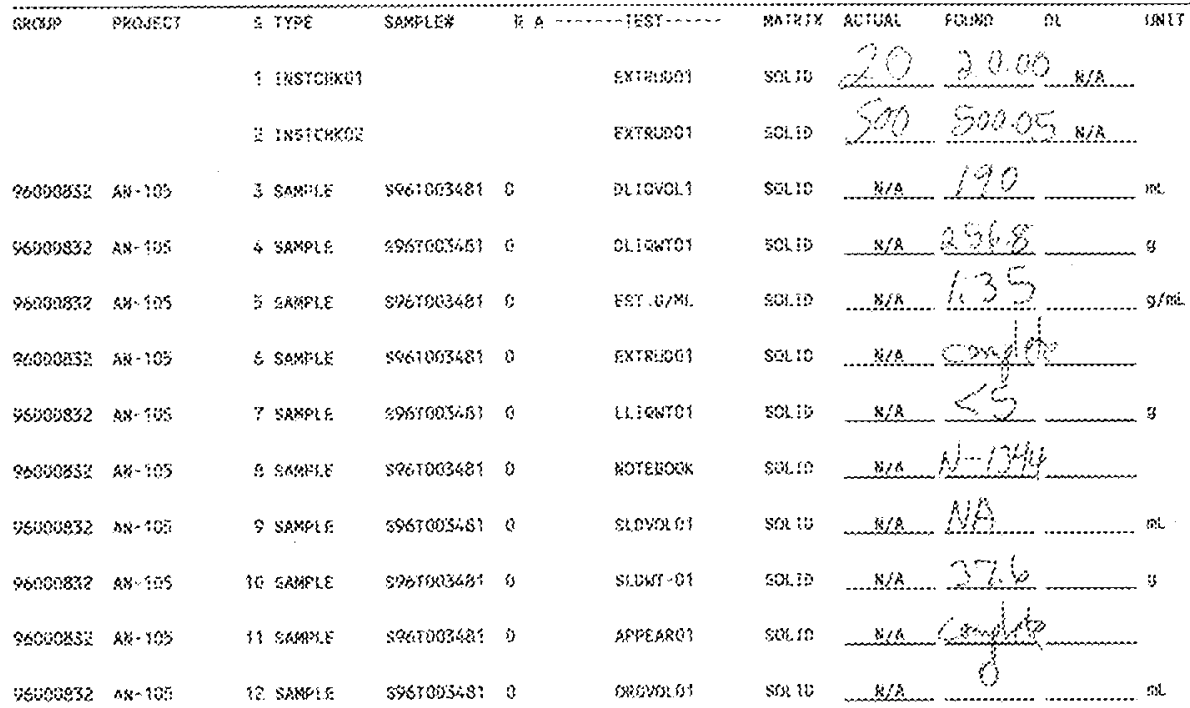

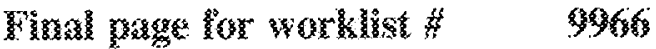

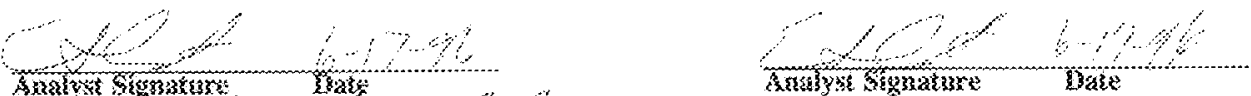

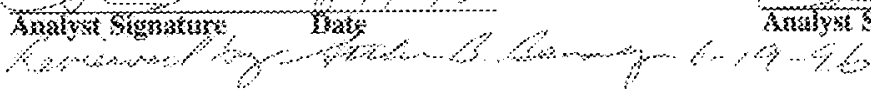

Busa Dron Sommen:

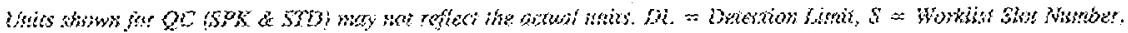

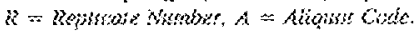


और

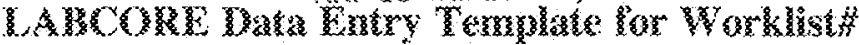

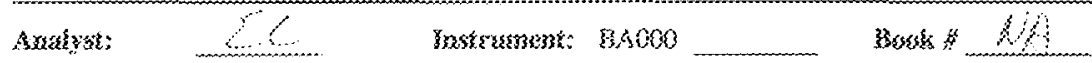

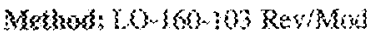

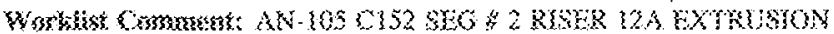

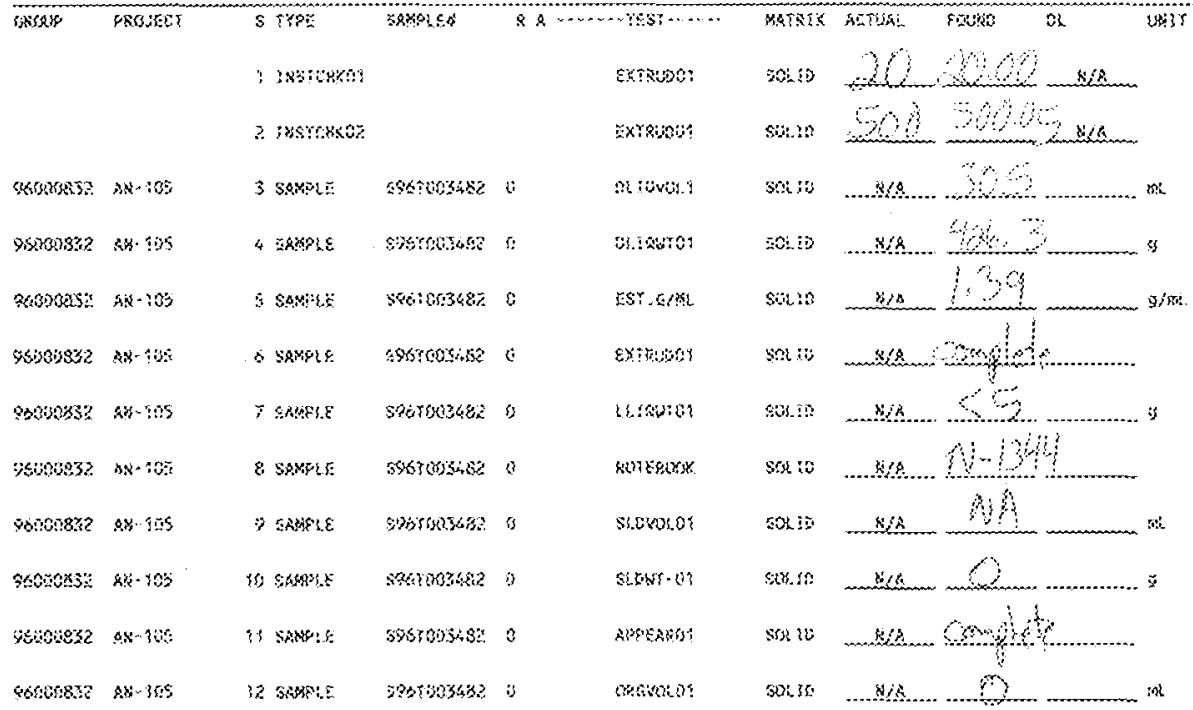

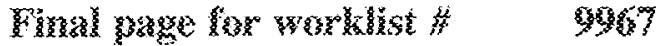

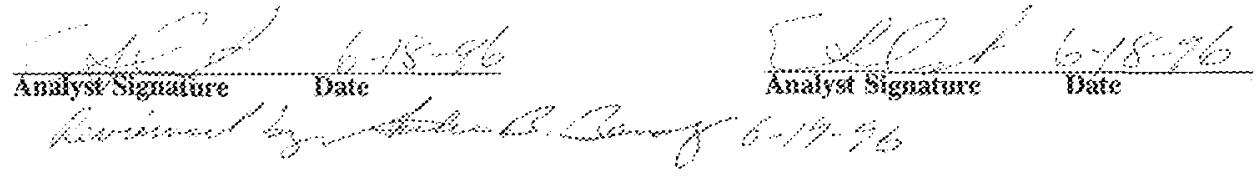

Eotus Ensy townonts:

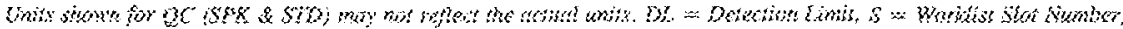

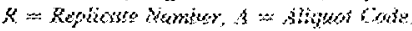




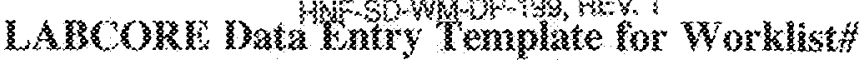

Anakss:

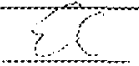

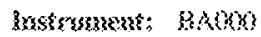

3yissil : :

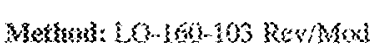

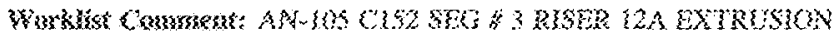

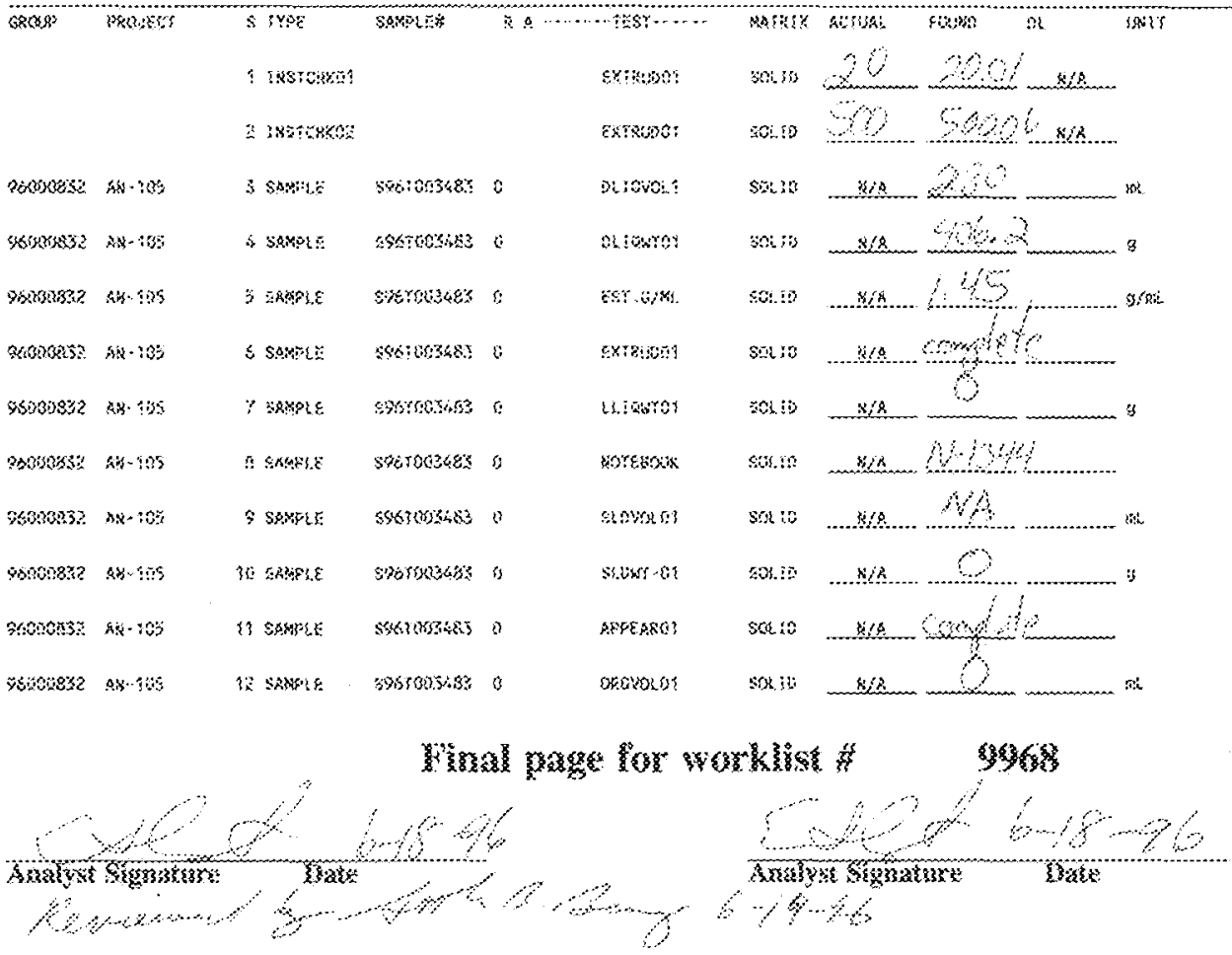

Thuse Erat: Gommens:

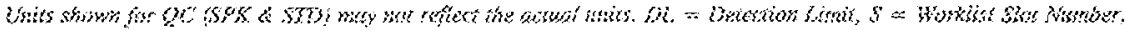

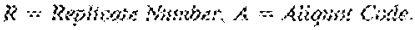




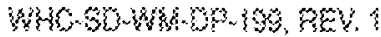

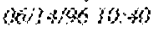

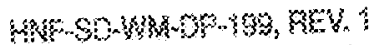

Page: $\quad$,

\section{W}

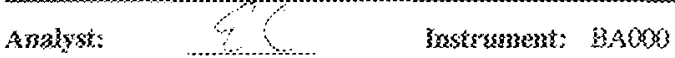

Brosk is is

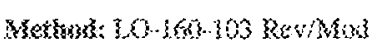

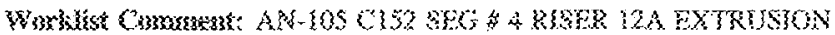

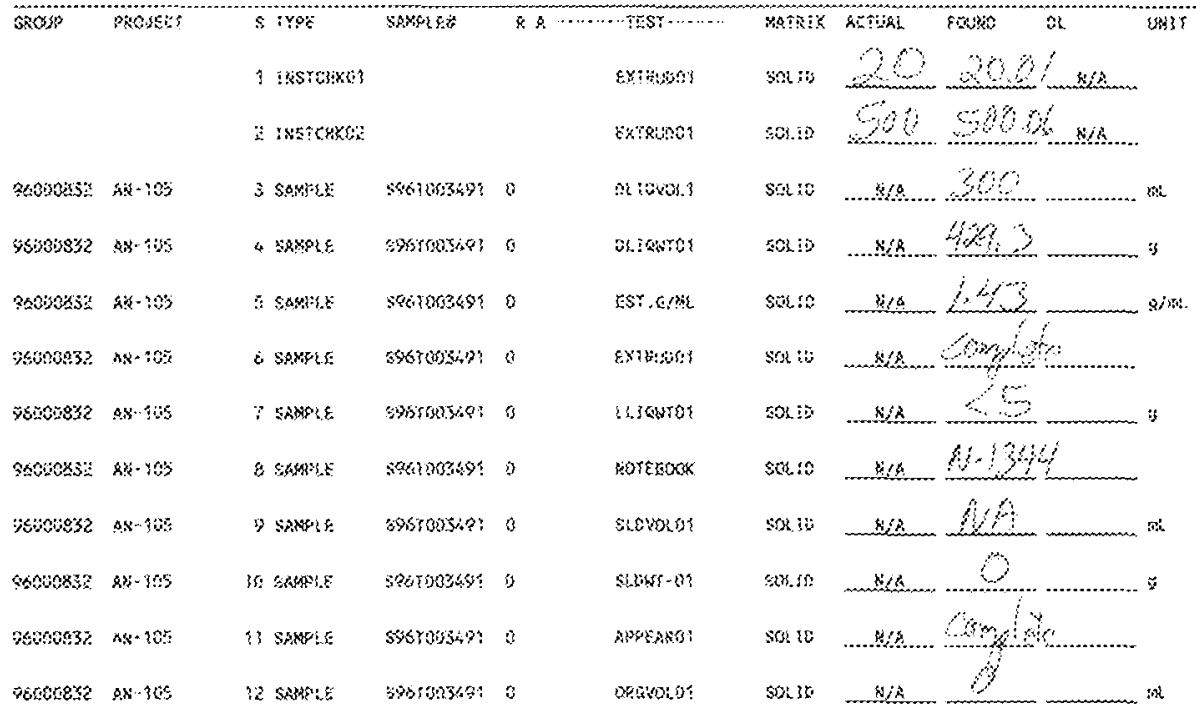

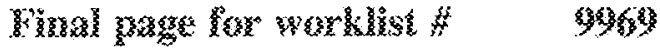

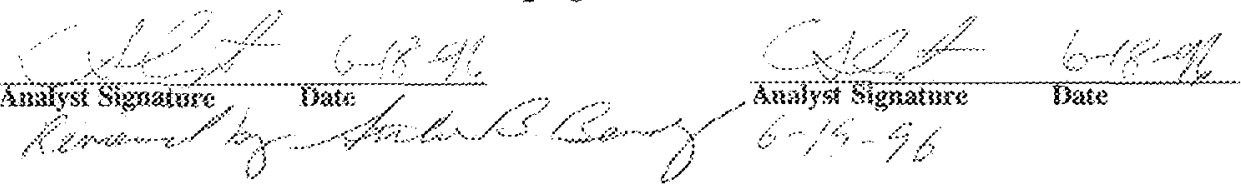

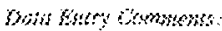

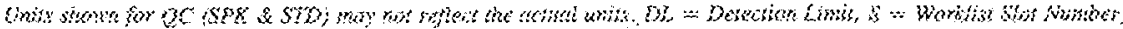

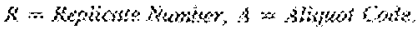




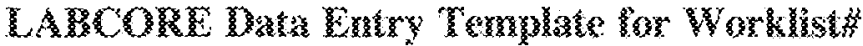

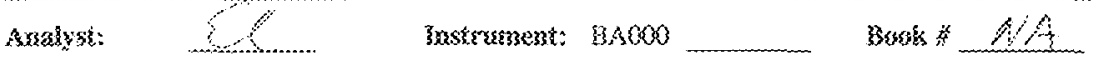

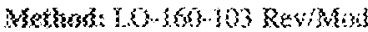
0

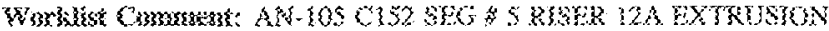

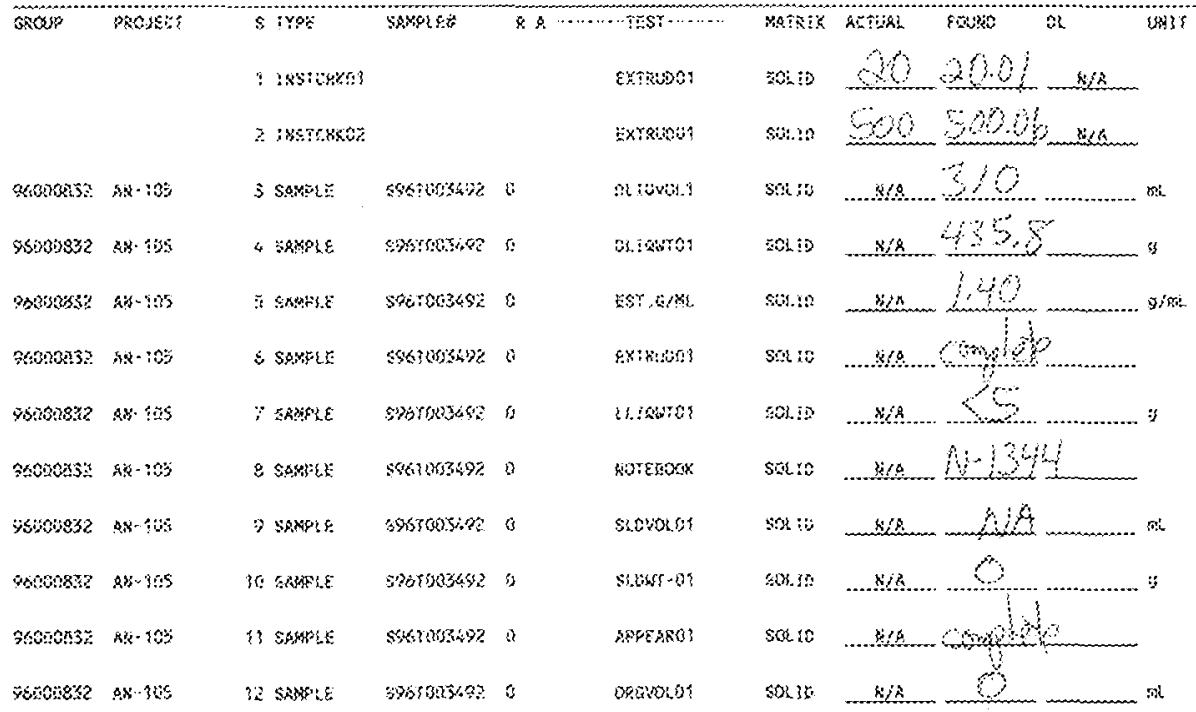

Ther yoge

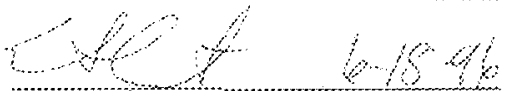

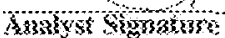
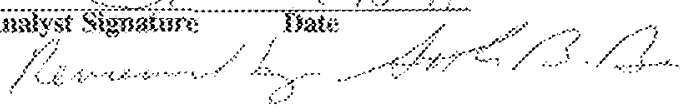

Anstyot segatisto

$\$ 3 \%$

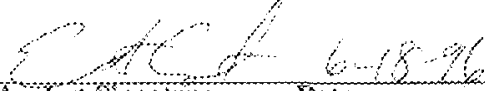

rats

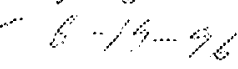

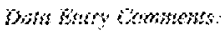

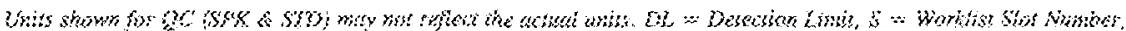

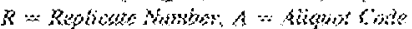




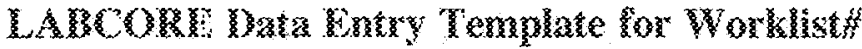

Anityst:

Bastrument: BA\{XYS

Sook $: \ldots$

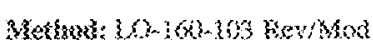

3

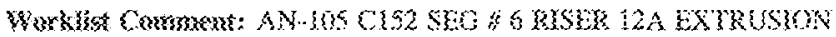

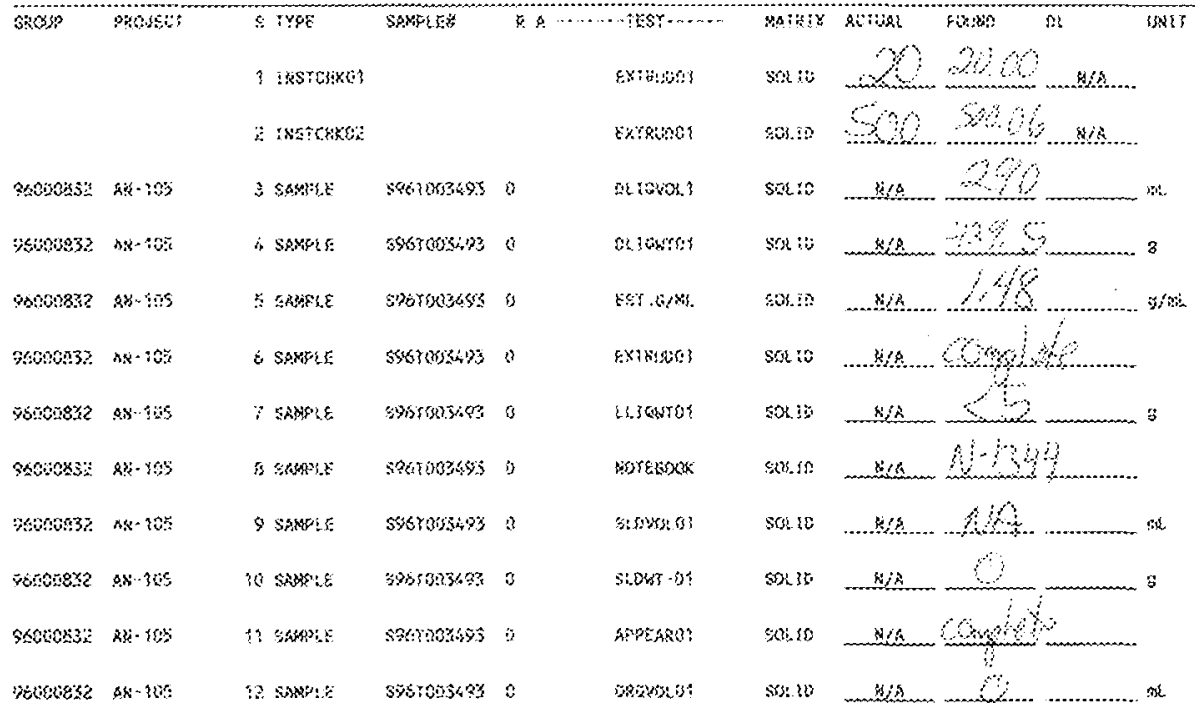

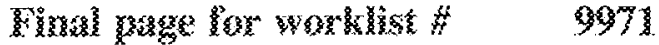

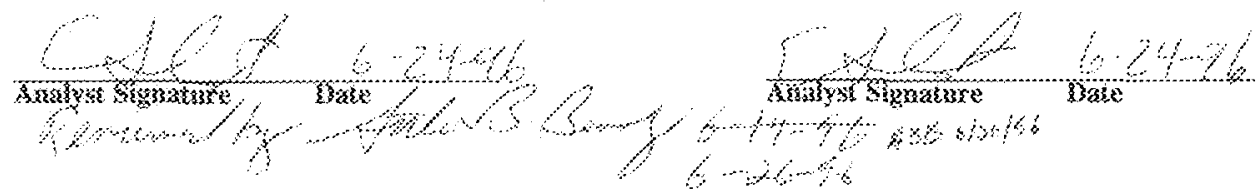

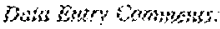

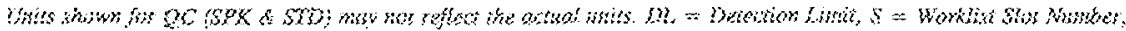

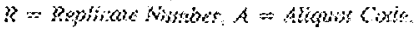




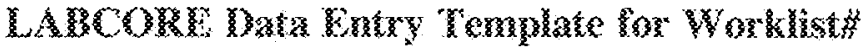

Ansulys:

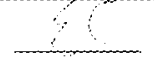

3rstirsmen: RA(X)

i

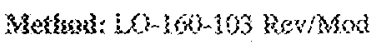

$330 \%$ \%

4

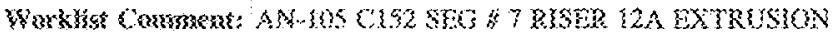

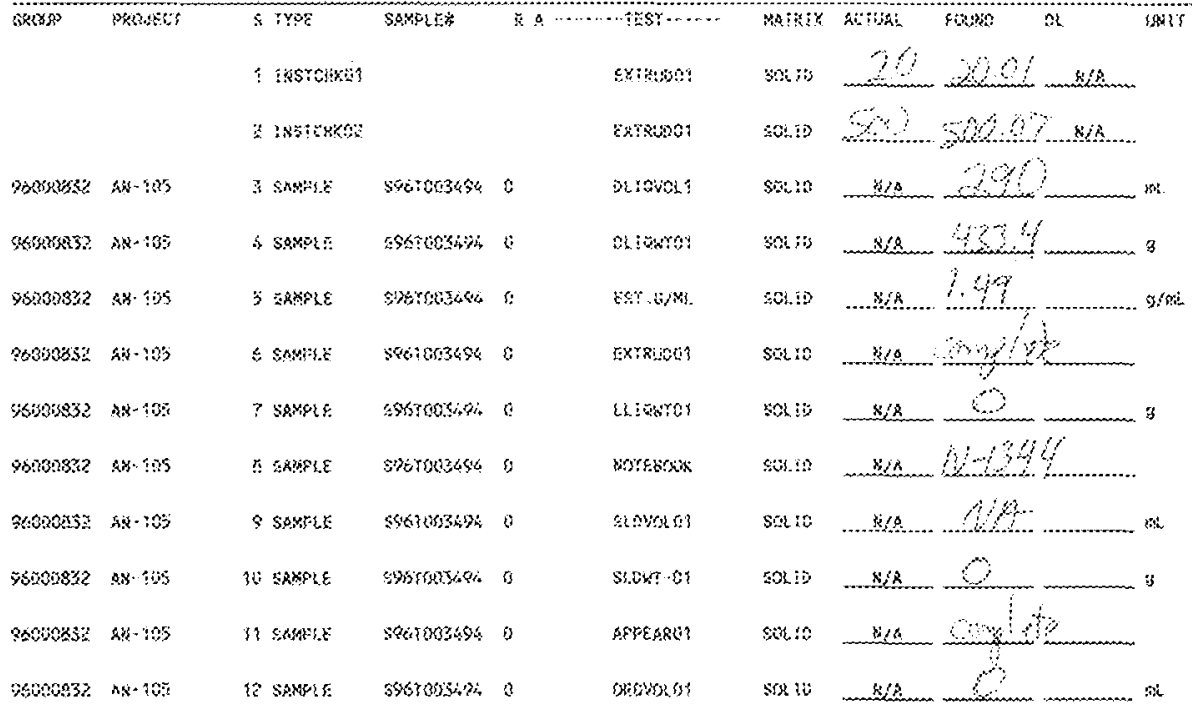

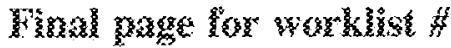

\section{2}
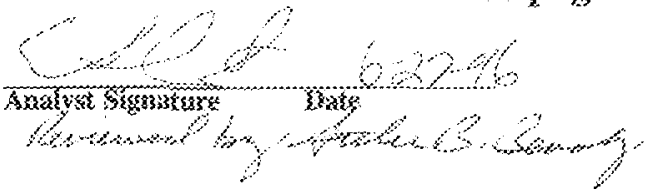

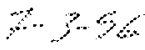

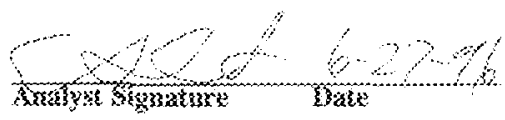

Exwa Erang somenest:

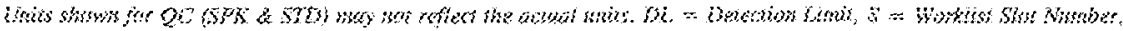

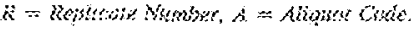




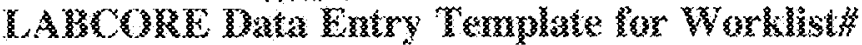

\begin{tabular}{|c|c|}
\hline Aurelyxi: & 2 \\
\hline
\end{tabular}

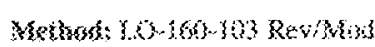

3

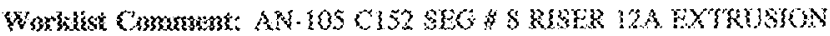

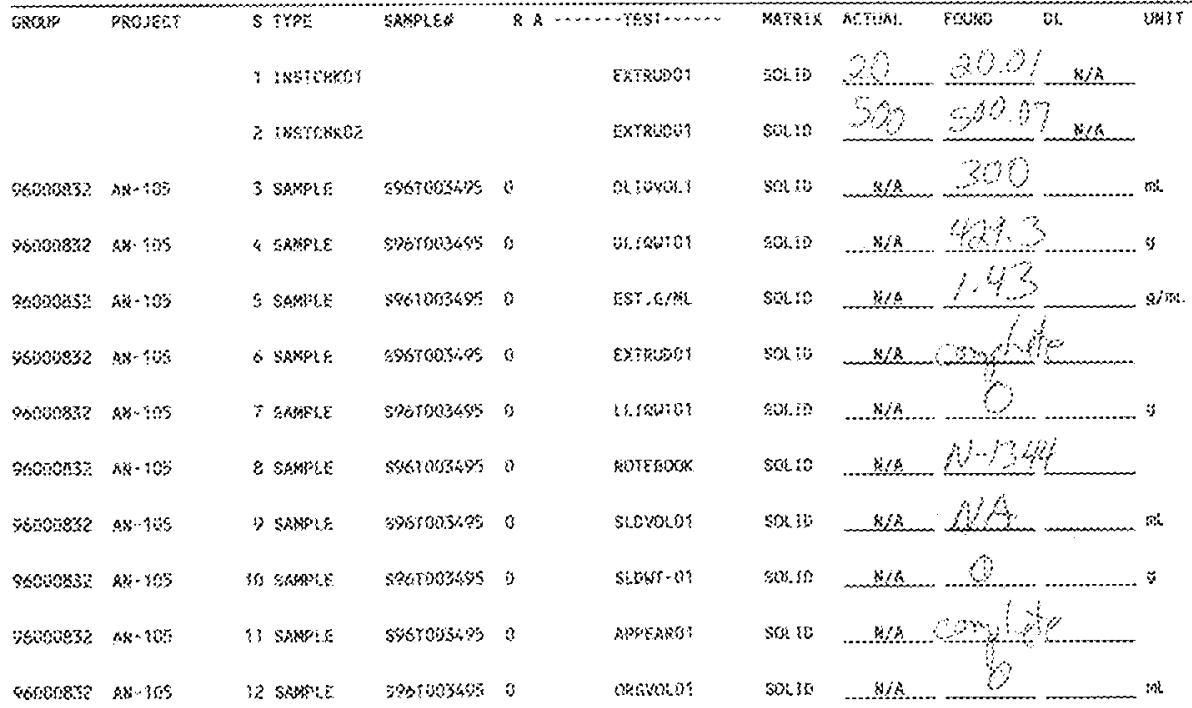

Fo

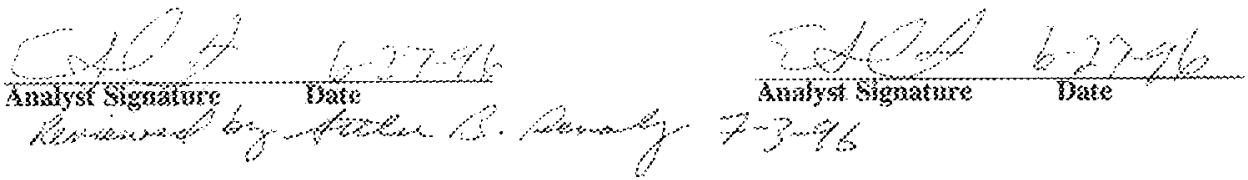

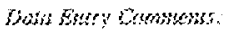

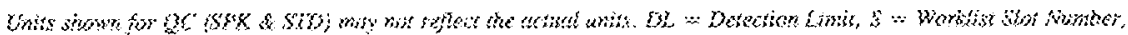

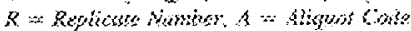




\section{D CORg}

Analyst:

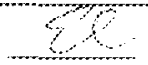

3rstrusuent; BASXX

smok $* 3 ;$

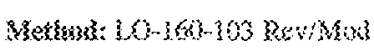

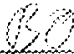

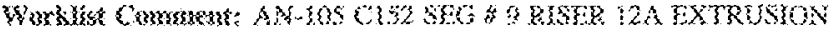

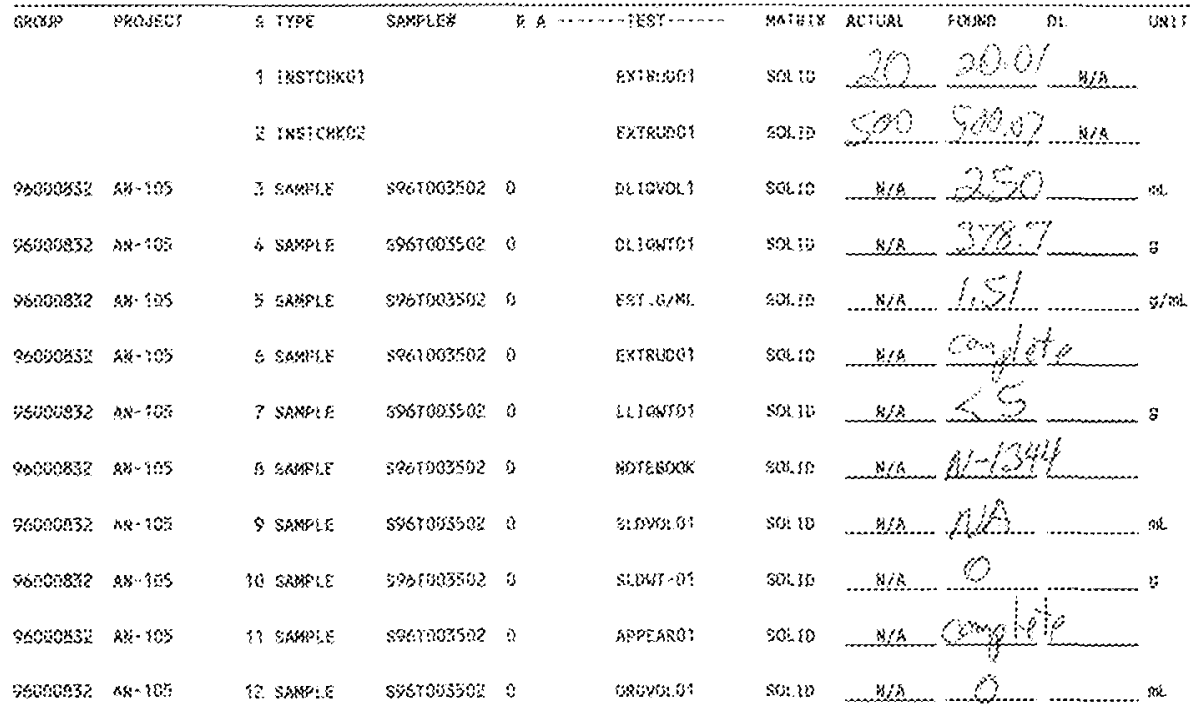

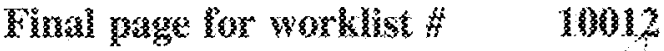

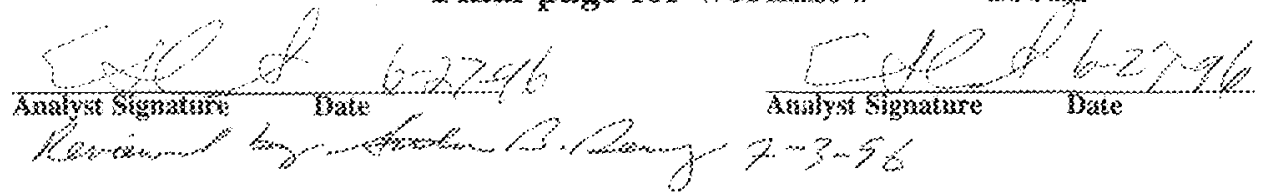

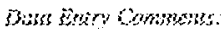

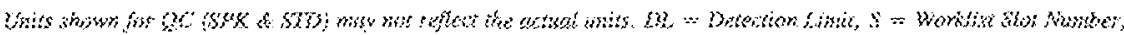

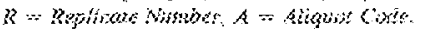


Analys:

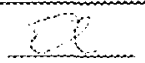

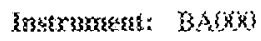

3arks ith

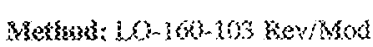

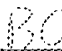

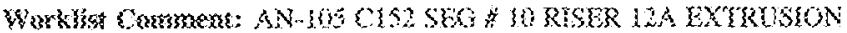

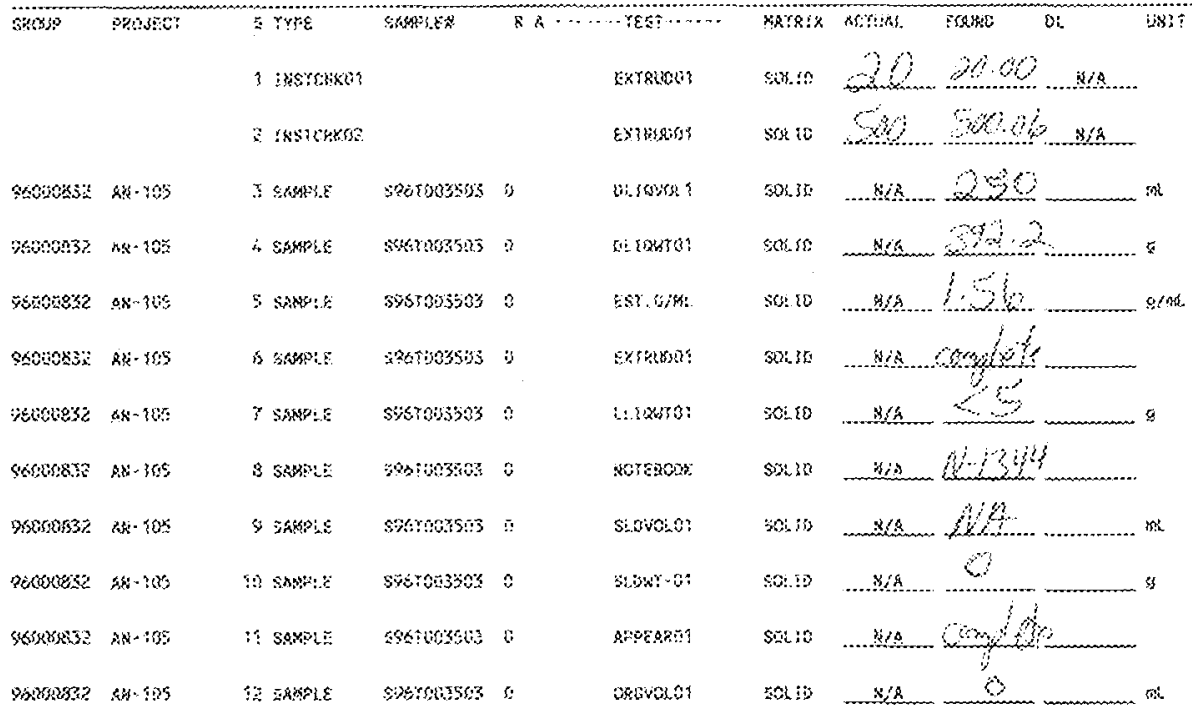

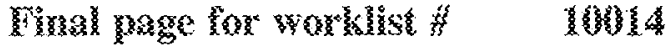

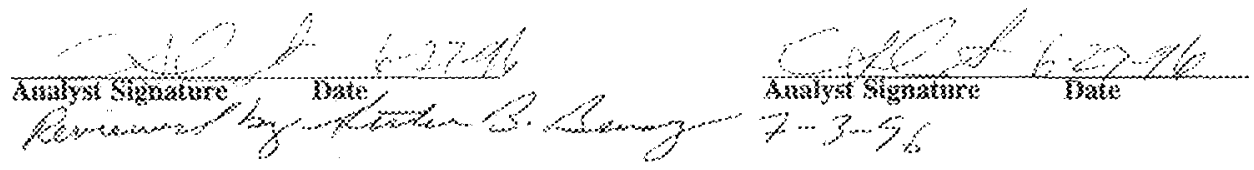

Dota binto tomments

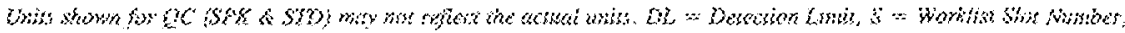

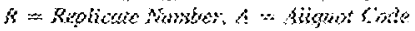




\section{F}

Axalys:

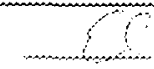

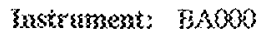

3robs:

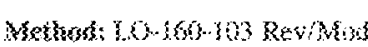

$\because$

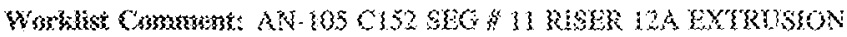

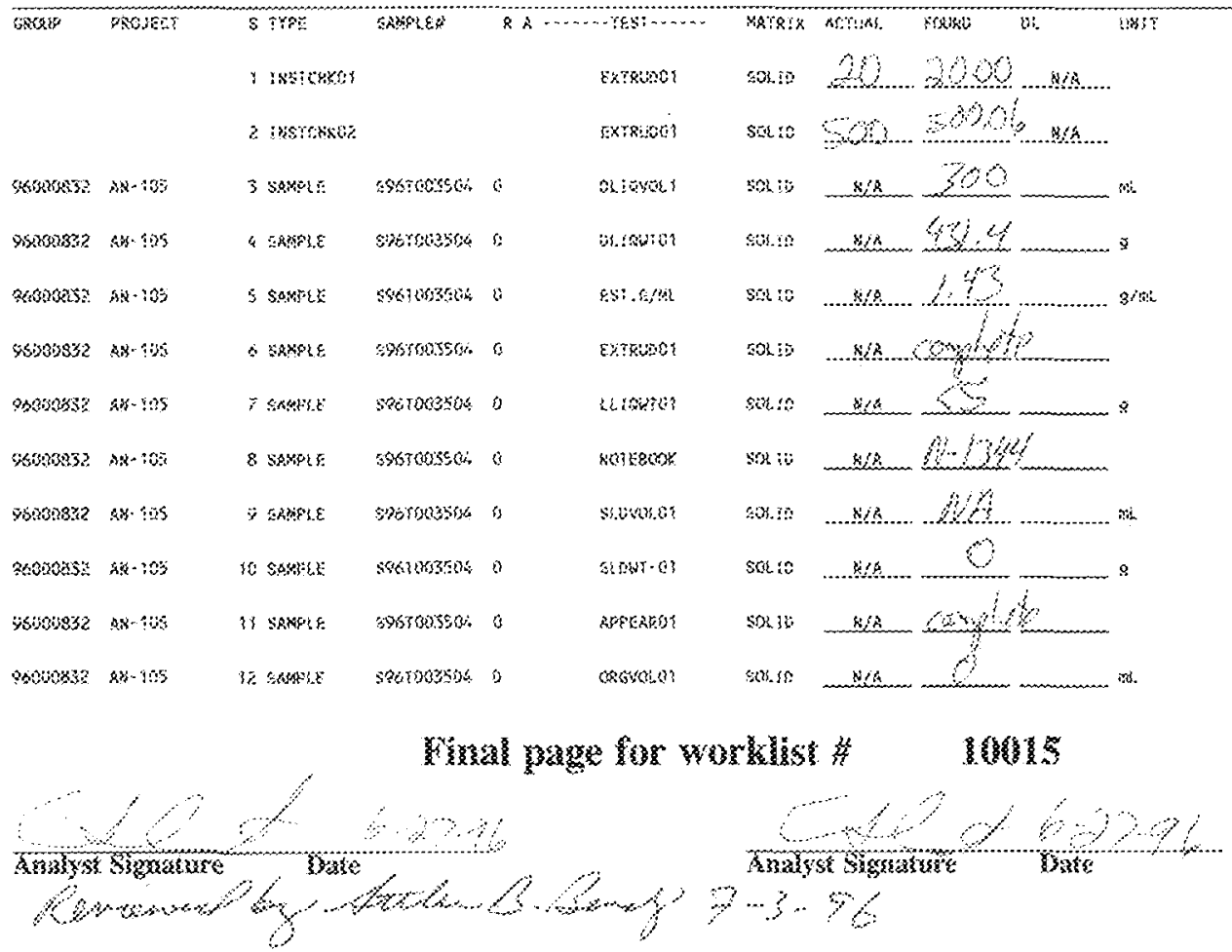

Dow Ensy chmonts.

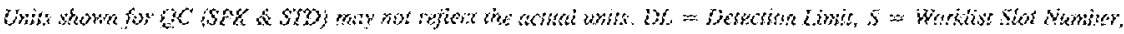




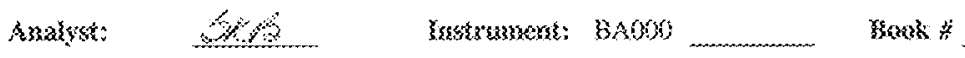

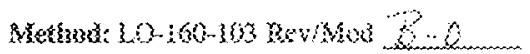

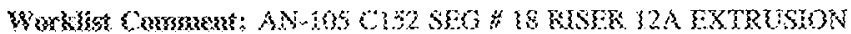

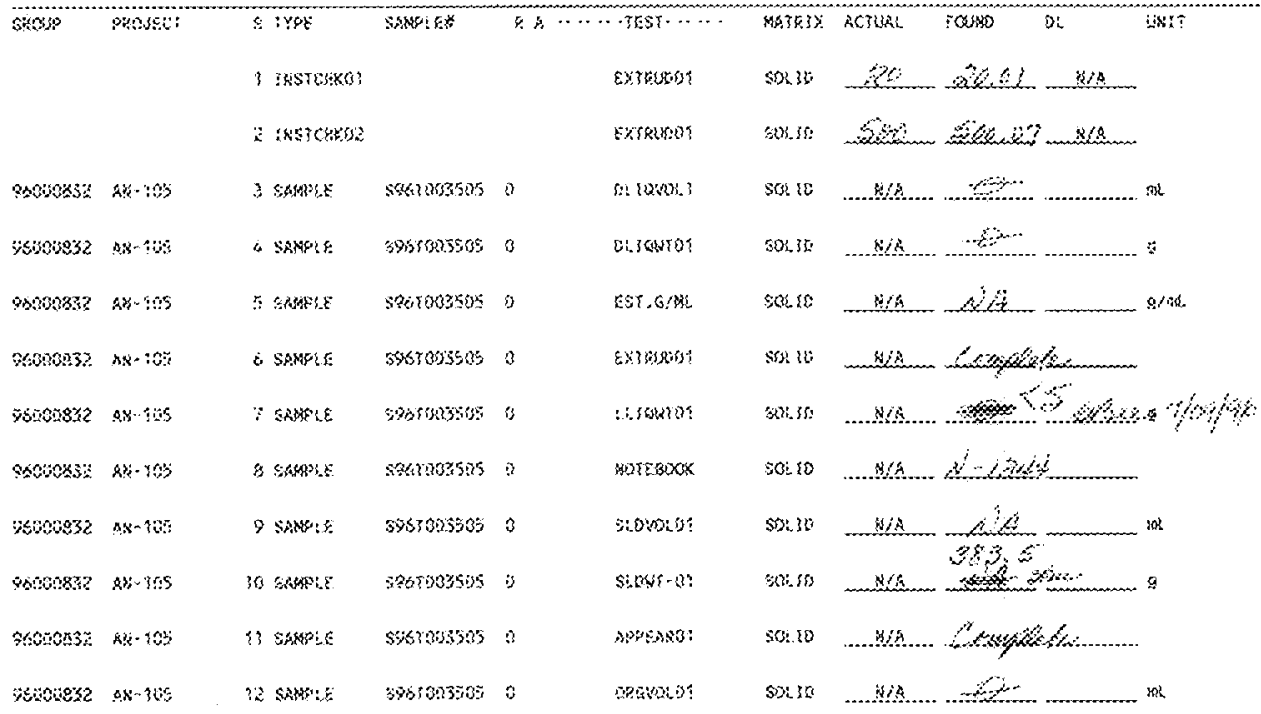

\section{3.}

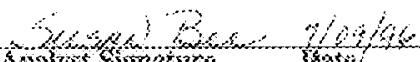
Sisiys signatust: knto

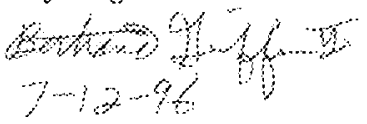

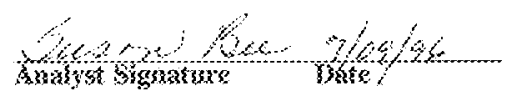

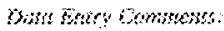

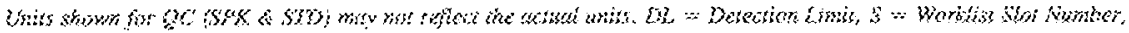

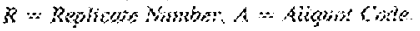




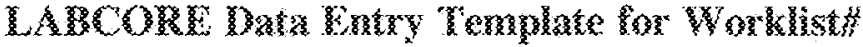

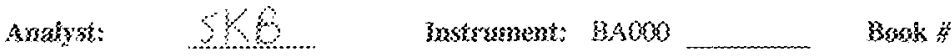

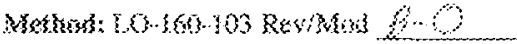

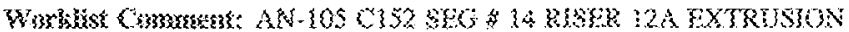

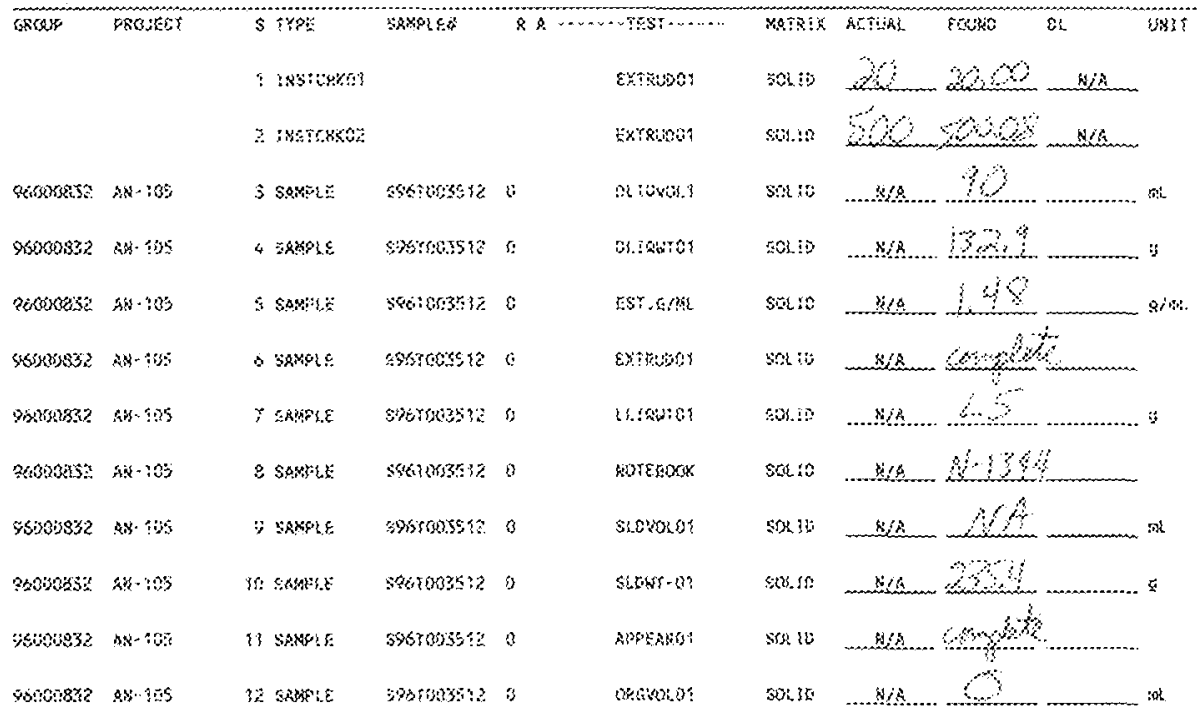

\section{gind}

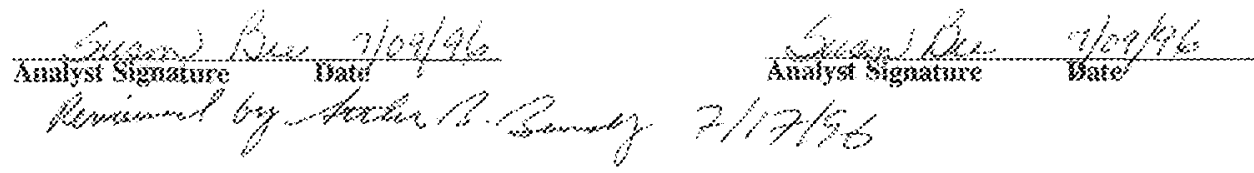

Buts Gury Eomstests:

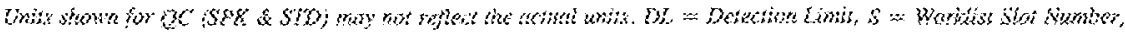

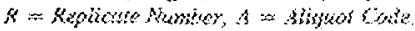




\begin{tabular}{|c|c|c|}
\hline Anakgs: & Irsstrsyment: & $3 \mathrm{~A} 000$ \\
\hline
\end{tabular}

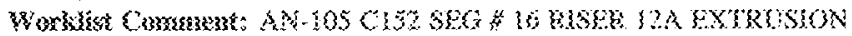

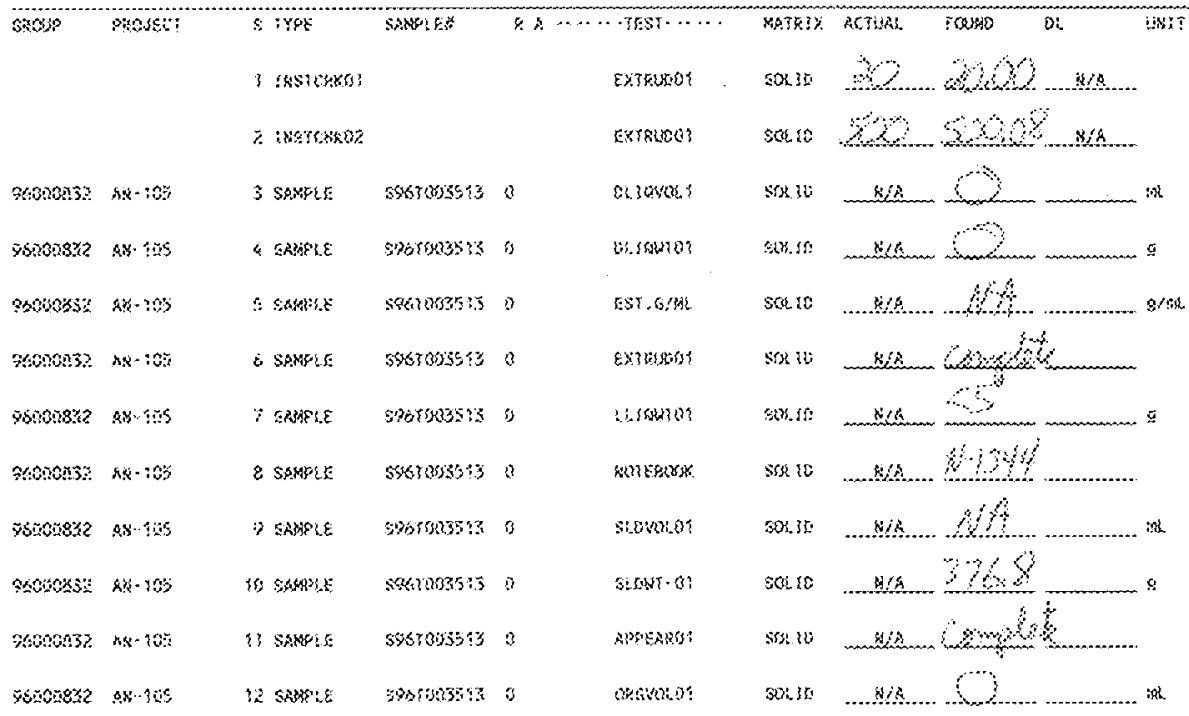

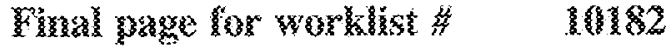
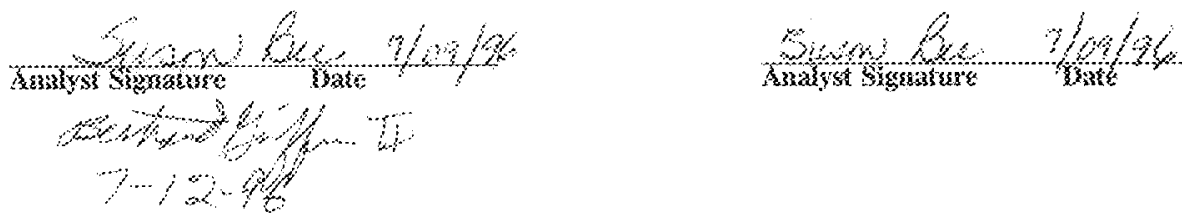

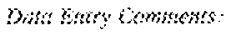

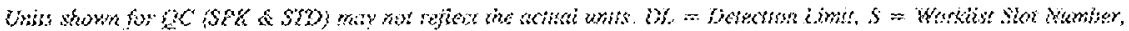

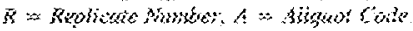




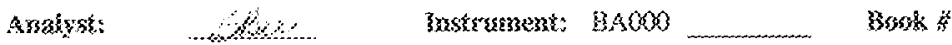

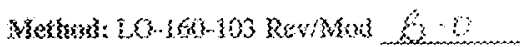

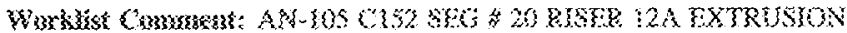

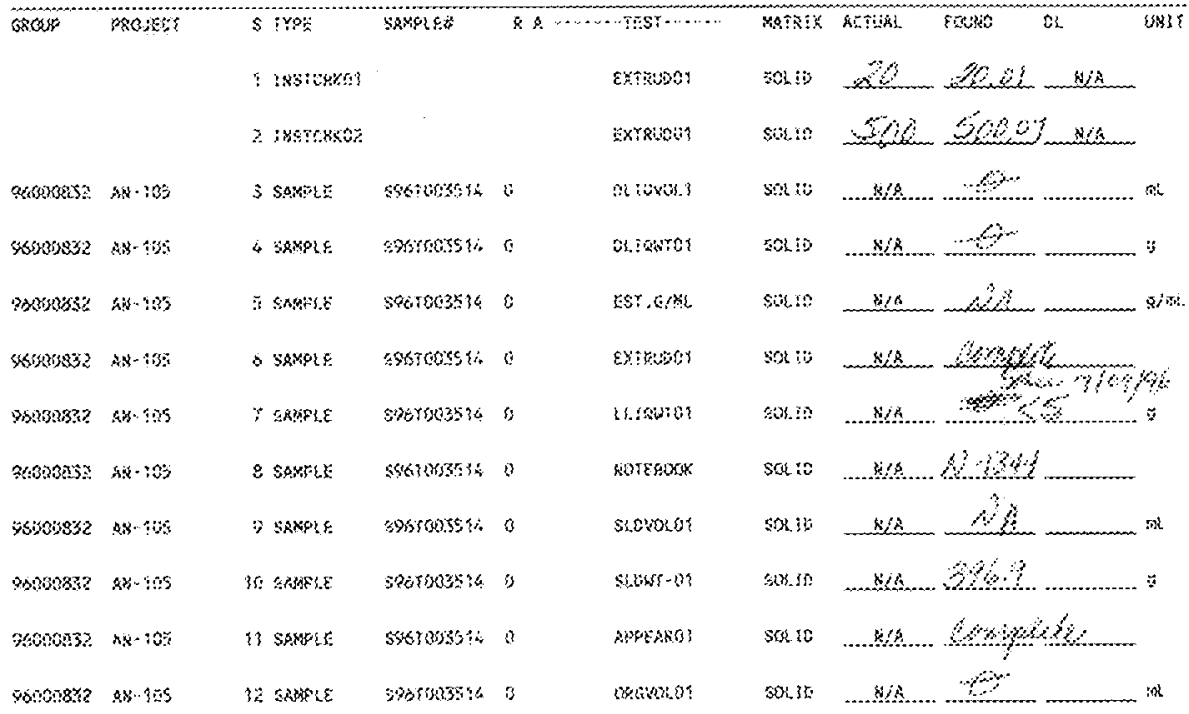

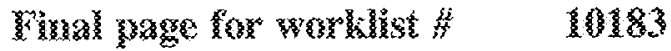
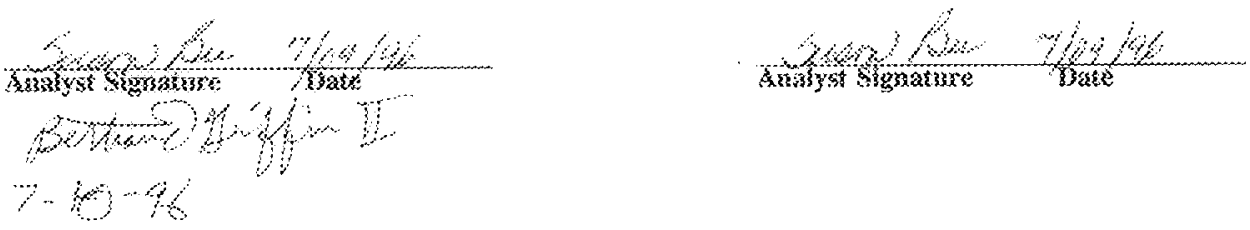

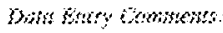

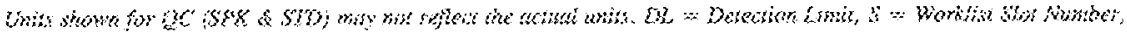

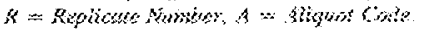




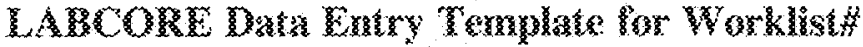

Ansaksst: $\quad 8$.

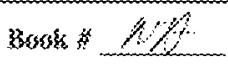

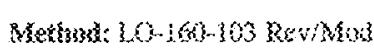
2)

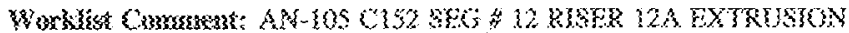

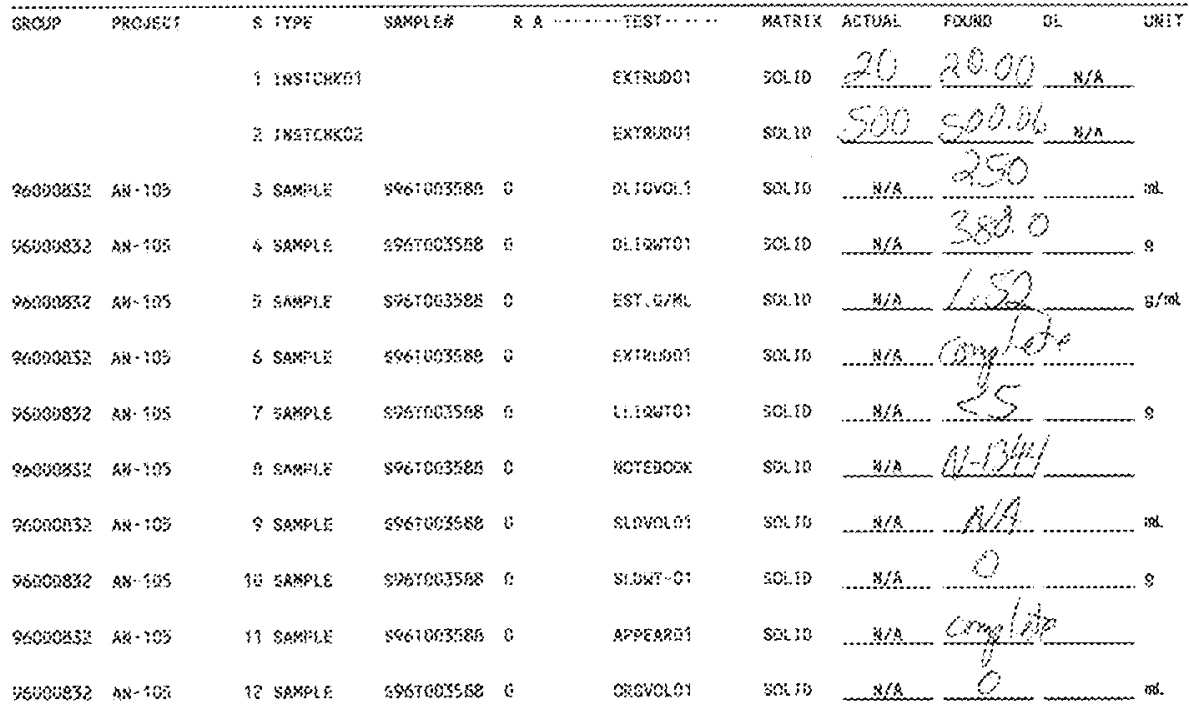

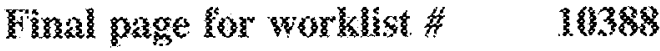

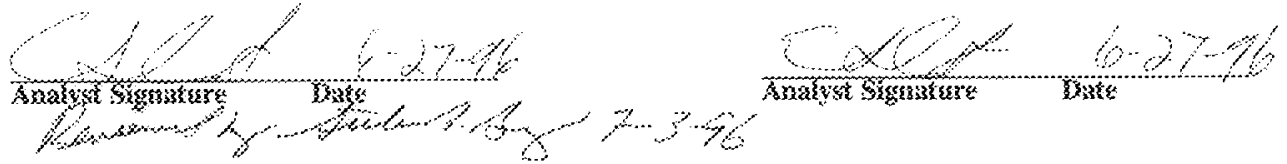

Enu Exrry Companis:

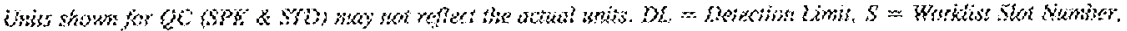

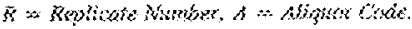




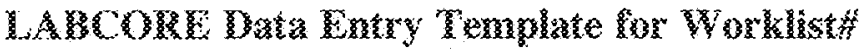

\begin{tabular}{|c|c|}
\hline Anaksh: & 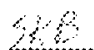 \\
\hline
\end{tabular}

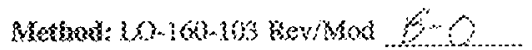

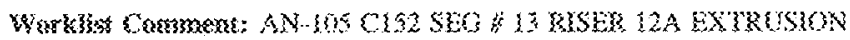

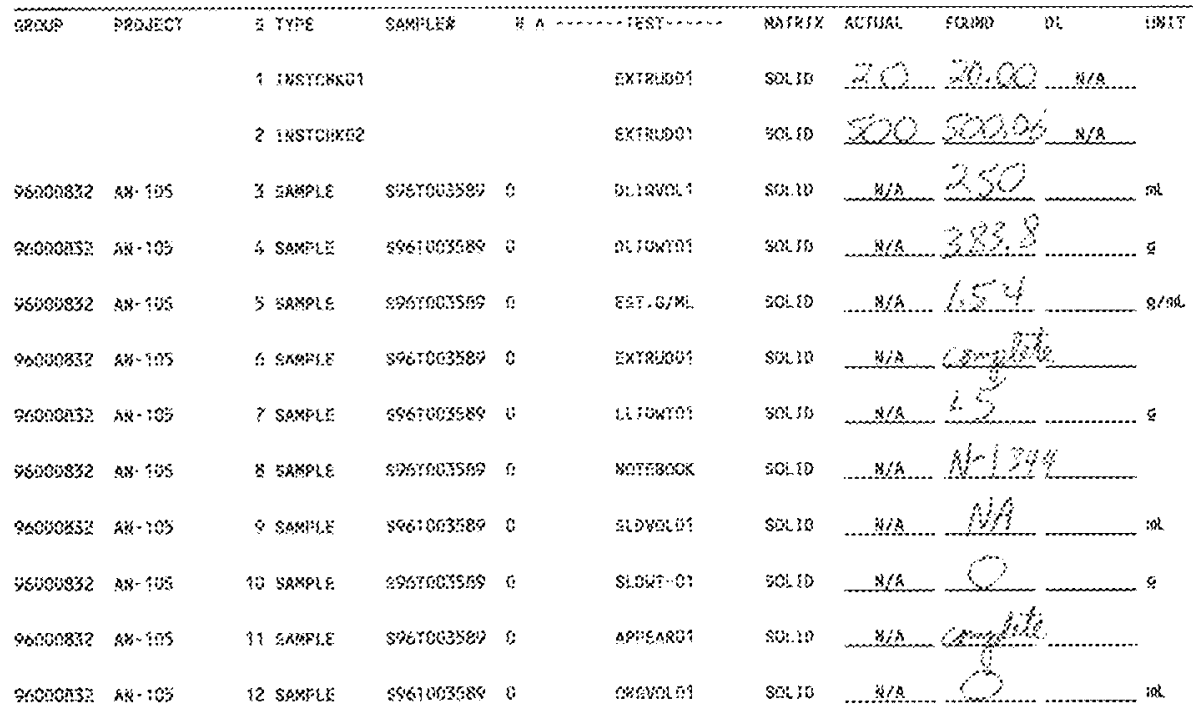

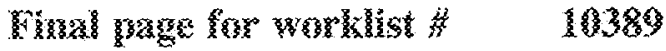

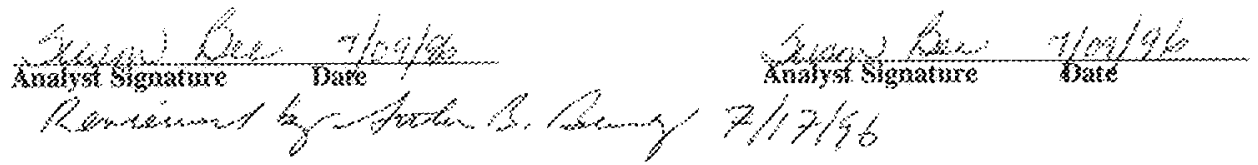

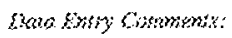

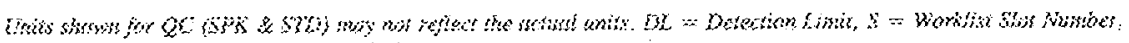

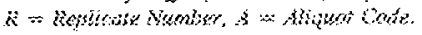




\section{Analys: 18 \\ $306 \%$ is}

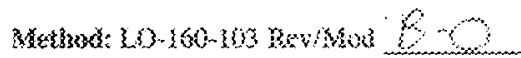

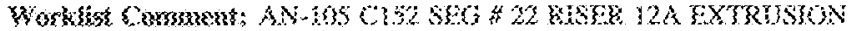

\begin{tabular}{|c|c|c|c|c|c|c|c|c|}
\hline \multirow[t]{3}{*}{ ercuó } & \multirow[t]{3}{*}{ QResect } & & TYFE & 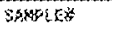 & \multicolumn{2}{|c|}{ f. } & \multirow{2}{*}{$\begin{array}{l}\text { woth:K } \\
\text { soi:is }\end{array}$} & \multirow{2}{*}{$\begin{array}{l}\text { ALIBAL } \\
\therefore y\end{array}$} \\
\hline & & & issretixe? & & & Ex:3000y & & \\
\hline & & $\ddot{z}$ & Natchoo: & & & Exrabos & sion & \\
\hline soososi: & $x \leqslant-y 03$ & 3 & $\sin \theta+1, \xi$ & 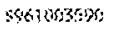 & 8 & a:3vor? & 30 ; & $3 / 8$ \\
\hline 09000832 & $n x+90 x$ & 4 & $\sin \times 4 \times 4$ & zsstowstos & 6 & Dindro: & 20, id & $\ldots / A$ \\
\hline $900008 \times 2$ & axus & $\%$ & $\operatorname{sMPS.E}$ & shereossed & 6 & $\Sigma S T, \Delta / B L$ & $\sin : 3$ & nis \\
\hline$\{0000 \times 3$ & $8 x-103$ & 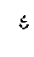 & Somste & 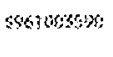 & : & extandel & sotis & mansisanom \\
\hline 36000932 & $3 x-30 \mathrm{~A}$ & $?$ & SAriple & serssosses & s & 24.00\%: & $50: 0$ & BiA.... \\
\hline 90000852 & abos & 4 & $\sin \theta+2 x$ & seongossse & 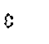 & boreyogr: & $\sin 10$ & xis \\
\hline S40nonsz & $x+90$ & $s$ & SAMELE & 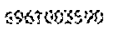 & 0 & seoves & $39 ;$ & 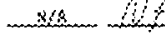 \\
\hline 4808832 & $A S \cdot 305$ & 30 & $5 \times>P<\varepsilon$ & shrsoss: & 5 & 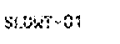 & SG: & Wra \\
\hline $\sec 60 \times 34$ & $82-y 03$ & 13 & $\sin \theta=15$ & SQR & 8 & AFockns? & $\sin 10$ & sts \\
\hline zasotons? & $x \mathrm{x}-90 \tilde{z}$ & $\hat{z}$ & samels: & $456 r 00 s+2$ & 4 & arvelay & 20,30 & $\frac{s_{i}}{2}$ \\
\hline
\end{tabular}

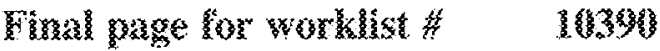

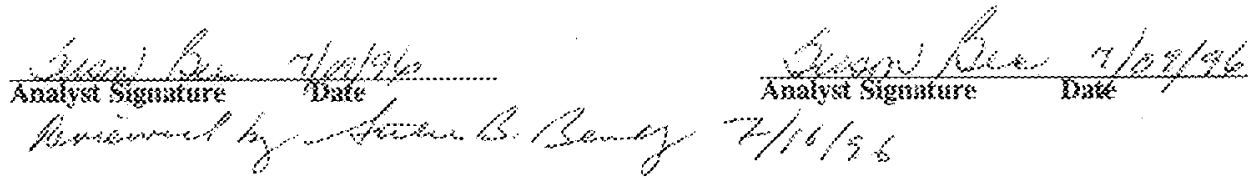

Gow Enst Sisnmentis:

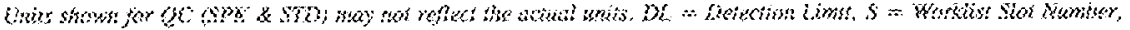

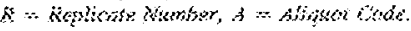




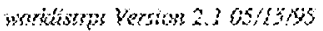

[Mosks 30:s?

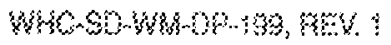

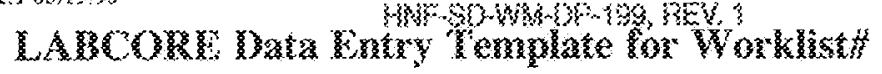

Poge: ;

S6\%

Anatyst:

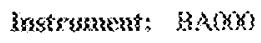

3 sssk : :

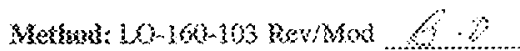

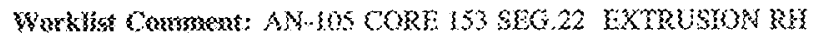

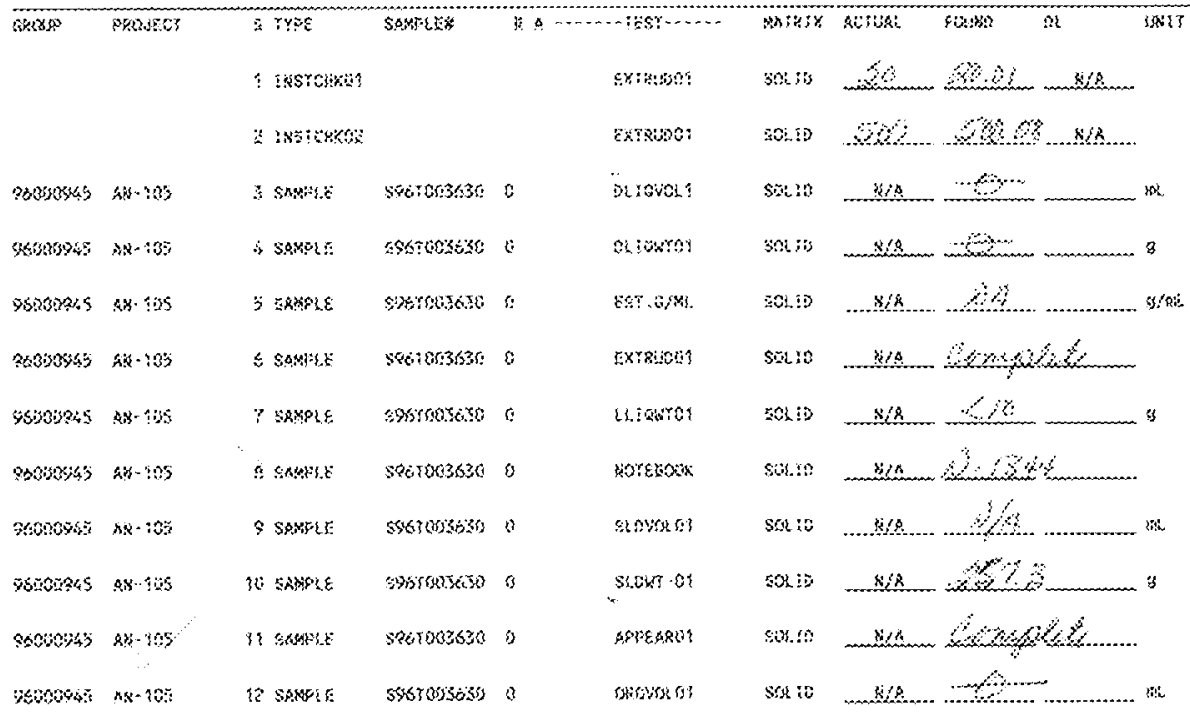

Fis

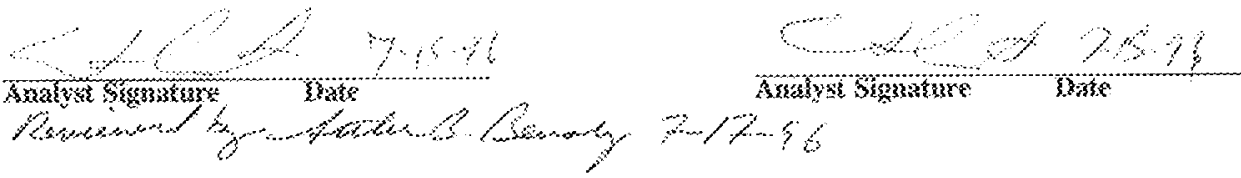

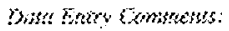

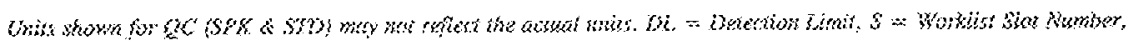

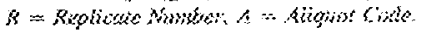
$2 \times 3$ 
Assalyst:

3rastrususes: $\quad M R(K X)$

35030 :

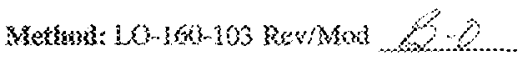

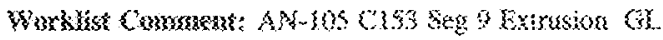

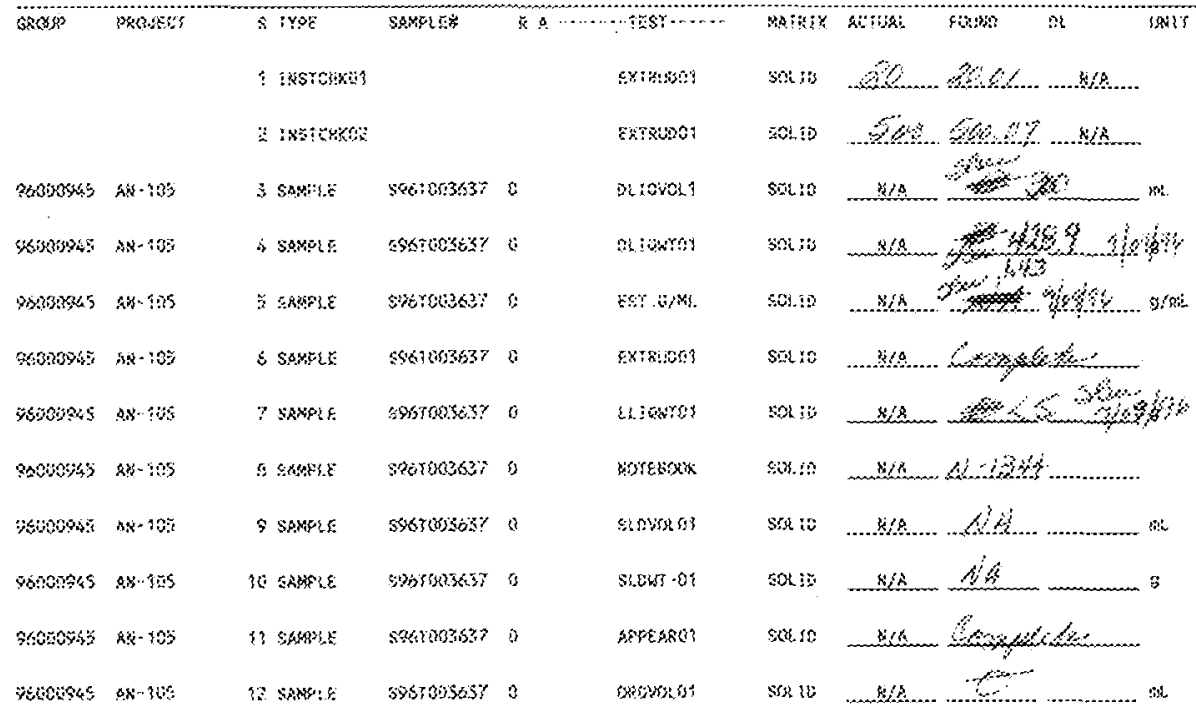

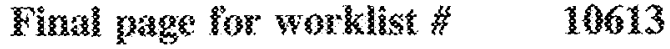
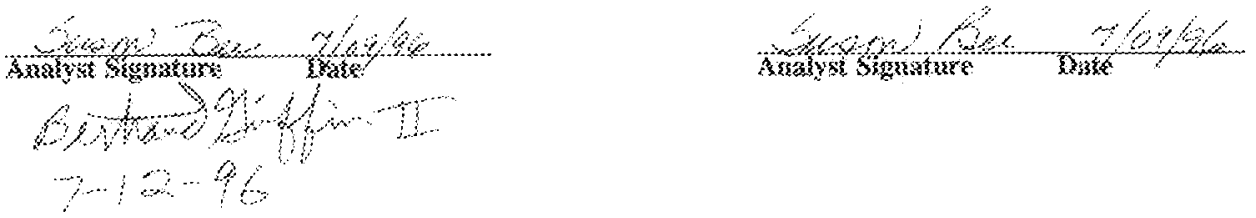

Fins Etory Sompens:

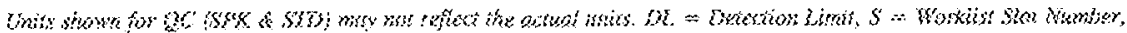

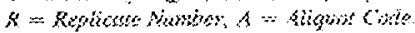


Analyst: $\quad \%$

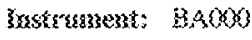

ssissk is

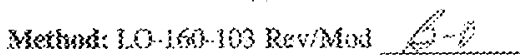

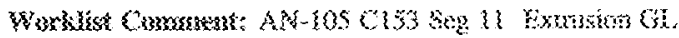

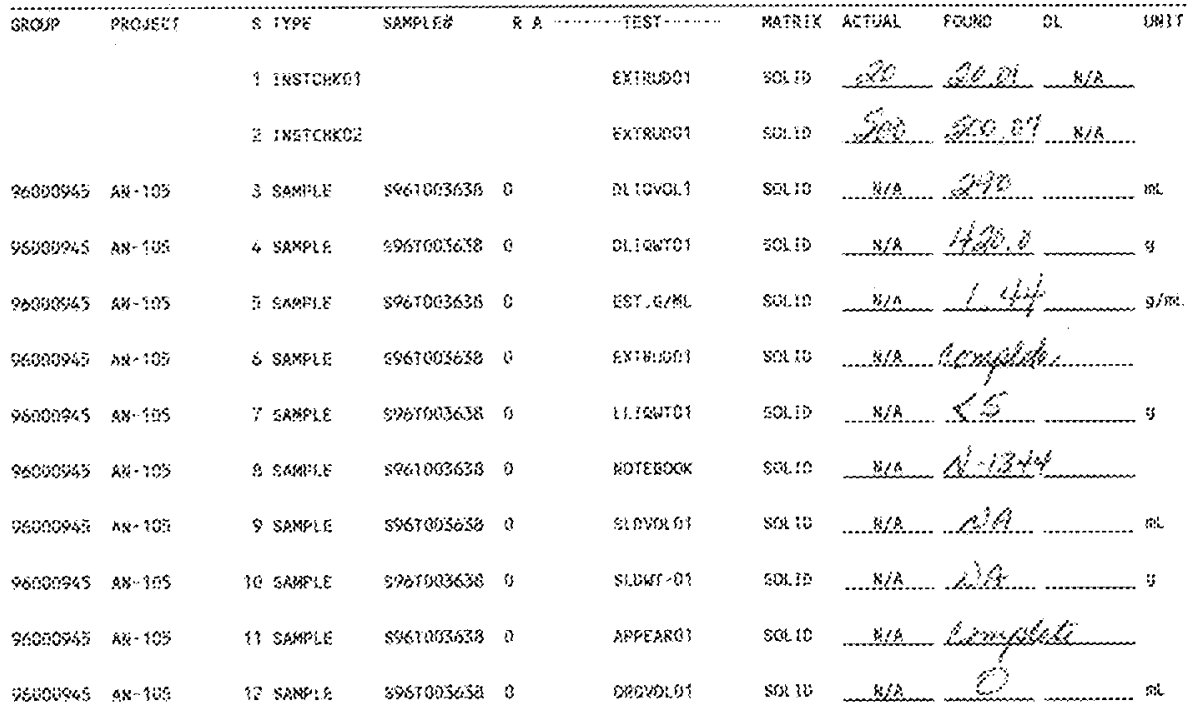

\section{ร)}
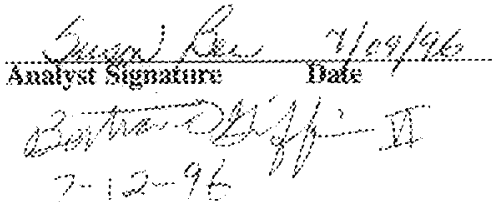

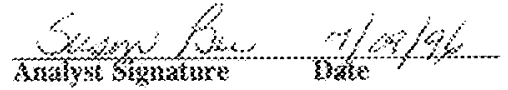

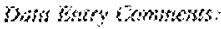

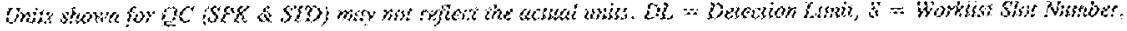

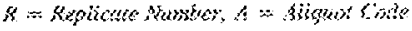




\section{Anabys: \\ Saxtroment: DAO00 \\ 35ses: is}

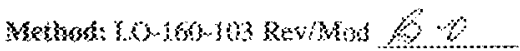

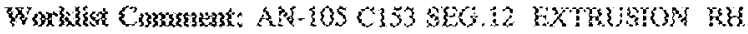

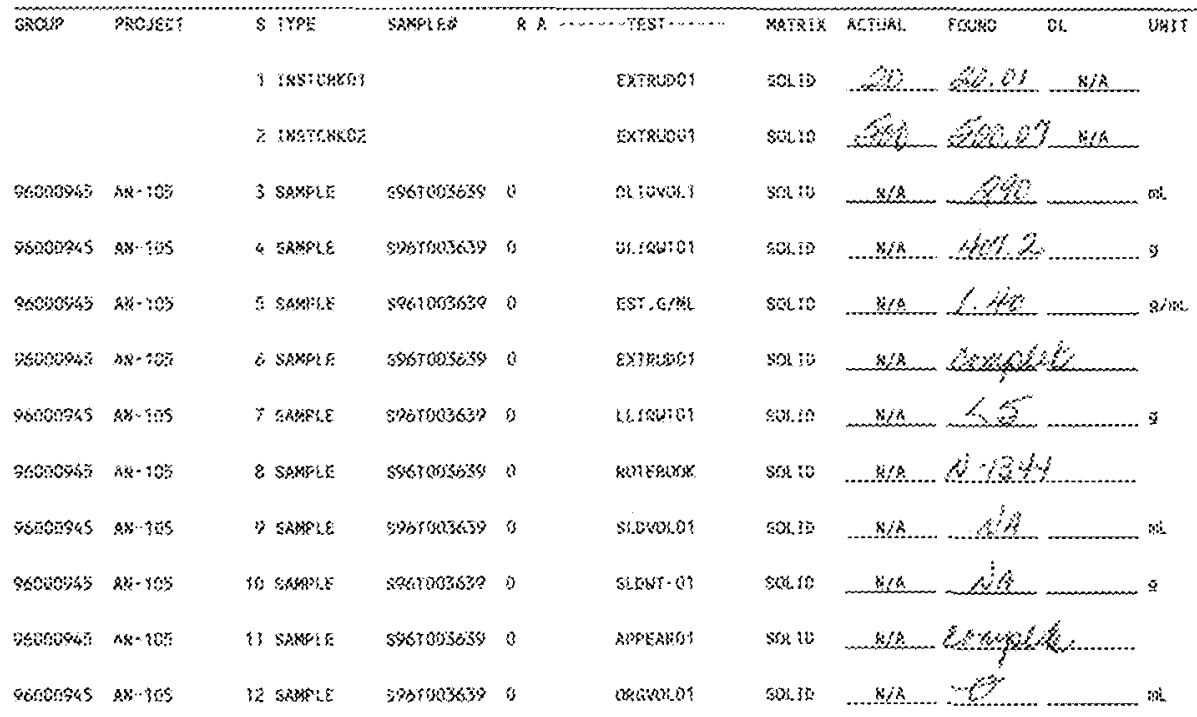

\section{Fura page}

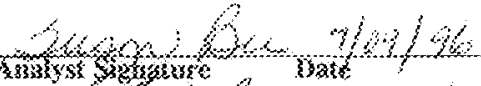

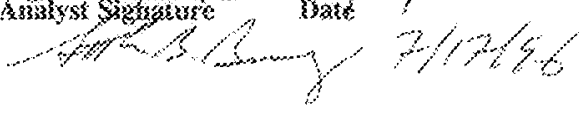

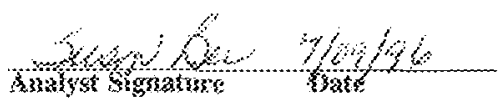

Dun tarty inomems:

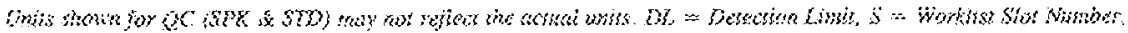

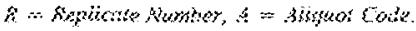




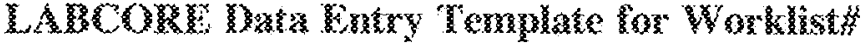

A3styst $\quad$ Het.

Srastruasewt: $3 R \times K O$

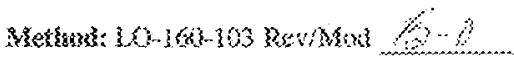

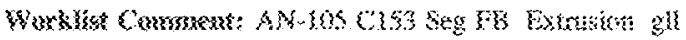

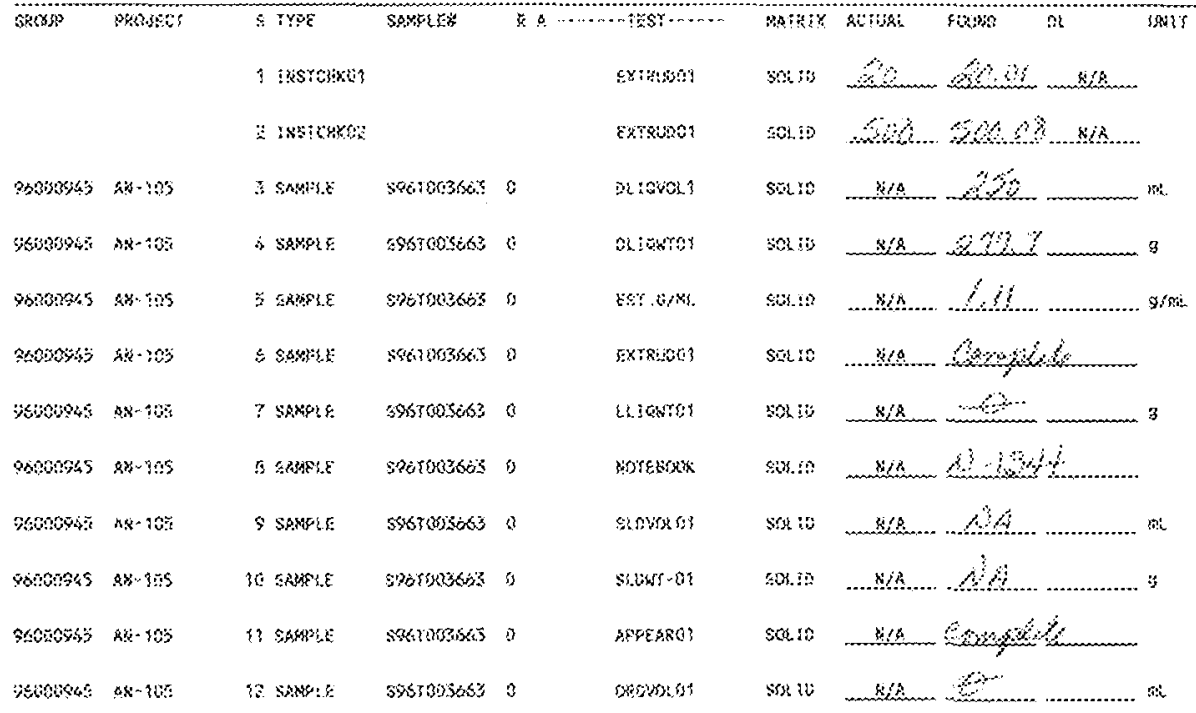

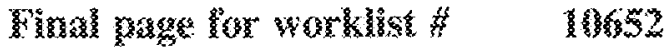

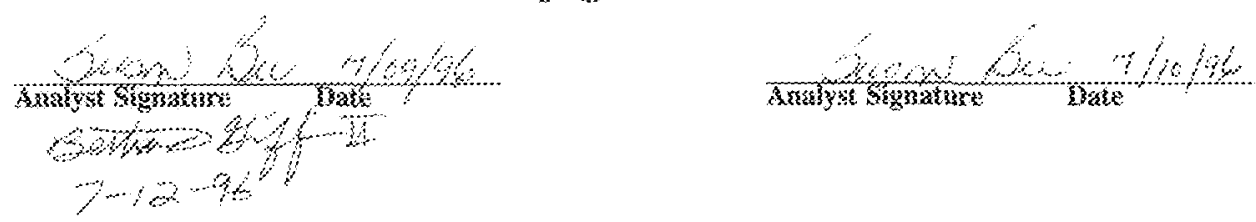

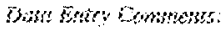

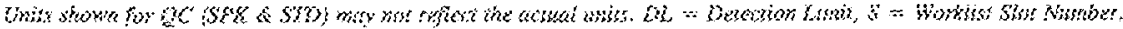

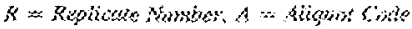




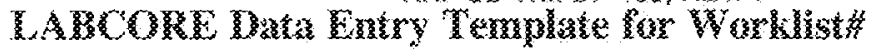

\section{Aralyst: \\ 20 \\ 3xascrument: B,S000

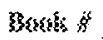

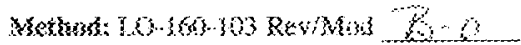

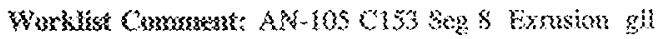

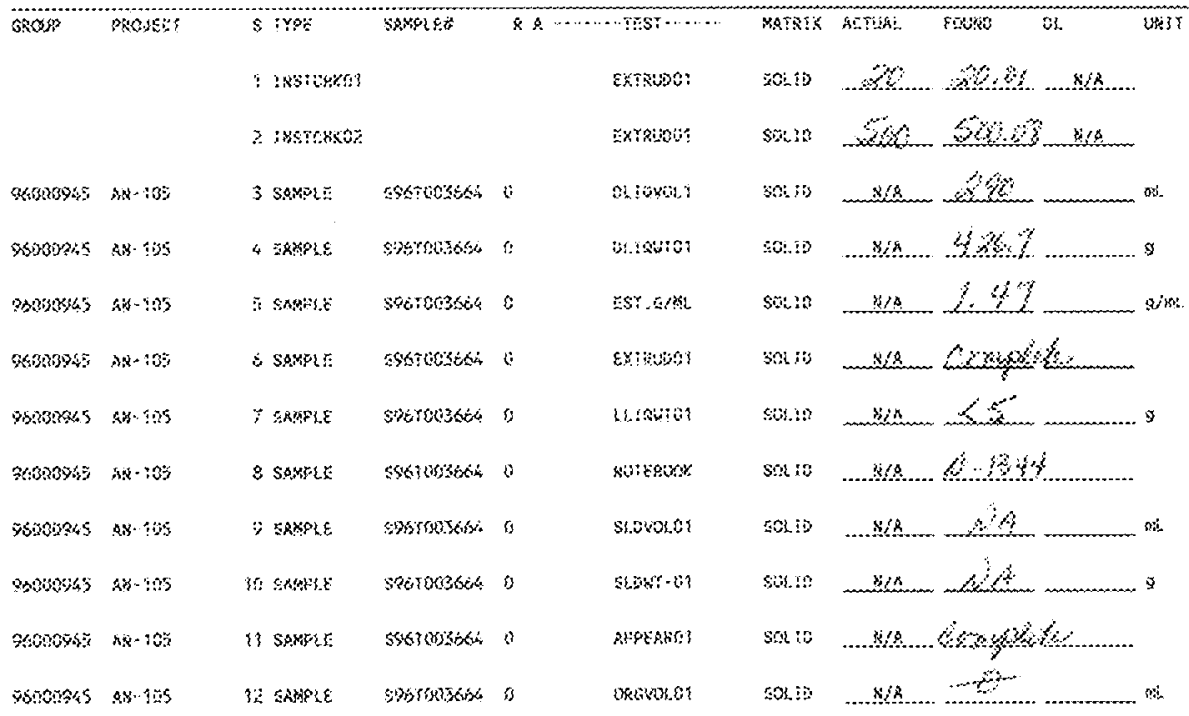

ming
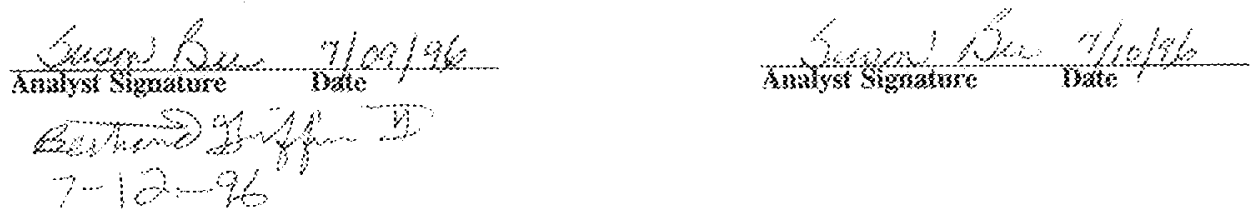

A3inlyse sigrasusec

roxto:

Doto Eusy comonosts:

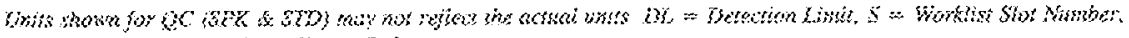

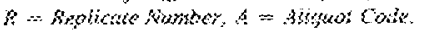




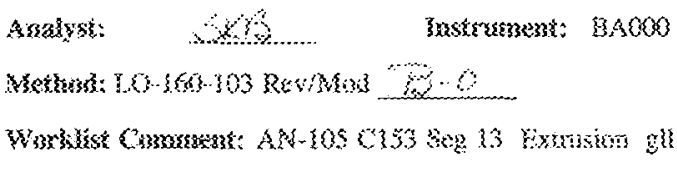

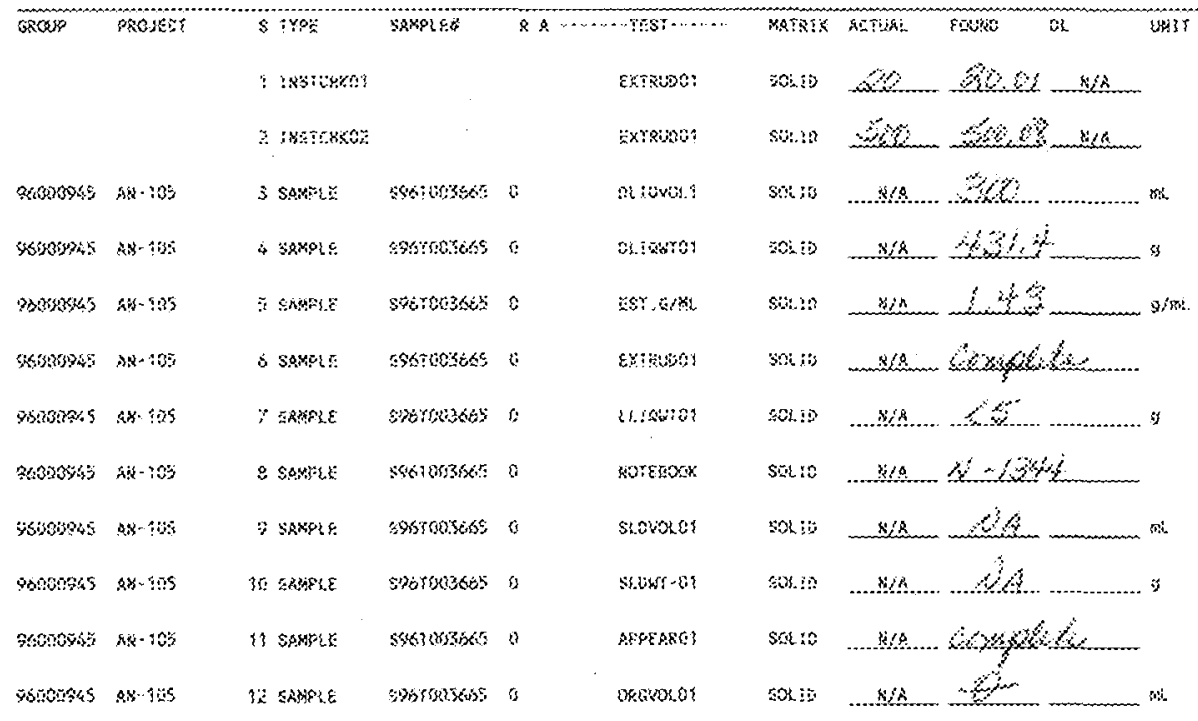

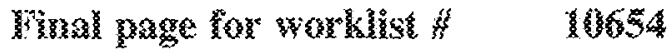
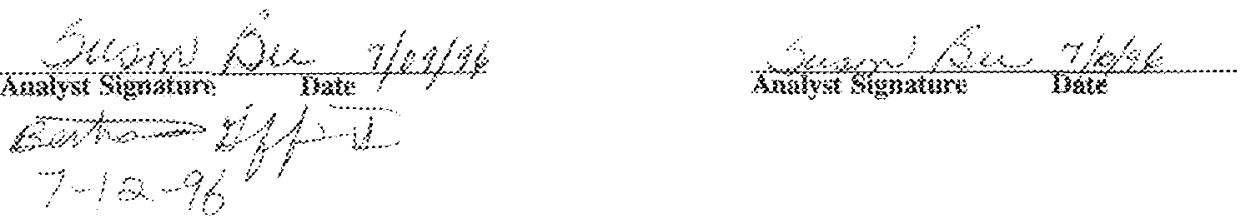

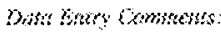

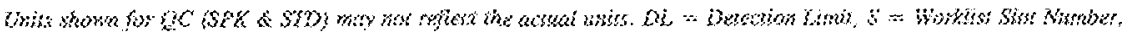

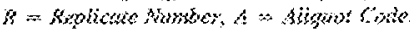




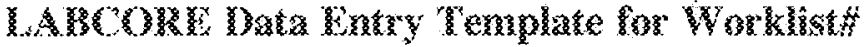

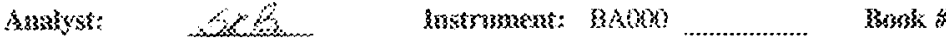

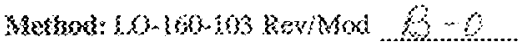

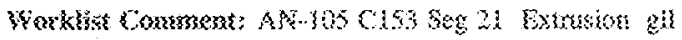

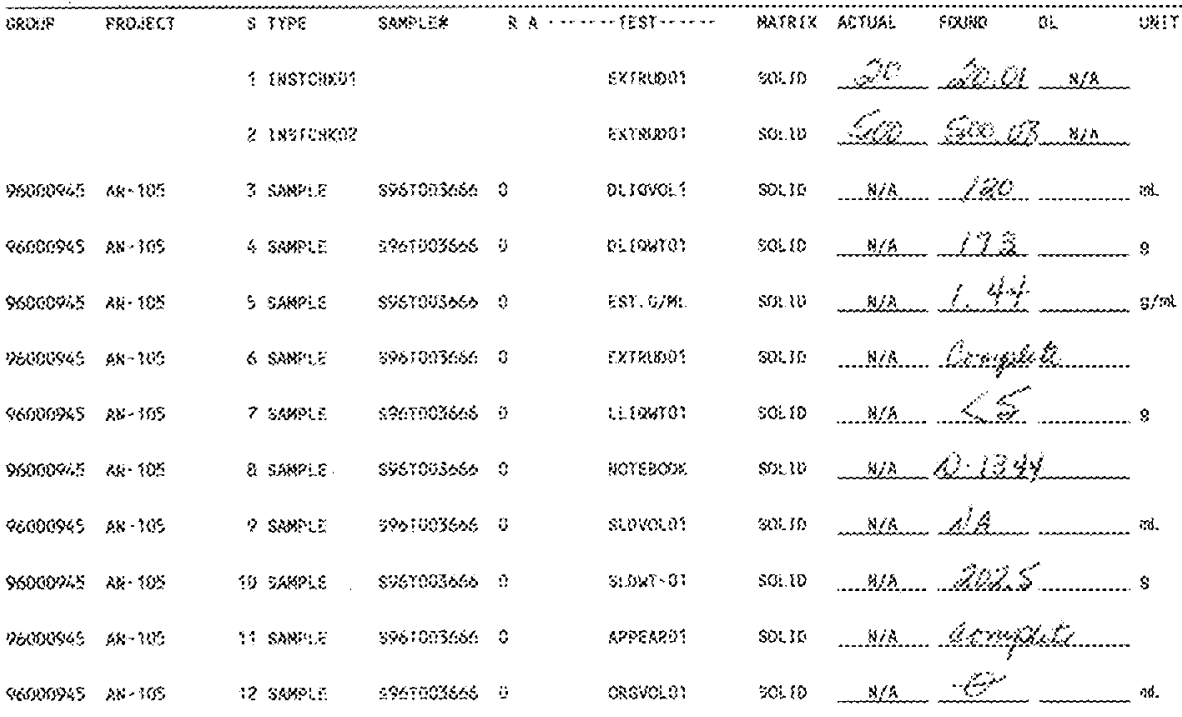

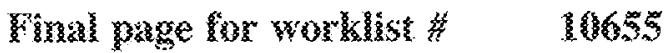
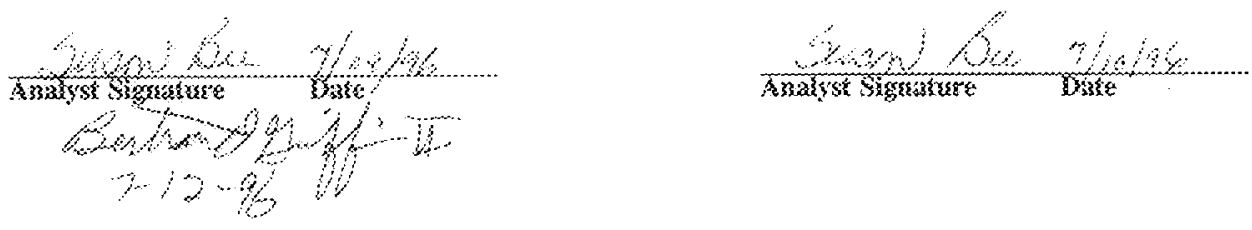

Doto Eny Commons:

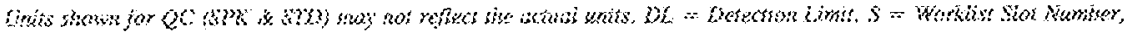

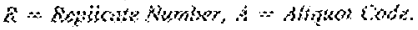




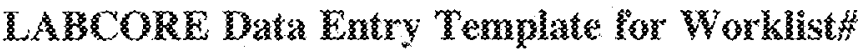

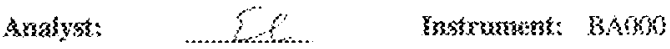

Sook $: 94$

Mothew: $\{\Omega-360 \cdot 109$ RewM:

8

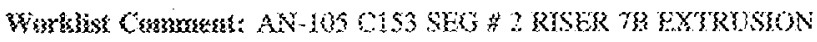

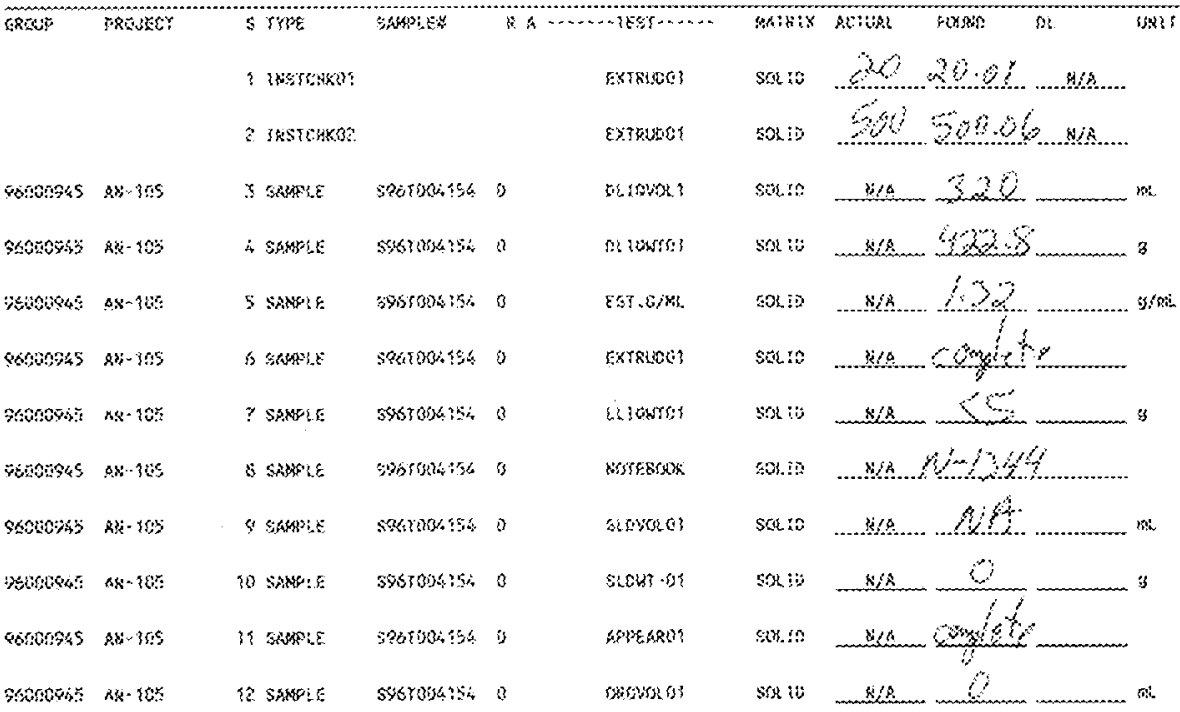

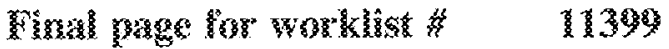
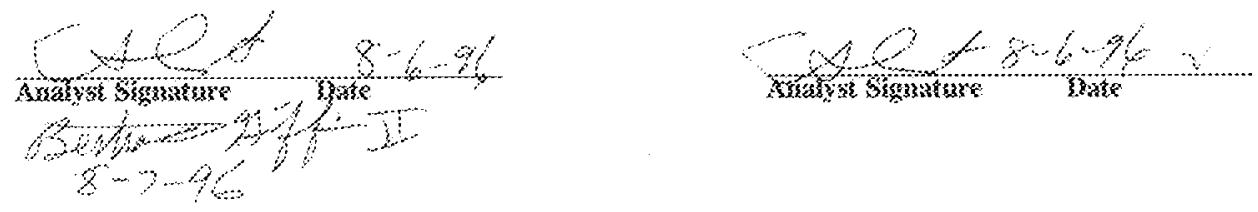

Skoto finy Cismomin:

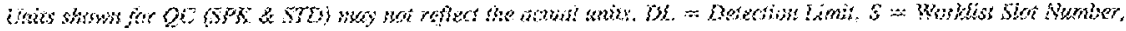

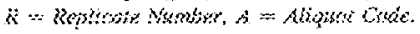




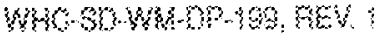

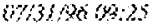

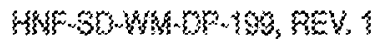

Fag:

\section{ABCORE Data Entry Tenulate wor Worklist}

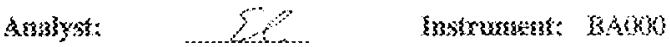

gook : Iy

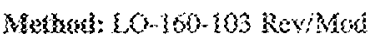

30

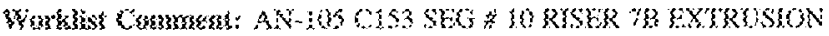

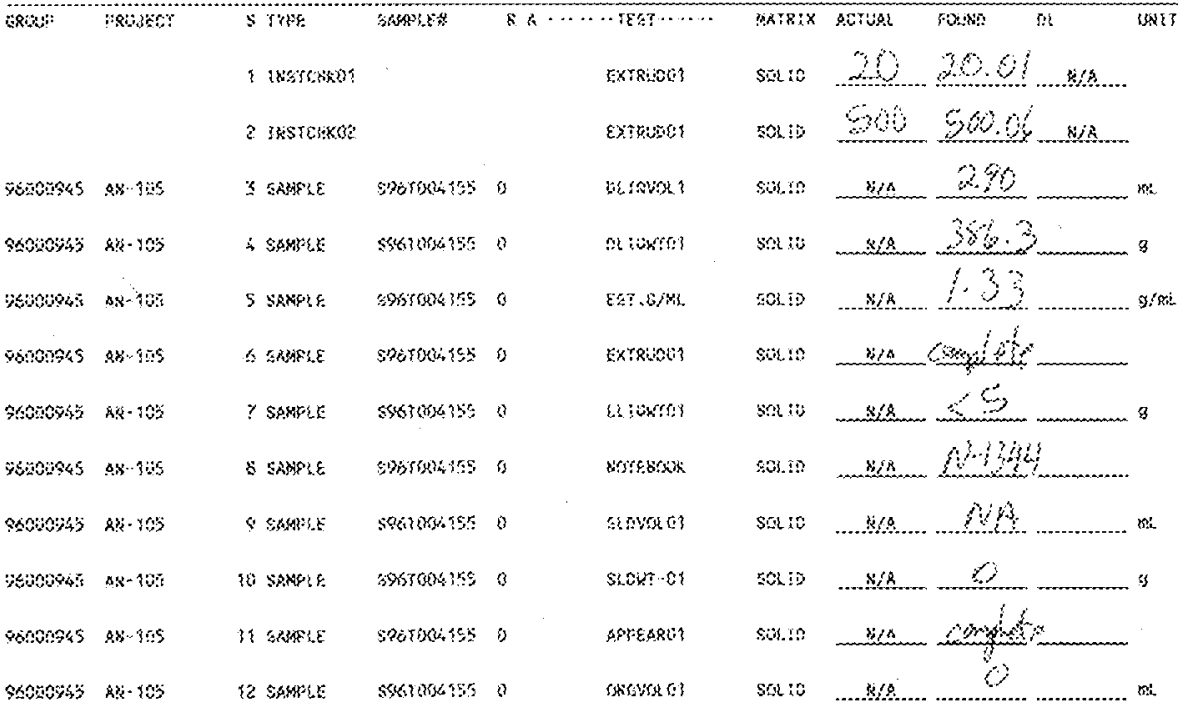

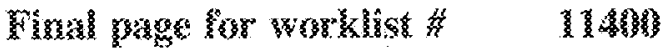

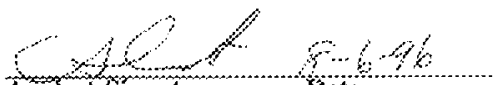

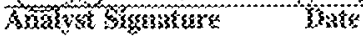

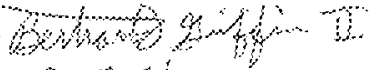

Q $-9+3$

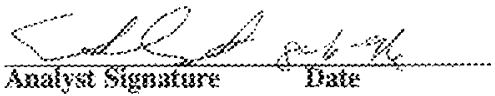

Sols Entry Sinumens:

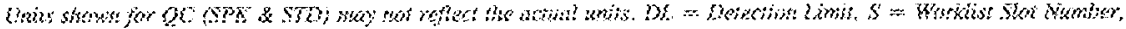

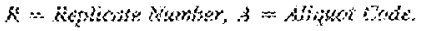

23 
WHৎD

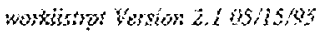

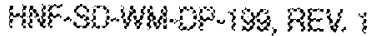

Euge: 3

(2) 3 )

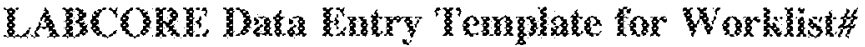

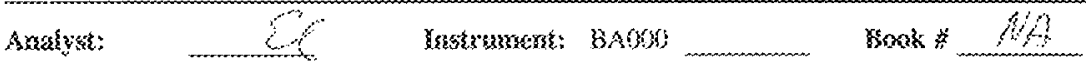

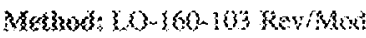

30

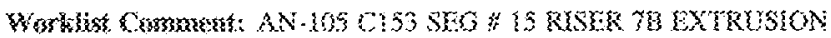

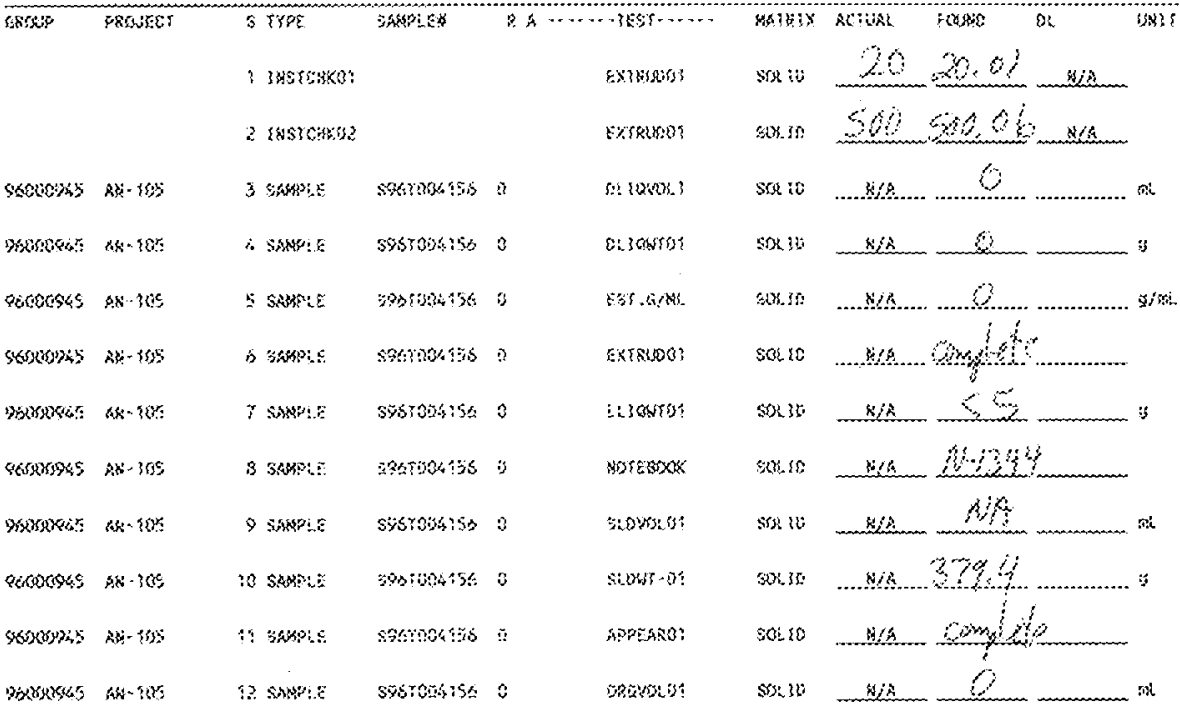

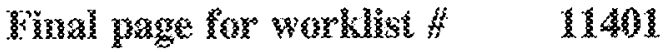

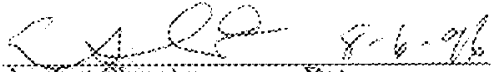

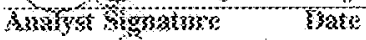

करी

$\therefore-6$

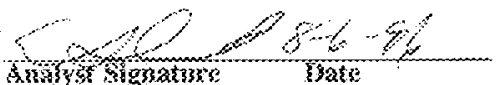

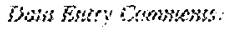

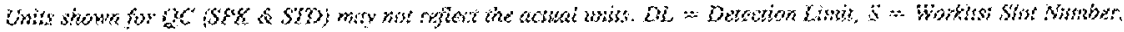

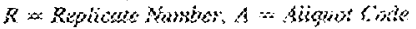

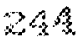




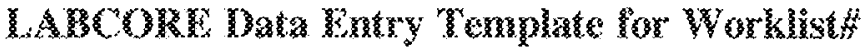

kaksi3yst:

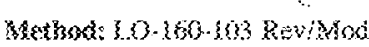

mascrmens: BAwor

gooks:

(3)

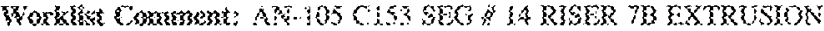

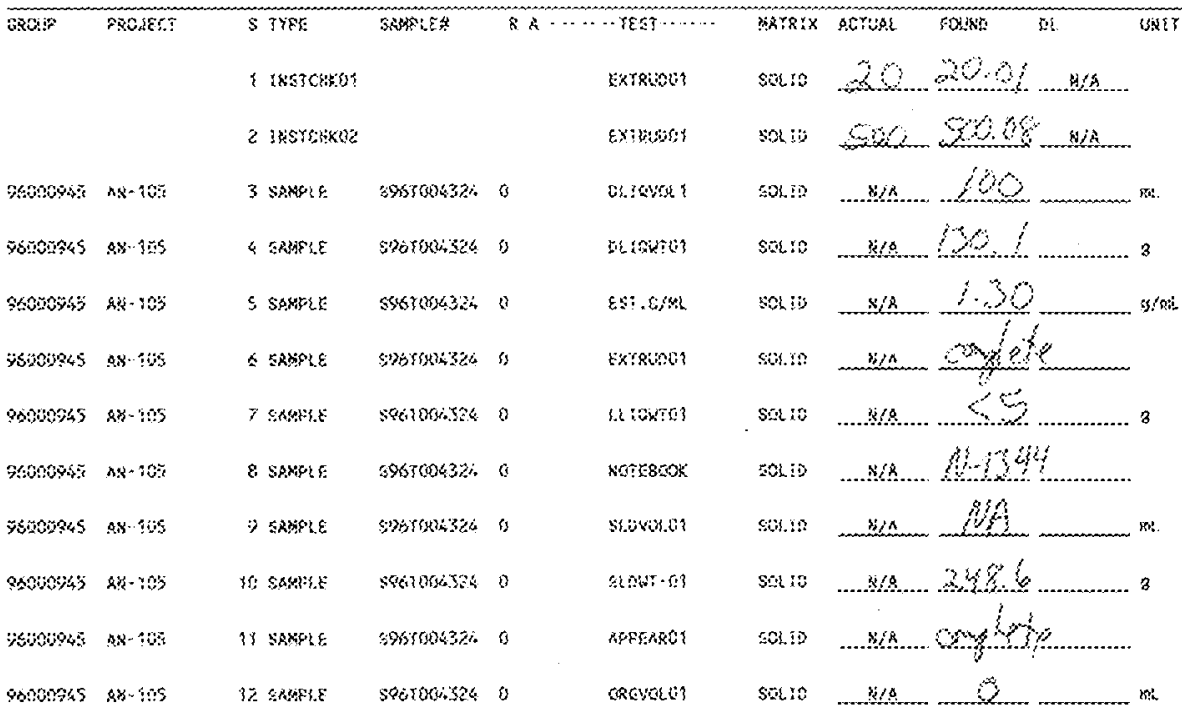

\section{Fing}

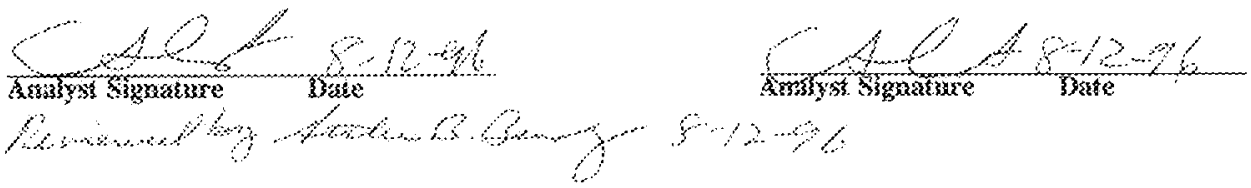

Dari Den Camswas:

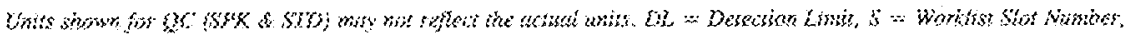

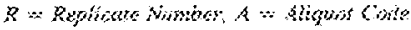


WG-OWMOF-49,

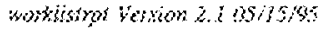

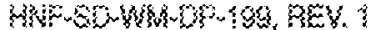

lage:

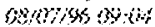

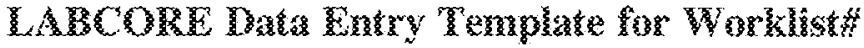

Anakgst:

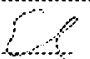

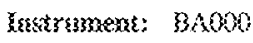

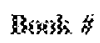

30

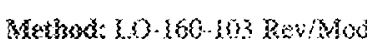

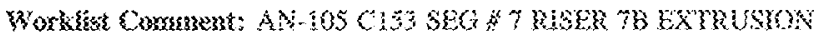

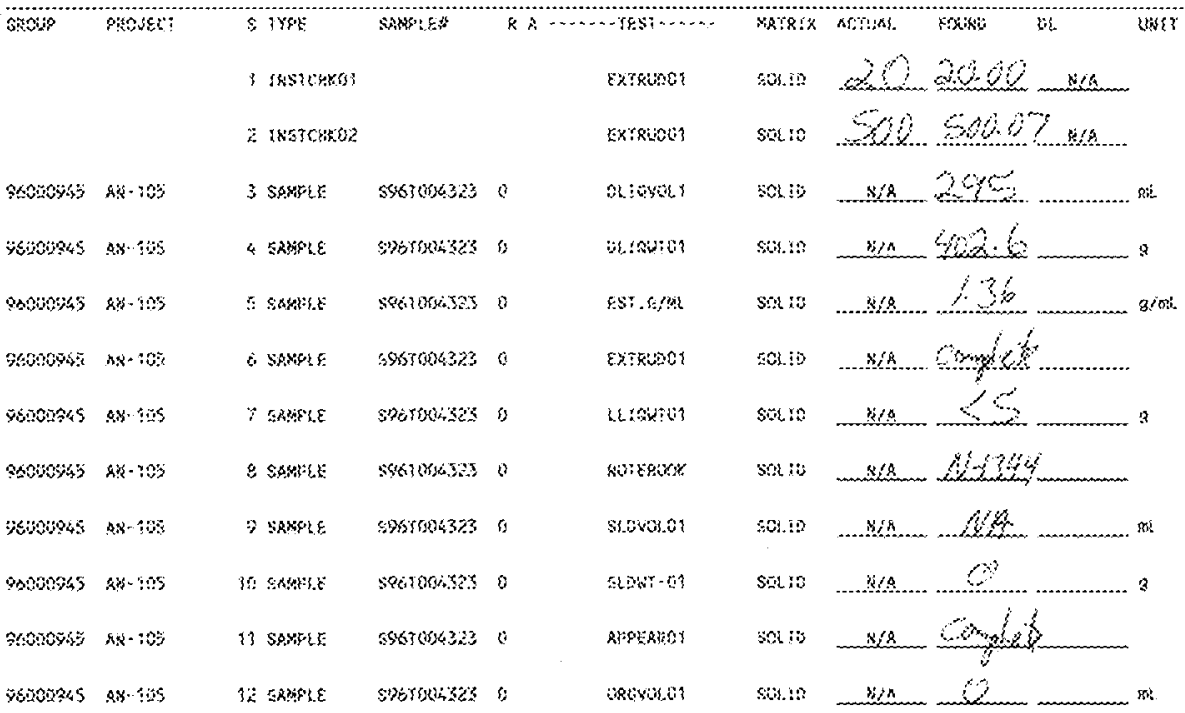

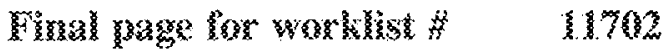

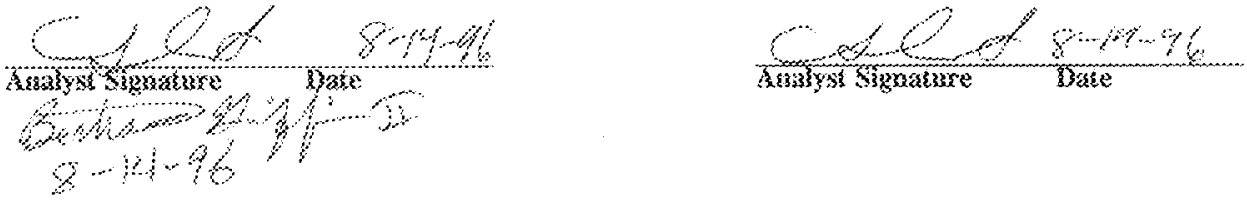

Wow Dons Commerss:

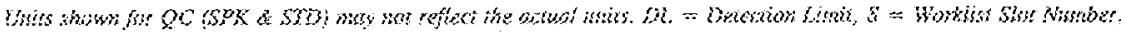

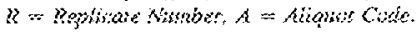




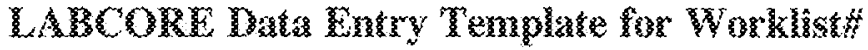

Analyst:

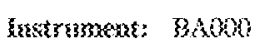

karosh $4 A$

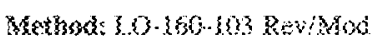

$\$ 0$

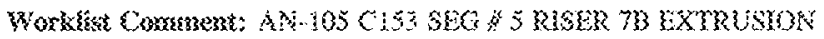

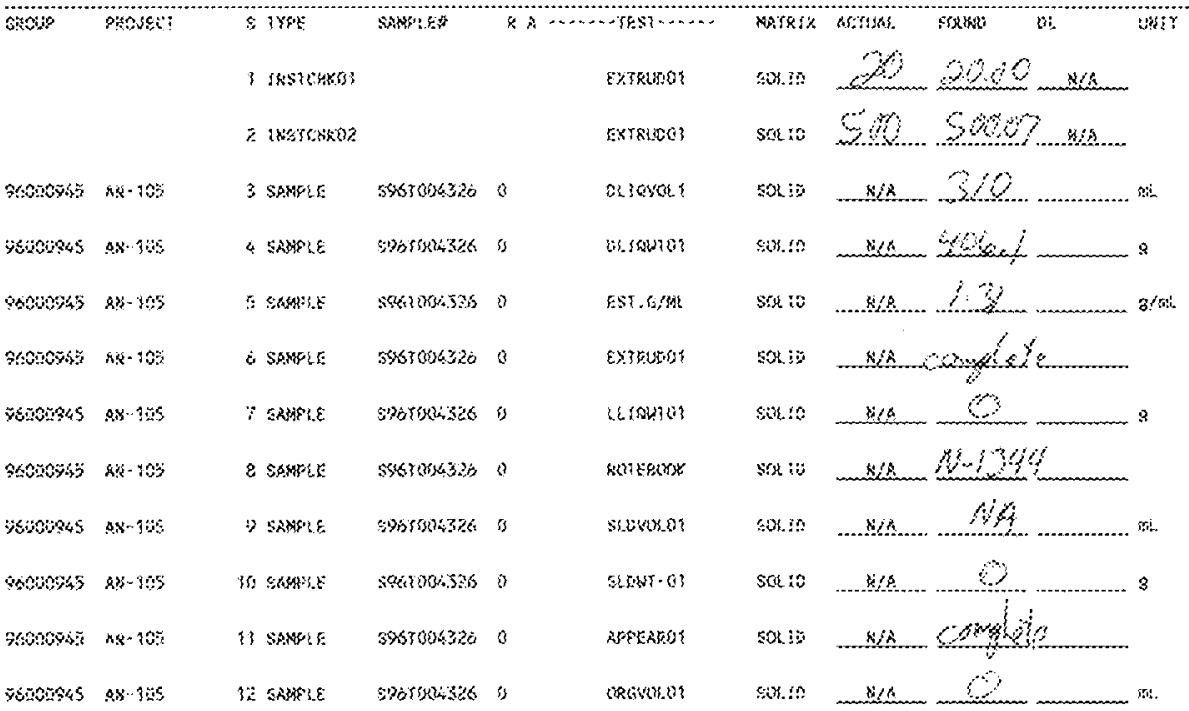

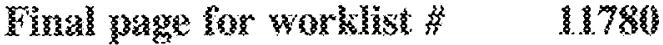

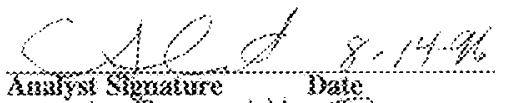

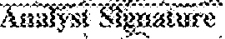
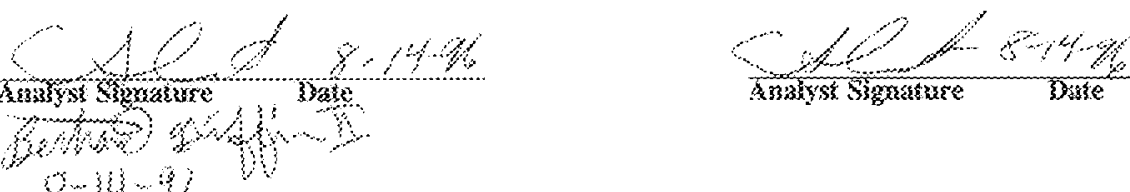

$$
x+3\} \cdots
$$

Dost Gory Comsess:

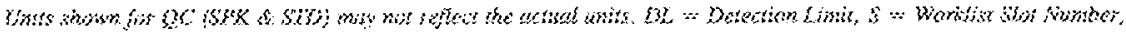

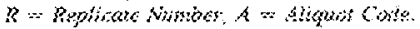




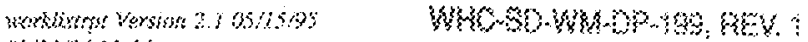

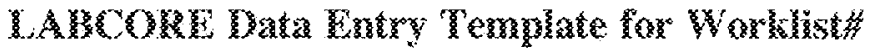

\section{Anakyst;}

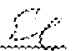

Yastrumert: 8AOKO

Sork $\quad K A$

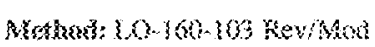

13

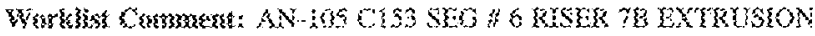

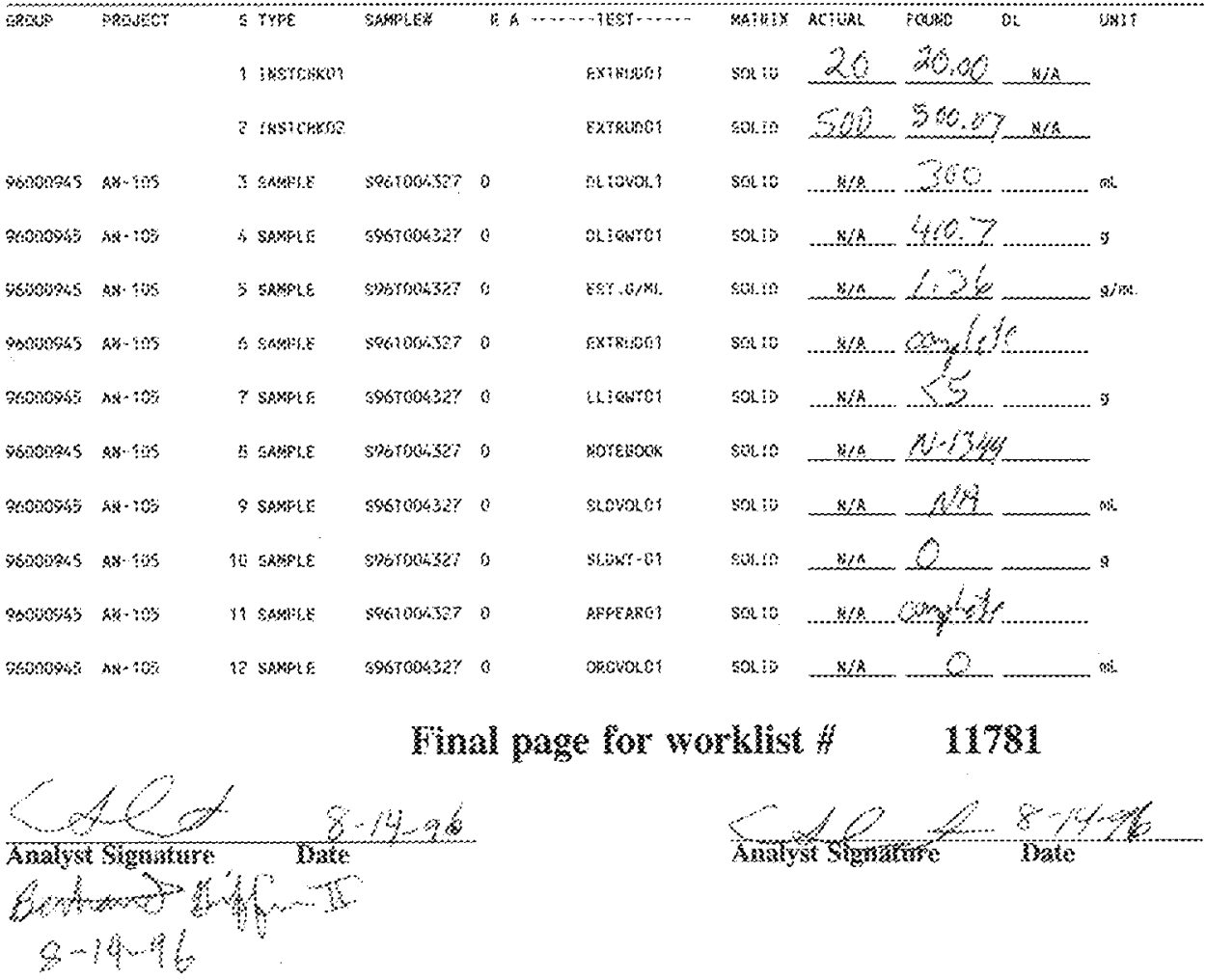

Dora Enry Conmeras:

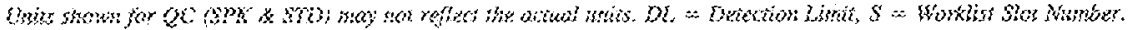

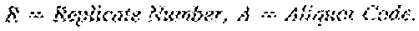




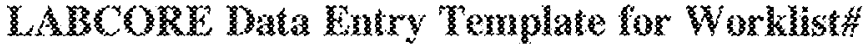

Anatys: $\quad 58$ Testrumear: $33 \mathrm{~A} 000$

Wä3: $\# / 4$

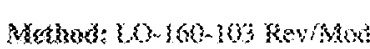

91

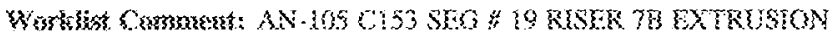

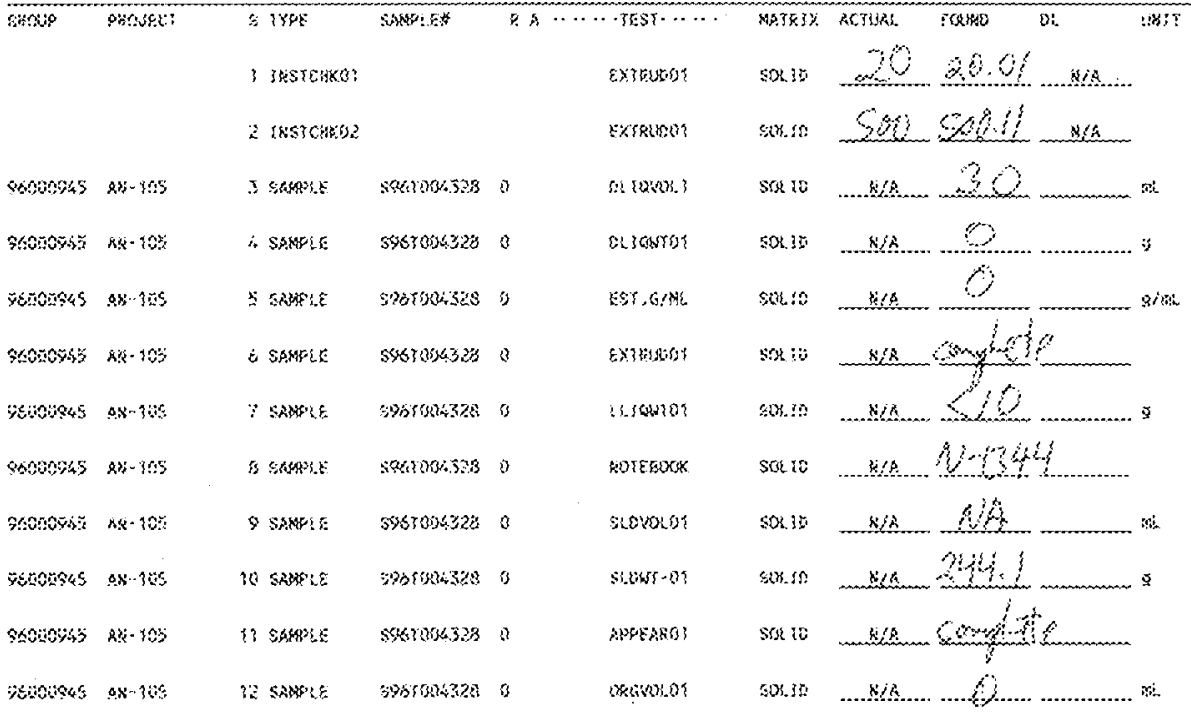

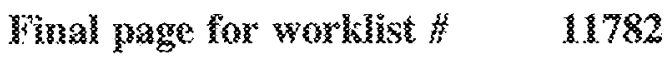

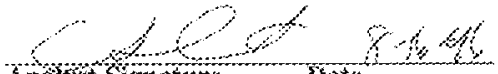

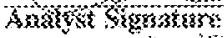
bates की

$\times+4$

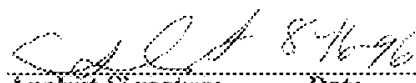

misulst signakse

3)

Orw Shiry Gownews:

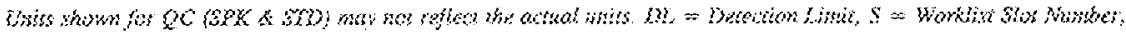

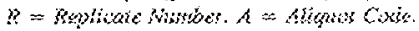


MH

whisnot Yerson 2,1 osissis:

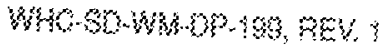

Page: ;

$0313,54: 3.56$

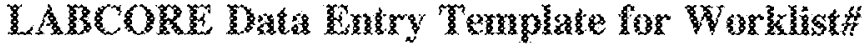

Anatyst:

et

Iแsstrumem:; $33 \mathrm{~A} 000$

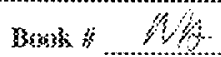

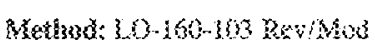

if

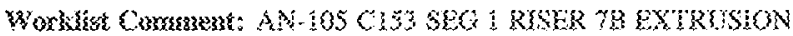

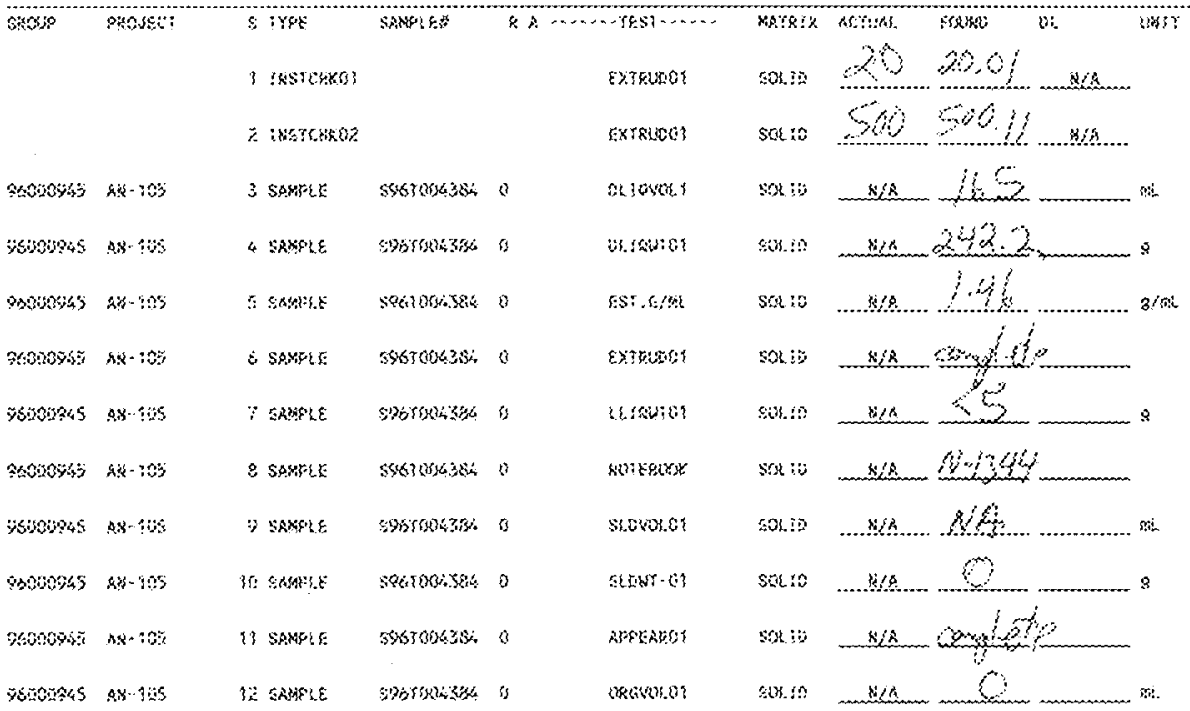

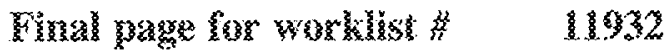

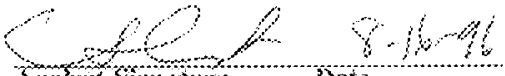

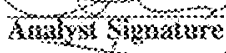

से।

Q. 46

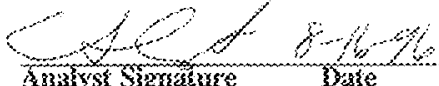

Dota Lusy Camsans:

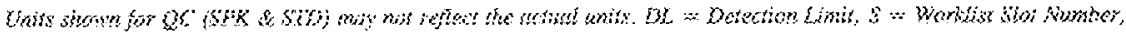

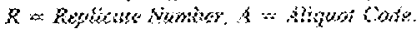




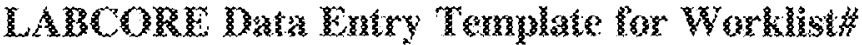

Anolyst:

3astrugnent: 339000

3 3sst: $4 X$

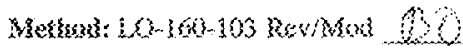

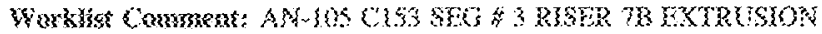

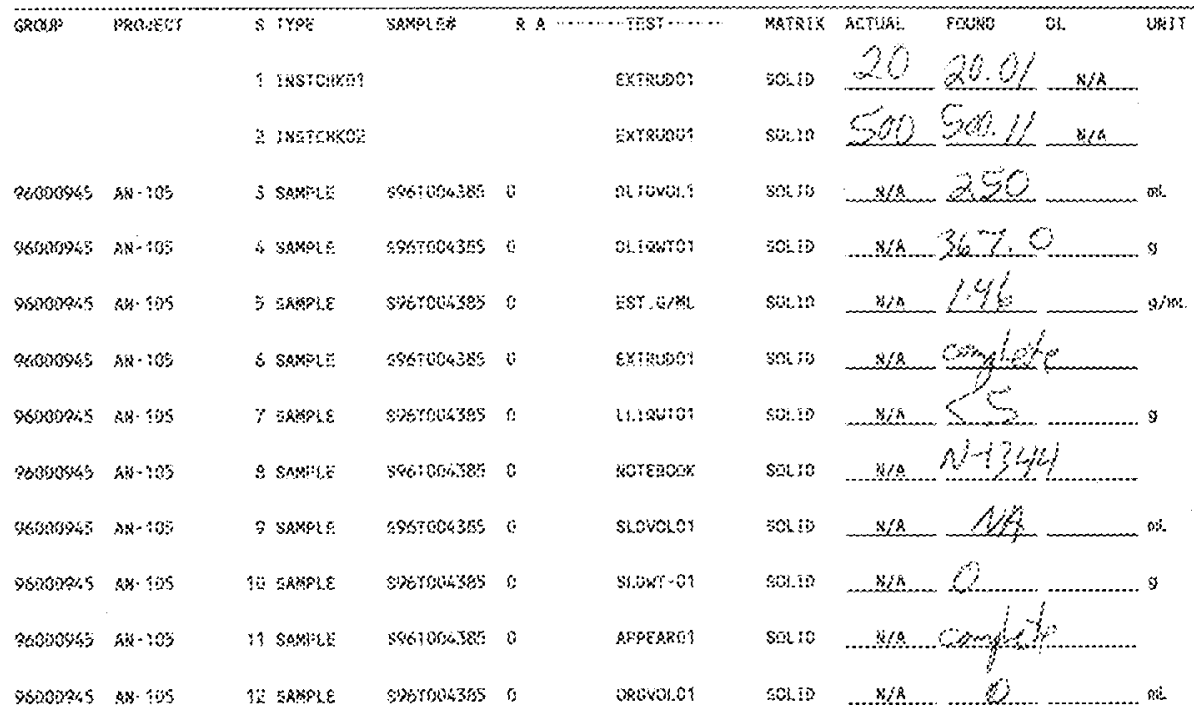

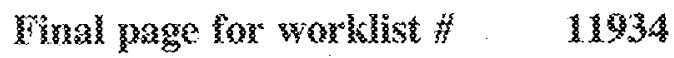
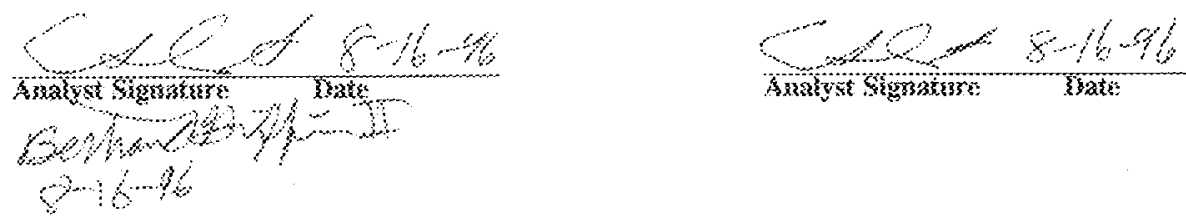

Dowa Enry Commens:

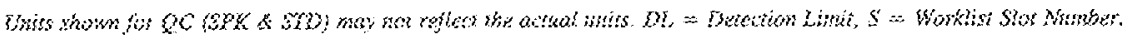

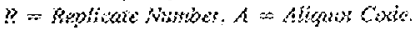

$$
\text { as }
$$




\section{Analyss: \\ Bestresszent: 94000

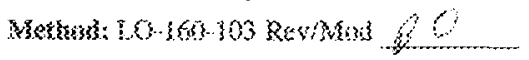

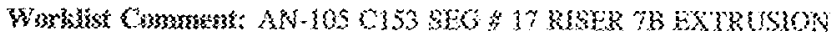

sessis $24 \%$

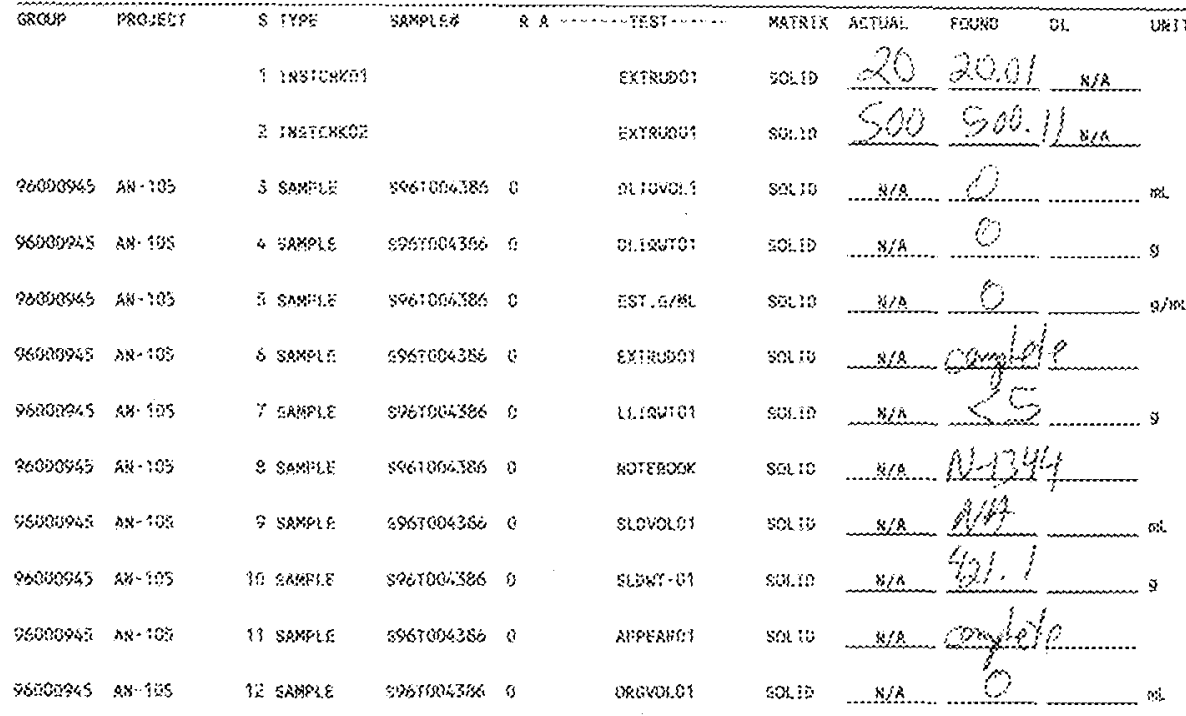

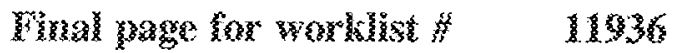

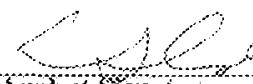

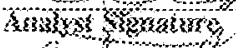

ant

$3 \ldots+\cdots$

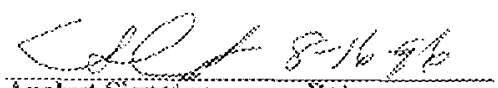

Anslys simatise
Trate:

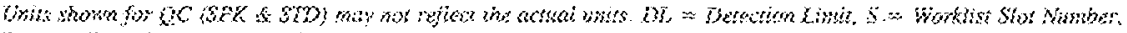

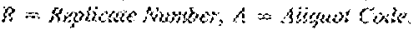




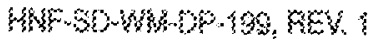

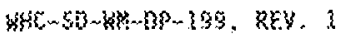

GAMPE WEERXKR IONS

$2 x, 3$ 


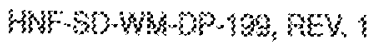

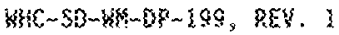

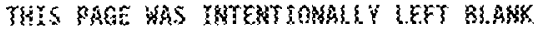

253 
Analyst:

$3)$

E3strumbers: Fiso!

Fook : :

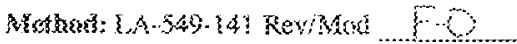

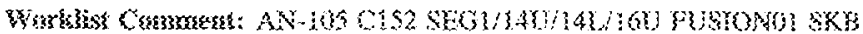

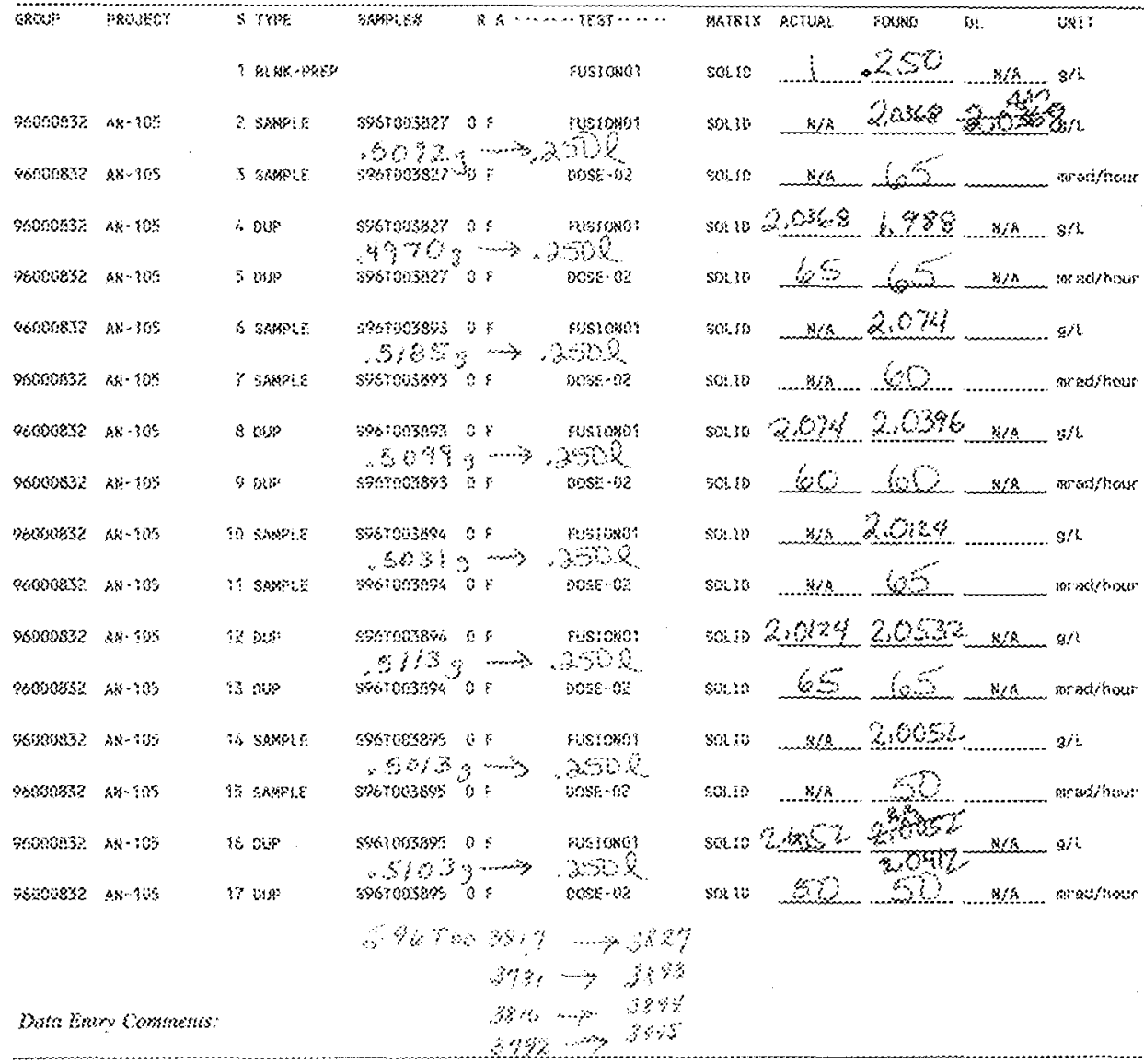

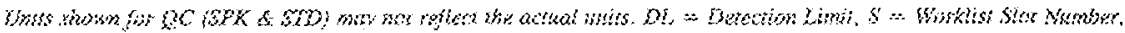

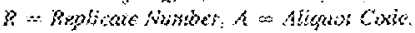

$$
\begin{aligned}
& \text { ase } \\
& 3 / 24 ?
\end{aligned}
$$




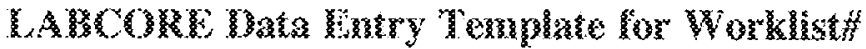

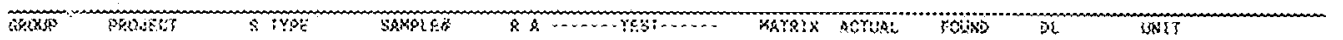

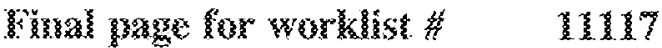

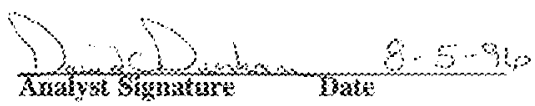

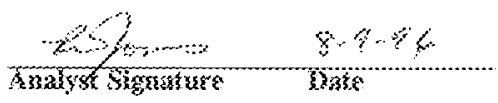

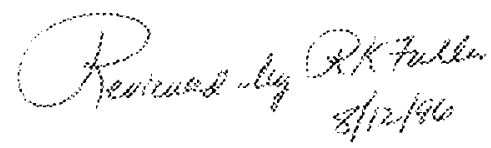

Lata entry Cowmenas:

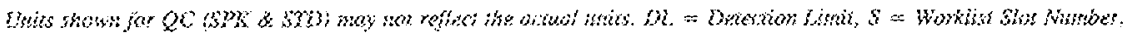

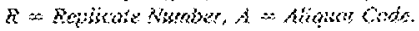


Srestye:

$\infty \mathrm{s}$

T8m:rowarest: resSot

Y3ishik : :

Methoss: YA-SAQ-3AS REVIRGOS

C

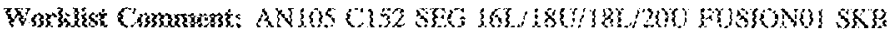

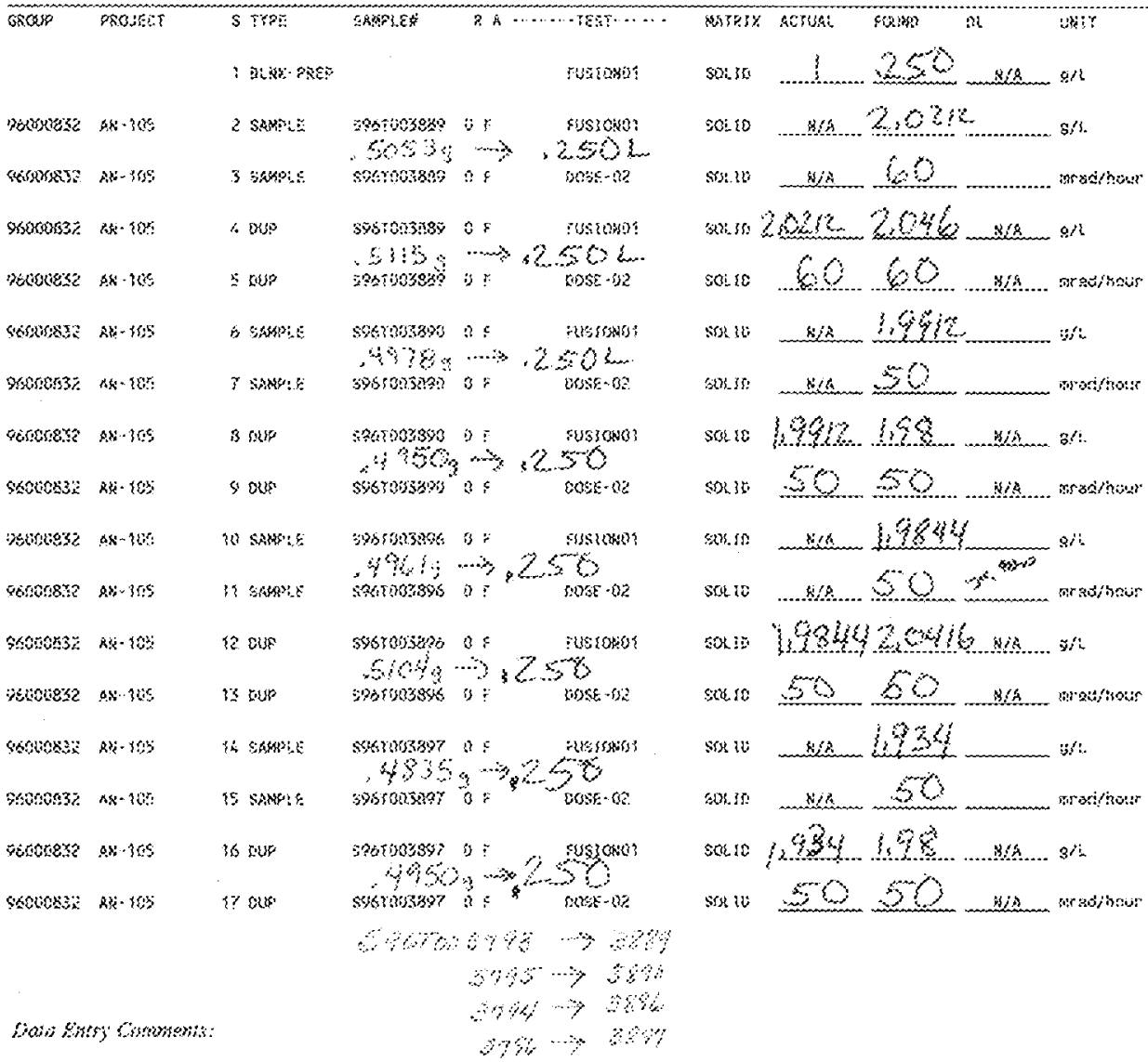

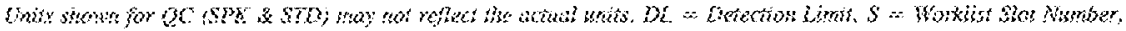

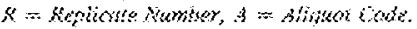




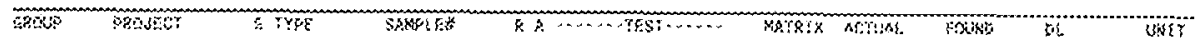

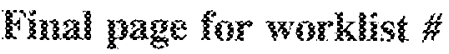

1.
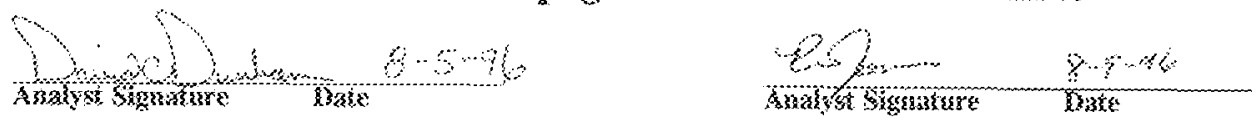


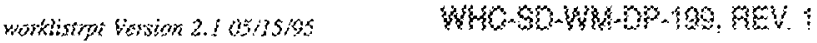

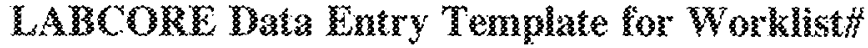

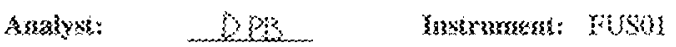

Kasok

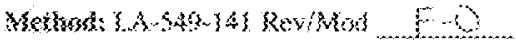

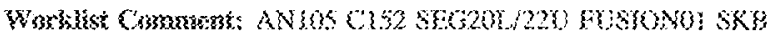

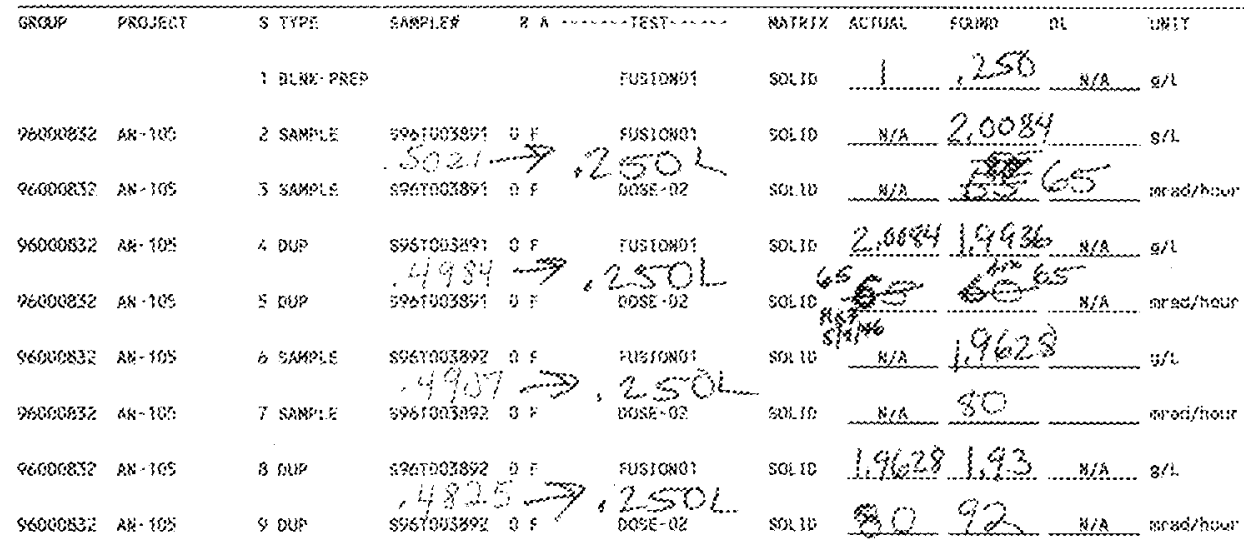

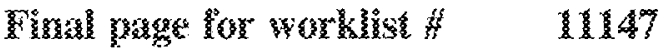
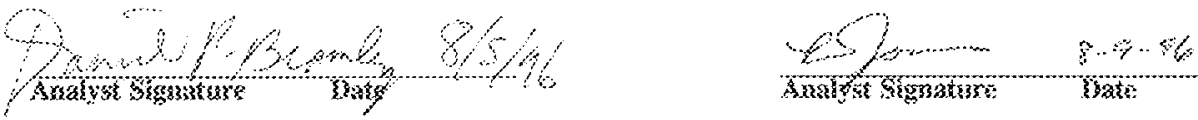

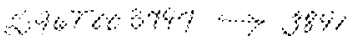

$$
\begin{aligned}
& \text { sक }
\end{aligned}
$$

Eua Eary Gommonis:

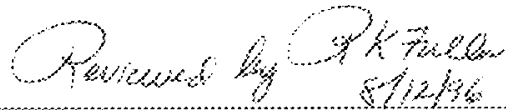

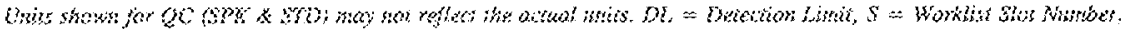

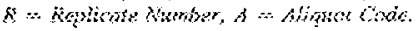




\section{Anakyst:

al

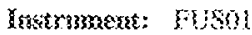 \\ Bsssk "3.}

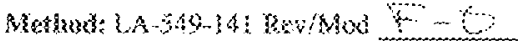

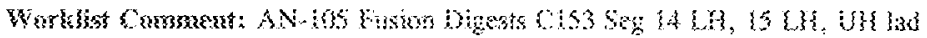

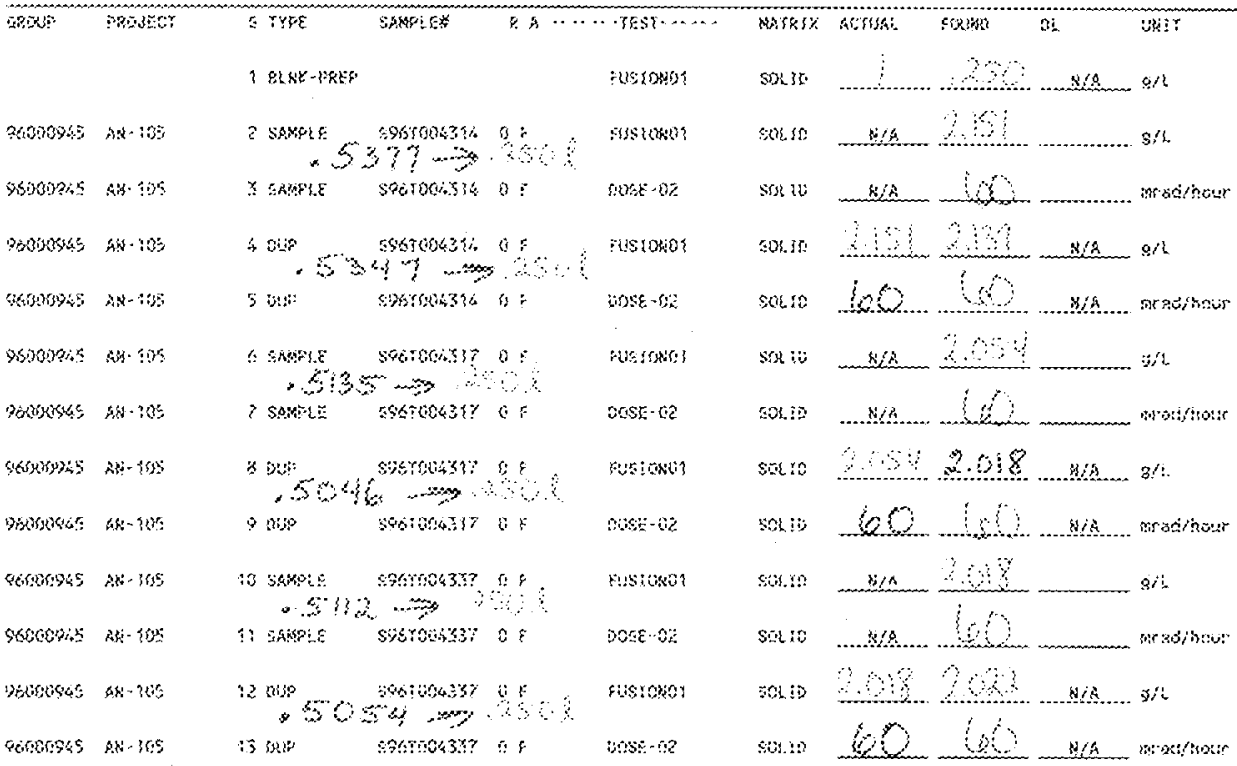

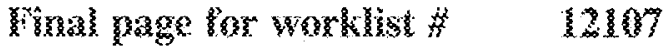
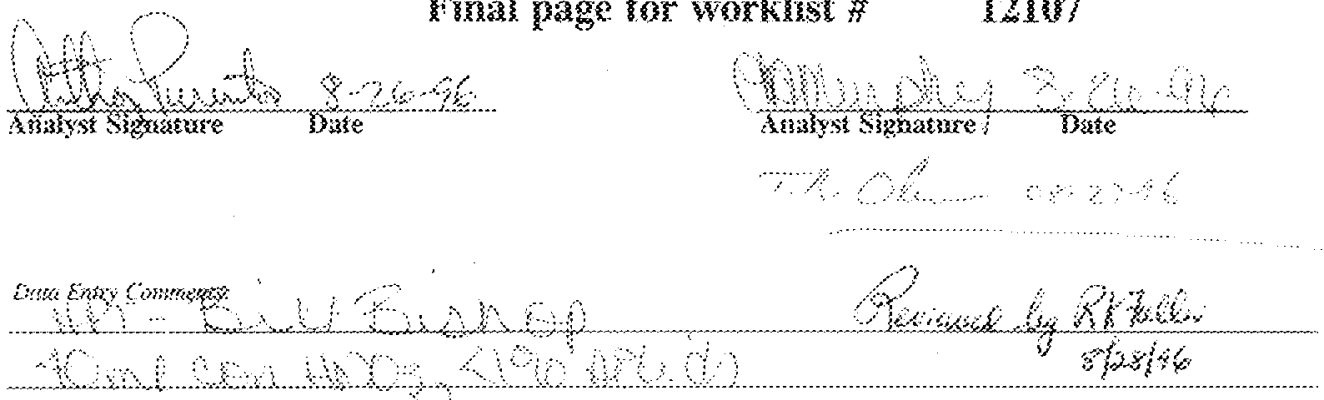

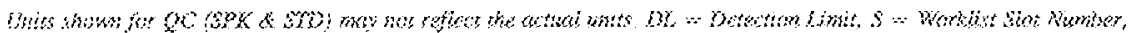

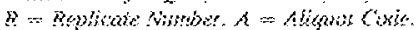




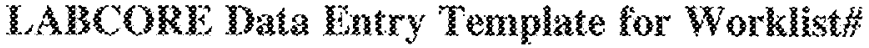

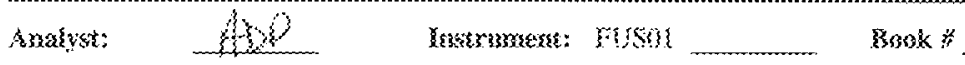

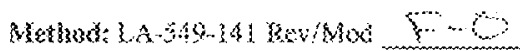

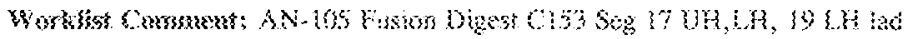

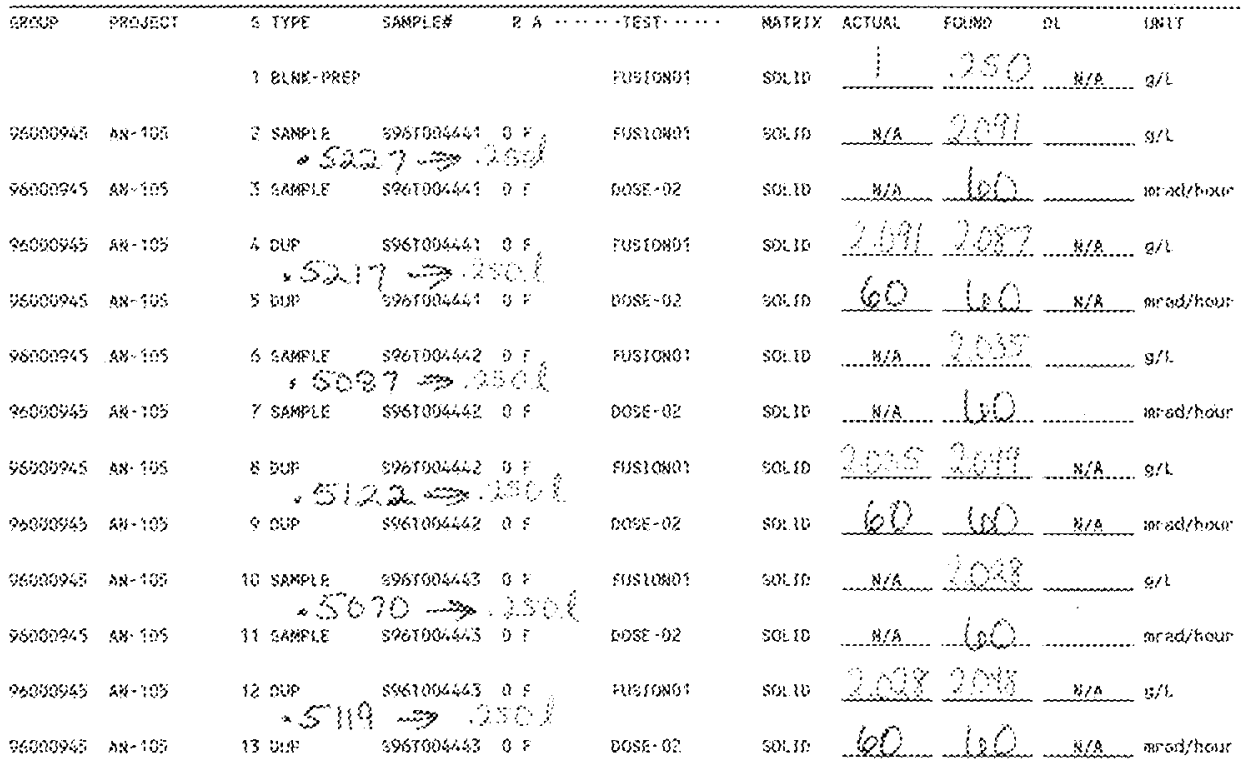

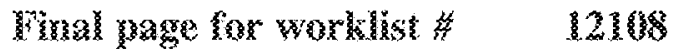
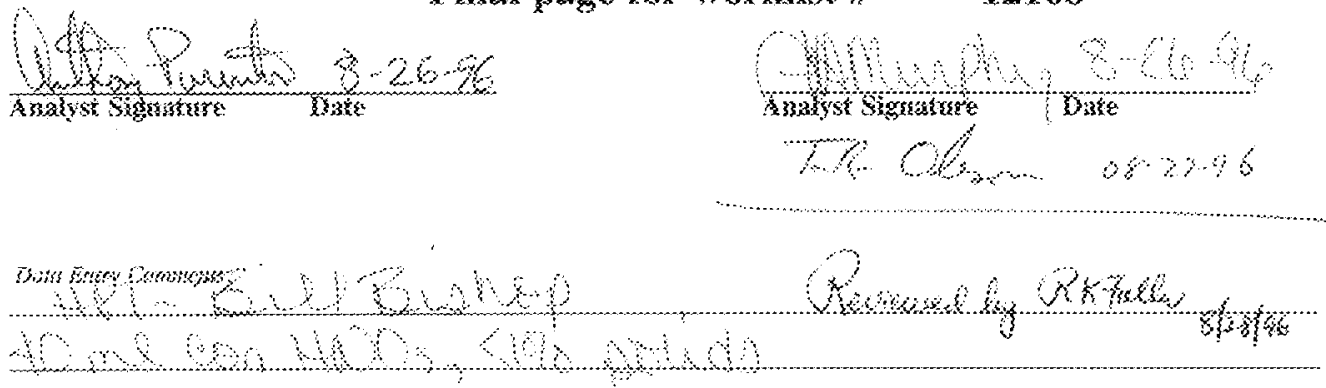

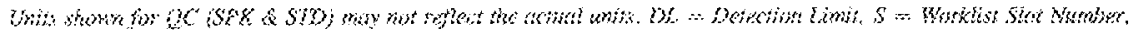

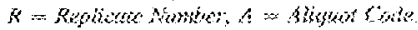




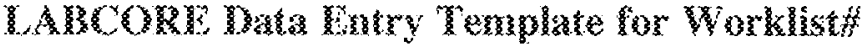

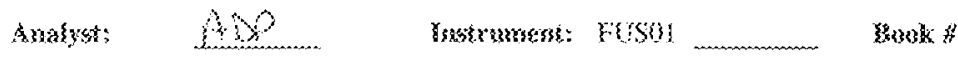

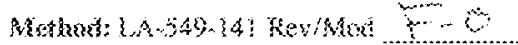

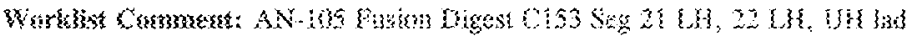

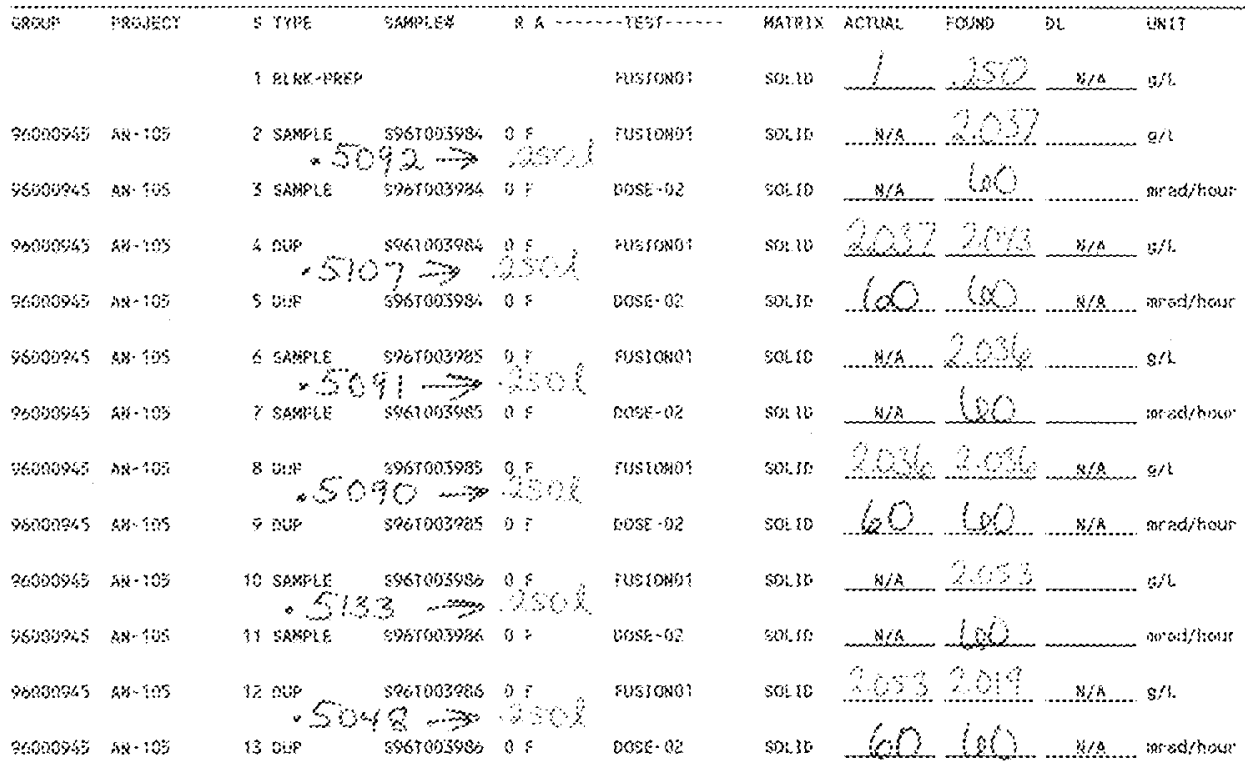

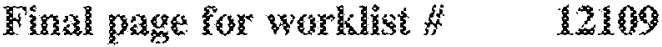
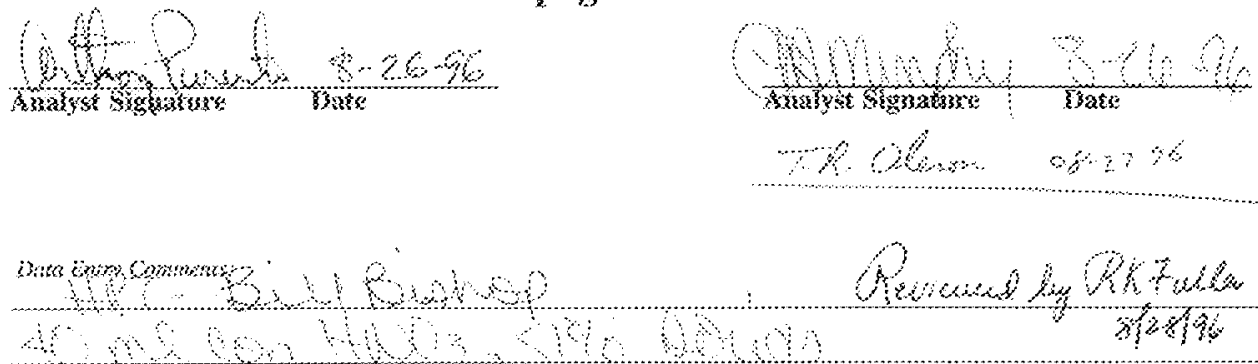

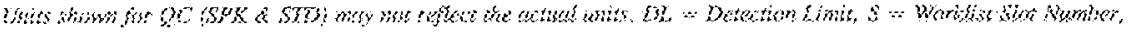

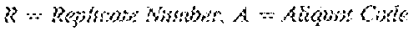




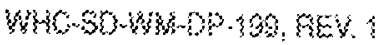

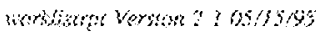

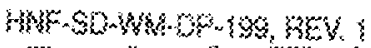

Eves: ?

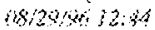

LA A

Anatys:

knstrunzent: $\quad 87350 ?$

Brogk

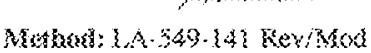

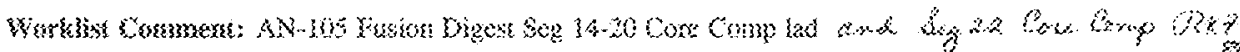

nowes

Fonos? $+x-16$

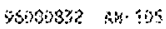

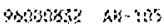

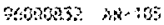

95osis

$9004032 \quad 40100$

700032 $30-40$

4songs:2 $\quad \%+19$
2. ryes

; $R L K R+Q \operatorname{Ros}$

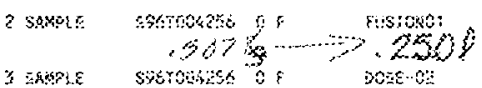

$4: x$

S s::

a Ektrite

isonitis

8 3:;:

צे Dus

Sositet if 4

sogindthes ak

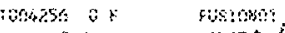

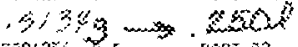

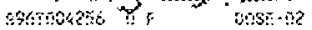

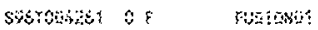

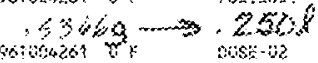

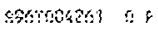

sorathis
Natix nitikl

Fitop

ol.

Sis!

Fis

3atis 3.205

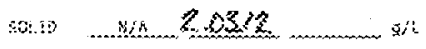

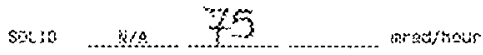

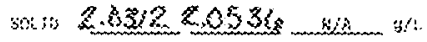

STit)

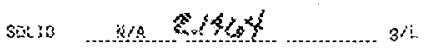

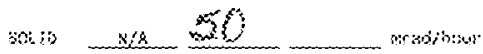

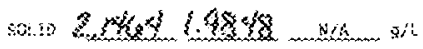

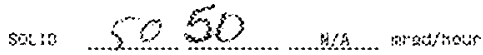

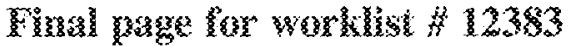

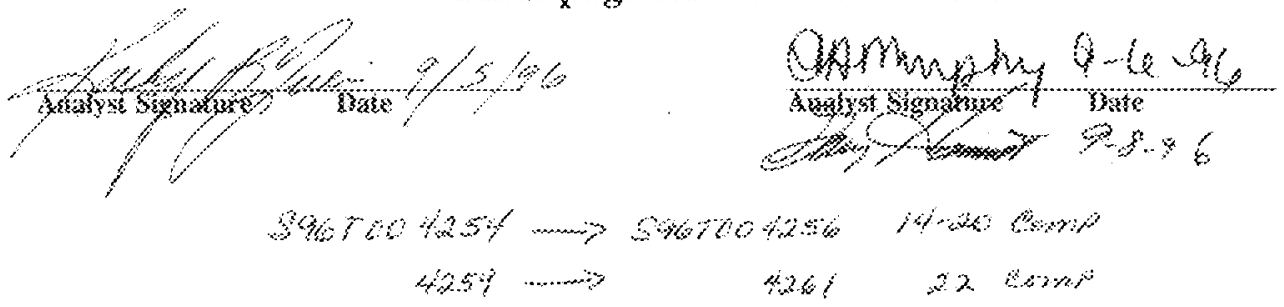

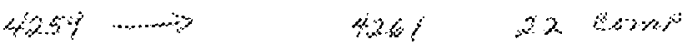

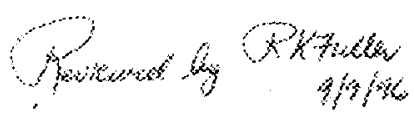

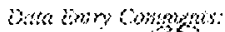

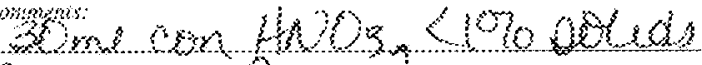

Ifo $\sec (\cos 6 a)$

6sinsos

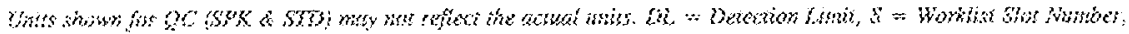

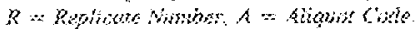

$26 \times$ 
Aranges:<smiles>[As]</smiles>

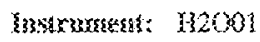

sook:

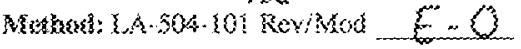

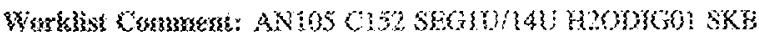

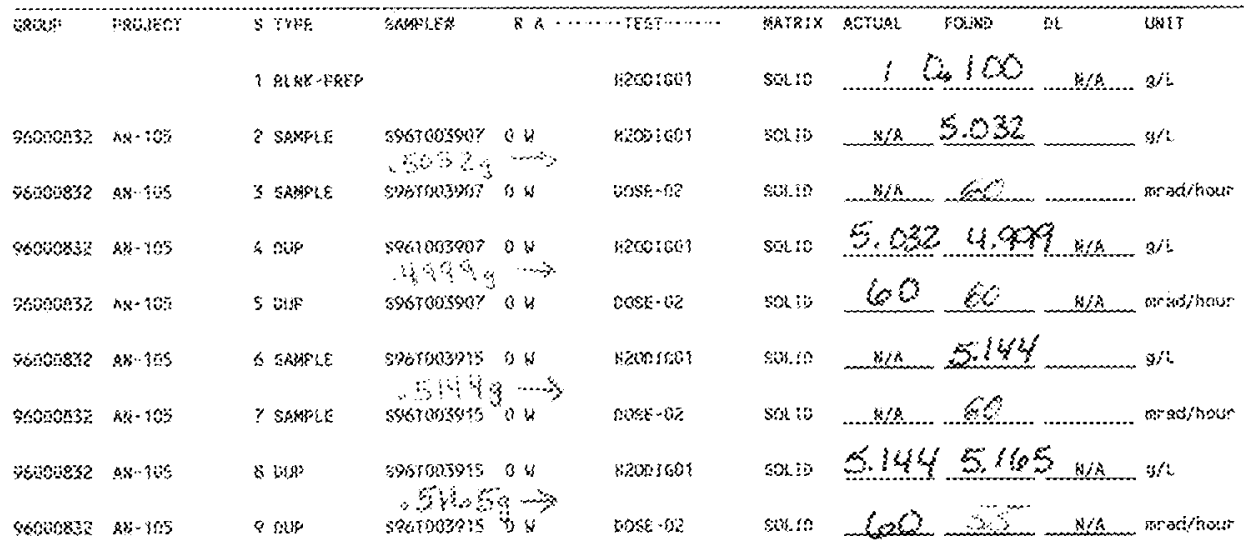

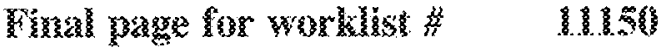
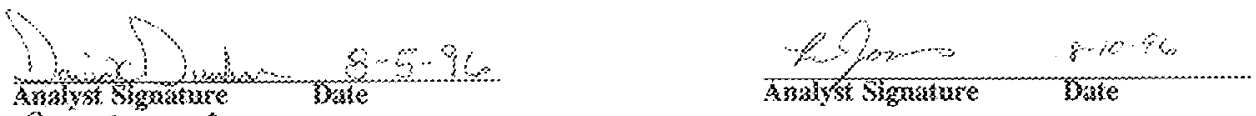

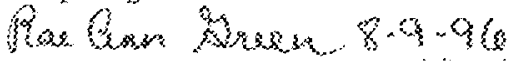

$$
\begin{array}{r}
\text { कोक } \\
\text { कर }
\end{array}
$$




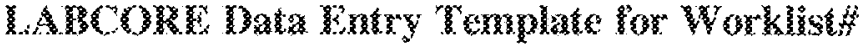

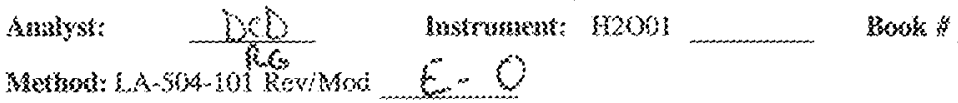

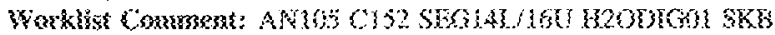

\begin{tabular}{|c|c|c|c|c|c|c|c|c|c|}
\hline \multirow[t]{2}{*}{ KRo:" } & \multirow[t]{2}{*}{ Eesoses:r } & S TPPE & $\operatorname{secsin} \theta$ & \multicolumn{2}{|c|}{ 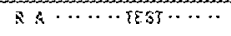 } & 36roIK & s. Trush & FEXSN: & WKIT \\
\hline & & S SSBSAX-PREP & & & secoran? & sotio & $\ldots$ & WAS $B A$ & $s^{\prime}$ \\
\hline $5606083 \%$ & $2 x) \cdot\{\xi x$ & 2 SARBDE & 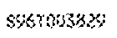 & $3:$ & arongas & ssin & s/s & $20 i 3$ & wit \\
\hline 1200632 & $\Delta x-180$ & $3 \sin 80$ & 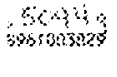 & 34 & WSE- 12 & Sh.m: & $\mathrm{N} / \mathrm{A}$ & $\frac{40}{3.92}$ & worithour \\
\hline WOSOS: & A2:-345:5 & 458 & $\begin{array}{r}\sin 6382= \\
4363\end{array}$ & קis & nooras? & solit & 96 & $\operatorname{mos}_{x}$ & 34 \\
\hline 96050932 & 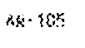 & 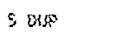 & sostoss & 38 & $000 \times \cdot 02$ & 50.35 & $8 x$ & Ui: Nis & madhow \\
\hline Ponsto: & 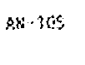 & 6 sasts:5 & $\begin{array}{l}30+303088 \\
.4921\end{array}$ & 3 & Hexsions & sains & Dis & म. 30 & sit \\
\hline 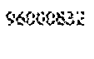 & 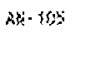 & P SASBi: & sarnossos & $n x$ & $50 \times 5 x-62$ & $\operatorname{son} i c$ & 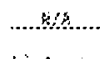 & 5,13 & garestitavir \\
\hline $506863 \%$ & $46-186$ & a $x y$ & $\begin{array}{l}5 \times 6 r 005 \% 0 \% \\
.535:\end{array}$ & 0 & $\{20 \times 130\}$ & $\operatorname{son} 16$ & & $y i s^{3 x-1}$ & $y^{\prime}$ \\
\hline 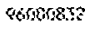 & $A x-6 c 8$ & $?$ PWP & $3067039 \% 98$ & $3 \dot{x}$ & $605-132$ & shiss & $8 \mathrm{a}$ & 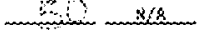 & wothent \\
\hline
\end{tabular}

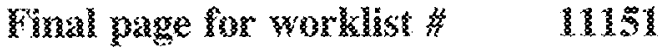

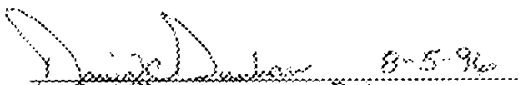

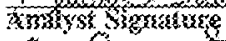

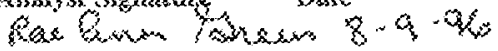

Doto Ensy Cowmorks:

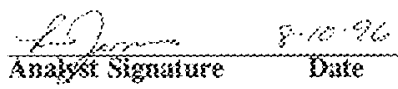

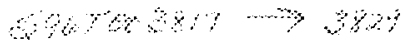

$$
\begin{aligned}
& 3 \% 2 \quad 3 \%
\end{aligned}
$$

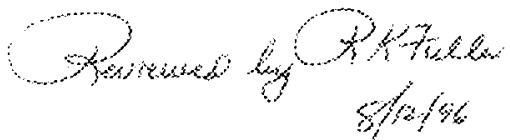

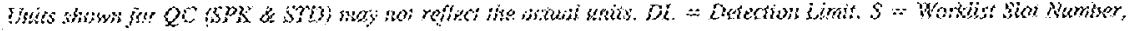

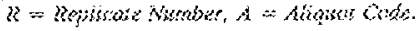




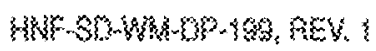

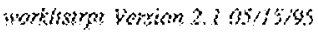

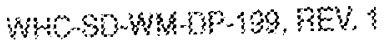

Poige: I

$0 ?: 20,50: 1: 23$

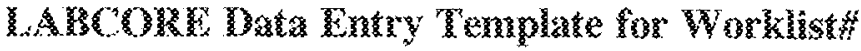

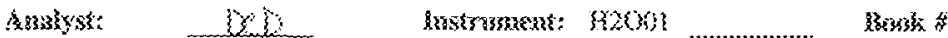

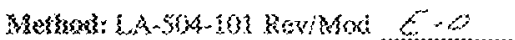

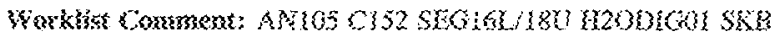

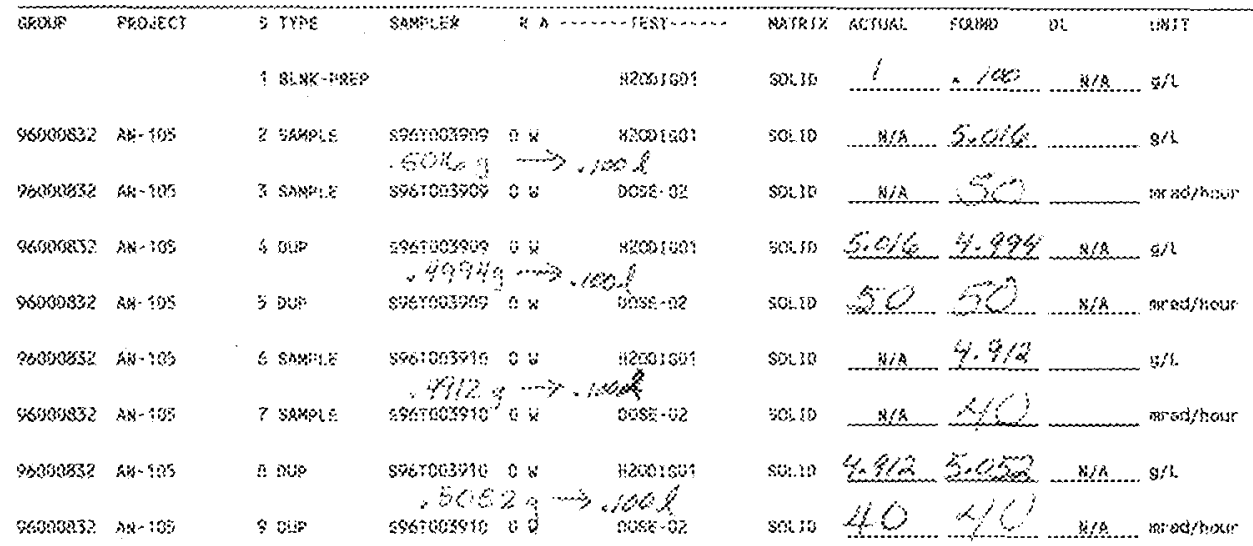

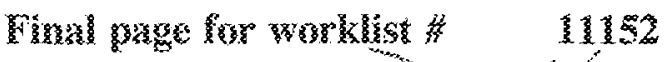

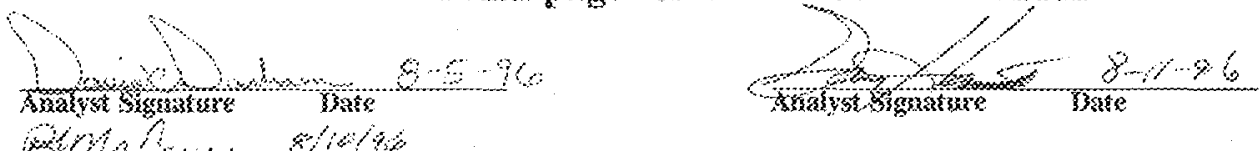
why/pस\% $8 / 4 / \%$

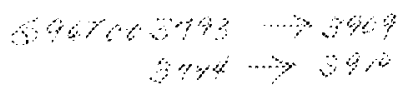

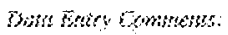

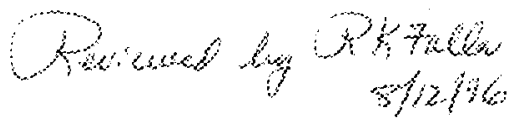

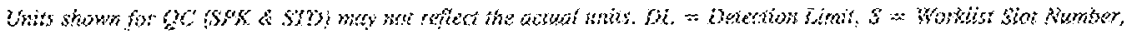

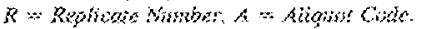




\section{Arastist: \\ Bastrumbont: B200: \\ Kank tit}

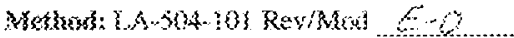

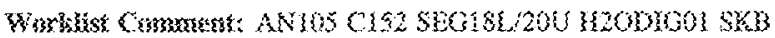

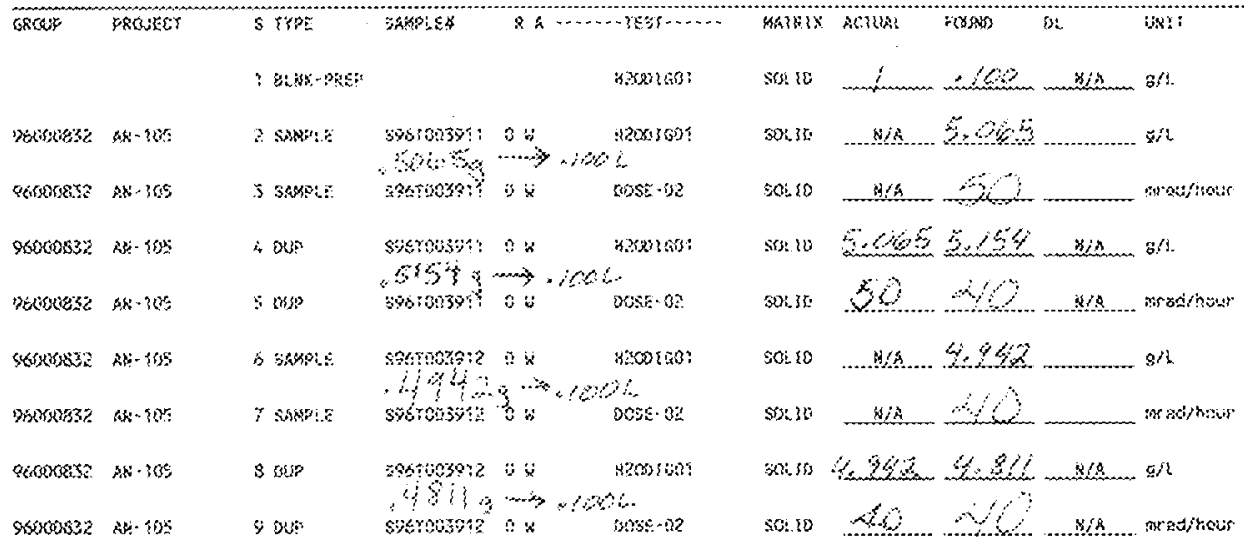

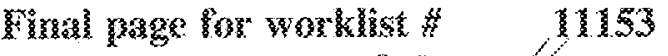
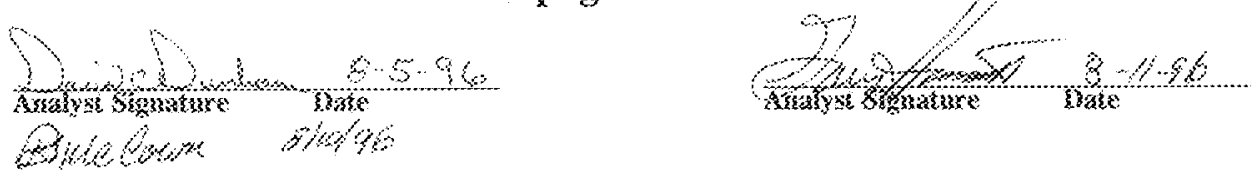

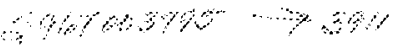

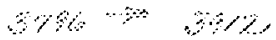

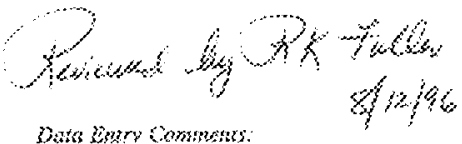

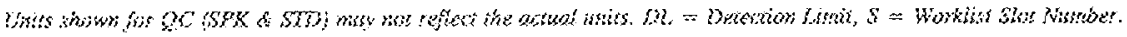

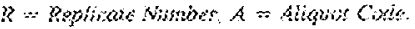




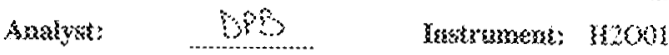

Sraks

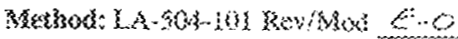

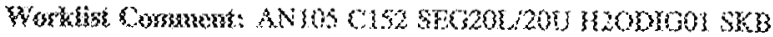

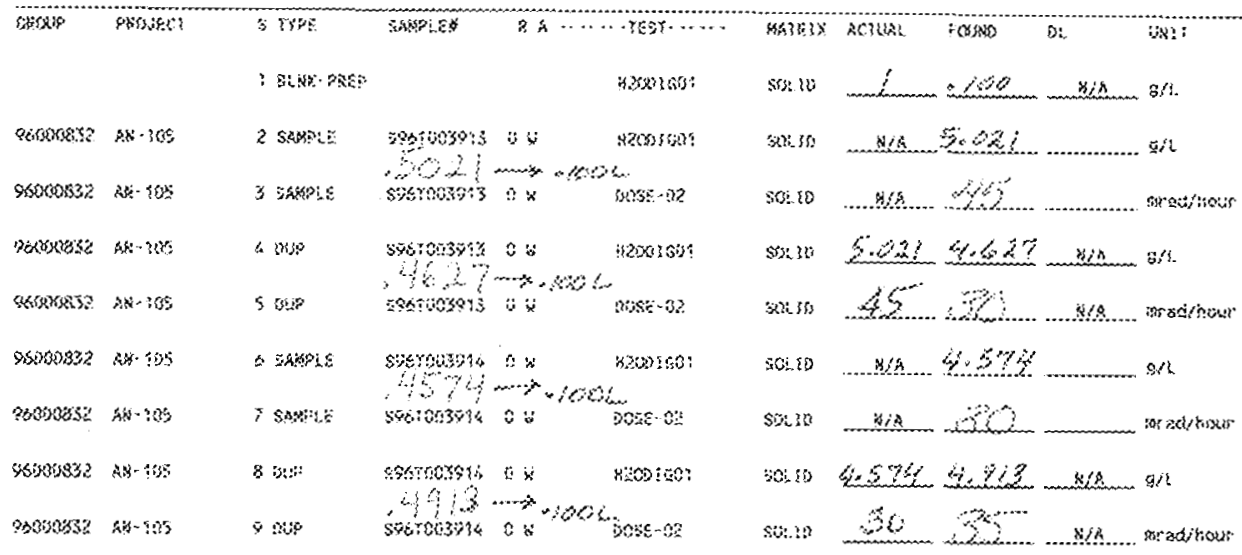

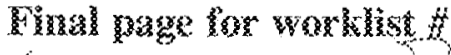

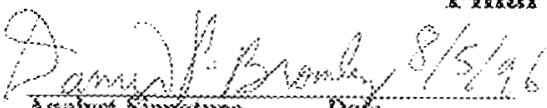

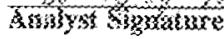

Base

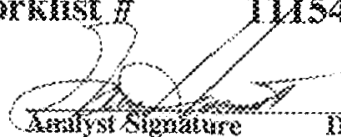

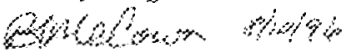

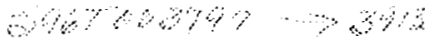

$$
\begin{aligned}
& \text { अध一 }
\end{aligned}
$$

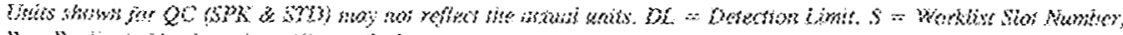

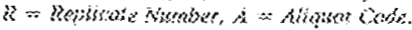




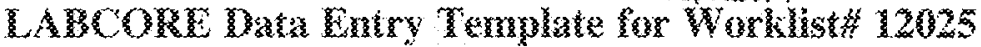

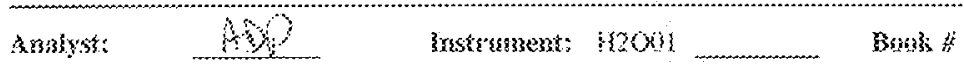

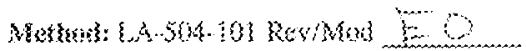

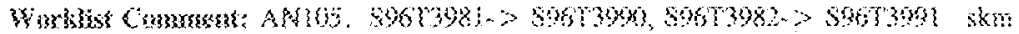

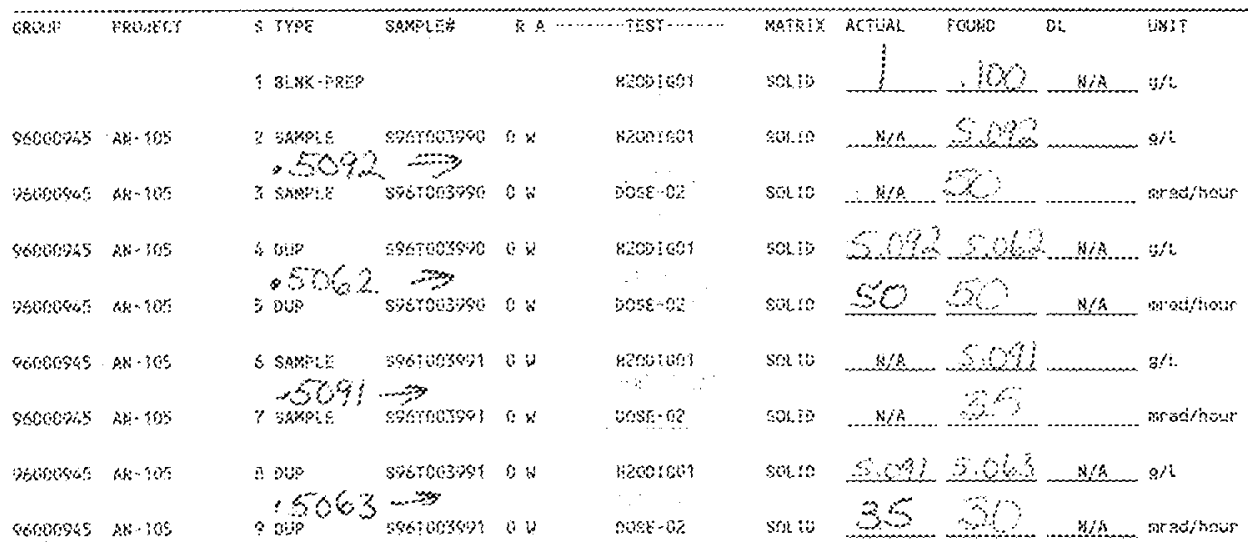

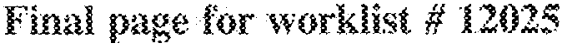
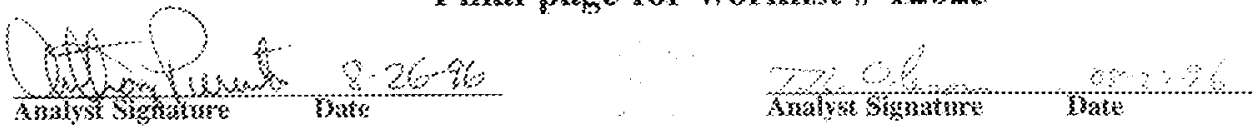

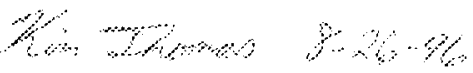

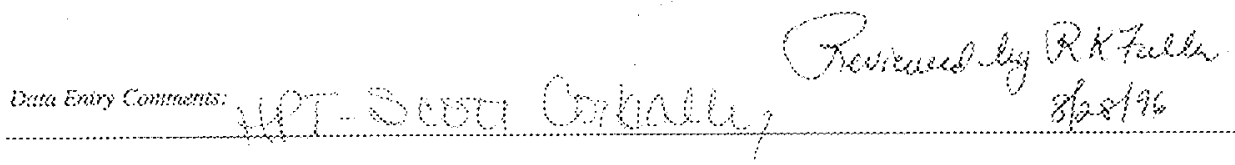

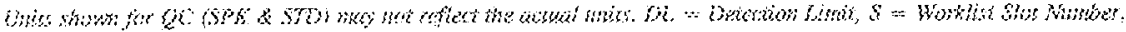

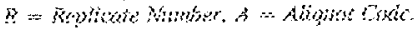




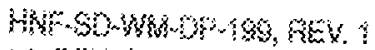

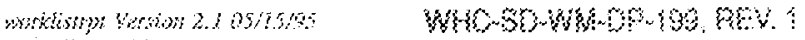

Pine: $\quad$ i $0 \pi / \% 6 \%$

4. A

Aralyst: tis 3astrument: YOOM kisssik

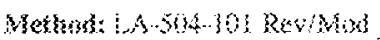

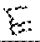

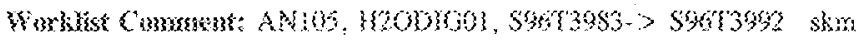

GRos?

Mathes:s

$S$ iros

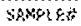

$-1651$

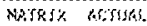

Erxise:

i!

Whi?

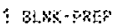

How ifos

SOR.

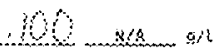

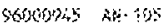

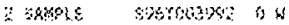

titangsis?

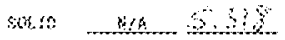
sit.

$70000545 \quad 4 k+36$

$+6,3: 3$

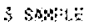

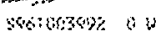

axter

sot io 5 se argothow

sobosis ak-165

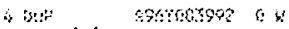

Hax?

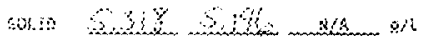

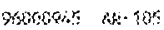

, sites

; bis?

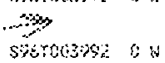

$\sin i x$

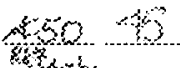

B.t... arazkats

xil

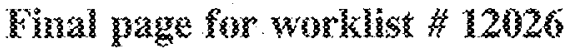
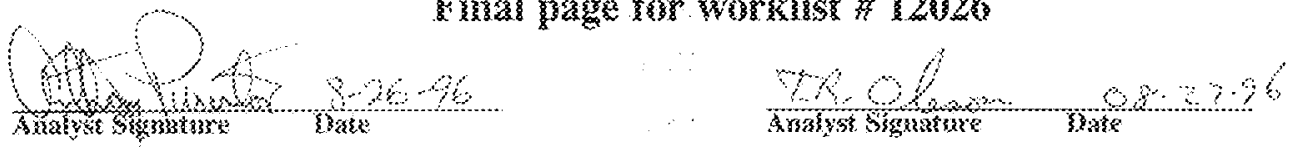

ध:

...

$2.2 \%$

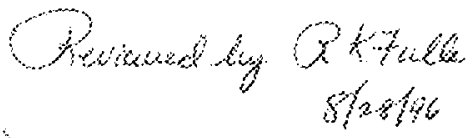

Erat Entry Conmera:

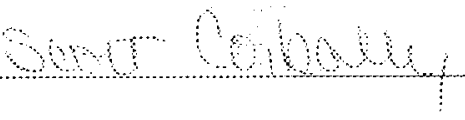

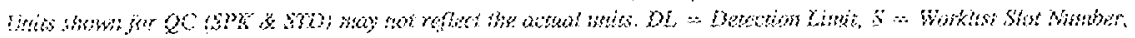

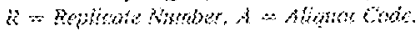

$2 \times 63$ 


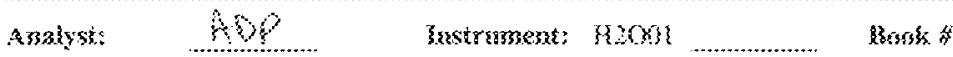

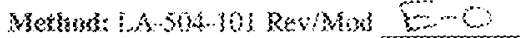

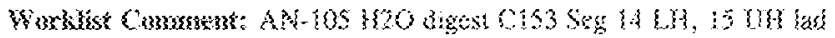

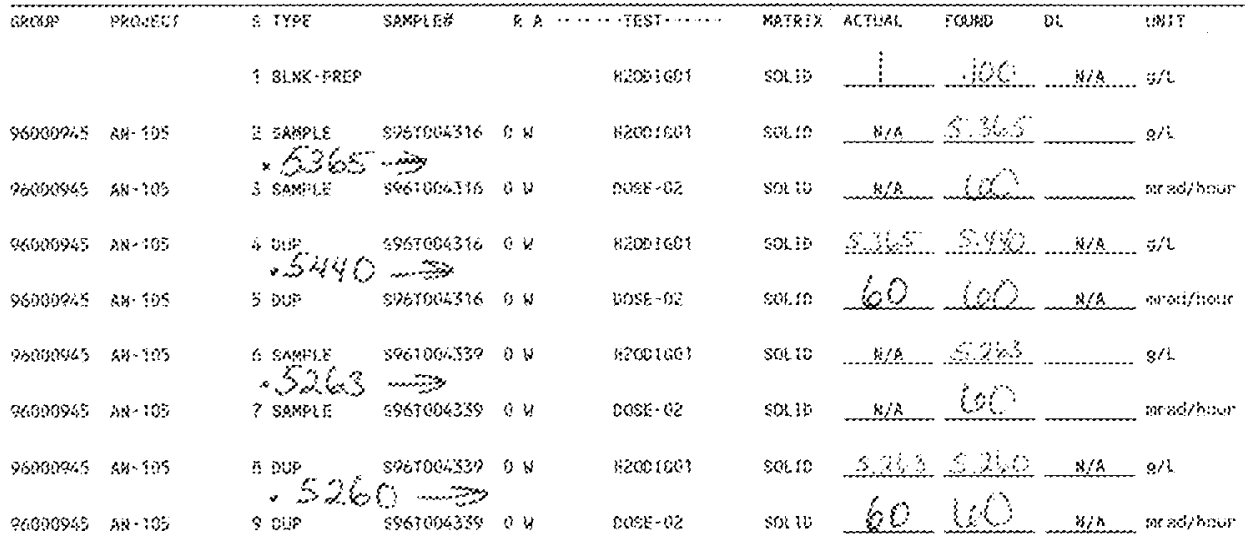

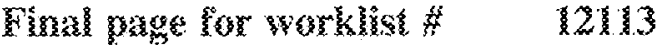
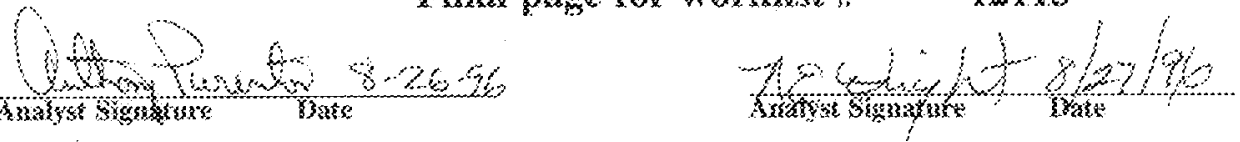

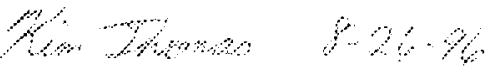

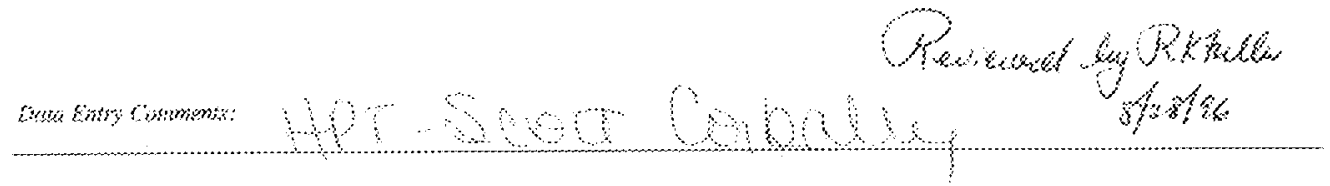

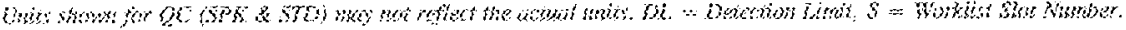

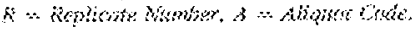

$2 \% 3$ 


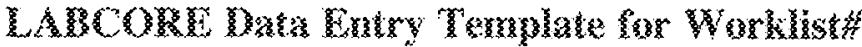

disulyst:

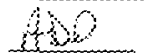

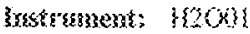

Bowsis it

Merknu: L.4-504-101 Bewhod.

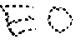

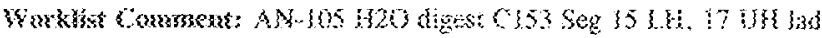

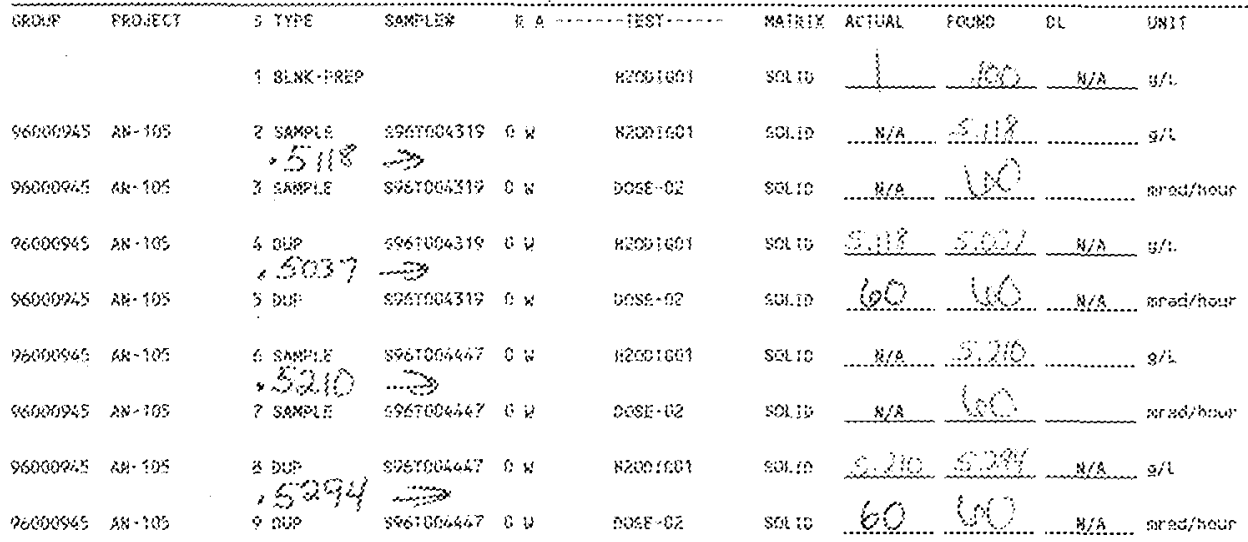

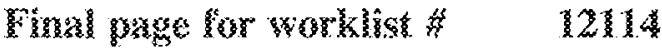

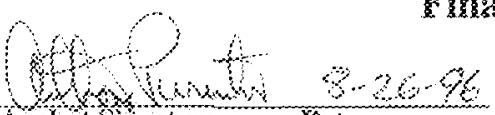

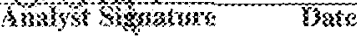

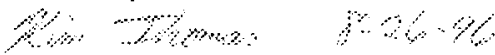

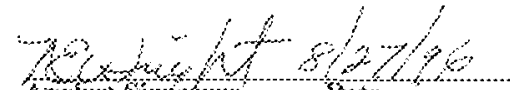

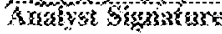

bate

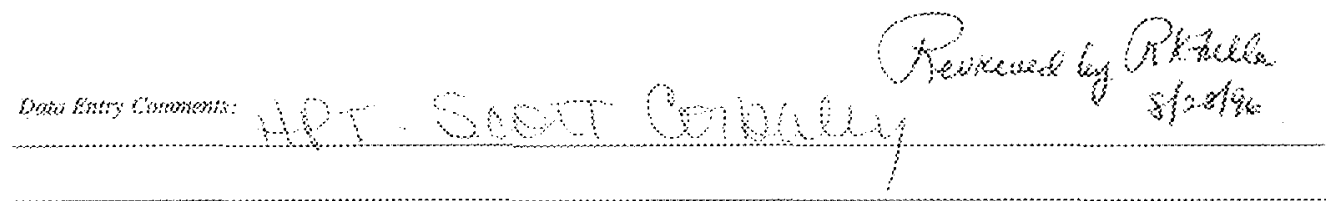

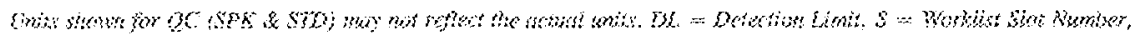

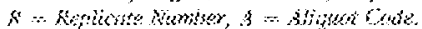


Arstyo:

$3 x$

\{3ะ×rumsens: $\{200\}$

Book:

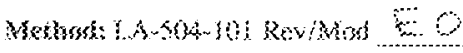

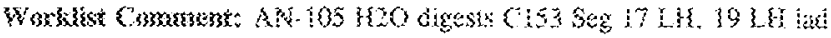

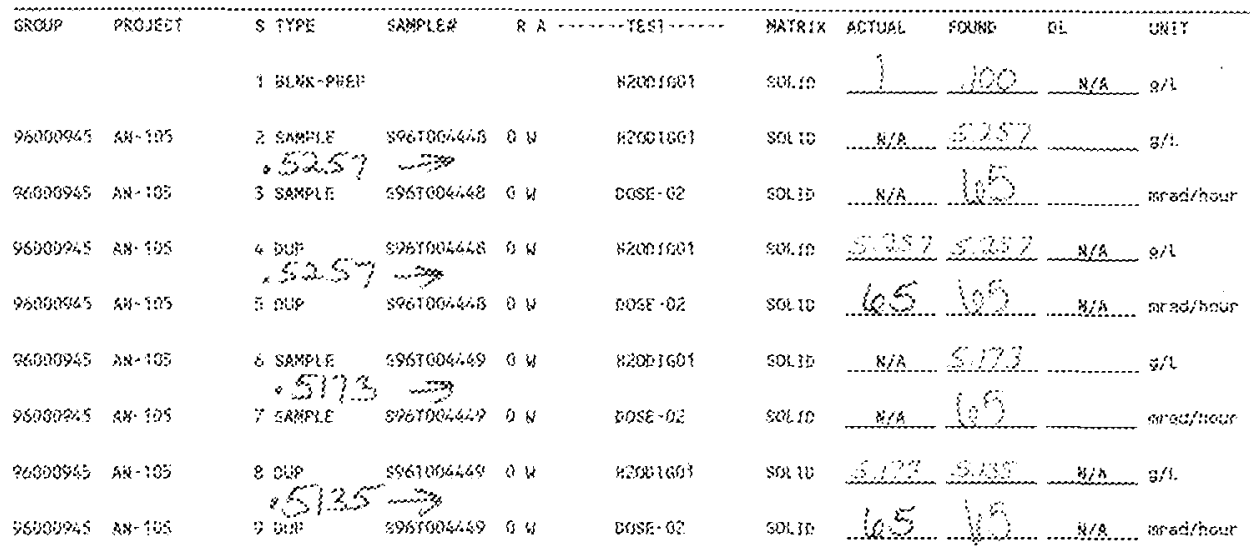

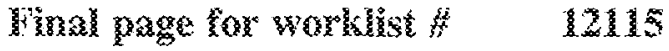

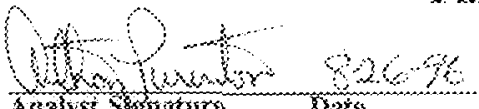

Andyst shoming

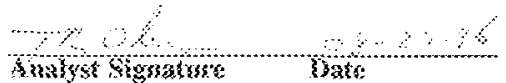

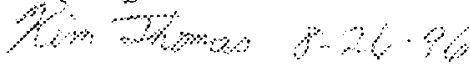

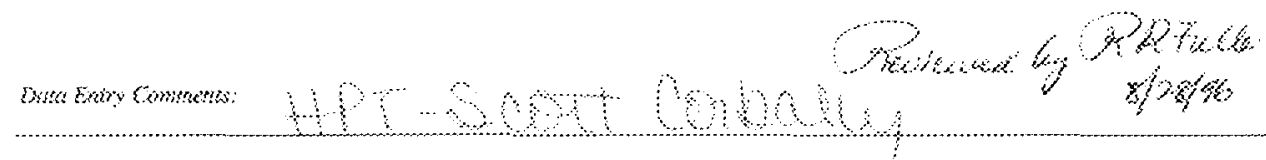

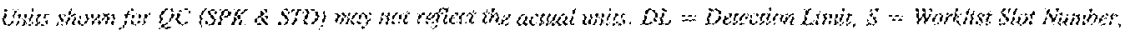

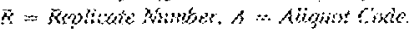




\section{LA}

Arasyst:

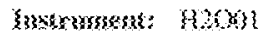

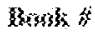

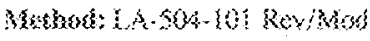

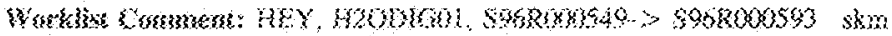

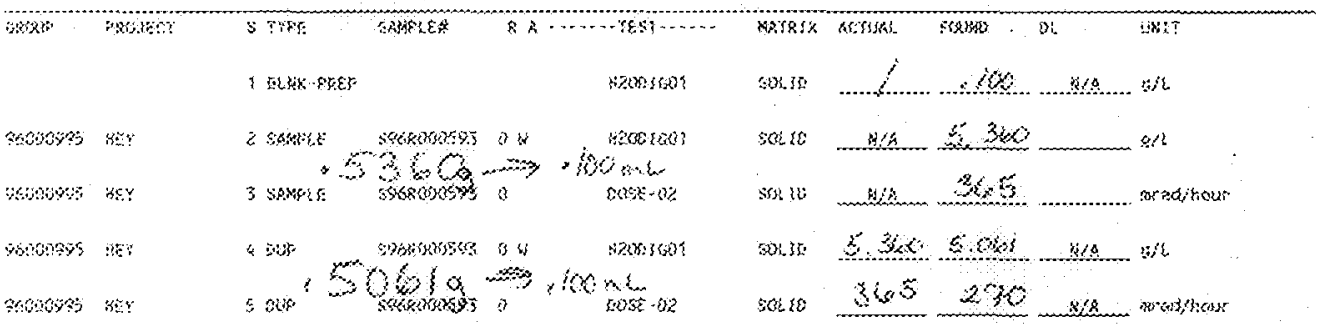

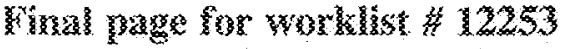
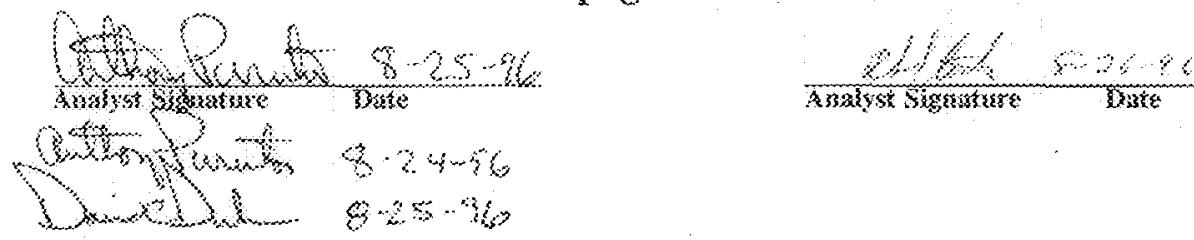

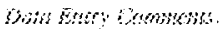

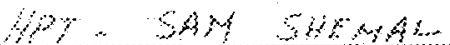

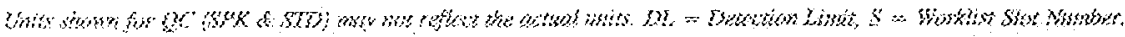

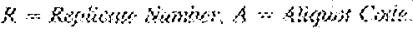

$x+8$ 


\section{LA COME}

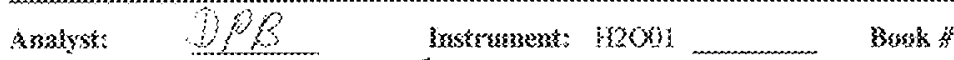

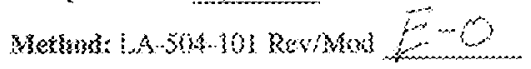

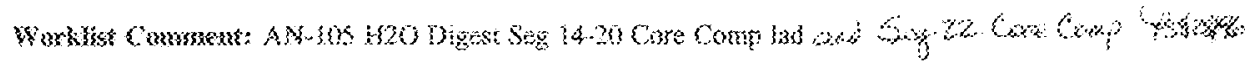

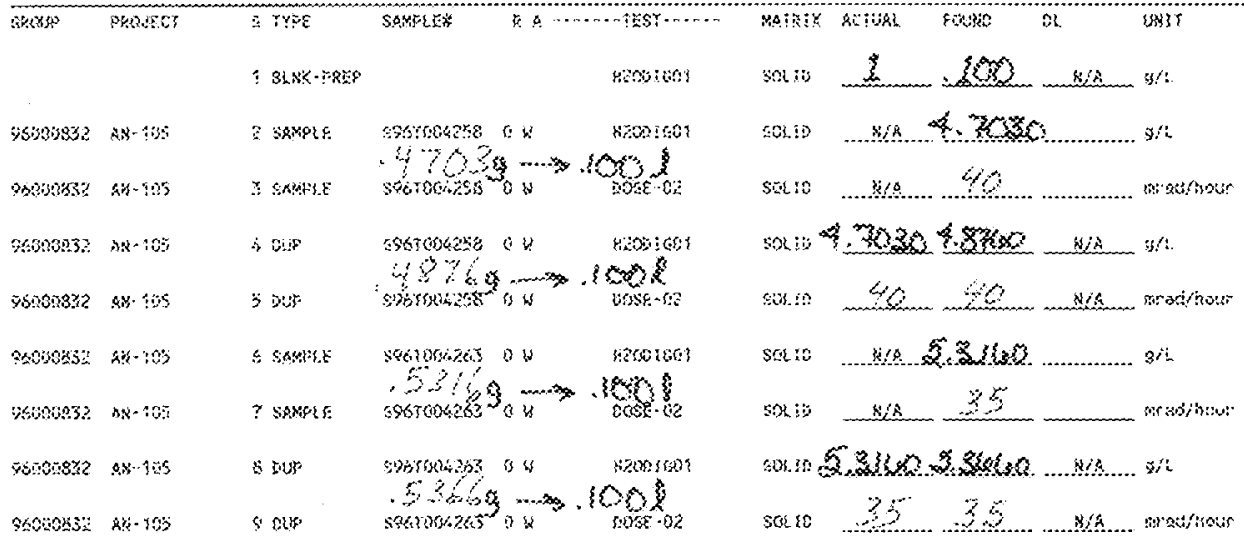

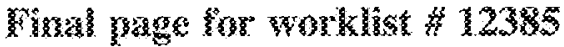
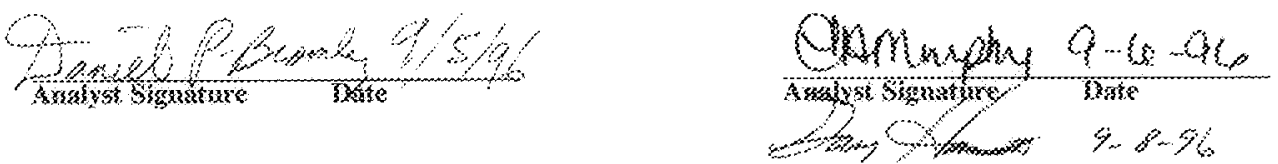

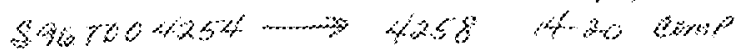

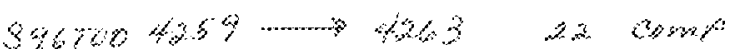

Nan enisy romomi:

sond by

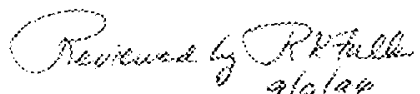
q/q/ir

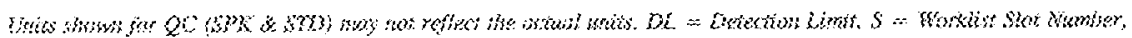

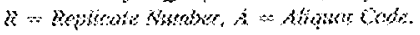

rem 
M40.5.6\%

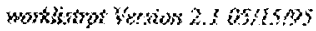

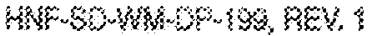

towe $\quad t$

$50,03,36,262$

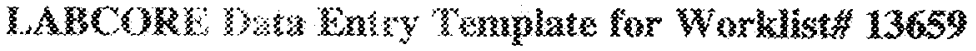

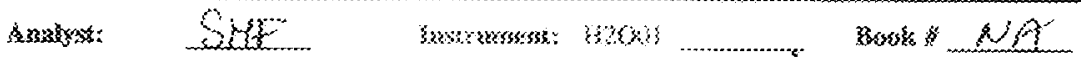

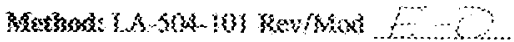

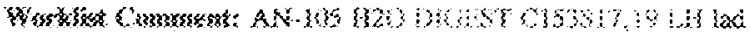

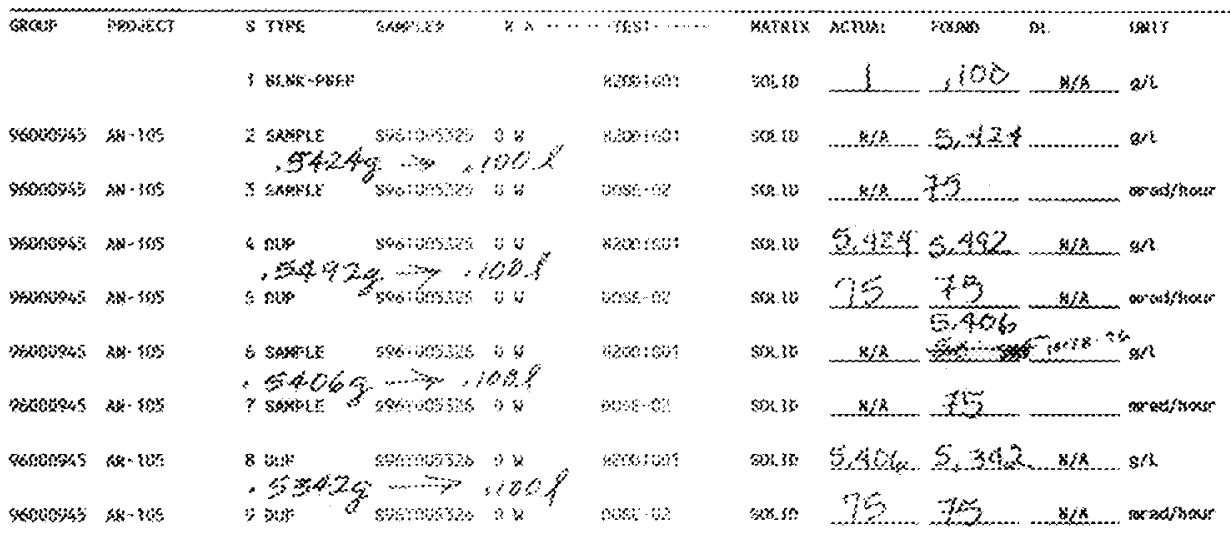

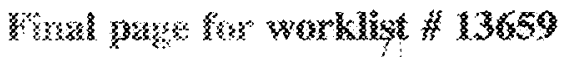

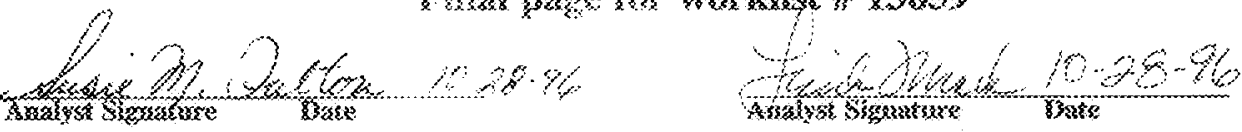

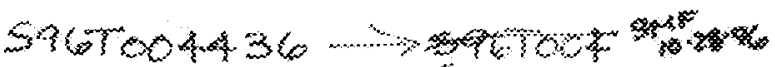

20670053

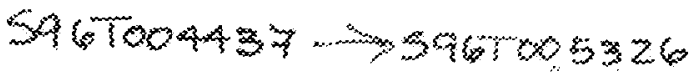

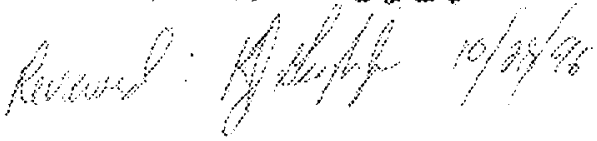

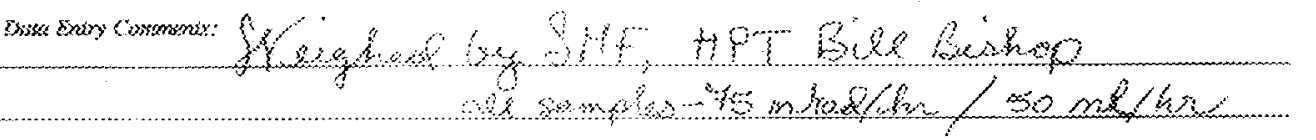

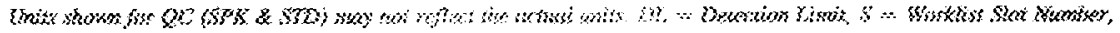

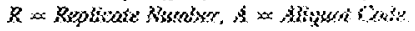

20 


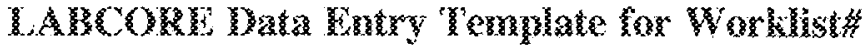

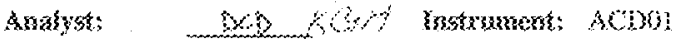

\author{
soms if
}

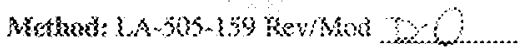

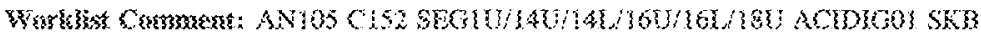

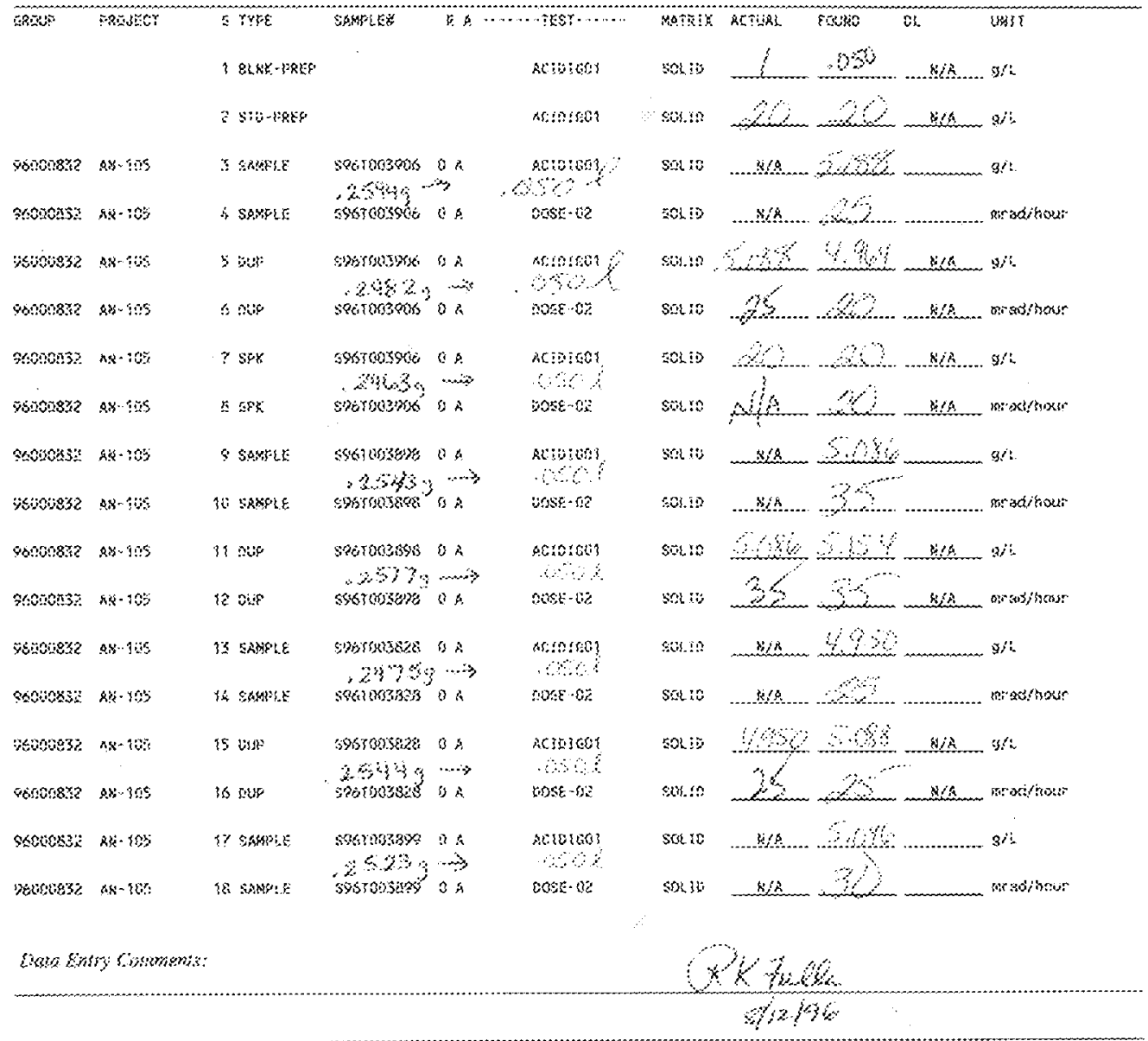

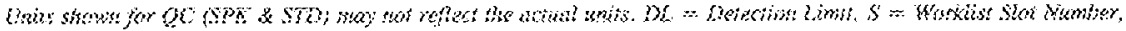

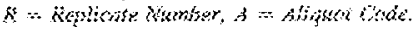




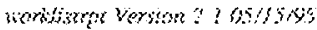

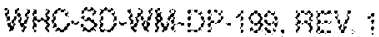

mp5s:35:05

\section{I.}

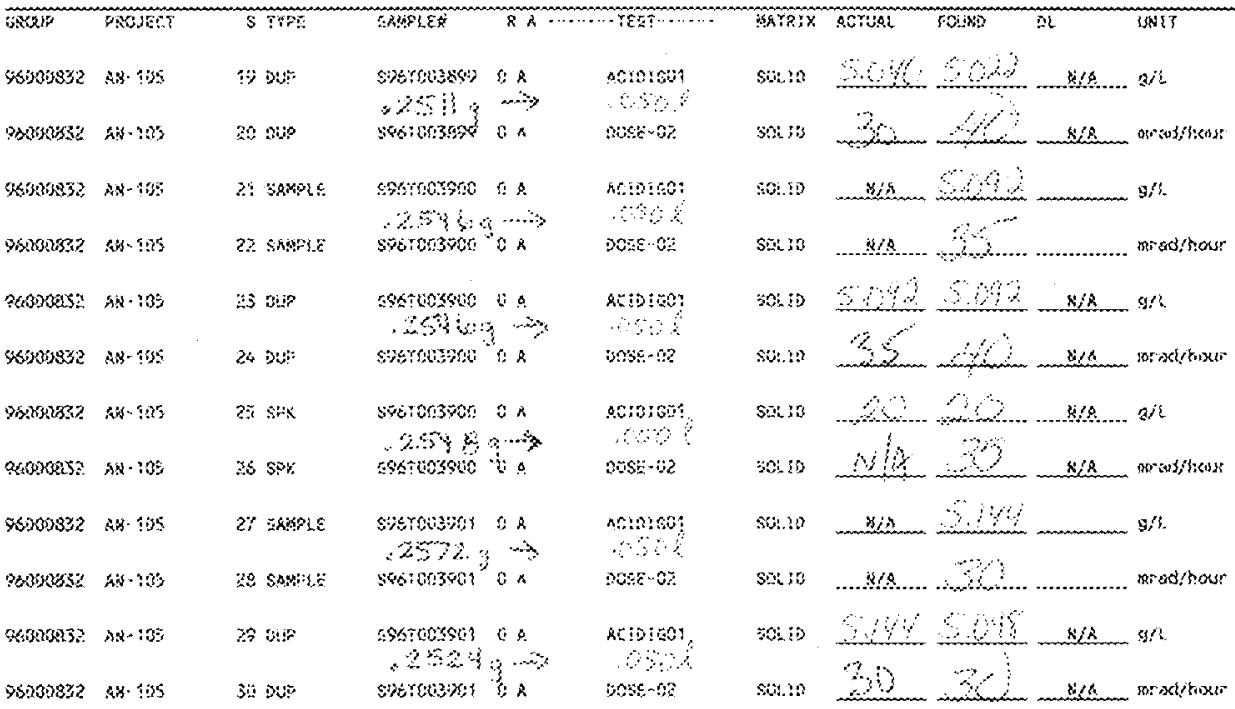

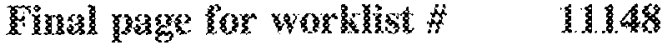

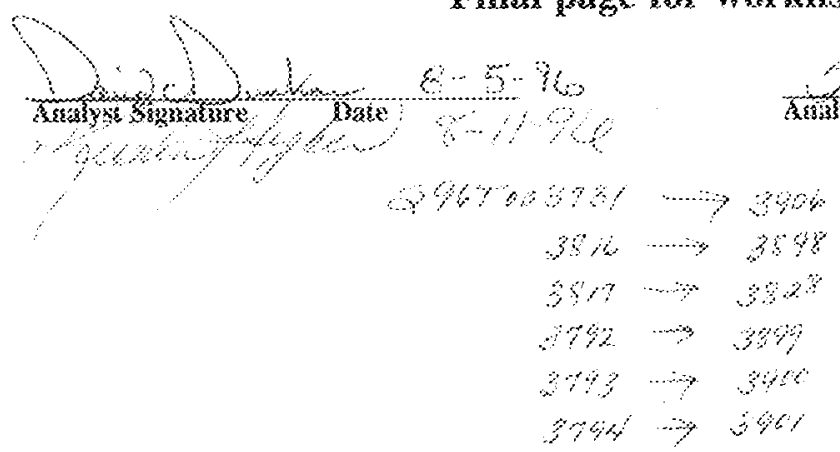

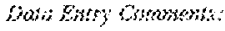

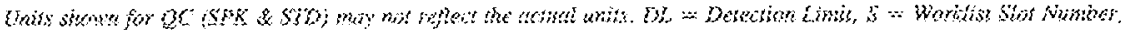

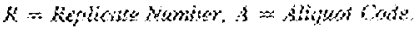




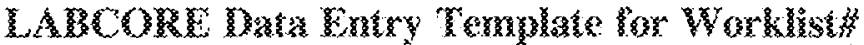

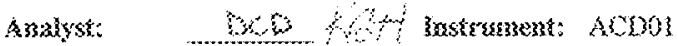

3)

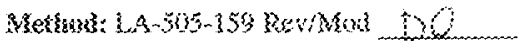

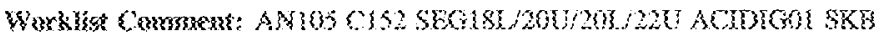

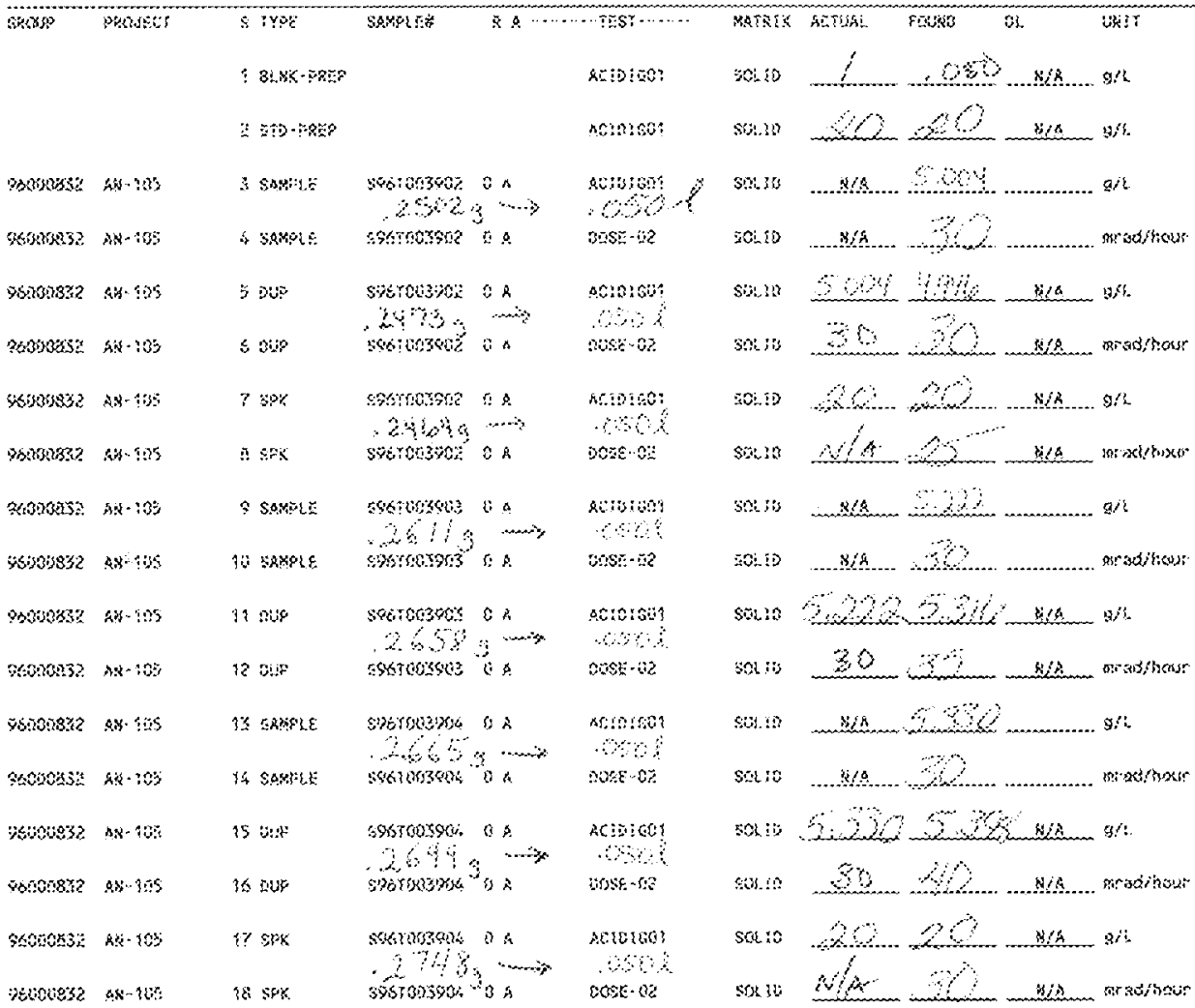

Lota Enty Sintwonis:

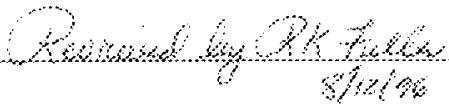

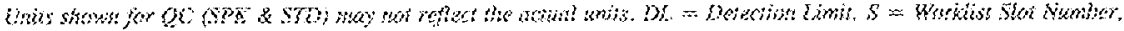

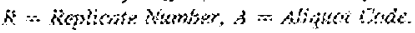




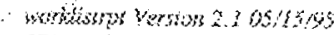

Ha.

whosh 1100

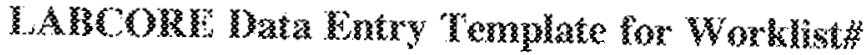

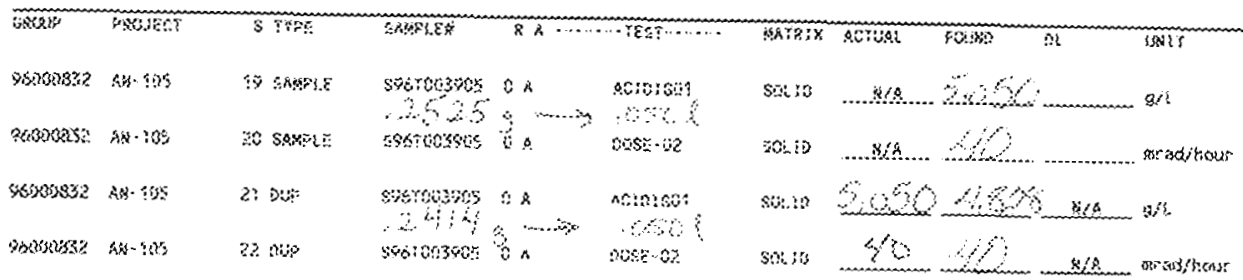

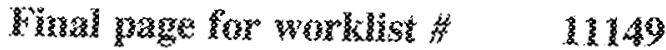
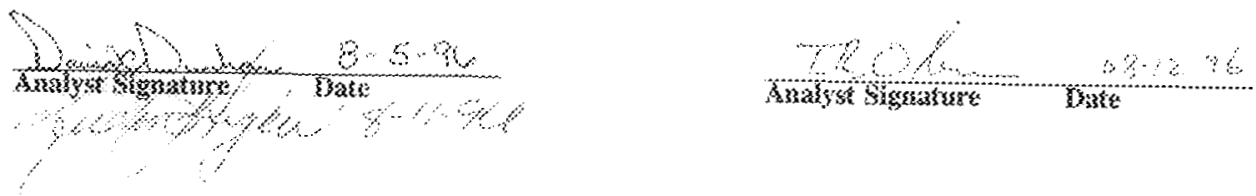

$$
\begin{aligned}
& \text { कमा } \\
& \text { कास } \cdots \text { भे } \\
& \text { का } \\
& \text { तो का का }
\end{aligned}
$$

incia Entry Conmeris:

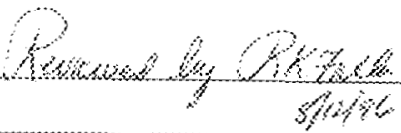

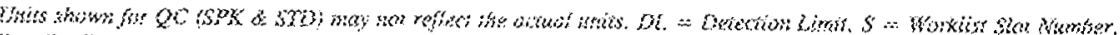

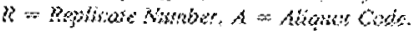


Rowak

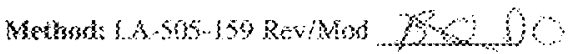

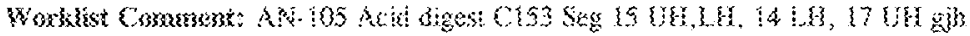

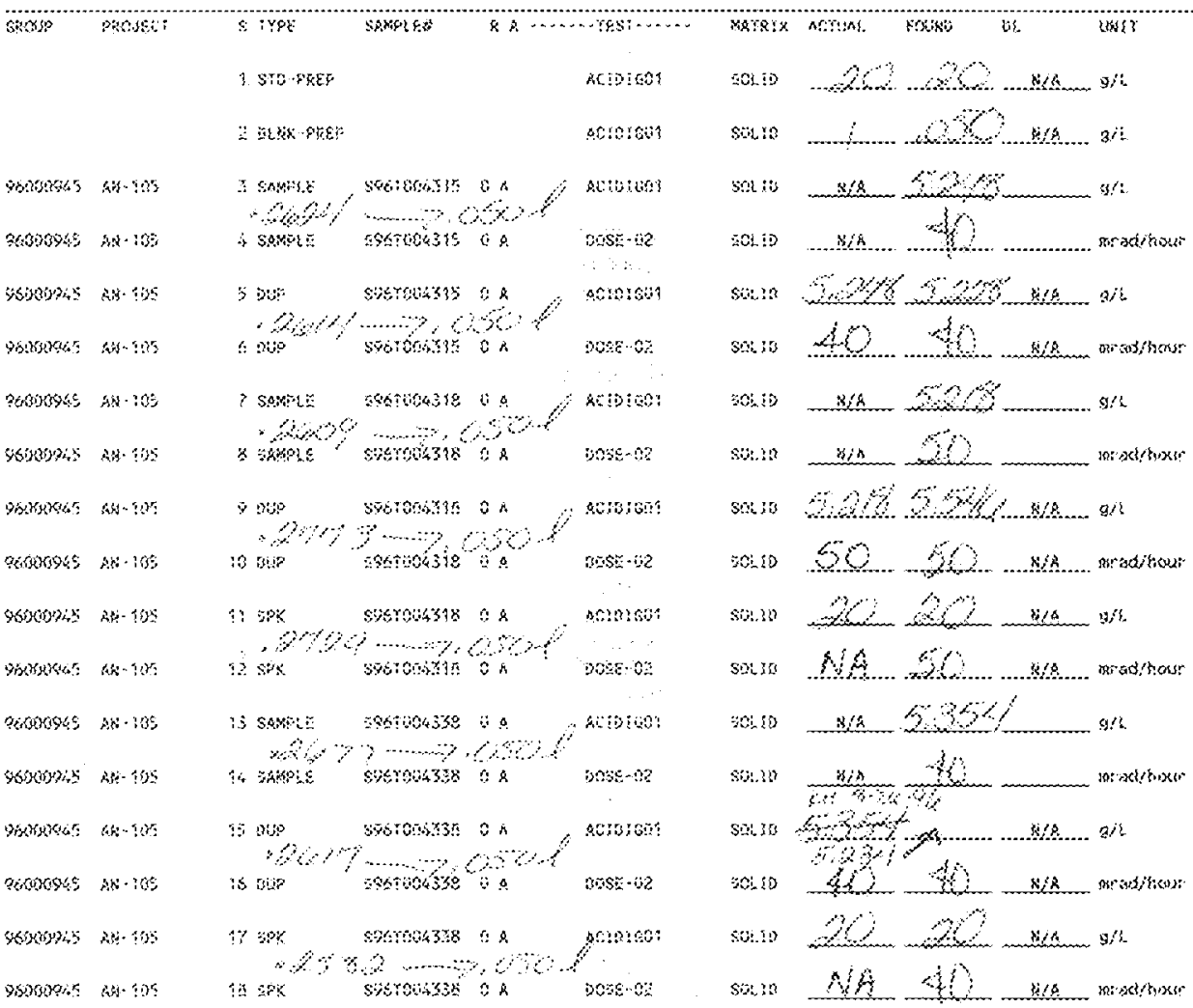

Dato sum cimmon

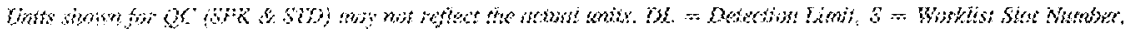

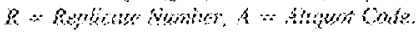




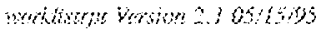

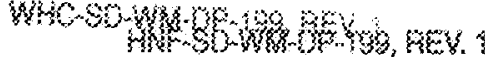

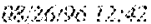

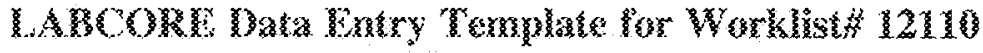

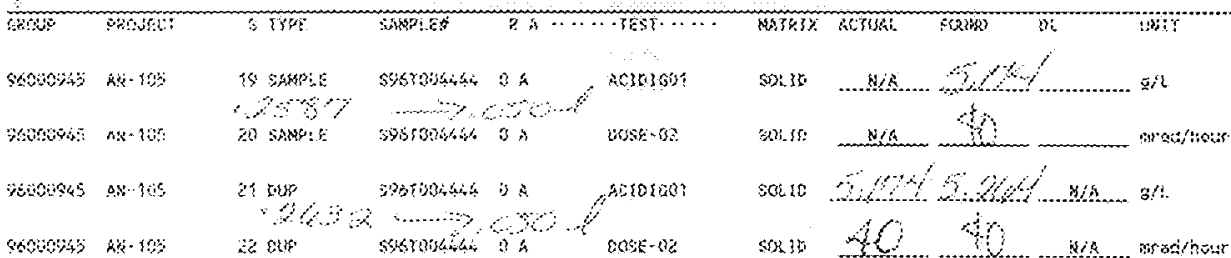

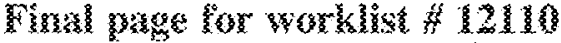
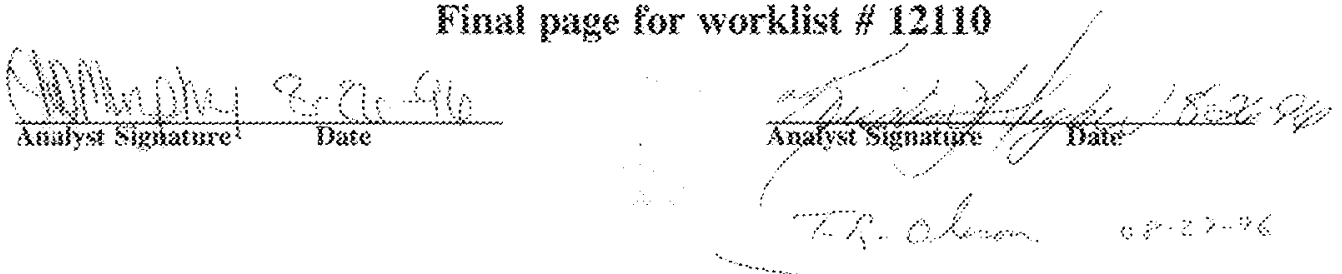

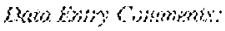

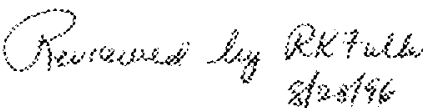

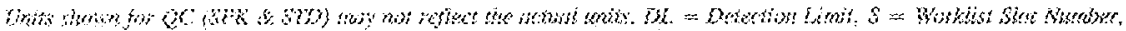

$R$ a fow 


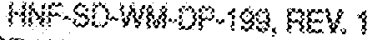

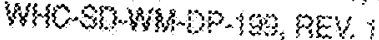

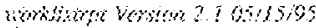

18\% $20 \%$ is

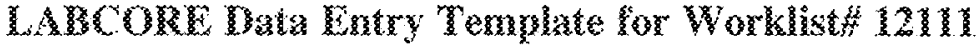

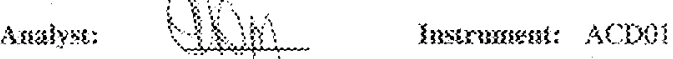

Eosk

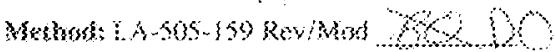

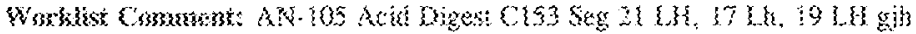

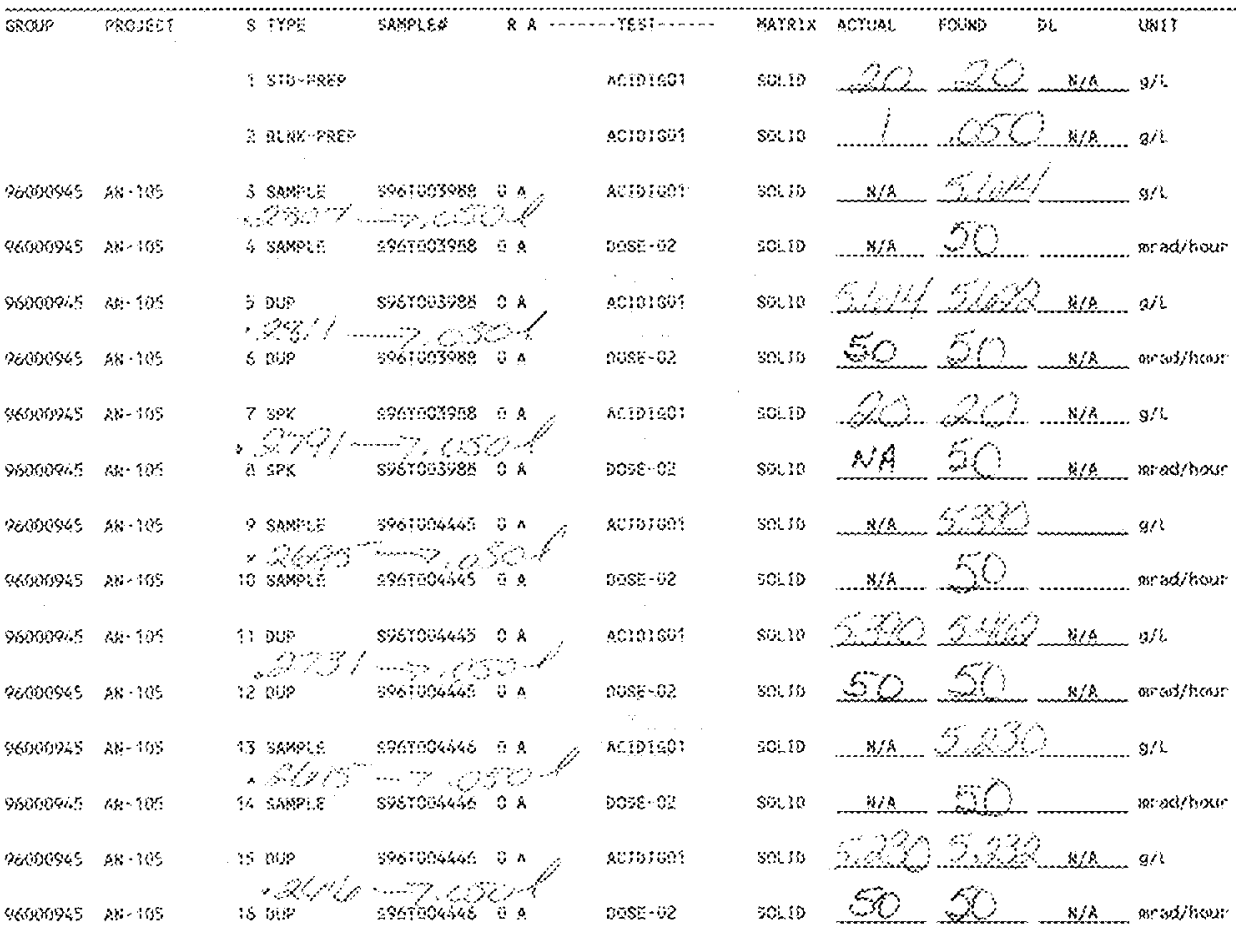

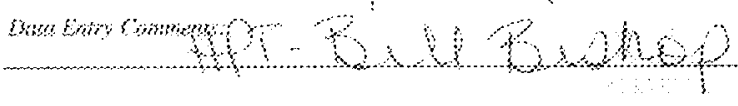

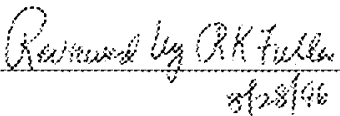

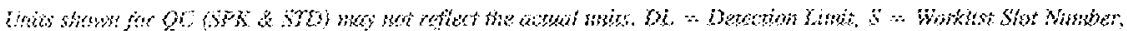

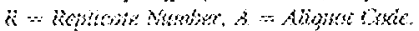




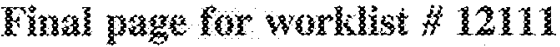
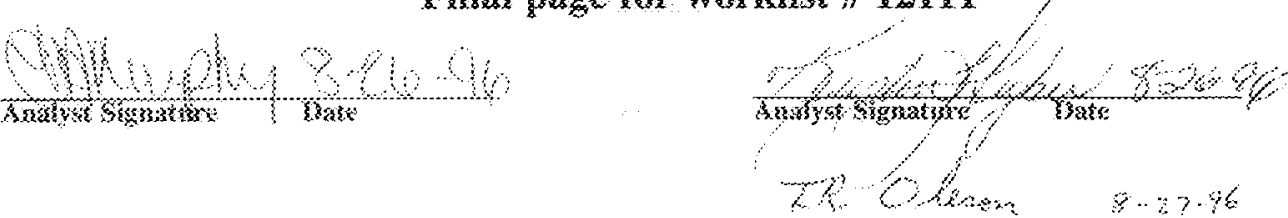


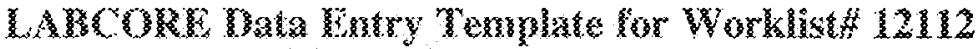

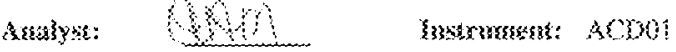

gook

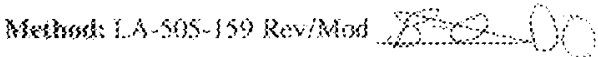

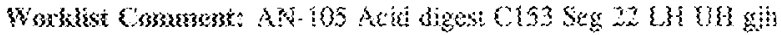

\begin{tabular}{|c|c|c|c|c|c|c|c|c|}
\hline \multirow[t]{3}{*}{ Gxosp } & PAOYE: & 5 iYot & SABSLE⿱一兀 & -resin-.... & Sorkix & ACYUAL & bl & BSEST \\
\hline & & $5 \mathrm{sij}+\mathrm{Ne}$ & & krtotso1 & Eod. & & $N / 2$ & $9 / 4$ \\
\hline & & 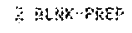 & & otarsol & $\sin 10$ & & $4 / 8$ & ari \\
\hline 96000565 & $\operatorname{sid}-98$ & 3 son:ti & sobineses? d 4 & 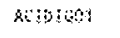 & 30 is & $8 / 8$ & & 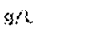 \\
\hline Siktiph: & $22-35$ & $438 \times 015$ & sgargusa? in & $\operatorname{sisin-12}$ & $\$ 0 . .93$ & $\forall i n$ & & norstiknser \\
\hline $96 \times 1204.5$ & $4 i \cdot 95$ & $50 \%$ & \$シErouz:8; OA & Acrorsit & sexeso & & H/A... & $a i$ \\
\hline poroses & $A s-3$ & 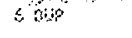 & $5000038 \%$ o & ase-b2 & 30,5 & & sis & aroubihens:- \\
\hline 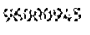 & $A B-: A B$ & $7 s \%$ & Astronst? of & NED:Go & ESTD & & NiR. & sh. \\
\hline S6,900\%:x & $4 x^{3} \cdot 19$ & IA $\quad \mathrm{AR}$ & sysrow3\%8\% of & 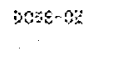 & sonen & & $B 18$ & notstroser \\
\hline $9600 \times 345$ & 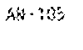 & $\begin{array}{c}9 \sin x \\
\%\end{array}$ & 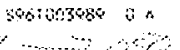 & sobitos & sotis & & & gil \\
\hline 6600945 & 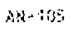 & 10 3axpl & SSTEOTStS d A & $\operatorname{mos}-12$ & $5 \infty: 10$ & & & seresiksus: \\
\hline 9600004 & $44+395$ & I: & s\%cro43:8; ok & Acionsis? & sals & & سن & $3 / 6$ \\
\hline poovoses & $503-290$ & $12 n i p$ & $50000380^{\circ}$ on & $208+62$ & 30 is & & .M..... & Bead/hour \\
\hline
\end{tabular}

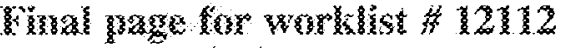
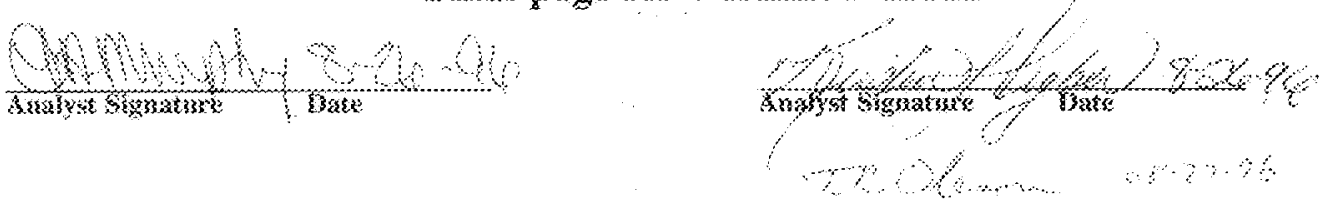

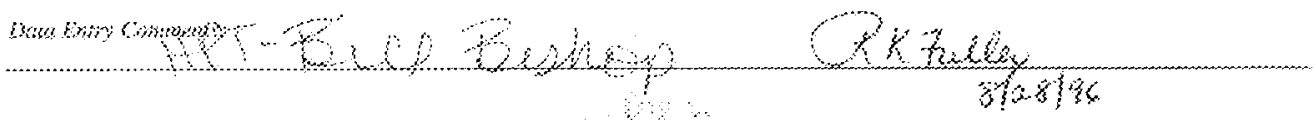

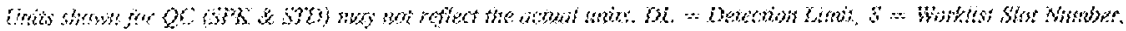

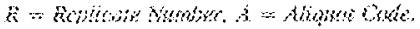




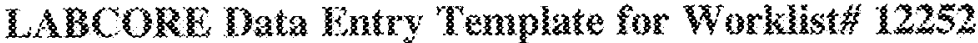

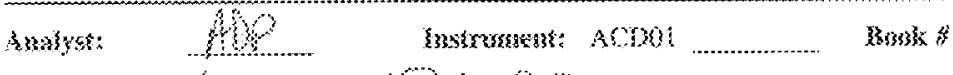

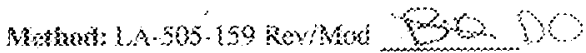

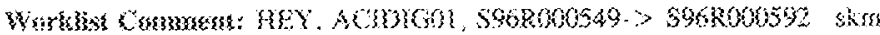

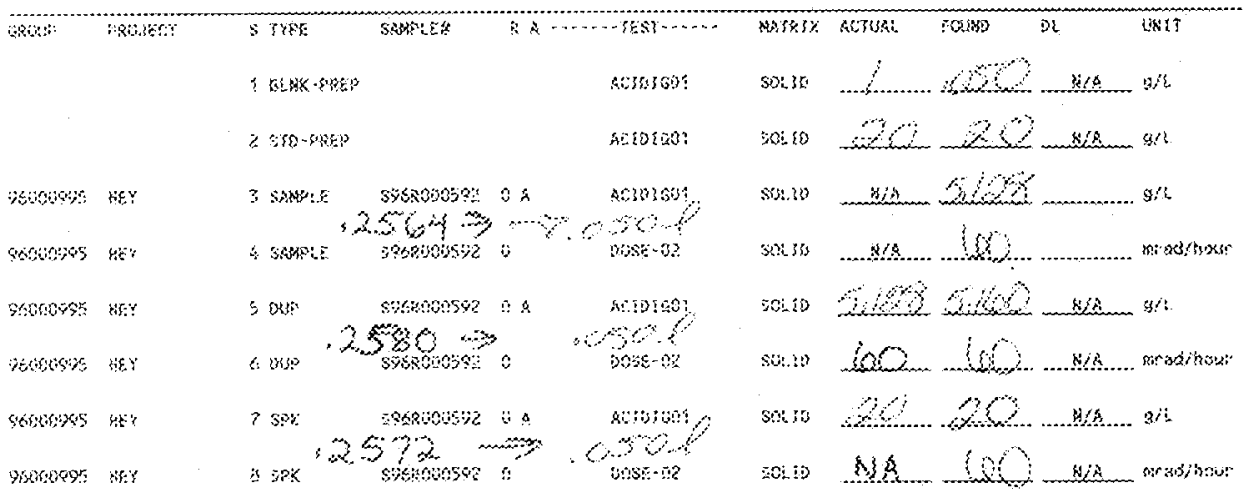

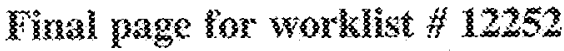
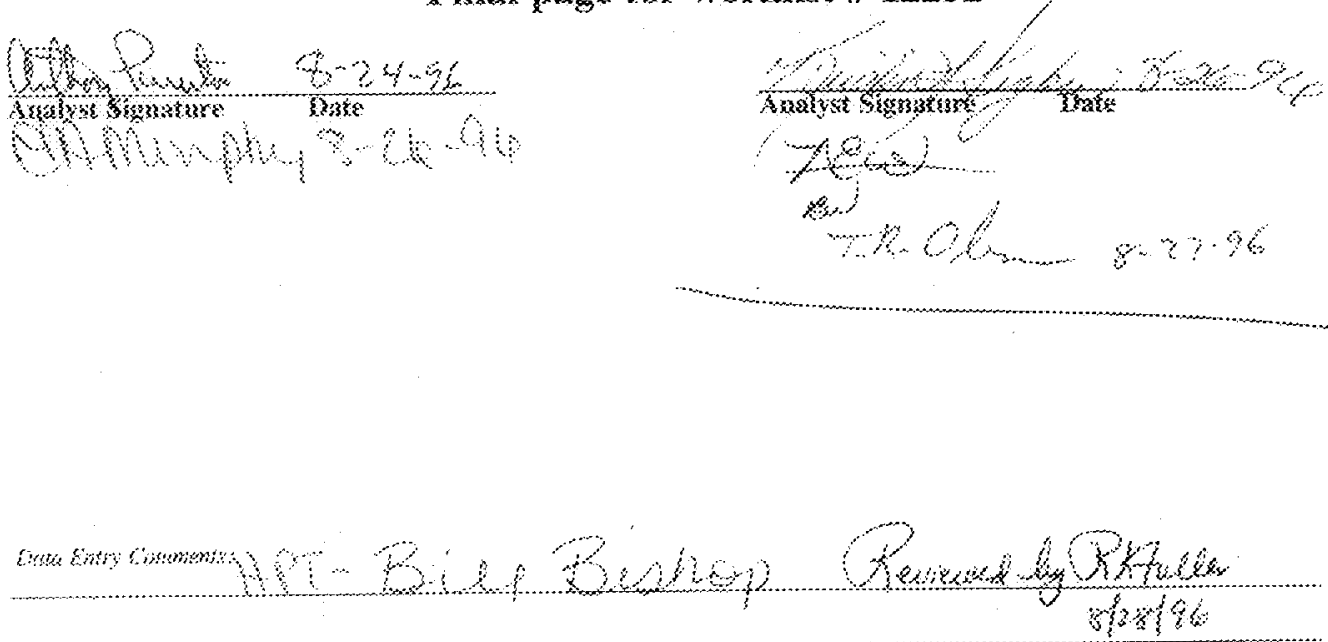

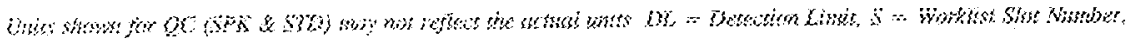

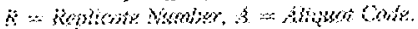




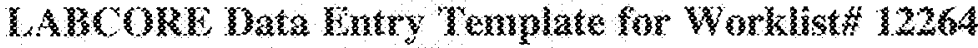

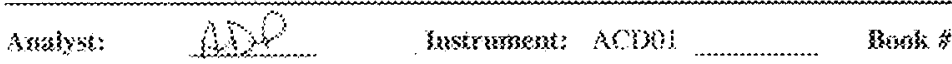

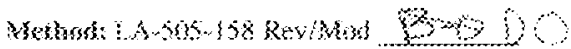

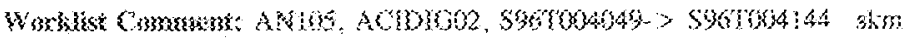

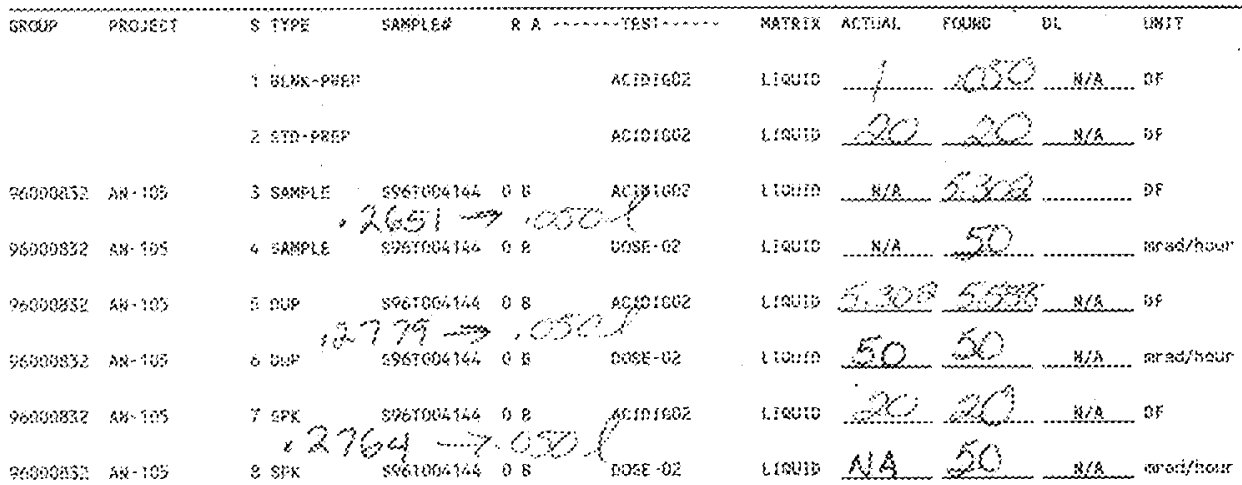

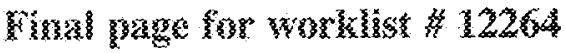
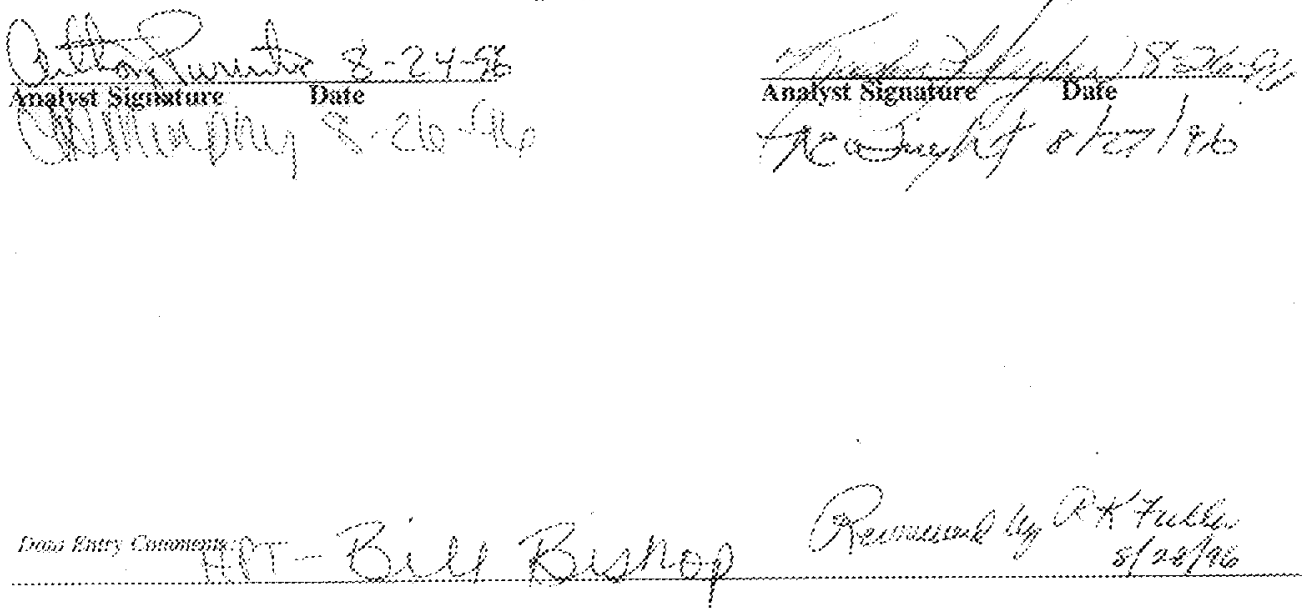

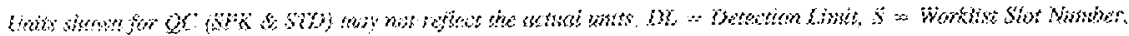

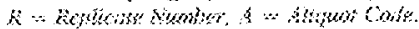




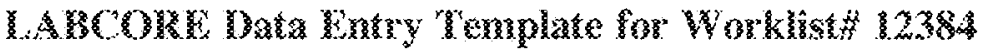

ArRatys:

knstriment: $\mathrm{Elist}$

tit

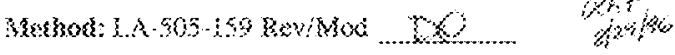

13006

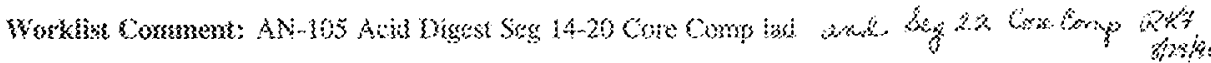

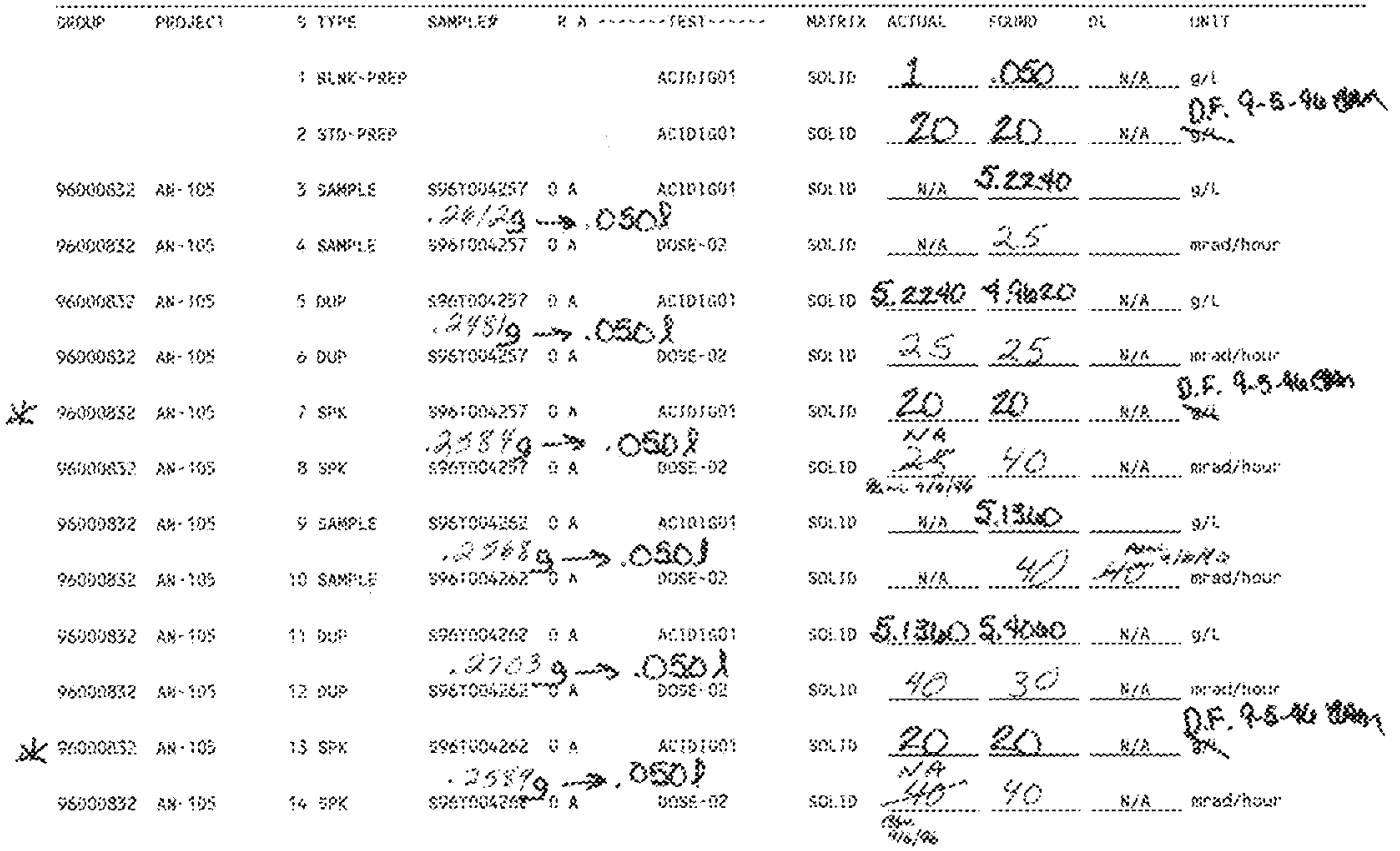

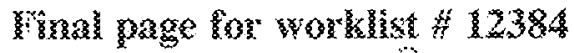
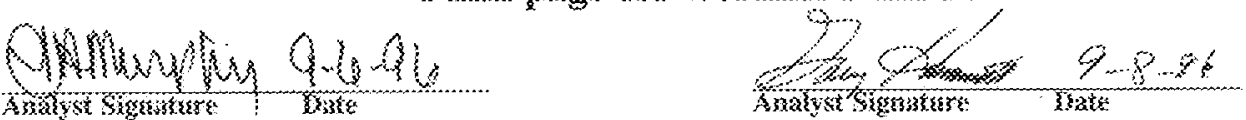

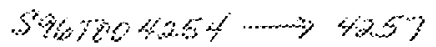

Qua lom compens:

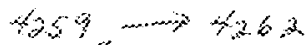

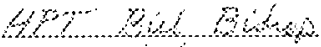

\section{$4<\alpha<2<x+\infty$

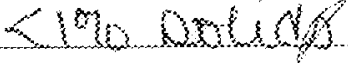

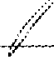

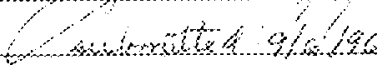

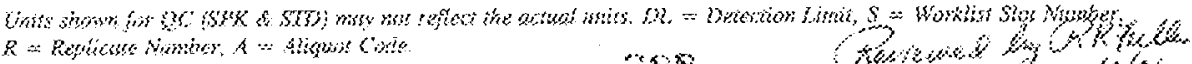




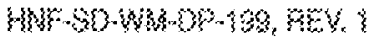

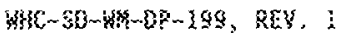

AK -1OS BULX DENSETY WORSAEETS 


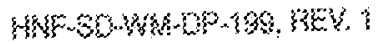

Whe-55,

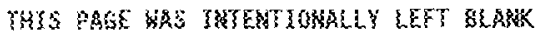




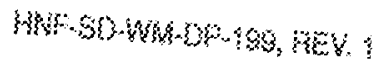
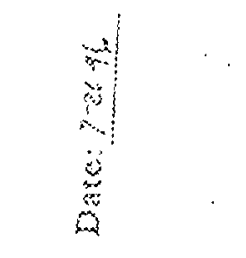


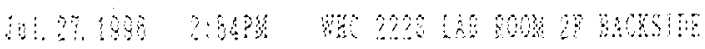

Ho. $34 \%$ ?

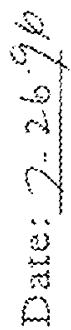

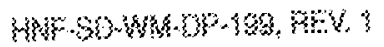

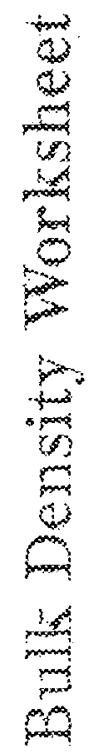


(3)

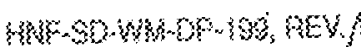

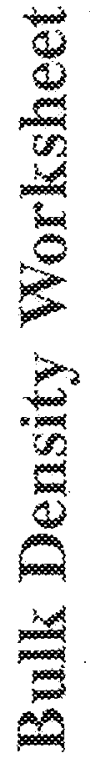

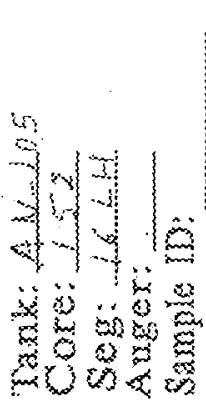
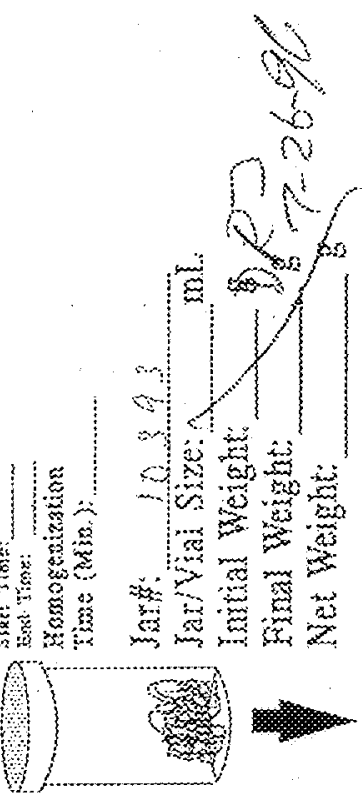


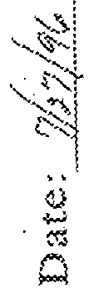

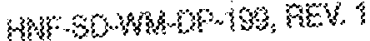
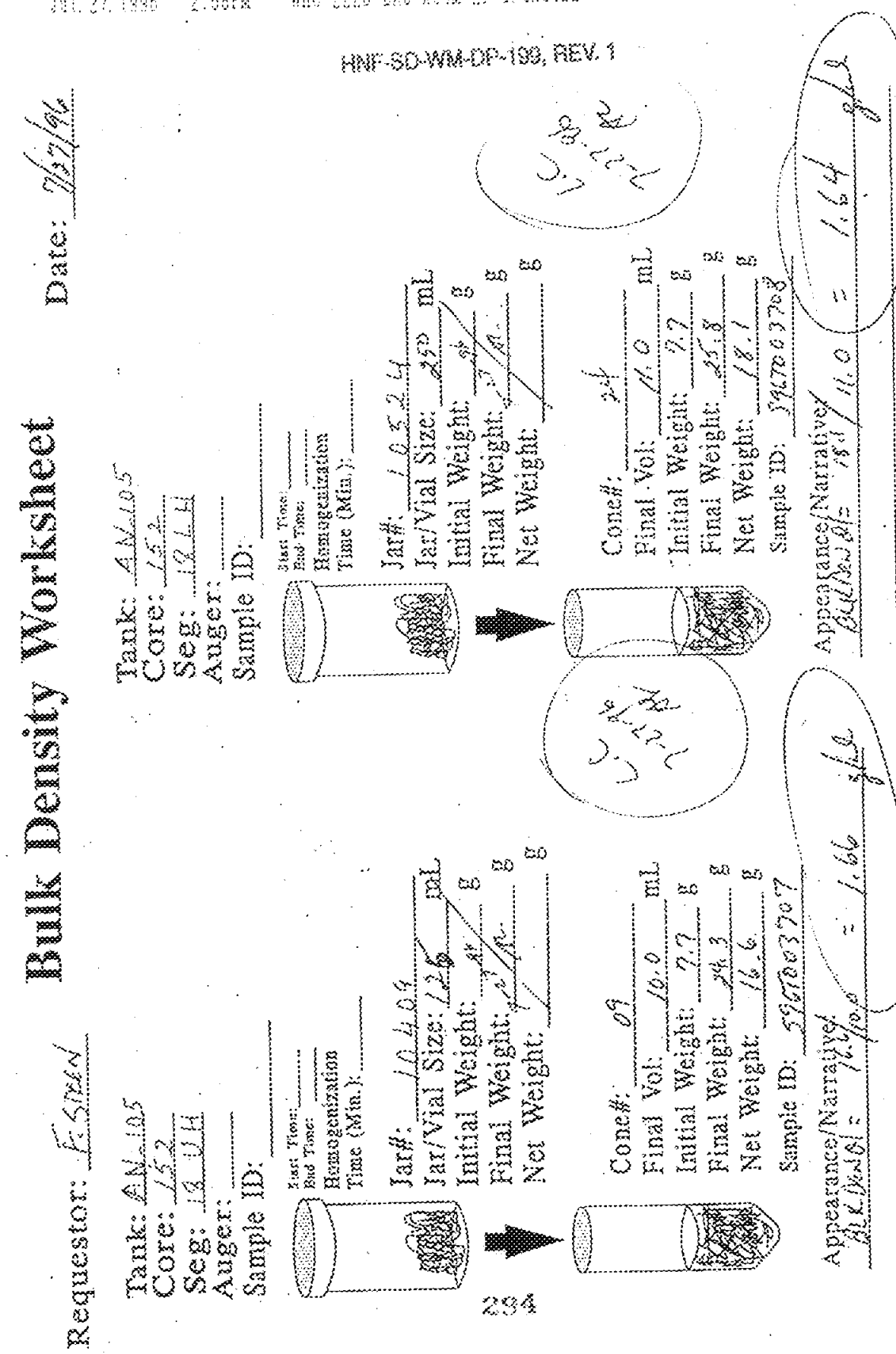


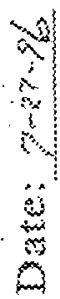

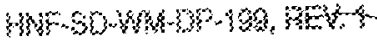
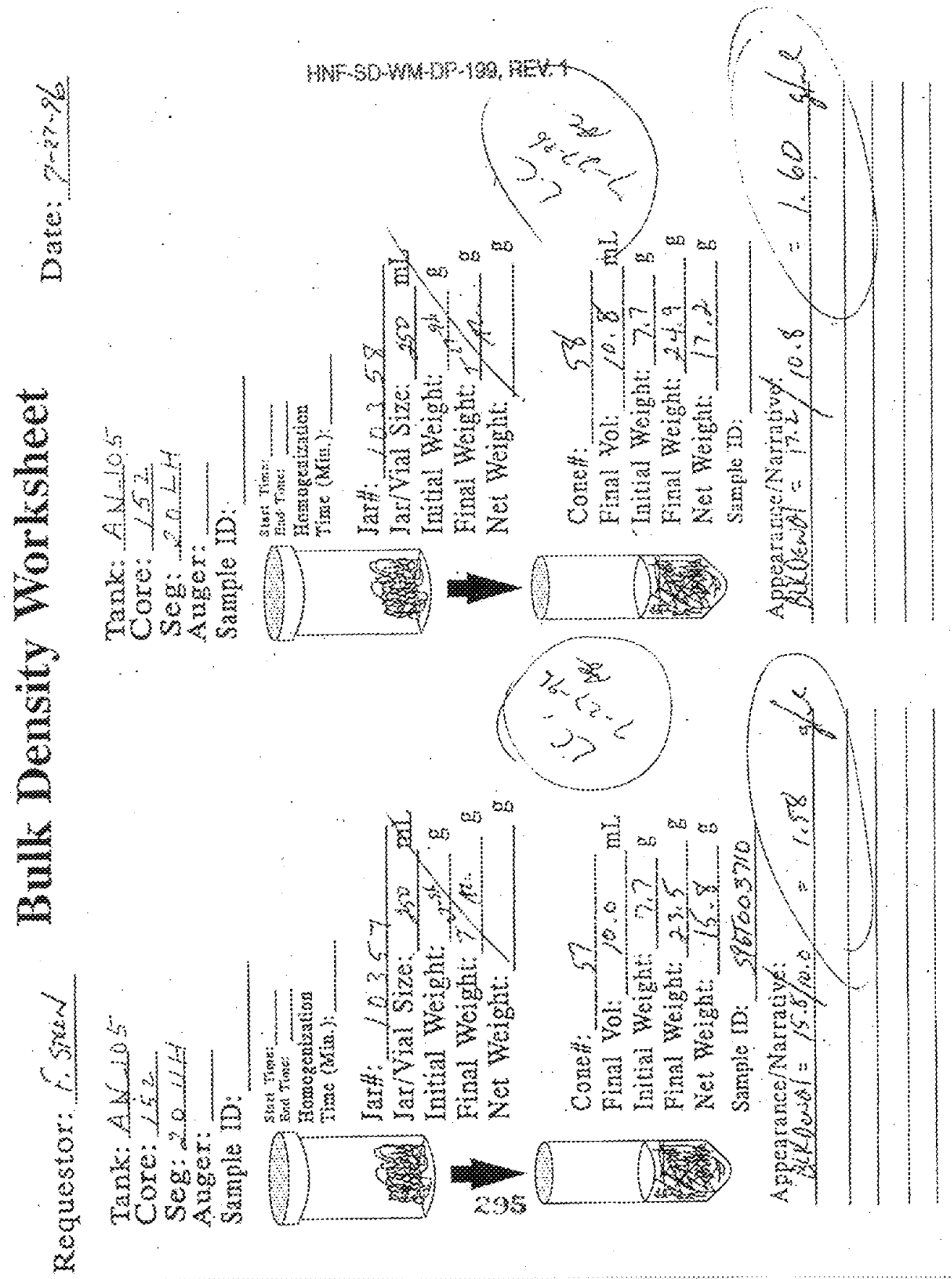
M.
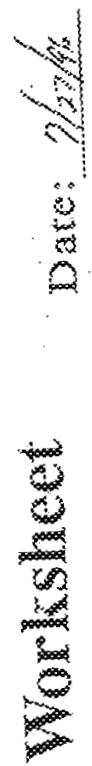

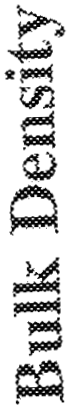
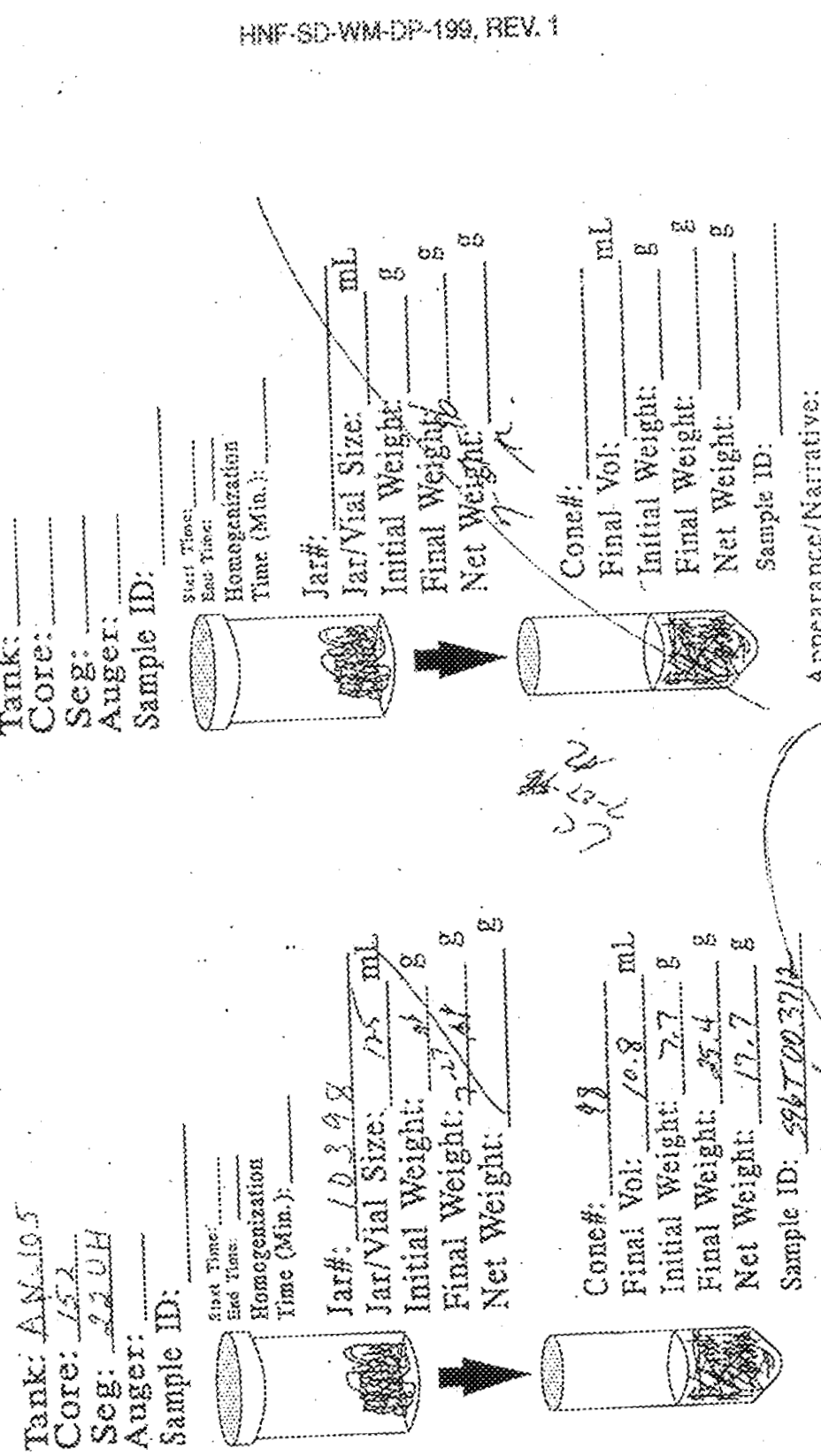

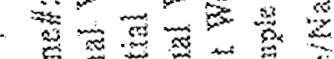

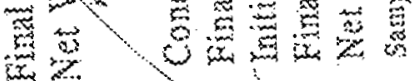

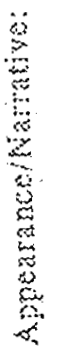
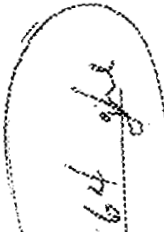
2
0 


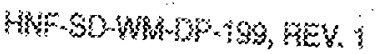
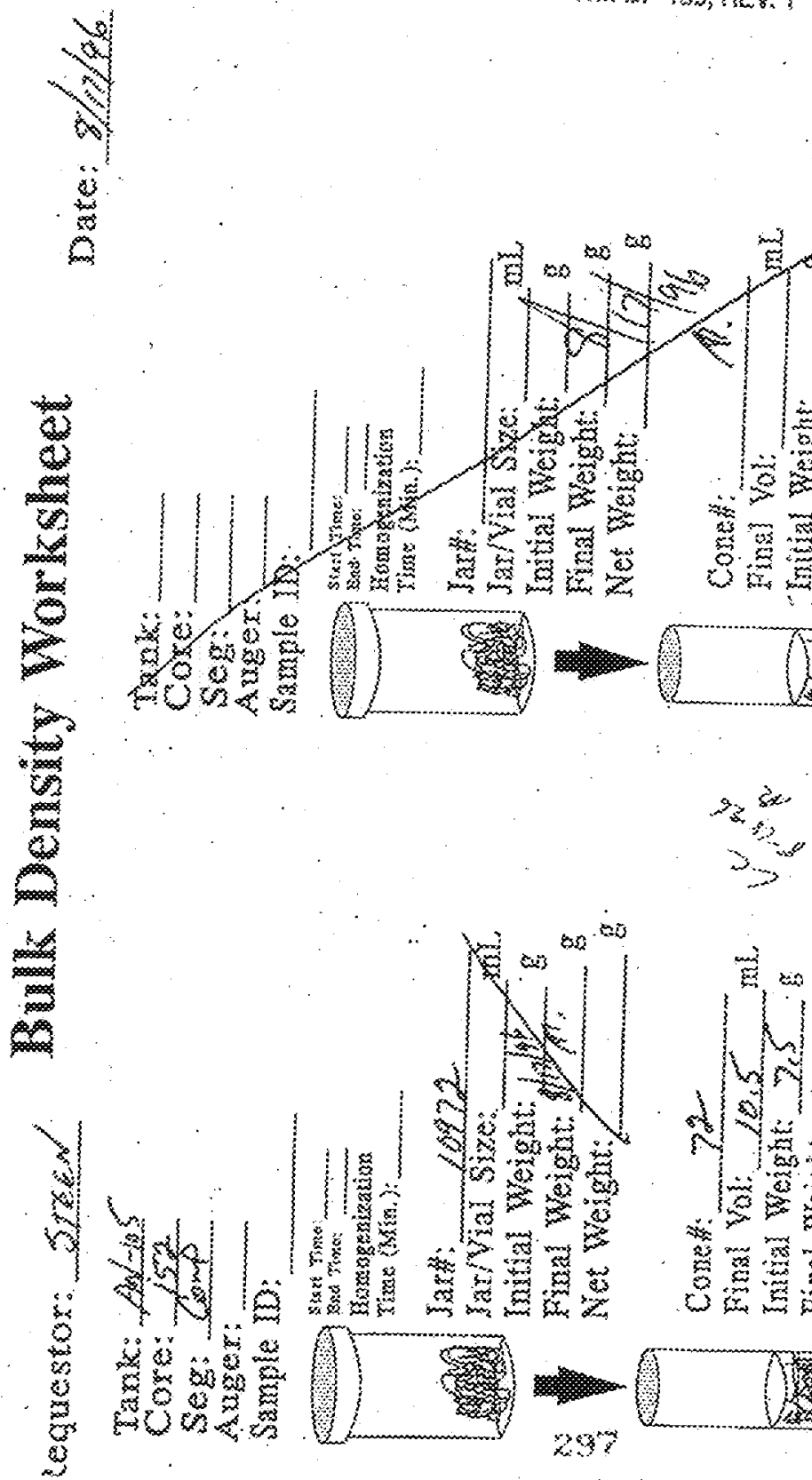
How
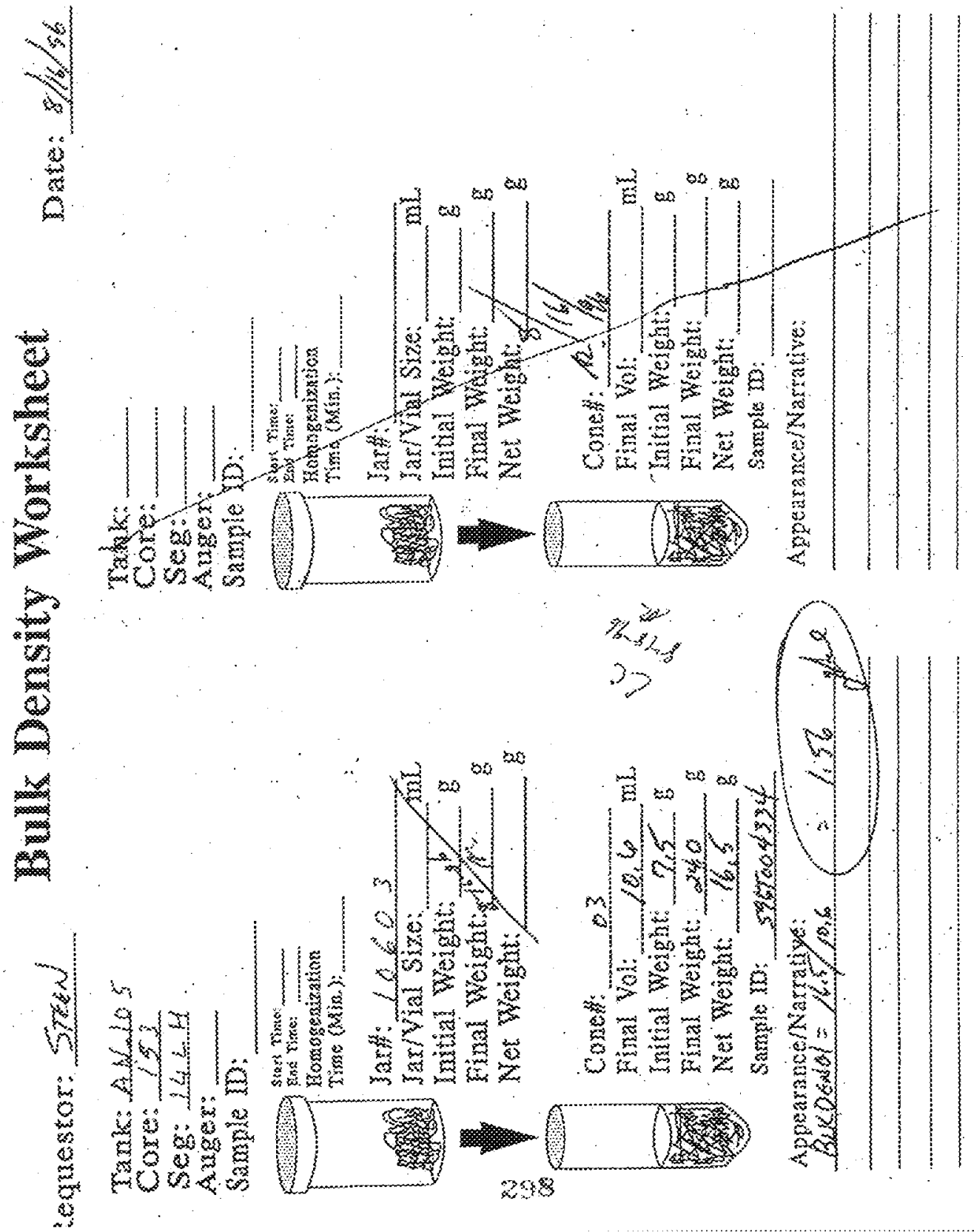


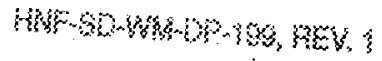
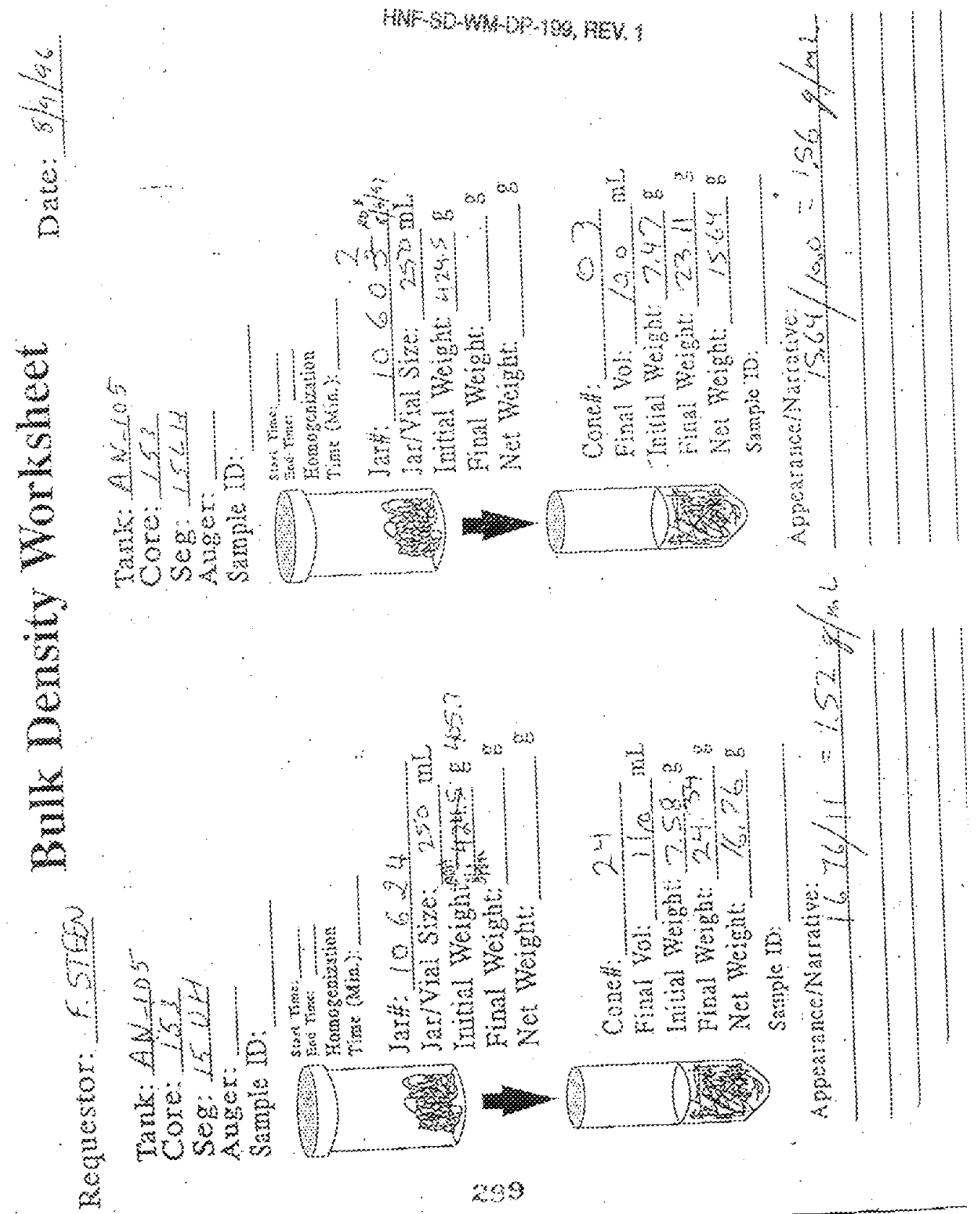


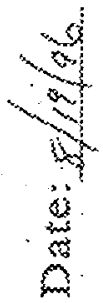

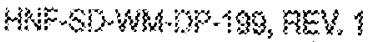

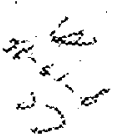
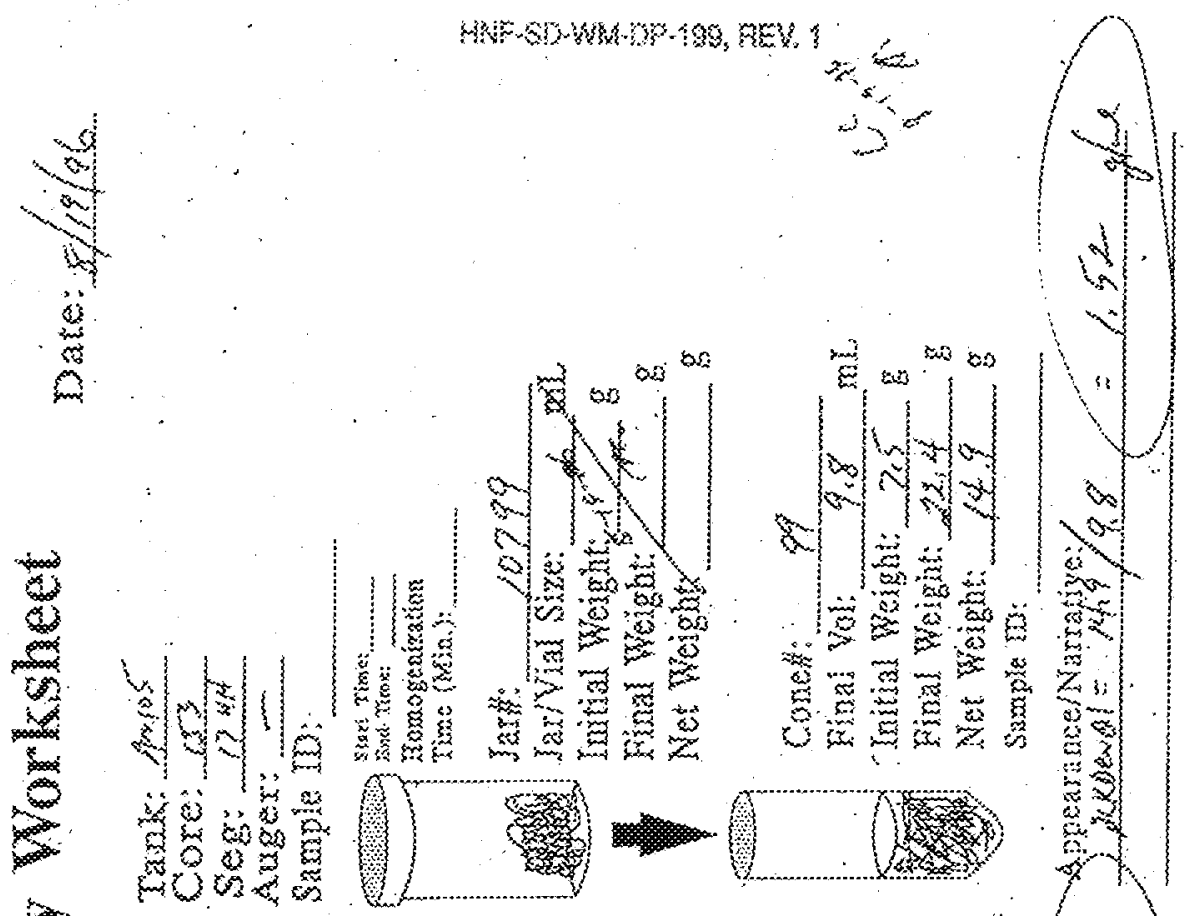

$v_{2} w_{2}$

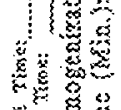

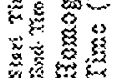
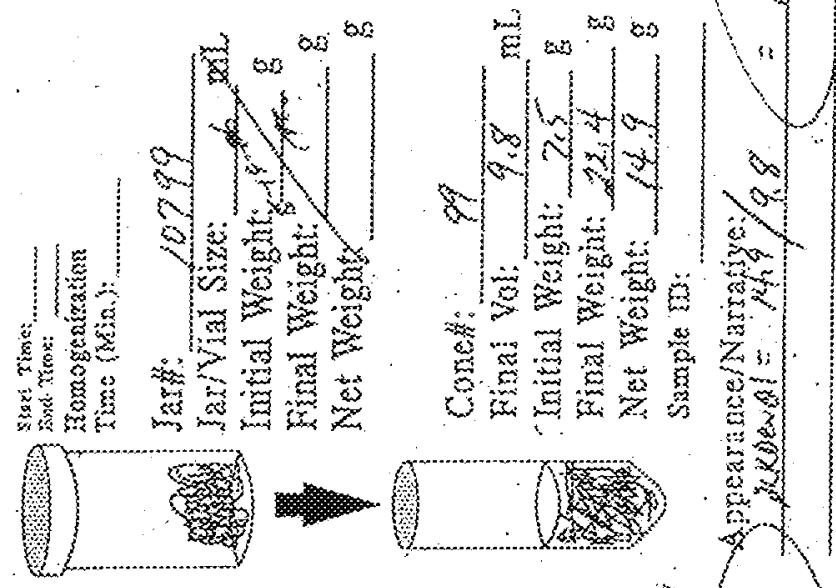

(2) $\left.x_{0}\right)$
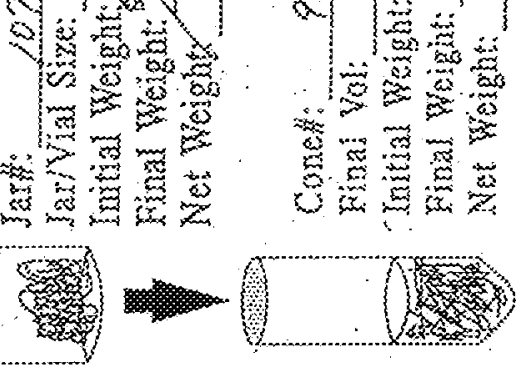

$\min 20$

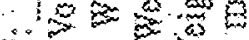

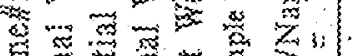

jon
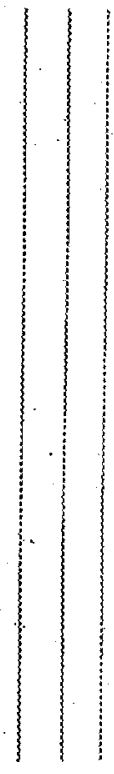

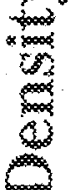

:

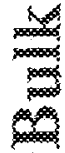
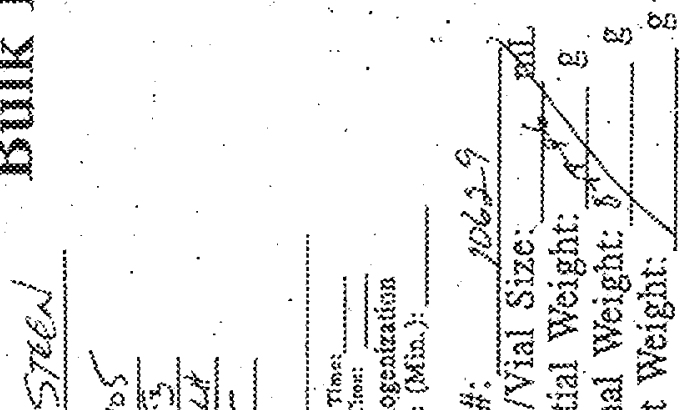

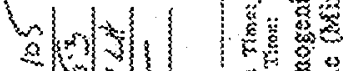

$<$ 皮

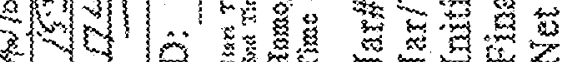

$\because 4), \lim$

然然

ins

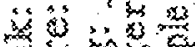

in thos

1 样
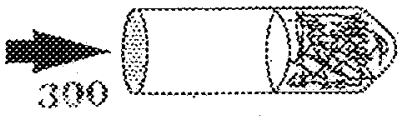

5

$\sum_{\pi}^{\infty} \operatorname{sos}^{\infty}$

sis bs

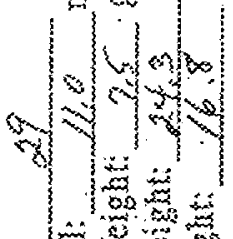

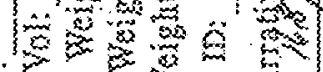
$\therefore$ os os

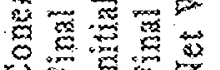

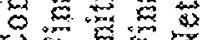

要

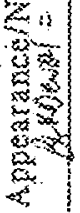




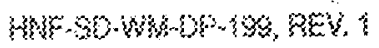

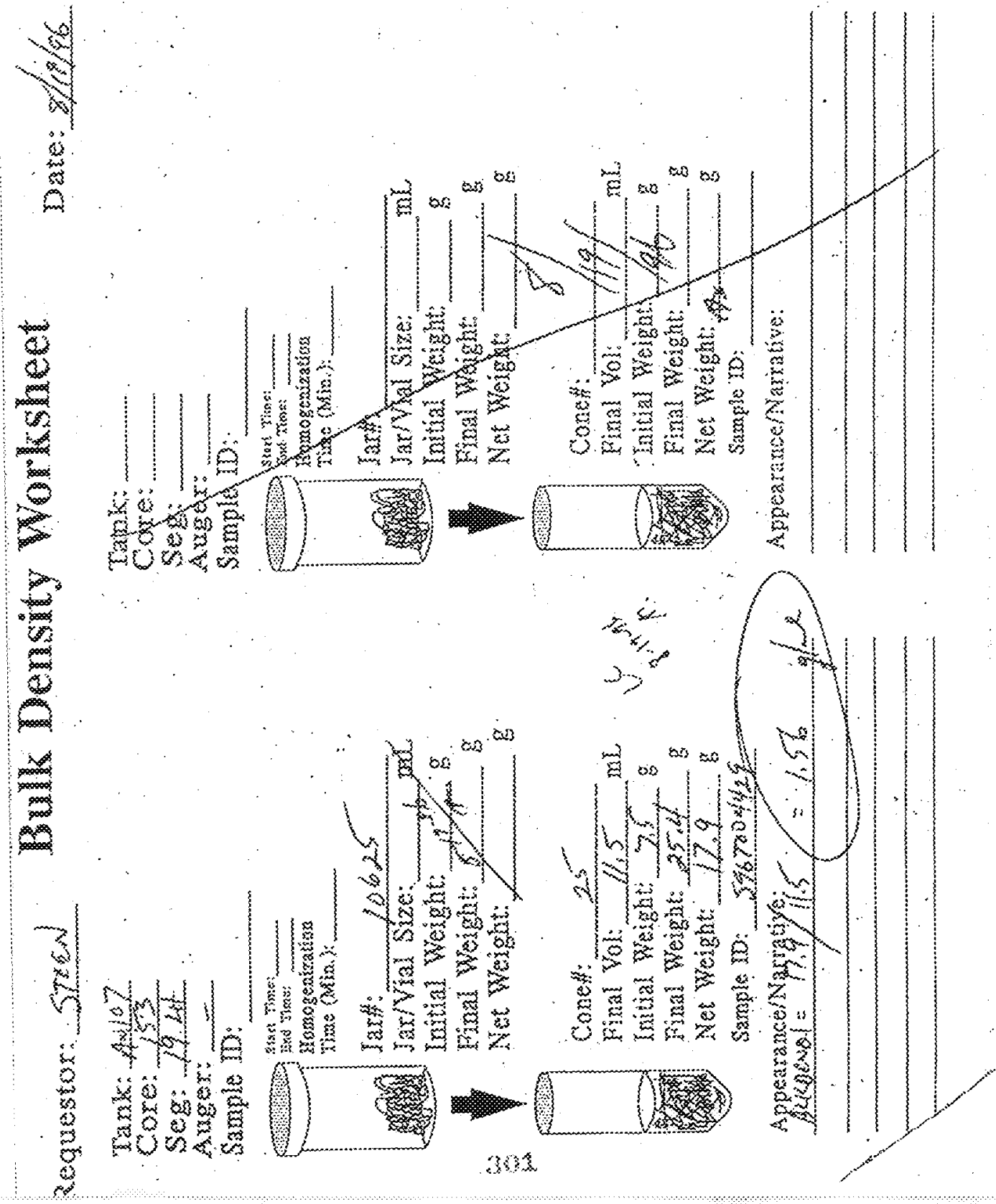




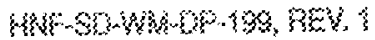
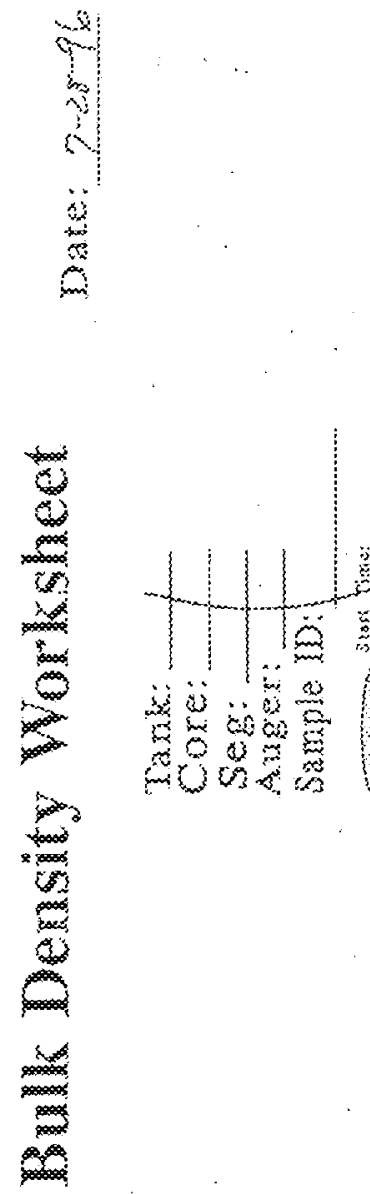

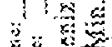

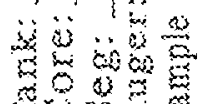

in 030

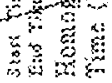
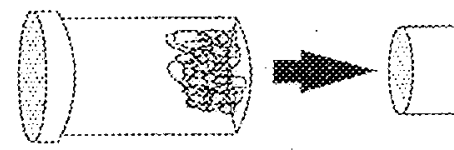

on

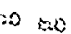

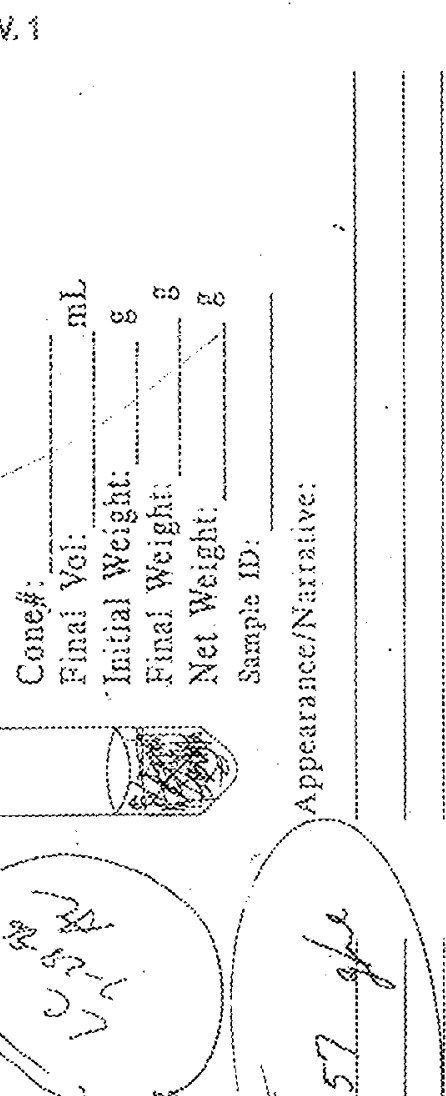

T.

1... os an an

$(m \infty) \cdots$

की

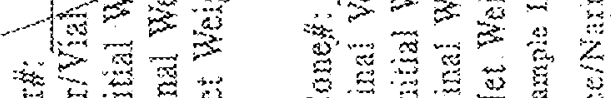

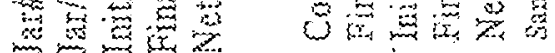
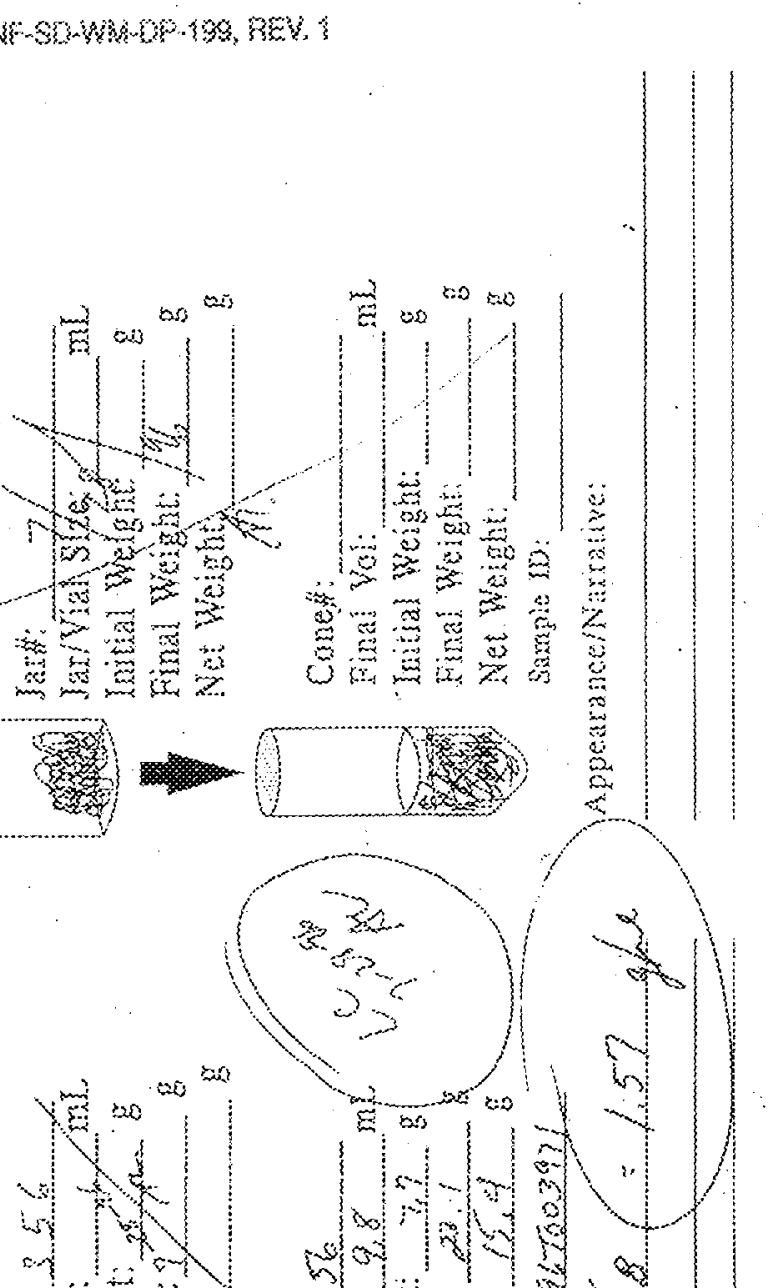

(4)
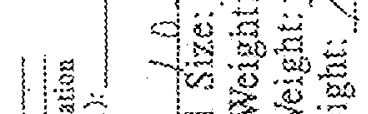

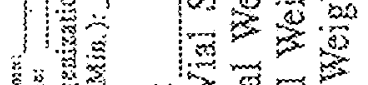

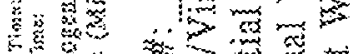

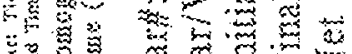

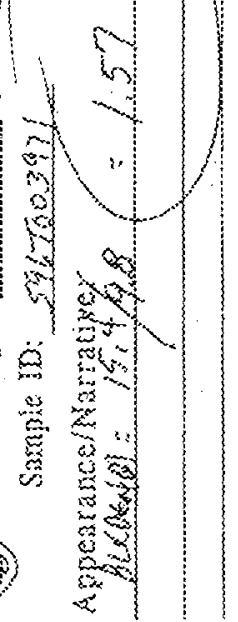

2
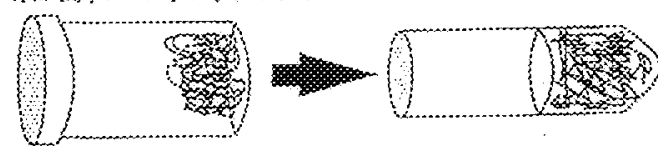


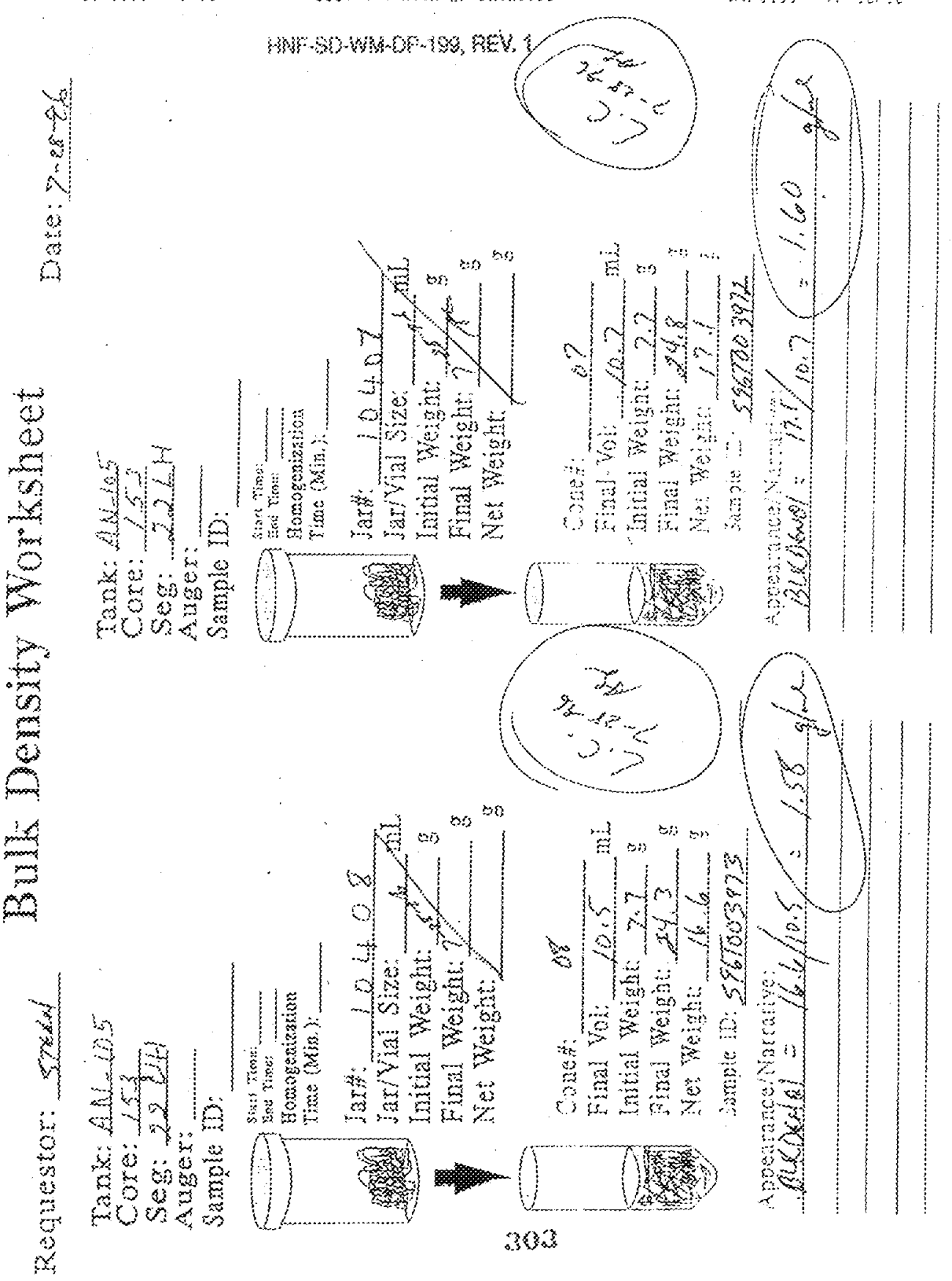




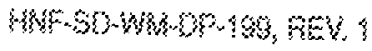

WHE - SO - HW

JHMRARYE XKRLYSES 


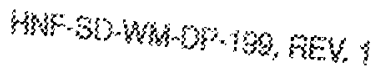

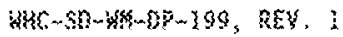

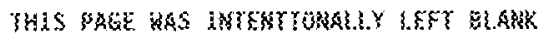




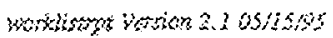

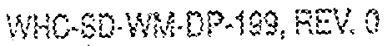

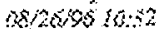

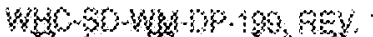

Pegs: :

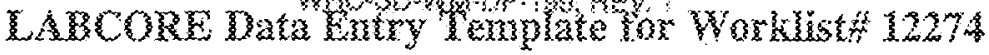

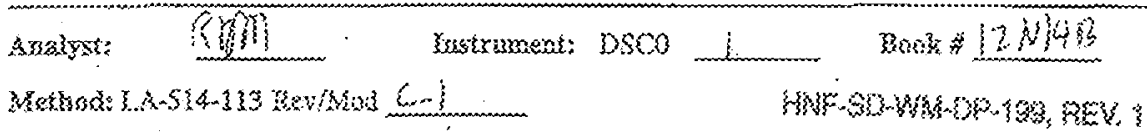

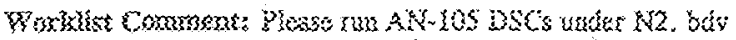

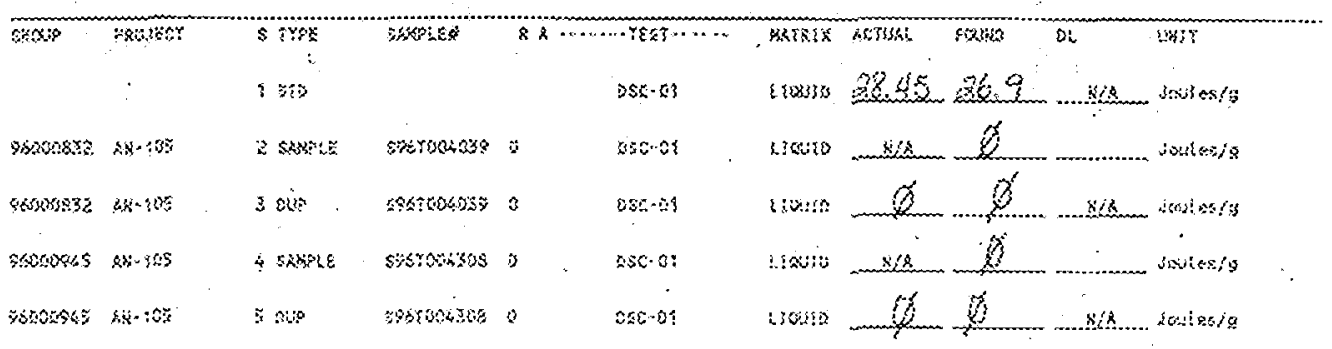

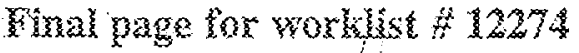
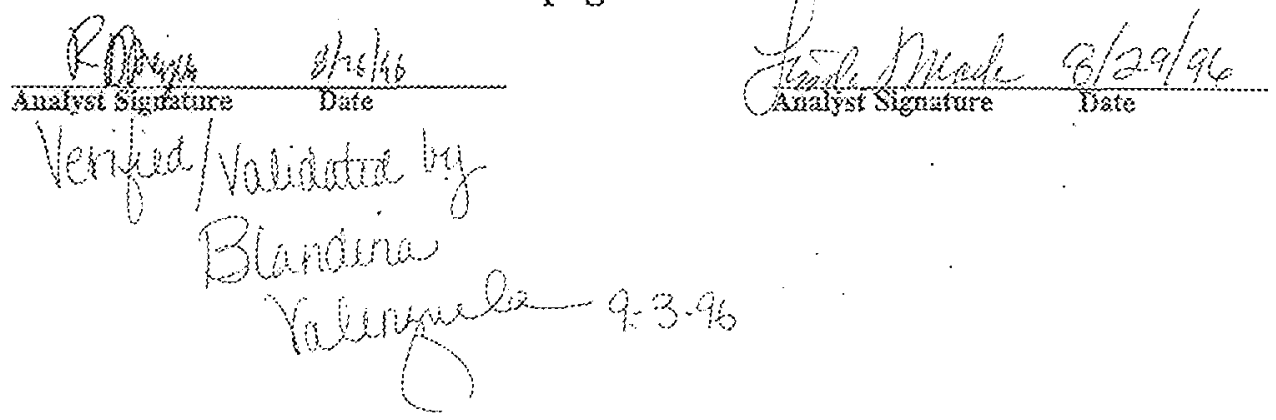

Daxi Exs? Comenes:

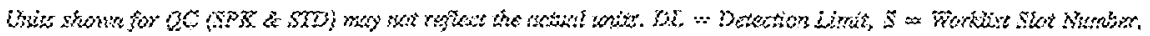

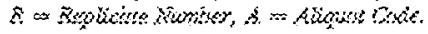

396 


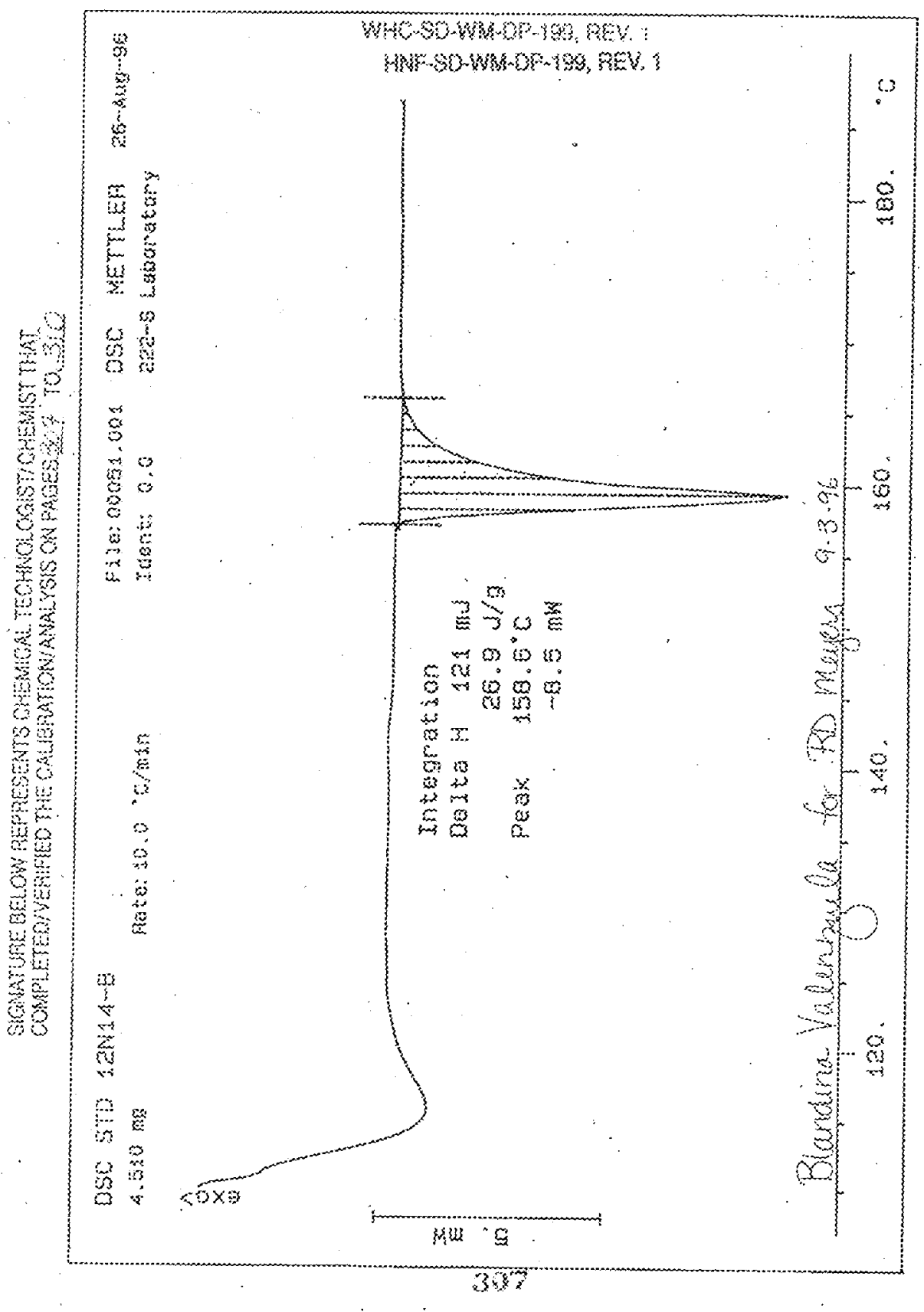




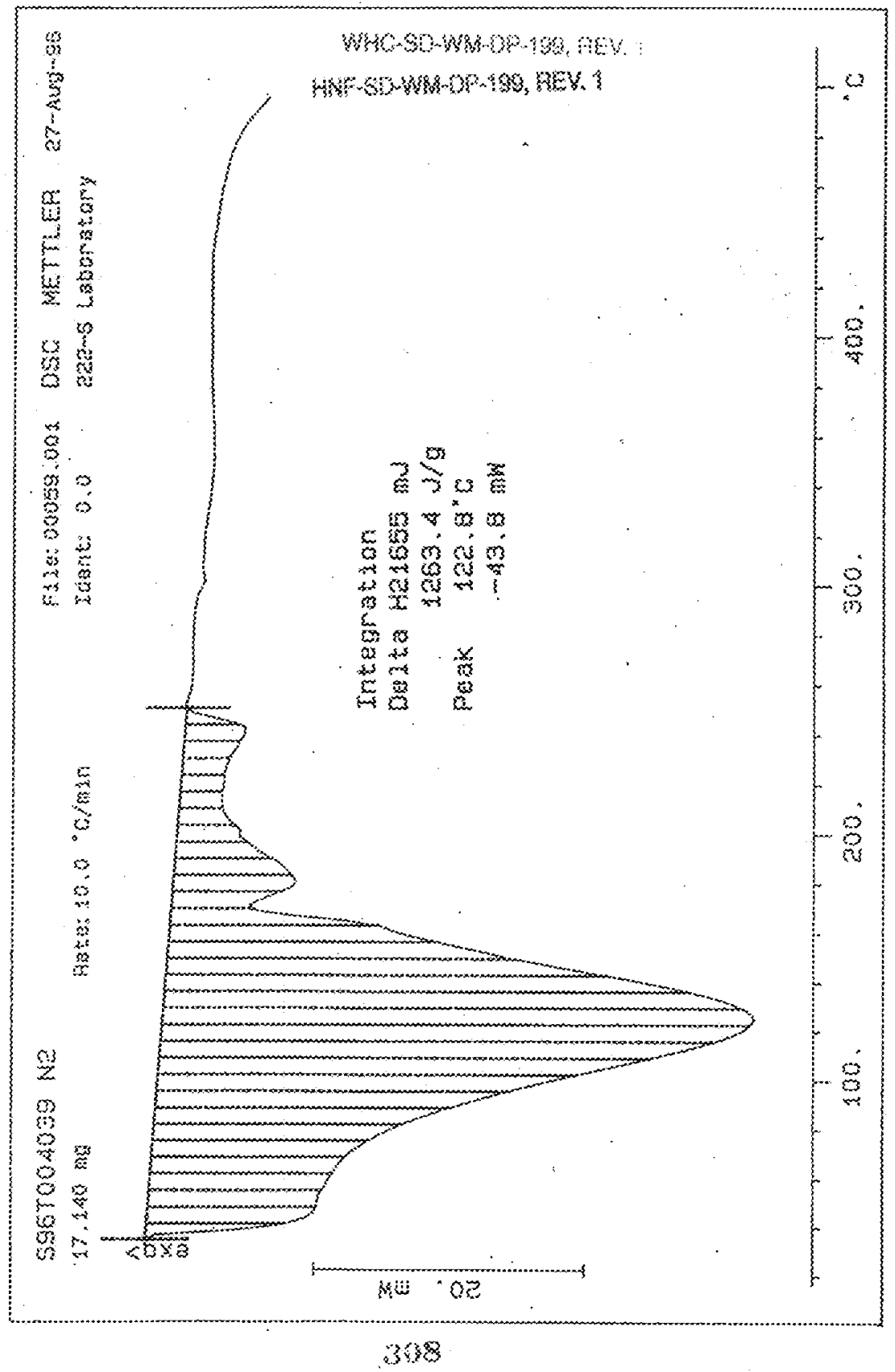




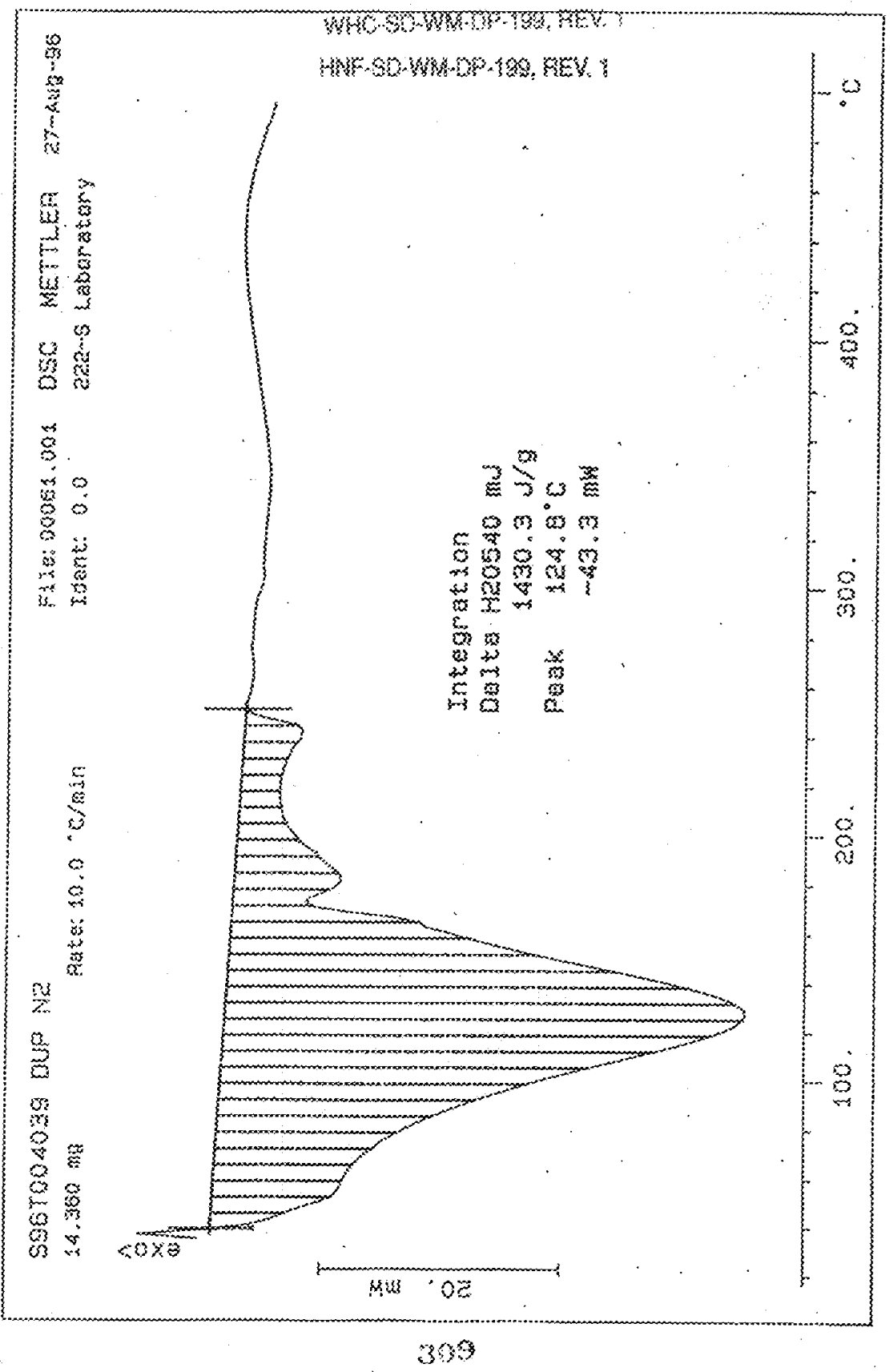




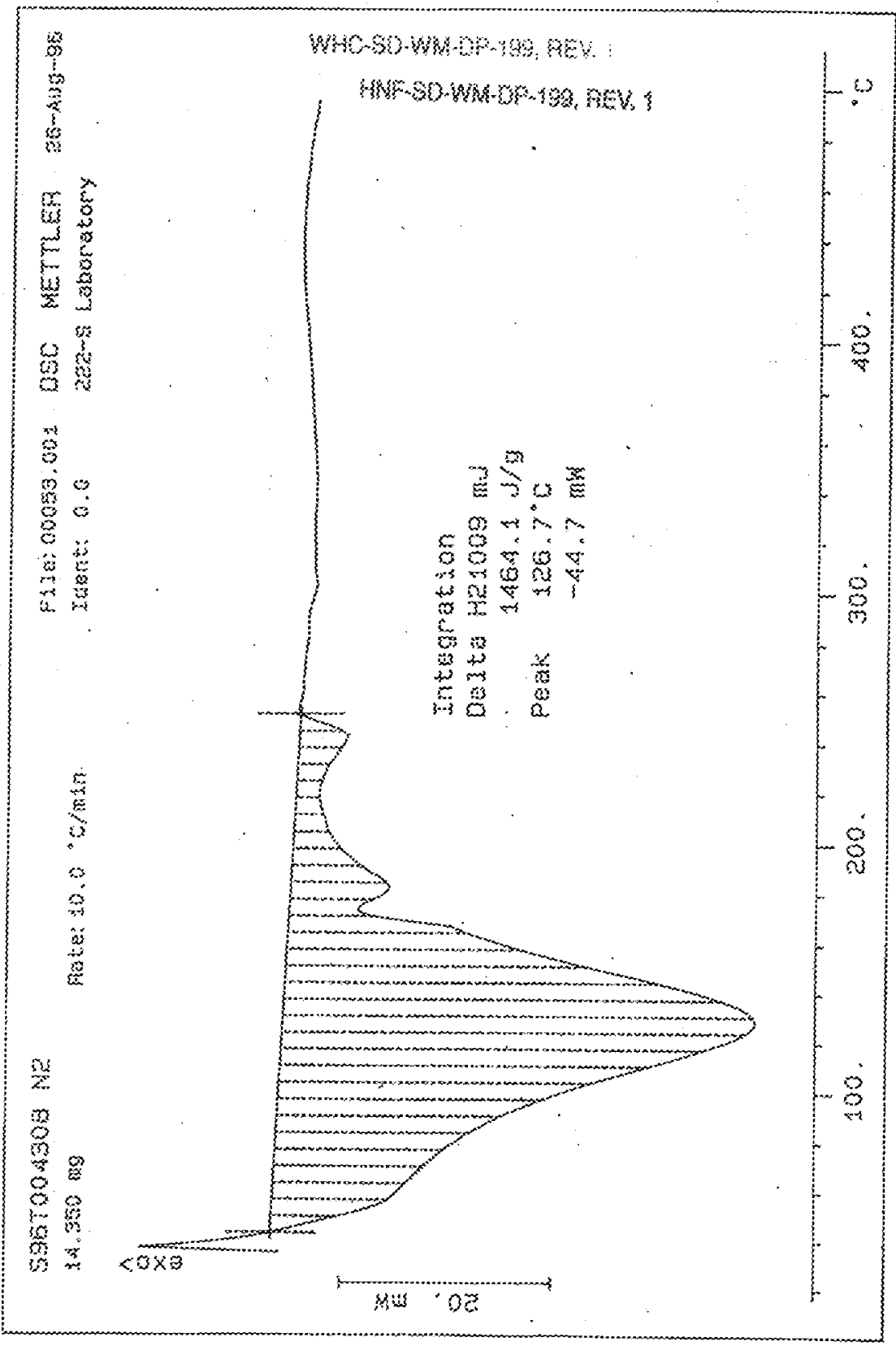




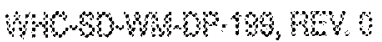

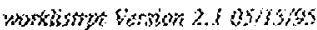

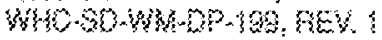

ô,

1.

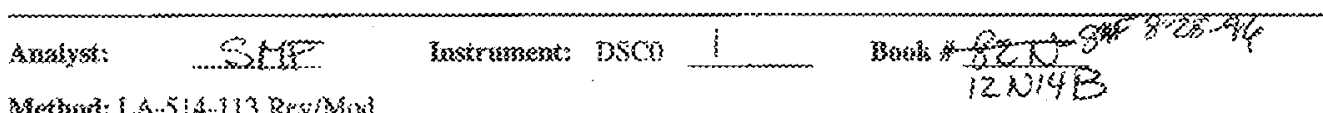

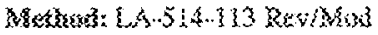

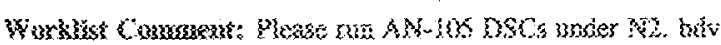

और:-5\%

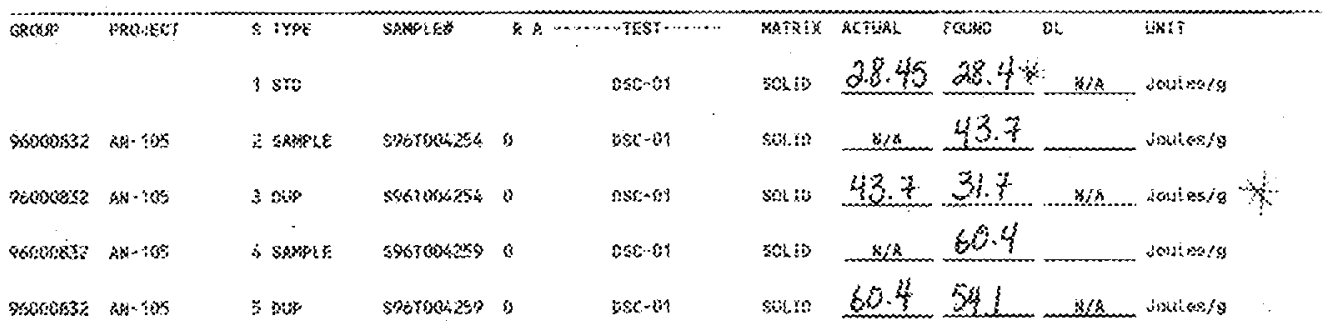

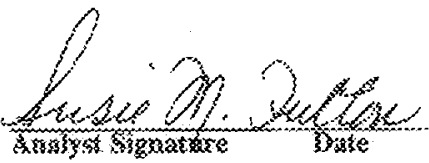

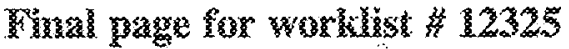

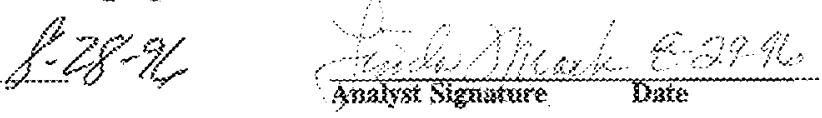

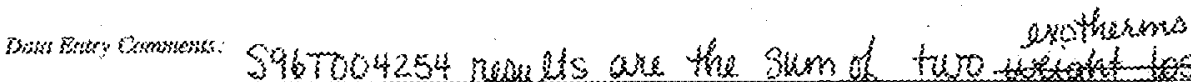

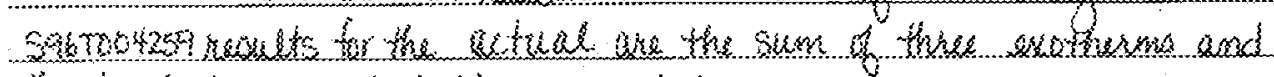

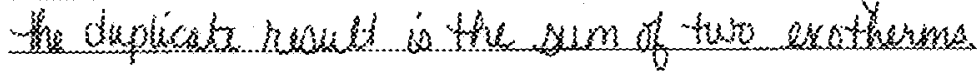

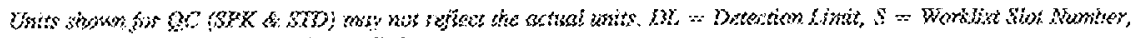

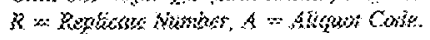

3.3 .3 


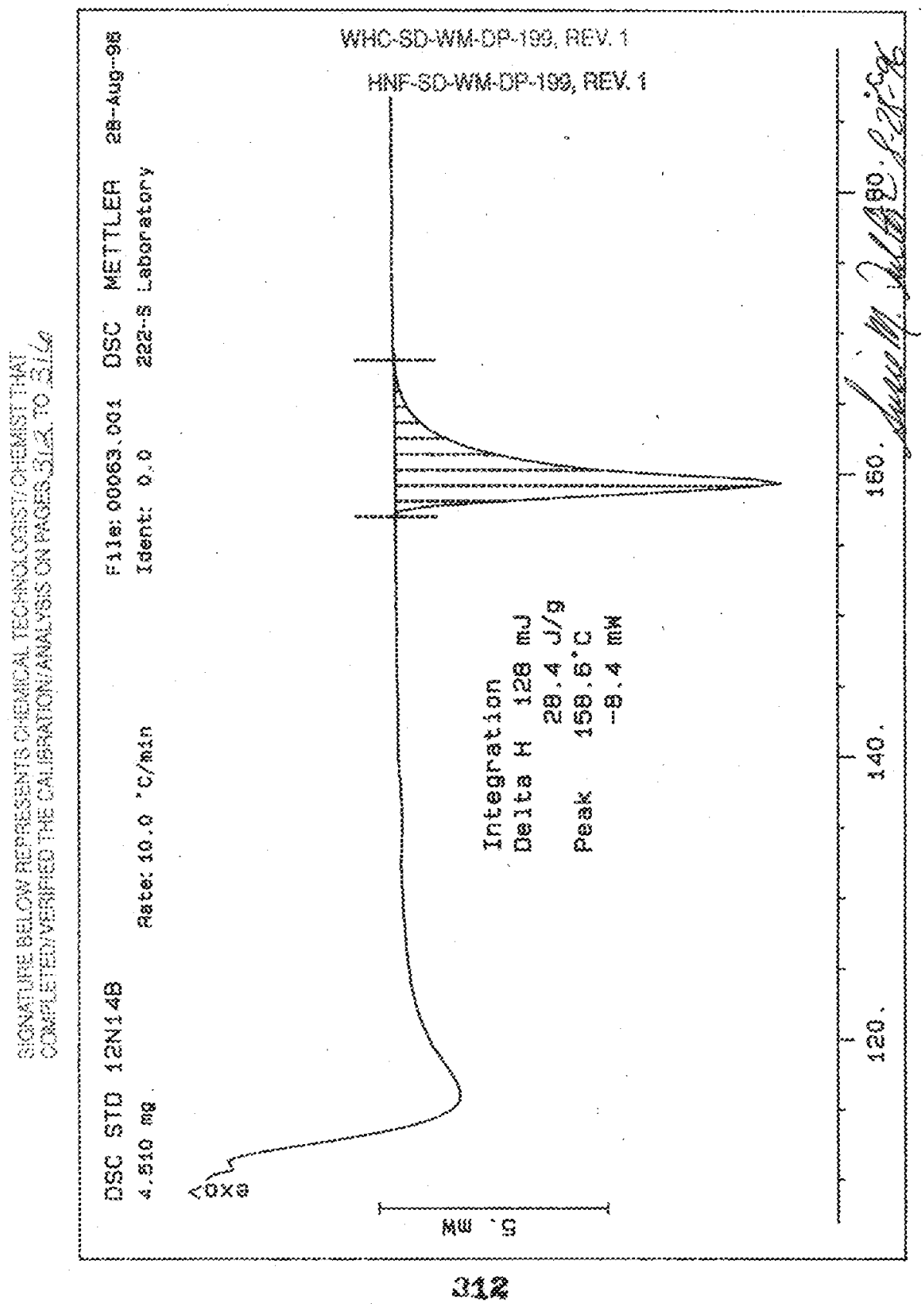




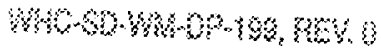

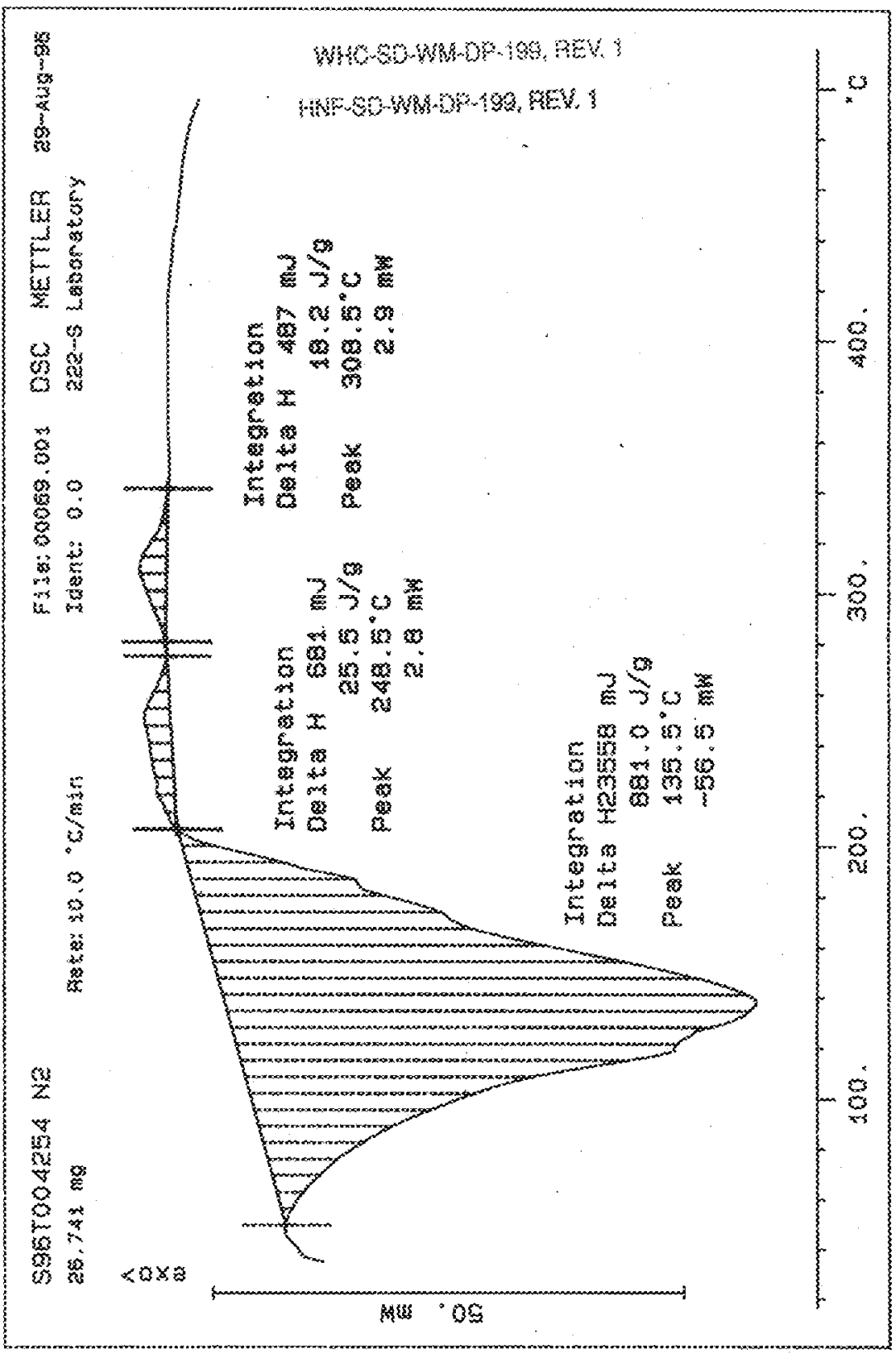




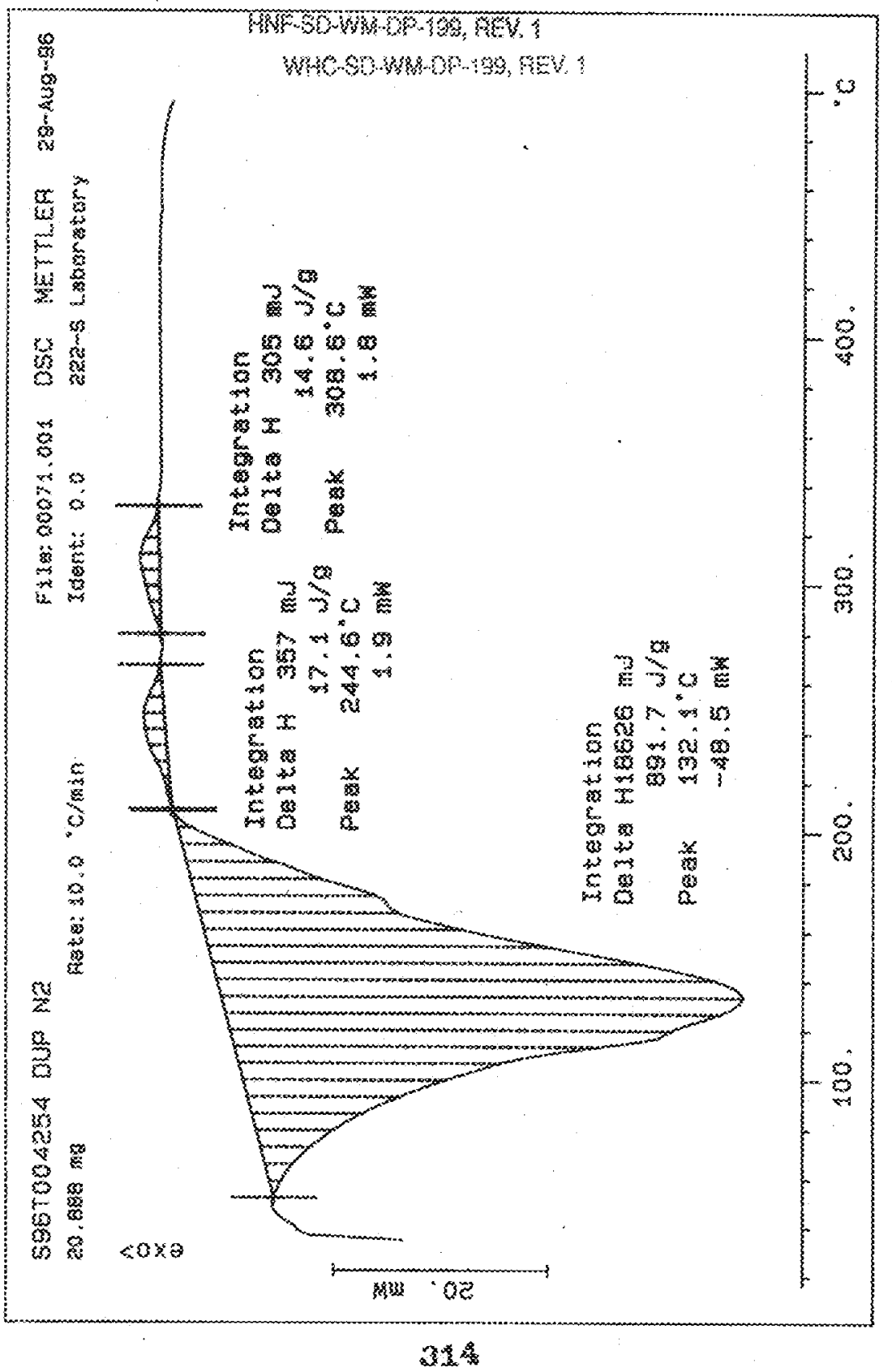




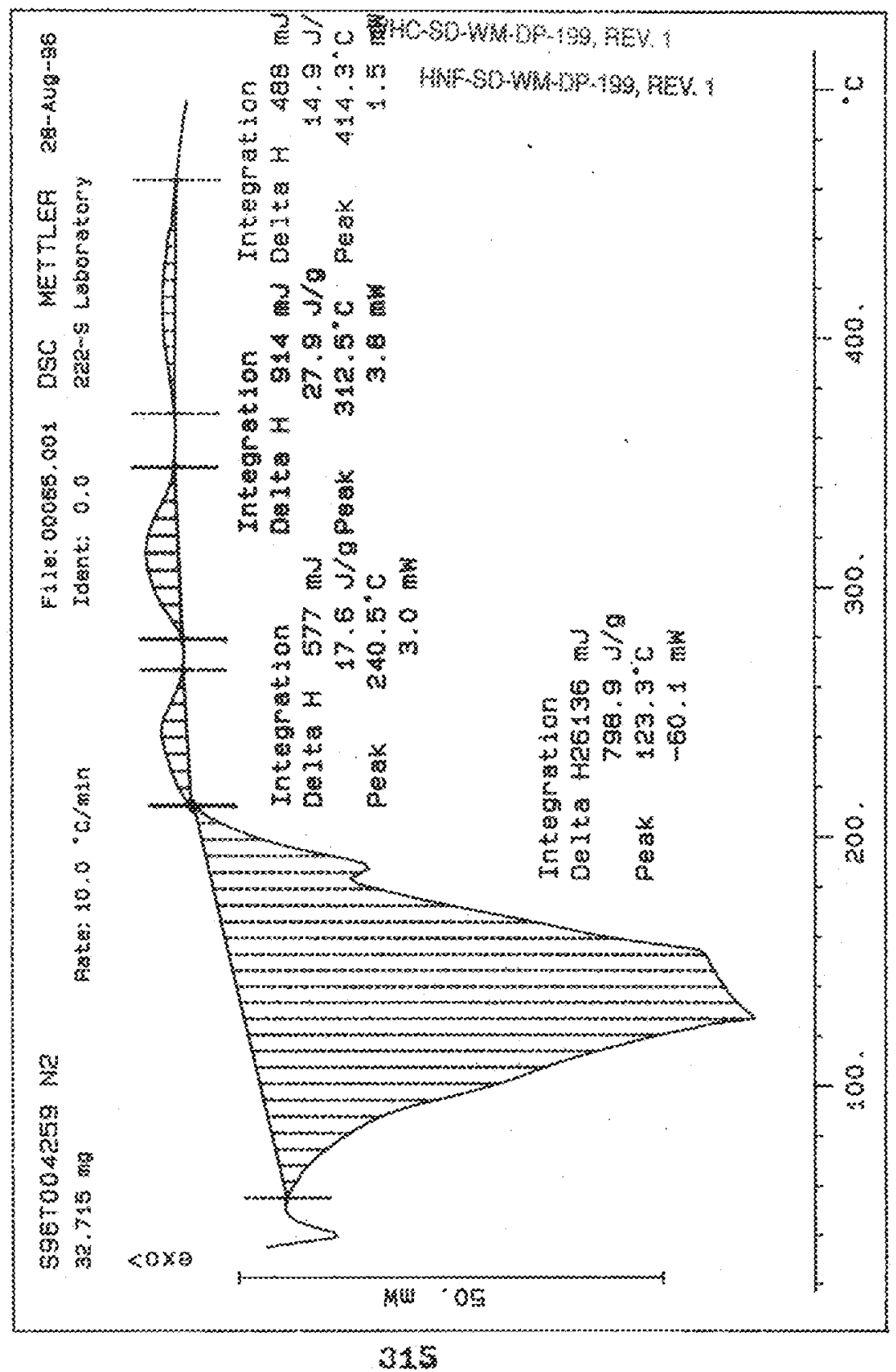




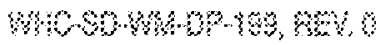

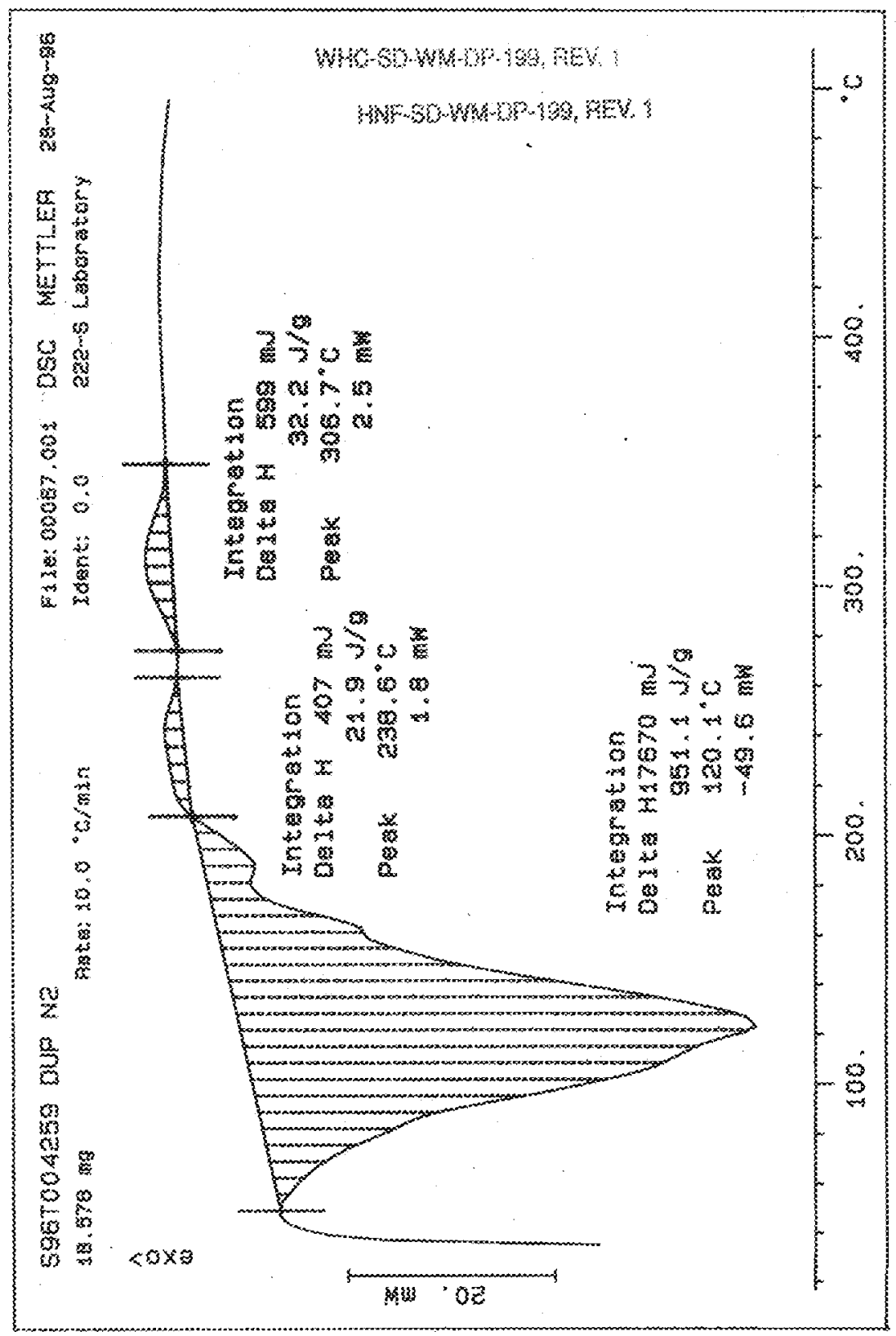




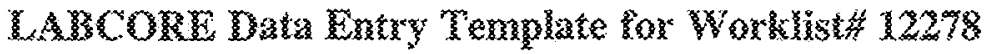

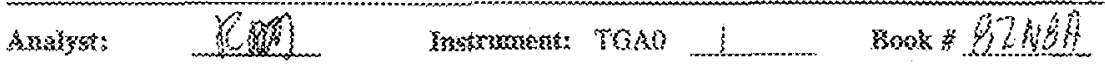

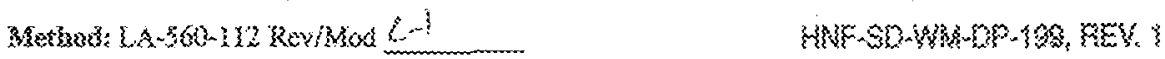

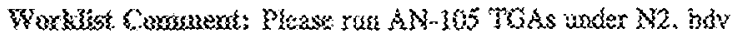

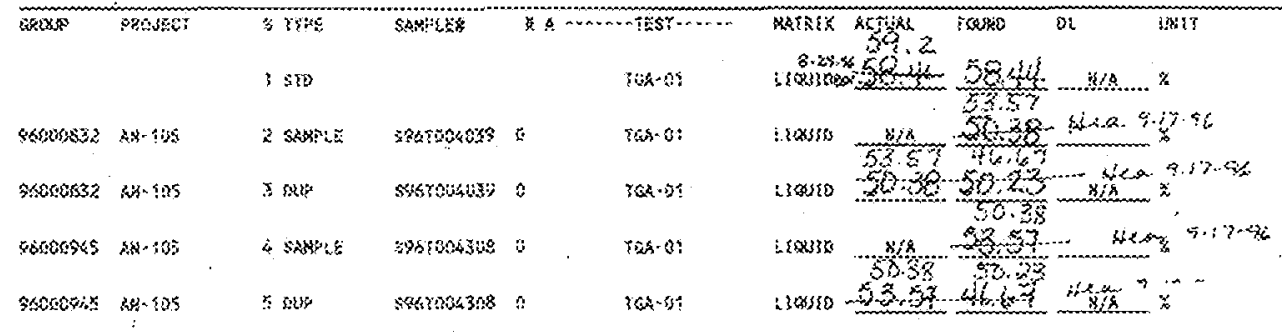

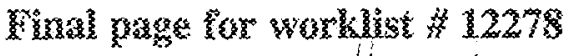
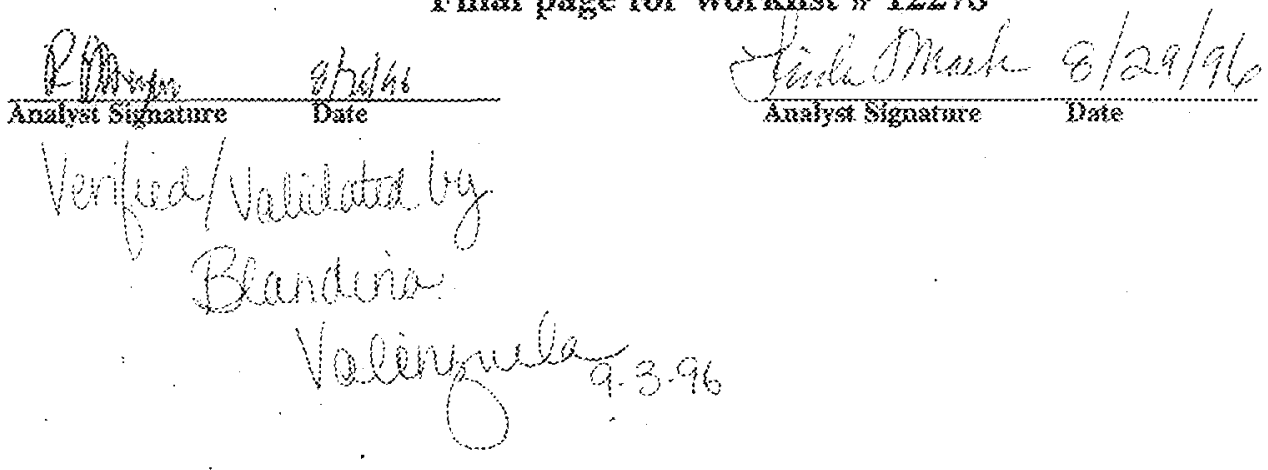

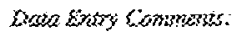

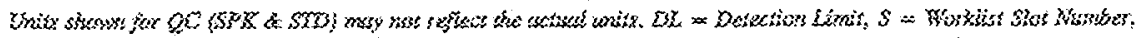

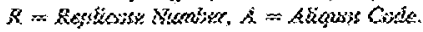




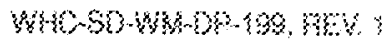

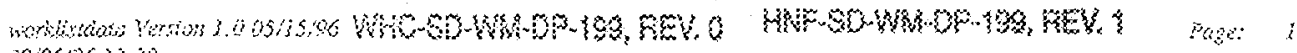
$0,06, \$ 6,3,30$

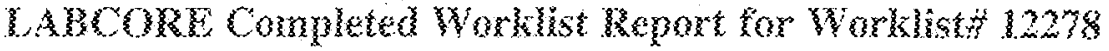

Analyst: rim

Yewnment: TSAOSI

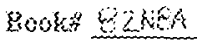

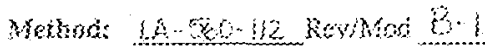

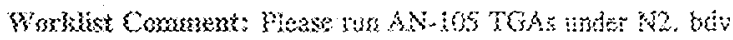

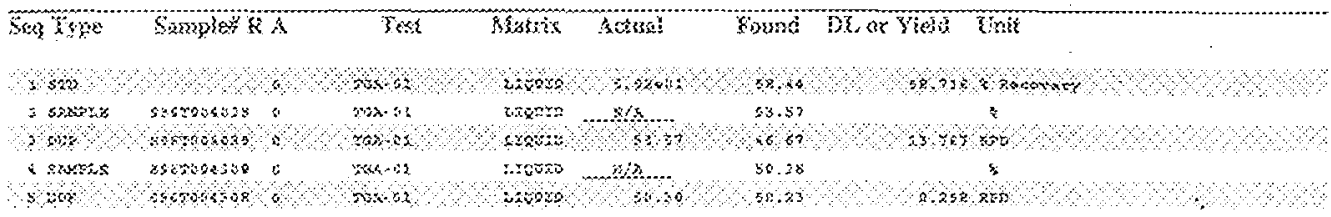

\section{Final wage for worklist 12278}

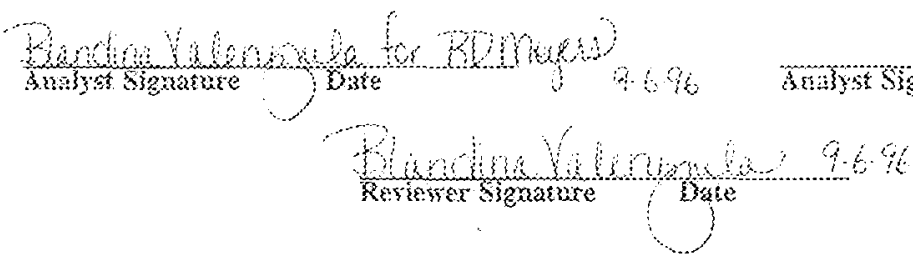




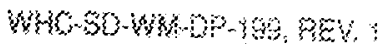

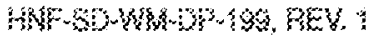

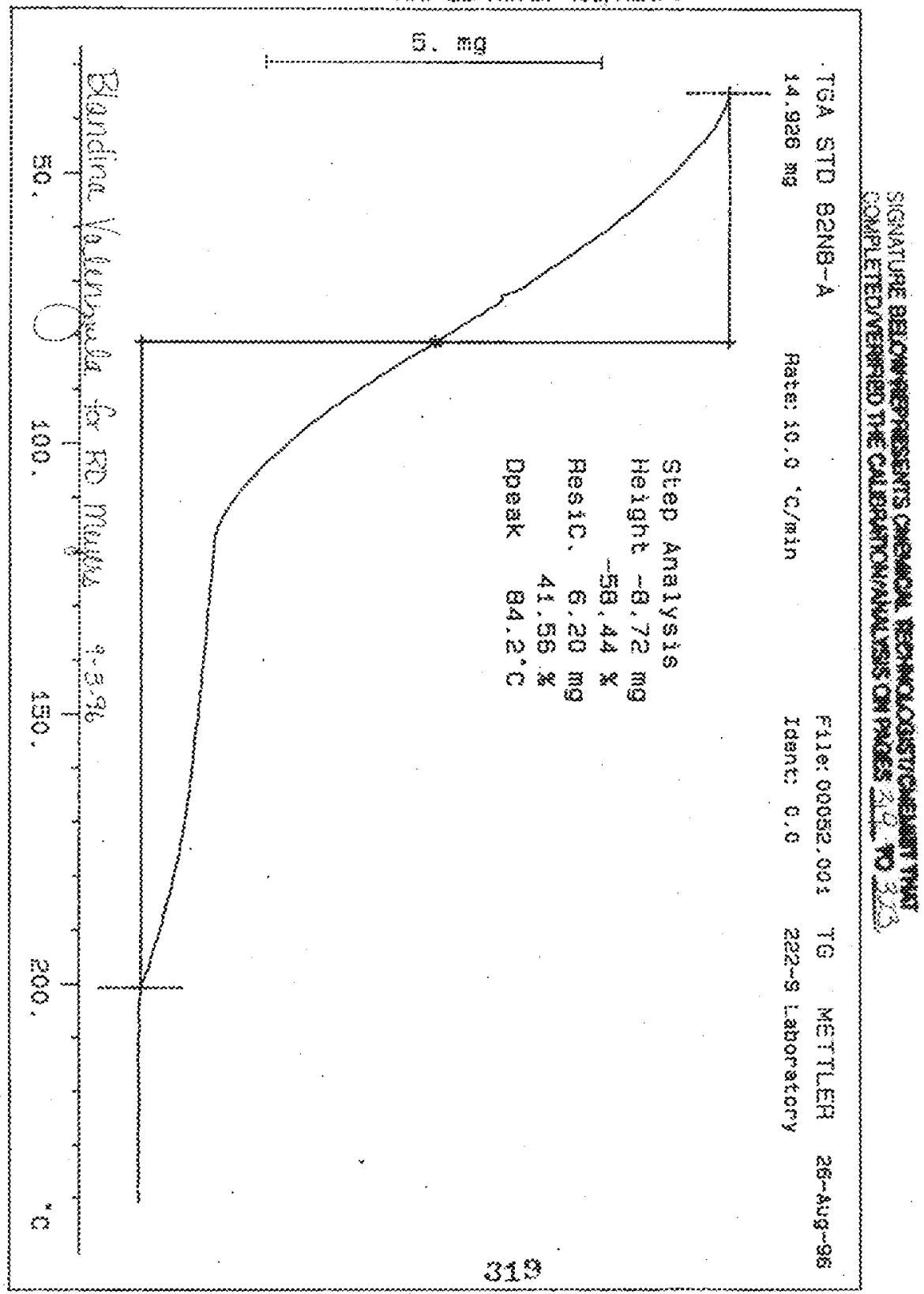




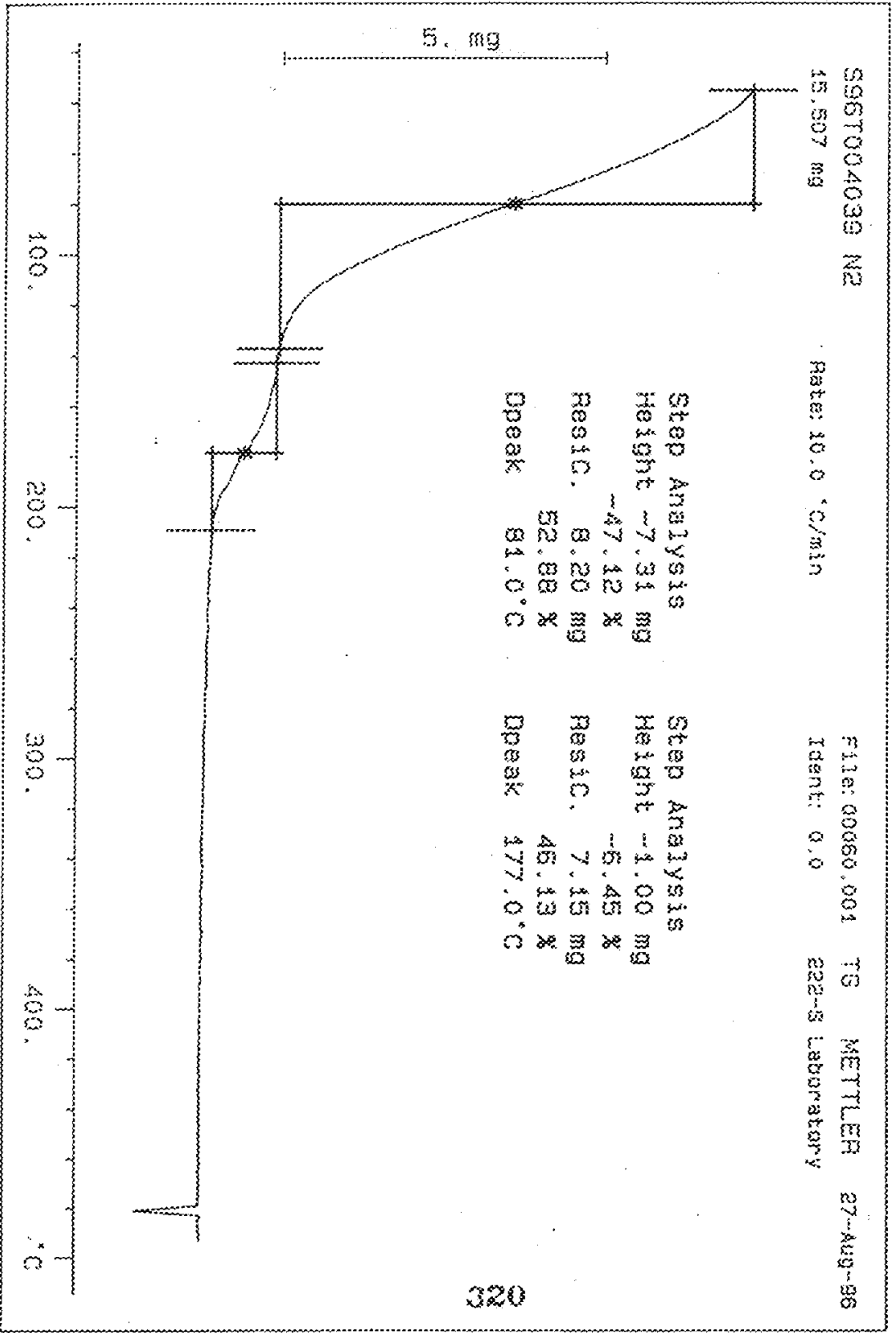




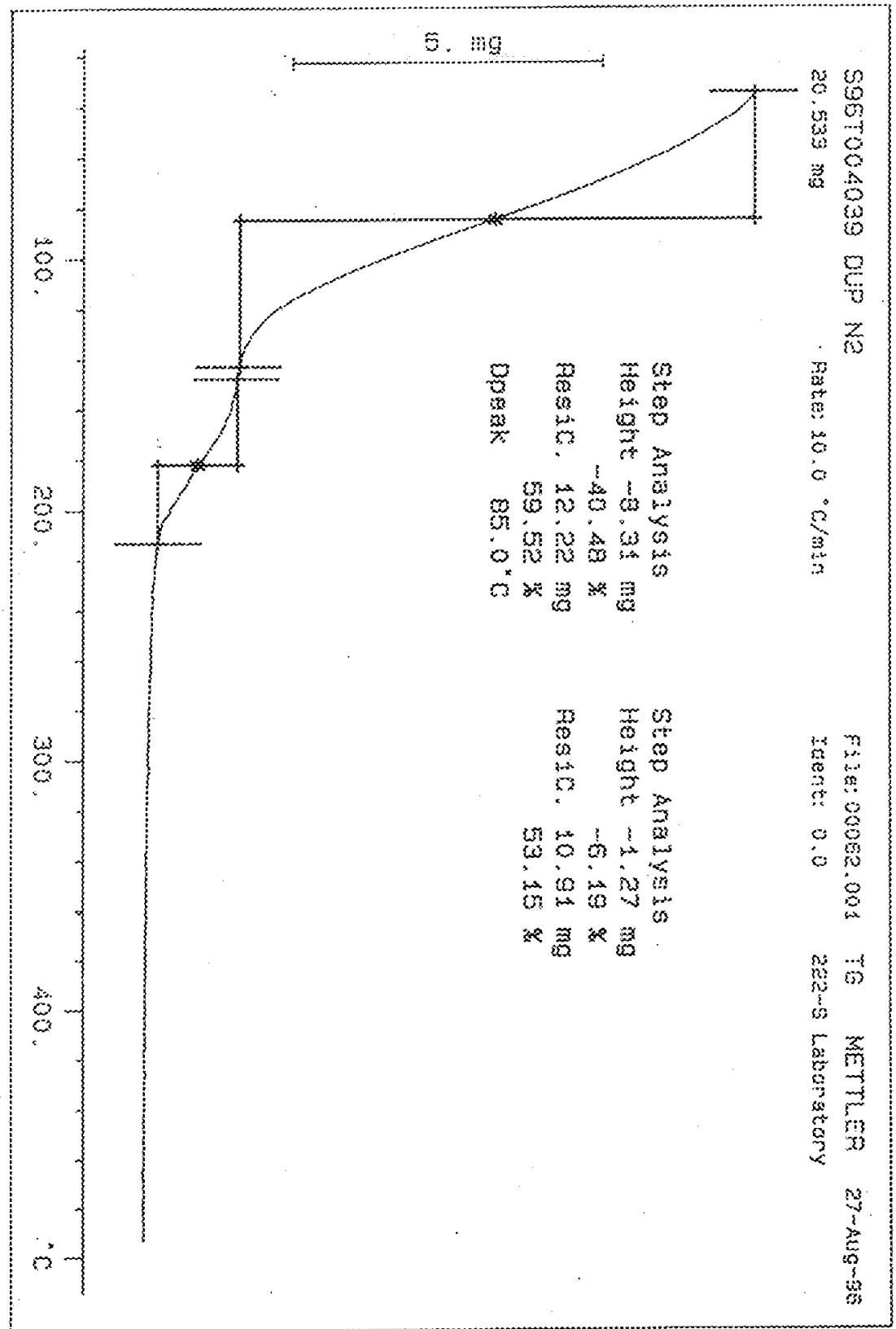




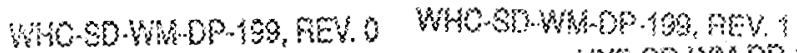

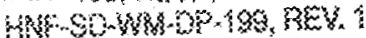

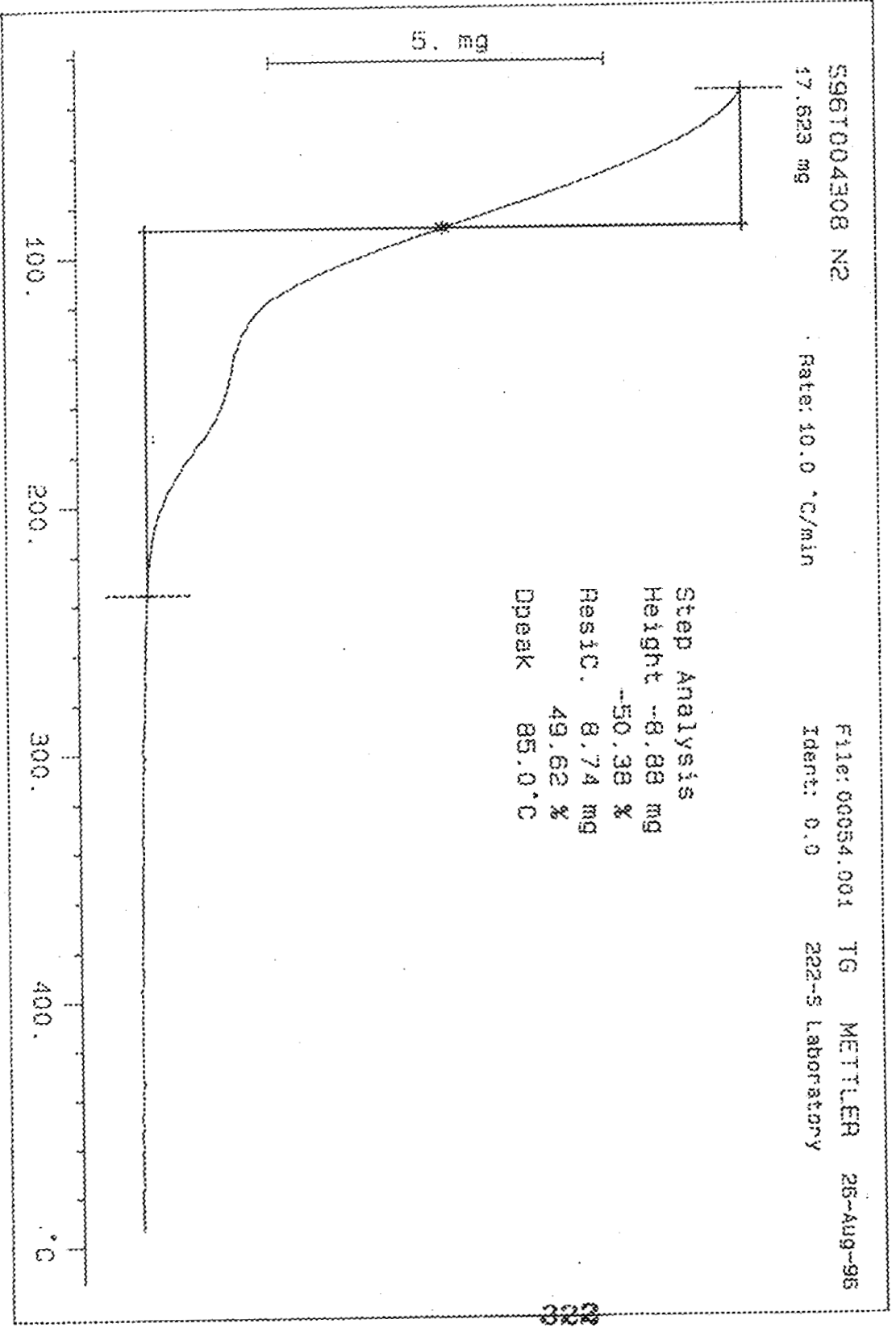




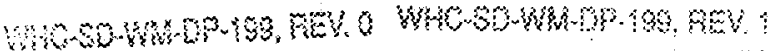

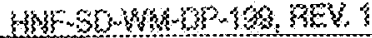

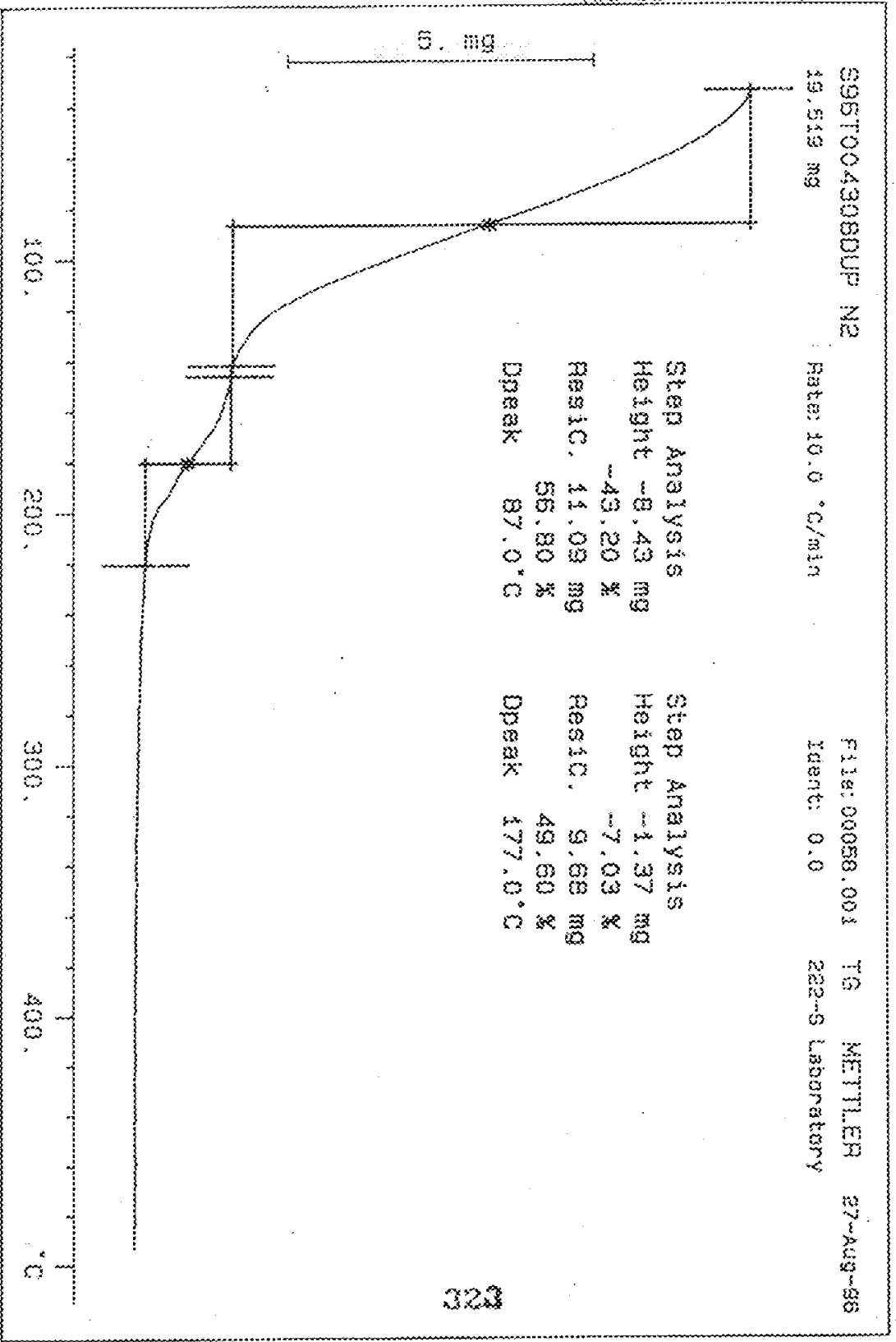




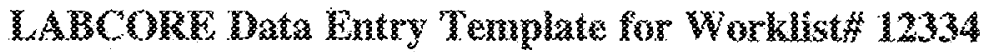

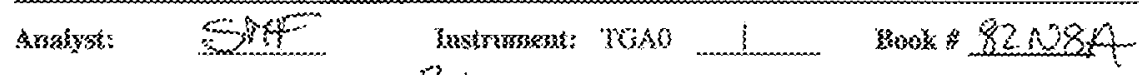

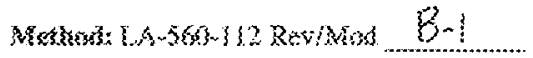

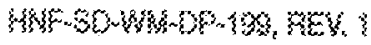

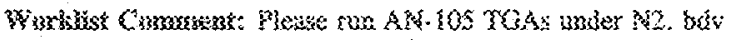

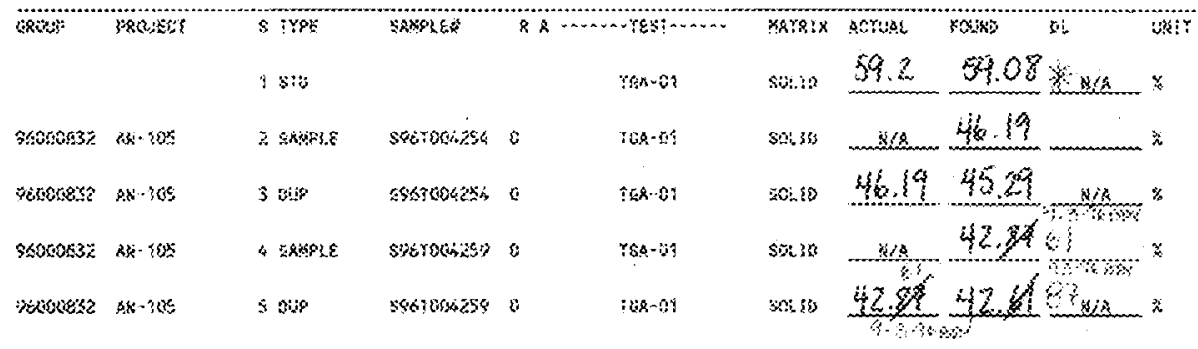

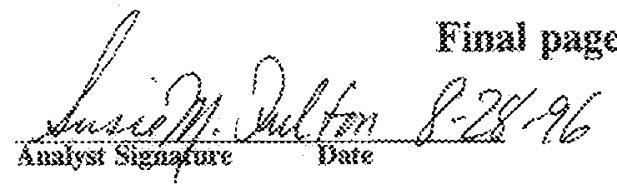

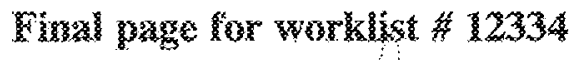

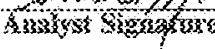

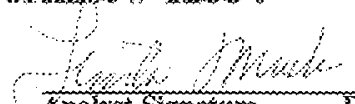

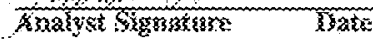

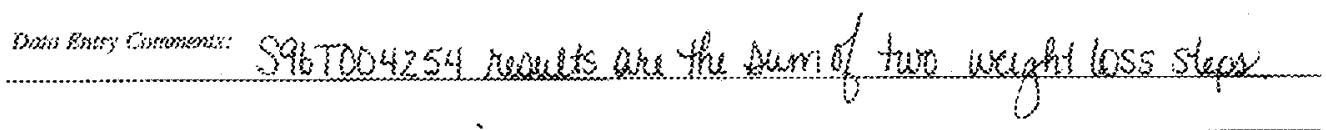

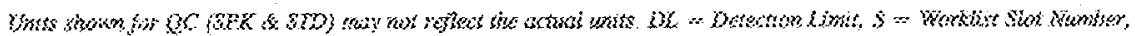

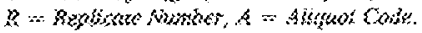




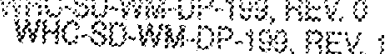

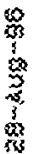

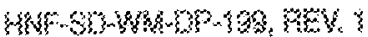

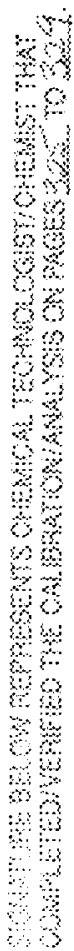

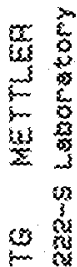

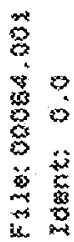

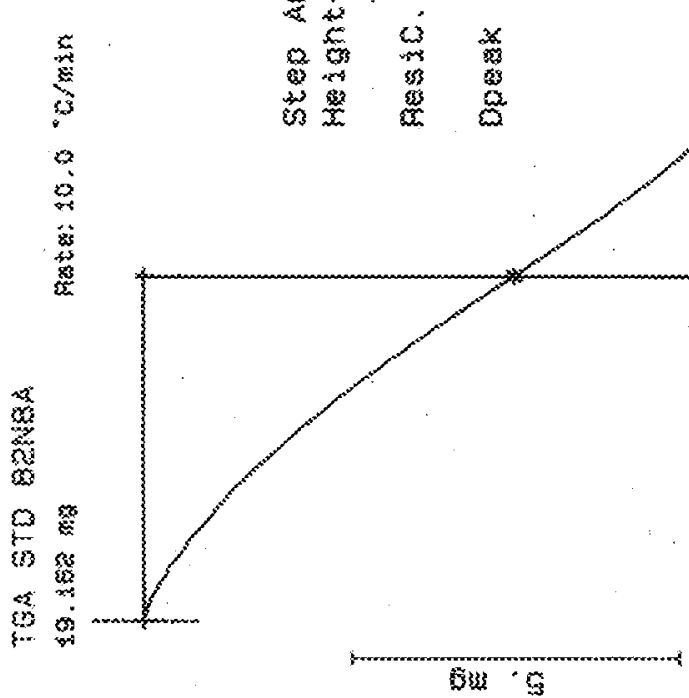

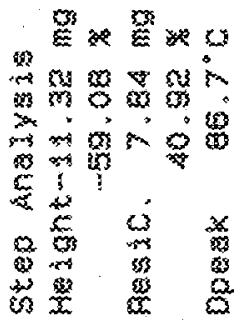




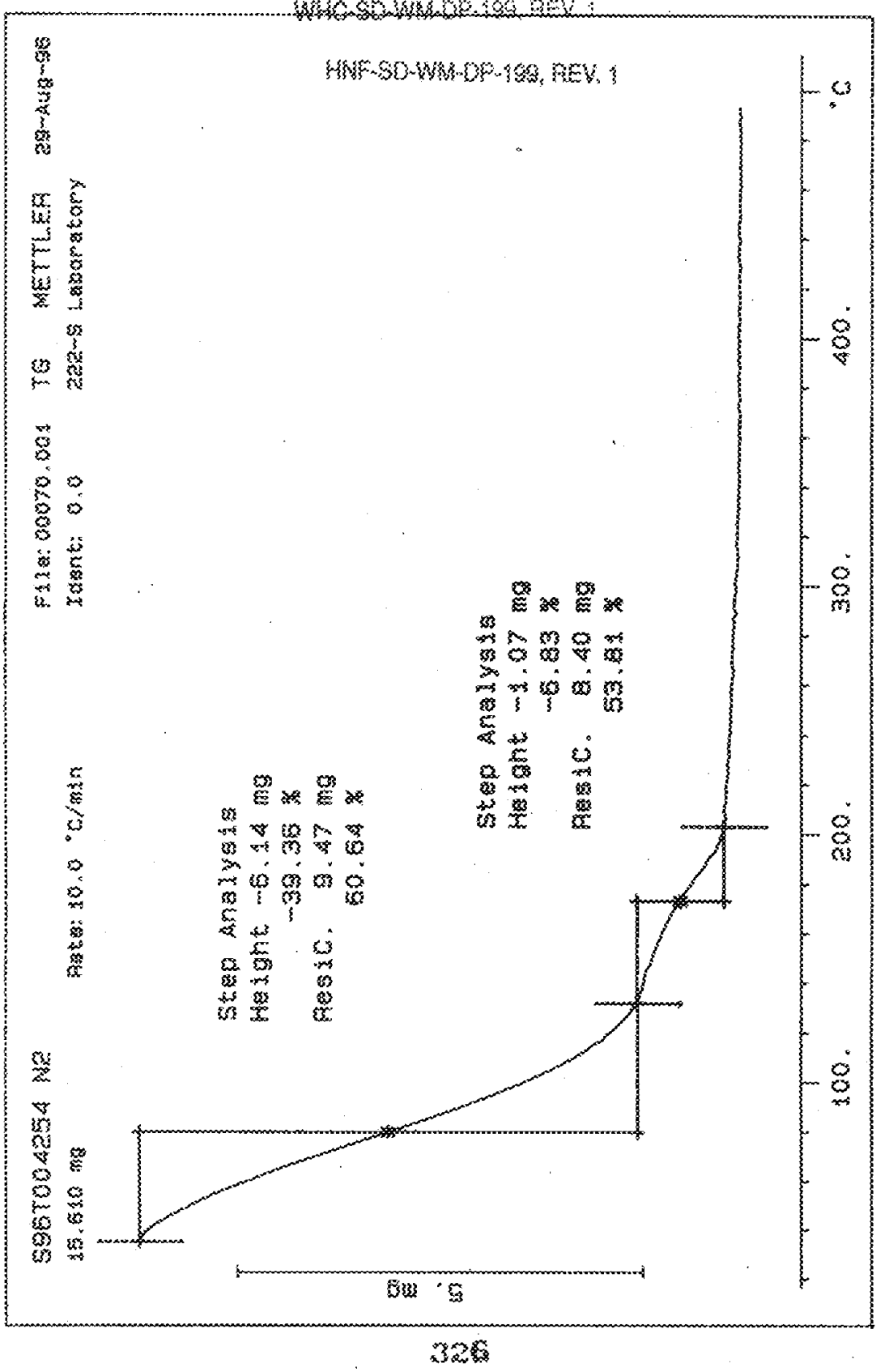




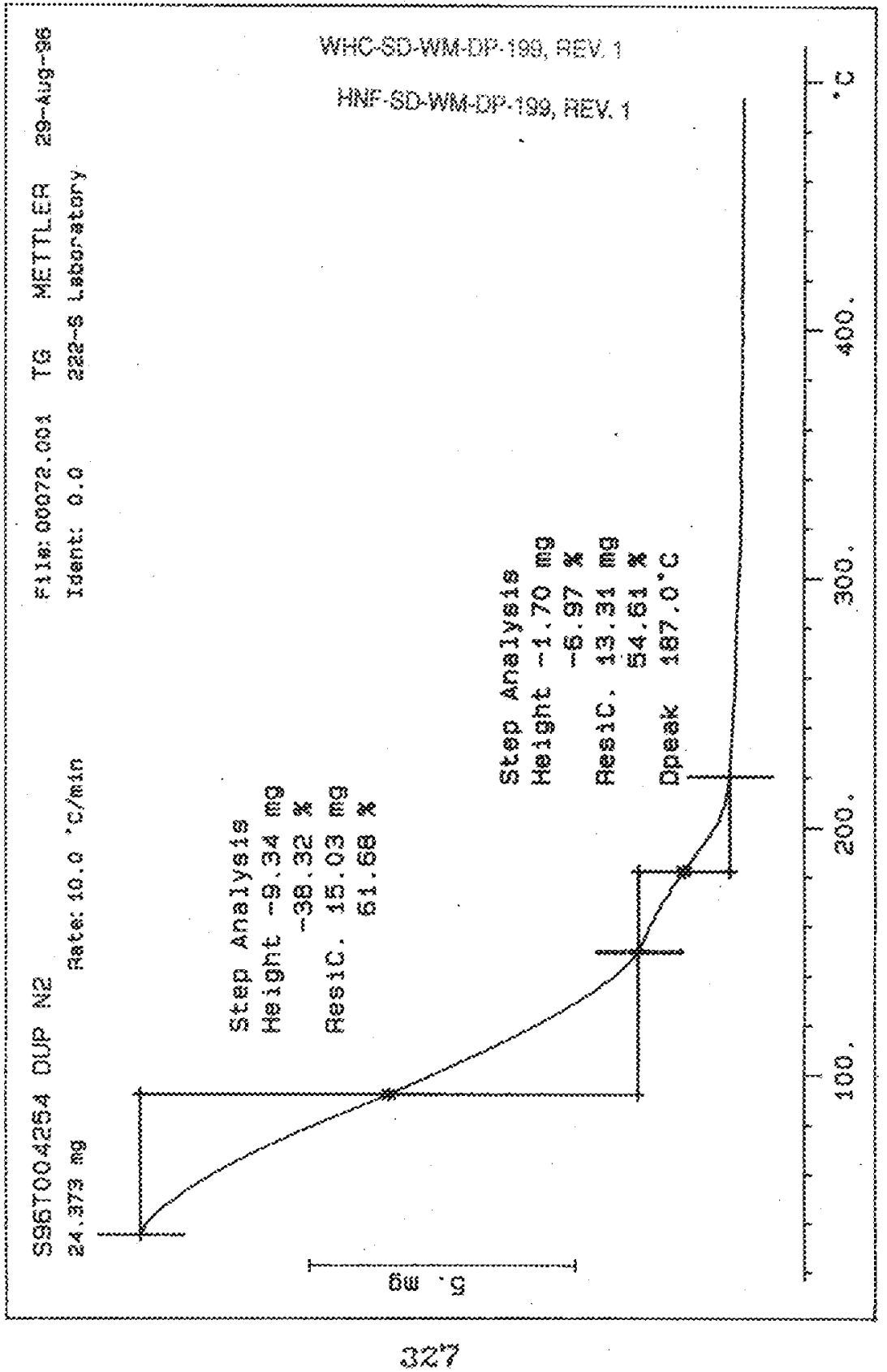




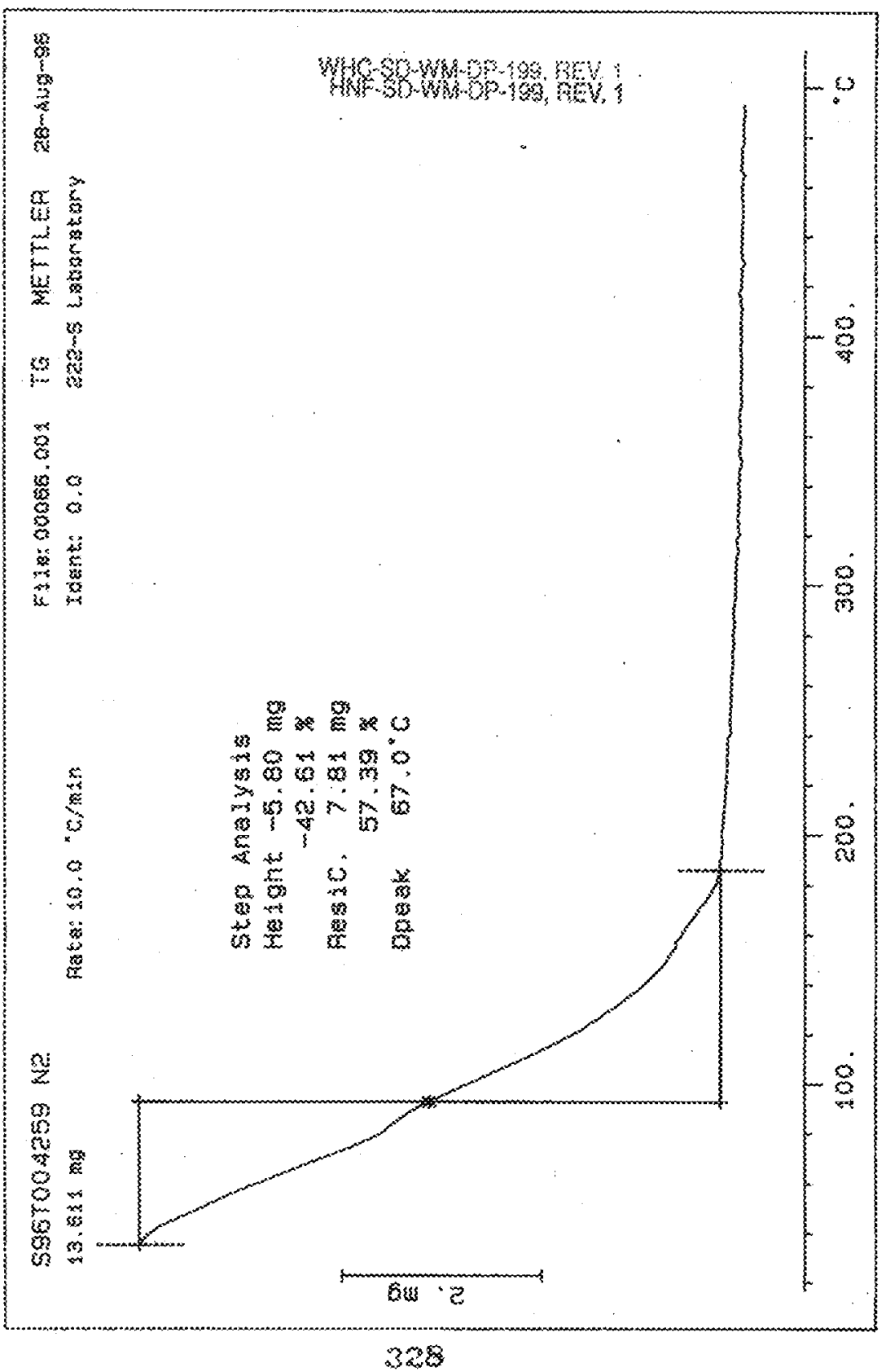




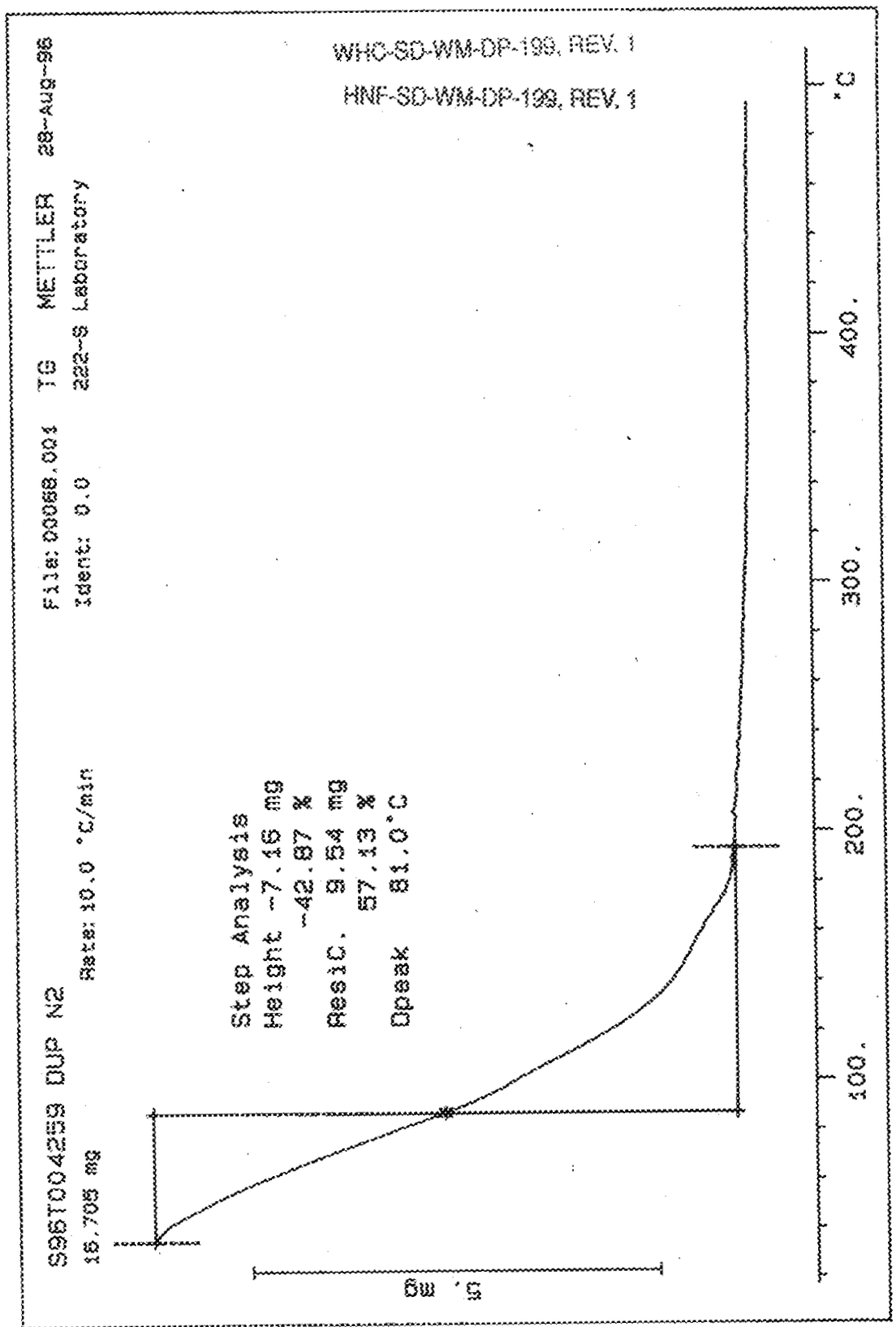




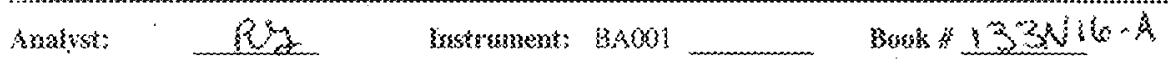

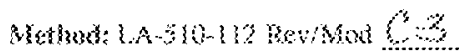

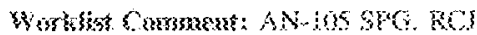

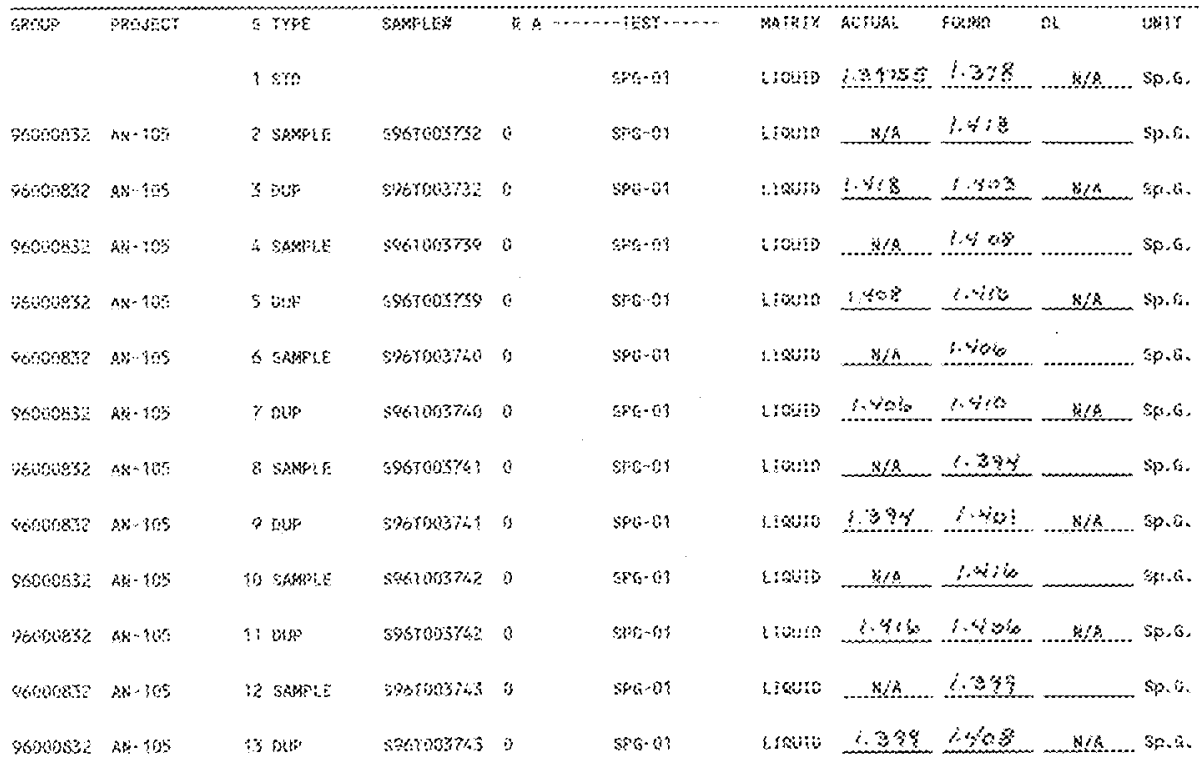

\section{What 11273}
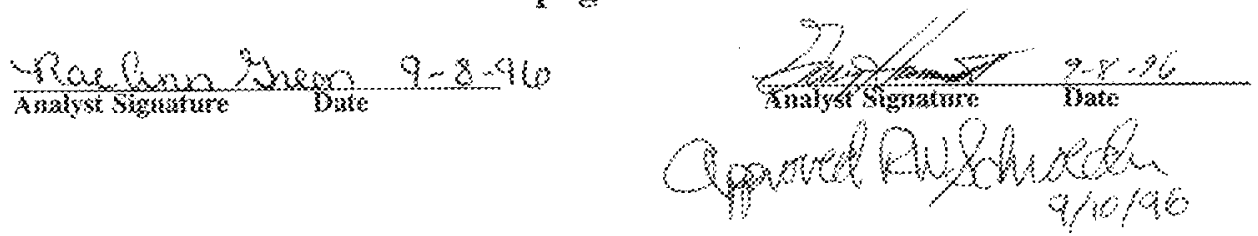

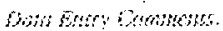

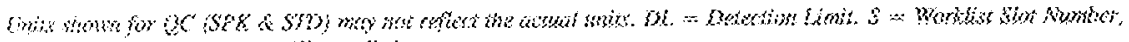

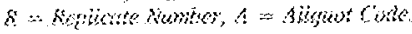




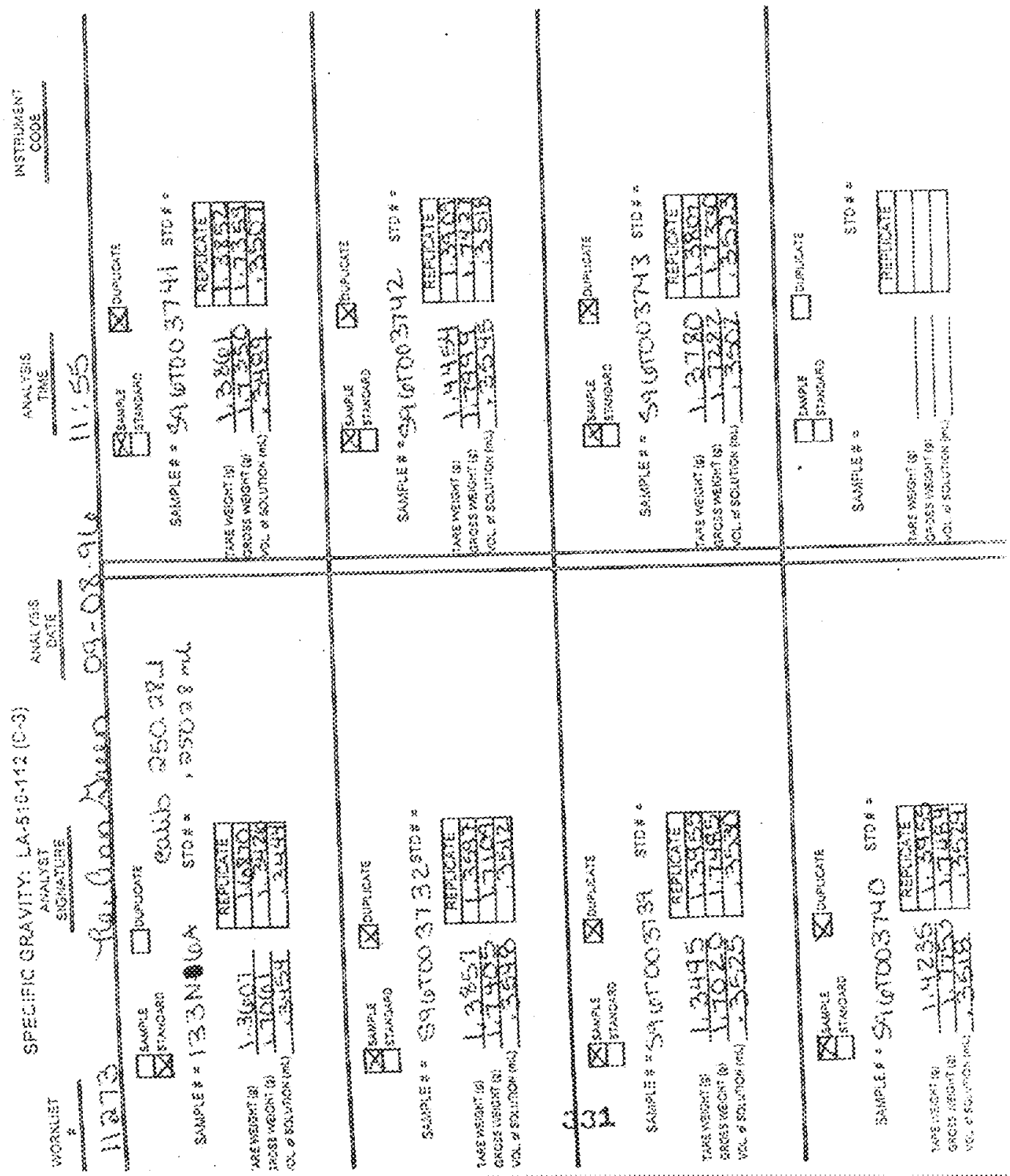




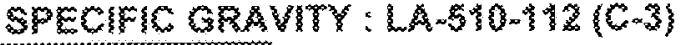

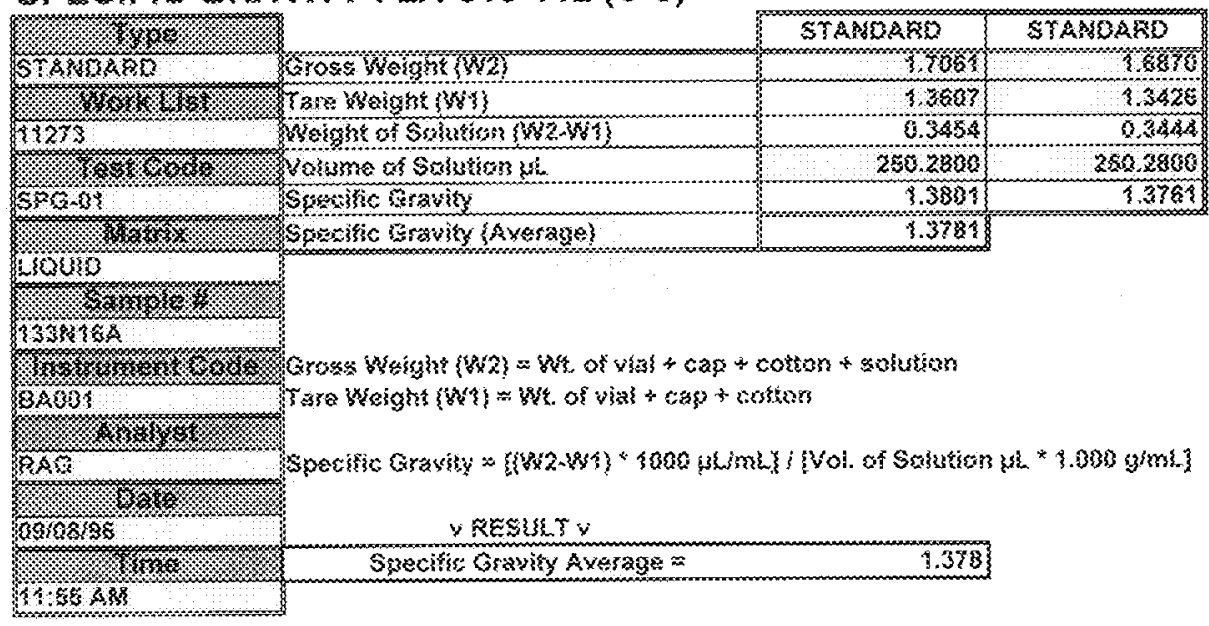

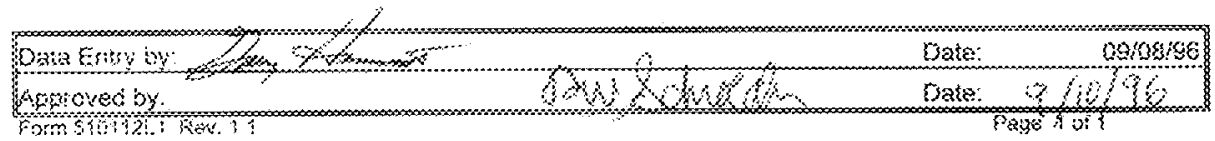




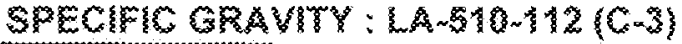

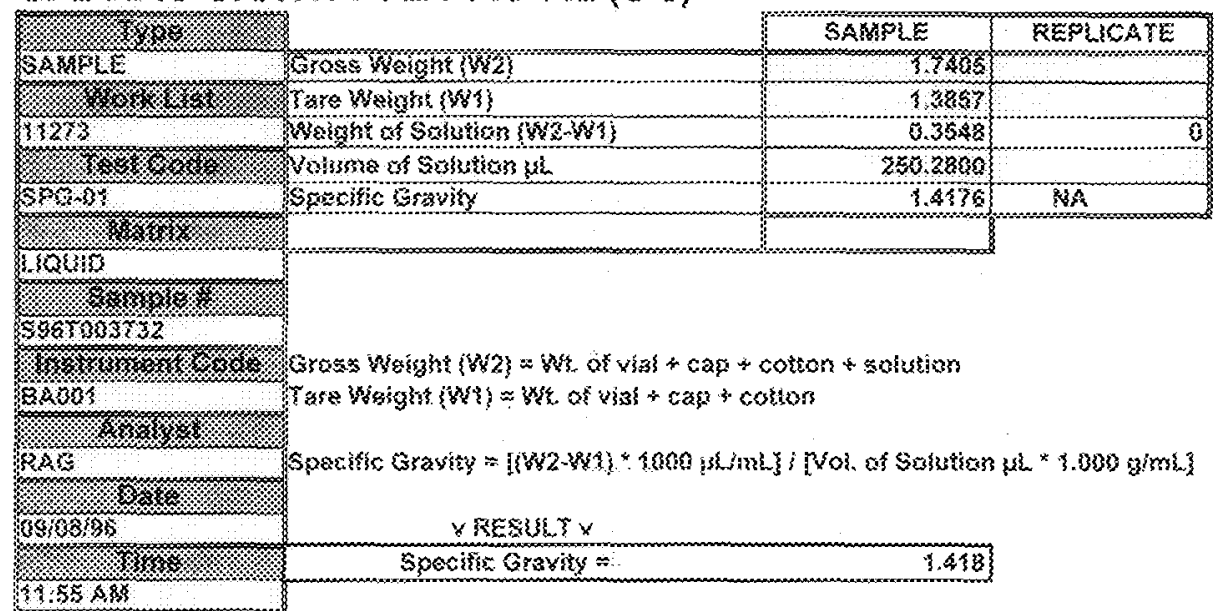

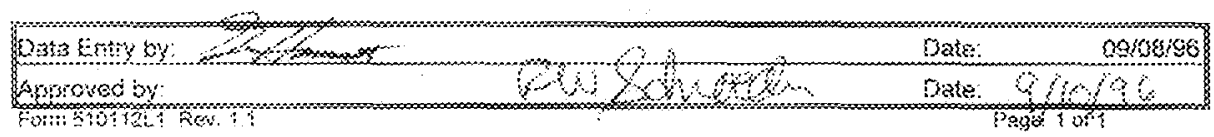




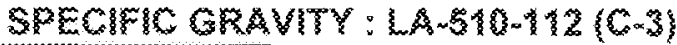

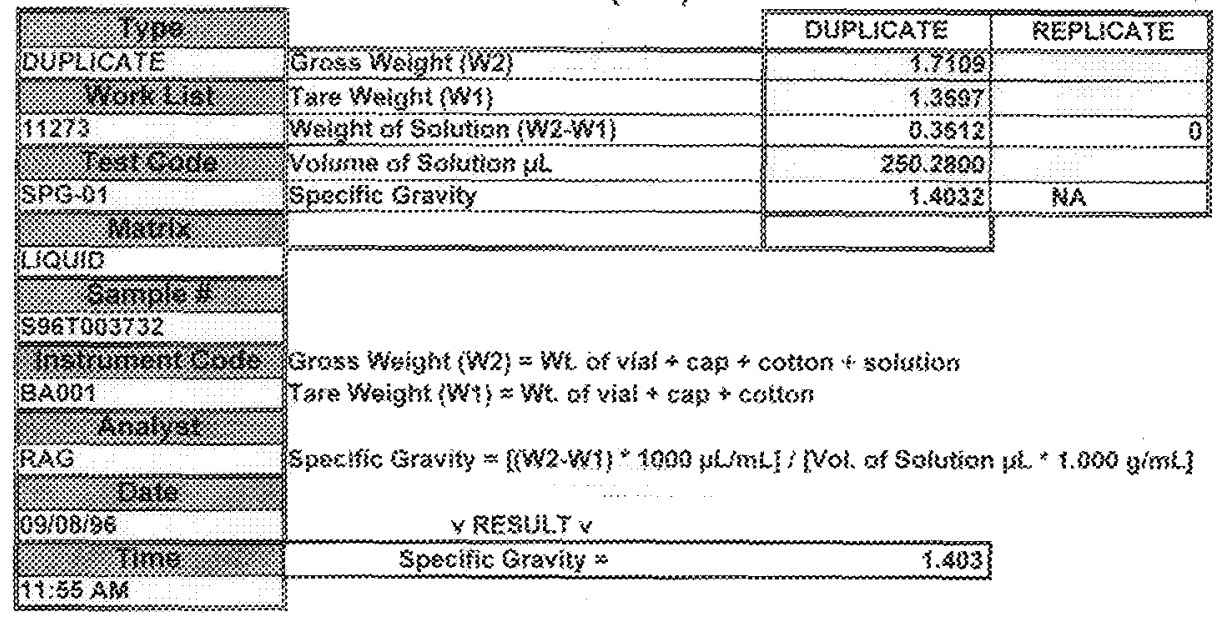

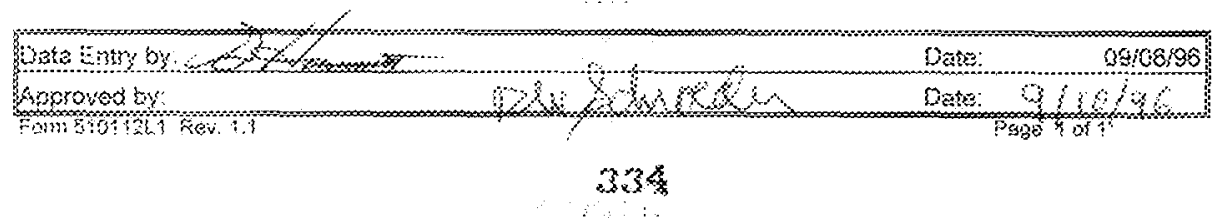




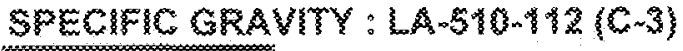

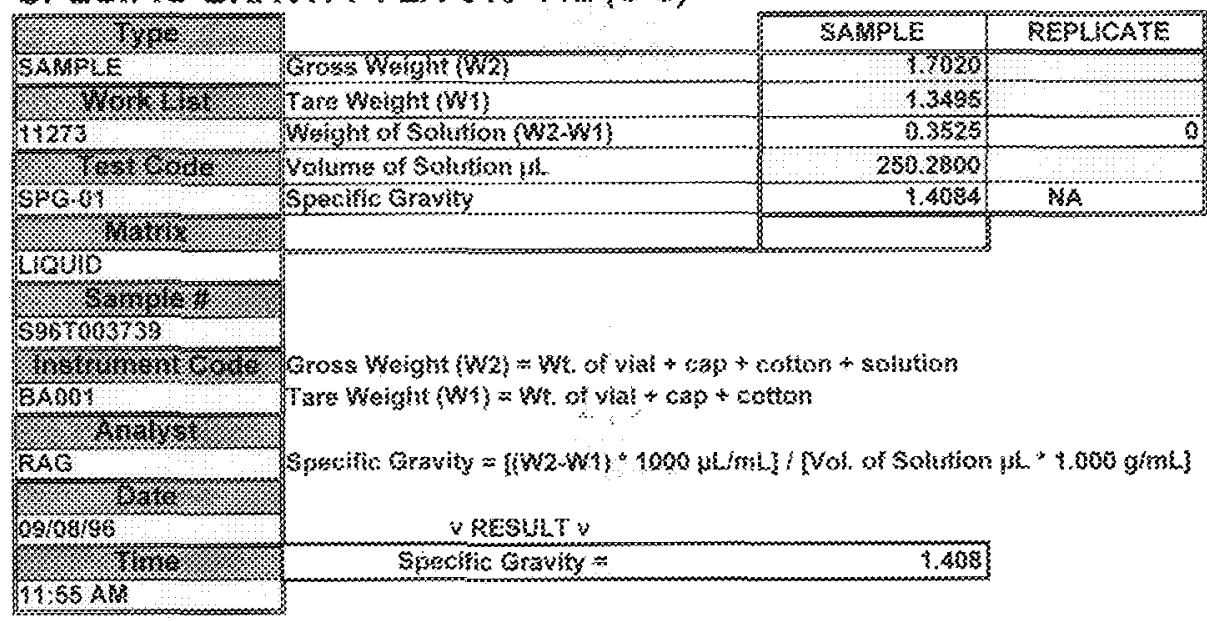

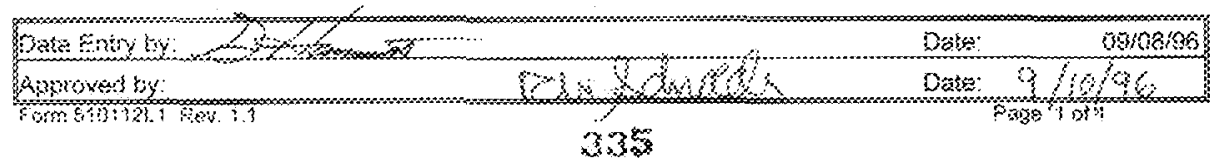



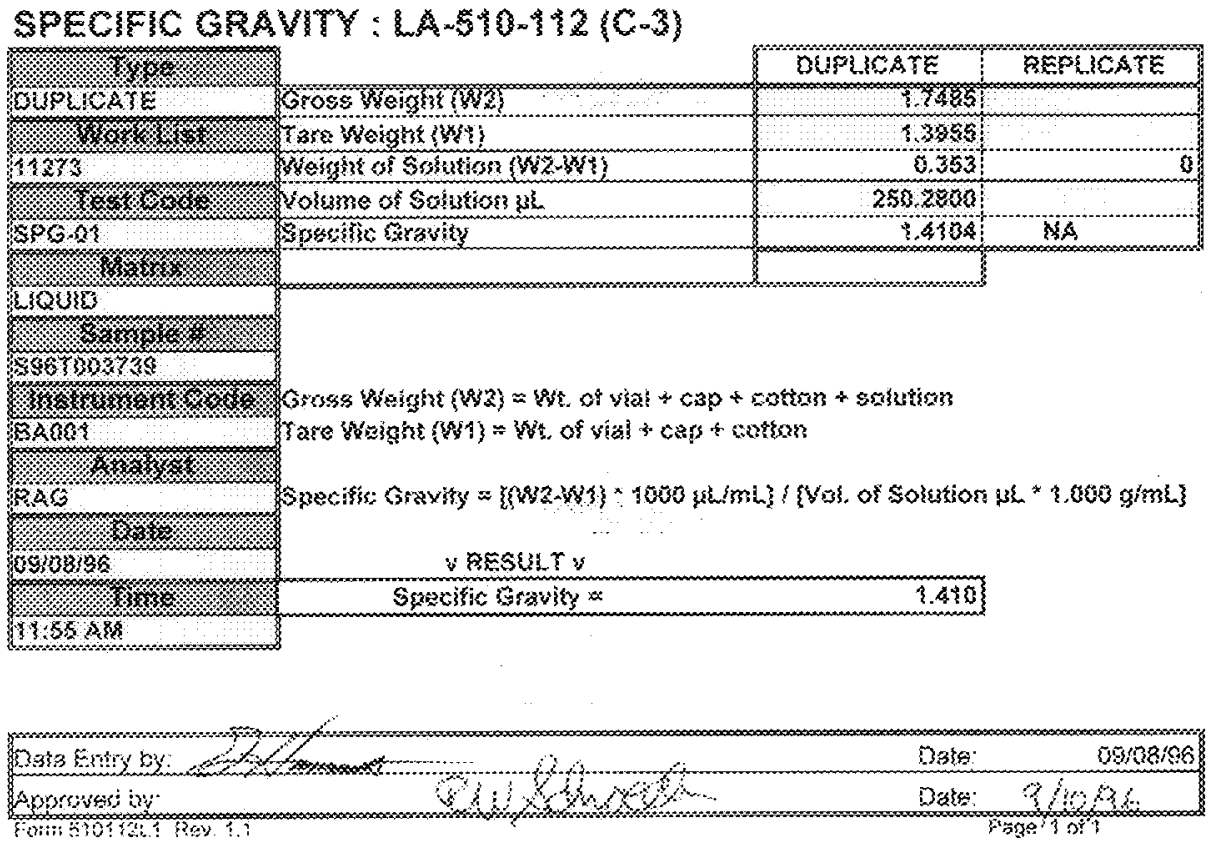

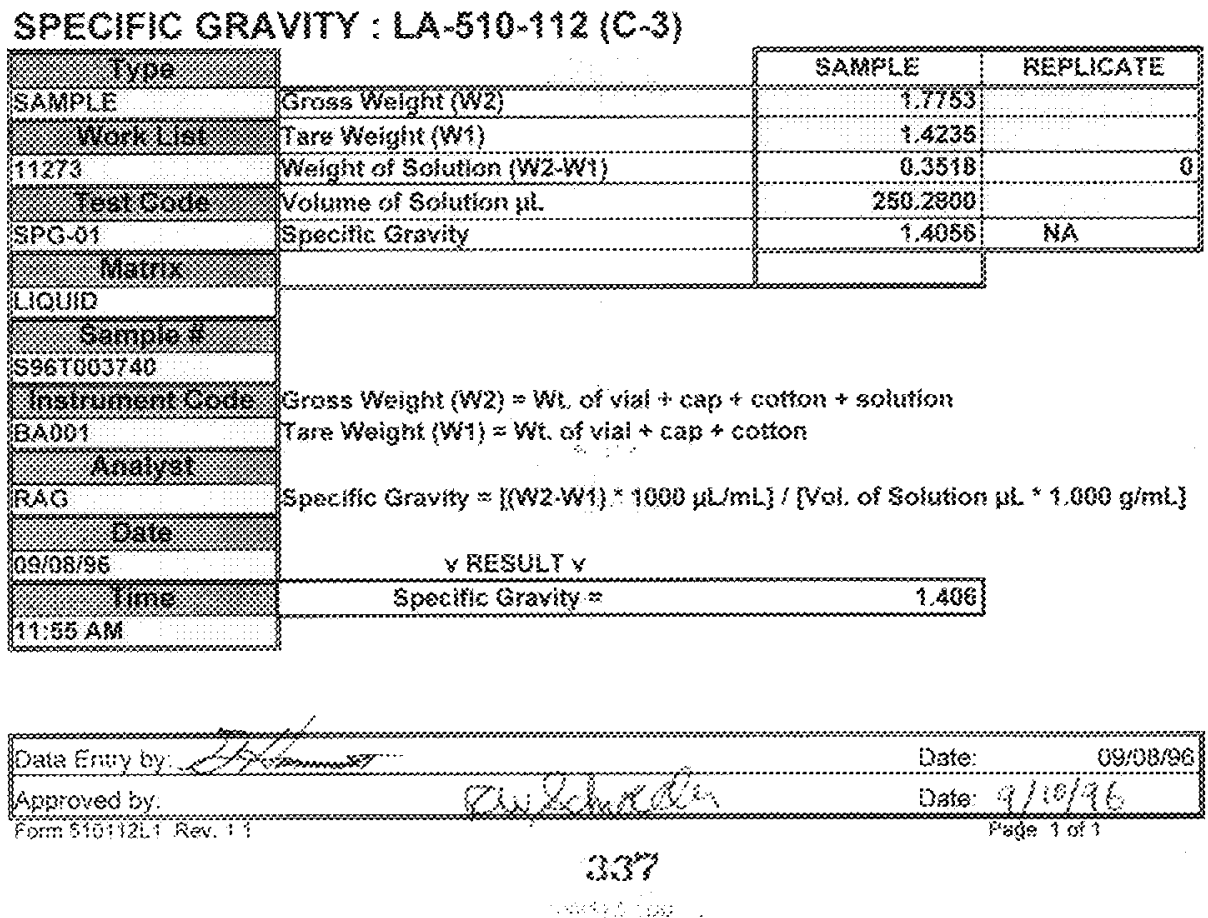


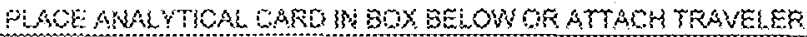

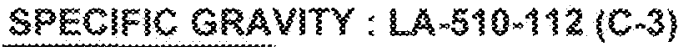

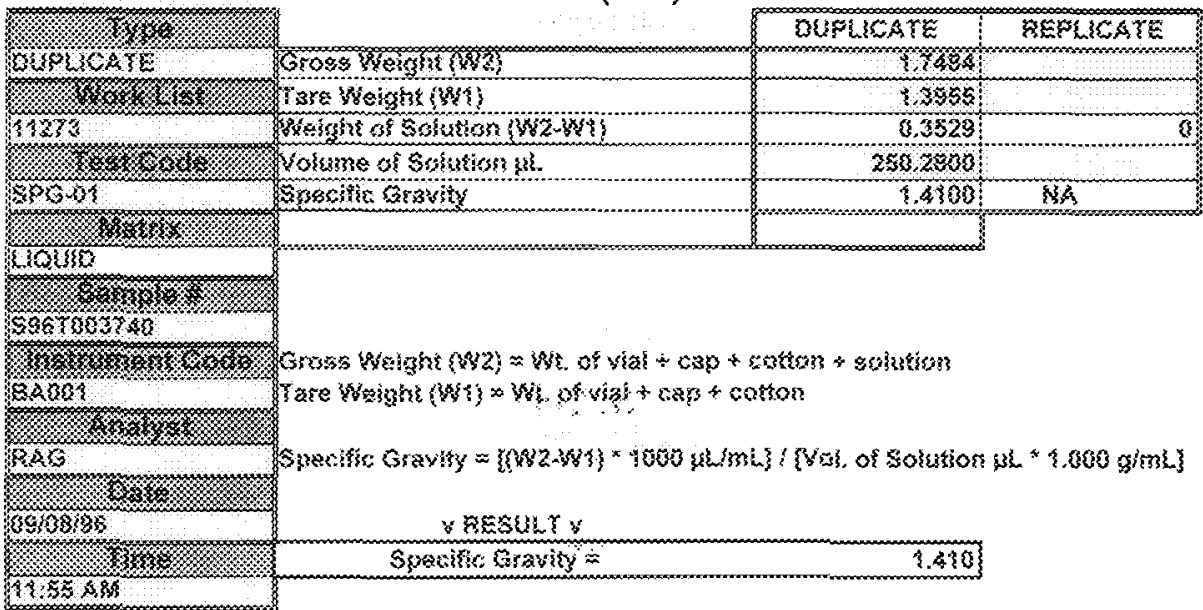

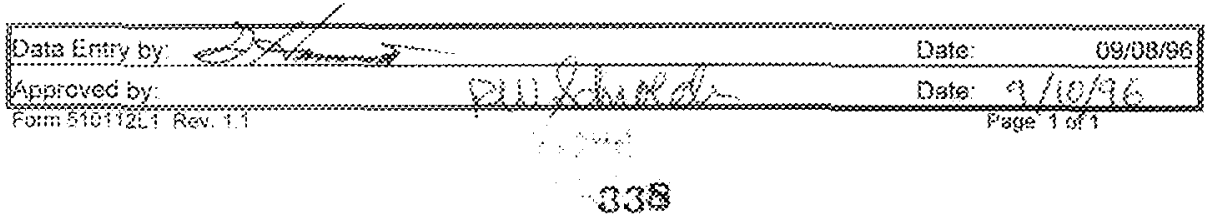




\section{S}

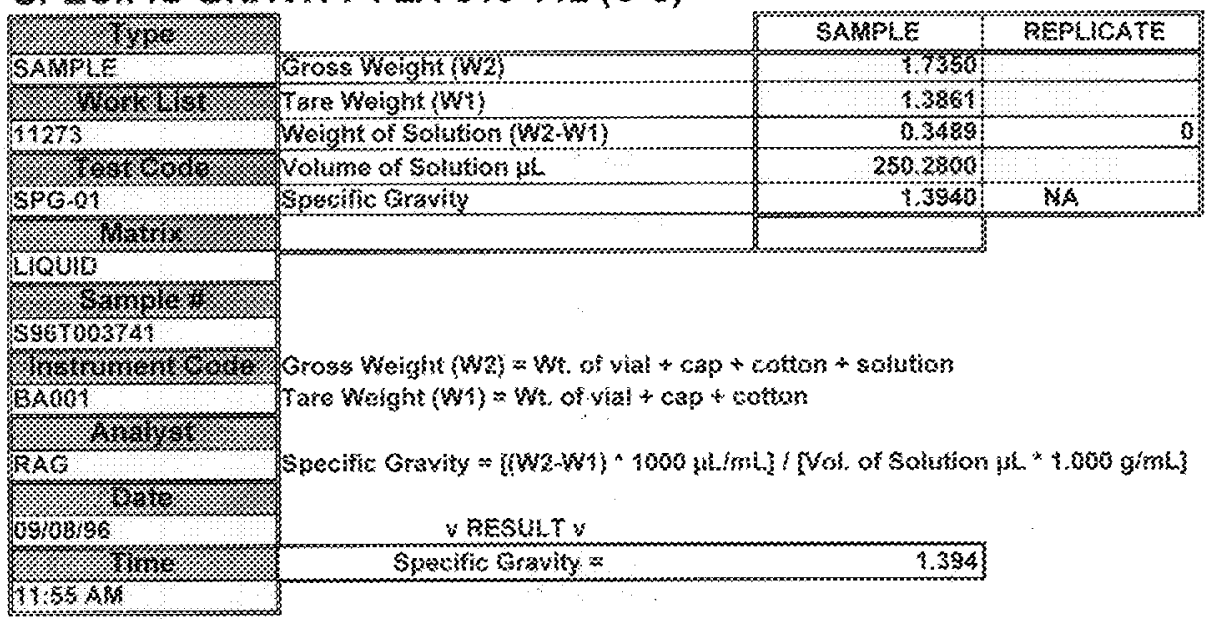

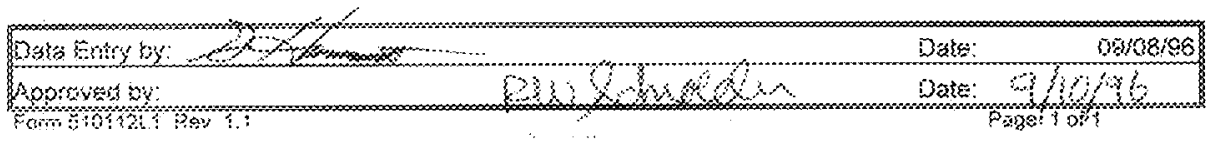



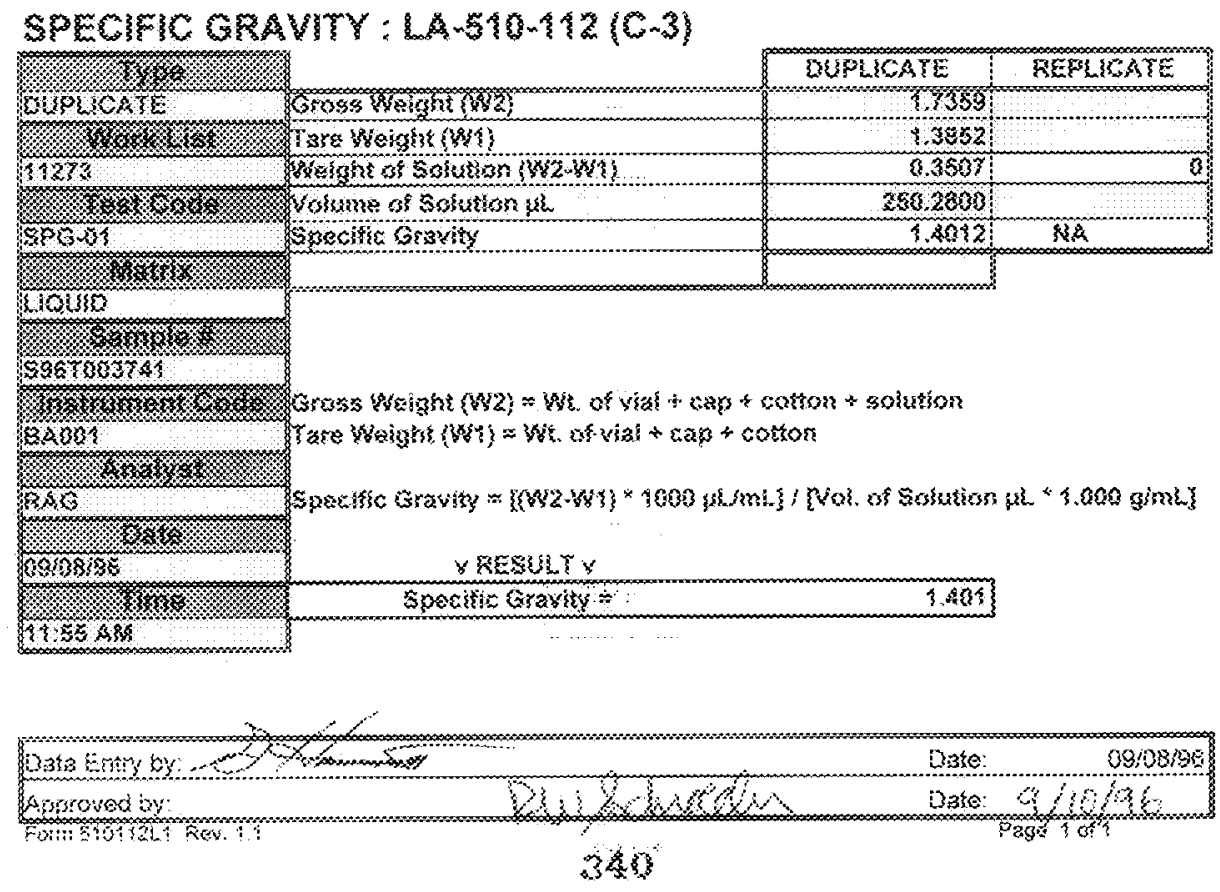

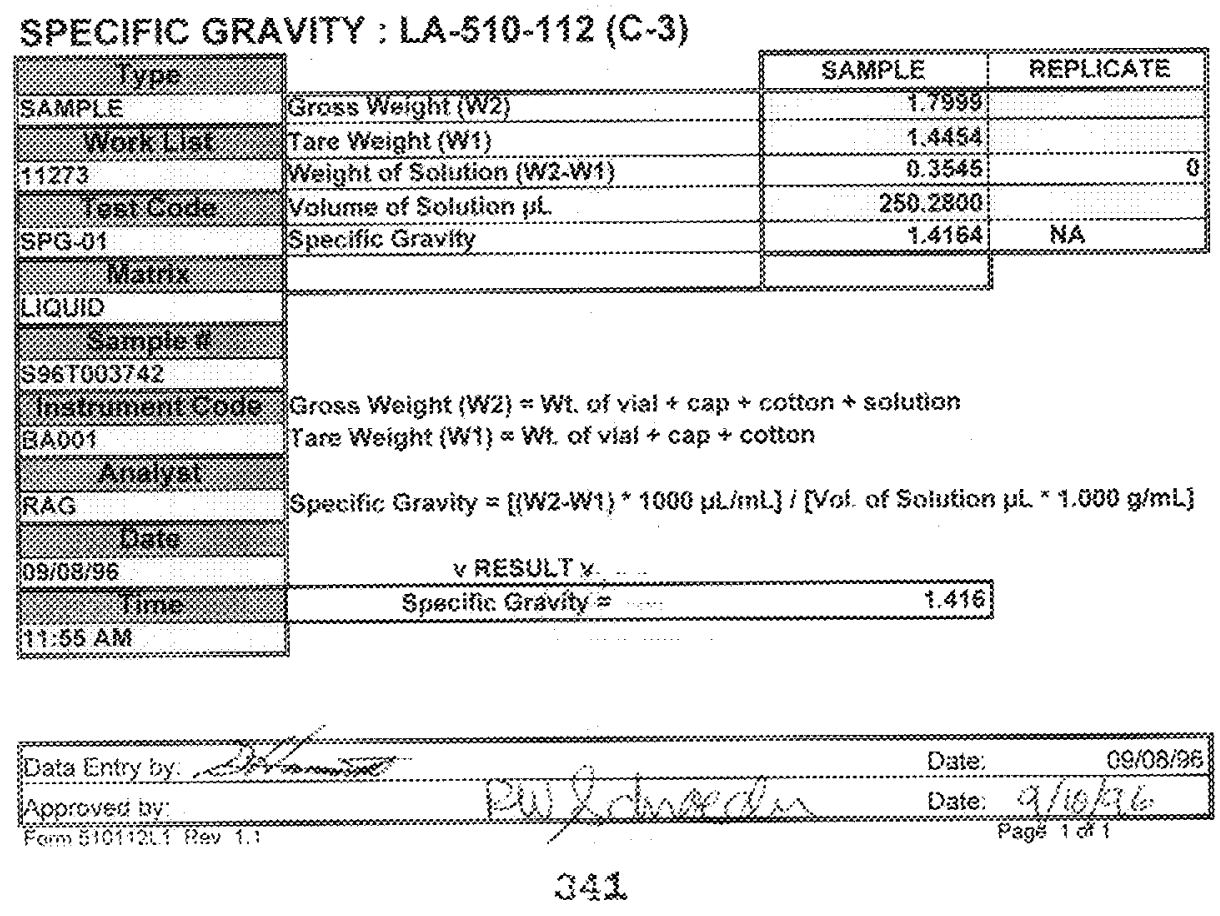

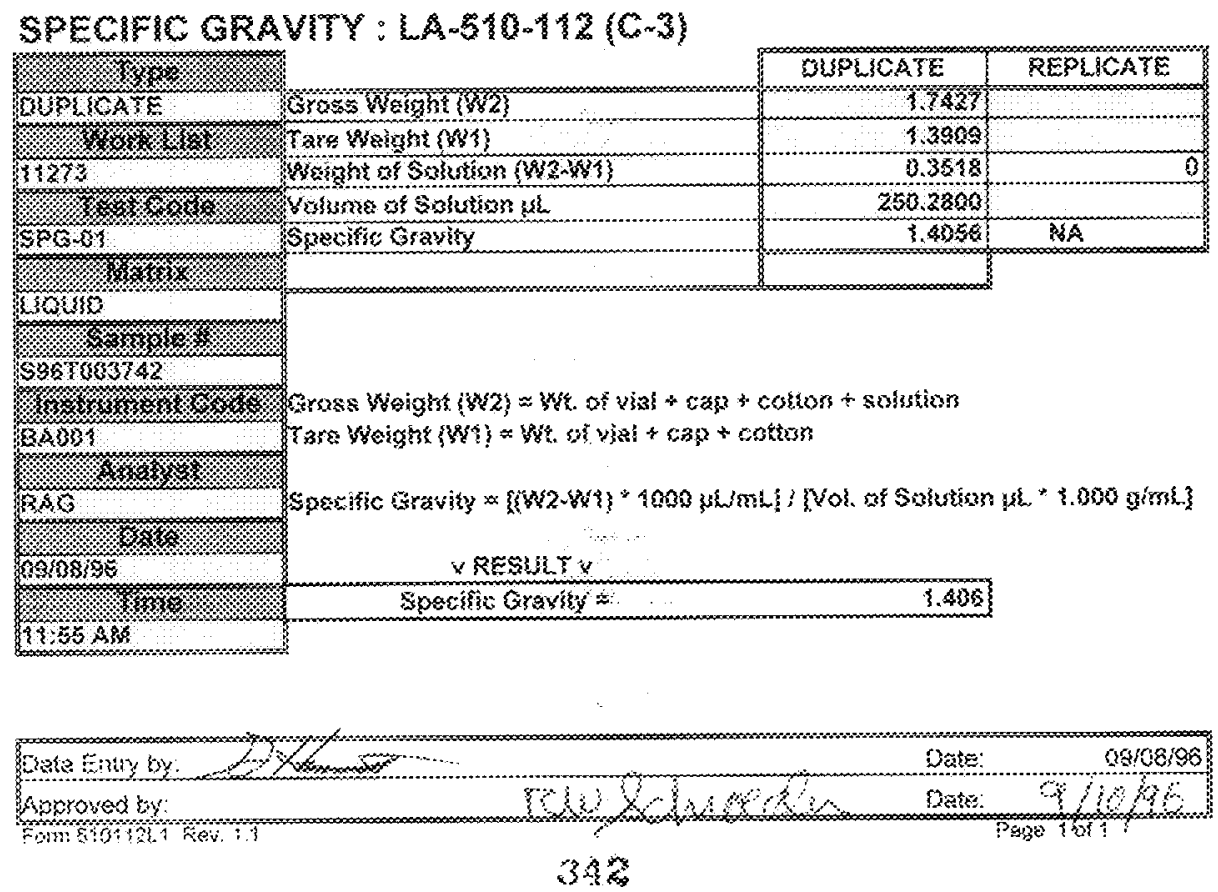


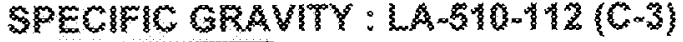

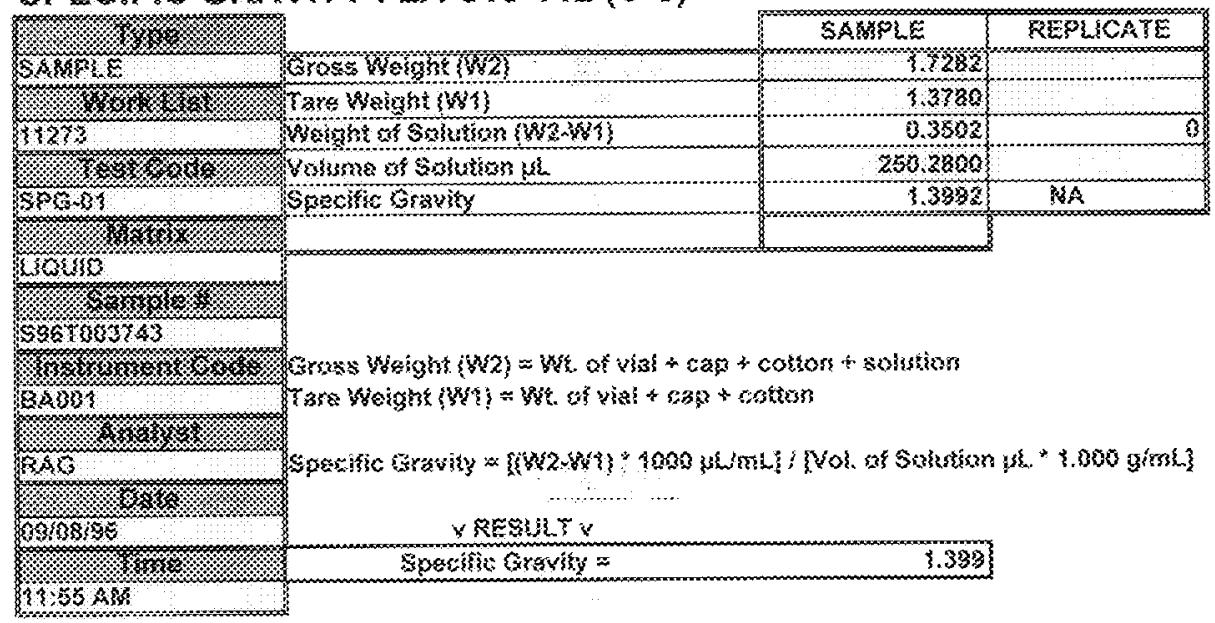

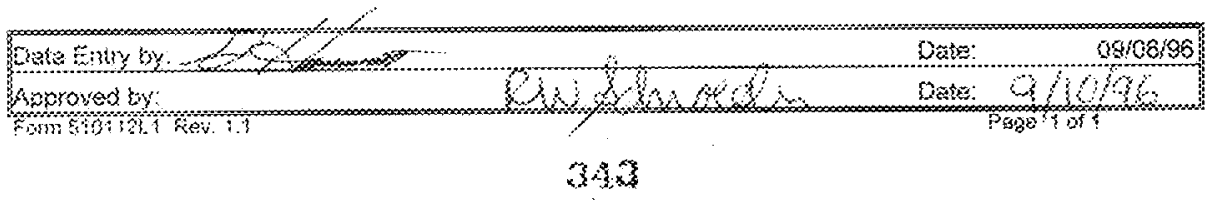




\section{SRECHFIC GRAVTY: $\{\mathrm{A}-5,10-112\{\mathrm{C}-3\}$}

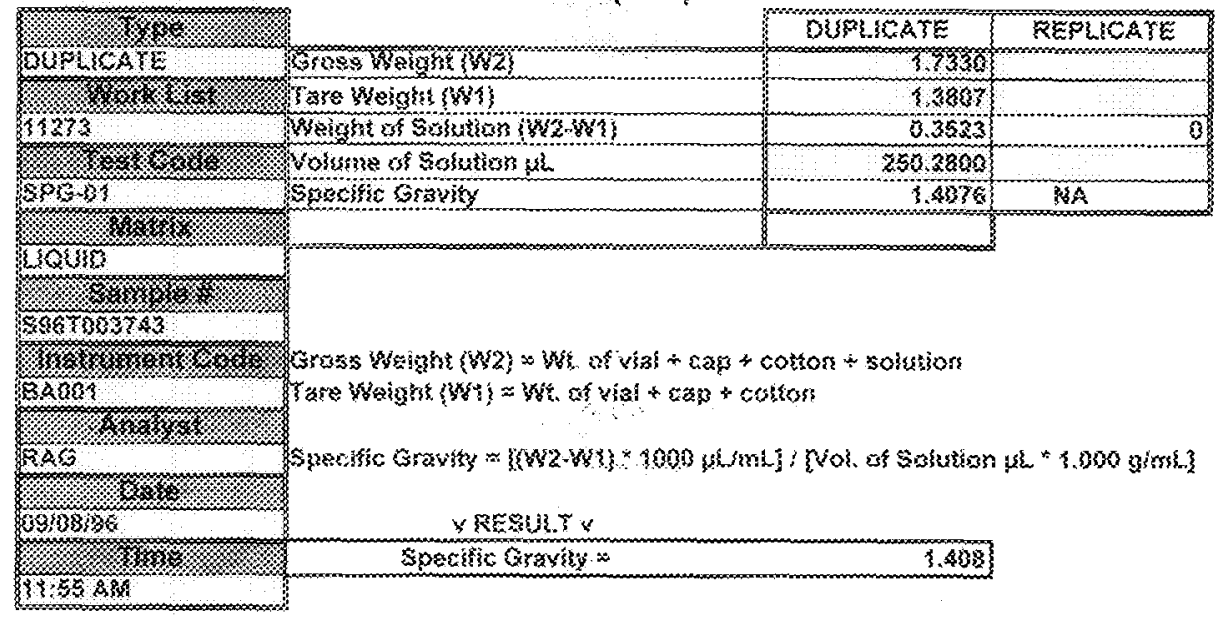

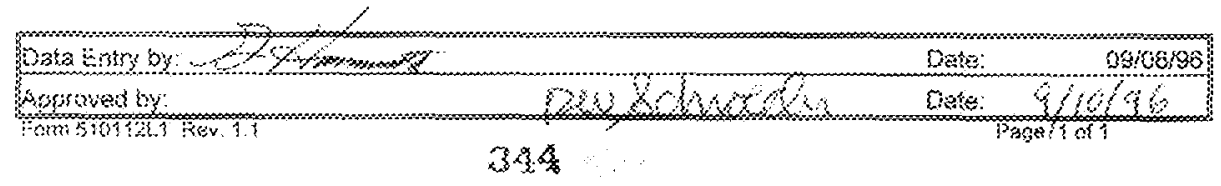




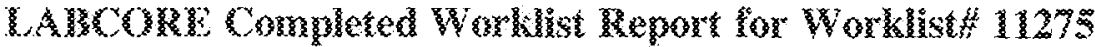

Aragkys: kis

Enstruemina: BAOS

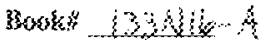

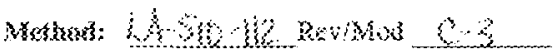

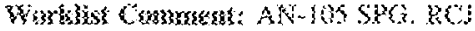

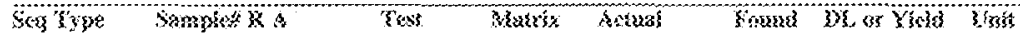

\begin{tabular}{|c|c|c|c|c|c|c|c|}
\hline $25 \mathrm{~s}$ & & 4 & $\theta x s$ & rovess & $2,25 \%$ & 2,32 & 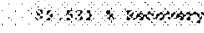 \\
\hline s sersons & 3960003934 & $\therefore$ & $800 \cdot 3$ & axwss & ris & 2. 233 & $1 \cdot(00 x-20\}$ \\
\hline $30 \mathrm{xt}$ & 6estopsos & $\theta$ & $3 \times t<02$ & iscons & 325 & 233 & n, n ph \\
\hline $4 \operatorname{sing} x$ & 5865053745 & 0 & $\sec 0.2$ & iscoros & 84 & 3.387 & $2.0 x+5+3063$ \\
\hline nos & osooosos & 8 & +806 & mingen & 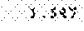 & 1.595 & $0,5 \times<\times 0$ \\
\hline \& soxper & $854 \div 2537 \div 8$ & s & $450-63$ & yegres & mis & $1 . \operatorname{sos}$ & 1.000 .605 \\
\hline$\% \$ \%$ & Assrosors & $a$ & opor & axussis & 200 & 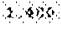 & 15०<< \\
\hline 8 sesson & 8964003537 & ? & sentis & nextsys & $H$ & $1.36 \%$ & $2,00-400$ \\
\hline 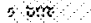 & b9otusis? & sis & 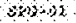 & $\mathrm{x} \| \omega \mathrm{J}$ & 16\% & 6,32 & $60 \% 230$ \\
\hline
\end{tabular}

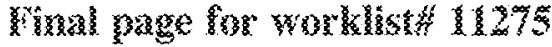

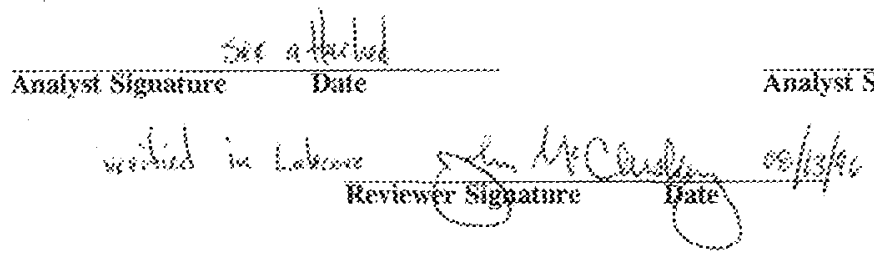




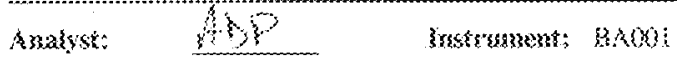

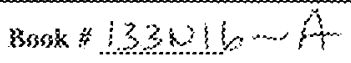

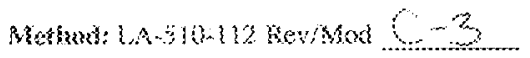

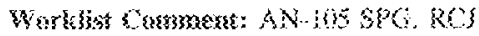

\begin{tabular}{|c|c|c|c|c|c|c|c|c|}
\hline $\sec$ & $580360 \%$ & Yrite & 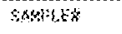 & $\because A$ & and & SSATEYY & ACrus: fol ot & WRIT \\
\hline & . & $1 \operatorname{sen}$ & & & $560 \cdot 01$ & $\therefore 2010$ & $3996 \angle 34$. BA & sp.s. $\quad 0 \%$, \\
\hline$\% 00032$ & $n \times-9: 5$ & 2 SAM & ststocstat & $:$ & $5: \Leftrightarrow-0 y$ & :30:28 & $3 / 3-\alpha z<$ & sp. \\
\hline $760608 \% 2$ & QN : : :S & $\because$ ids & sorowatis & : & $\operatorname{ses} \theta 01$ & axprs & 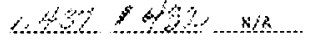 & sp.s. \\
\hline $900008:$ & $x y-4$ & $\Leftrightarrow$ EAGHSE & serocosis & 0 & $586 \cdot 03$ & L:obib & $3 / x-3$ & 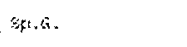 \\
\hline 9000003 & $n k+3$ & $5 \mathrm{BuF}$ & 5667000745 & $\therefore$ & $\sin +4$ & Lyips & 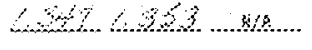 & sp.6. \\
\hline $56068 \times 2$ & Ax Tus & $S \operatorname{SADPLE}$ & S1955007: & 3 & sog.6\% & Liono & 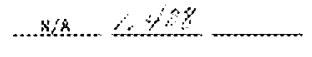 & $s p \cdot i$ \\
\hline 56000832 & $k<-10 b$ & 7 nur & sentonstis & 6) & $596 \cdot 63$ & Lavos & mann & 30.6 \\
\hline 29060637 & $n B-10 !$ & 8 SALERE & $556305376 \%$ & 8 & $s:\{1 ;-\{13$ & $100 i p$ & 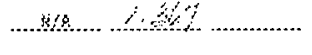 & 30.6 \\
\hline $70 n 0 n 8 \times 2$ & $9 x-105$ & 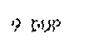 & 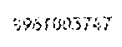 & 3 & $\$ F B \cdot a\}$ & Liguto & $13 \%$ in & sprur \\
\hline
\end{tabular}

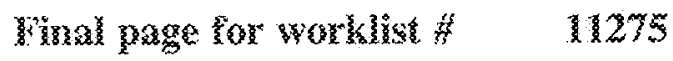
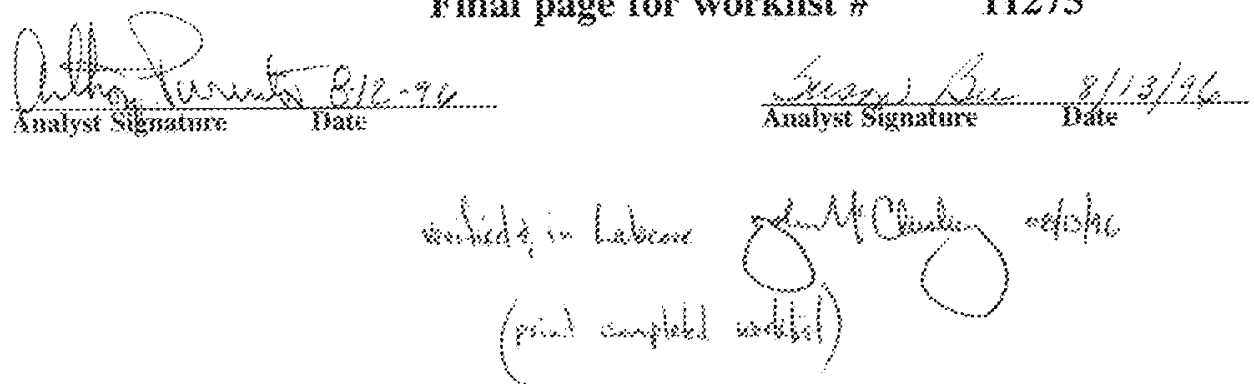

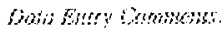

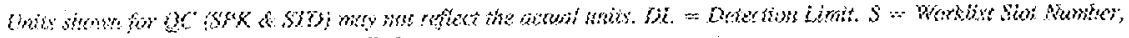

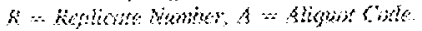




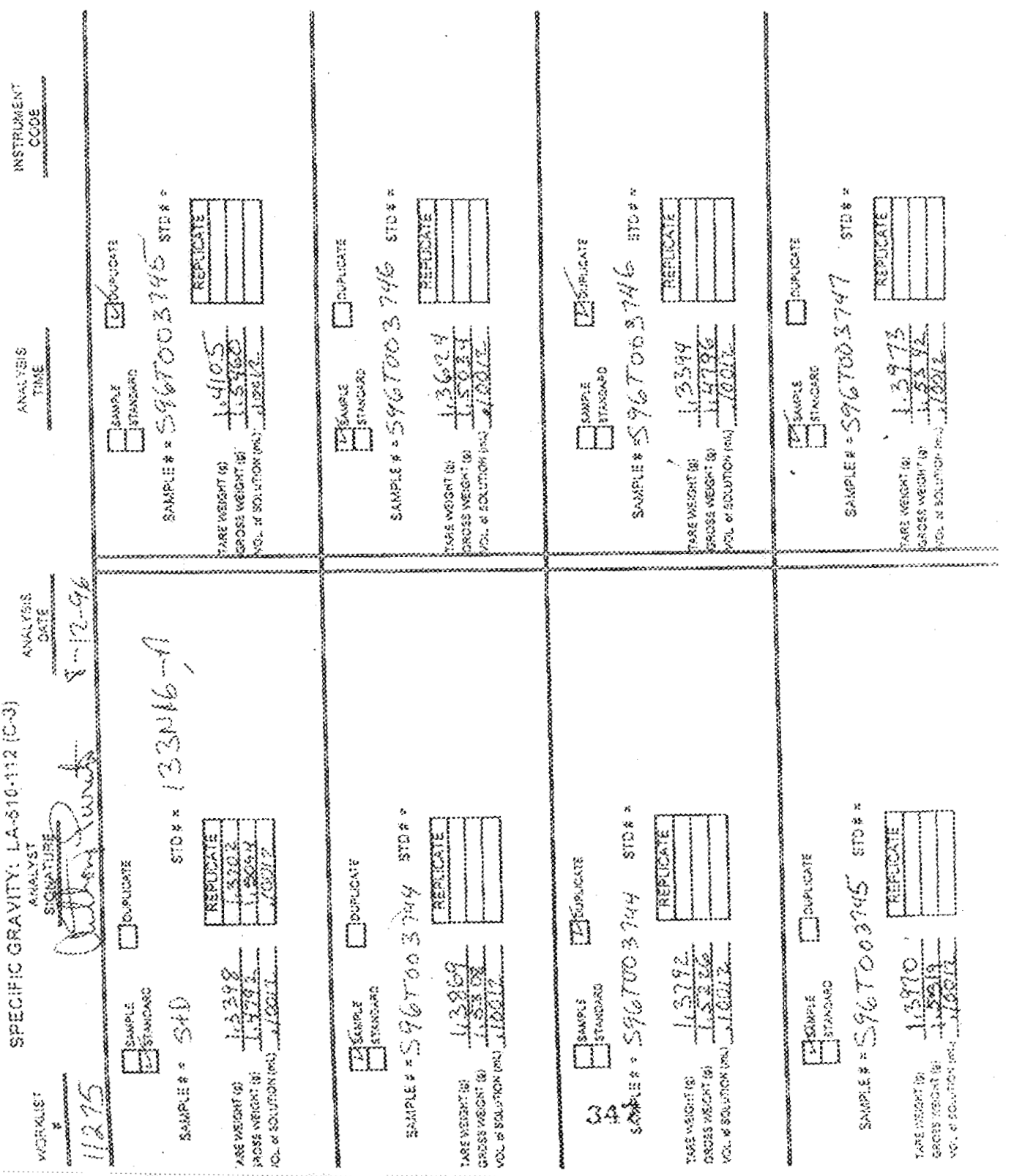




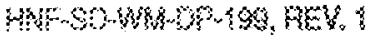

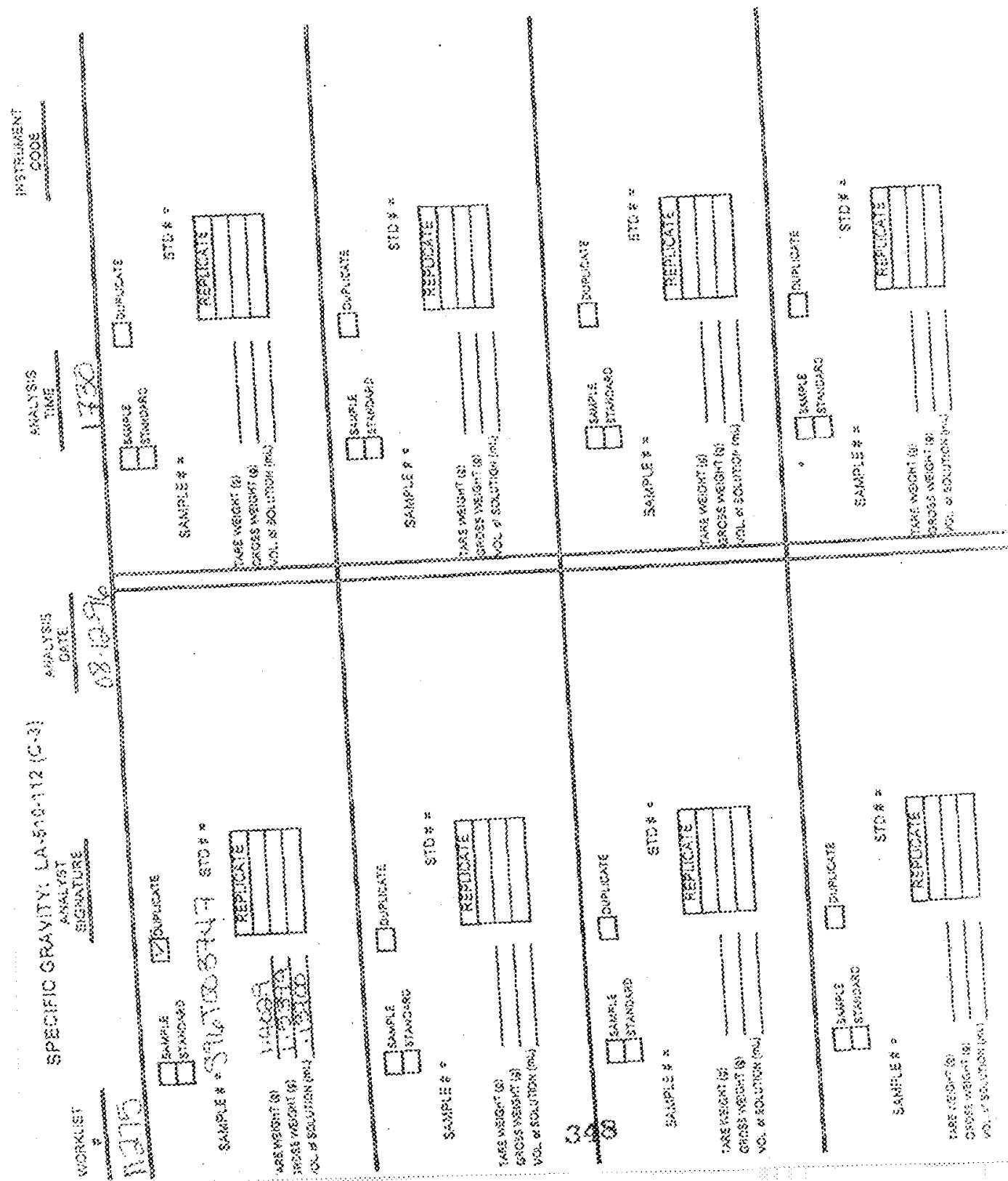




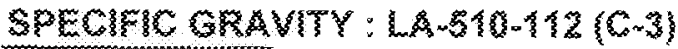

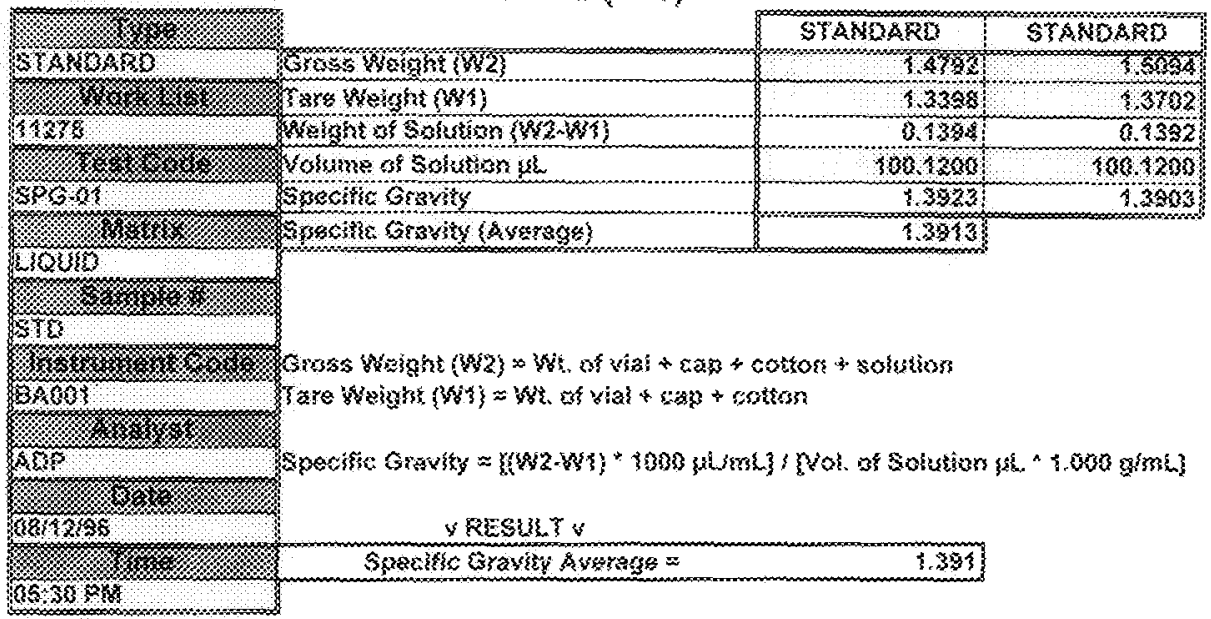

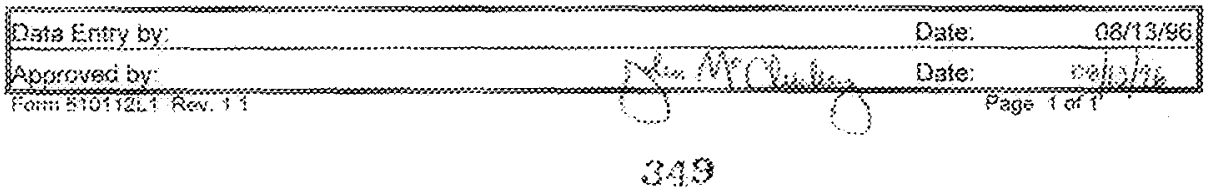



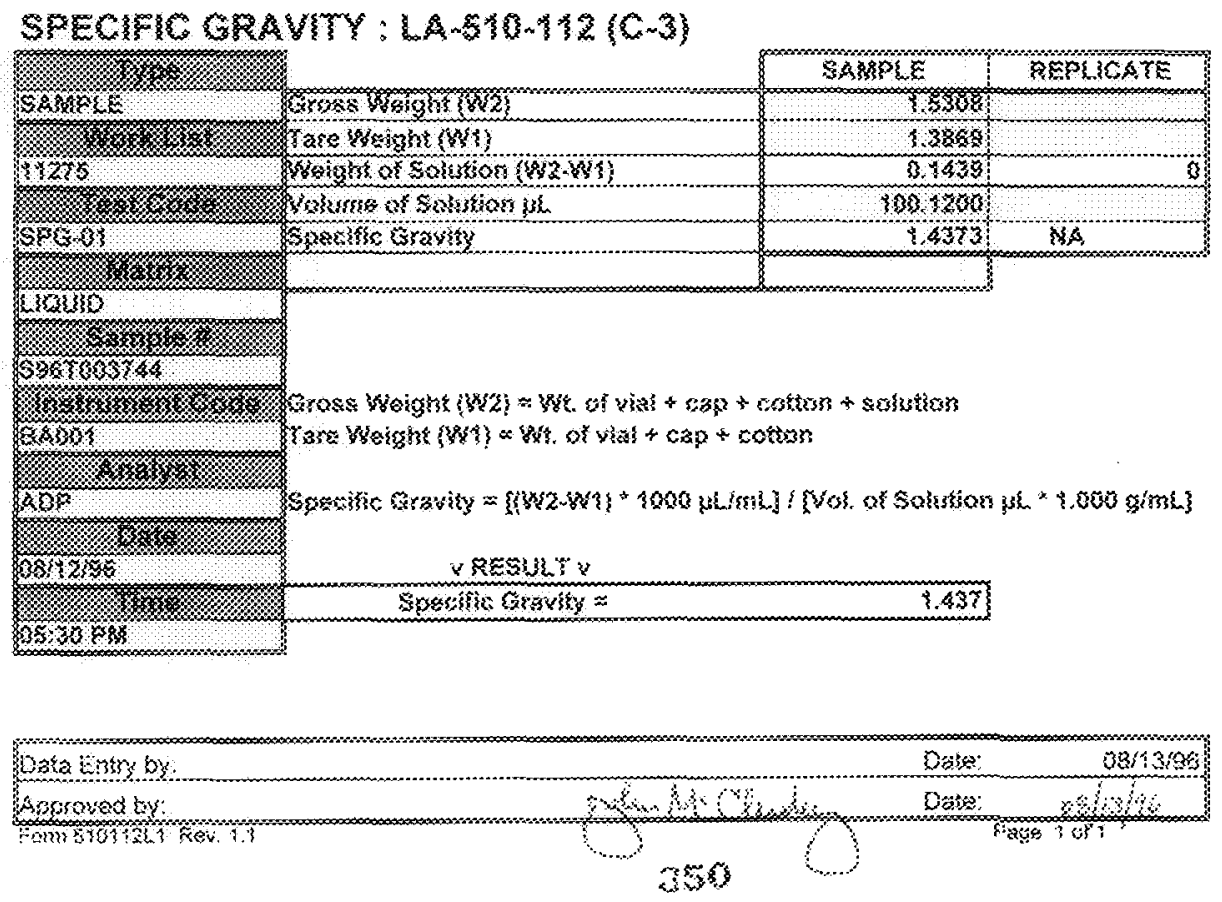


\section{SPECHIC GRAVET: : L}

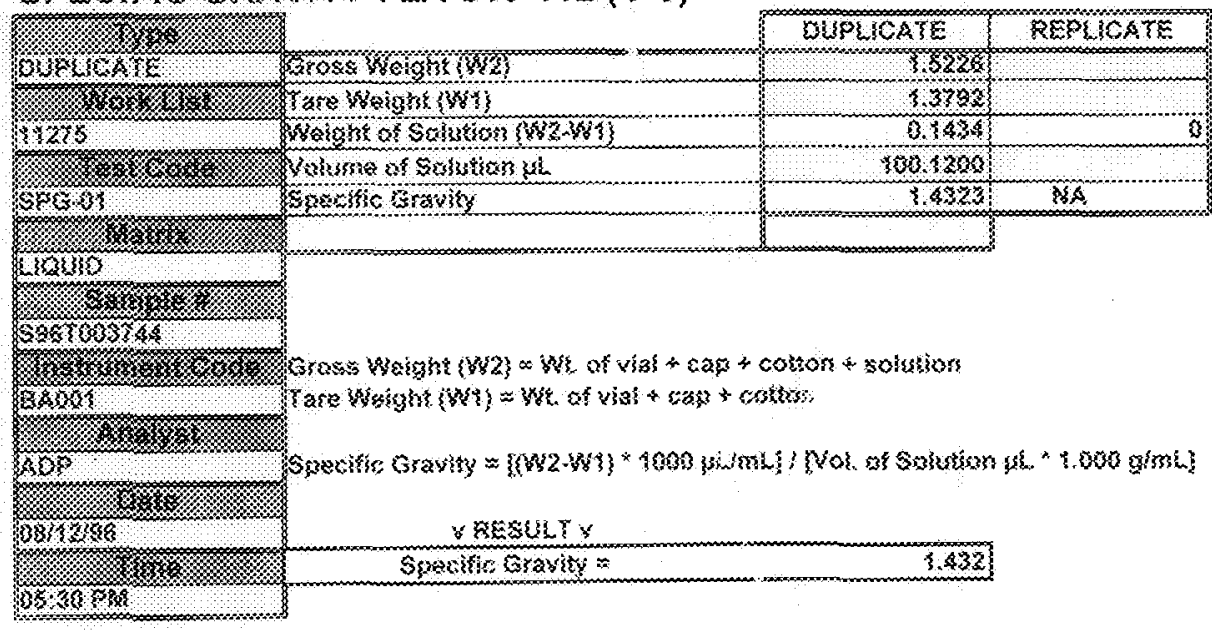

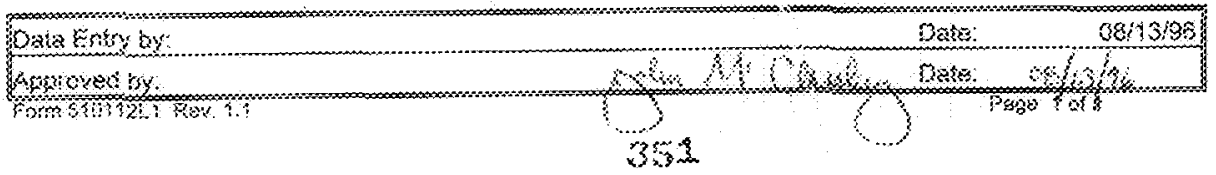




\section{SPECIFIC ORAVITY : LA-S1)}

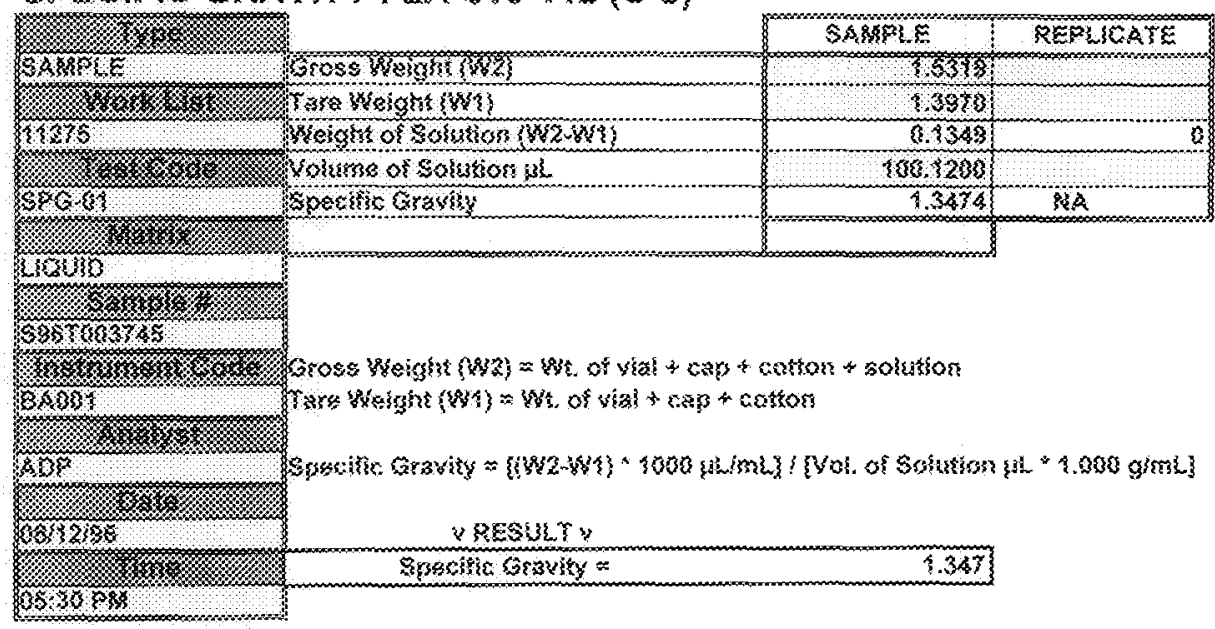

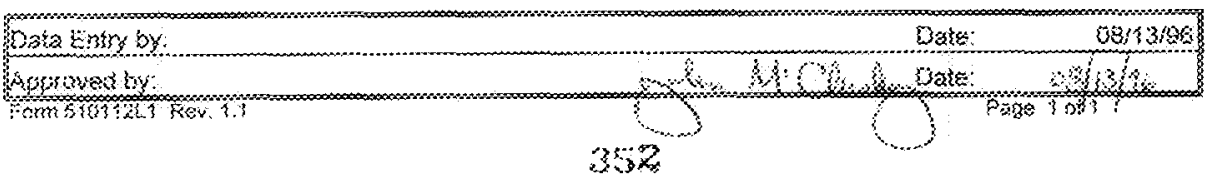




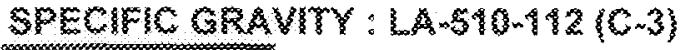

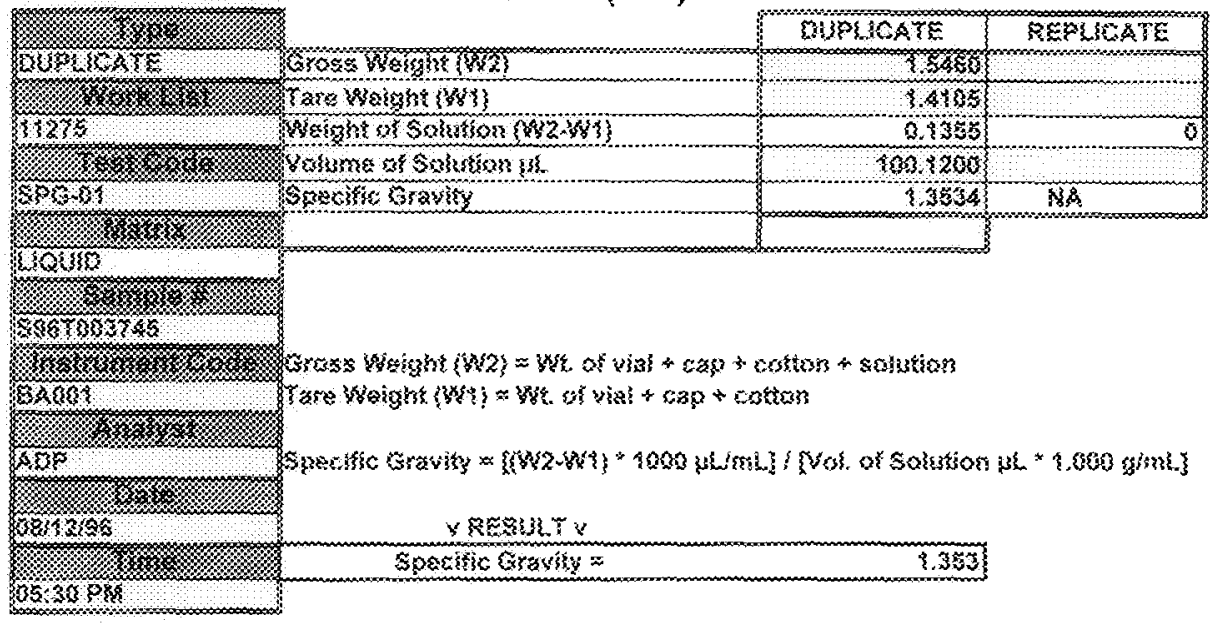

\begin{tabular}{|c|c|c|}
\hline Oats Ertry $6 y$ & Date: & $08 / 1386$ \\
\hline Ayroved by: & Date: & $\otimes<+1$ \\
\hline 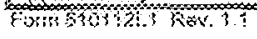 & & 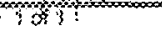 \\
\hline
\end{tabular}




\section{SPECIFIC CRABU: : L}

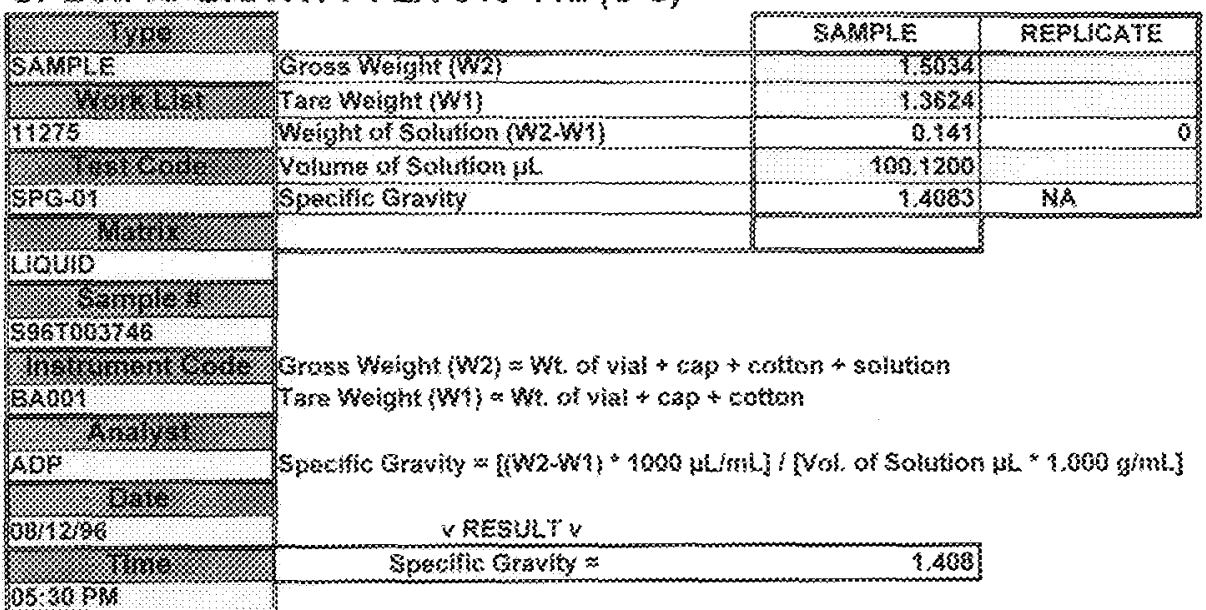

\begin{tabular}{|c|c|c|}
\hline Oata Entry by & Gata & $08 / 1386$ \\
\hline Agroved by. & 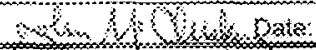 & 34 \\
\hline
\end{tabular}




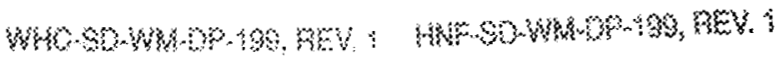

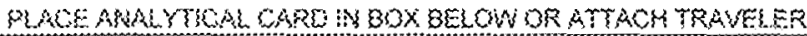

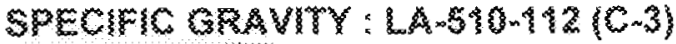

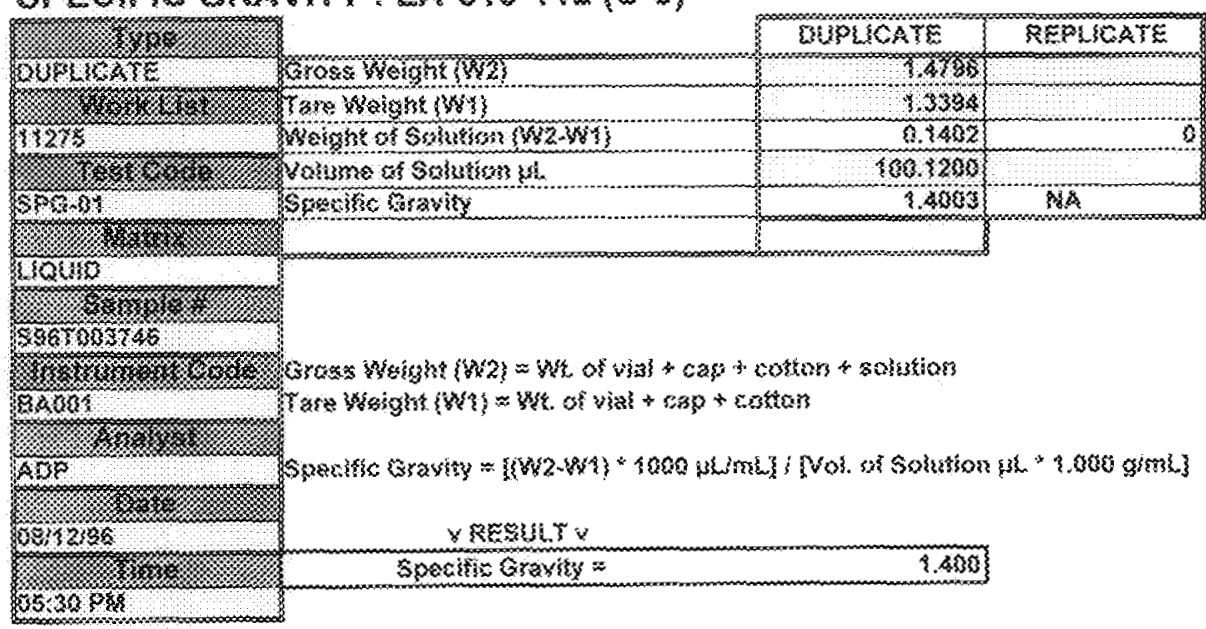

\begin{tabular}{|c|c|c|}
\hline Data Entry & Dele: & $33313 / 46$ \\
\hline ABoroves by: & Qsis: & 201 \\
\hline 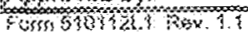 & & 1 \\
\hline
\end{tabular}




\section{SPEC}

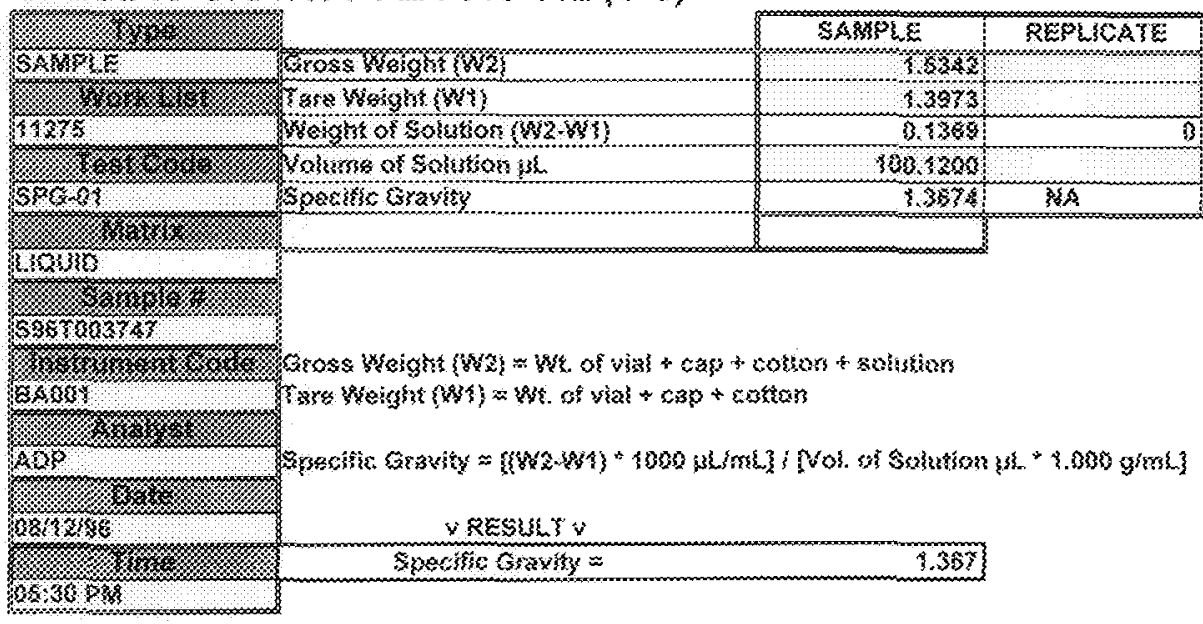

\begin{tabular}{|c|c|c|}
\hline bate from by: & Date: & 60,3368 \\
\hline Approved by & 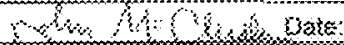 & $4 / \infty 96$ \\
\hline
\end{tabular}




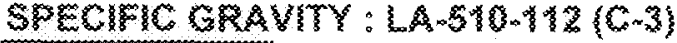

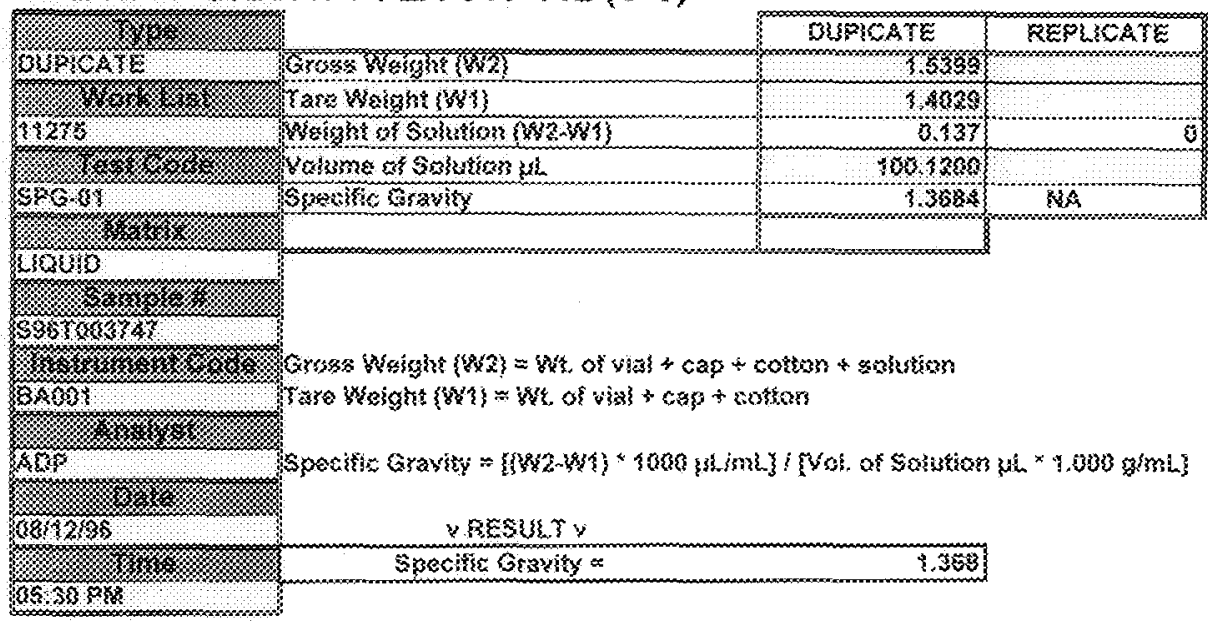

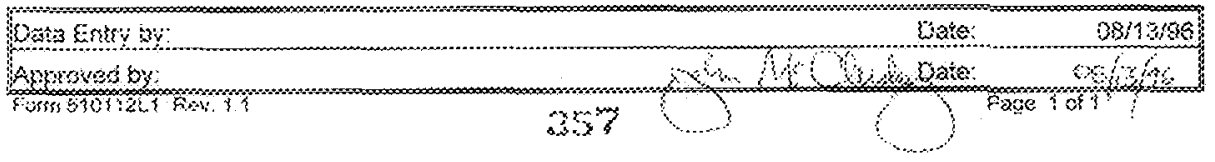




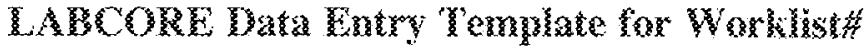

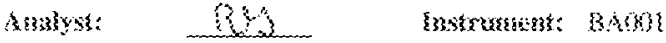

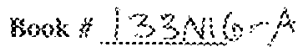

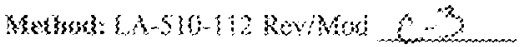

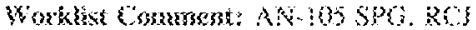

\begin{tabular}{|c|c|c|c|c|c|c|c|c|c|c|}
\hline sko:? & PRoift? & $S$ TYRE & SAAPLE & $8 A$ & A $\cdots \cdots \cdot \operatorname{rgr}:-\cdots$ & ShIPIX & Acrual & Fut:? & $\mathrm{al}$ & isk3? \\
\hline & $\cdot$ & 150 & & & $590-63$ & Laibi & 939846 & $\therefore 350$ & $\ldots$ & sp.ti. \\
\hline so:008:2 & $46-505$ & as sesisis & secios:3ras & 8 & $5: 8-9$ & :;on:? & $3 / 2$ & 138 & & sp. a. \\
\hline sogoss & 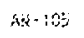 & $3 \mathrm{sidp}$ & 5967009748 & c & s.9.0: & movis & $\therefore 382$ & $\therefore 38$ & $y<s$ & sala. \\
\hline 3000832 & $4 k-435$ & i EXEPLS & socrousin & 8 & 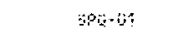 & 230450 & $13+4$ & $\{3 \theta \alpha$ & & sp.t. \\
\hline Q5nans?? & ans & 3060 & $85000937 i 5$ & 0 & stant & t3ons? & 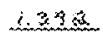 & 1383 & 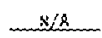 & Sp.e. \\
\hline 2roposs & $3 N \cdot 0 \%$ & $\therefore$ SOWOLE & 6967005730 & ia & s.0.6: & LESU3 & ...... & ¿3\%4. & & 40.6. \\
\hline 3500382 & Sis. 45 & 730 & sxyogst: & 9 & spi-je & Lisise & 4.38 & 638 & $\ldots$ & $\$ p, 6$ \\
\hline PStrots? & and 905 & a shar:ecte & $390: 00375$ & 0 & $5+5-9 !$ & sosits & $\ldots a$ & $1 \cdot 2 y 0$ & & sp.6. \\
\hline $96030 \mathrm{~s}:$ & $5 x-73 \vdots$ & Q bisp & 580063754 & i: & $\sin 6 \cdot 0:$ & :ERk3r & t. sing & $\therefore 395$ & 8 & ك. \\
\hline
\end{tabular}

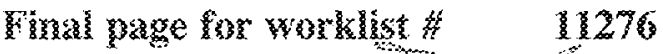

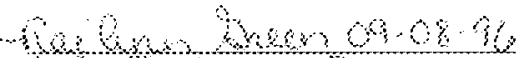

Ankyst angmass Tate:
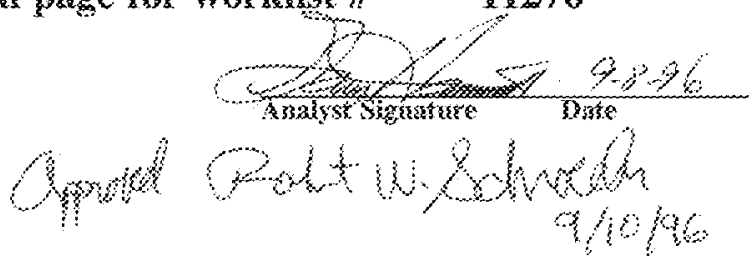

Hifa Entro Comments:

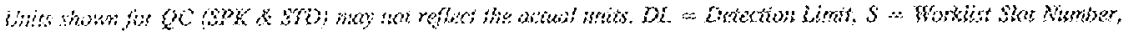

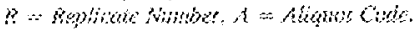




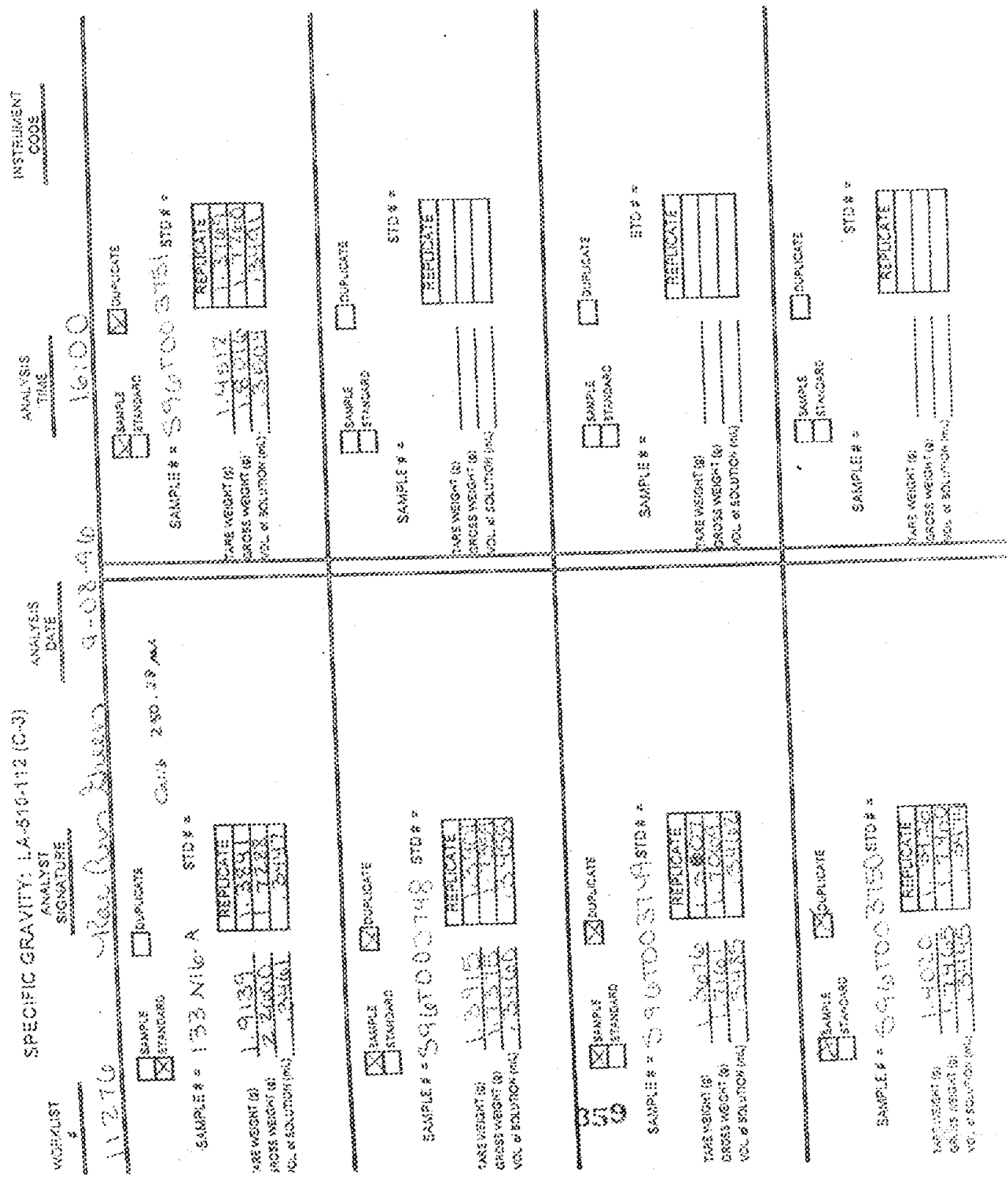




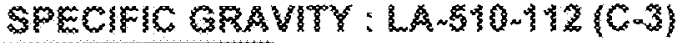

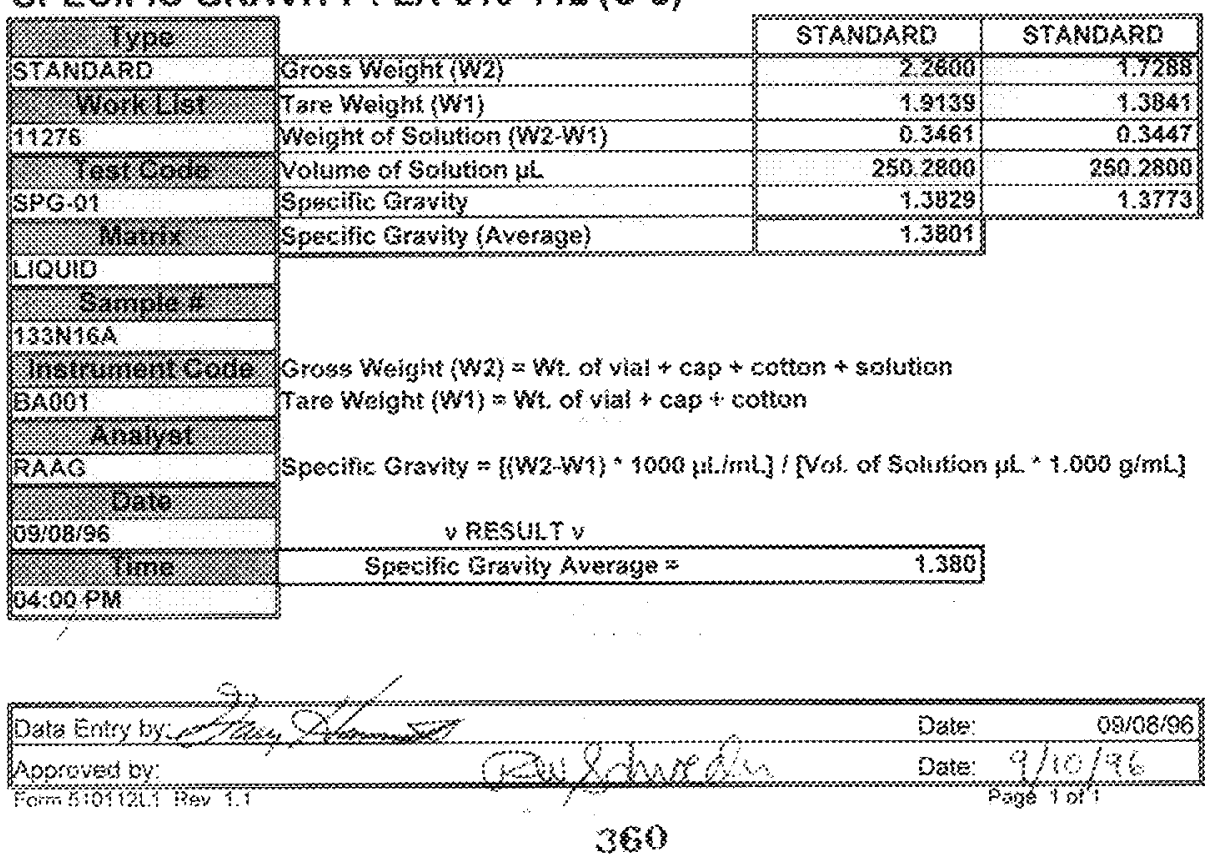




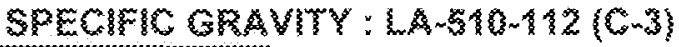

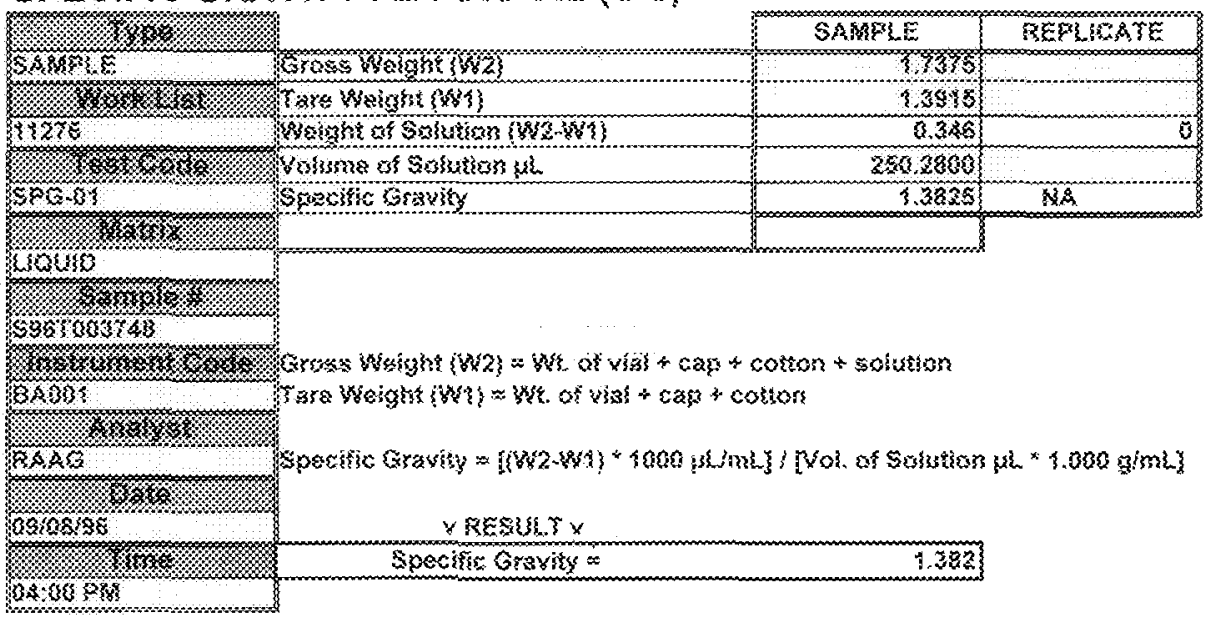

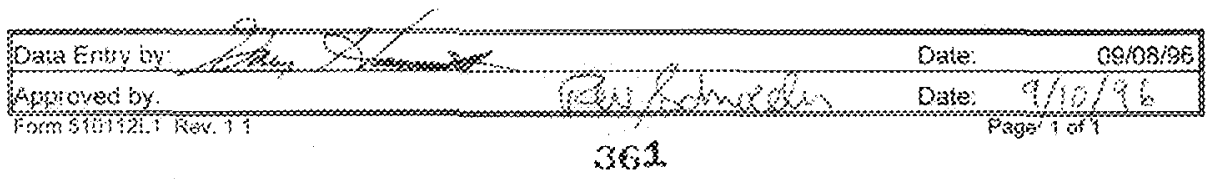




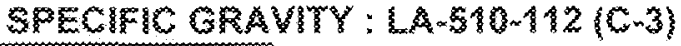

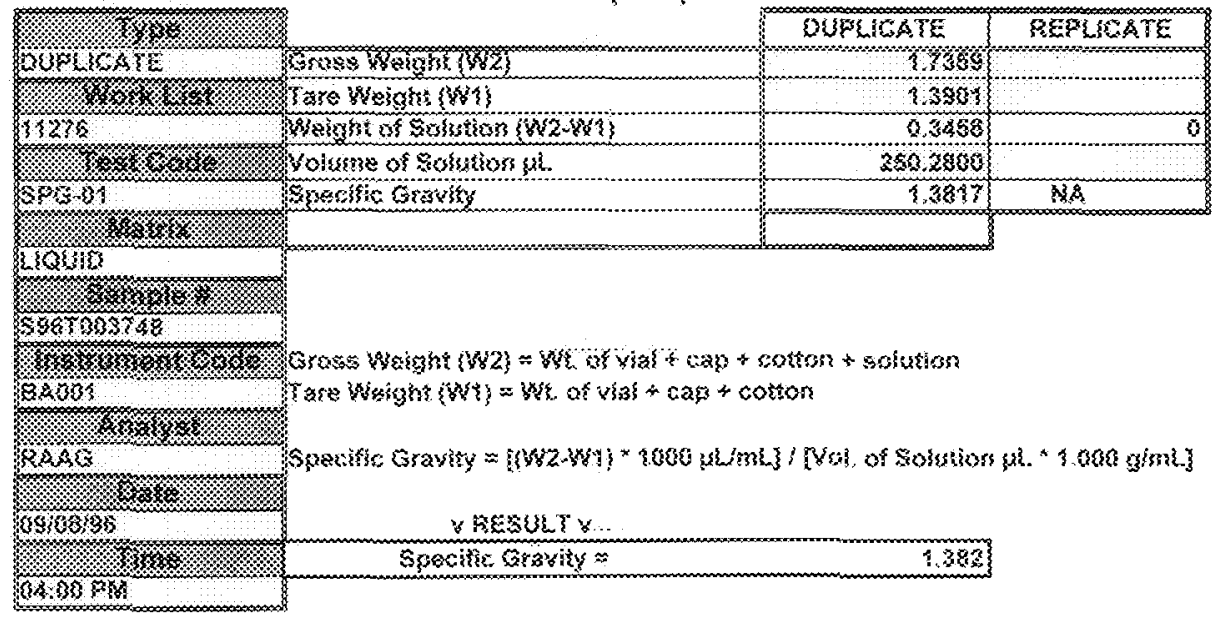

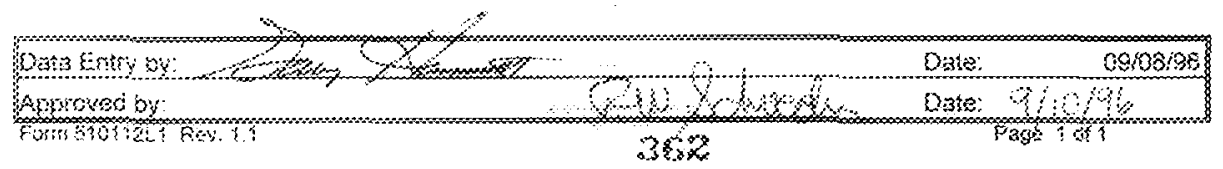




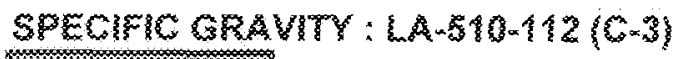

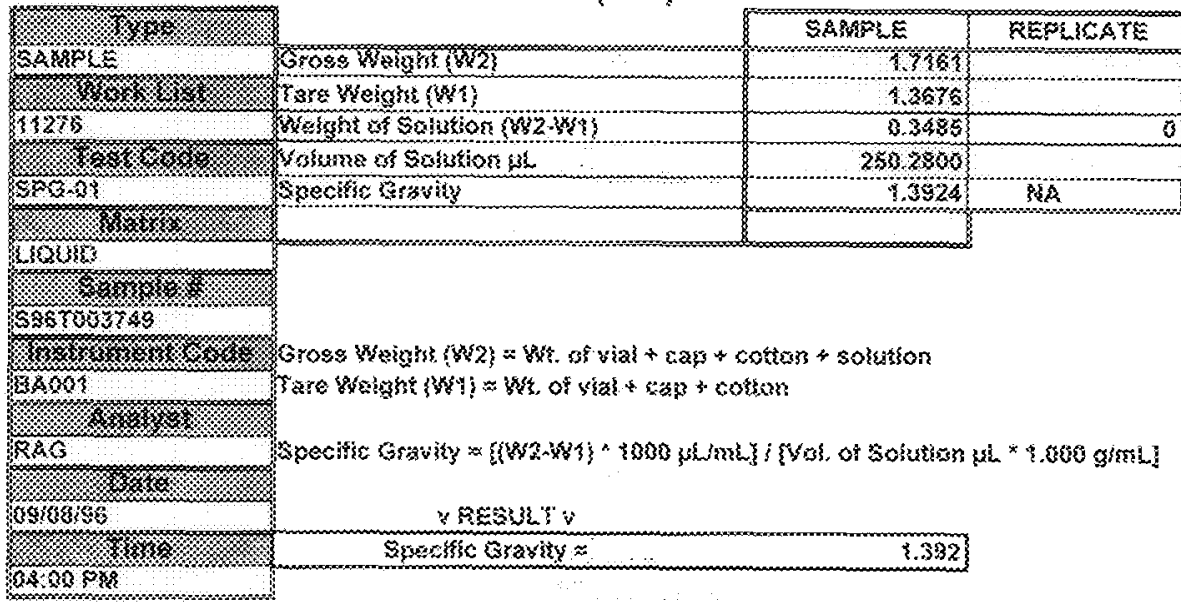

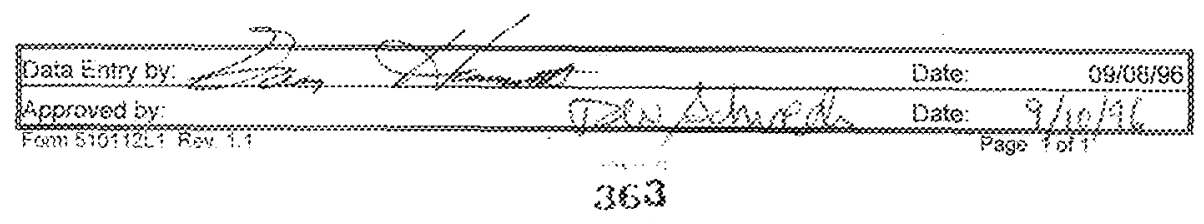




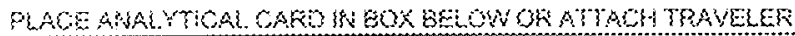

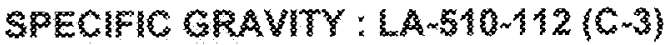

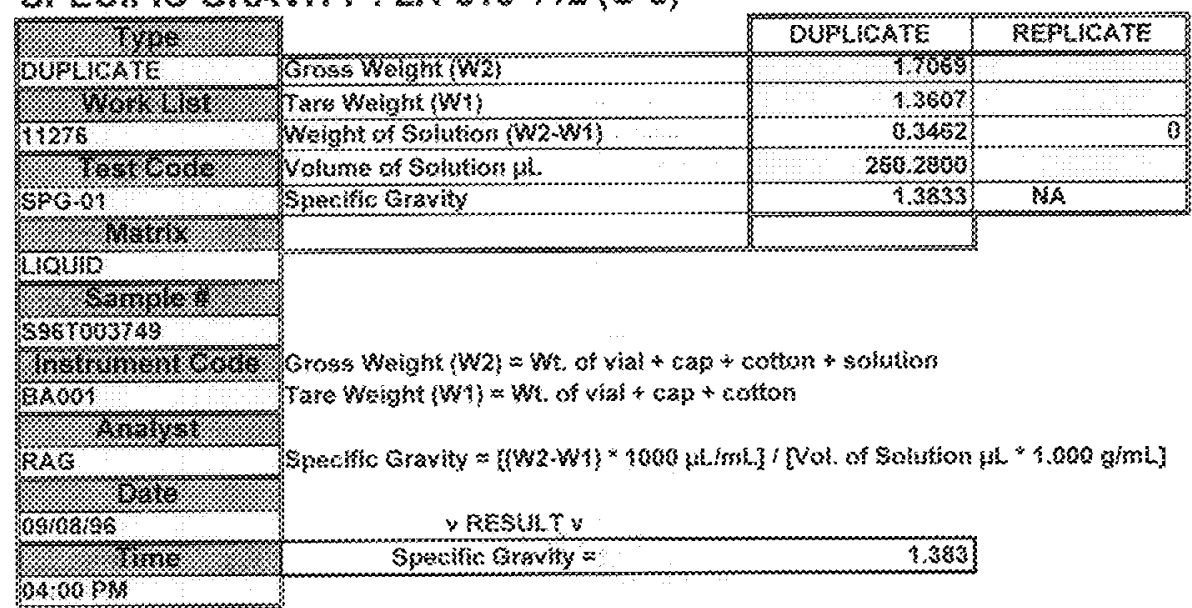

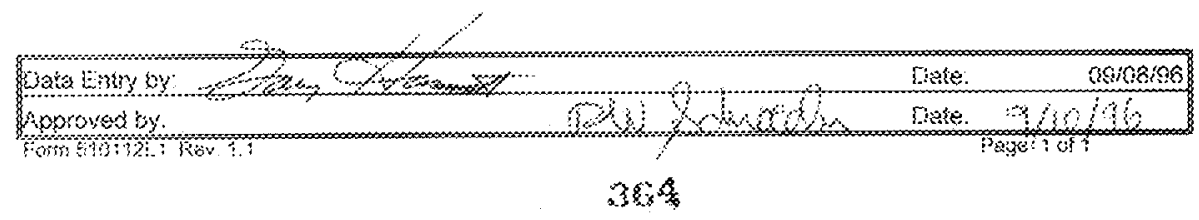




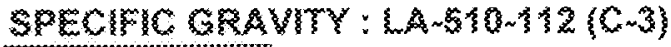

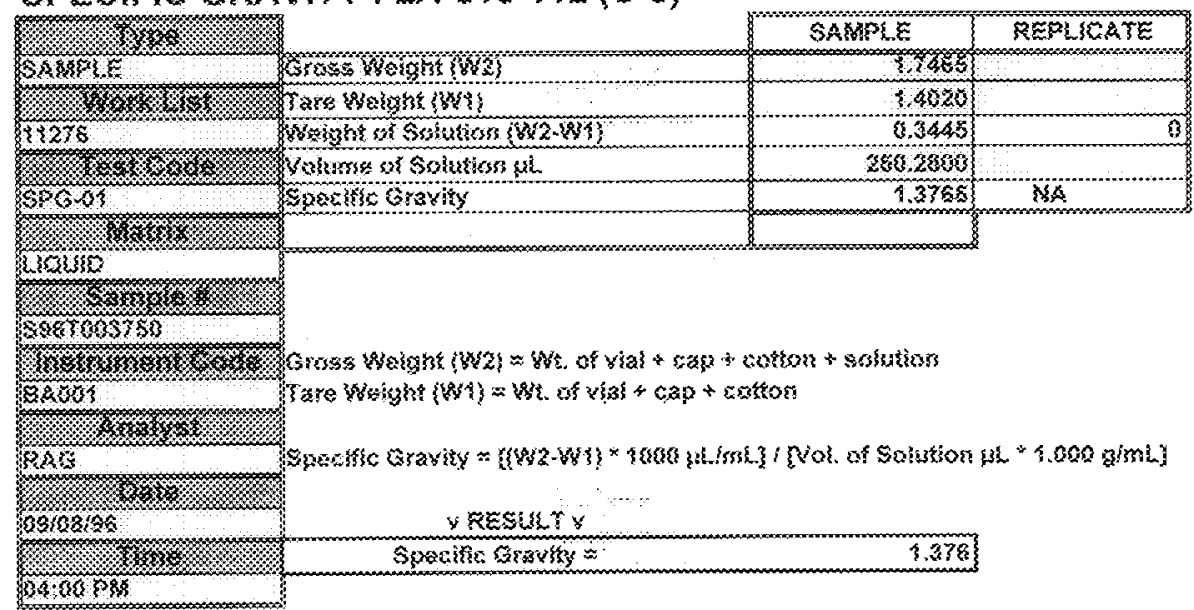

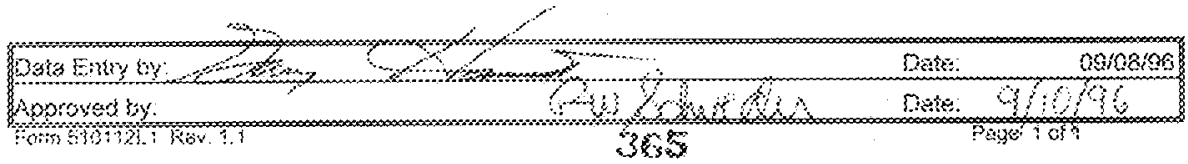




\section{SWE}

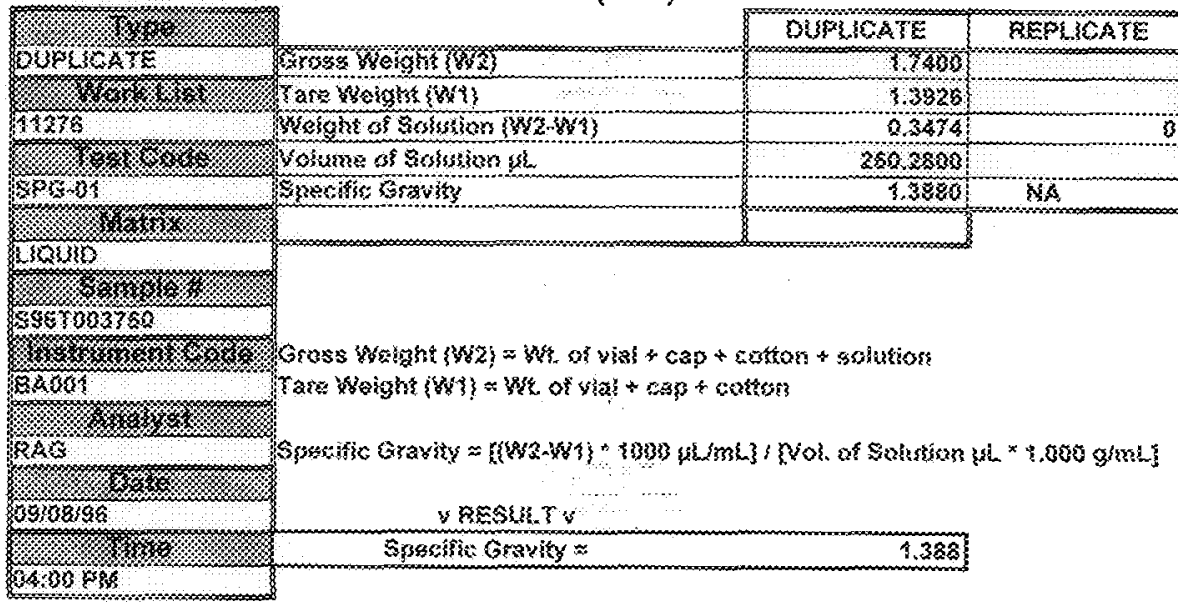

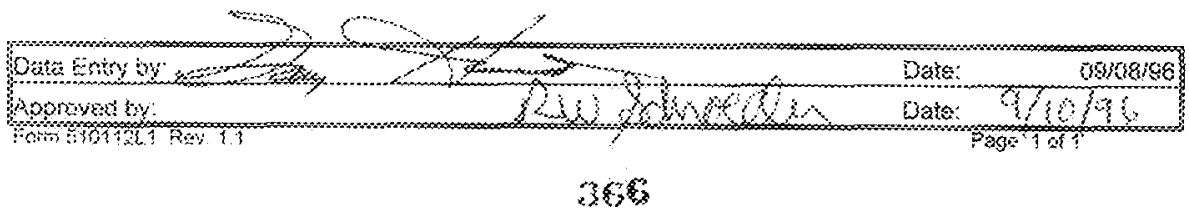




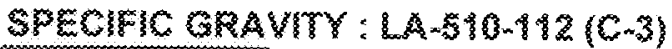

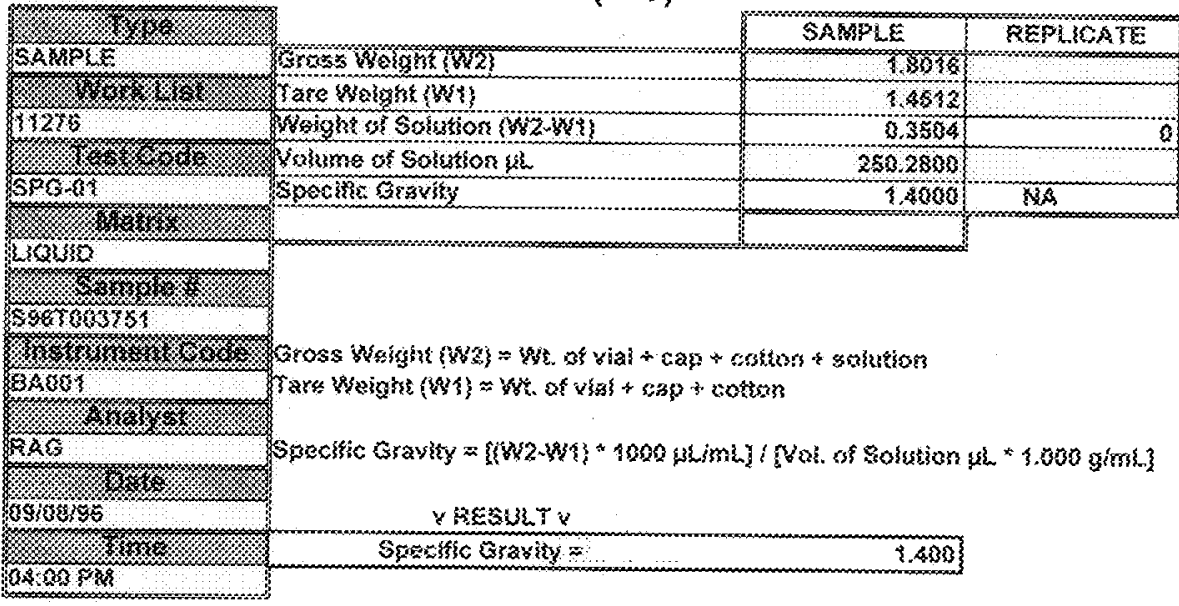

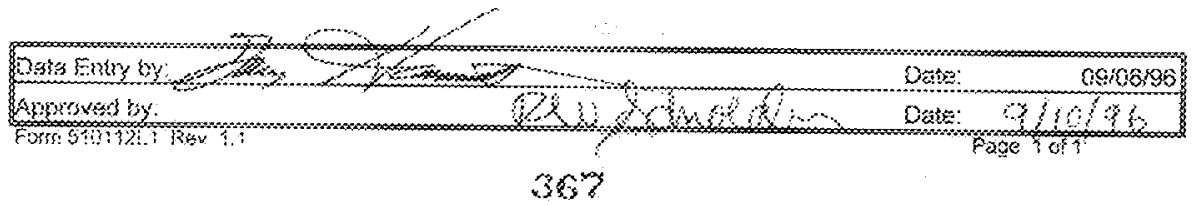




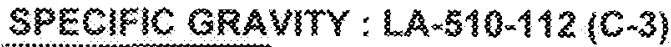

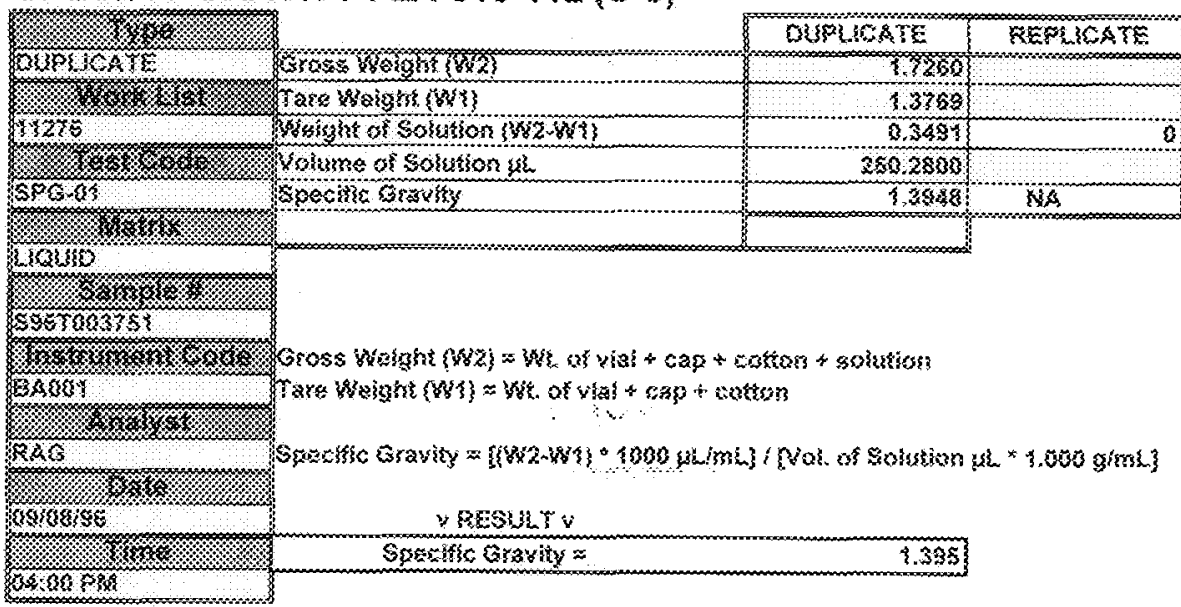

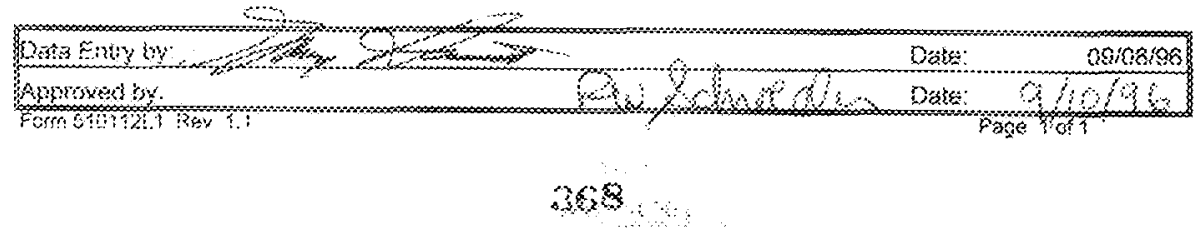




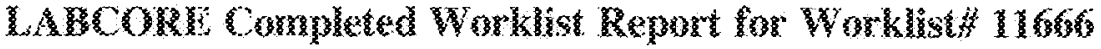

Arsalys: drat

3ass?wanent: BASB]

33 iso3k

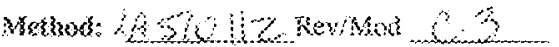

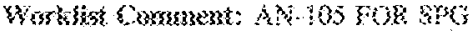

\begin{tabular}{|c|c|c|c|c|c|c|c|}
\hline $\operatorname{Soy} 3 y$ & \&sss5pso\% & $F B$ & lost & Mration & Xustos? & losusod & Thl w Kand Whit \\
\hline $2 x+x$ & & 3 & opos & 10000 & Iss & 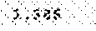 & $94,24<<$ 4रorosol \\
\hline $23 x+202 x$ & s5expossts & $\therefore$ & $\Delta x+20$ & išraro & $x / x$ & $\therefore 28$ & 2. 300.002 so.t. \\
\hline$s x$ & 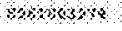 & $\mathrm{a}$ & sean & $+5 \times r k$ & oss & $2 s$ & a, so \\
\hline $4 \sin 28$ & sogrossing & 6 & son. 5 & $\cos x \cos$ & $4 x$ & 3.423 & $3.606-003$ as. \\
\hline$s+\infty)$ & $6000, s s \mathrm{~s}$ & \& & sese & tosss & $3 \times 3$ & shss: & $\sigma_{\alpha} \sin \phi$ \\
\hline $6 \operatorname{sinys}$ & 2500003578 & 8 & sod -4 & $\operatorname{sic}(0) 120$ & $x$ & $2 \sin 6$ & $\therefore \operatorname{cosec} 062$ sect \\
\hline ios & sssos, ss & a & sents & 20000 & $x<16$ & 483 & 2 psoss \\
\hline$a \sin 0$ & s)6rows? & $v$ & $\operatorname{sos}-6$ & $\operatorname{singat}$ & ols & 1.482 & $5.80 \mathrm{~m}-802$ 50.s. \\
\hline $2 \pi x y$ & 49000009 & 3 & $60-0$ & 3000 & 1402 & ats & $2, \alpha 2 \pi x^{2}$ \\
\hline is zatenses & zastoress & 3 & $2 y+0$ & wismen & Xis & 2. 32 & 2.390-392 so. \\
\hline$x h$ & \%or $\leqslant<\infty$ & 9 & $x+2-2$ & hot & $2 \mathrm{~s}<$ & 2,8 & s pr pps \\
\hline is $\$ 3 x+2 y$ & saropsos & 3 & $\operatorname{sis} \cdot n t$ & oxun & six & 2.254 & $3040-30$ son: \\
\hline 23,20 & 606064848 & $a$ & $x<<$ & $x<0<s$ & $30 s$ & $4<$ & 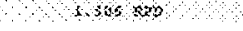 \\
\hline $2 x 6 \times 20 \times 2 \pi$ & 500000503 & 5 & 600-iss & rxoss & sis & 3.423 & $1.604-605$ \\
\hline shos & gs rrossos & 3 & toys & 0,50220 & ss $x=$ & $1 x 0$ & a, s o w \\
\hline
\end{tabular}

Analys Sigenture




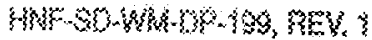

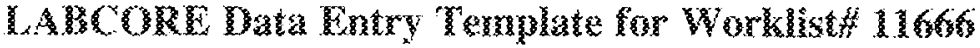

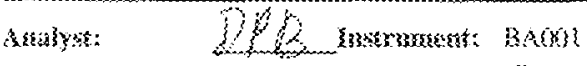

3ookt: $(33), 6 \cdots$

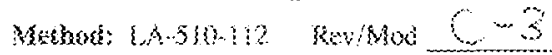

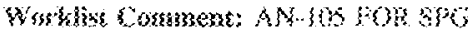

\begin{tabular}{|c|c|c|c|c|c|c|c|}
\hline$s$ & $79 \mathrm{se}$ & Bagket & 328 & TASE & xarrix & Gsoug & Projent \\
\hline$\vdots$ & $\omega$ & & & $31 \times 0 \cdot 6 i$ & W00\% & & \\
\hline 2 & SNRE: & 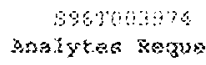 & 0 & $\begin{array}{l}5,001 \\
=860-53\end{array}$ & $3 x \times 0$ & 36000945 & $2 \mathrm{Nr} \cdot 206$ \\
\hline$\therefore$ & $m e$ & $5900039 \%$ & 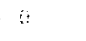 & $\sec \cdots 2$ & 4. & & \\
\hline+4 & $\operatorname{santR}$ & 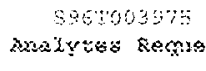 & $\operatorname{sostax:}$ & 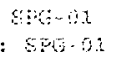 & 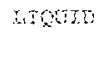 & 90000985 & $288-205$ \\
\hline$\because:$ & $n$ & 596000975 & 0 & $5390-0 !$ & monts & & \\
\hline 6 & SAME: & 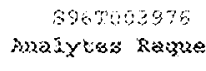 & ogtga: & 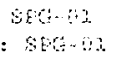 & 3.0\% & 96000945 & An- $26 \%$ \\
\hline$\because$ & 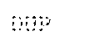 & Eorogong & $\dot{0}$ & $8 \times 3 \cdot 0$ & $6,1 \infty \mathrm{m}$ & & \\
\hline 8 & SANE SE & $\begin{array}{r}586006 \% \\
\text { snarytas Regue }\end{array}$ & $\theta$ & $\begin{array}{l}5603 \\
=80003.03\end{array}$ & Lipon & $2 x 6000935$ & $23-205$ \\
\hline 8 & oc: & 856000397 & 0 & and & E.\$OLE & & \\
\hline & \$लि̈ & 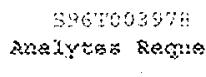 & Ostat: & $\begin{array}{l}4 \mathrm{sen} \\
=\mathrm{spot}\end{array}$ & rrom: & 36000965 & 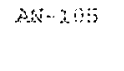 \\
\hline & WOE & s5emono?:3 & 0 & $5 n-0$ & Suptos & & \\
\hline$\%$ & SWMEs & 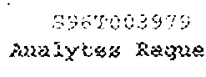 & estaci: & $\begin{array}{l}5 \mathrm{e}-43 \\
=\sec -\mathrm{at}\end{array}$ & 30000 & 36000945 & $\sin -268$ \\
\hline 3 & $m$ & $\operatorname{sectos979}$ & 0 & $(2: 3 \cdot 0 !$ & 600\%: & & \\
\hline & SAxtre: & $\begin{array}{r}396000600 \\
\text { malytag Rewae }\end{array}$ & ouked: & $\begin{array}{l}80001 \\
=\$ 80.03\end{array}$ & 2000 & 00000045 & $26-.205$ \\
\hline$\therefore$ & $6 !$ & stmonseso & & $200-01$ & EWOEL & & \\
\hline
\end{tabular}

Bera Enty Comowns:

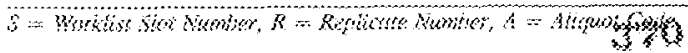




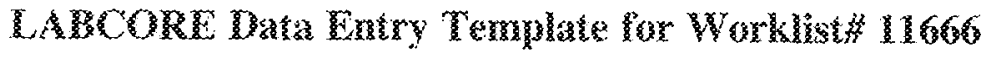

s xyo

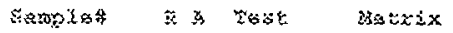

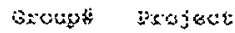

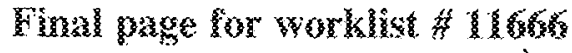
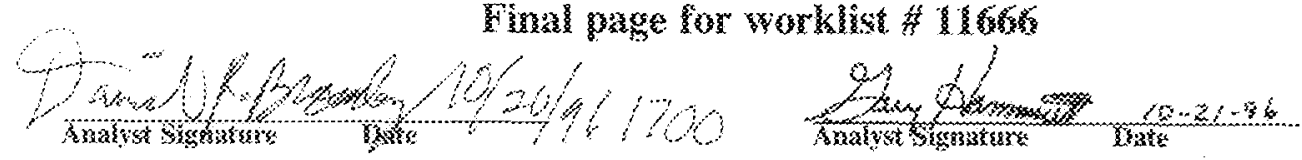

Dou chry Cownoma: 


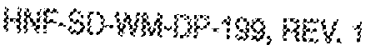
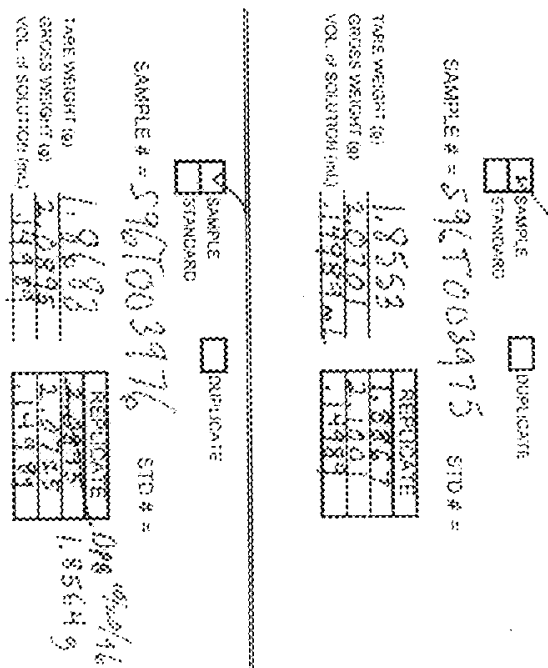

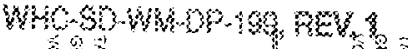

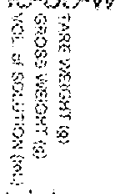

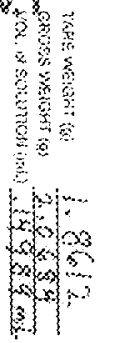

(a)

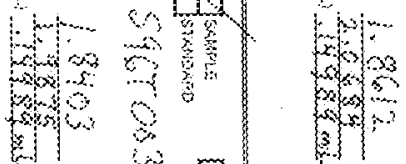
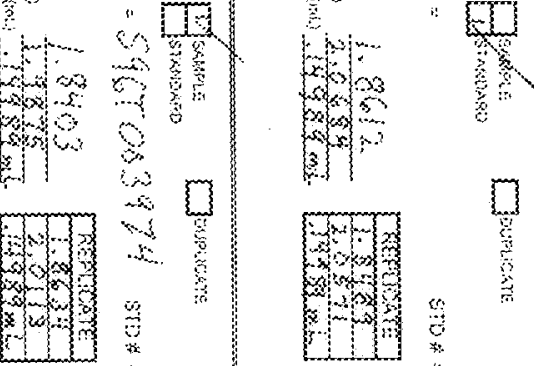

(n)

8

空

H.

(x)

$x\} 2,3)$

0

\%

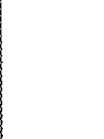

$\frac{1}{2}$

in

$\frac{1}{6}$

\%

ard

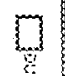

3

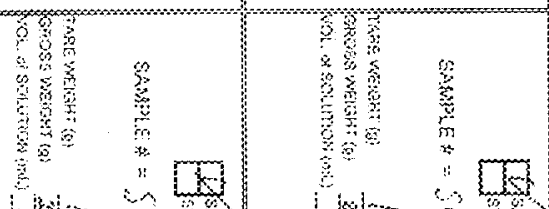

i.

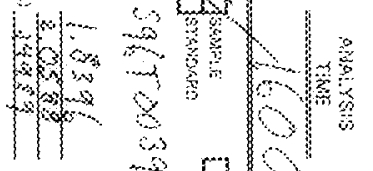

lwen tos

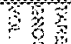

$\therefore<$

85

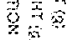

$\begin{cases}\infty & 4 \\ 0 & \infty\end{cases}$

6x

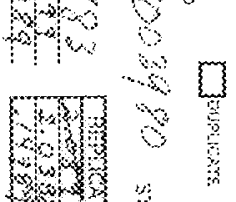

\section{.}

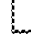

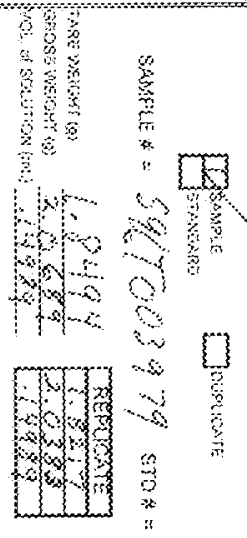




\section{SW}

\begin{tabular}{|c|c|c|c|}
\hline $\sec x \sin$ & & STANSSARO & STAMBSARA \\
\hline $6 r \times k \alpha$ r & 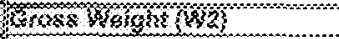 & onomoxom & 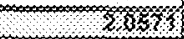 \\
\hline \% & 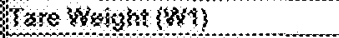 & 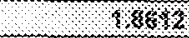 & os \\
\hline 1365, & 8 Waighs or solusiom $|X| 2-1 / 3$ & 3.3032 & 0.2082 \\
\hline 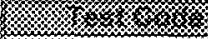 & Solussig of Solutson yl & $18,8,30$ & 48,880 \\
\hline $3 \% 31, \ldots$, & oboctsc Grarts & 3323 & 1,3850 \\
\hline Ky.x & 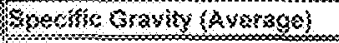 & $3.35 y$ & \\
\hline
\end{tabular}

\section{US 3 ins \\ \%}

H3
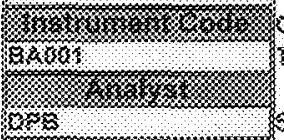

30

$\% \% \% \%$

atosis

$8 \%$ on

$3.33)$

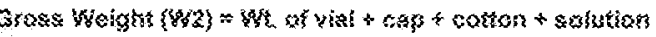

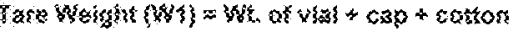

Y HESULTY Spexiflc Gravity Averame :

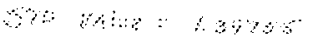

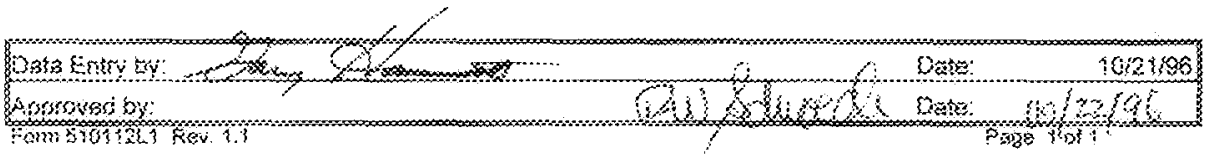


SPECIFIC GRAVTY : $\{$ A

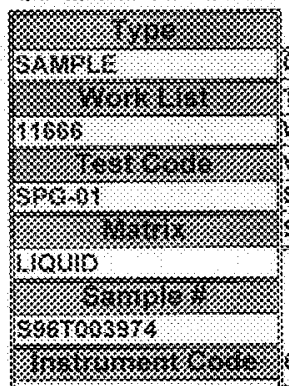
(3.003 $1000 \% \% 0 \%$

(o,

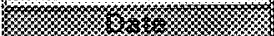
102015

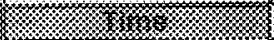

S61015 \%

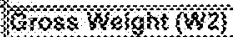

sure Woighe (AA)

Wang of solution $\{(12-64\}$

vensme of solution yh

Snowisc Graving

Swoths bravity (Avarage?

YRESULT

Speriffe Grovity Average :

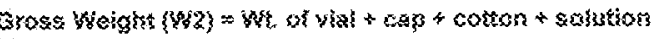

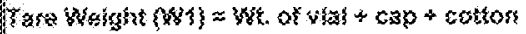

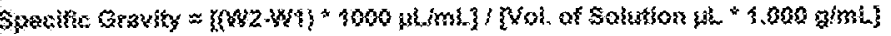

0.38 .8

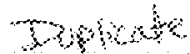

pxis \%

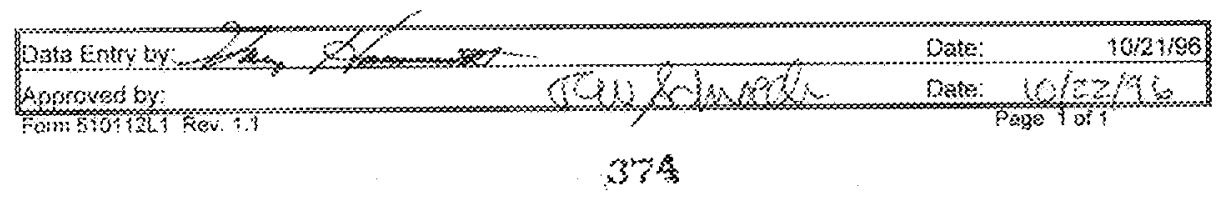




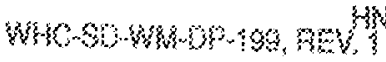

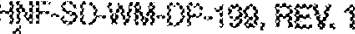

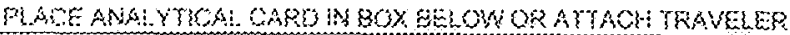

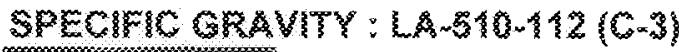

\begin{tabular}{|c|c|c|c|}
\hline $3 \mathrm{CHFEG}$ & 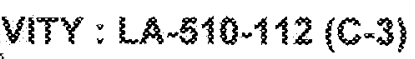 & & Ponses \\
\hline 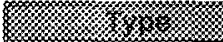 & & SAMPLE & Heptonth \\
\hline $2188 \times 4=$ & 篗 & 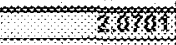 & / 101 \\
\hline res & (sore Wosight (WOS & 3888 & $138 \%$ \\
\hline (1) S S S , & Selph of solution $($ W $2+161\}$ & $323 \times 8$ & 0.2130 \\
\hline 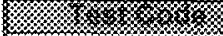 & Voriusse of zolustom pl & 108,850 & 148.8580 \\
\hline $5 /, 31, \ldots$ & 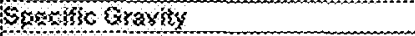 & 3.8331 & 3.424 \\
\hline 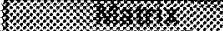 & 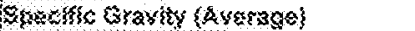 & 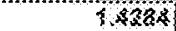 & \\
\hline
\end{tabular}

Susus

$10 \%$ os

235013335

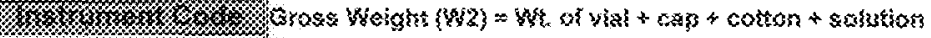

82005

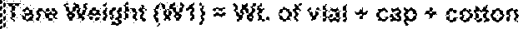

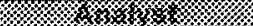

S0:

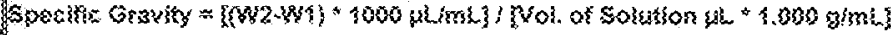

10308s

$\checkmark$ REQSLT Y

\$ $50 \%$ \%

Epesitic Gravity Average :

3.423

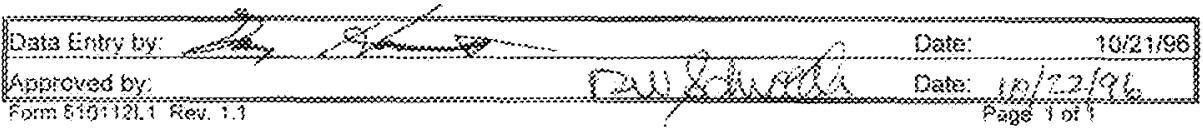




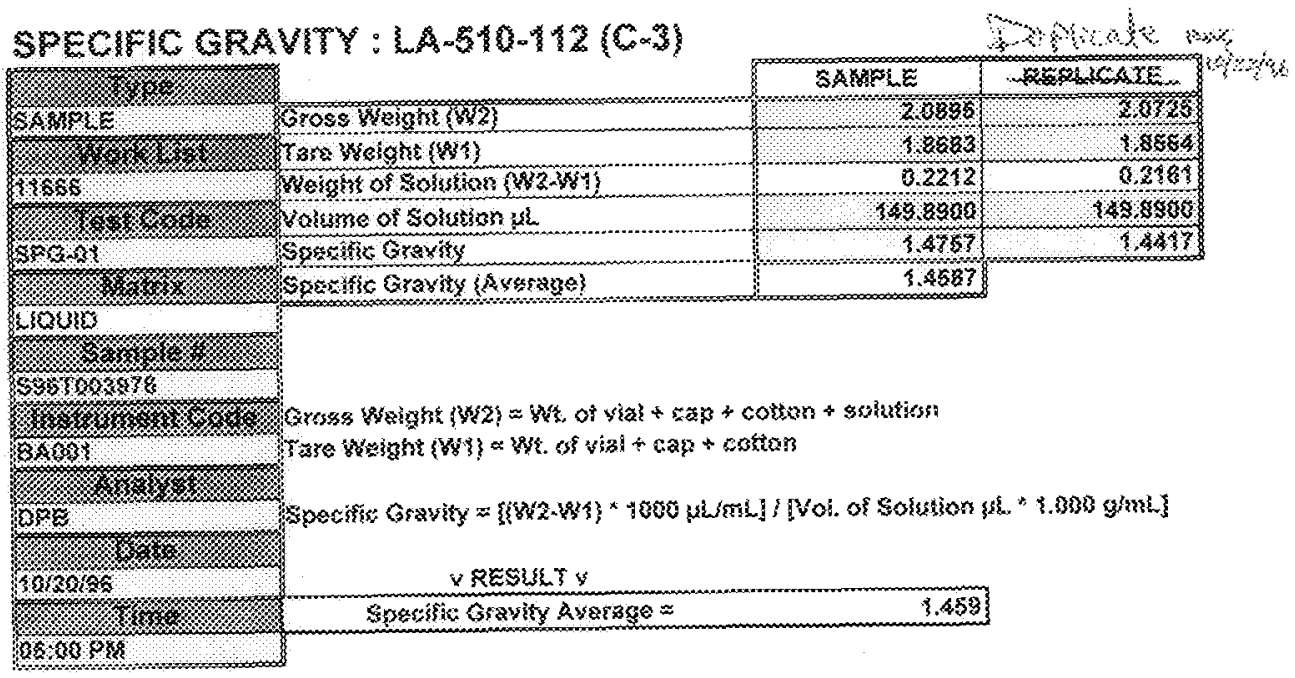

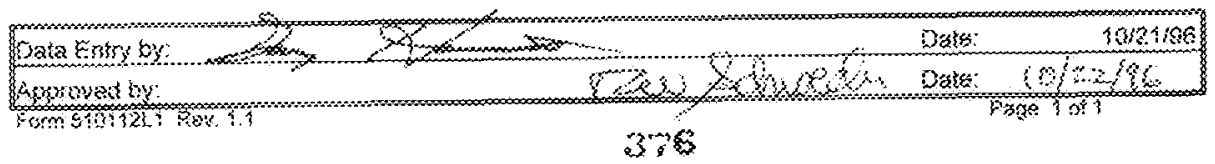


SPECIFIC GRAVTY : LAASTOH.12 $\{C-3\}$

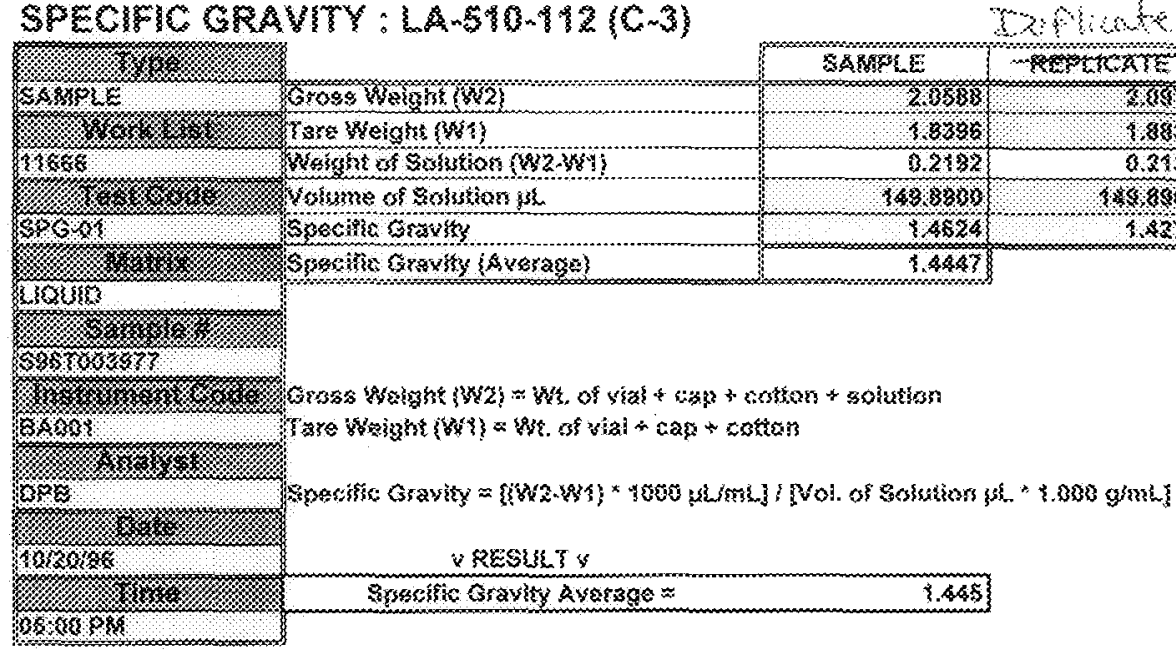

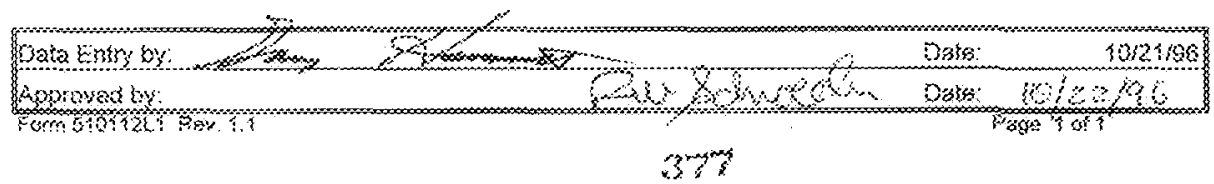


SPECIFIC GRAVIS: :

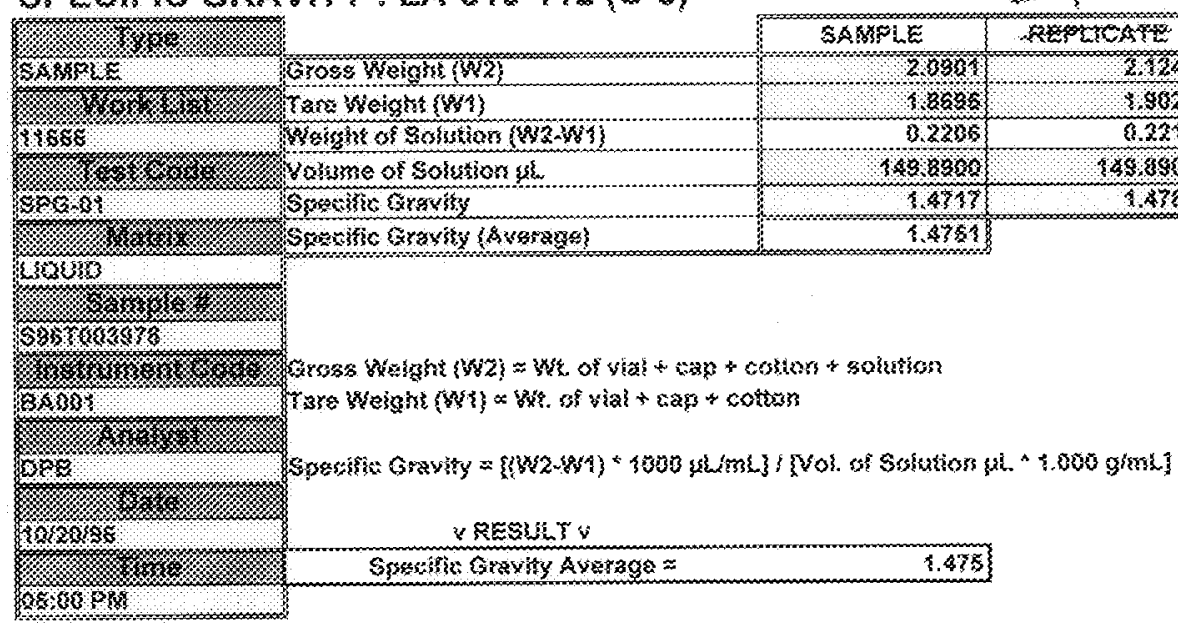

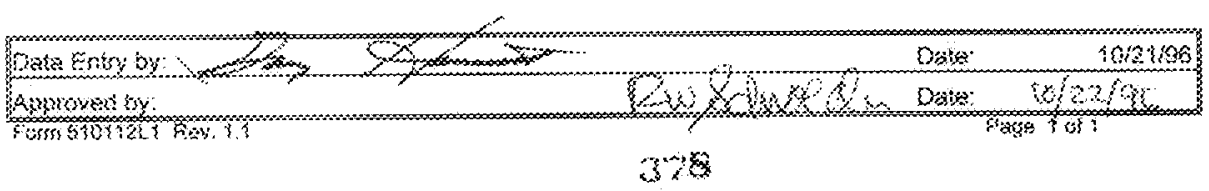



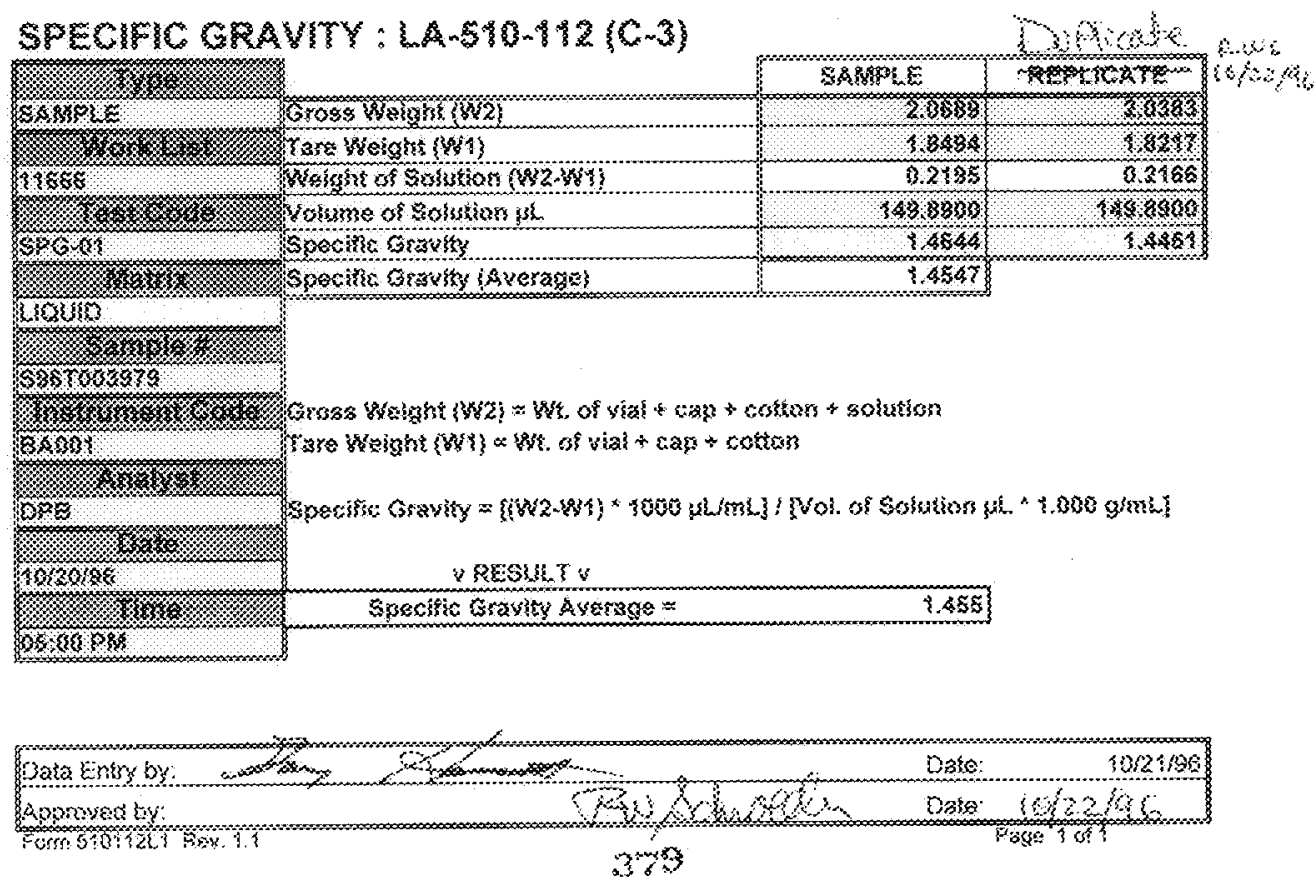


\section{SPECHFC GRAVIT: :}

\begin{tabular}{|c|c|}
\hline \$ 8 BP, & Gose Weingt (r) \\
\hline \%) & 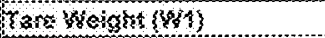 \\
\hline S 8 \& & 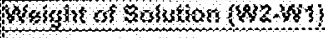 \\
\hline 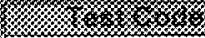 & golume of Solspiom gh \\
\hline $80 \%$ & Sgocinas grovss \\
\hline 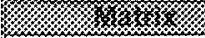 & Syoukic Qrovity (Averages \\
\hline
\end{tabular}

\section{3}

\%

35150\%6580

\%

s.001

\%. . $\ldots \ldots$

s.

39

\%

106008

\%

W.

8

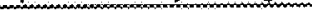

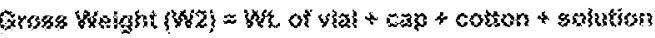

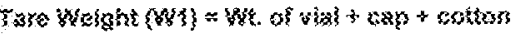

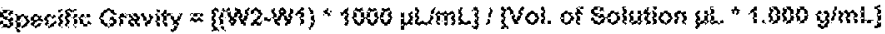

V RESUL' 4

Sostsilie Gravity Aversae $\approx$

3.413

\section{(1)}

mol

SAAYHFL

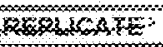

2033!

38383

B.115

38,500

36113

1.453\%

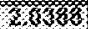

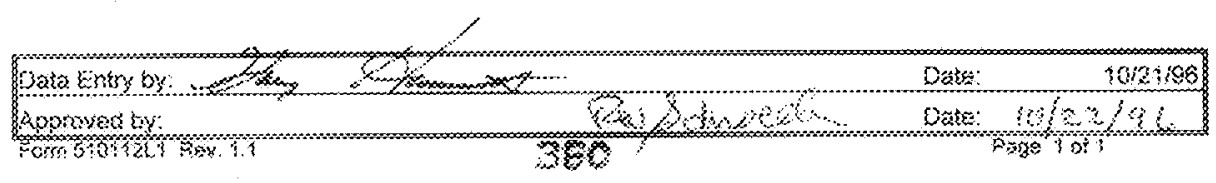




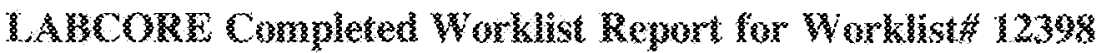

Ansight: ear

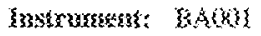

Powstrin

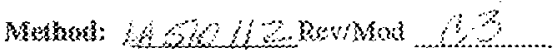

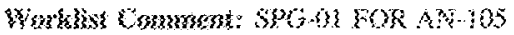

3rs?

\begin{tabular}{|c|c|c|c|c|c|c|c|}
\hline $\operatorname{seg} s y 5$ & Samples & $3 \mathbf{B A}$ & $3 \cos$ & Matrix & Antwis & Fossme & M or rete lasst \\
\hline $\mathrm{ses}$ & & 2 & $282-8$ & boos & 2390 & 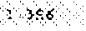 & $96-898 \times<0 \times m$ \\
\hline is 36002 & $390 \times 306000$ & $n$ & stong & buxtres & $\% / \beta$ & 2.230 & :0ne-0nz so. \\
\hline$\geqslant 0 \times 0$ & 800000,00 & 3 & $300+2$ & stक & $x_{0} \alpha_{2}$ & Ass & n $25 x$ s \\
\hline 4 suspes & 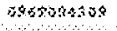 & $\sigma$ & $58 i-62$ & $\sin \alpha \cos 20$ & $x / x$ & 5.368 & 2.nEस-002 $520 \%$ \\
\hline $000 \%$ & 000000 & 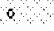 & $60-0 \mathrm{~s}$ & issorite & sos & 2068 & n n>p \& \\
\hline s soverty & sustost3ys & 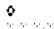 & $090-62$ & $5 \times 000$ & $\$<\ldots$ & 2.500 & $2.200-203$ so.s. \\
\hline 7 ाण & Arsorosss & 2 & $28<0$ & 2400 & 080 & 48 & 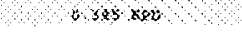 \\
\hline 8 SA6S:; & 32505064t & $n$ & stend & zapers & $x / 3$ & 1. 454 & : one-ose spo. \\
\hline 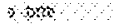 & tojobnsas & 6 & $800+2$ & $3+\cos 2$ & 648 & ass & $0,0<\operatorname{sos}$ \\
\hline
\end{tabular}

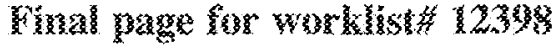

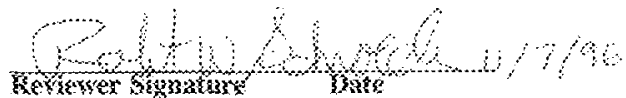




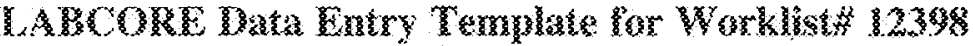

Assilyst: $\quad 2 / 6$

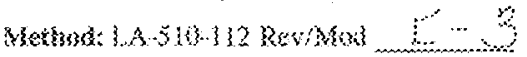

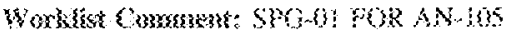

QIS\}

\begin{tabular}{|c|c|c|c|c|c|c|c|}
\hline \multirow[t]{2}{*}{ SसQ } & Basen: & $S$ iYse & 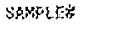 & $* 0$ & 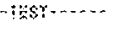 & mink & setse: \\
\hline & & $15 r 0$ & & & 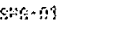 & :OWis & 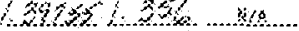 \\
\hline $9800 \% 45$ & $9 x \cdot 405$ & 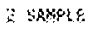 & $599 \times 004368$ & 6 & $5 \% 0 \cdot 01$ & aivan & $\frac{3<}{2}<2,200$ \\
\hline aobous & $20-40$ & $300 \%$ & serow300 & ; & $\sec 6$ & a:s:3:3 & 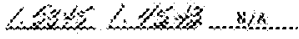 \\
\hline $200686 . \overline{1}$ & $n N \cdot 36 t$ & 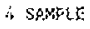 & 0560004305 & 8 & 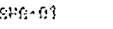 & WOMT & 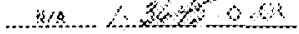 \\
\hline 5600045 & a) $\div$ : & 30 & $\operatorname{sisrog} 4309$ & 0 & $596 \cdots 1$ & 1iono & 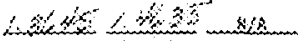 \\
\hline $56060 \% 3$ & $2 k-10 \%$ & $5 \sin 2: 25$ & Spirnoisss & b) & $\$ \$ 6-81$ & mons & $x+\alpha \beta b \theta$ \\
\hline 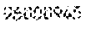 & $\mathrm{AB}-3 \mathrm{OS}$ & $7 \mathrm{DIF}$ & 5563034333 & 0 & 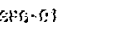 & Lois & 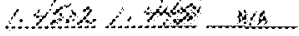 \\
\hline Geromsus & $B x-30 s$ & $8 \sin 40 . \varepsilon$ & 926500649 & 0 & Swa & k:ones & 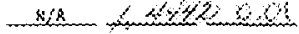 \\
\hline $56060 \%$ & $A 3-15 \%$ & aste & sostoust? & is & $\operatorname{seg}-83$ & s.sine & 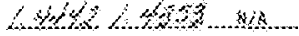 \\
\hline
\end{tabular}

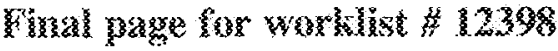
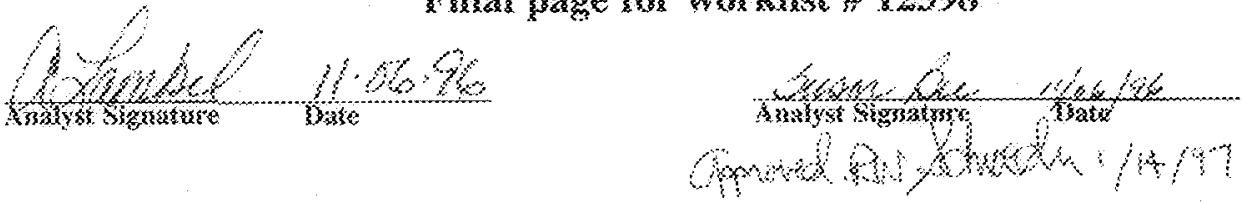

Trac knty Cownons:

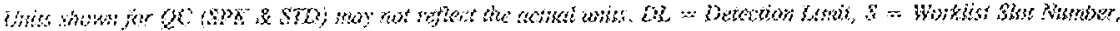

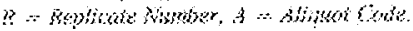




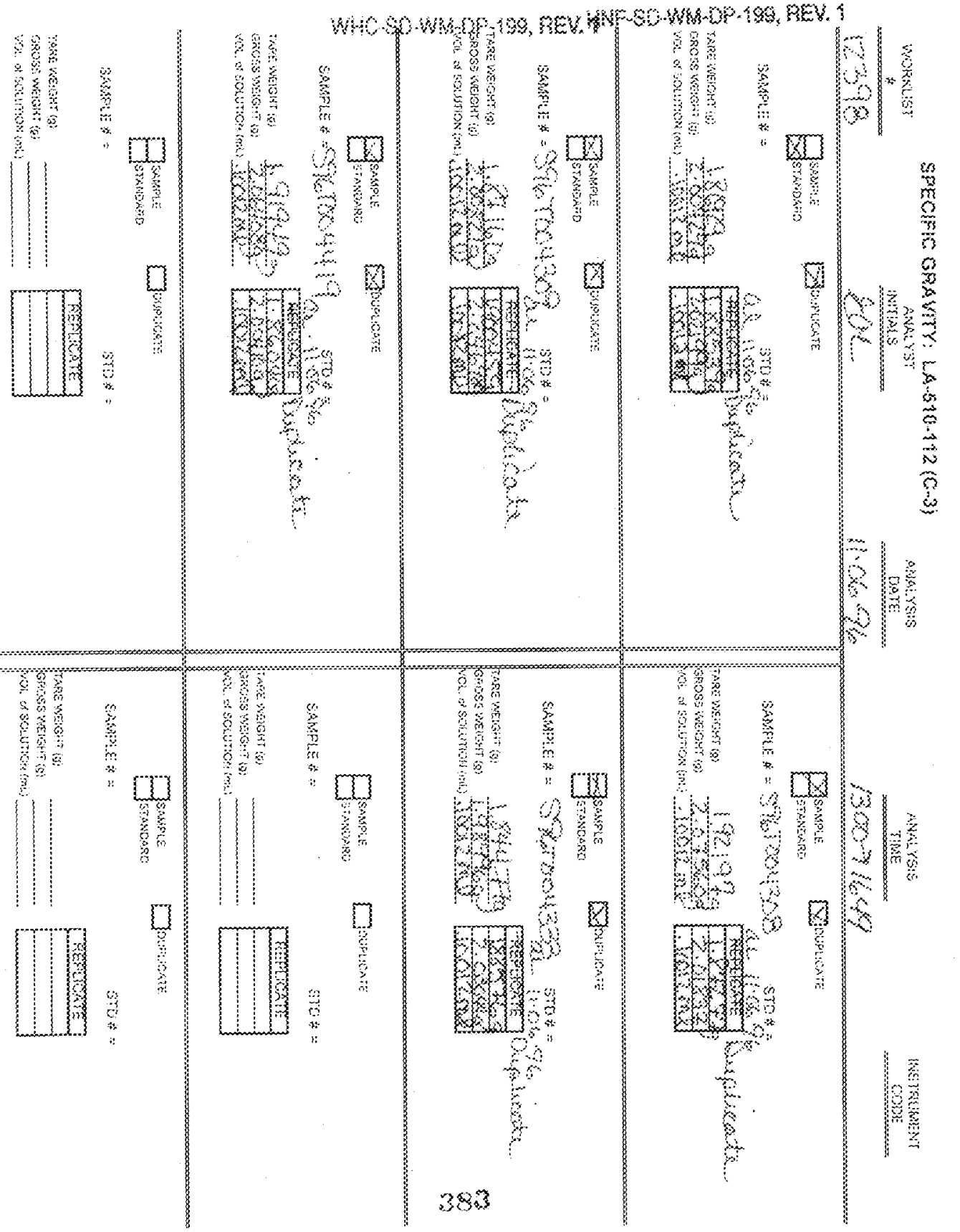


$\frac{1}{169}$

: $3 \% \& \mathrm{M}$

:

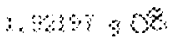

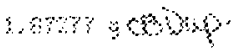

$\therefore \operatorname{xखs} 30$

अ.

सेखी 33

a.8. $3 \mathrm{a}$

म. केष $\because$ ?

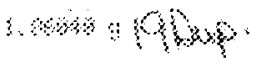

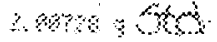

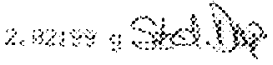

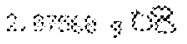

2. $84080 \%$

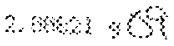

2. मूल

$\therefore$ क 23

ब. बक्ये

2.638 \&

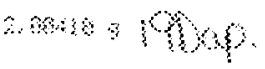




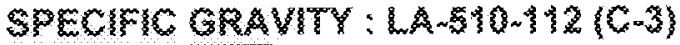

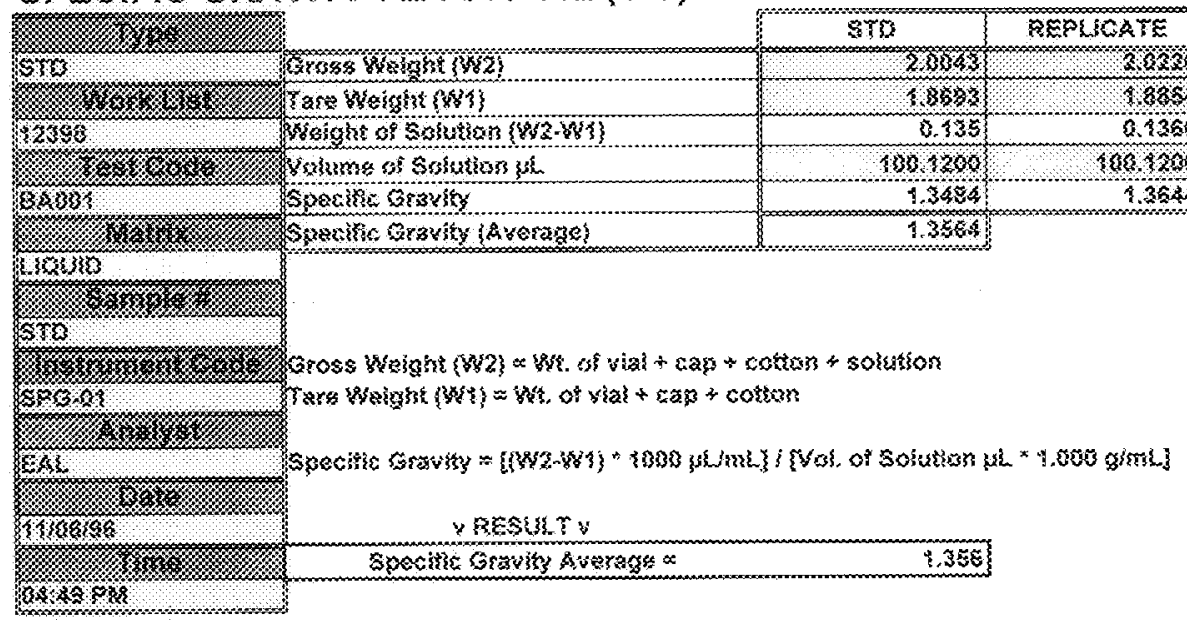

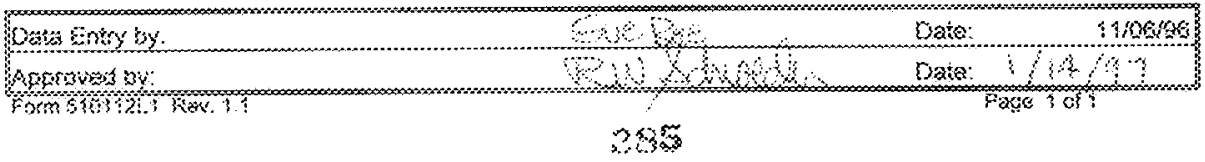




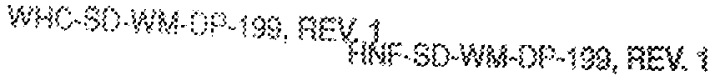

PACE ANAE YBCAL CARD IN BOX BELOR OO ATTAOB TRAVELEK

\section{SEX}

\begin{tabular}{|c|c|c|c|}
\hline צr & & GABYPLE & REPLLATE \\
\hline 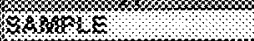 & 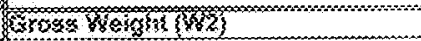 & .... $20 \% 8$ & \\
\hline 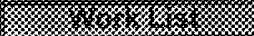 & Trase saight (by) & $18<8$ & \\
\hline C488 8 & 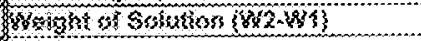 & 2.38363 & \\
\hline 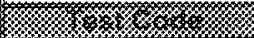 & Wossme of Solustion ig & 001300 & \\
\hline shom & Spesifo Braplo - & $\frac{1848}{164}$ & Wh \\
\hline 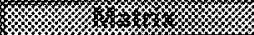 & & & \\
\hline 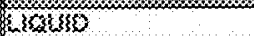 & \multirow{6}{*}{\multicolumn{3}{|c|}{ 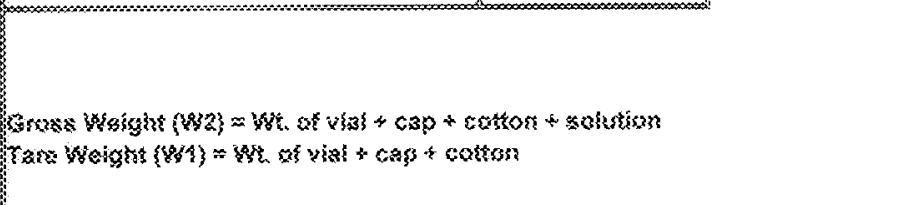 }} \\
\hline 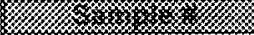 & & & \\
\hline 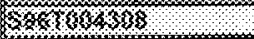 & & & \\
\hline 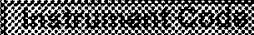 & & & \\
\hline 403 S & & & \\
\hline 济 & & & \\
\hline $36, \ldots$ & \multirow{2}{*}{\multicolumn{3}{|c|}{ 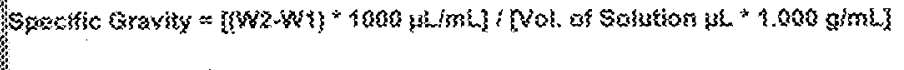 }} \\
\hline 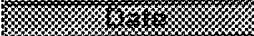 & & & \\
\hline $1108 \% 5$ & \multicolumn{3}{|l|}{ Y Besul $T y$} \\
\hline ry & Spreatifo Grspriky $\approx$ & 1.634 & \\
\hline $4 \times 63 \%$ & & & \\
\hline
\end{tabular}

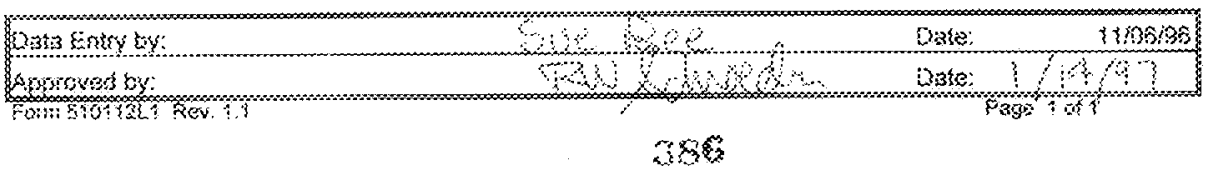




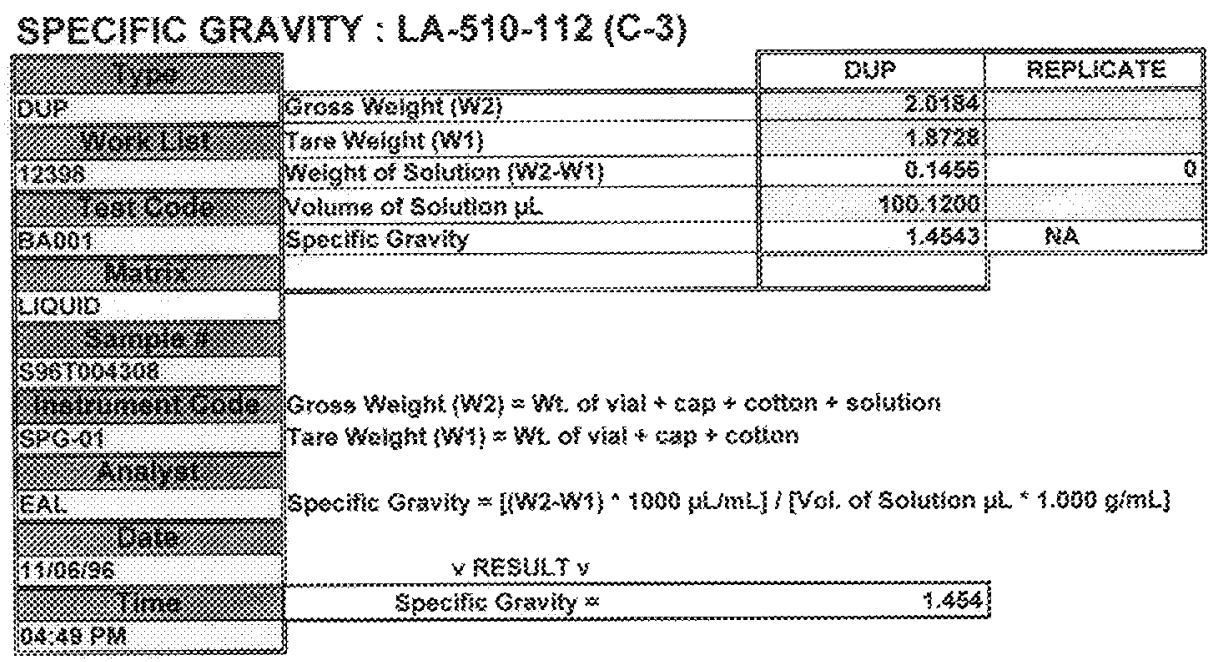

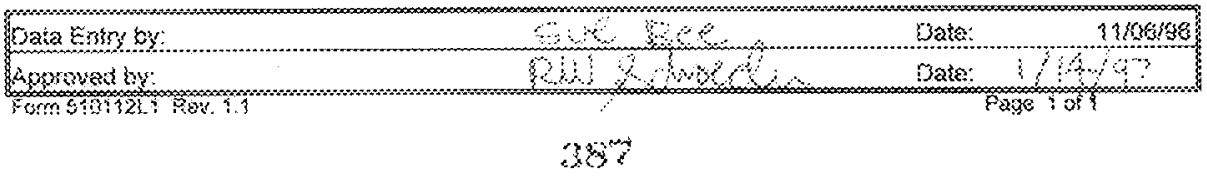




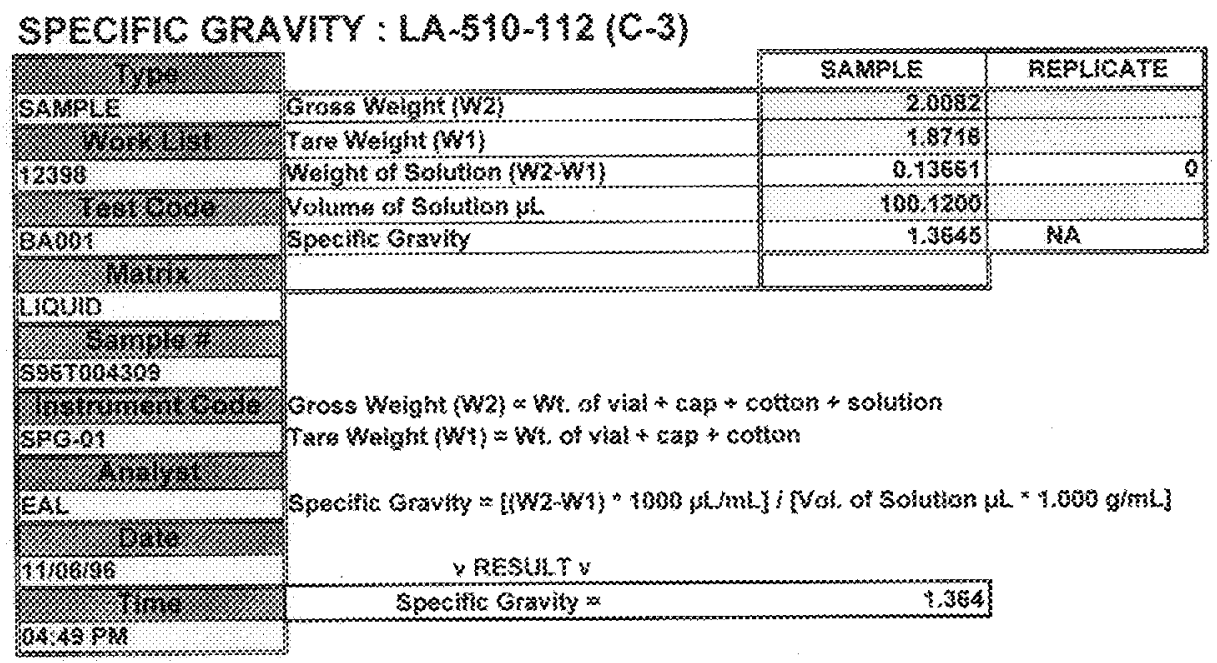

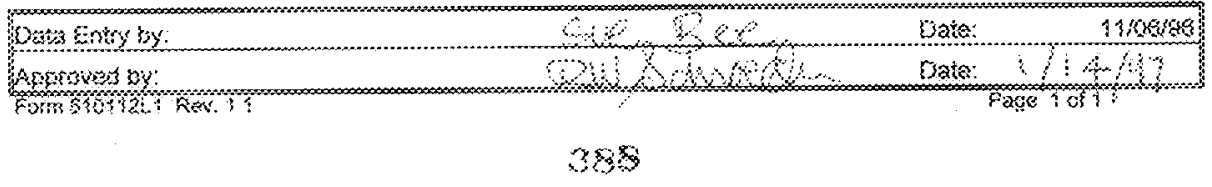




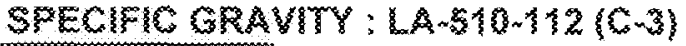

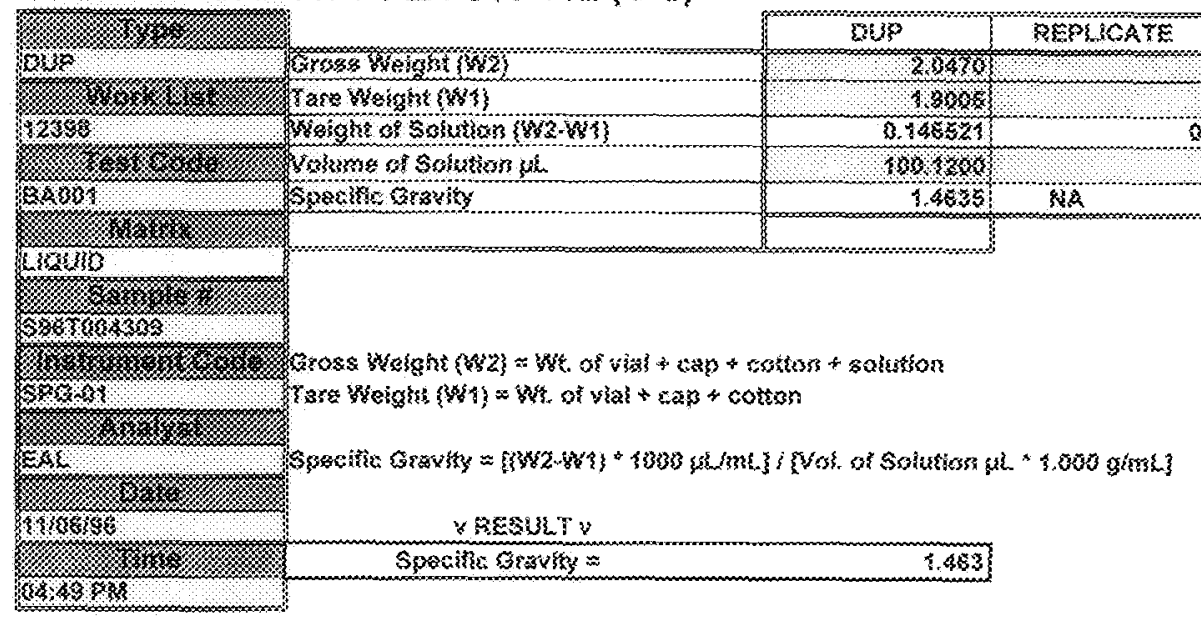

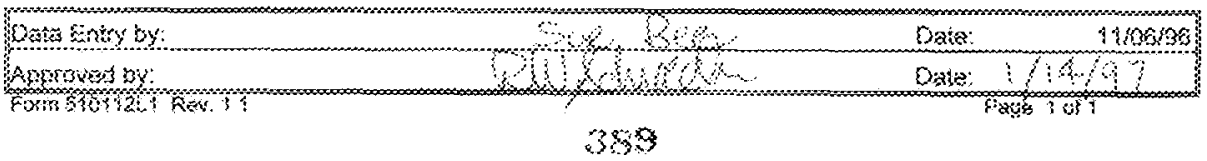




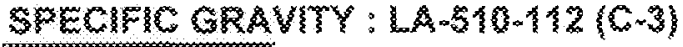

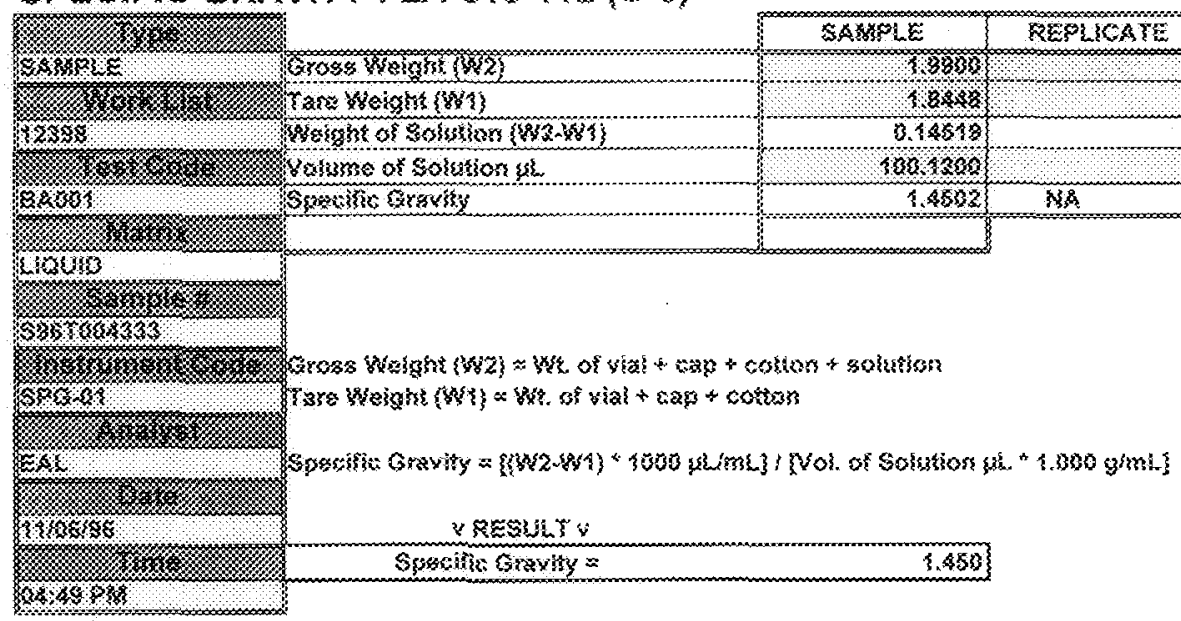

\begin{tabular}{|c|c|c|c|}
\hline Data ensy by: & 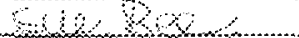 & Oabs: & sitgses \\
\hline comoves by & 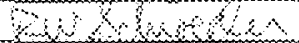 & Oat: & $1 / 4, y^{\cdots}$ \\
\hline
\end{tabular}




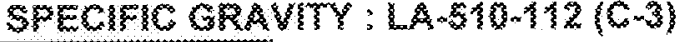

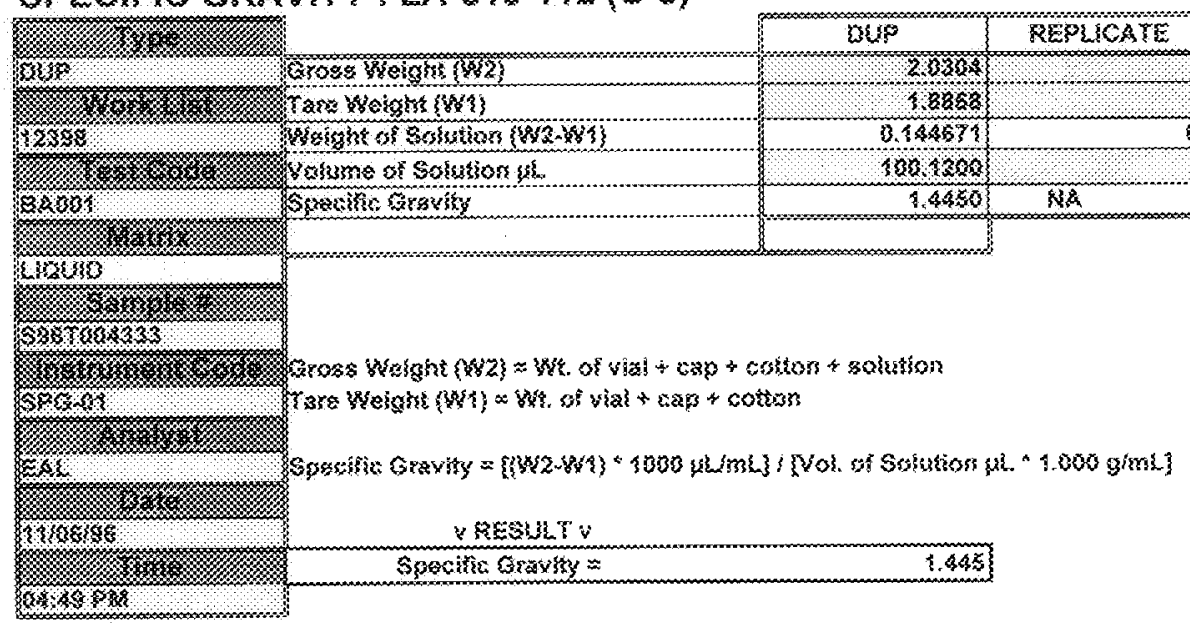

Oxtantey:

Apowsiged by:

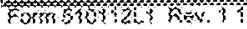

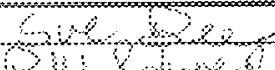

Oate: $11 / 8008$

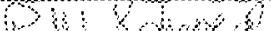
$\therefore$

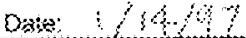
fists 3 if?

$$
303
$$


SPECIRG GRAVITY:

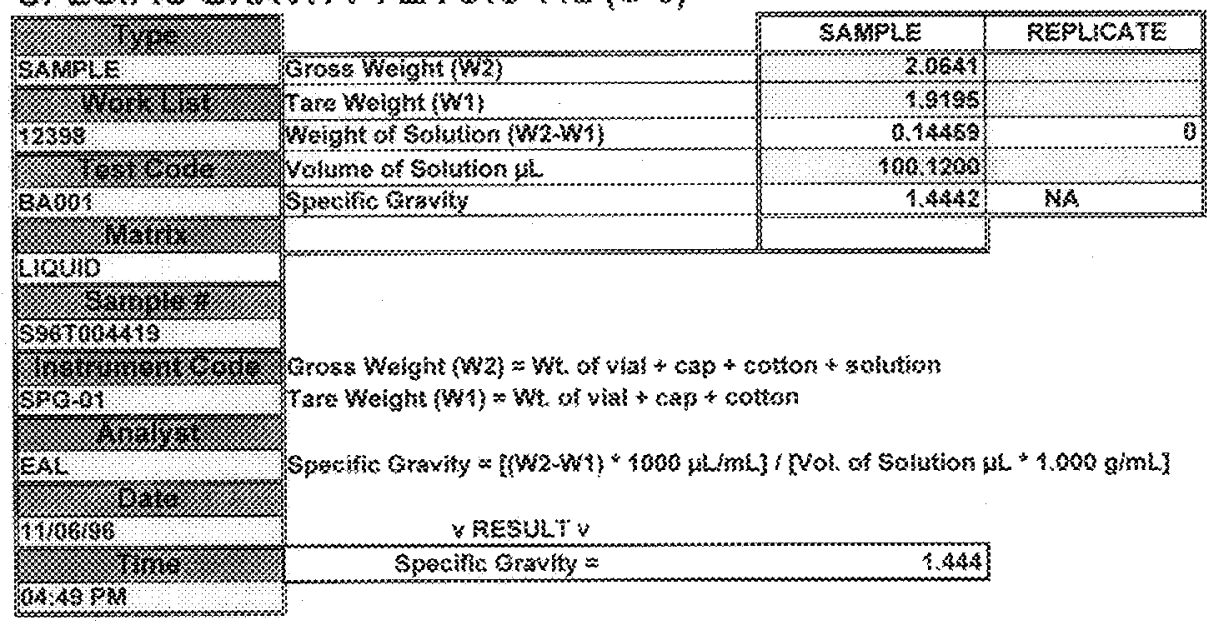

\begin{tabular}{|c|c|c|}
\hline Oous frnes by: & $2 y$ & $1 / 106 / 36$ \\
\hline Aqqroves oy: & $3: Y, 3<+4$ & 9 \\
\hline
\end{tabular}




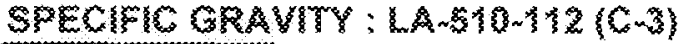

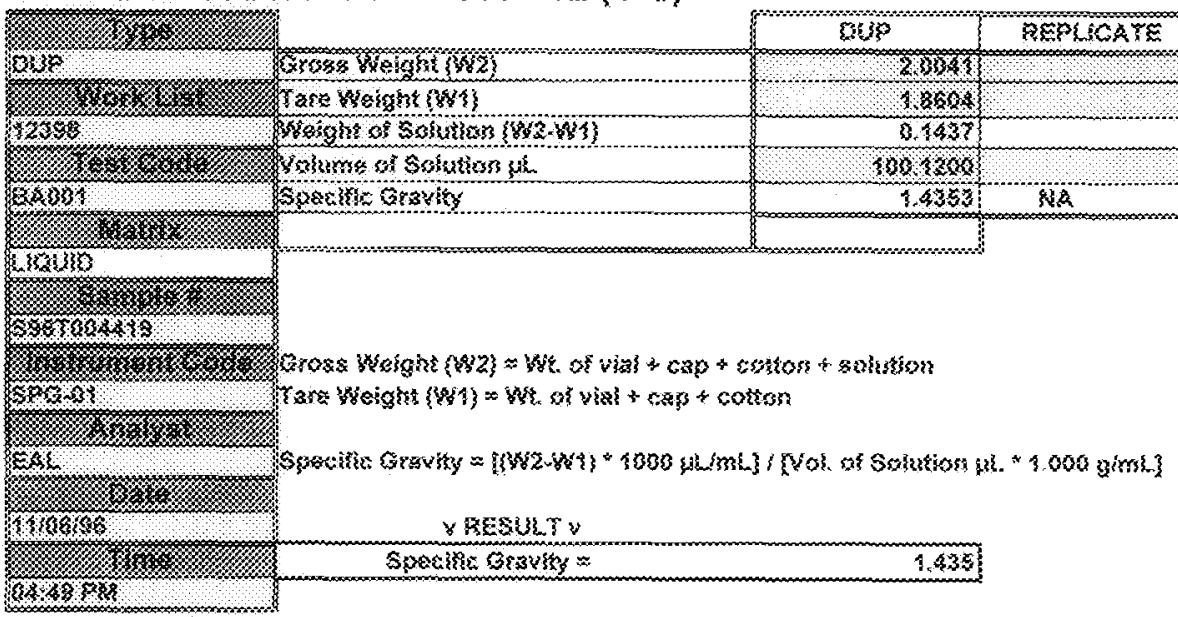

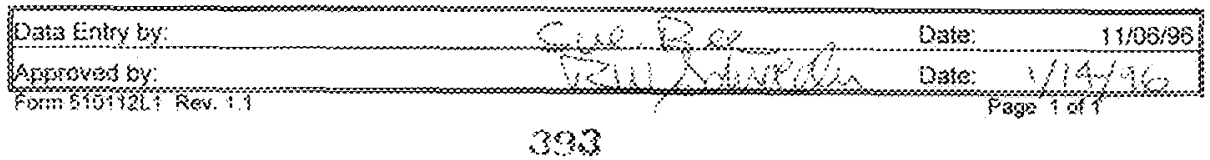




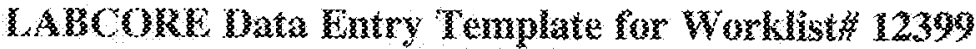

\section{B.tuksy:

$$
\Leftrightarrow>
$$ \\ Sustrsment: BSA601}

36r: 3323160

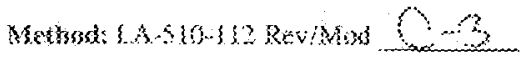

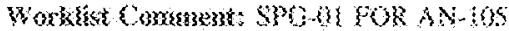

ETS?

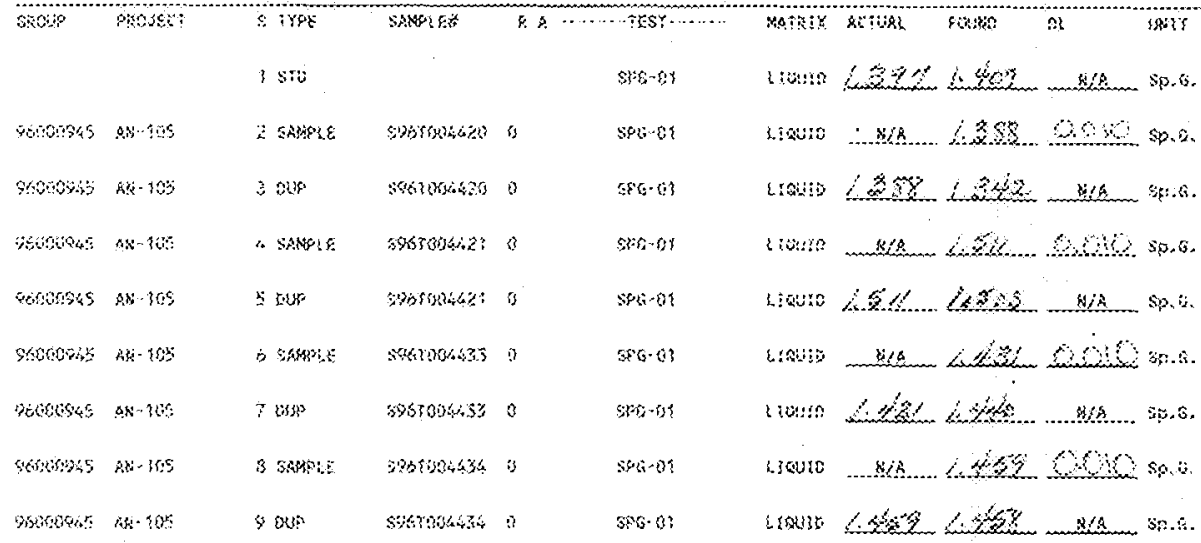

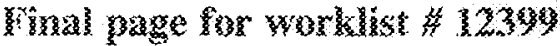
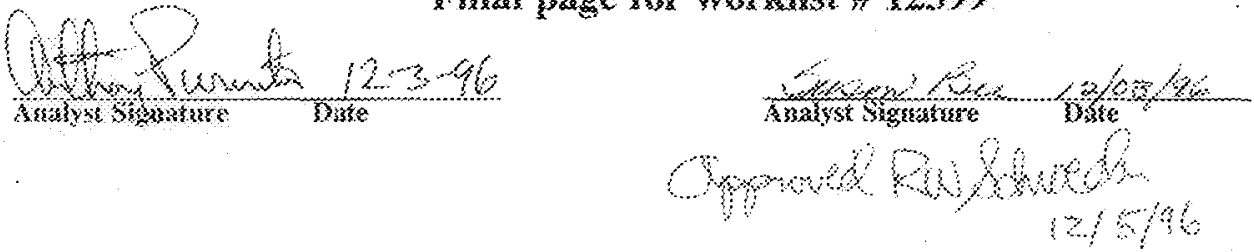

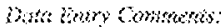

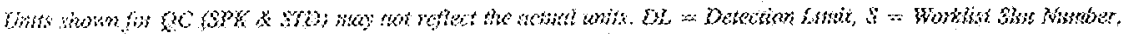

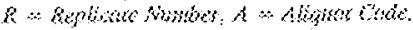




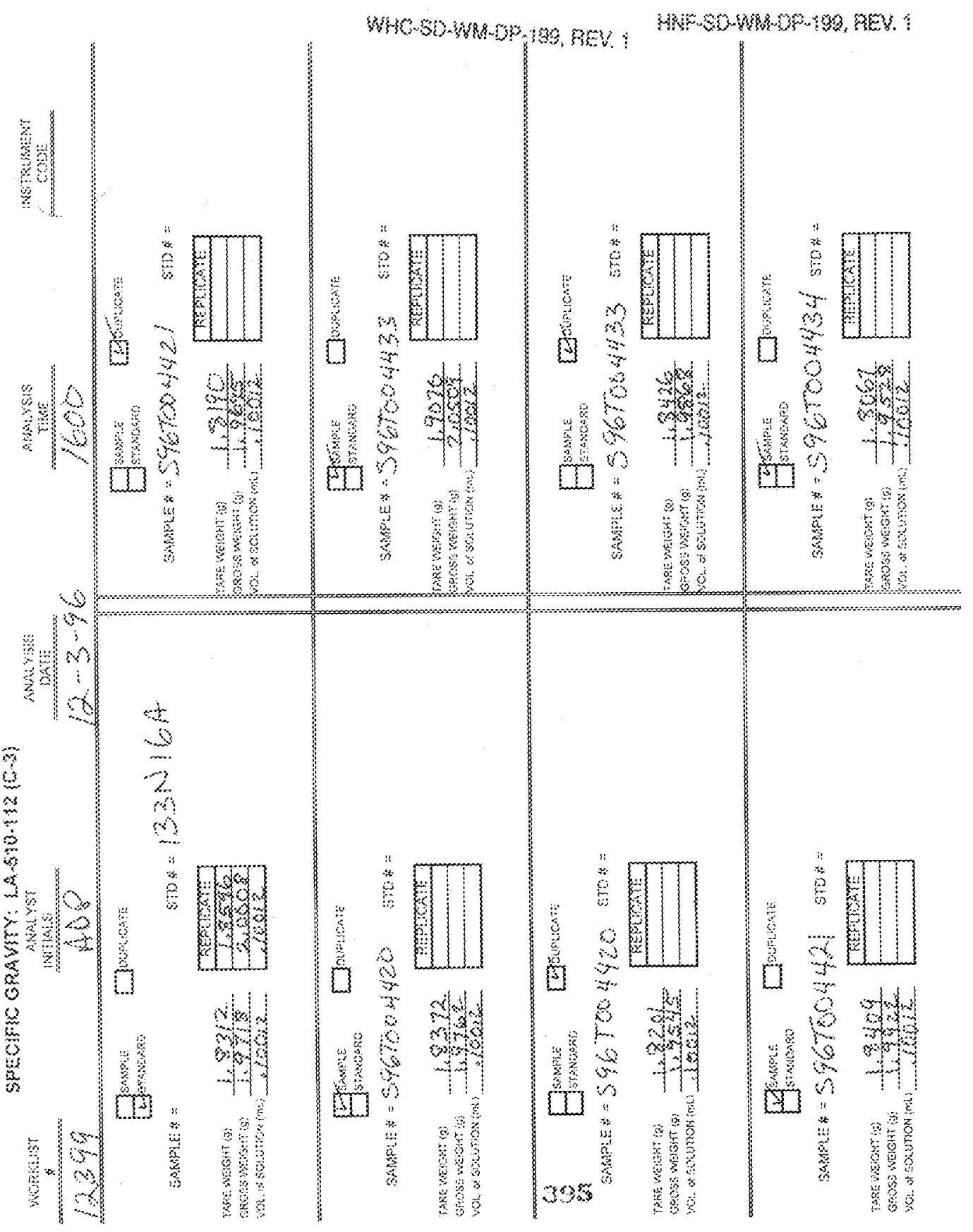




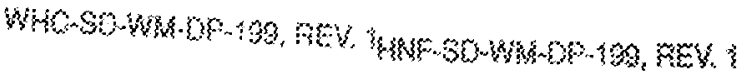

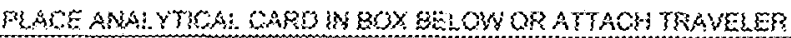

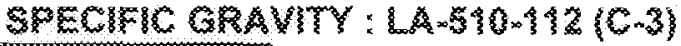

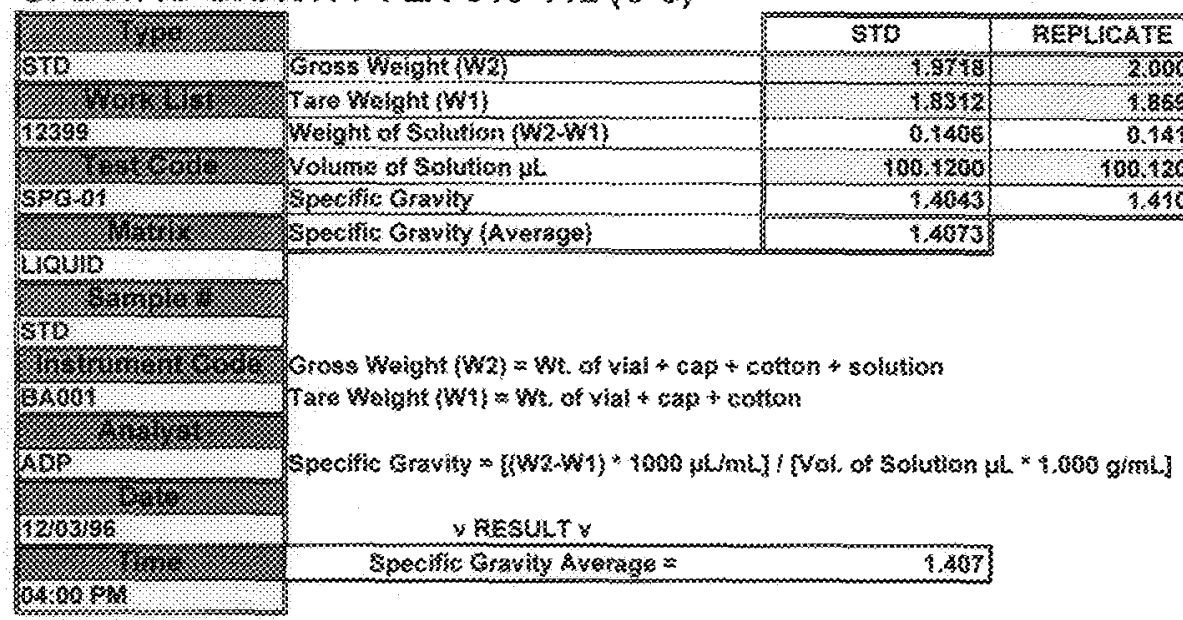

\begin{tabular}{|c|c|c|}
\hline osses sno sy: & Gare: & 12030 \\
\hline sponoses by: & Sain: & $3 / 5 / 4$ \\
\hline
\end{tabular}




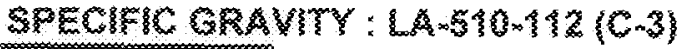

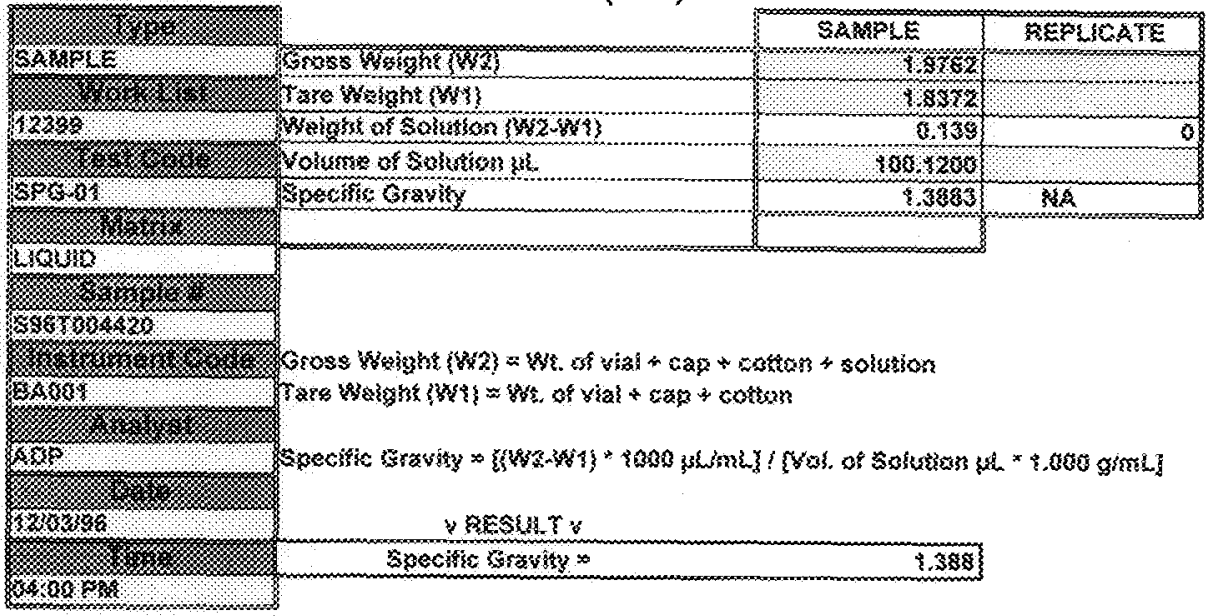

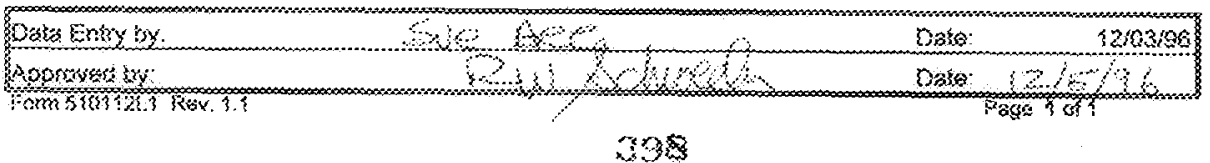




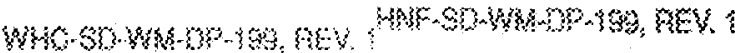

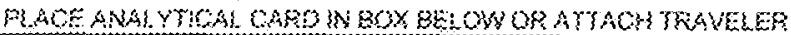

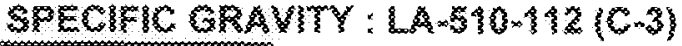

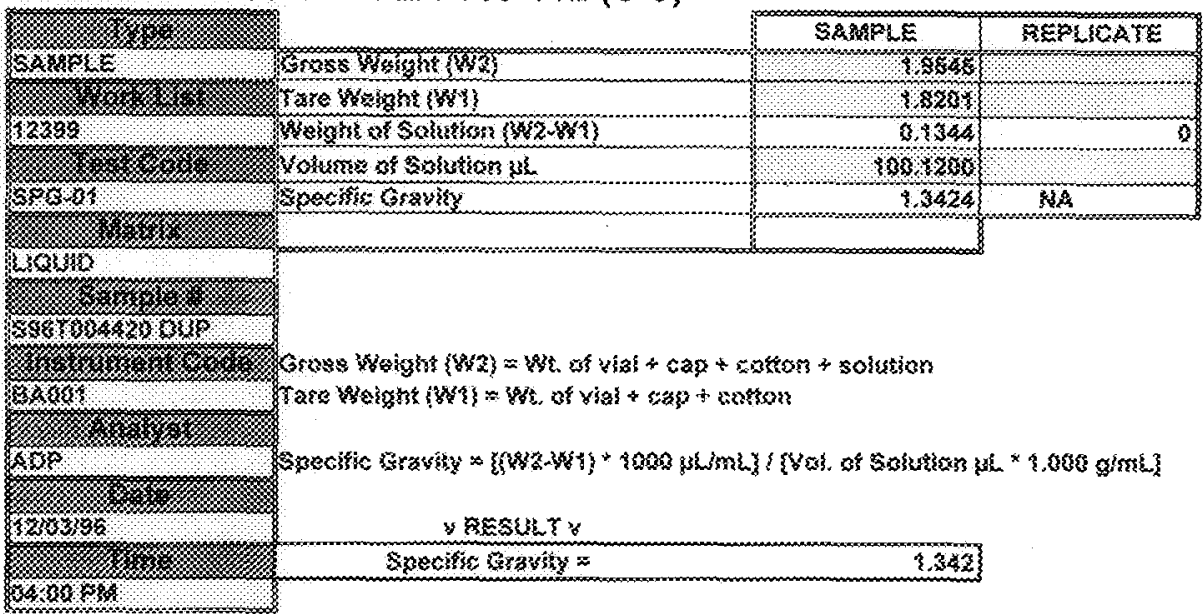

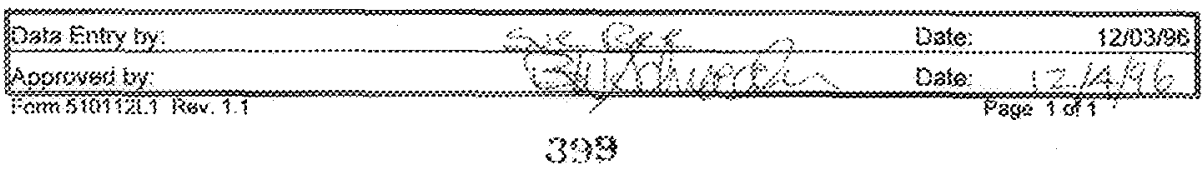




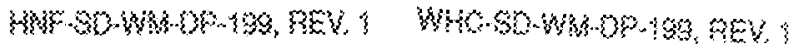

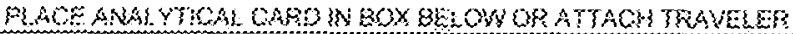

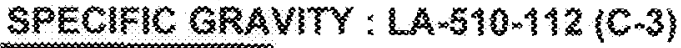

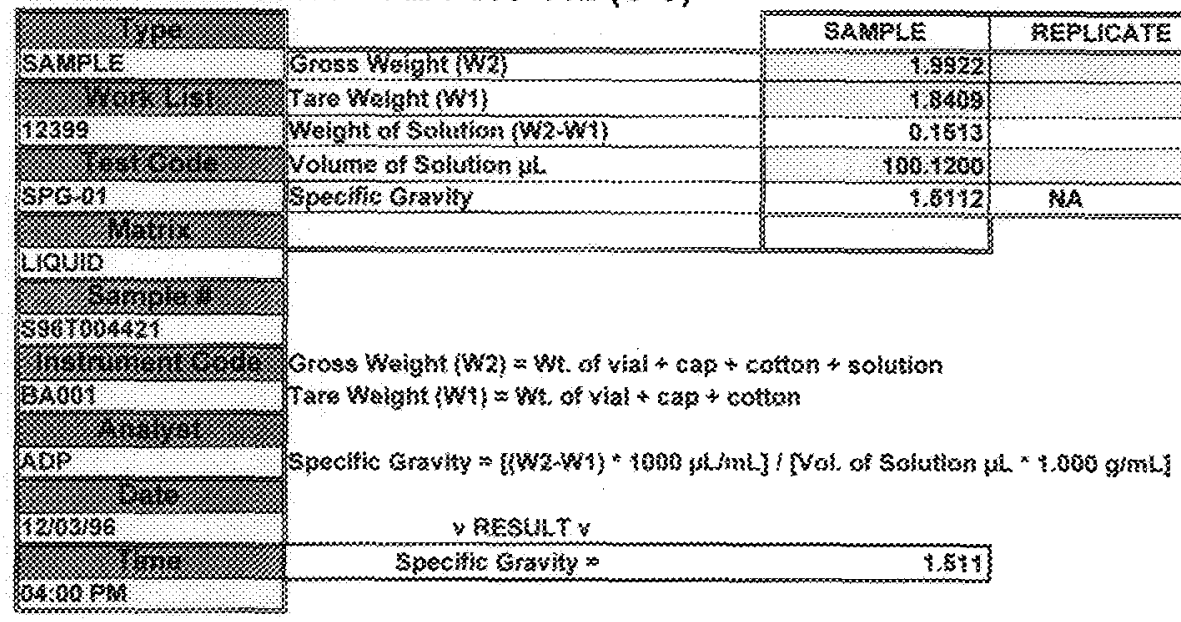

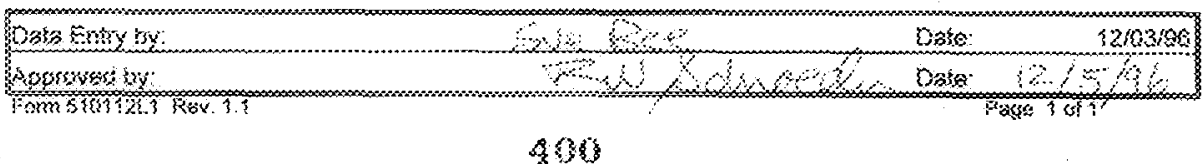



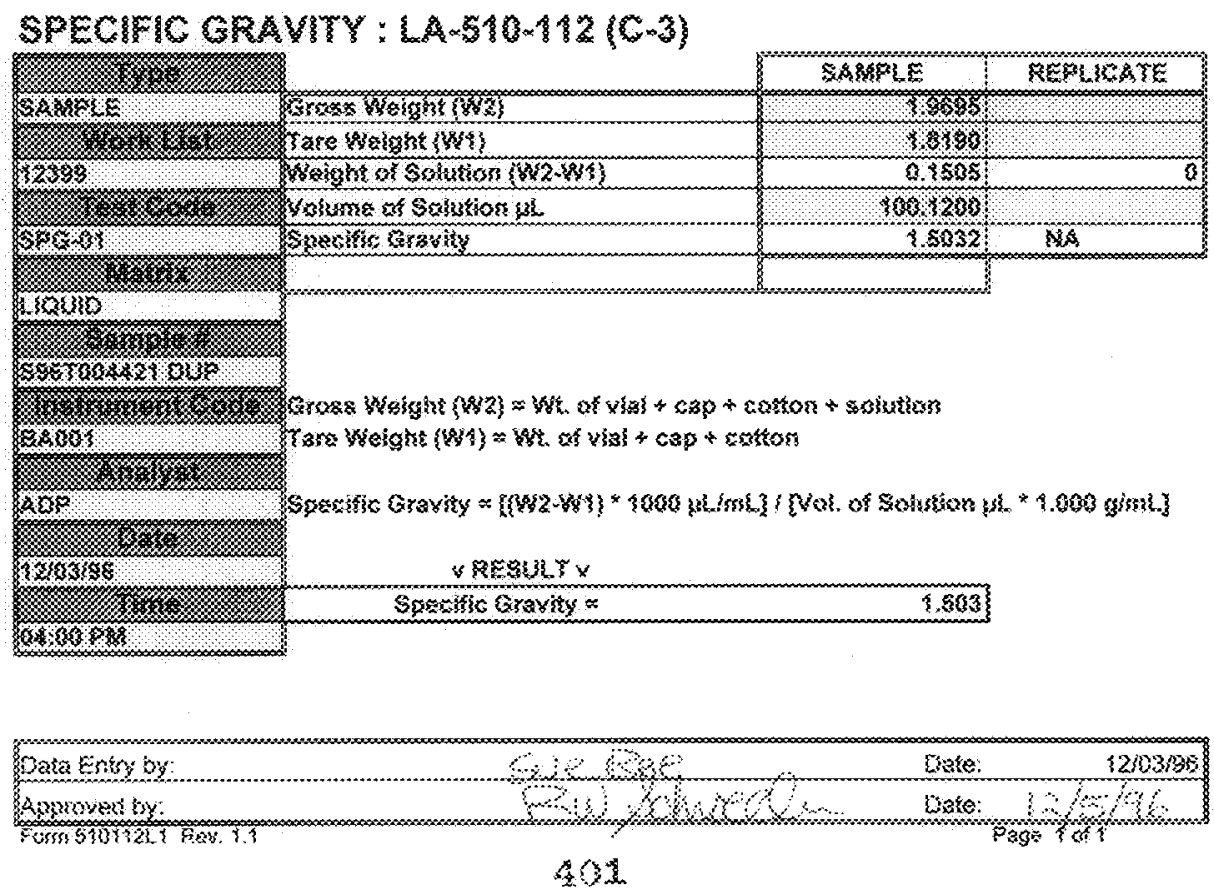


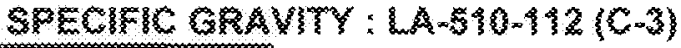

\begin{tabular}{|c|c|c|c|}
\hline 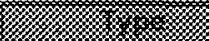 & & \$MMPLE & REPLLATE \\
\hline 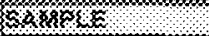 & 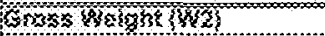 & 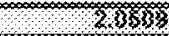 & \\
\hline 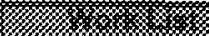 & 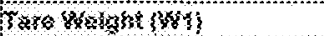 & 3333 & \\
\hline 32393 & 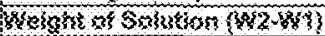 & B.1483 & 3 \\
\hline 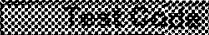 & Yoluma as Solutions ob & $36010 \%$ & \\
\hline 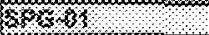 & Krochiss $G r a$ sify & $B A B A$ & Na \\
\hline 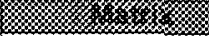 & & & \\
\hline
\end{tabular}

\section{3 s.3.}

\begin{tabular}{|c|c|}
\hline 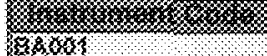 & * cas + softor \\
\hline
\end{tabular}

\begin{tabular}{|c|c|c|}
\hline (8) & \multirow{2}{*}{\multicolumn{2}{|c|}{ 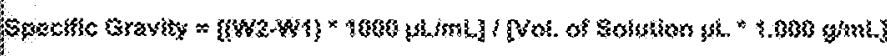 }} \\
\hline s & & \\
\hline 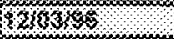 & \& R R & \\
\hline ry & Satecifoc oravity s & \{A3 \\
\hline
\end{tabular}

\begin{tabular}{|c|c|c|c|}
\hline got Enty & 1० $\quad 8$ & bate: & $120 \mathrm{ges}$ \\
\hline Aporoved by: & $3+3<<1<1<$ & Sata: & 96 \\
\hline
\end{tabular}




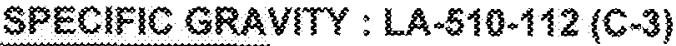

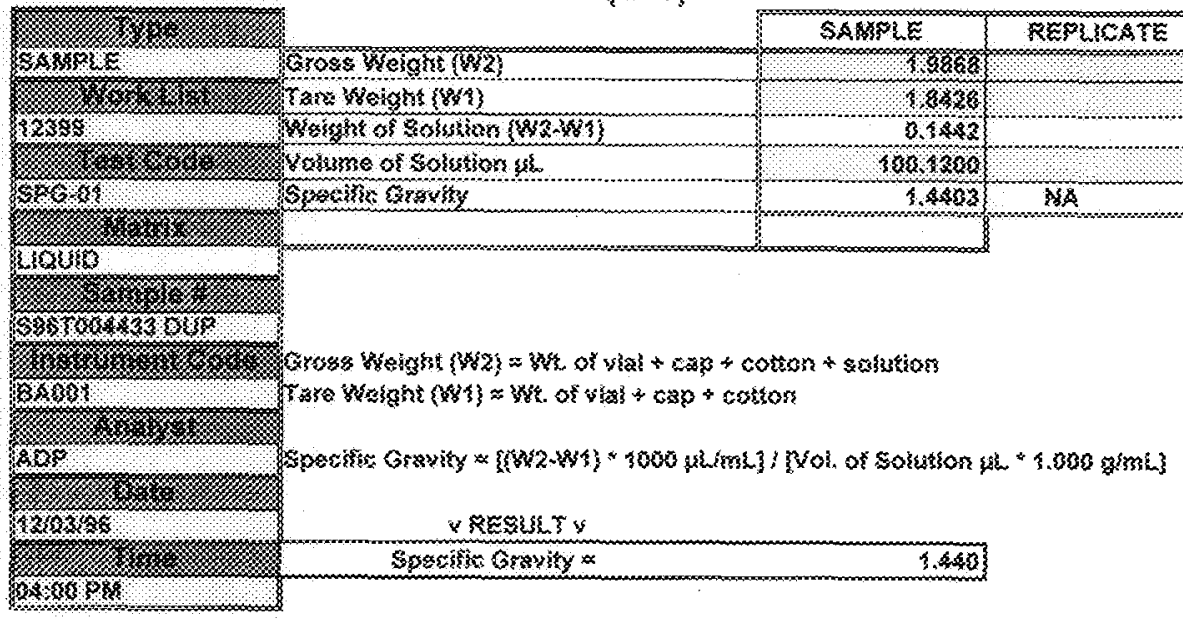

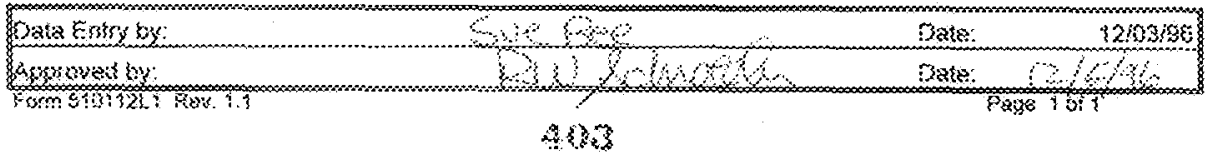




\section{Sp}

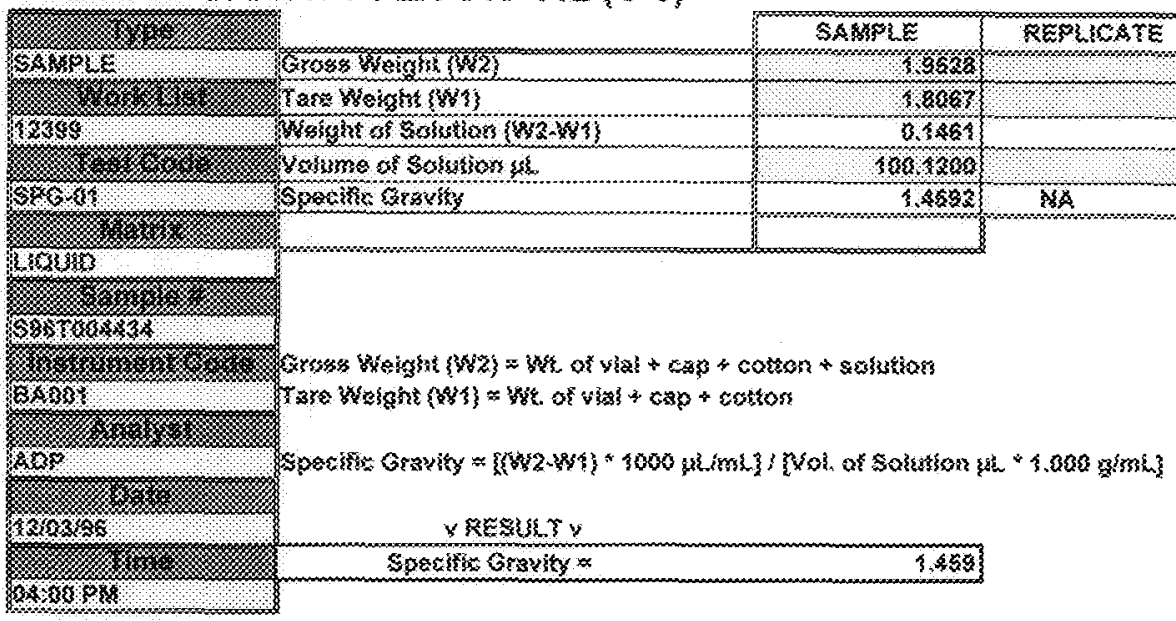

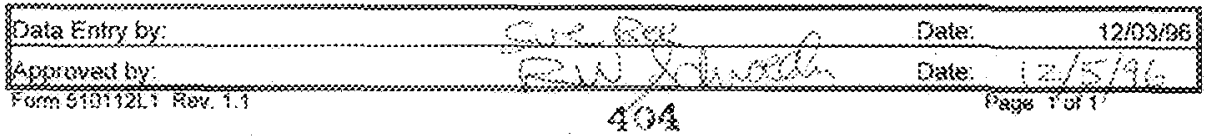




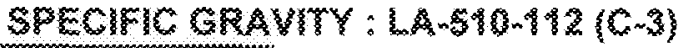

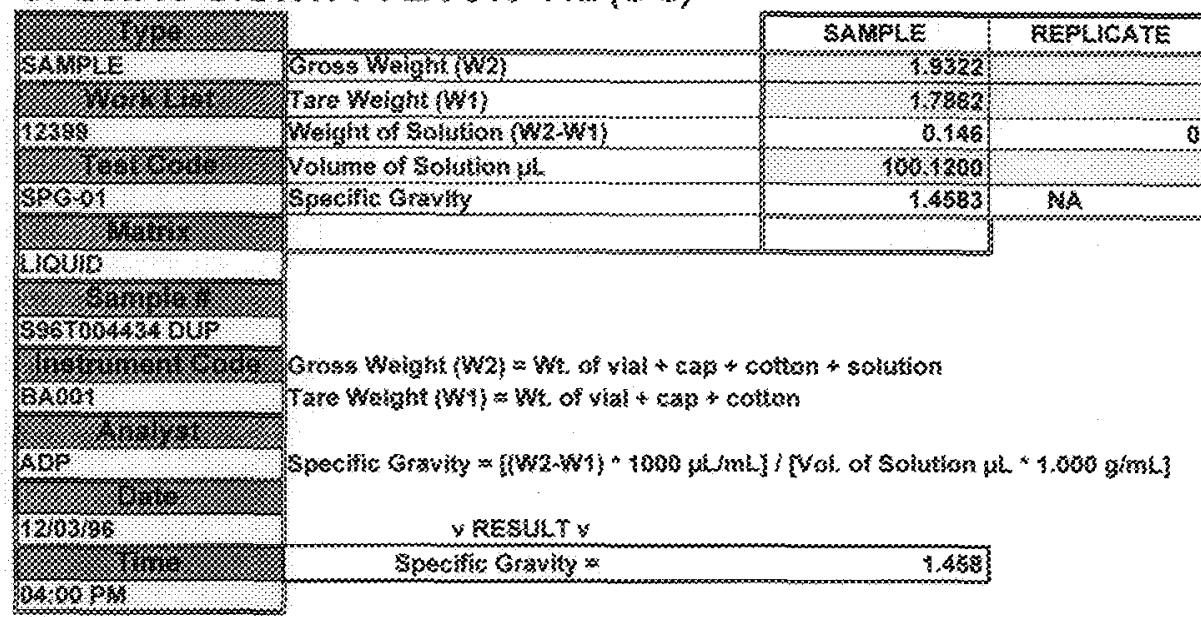

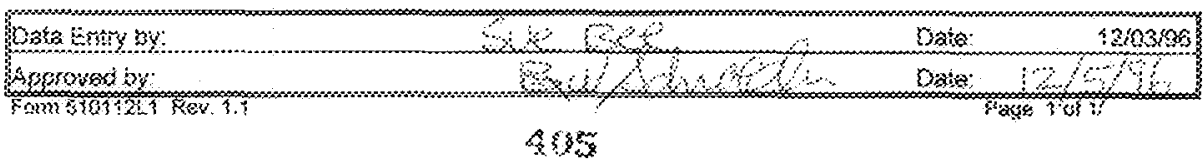




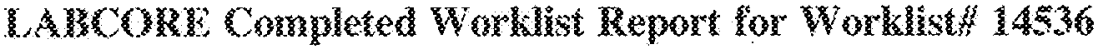

Axafys: cal

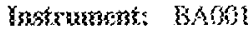

Borkt:

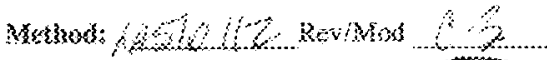

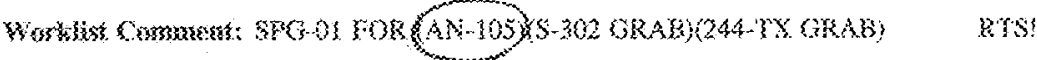

\begin{tabular}{|c|c|c|c|c|c|c|}
\hline Sxus 5 Y32 & 3rostoses & $B A$ & 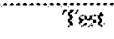 & Mutrix & setexs) & Eorus \\
\hline$\therefore 3 x$ & & s & mess & thessos & 1254 & 2,38 \\
\hline$=2005020$ & 59020050se & 0 & 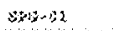 & sxoms & Sis. & i. 452 \\
\hline 100 & $800000 s 00$ & 8 & $820 \mathrm{x}$ & Hosov & $3 \times 2$ & 3308 \\
\hline a sounser & 2500042525 & $a$ & 800.63 & ssigrter & $v k$ & $\mathrm{Sez}$ \\
\hline nos & 8500000580 & 8 & owans & $20 x a s$ & n्य & का \\
\hline os simpux & 8250005507 & ? & 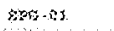 & xxossos & $4 / 3$ & $\therefore$ \\
\hline $\mathrm{\gamma}_{\mathrm{O}}$ & sorkossos & c & sor & noors & 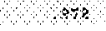 & $\checkmark s a$ \\
\hline - sknor & 800000505 & 6 & $500-02$ & $\cos \cos 20$ & Nis & .058 \\
\hline 900 & 040000020 & 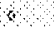 & $0<0$ & sisoiver & का & $3 s$ \\
\hline sexter & S5EYCDEBES & 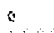 & sorses & 5.5xख & Nor & $x .693$ \\
\hline का & 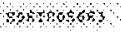 & 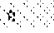 & $2 n+\infty$ & moress & 20 & $1,0 t$ \\
\hline spsers & sotuvess & 8 & ans.ns & xactoss & Ex.... & 2.057 \\
\hline $0 \mathrm{~d}$ & $88 \sigma \times 0548 \%$ & 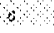 & 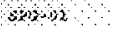 & $x(x)$ & ans & 1060 \\
\hline $2 x 000<x$ & $006001506 \%$ & 6 & soan? & ixpors & $\forall 4$ & 3.062 \\
\hline sos & $8,500,0,0$ & 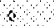 & करता & $\rightarrow$ rown & a,bs & $x, 03$ \\
\hline
\end{tabular}

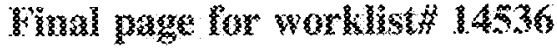

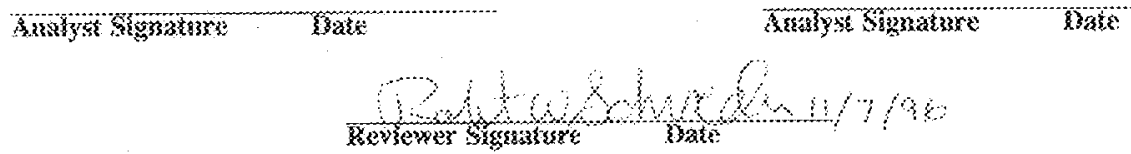




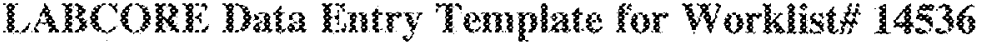

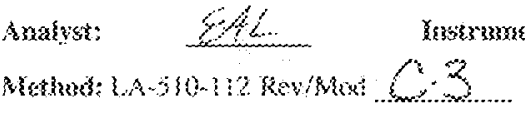

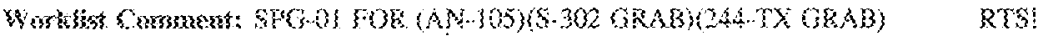

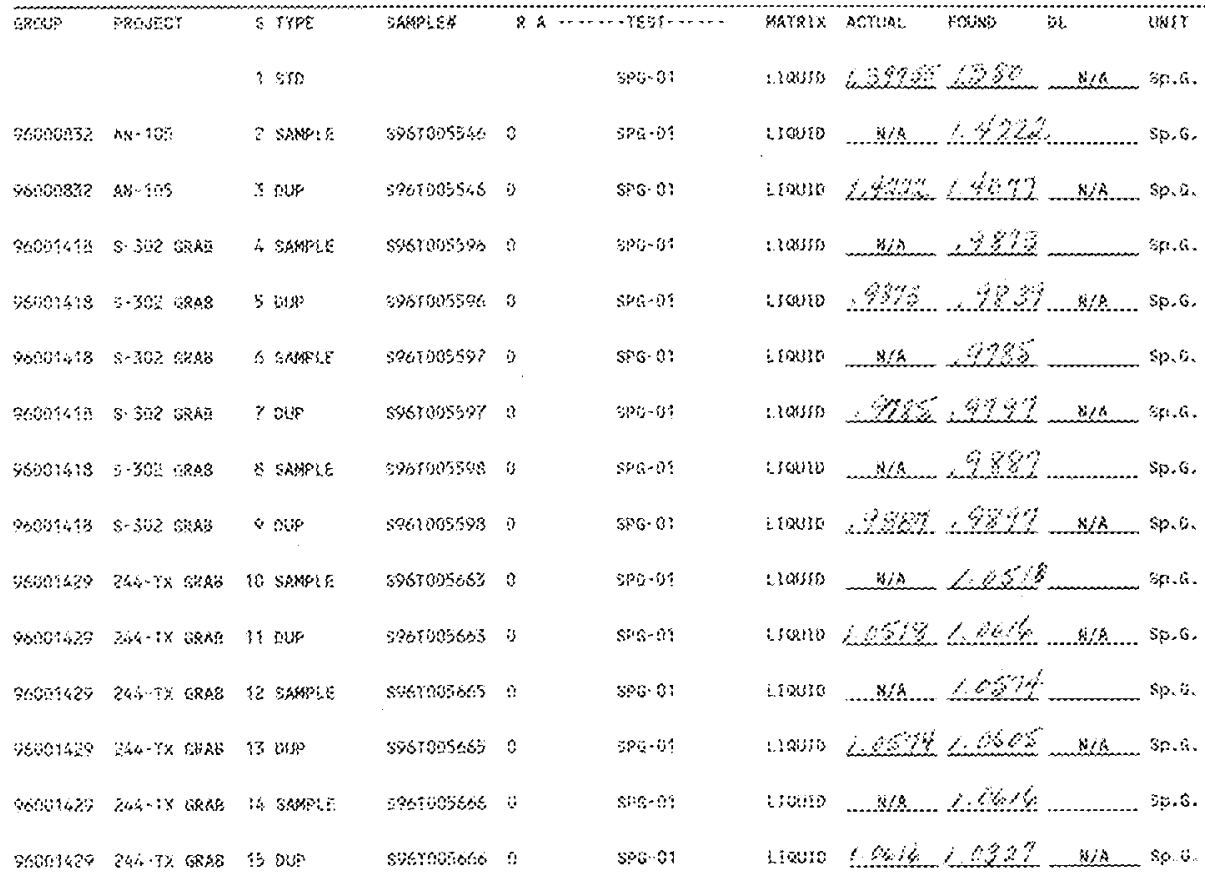

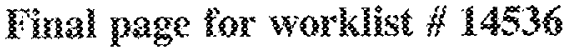
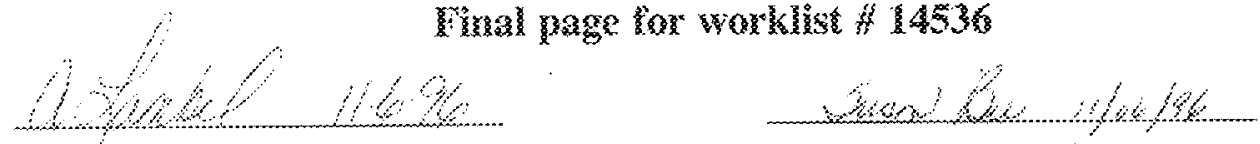

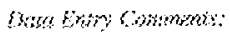

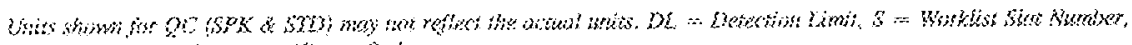

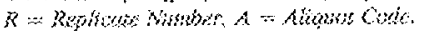




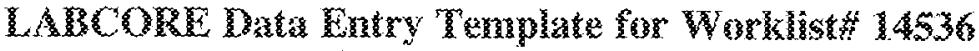

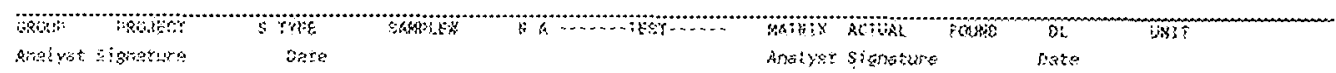

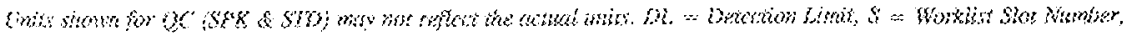

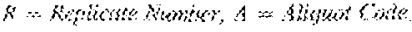




$$
\begin{aligned}
& \alpha
\end{aligned}
$$

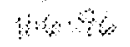

$$
\begin{aligned}
& \text { बि्ब बे } \\
& \text { ख) } \quad, \% \% \\
& \text { सा क्ष }
\end{aligned}
$$

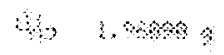

$$
\begin{aligned}
& \text { औरका का } \\
& \text { कै क्षाओ } \\
& \text { आए। },+\infty
\end{aligned}
$$

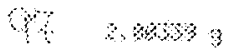

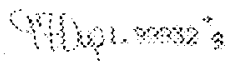

$$
\begin{aligned}
& \text { क } \quad 2 \times \text { के }
\end{aligned}
$$

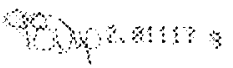

$$
\begin{aligned}
& \text { अ } 2, \quad \alpha \\
& \text { बम } 2 \text { का } \\
& \text { औ } \quad \text { आसे }
\end{aligned}
$$

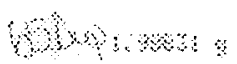

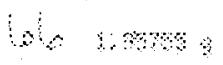

$$
\begin{aligned}
& \text { औद्य }
\end{aligned}
$$

$$
\begin{aligned}
& \text { का } \\
& \text { क्रा क्ष } \\
& \text { से } 1 .+4 \% \\
& \text { \% } 10 \mathrm{~s} \\
& 4 t_{10}+4 \\
& \text { topos } \\
& \text { का } \$ \# \\
& \text { क्सा कीस } \\
& \text { \% } 630 \% \\
& \text { मान्या का } \\
& \text { \% }, 6 \% \\
& \text { और } \\
& \text { a - i. }
\end{aligned}
$$

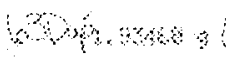

$$
\begin{aligned}
& \text { a } \\
& \text { का } \\
& \text { कर } \quad 4.8 \%
\end{aligned}
$$

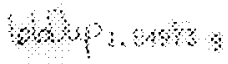




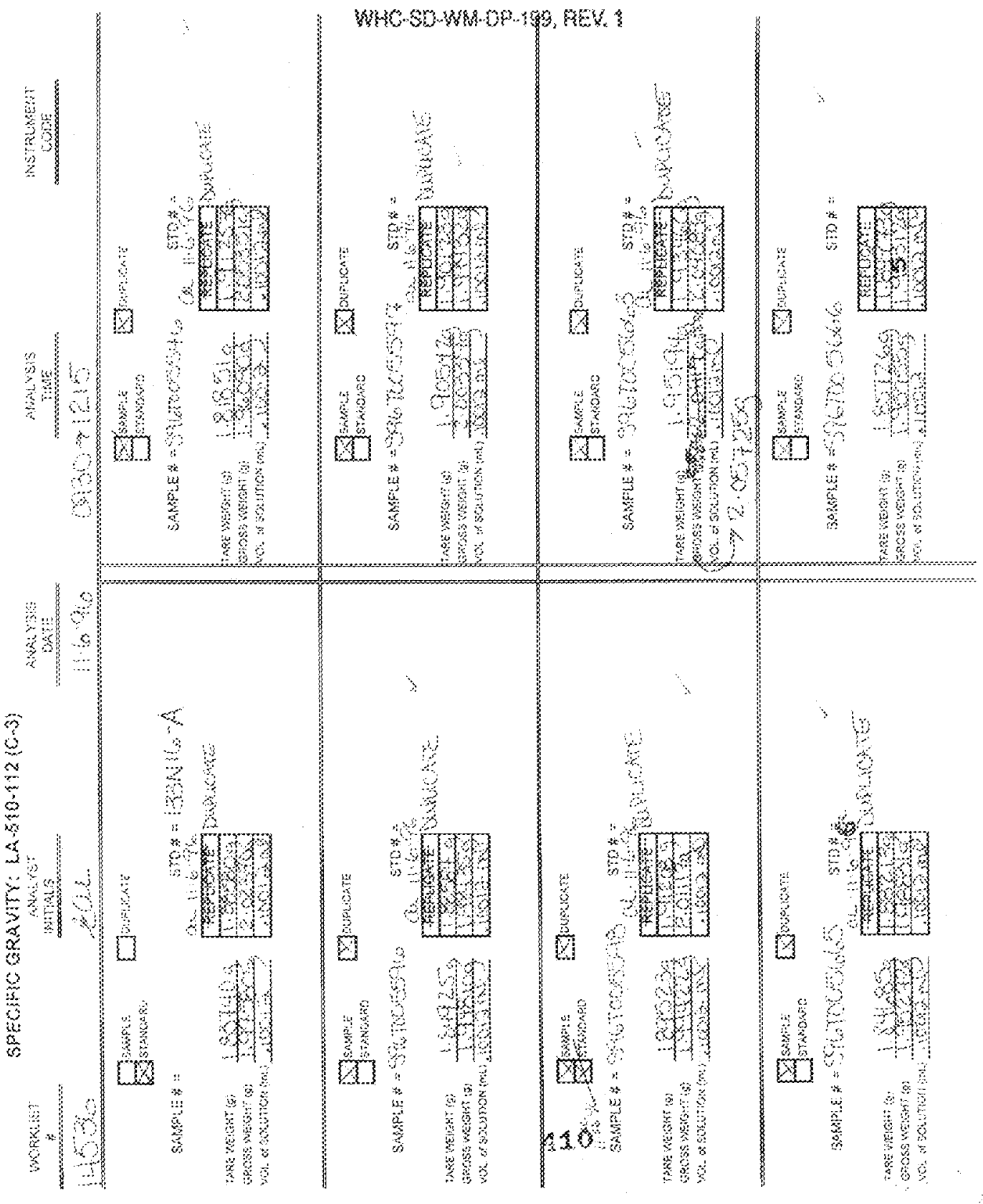




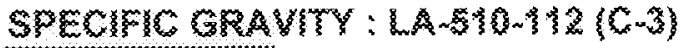

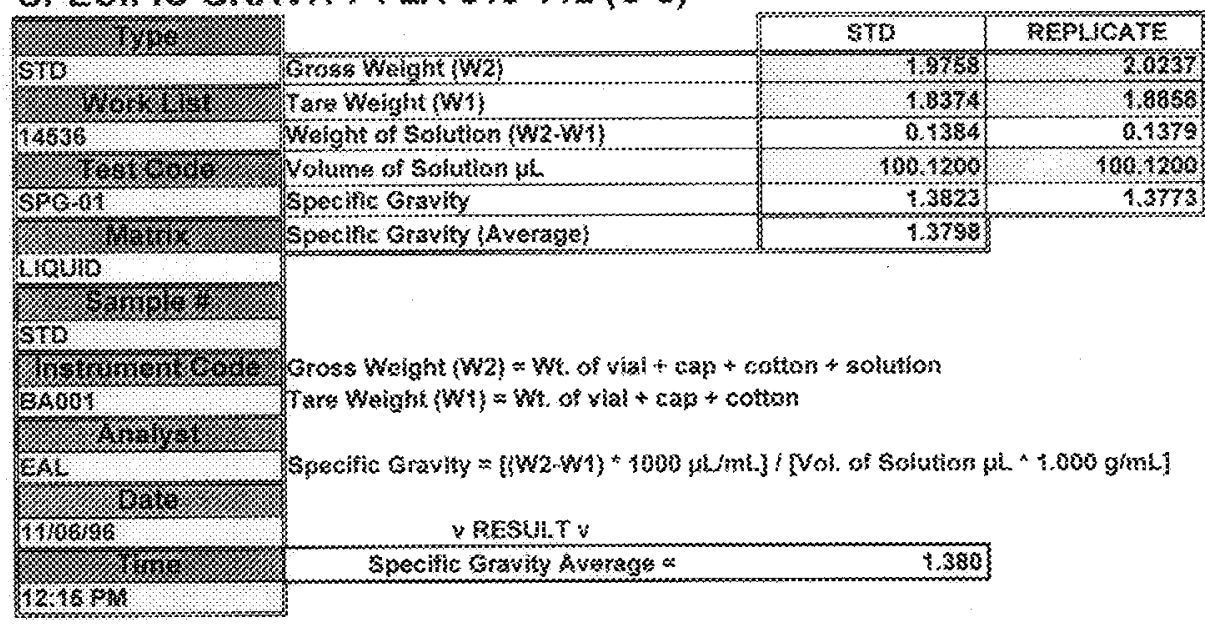

\begin{tabular}{|c|c|c|}
\hline Pata Ensy by. & Oste: & 146606 \\
\hline poposvess by: & Dew: & tis \\
\hline
\end{tabular}




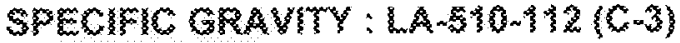

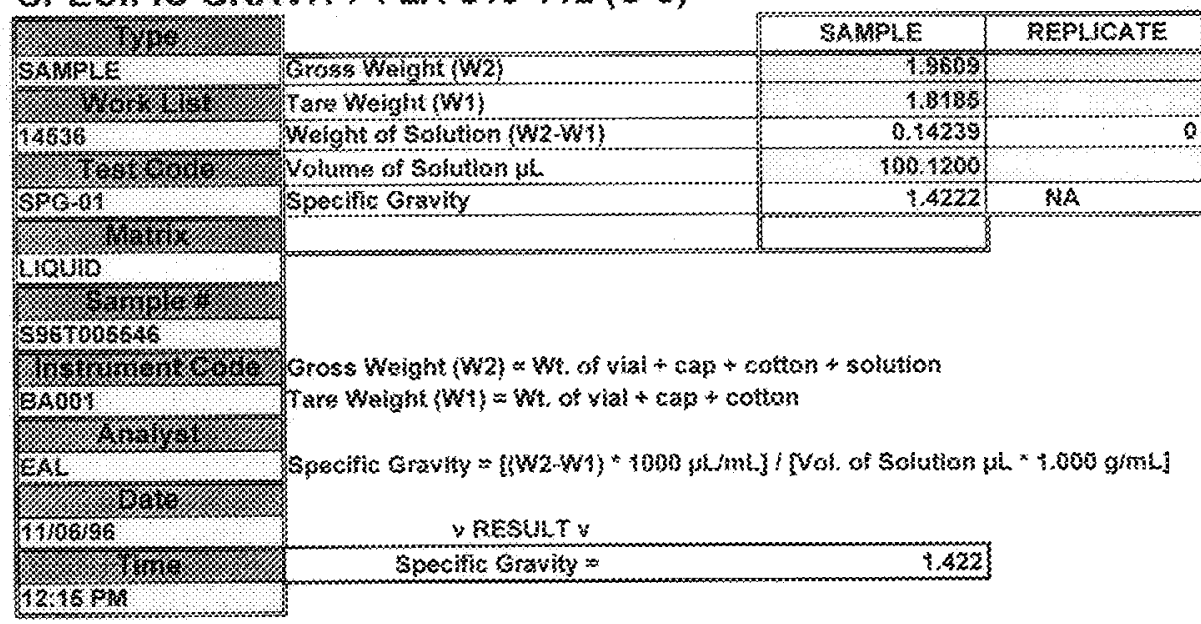

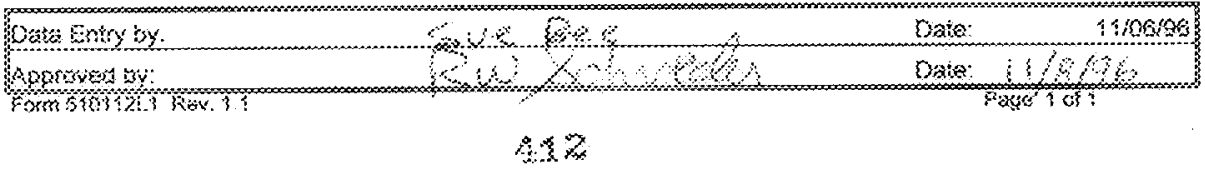



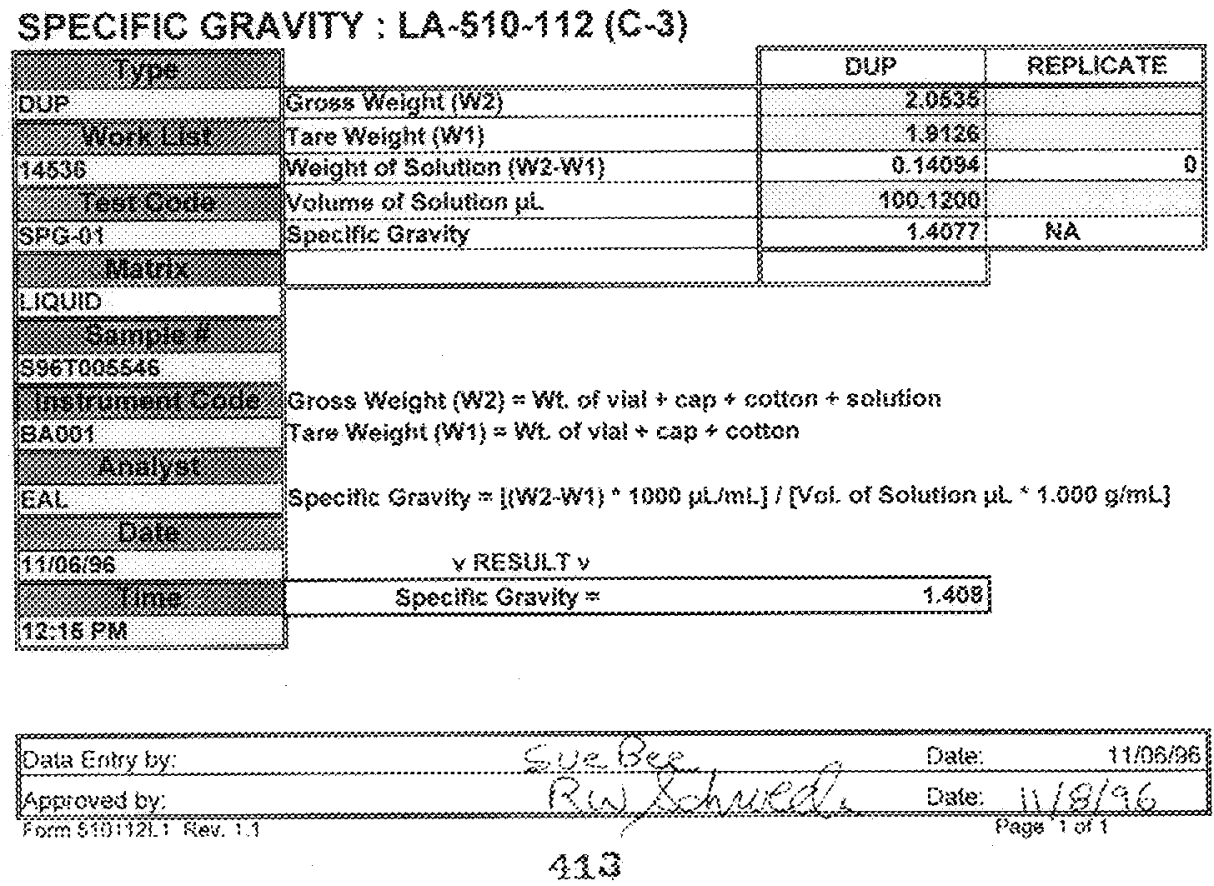


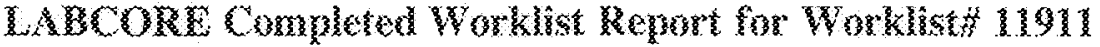

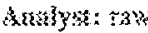

Bustrment: PBO

Bosk $79 \mathrm{~N} 8$

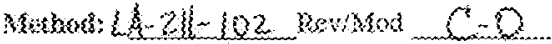

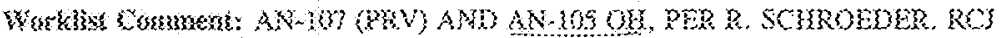

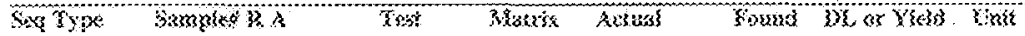

$2 \pi x$

$22 \mathrm{mos}$

20

$\leqslant 2 \times ?$

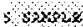

6 ans

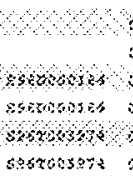

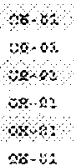

\begin{tabular}{|c|c|c|}
\hline tixpess & 2.56sos & 2.0204 \\
\hline on, & $x_{x}$ & 30 \\
\hline 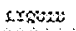 & 425 & 250 \\
\hline & 30 & 43 \\
\hline $2: 4000$ & $\therefore 93$ & $\angle 23$ \\
\hline
\end{tabular}

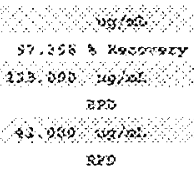

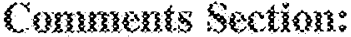

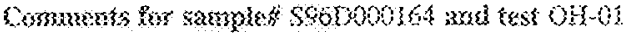

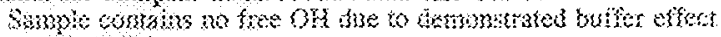
riws: 813,3

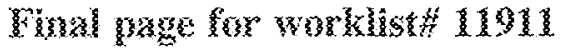

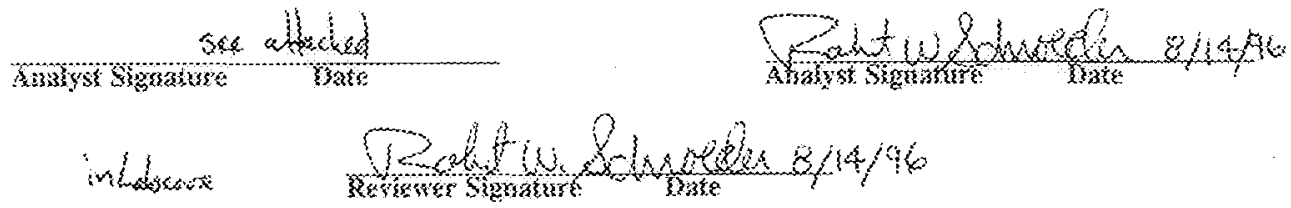




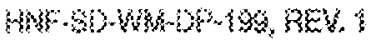

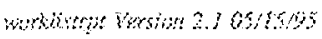

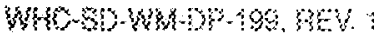

Fuge: ?

(20) $3,5633.53$

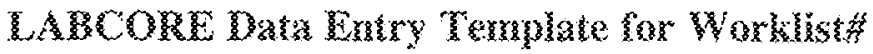

11938

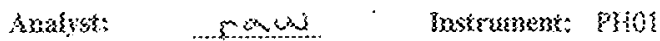

Somb * $73 \sqrt{8}$

Mabud: LA-21:-102 Revihui\}

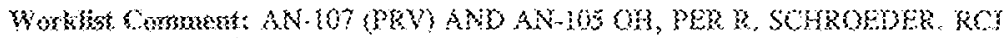

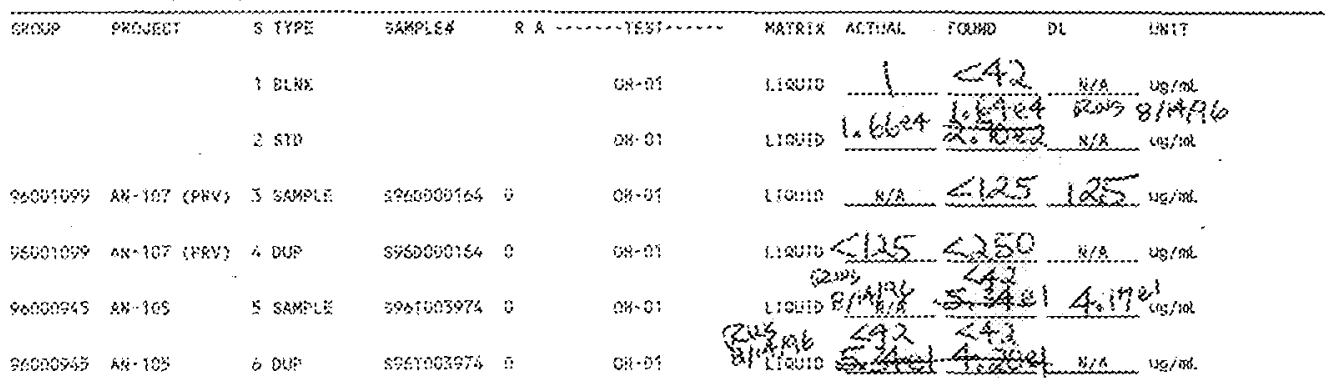

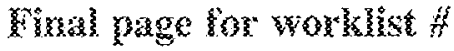

1818

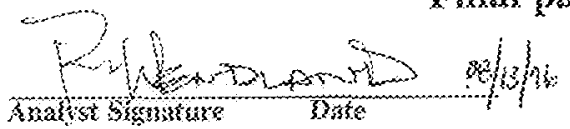

Analust Grzzature Bate

Dins Entor Dromons:

Crape spoboodot coutains wo free of due to dewastrated ks fereffect $8 / 4 / 96$

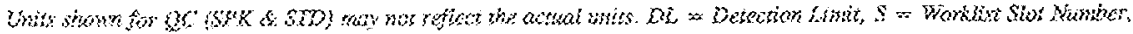

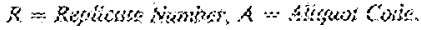

Ast 
moso

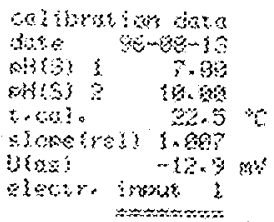

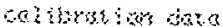

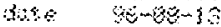

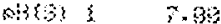

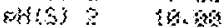

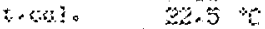

Stonetras\} \{.89?

bras -

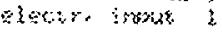

$x+x+x=$
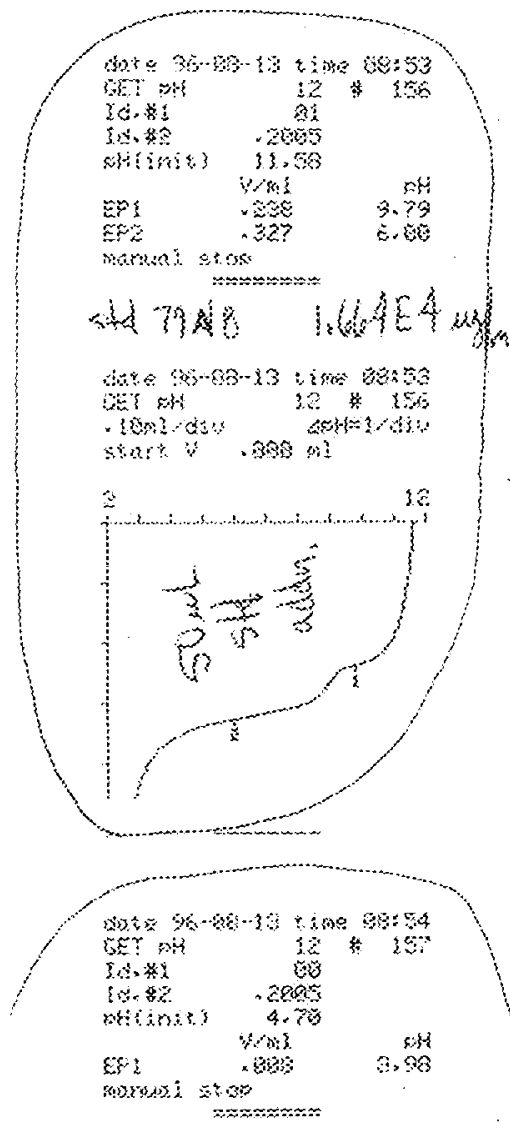

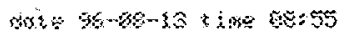

बET रो क स

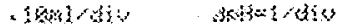
start $Q$, bon की

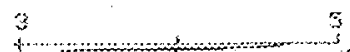

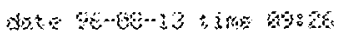

bet कi

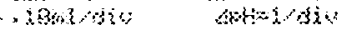

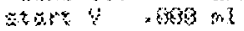

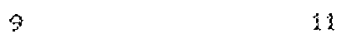

13
Wre $3 \%$ - $3-3 \%$ \&

उस

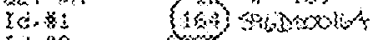

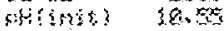

moruts otos

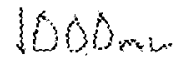

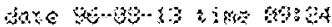

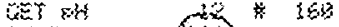

ond

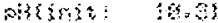

stop ienches

2rimin:m

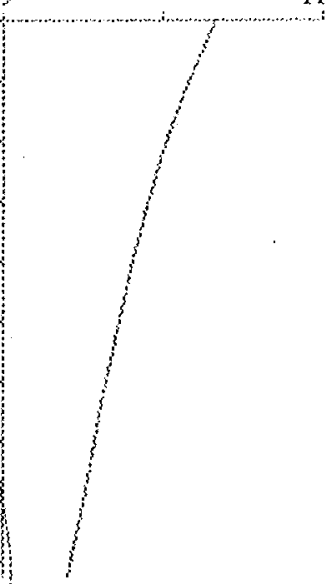

dus

जF

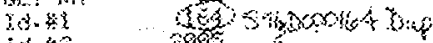

isus

misnis: zeopted

sop trached

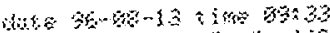

Q⿱艹

-

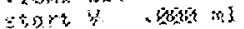

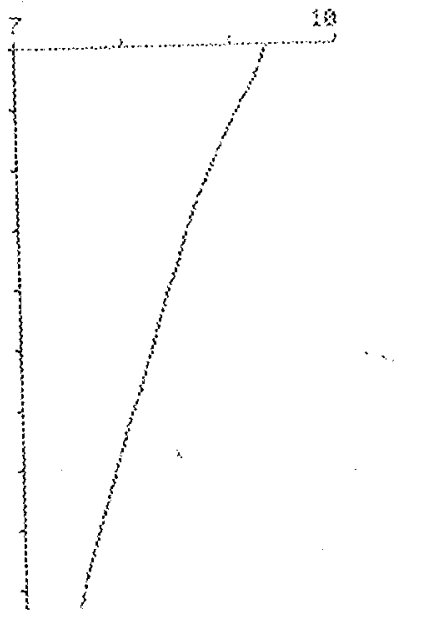

\section{6}




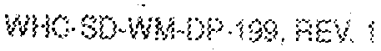

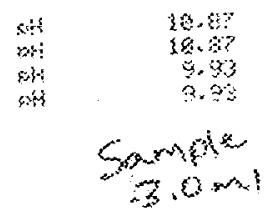

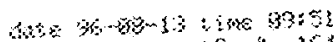

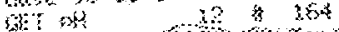

is. $x$

3.5. 5 की.

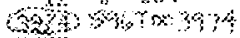

artivis

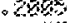

Detures

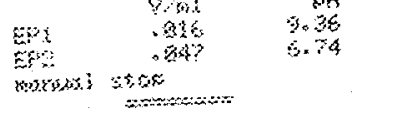

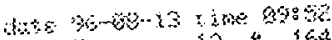

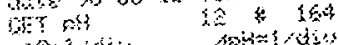

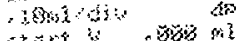

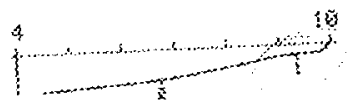

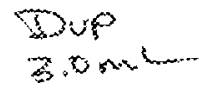
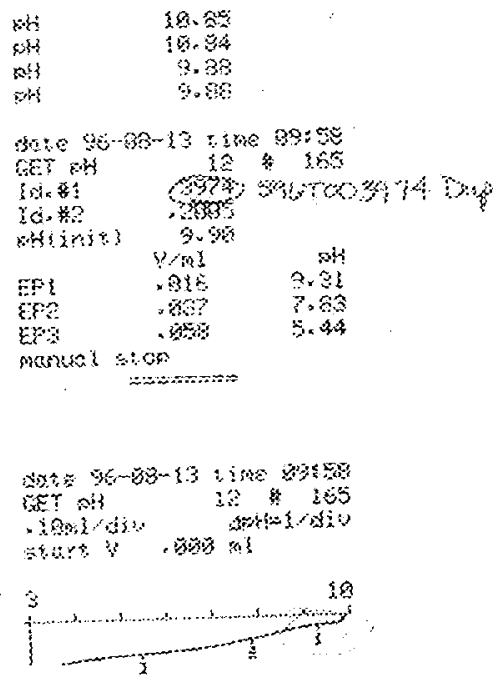


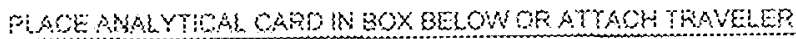

\begin{tabular}{|c|c|c|}
\hline \multirow{2}{*}{\multicolumn{2}{|c|}{ 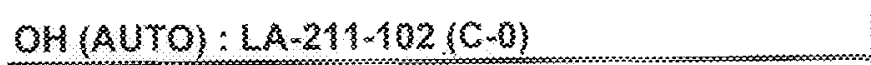 }} & \multirow[b]{2}{*}{ Bik } \\
\hline & & \\
\hline 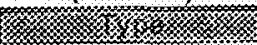 & Comple $3 \mathrm{mg}(\mathrm{mil})$ ss & 3001 \\
\hline B $3 k$ & 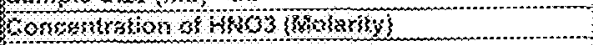 & 0.2000 \\
\hline 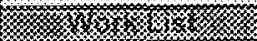 & 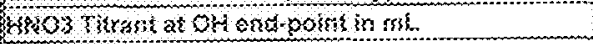 & 3.008 \\
\hline ISBSY & Gilimson $F=0$ o $O F$ & \\
\hline 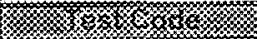 & Konsenirstion of on in Sarmple (solotity) & $\$ .35 E-64$ \\
\hline What & 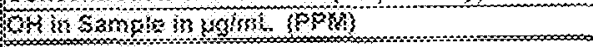 & Q096? \\
\hline 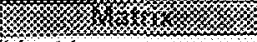 & & \\
\hline \multicolumn{3}{|l|}{ 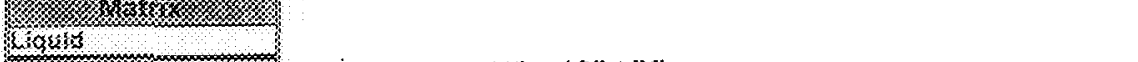 } \\
\hline 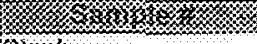 & Wetection Limit $=12$ sing is $\$$ * of & \\
\hline \multicolumn{3}{|l|}{83 nh } \\
\hline$\gamma / x_{x}$ & 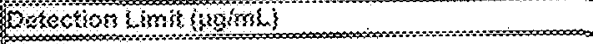 & $417+2+2$ \\
\hline \multicolumn{3}{|l|}{ SPST , } \\
\hline 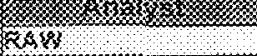 & \multirow{3}{*}{\multicolumn{2}{|c|}{ 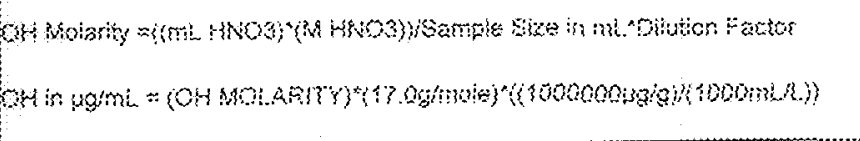 }} \\
\hline 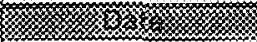 & & \\
\hline \multirow{2}{*}{\multicolumn{3}{|c|}{ 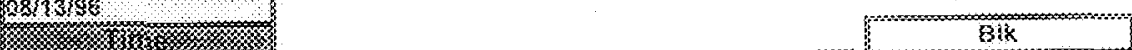 }} \\
\hline & & \\
\hline & 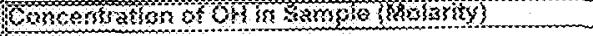 & 538500 \\
\hline & 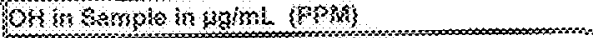 & $\$ 2$ \\
\hline
\end{tabular}

Tats

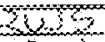

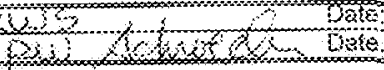

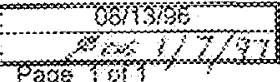




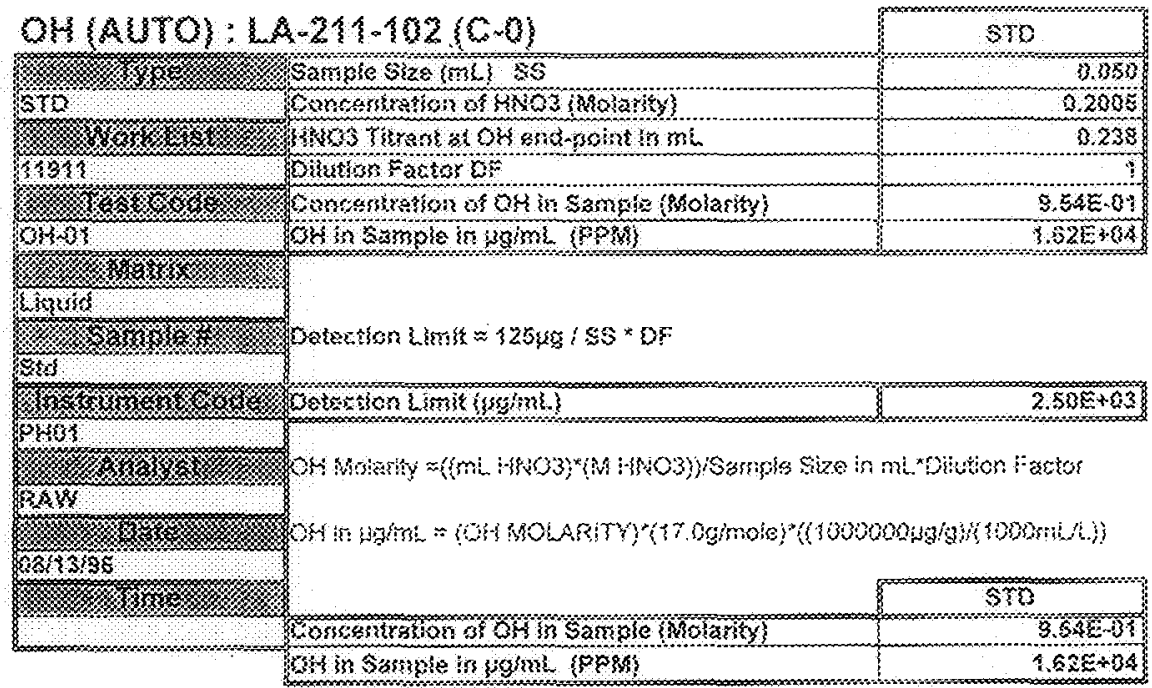

Wrajod:




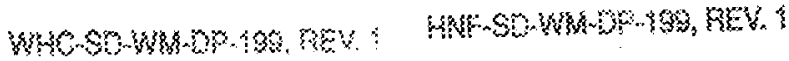

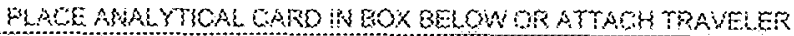

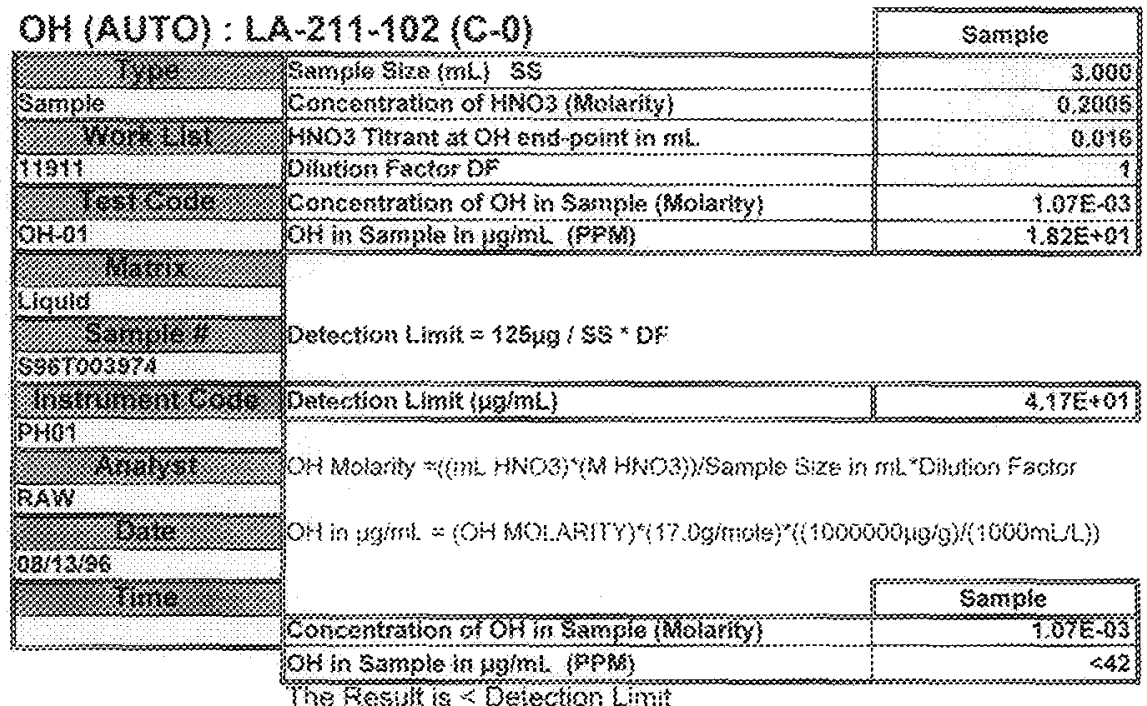

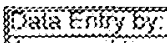
Shatowed by:

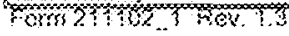

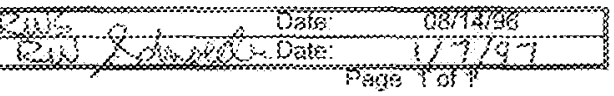




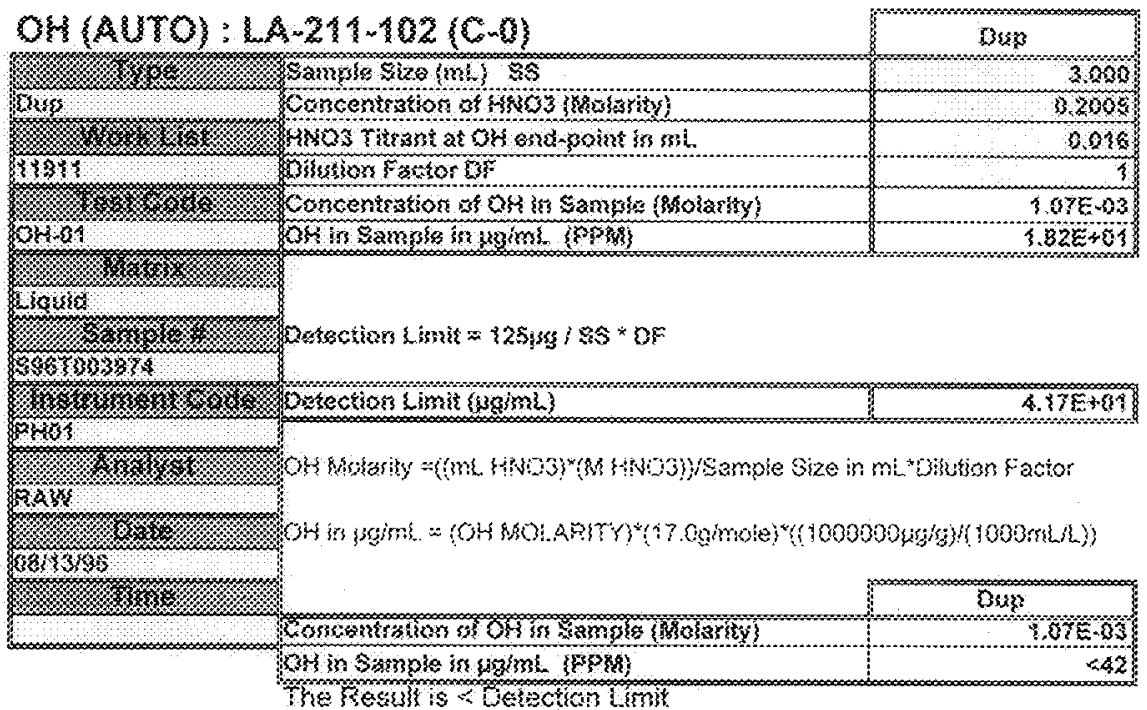

AQYoveO OY 


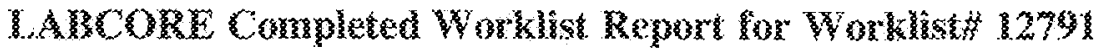

snatyst: ratk

3xstrumbert: MH1

sookt $29 \mathrm{k} \mathrm{k}$

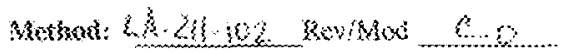

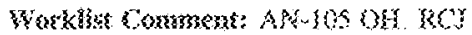

\begin{tabular}{|c|c|c|c|c|c|c|}
\hline Bes ryss & Sarmghts $\mathrm{R}$ \& & Tess. & Misterk & setersis & Fossus? & BS. $2 x$ Yiess \\
\hline 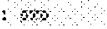 & 6 & $60-2$ & togros & trosan & $1600 \mathrm{~s}$ & 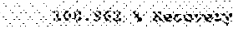 \\
\hline$=0 \sin x$ & c & $\Delta x_{i}-6 \lambda$ & xhonss & 3 & $2, x$ ses. & 23.586 \\
\hline 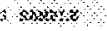 & onsorot $s$ & os & $\operatorname{sos} \alpha 2$ & $x x$ & $40 x<$ & sos 0000 roros \\
\hline$\therefore \mathrm{sor}$ & $305 \times 30003: 0$ & $x x-6 y$ & smovit & S. $5.6 \times 4$ & 6.53est & $6.6520 \times 0$ \\
\hline
\end{tabular}

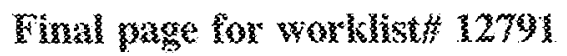

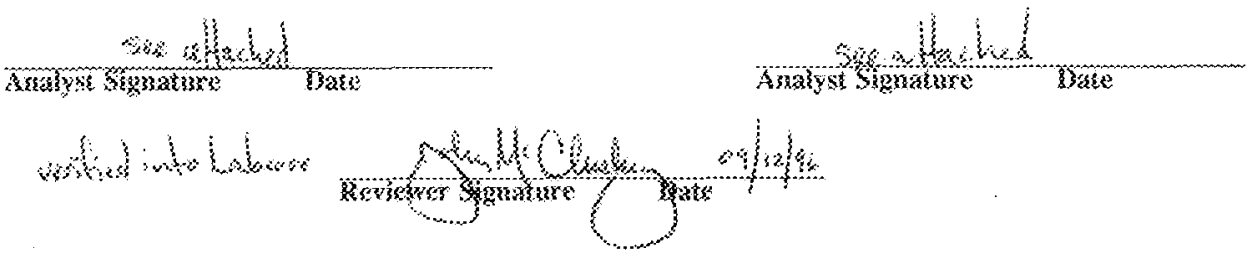




\section{LA}

sumbs: $\quad 2 / 2$

Gustrament: mob!

Rosis 2913

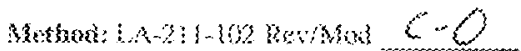

Workist Comment: AN.BS OH, RS?

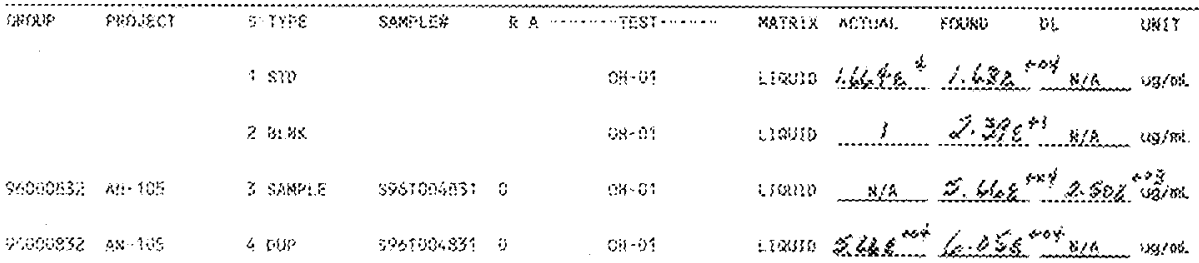

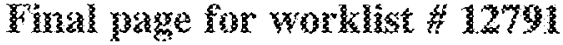

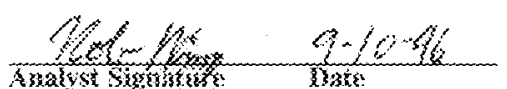

Analgst Sights

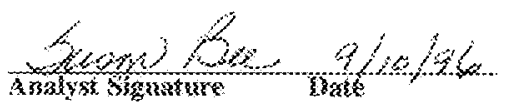




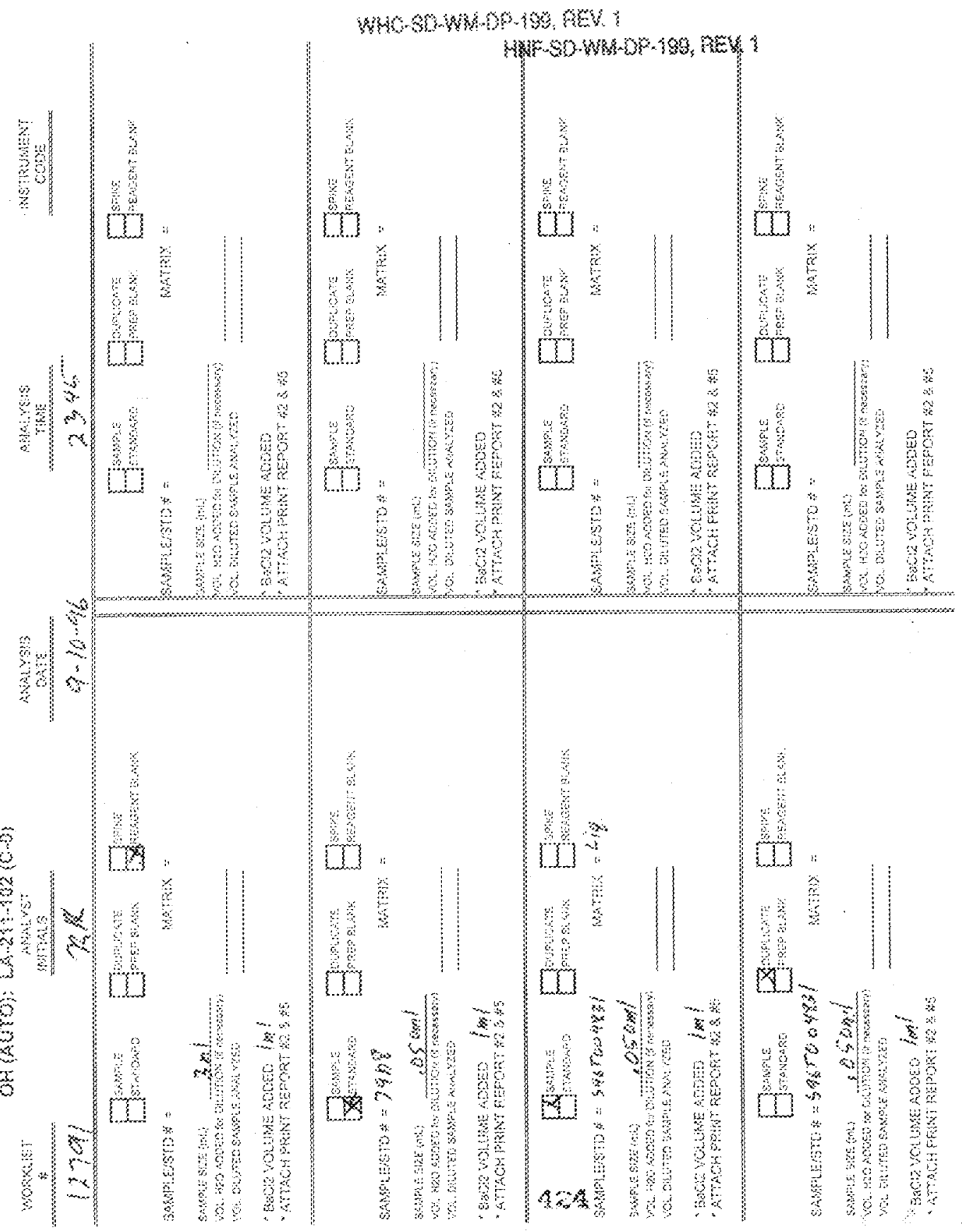




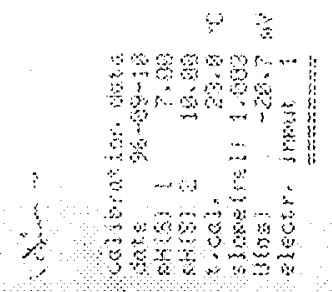

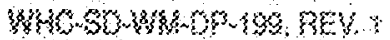

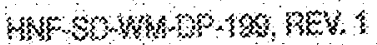
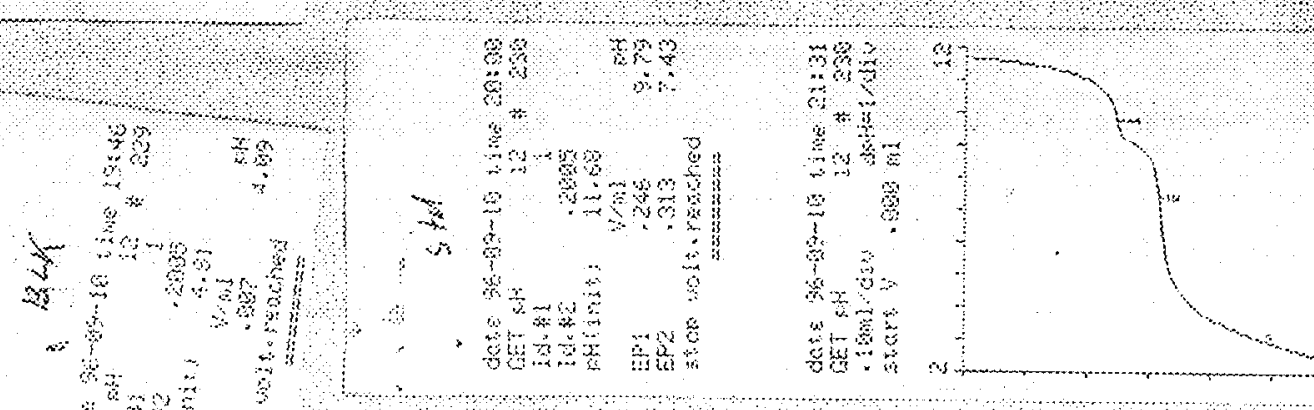

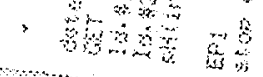
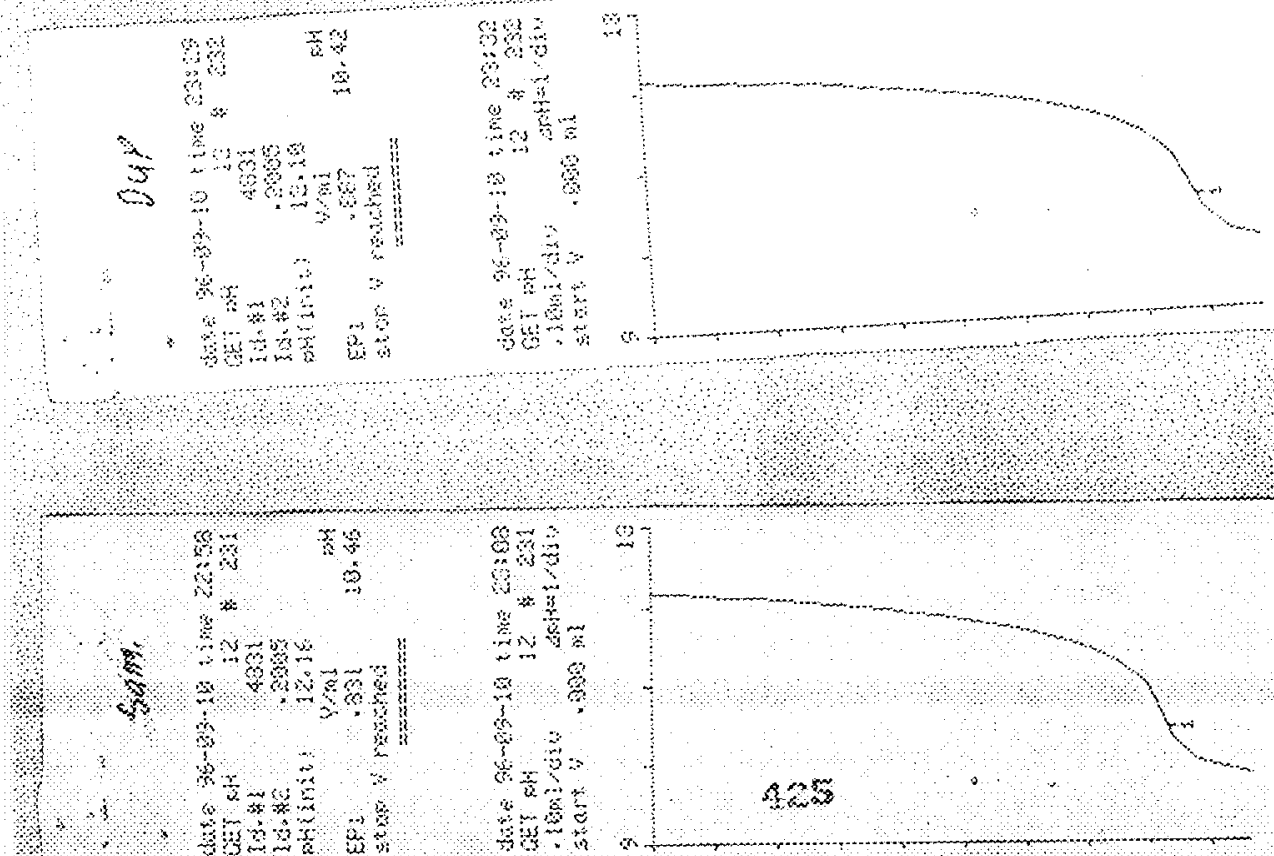


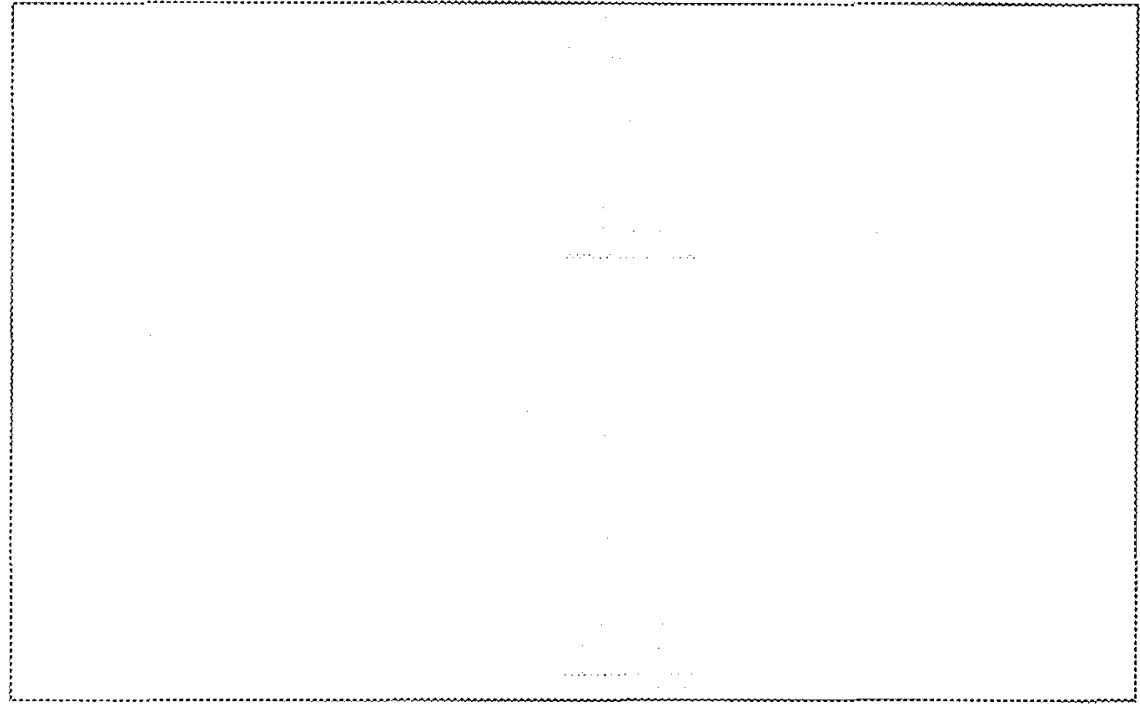

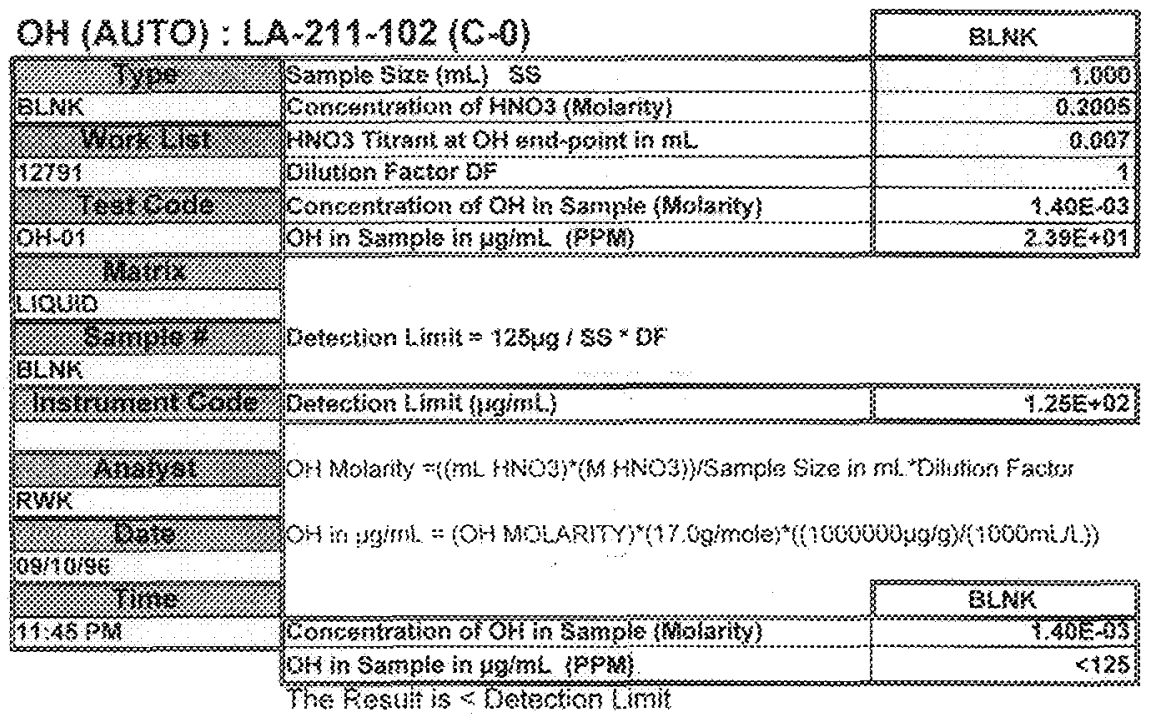

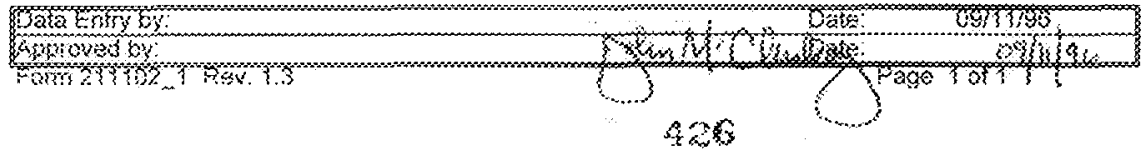




\begin{tabular}{|c|c|c|}
\hline \multicolumn{2}{|c|}{ 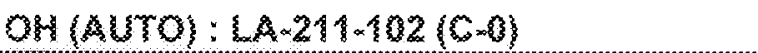 } & \multirow[t]{2}{*}{ sto } \\
\hline 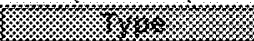 & Sample $3 i z q\{\mathrm{~m}\}$ \&S & \\
\hline 3ro & 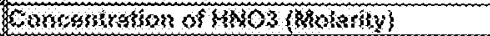 & 0.2005 \\
\hline 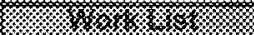 & 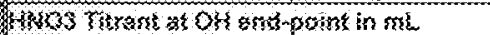 & $0.2+6$ \\
\hline X7\% 1 & Whaton $p$ actor of & \\
\hline ry & 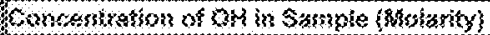 & $2.806-34$ \\
\hline $0 \leqslant 1,01$ & 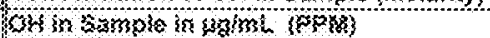 & $3.68 E+334$ \\
\hline \multicolumn{3}{|l|}{ \% } \\
\hline WOWH. & \multirow{3}{*}{ 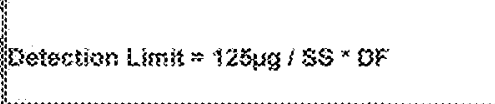 } & \\
\hline 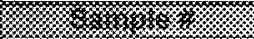 & & \\
\hline \multicolumn{2}{|r|}{ ( } & \\
\hline 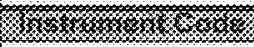 & 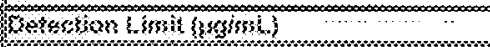 & 2602403 \\
\hline 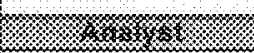 & \multirow{4}{*}{\multicolumn{2}{|c|}{ 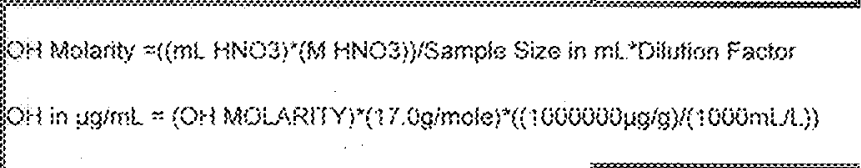 }} \\
\hline KWK & & \\
\hline \multirow{2}{*}{ 6 } & & \\
\hline & & \\
\hline 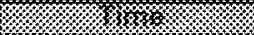 & & $\mathrm{Tt}$ \\
\hline $100600 \%$ & 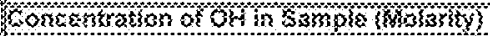 & 3.85 \\
\hline & 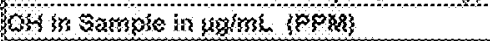 & 1.850+104 \\
\hline
\end{tabular}

Mat \&ns $\mathrm{Y}$

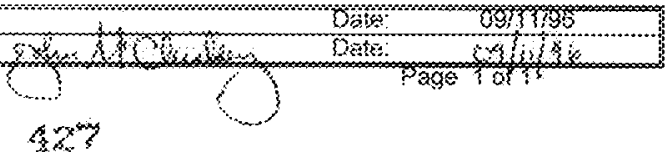




\begin{tabular}{|c|c|c|}
\hline \multicolumn{2}{|c|}{ 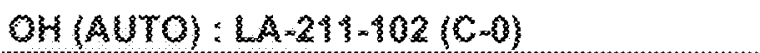 } & \multirow[t]{2}{*}{ a. } \\
\hline '/ / & Samples sion $\{m\}$ SS & \\
\hline SAm & 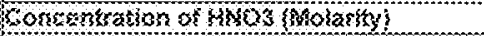 & 0.2035 \\
\hline 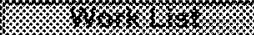 & 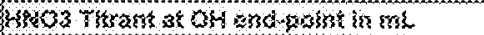 & $0.5 \$ 1$ \\
\hline 2731 & SHThtom Gagsor DE & \\
\hline 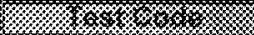 & 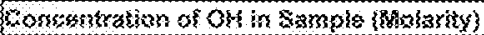 & $3.335+00$ \\
\hline WHOY . & 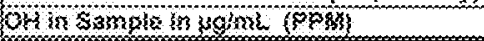 & S \& \&E: 64 \\
\hline צr/ & \multirow{4}{*}{ 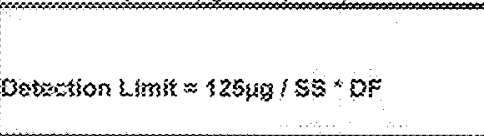 } & \\
\hline UQUUO & & \\
\hline 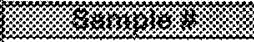 & & \\
\hline SYYYOQOKOSY & & \\
\hline xy & 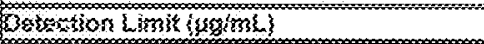 & 2.502403 \\
\hline r & \multirow{4}{*}{\multicolumn{2}{|c|}{ 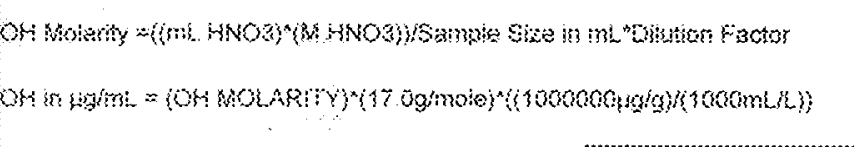 }} \\
\hline Kk\% & & \\
\hline 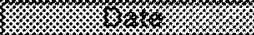 & & \\
\hline 09350968 & & \\
\hline 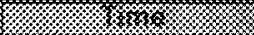 & & Am \\
\hline \multirow[t]{2}{*}{3144083} & 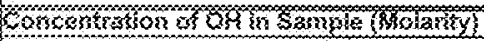 & $3 \sqrt{4}+30$ \\
\hline & 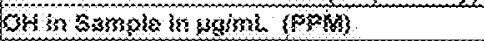 & $9,6 E E+3,8$ \\
\hline
\end{tabular}

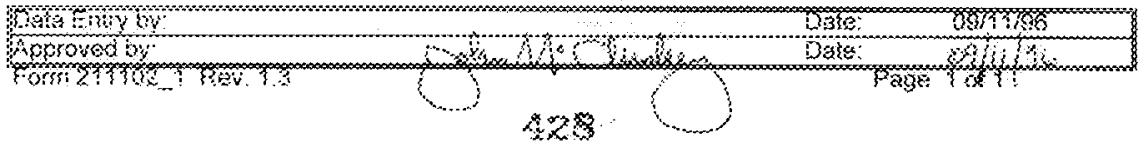




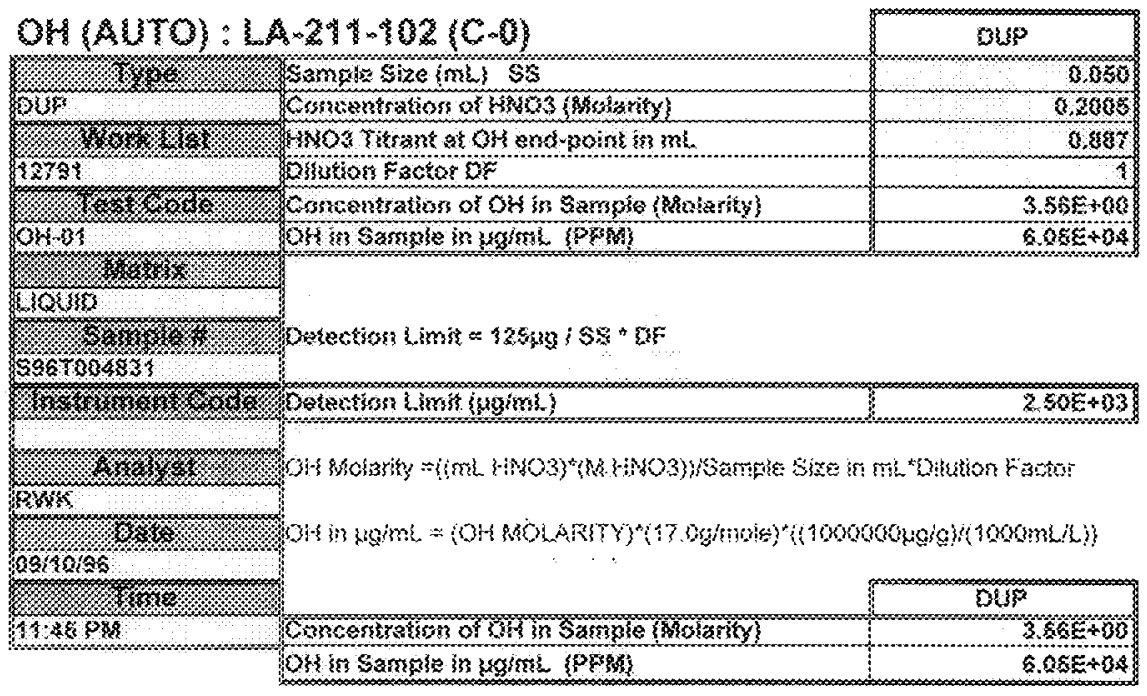

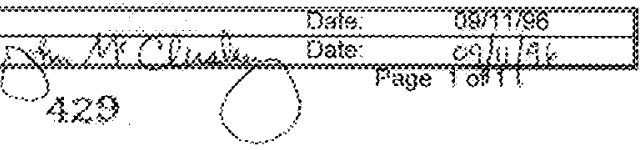




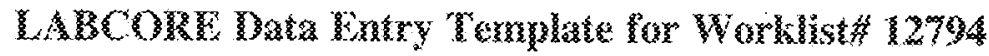

Analyst:

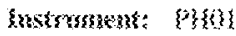

Bond :

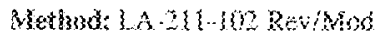

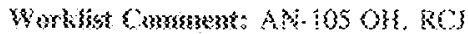

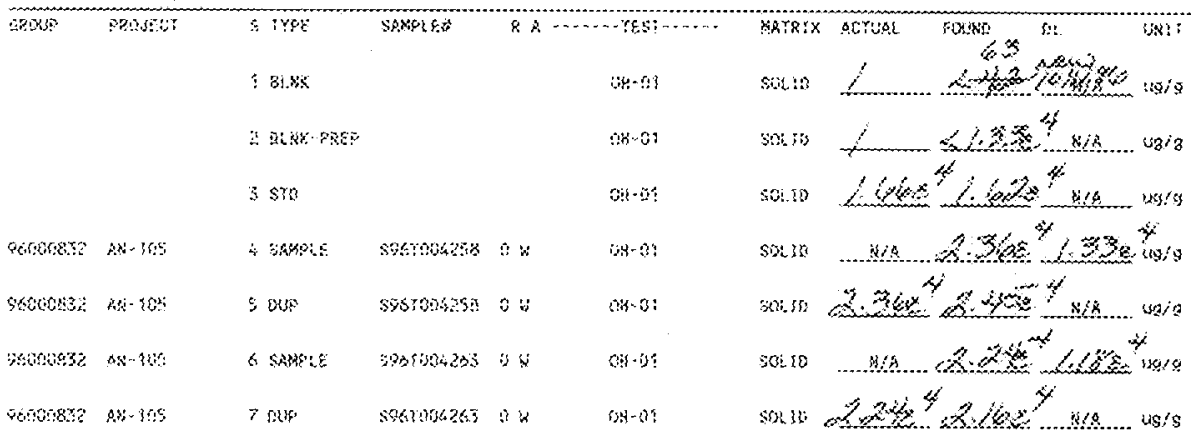

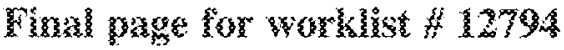

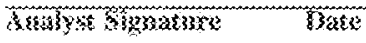
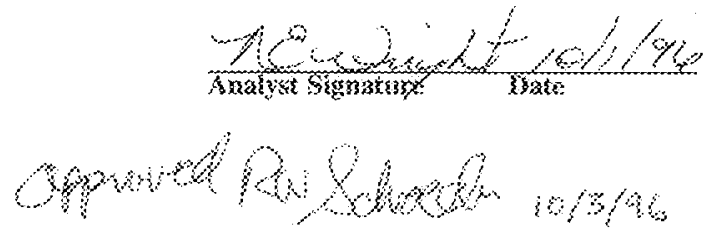

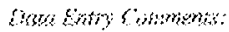

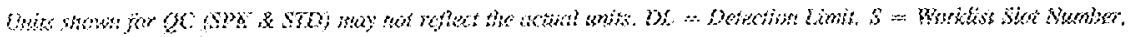

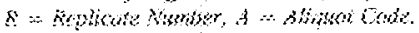




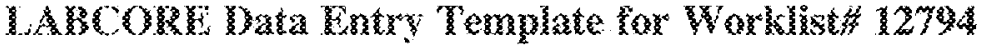

Aratgist:

S

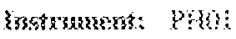

Book $\because k \times 8$

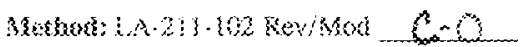

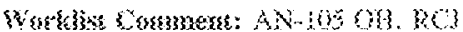

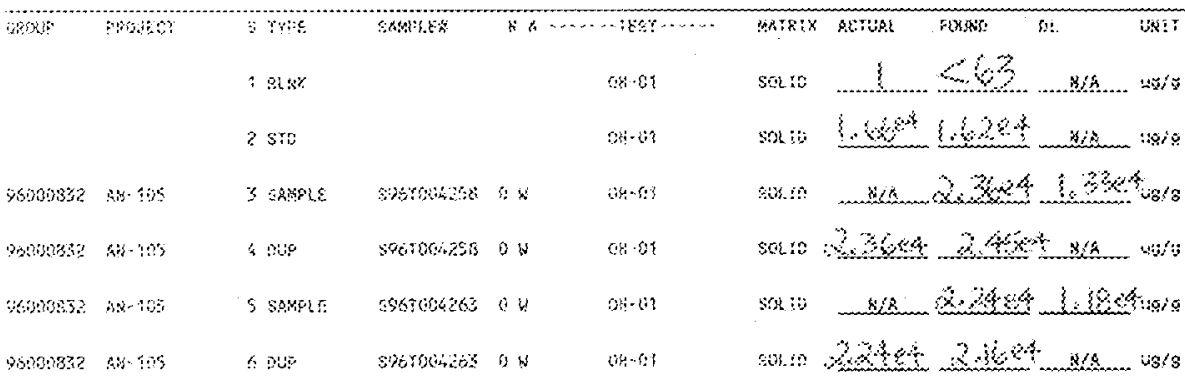

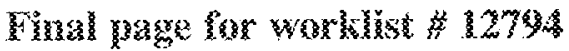

Paran whan anowas

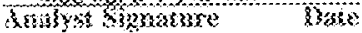

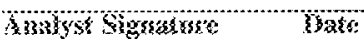




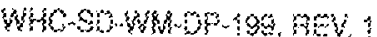

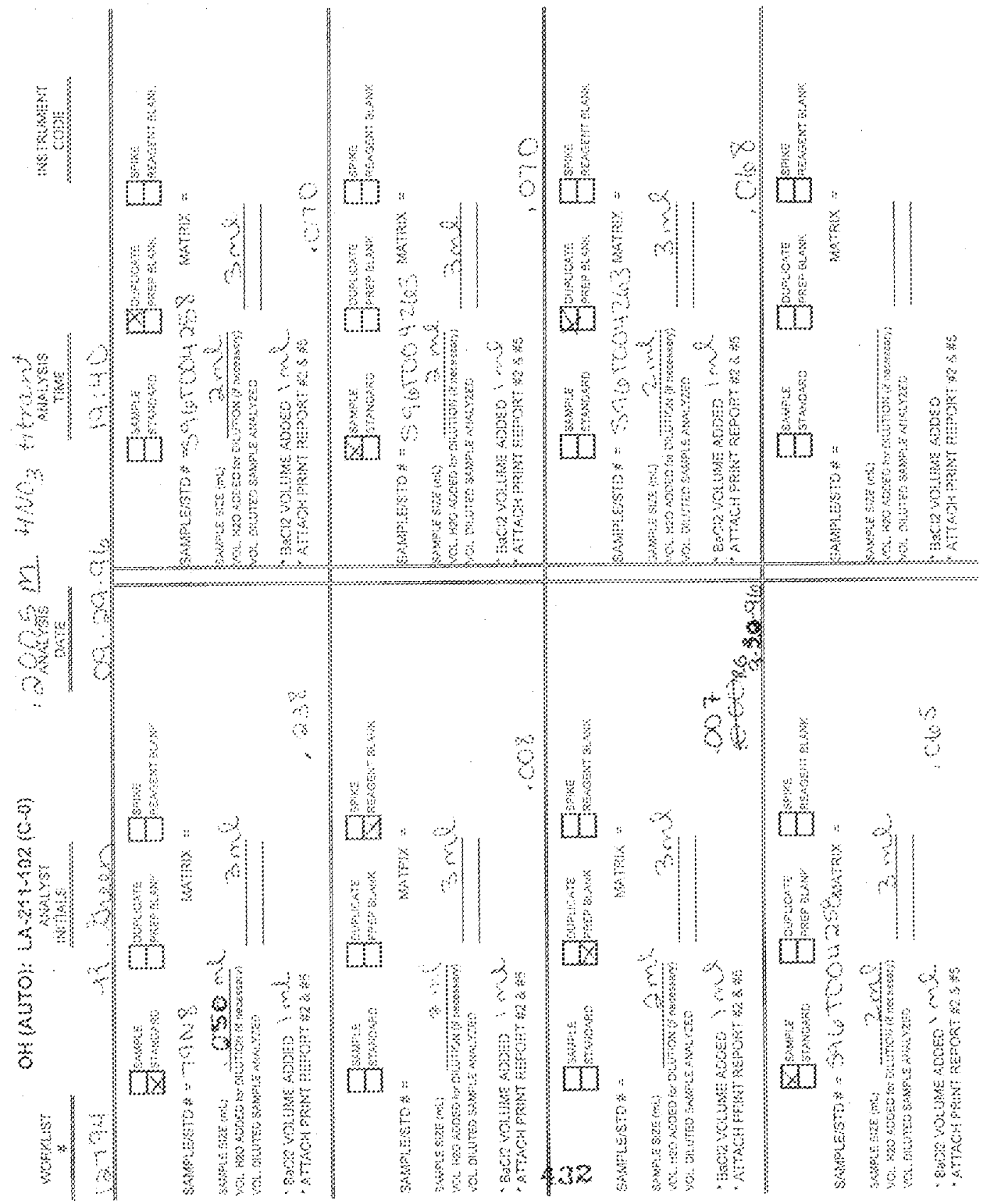




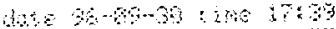

mith

32 म

vons?

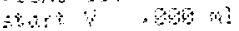

\section{equbiklwh}

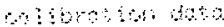

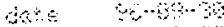

blat

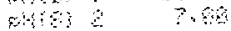

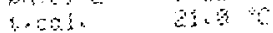

trotas: $1,6 \%$

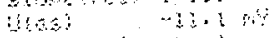

kagenz. book

मक्

$3+4:$

a.

100

mat at

E: - 5040

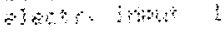

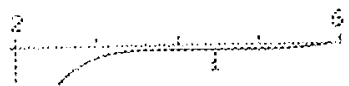

50

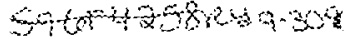

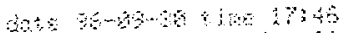

का की मे

l一

J 3 ats

Fition $\quad 30 \%$

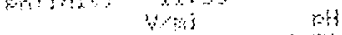

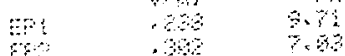

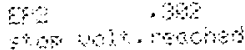

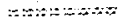

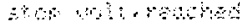
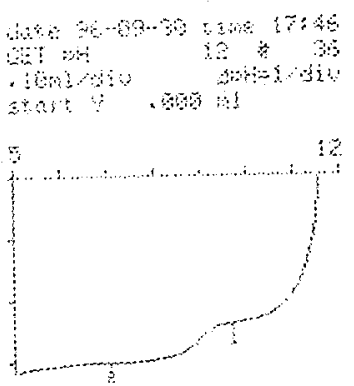


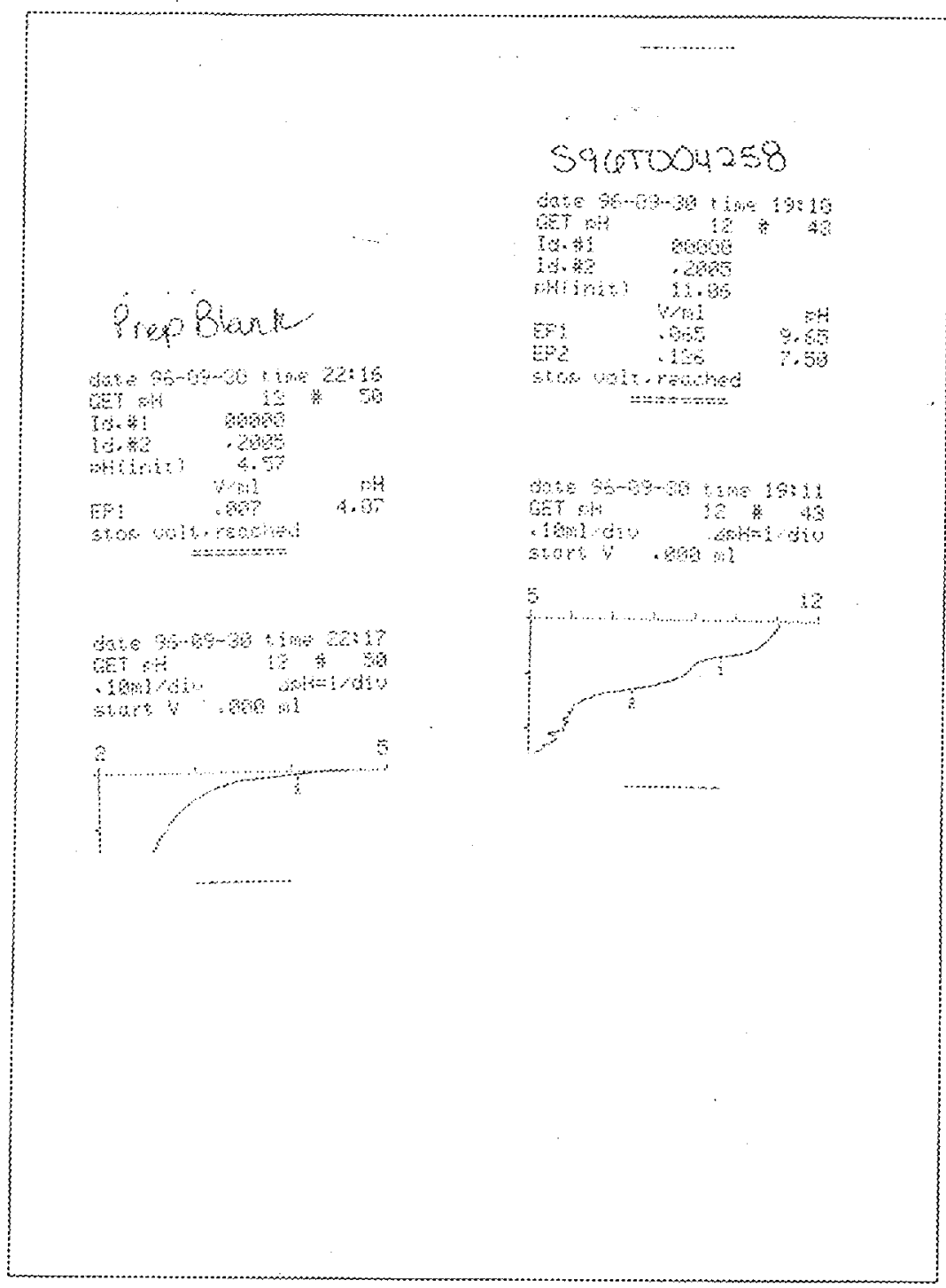




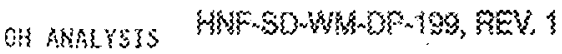
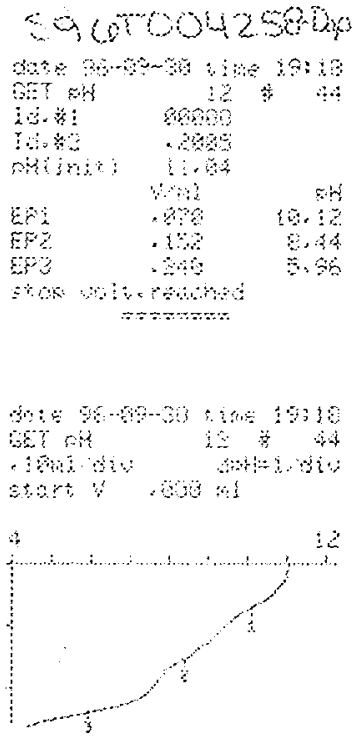

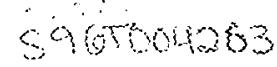

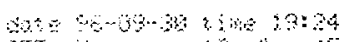$$
\text { AI }
$$

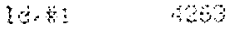

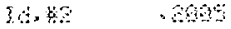

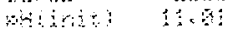

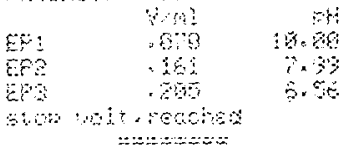
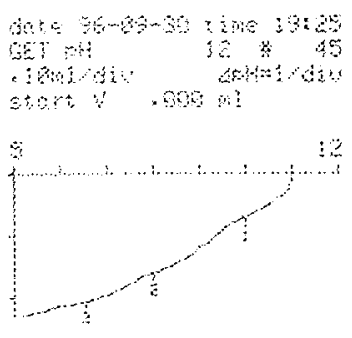

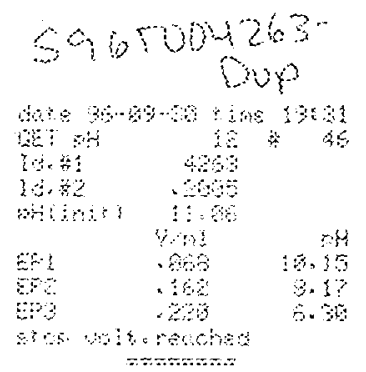

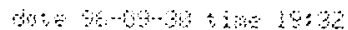

60 का मे

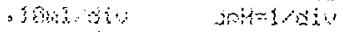

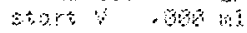

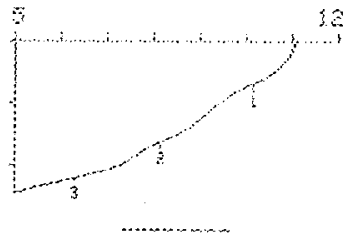




\begin{tabular}{|c|c|c|}
\hline \multicolumn{2}{|c|}{$0 F\{A\} T O\}:\{A-2\} 1 \times\{32\{\mathrm{C} n\}$} & PKEF-ZLAASK \\
\hline wow & Sample $3 z a \mathrm{~km}\} \leqslant S$ & 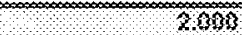 \\
\hline 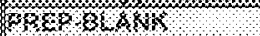 & Conconkration of swos molarivy & 0,3008 \\
\hline 4 y & 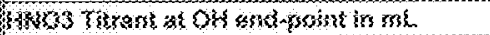 & 0,007 \\
\hline 3784 & Whuten fases of & 2186 \\
\hline 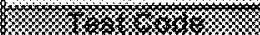 & 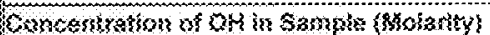 & $1,4 \$ 8,02$ \\
\hline $68 \times 3100$ & 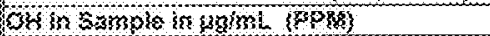 & $3 . \$ \$ 1003$ \\
\hline \multirow{2}{*}{\multicolumn{3}{|c|}{ 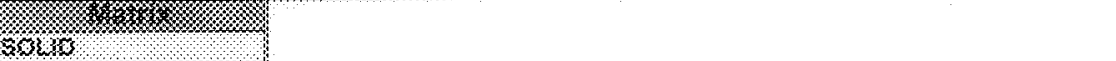 }} \\
\hline & & \\
\hline 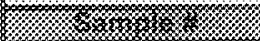 & \multirow{2}{*}{\multicolumn{2}{|c|}{ 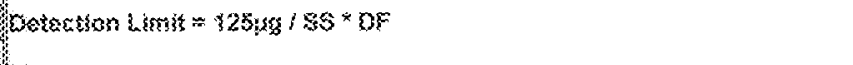 }} \\
\hline 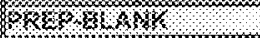 & & \\
\hline 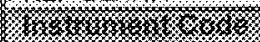 & 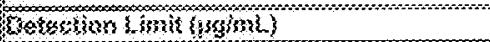 & $3.332+34$ \\
\hline 403 & \multirow{5}{*}{\multicolumn{2}{|c|}{ 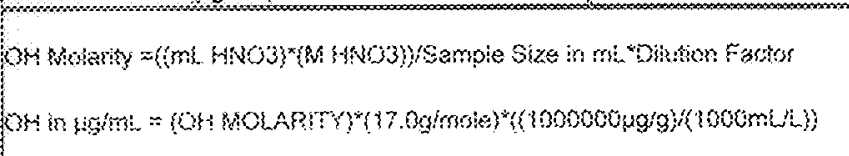 }} \\
\hline - / & & \\
\hline Q ok on & & \\
\hline y & & \\
\hline 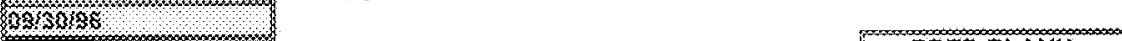 & & \\
\hline & & PREF $B$ BARK \\
\hline & 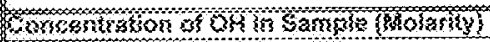 & $1,10 \% 01$ \\
\hline & 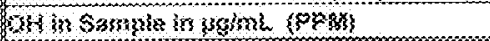 & $\leqslant 33288$ \\
\hline
\end{tabular}

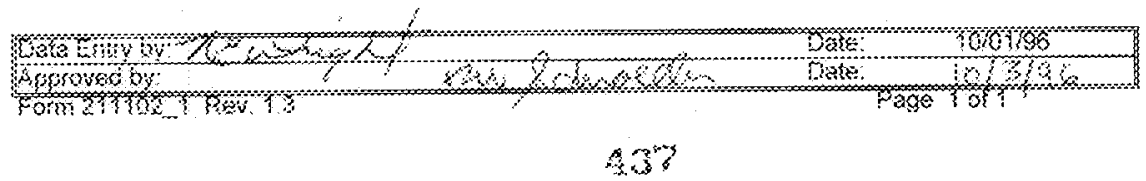




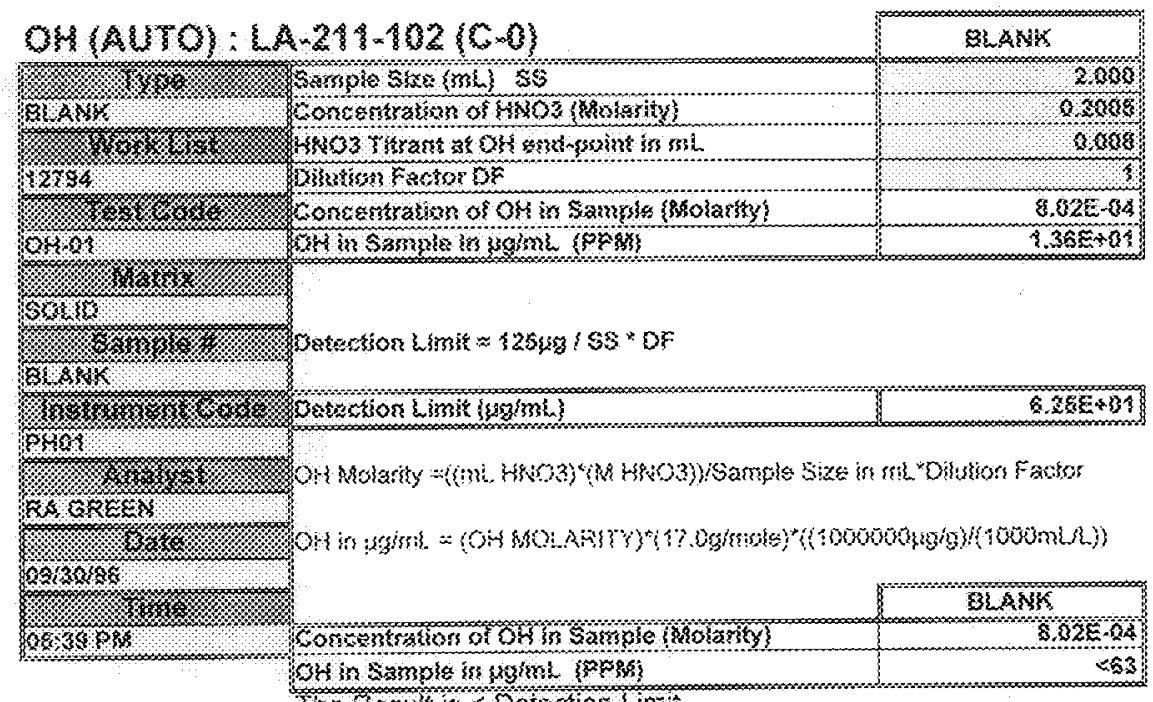

The kesibi \& $<$ Wetedon thin

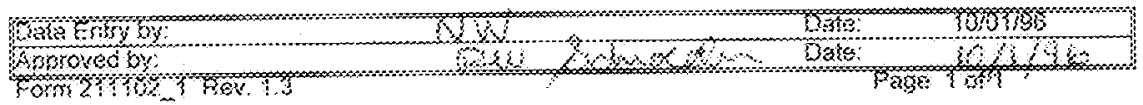




\begin{tabular}{|c|c|c|}
\hline \multicolumn{2}{|c|}{ 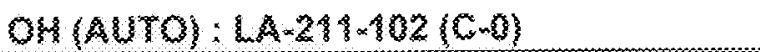 } & STANDAMD \\
\hline 辨 & 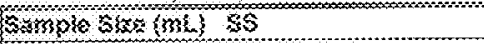 & \%OS\% \\
\hline 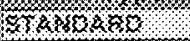 & 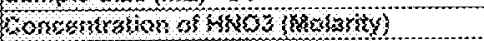 & 0,203 \\
\hline 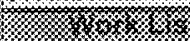 & 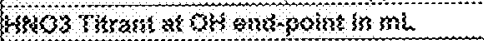 & on3a \\
\hline \%ros & 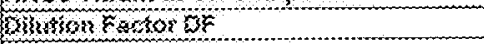 & \\
\hline 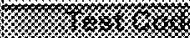 & 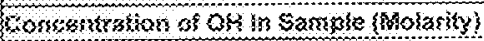 & $3.8 A E-31$ \\
\hline Sus 1 & 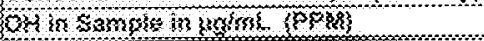 & 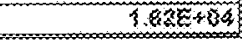 \\
\hline 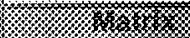 & & \\
\hline W\%, & & \\
\hline 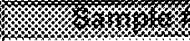 & & \\
\hline KSo & & \\
\hline 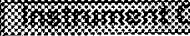 & & \\
\hline 3801, & & \\
\hline 歽原 & 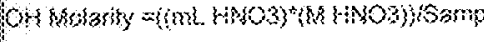 & i. Whituren Factor \\
\hline 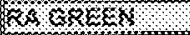 & & \\
\hline 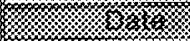 & 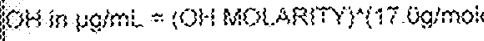 & 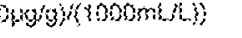 \\
\hline W & & aratenasos \\
\hline 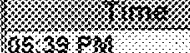 & 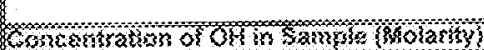 & $3.5+201$ \\
\hline & OH in sampse in yg/mL (PYs) & $3.52 \varepsilon+28$ \\
\hline
\end{tabular}

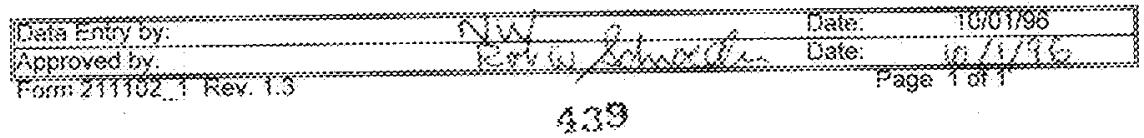




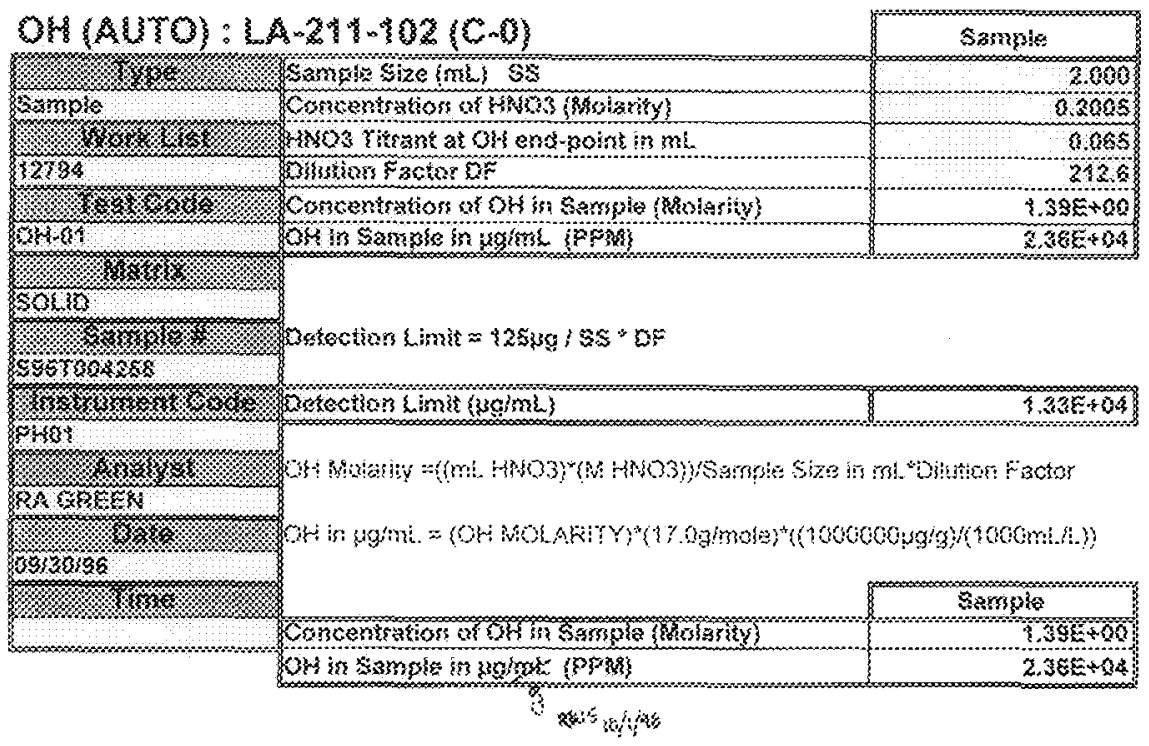

Baphy


PLACF ANALYTCAL OARD N EOK BELOWOA AYTACH TRAVEIER

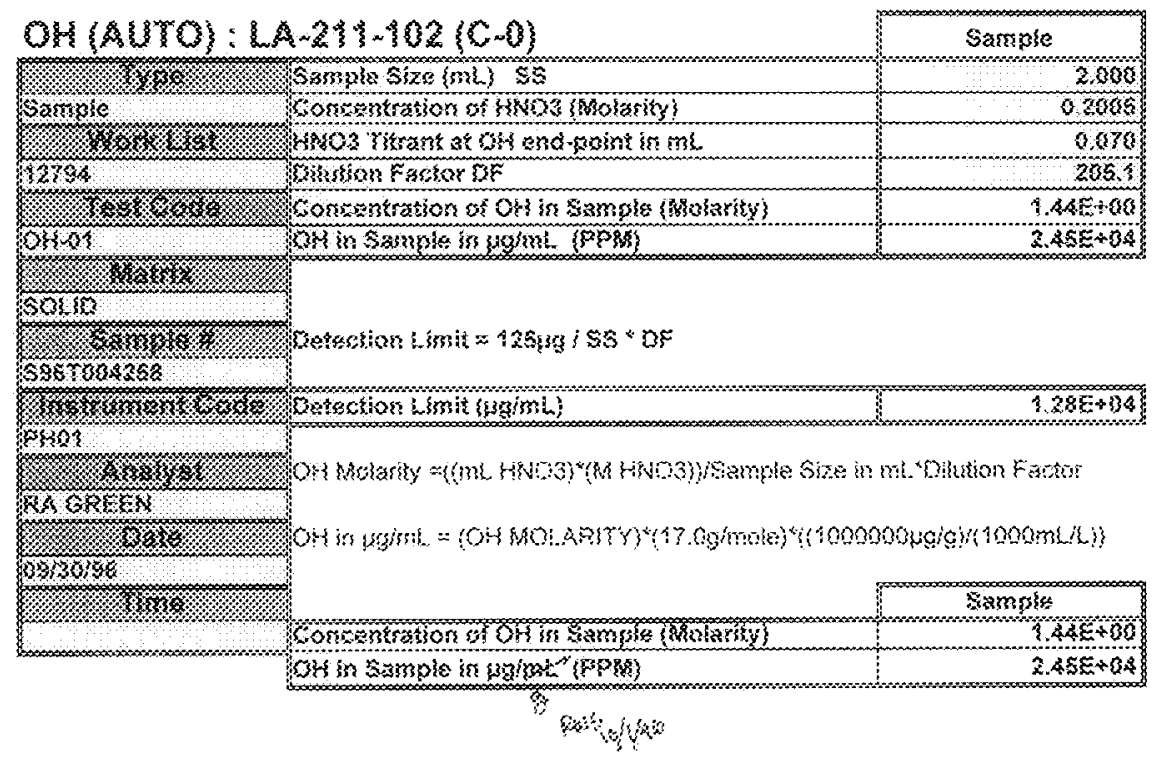

\begin{tabular}{|c|c|c|}
\hline 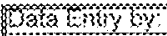 & $1 \times 1$ & Das ofos \\
\hline spowad & $4+1 \times 4<$ & $3 / 1110$ \\
\hline
\end{tabular}

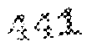




\begin{tabular}{|c|c|c|}
\hline \multirow{2}{*}{\multicolumn{2}{|c|}{ 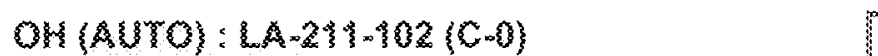 }} & \multirow[b]{2}{*}{ Sarmple } \\
\hline & & \\
\hline xym & asmpls afze $\{m h\} \leqslant$ & 2,000 \\
\hline Sample & Sormontration of HWOS Molatify? & 0,2083 \\
\hline k & 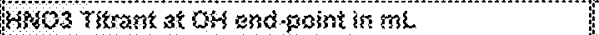 & norg \\
\hline 12704 & Giverion Faskex \&f & 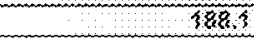 \\
\hline h / & Songentration of of in Sample \{molarity & $1.325 \% 66$ \\
\hline 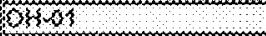 & 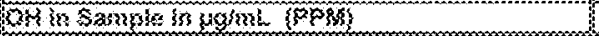 & $2.34 \times+34$ \\
\hline - & & \\
\hline SOLSO & & \\
\hline max & 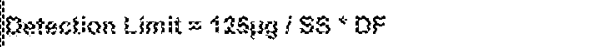 & \\
\hline $888780 \% 253$ & & \\
\hline 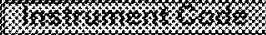 & 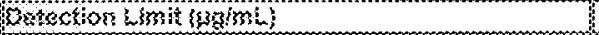 & $138 E+B 4$ \\
\hline S 303 , & & \\
\hline 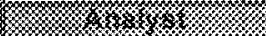 & 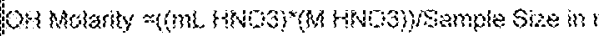 & M. Doution Farkor \\
\hline Ku s6kens & & \\
\hline 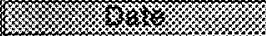 & 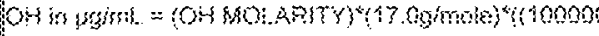 & Dopgig) $(1000 \mathrm{~m}$ Lhis \\
\hline 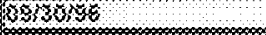 & & \\
\hline 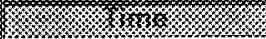 & & \$rmple \\
\hline & 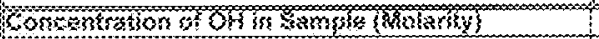 & $3 / 325+60$ \\
\hline & 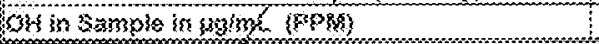 & $2.24 \varepsilon+64$ \\
\hline & ${ }^{3} x_{x}, x_{0}$ & \\
\hline 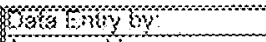 & 10 & 30 \\
\hline 8000100 & 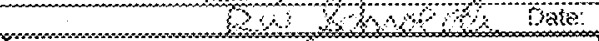 & 10 \\
\hline
\end{tabular}




\begin{tabular}{|c|c|c|}
\hline \multirow{2}{*}{\multicolumn{2}{|c|}{ 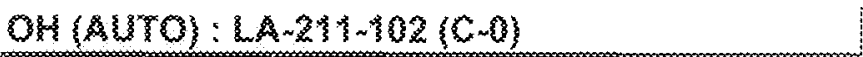 }} & \multirow[b]{2}{*}{ OUPLSCA TE } \\
\hline & & \\
\hline 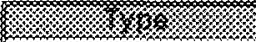 & 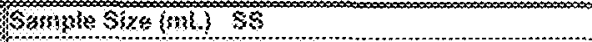 & 2000 \\
\hline Whilesie & Concantration ar mos [Wolarty & $0 \times 008$ \\
\hline 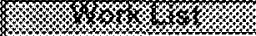 & bNo3 Thkant at oir gnd-posint in mh & 0,088 \\
\hline Y\% 64 & OWution pactor of & 388,4 \\
\hline kns & Concentration of on in sampla (Molarity) & $1.27 \%+50$ \\
\hline Q 45,03 & 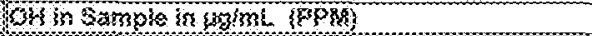 & $2.665+64$ \\
\hline 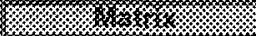 & & \\
\hline 5040 & & \\
\hline \% & 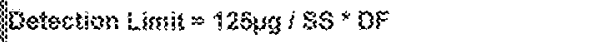 & \\
\hline 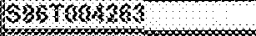 & & \\
\hline$\gamma_{3 x}$ & 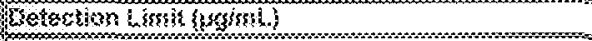 & $1.17=04$ \\
\hline 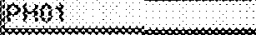 & & \\
\hline 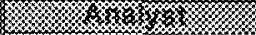 & 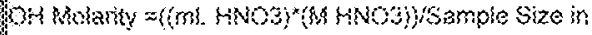 & mL tulution Factor \\
\hline SA SKE & & \\
\hline 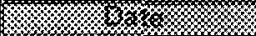 & 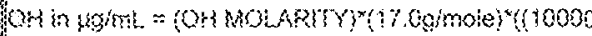 & 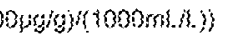 \\
\hline 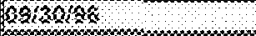 & & \\
\hline (1. & & DUPLISATE \\
\hline & 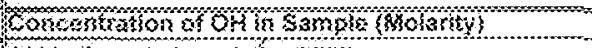 & 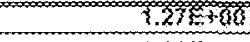 \\
\hline & 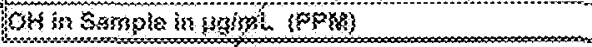 & $2.36 E+6 A$ \\
\hline & Fon & \\
\hline 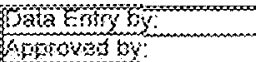 & 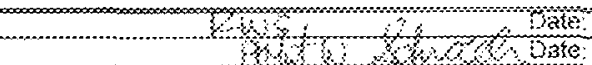 & 101010 \\
\hline mamps & $x_{3} \beta_{3}$ & 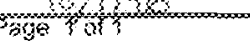 \\
\hline
\end{tabular}




\section{LAPCOR}

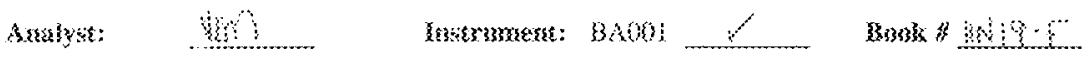

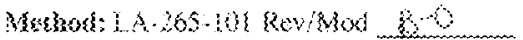

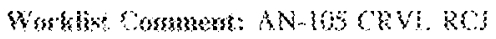

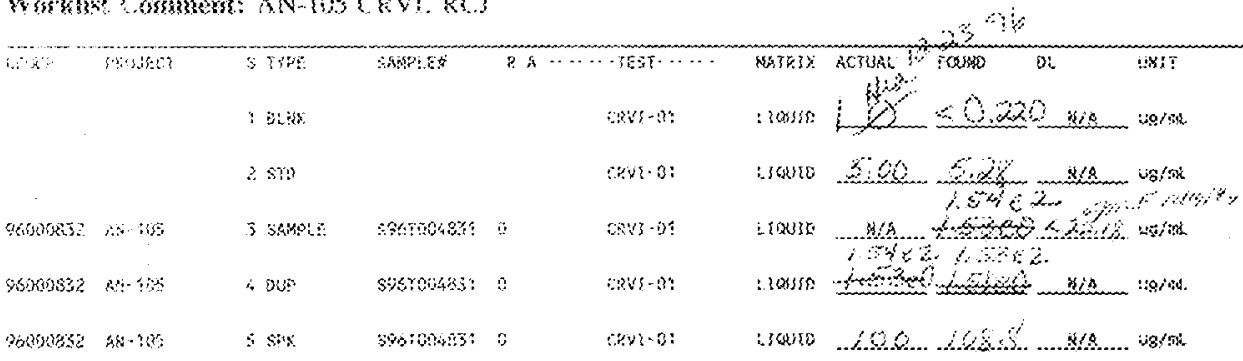

\section{8.}

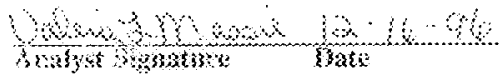

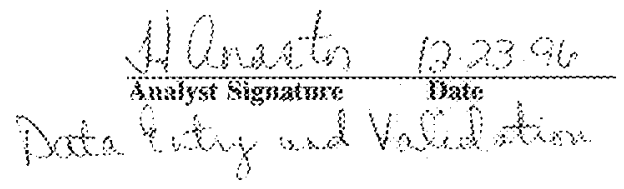

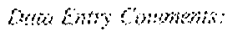

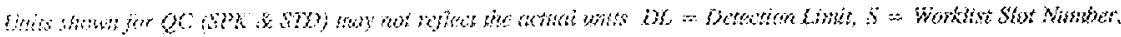

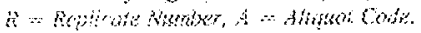




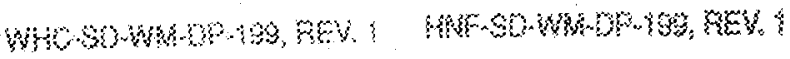

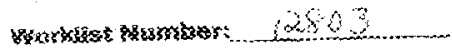

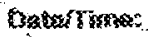

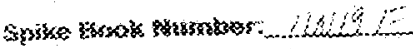

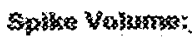

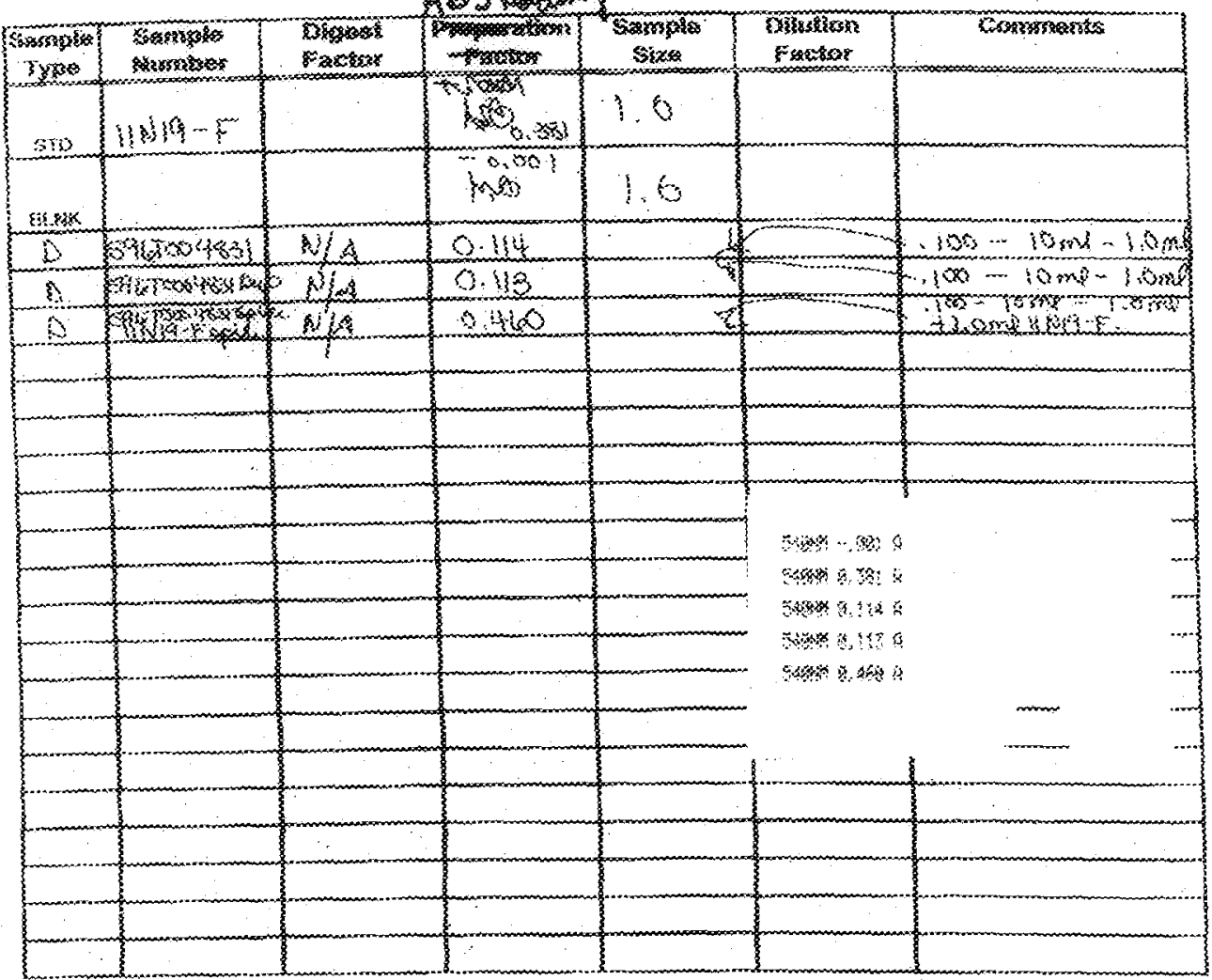




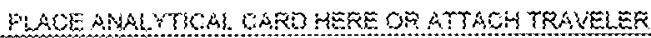

\begin{tabular}{|c|c|c|c|c|}
\hline$C_{5}+A_{3}=283$ & LIOUSOS S & & bink & DUPSSCATE \\
\hline r x & sample Assorbarre at $\$ 43$ nmen & [abss & H, & \\
\hline (3) 310 & Sxmpse volume in min & (Ussassmp) & 130 & \\
\hline 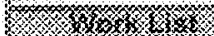 & 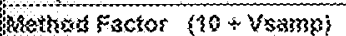 & 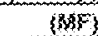 & 11 & 30 \\
\hline$(3803)$ & doludeson Factor & $\{\mathrm{BS}$ & 3 & \\
\hline 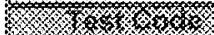 & Sorgest Dilutions fiscter & (ODF) & 3 & \\
\hline 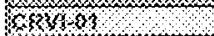 & 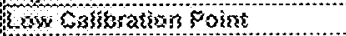 & & 0.021 & 0.021 \\
\hline 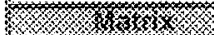 & Wish catussation point & & 0.773 & 0.773 \\
\hline SOUW & Yusnerces & (a) & 6.60537 & 0.68537 \\
\hline V. & slape & (b) & $0.7828 s$ & 0.73248 \\
\hline $50.7 \times 831$ & $C s+8$ B $3333 m$ & & $<0.220$ & 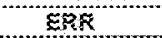 \\
\hline
\end{tabular}

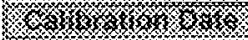

321336

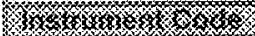

s333.

$100,000,0$

W 3 so

wim

$<\infty, \infty, 0,0,0,0$

a $20 \%$,

3 313635

s $\%$ or o

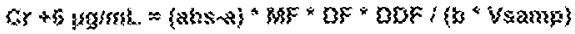

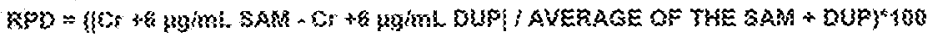

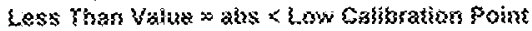

\begin{tabular}{|c|c|c|}
\hline$\forall$ RESULT $\forall$ & then & DPPUCAYE \\
\hline $\mathrm{Cr}+8$ surions & $<0,230$ & $\operatorname{En}$ \\
\hline
\end{tabular}

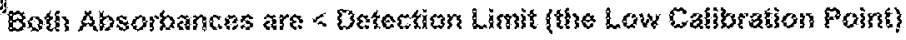

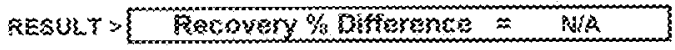

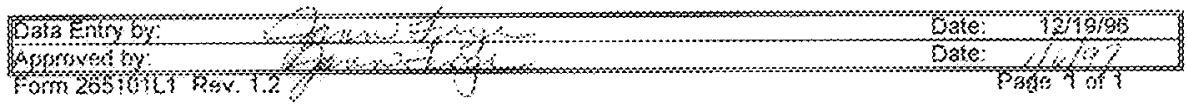




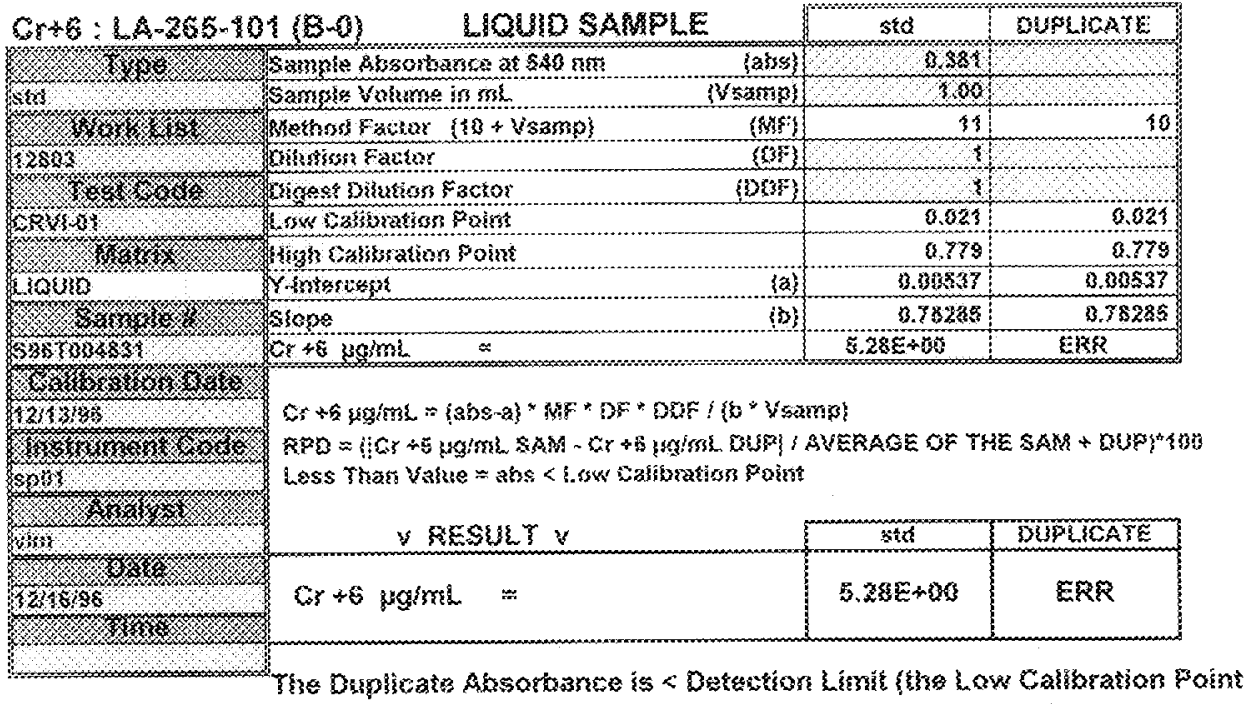

\begin{tabular}{|c|c|c|c|}
\hline BESul T $\times$ & Reonyors "s, Jiflerence & $\approx$ & W/A \\
\hline
\end{tabular}

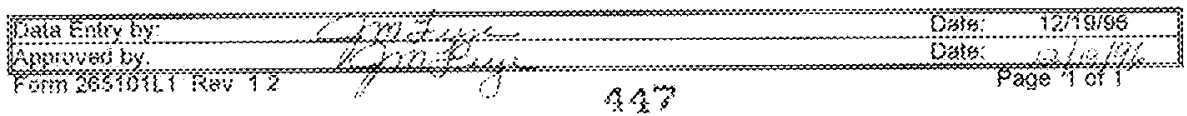


FUACE AWALTICA CARO MERE OR ATTAOHTRAVELER

\begin{tabular}{|c|c|c|c|c|}
\hline$\xi \xi+\xi=2 \%$ & $\{\{S\} S O) \leq 4$ & & sample & BUPS,SCATE \\
\hline $3 x \%$ s & Sample Absorbante at 540 nm & (abs) & $1,1,8$ & 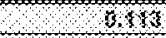 \\
\hline sonsis & sompse volume in nd & (Ysassing & 183 & 100 \\
\hline Kox & 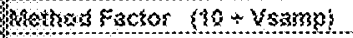 & 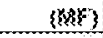 & 11 & 31 \\
\hline $180 \mathrm{3}$ & Dolutiost factor & 100 & S13 & $30 x$ \\
\hline x $x<z$ & Horosst Dilution Fostor & $\{00\}$ & +1 & 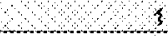 \\
\hline Q 31303 & Luw 0 Bissration polint & & 0.023 & 0.021 \\
\hline$\%$ \% & Whsh carizsation Point & & Q.739 & 0.739 \\
\hline U100h & Frinersespt & a & 0.00837 & 0.00537 \\
\hline 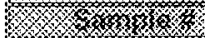 & Bope & (6) & 0.78285 & 0.78285 \\
\hline 3565005333 & $6 s+8$ sishm?. & & $3.5 A E+32$ & $53 E+32$ \\
\hline
\end{tabular}

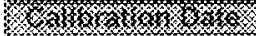

\section{$3+335$}

\%

$\$ 33$

3on ox ox

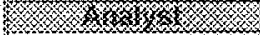

1ris

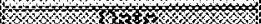

$10 \times 8,3$

3,3 s.

\%

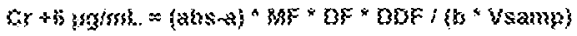

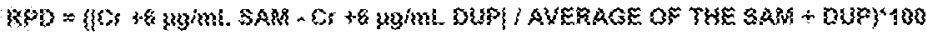
Less Then value $\approx$ als $<$ Lnw Gaskssation Point

\begin{tabular}{|c|c|c|}
\hline 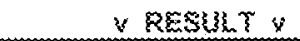 & sumple & DUPLEATE \\
\hline 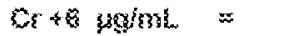 & $\{.545+62$ & $\{.53 E+62$ \\
\hline
\end{tabular}

+3.

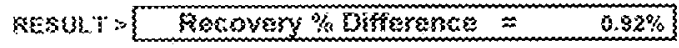

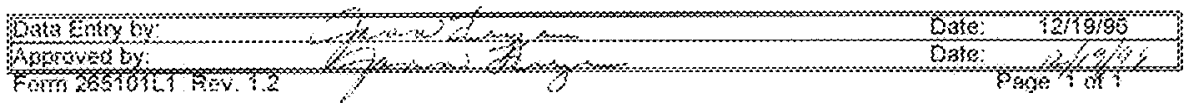


PLACE AWAYTEAL WARO IERE OR ATTAOH TRAVELER

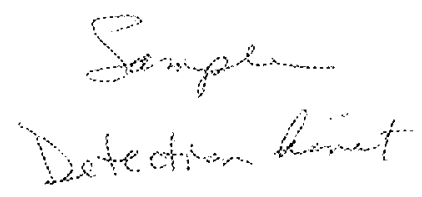

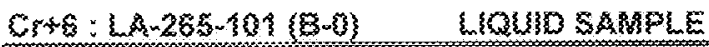

\begin{tabular}{|c|c|c|c|c|}
\hline $3 \%<3 \%$ & Sample Alosorbarse at 543 nirs & (ahs) & As & 2033 \\
\hline sansos & Somple jolume in $n \mathrm{~L}$ & Qyesssss3s: & 133 & 283 \\
\hline 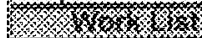 & 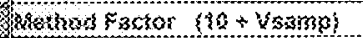 & $\{k\}$ & 11 & 11 \\
\hline 3833 & Sulustiss Fatros & (OF) & $\$ 3$ & 301 \\
\hline W $<<<<$ & Broger Shlution Facsos & (5OF) & 3 & 3 \\
\hline $4 \times 3 \times H_{n}$ & Low sobsssatims poins & & 0,023 & 0.021 \\
\hline 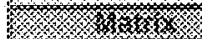 & Hiss Costinssation Paint & & 0.793 & 0.373 \\
\hline sulop & S-thssersesgk & ai & p.8gsa7 & 0.03537 \\
\hline$\%$ os & Stoze & $\{0\}$ & 0.79285 & 0.73285 \\
\hline $4,5>0,3<3<\}$ & 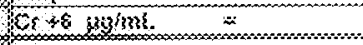 & & $\angle 2,+82$ & $<22,182$ \\
\hline
\end{tabular}

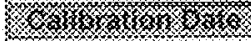

313130

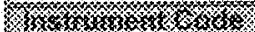
3313

W

whs

\% $\%$ $\%$

33605

\%

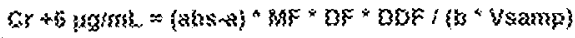

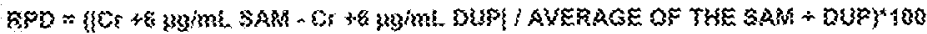

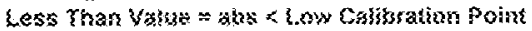

\begin{tabular}{|c|c|c|}
\hline$\checkmark \operatorname{RESU} T \mathrm{~T}$ & sample & BपPLCATE \\
\hline Cr \& kging & $<22,182$ & $<23.182$ \\
\hline
\end{tabular}

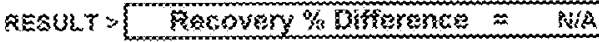

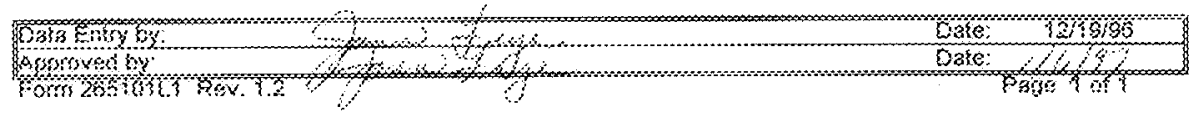




\begin{tabular}{|c|c|c|c|}
\hline $6 r+5:\{A-255-\{1$ & HEXAYALERT CHRONABSH & 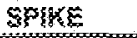 & SWIKE \\
\hline & 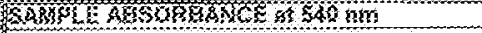 & (Shlo dos) & 1011 \\
\hline 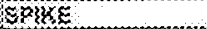 & 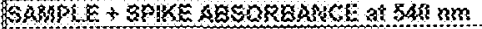 & $18+3$ ang? & 216 \\
\hline 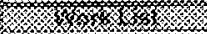 & 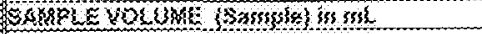 & $(8 \operatorname{san}\}$ & I66 \\
\hline $14 \% 3$ & 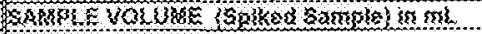 & 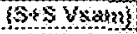 & 3.000 \\
\hline 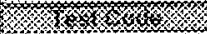 & 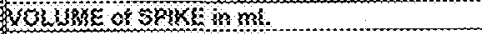 & (Xok S08\}) & 3033 \\
\hline 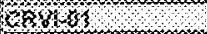 & 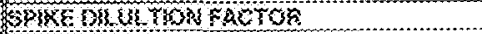 & $\{S \mathrm{tP}\}$ & נl \\
\hline $8 \%$ \% & SpSK Y YAUI & Sugmt & 3 \\
\hline Gos 100 & & & \\
\hline 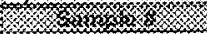 & LOW CASBSRSOAOS NOSNS & & 0021 \\
\hline 36 \% & 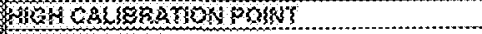 & & 0.738 \\
\hline 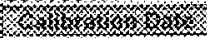 & YHAXSOS & (a) & $338053 ?$ \\
\hline 83,353$\}$ & $9 \mathrm{OPE}$ & (3) & 0.58285 \\
\hline
\end{tabular}

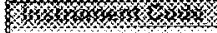

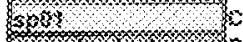

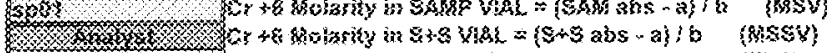

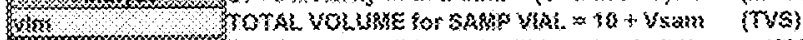

W

$12186 \%$

$\%$ \%

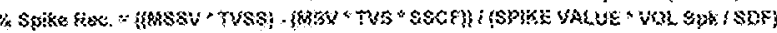

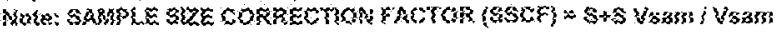

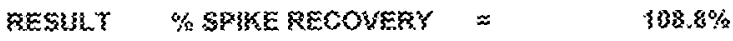

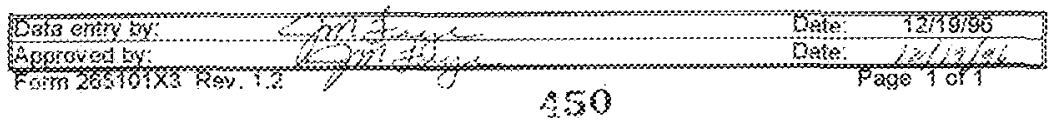




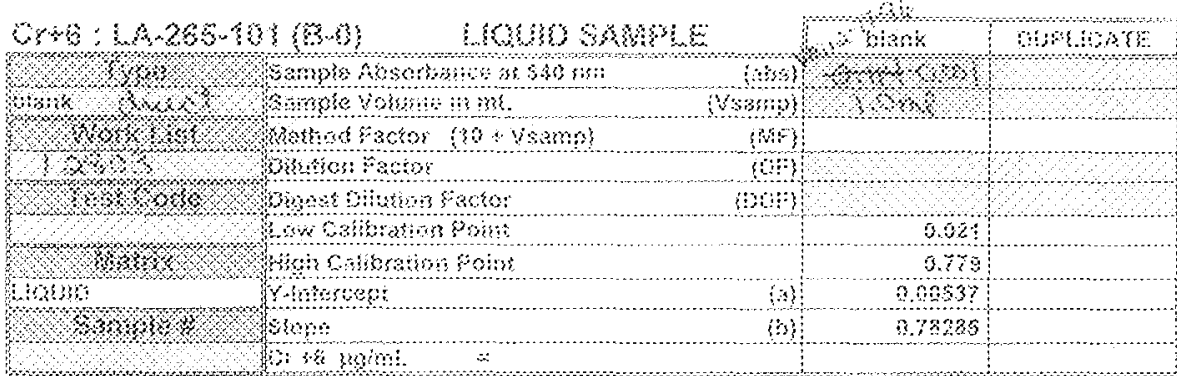

स

\section{1,6}

मेका

- का

+

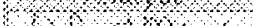

१००

\%०

४ \$ ४ +ork

-

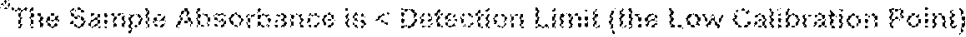

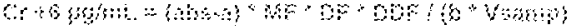

aph

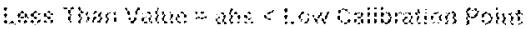

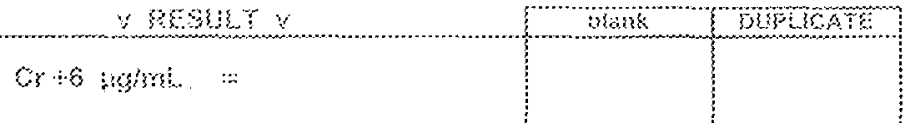

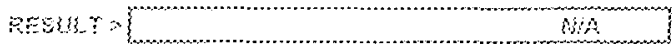




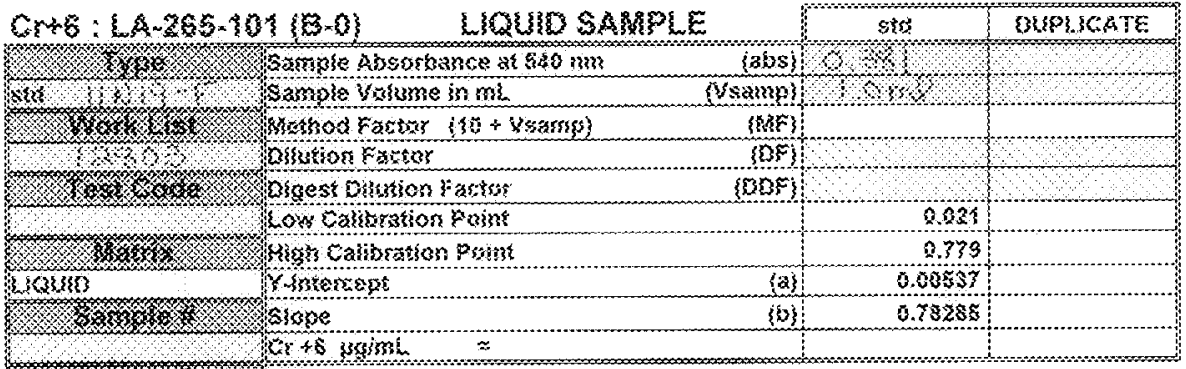

$\%$ 极

$323 \%$

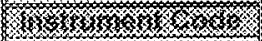

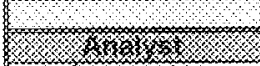

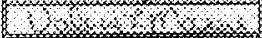

\%

, $3,+3$

\% $\%$ \% $\%$ \% $\%$

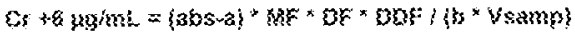

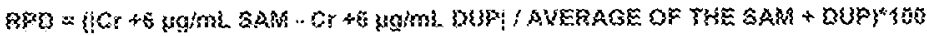

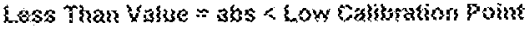

\begin{tabular}{|c|c|c|}
\hline$\checkmark$ FESU\}Y Y & stis & DOFLCA3E \\
\hline Cr +8 sgint $\quad \approx$ & & \\
\hline
\end{tabular}

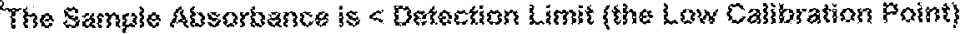

$$
\text { SEX }
$$

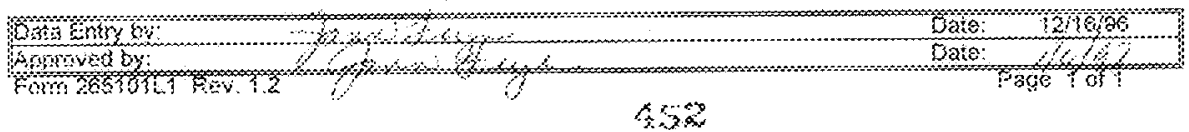




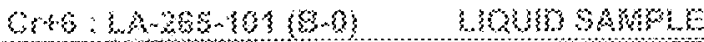

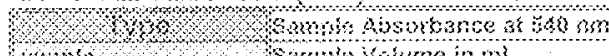

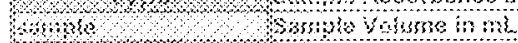

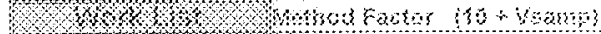
?townom petos ४४

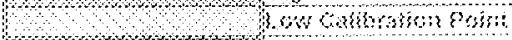

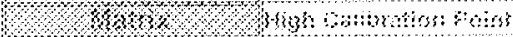
zin, 130

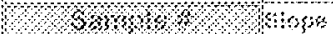

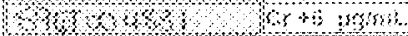
ओ $1 \%, \%$ sos \% $\$ \$ \$ \% ४ \%$ से , $, 2,1$, से $\$$ $+, \mathrm{l}$ सेसम

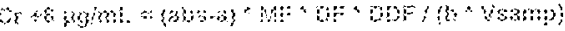

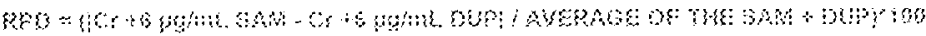

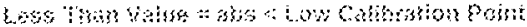

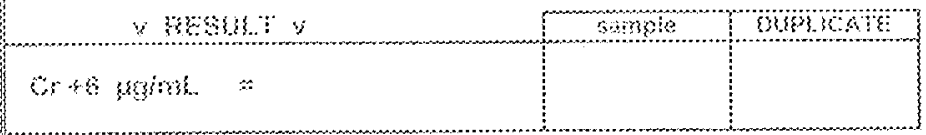

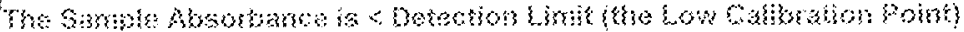

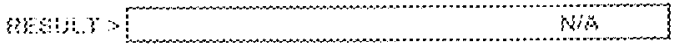

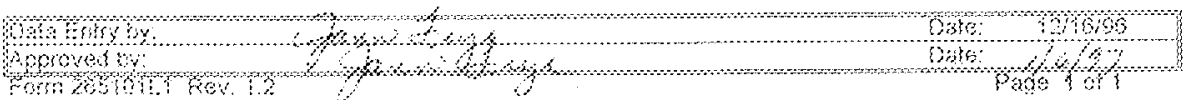




\begin{tabular}{|c|c|c|}
\hline 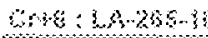 & 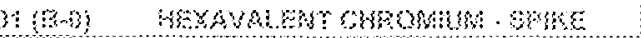 & $8 \%$ te \\
\hline +। & 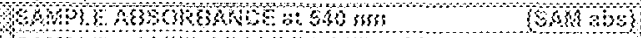 & +1 \\
\hline $\mathrm{b}, \mathrm{\alpha}$ & 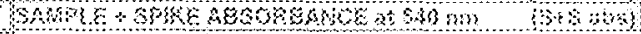 & +1, \\
\hline 10 0 & 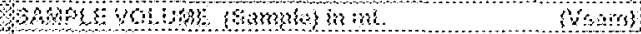 & $\$ 0,0$ \\
\hline ४४ & 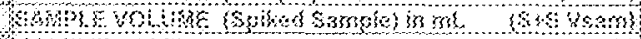 & \\
\hline & 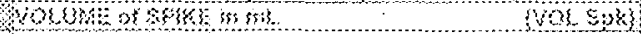 & 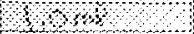 \\
\hline & WHEHUBOH FASOR & \\
\hline & ब⿰亻 vass & \\
\hline & 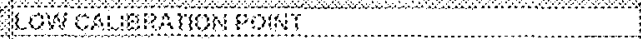 & 0.62 \\
\hline \% & HOH OA, anamos gont & 223 \\
\hline h & 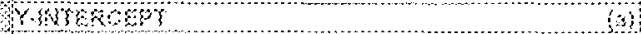 & 0.0083 \\
\hline 13,6 & Qझ! & $13,3,10 \mathrm{~s}$ \\
\hline
\end{tabular}

\% $\%$ \%

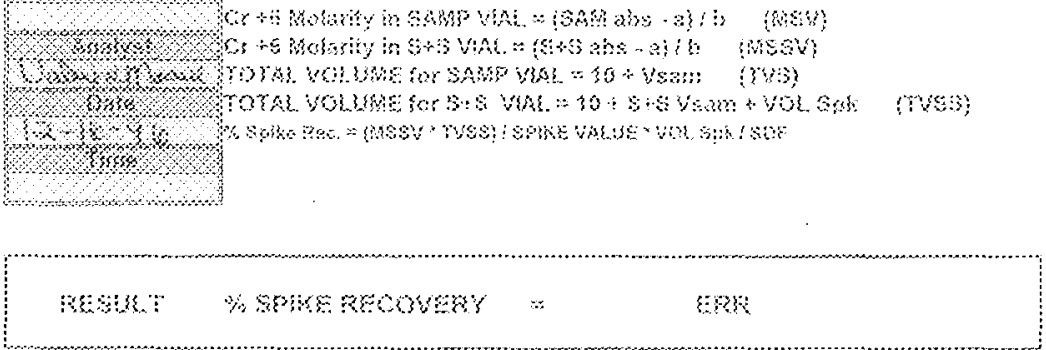

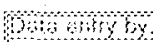

aisosats. and 


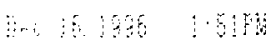

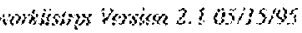

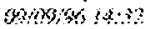

\section{4.}

ans: $\quad \&$

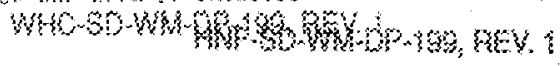

3996

massis $\ln 19 \%$

Asastyst $\quad$ Hen

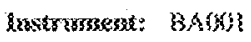

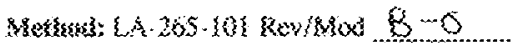

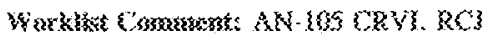

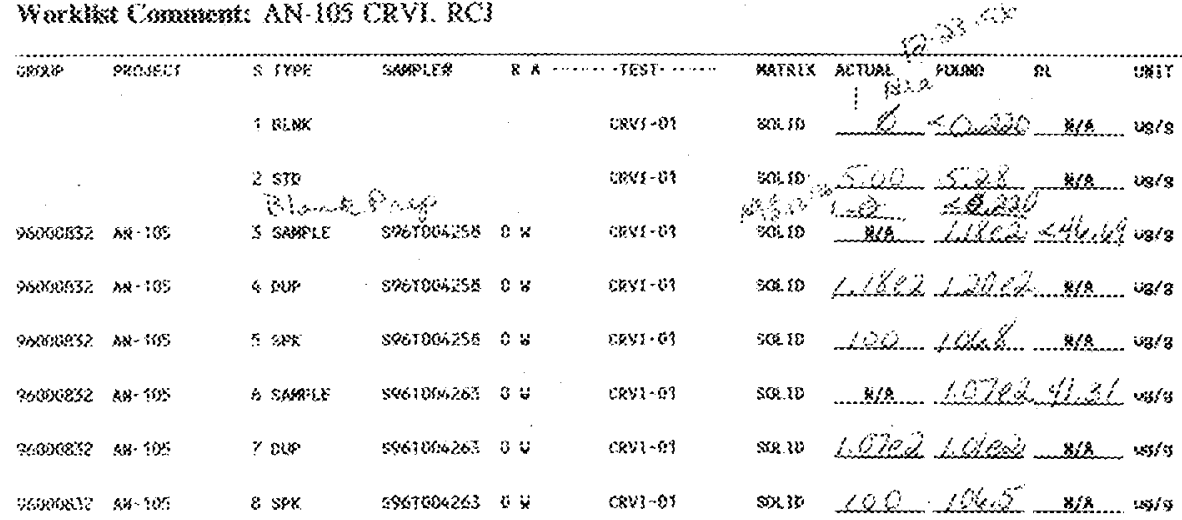

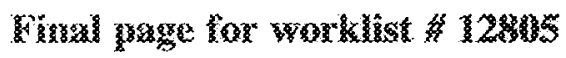

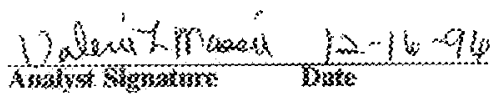

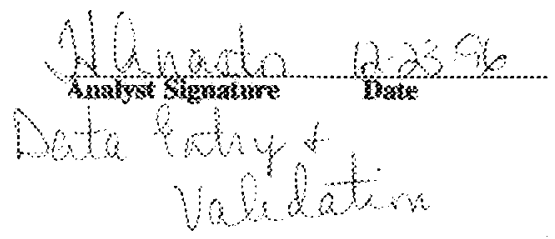

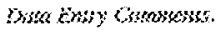

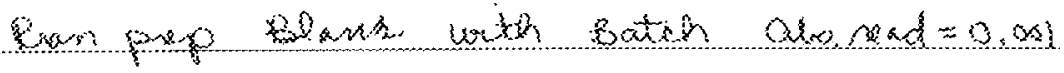

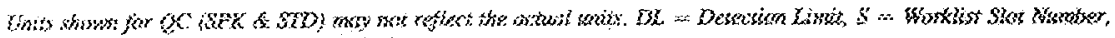

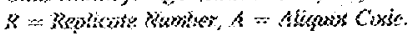




\begin{tabular}{|c|c|c|c|c|}
\hline $6 Y+5: L A-265$ & 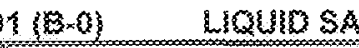 & & bink srep: & DUPS,CATE \\
\hline 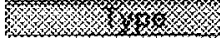 & 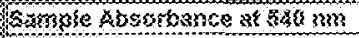 & (abs) & +, & \\
\hline sind oss, & Sanugse $y$ osume in mi & (Ysamm & to & \\
\hline 1/x & Matsoa factos iss + vsarns? & MS & II & 10 \\
\hline 12000 & Gubshian Fagtse & (O) & 3 & \\
\hline 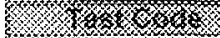 & Jussss ginsion fastor & Sors & n's & \\
\hline 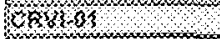 & Loo Calibraton point & & 3.142 & 3.321 \\
\hline$\%$ \% & Hion cialiburation Pan & & 3.734 & 3.779 \\
\hline 16110 & Hunkereent & (a) & $0.0053 ?$ & $0.0053 \hat{R}$ \\
\hline \% $/$ s 3 z & Sisges & (b) & 0,78235 & 0.78285 \\
\hline 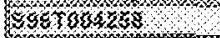 & $(s+65355 / 5 m$. & & 60,330 & ERR \\
\hline
\end{tabular}

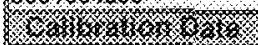

13330

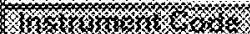

好3

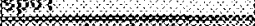
$8 \% 3$

\%3in

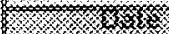

723535

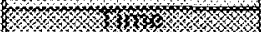

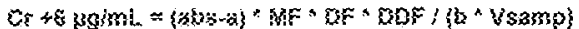

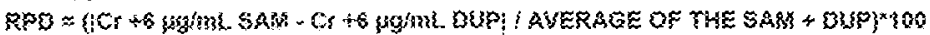

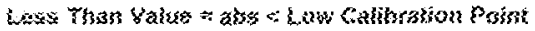

\begin{tabular}{|c|c|c|}
\hline$y$ KESIST Y & osnk prope & $03 / \mathrm{GKACR}$ \\
\hline Cr A & -20.220 & $E R P$ \\
\hline
\end{tabular}

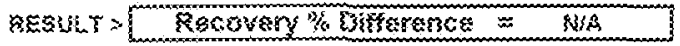


FACE ANATYTCA CAROHERE ORATTAC TRAVEIE

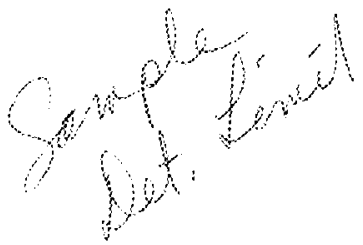

\begin{tabular}{|c|c|c|c|}
\hline $\operatorname{Cr}+5: 3,265-3,3$ & SOLUS SAMPLE & sample & BSSFILUSAGE \\
\hline 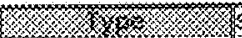 & 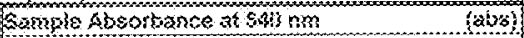 & 1000 & 1000 \\
\hline 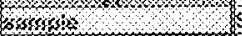 & Sample volume in m: & 300 & 3000 \\
\hline$\alpha z k$ K & 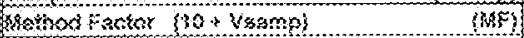 & 11 & 11 \\
\hline 3830 & Bihoton Facitur & 3 & 1 \\
\hline 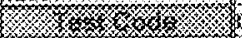 & gisest sik & 831 & 3,34 \\
\hline $6 \times 600 \mathrm{O}$ & cows salionation shs poist & 0.021 & 0.921 \\
\hline \% & Highs gatbration ass geint & 9.739 & $0.7 \mathrm{~g}$ \\
\hline Q QLSO & Hontarespe - 1 m & 0,0037 & Qmo3r \\
\hline 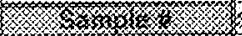 & sigho & 0.78385 & 0.78235 \\
\hline 0.3002313 & $135+5+\sin$ & 44,31 & 0.80 .328 \\
\hline 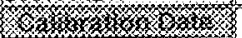 & \multirow{4}{*}{\multicolumn{3}{|c|}{ 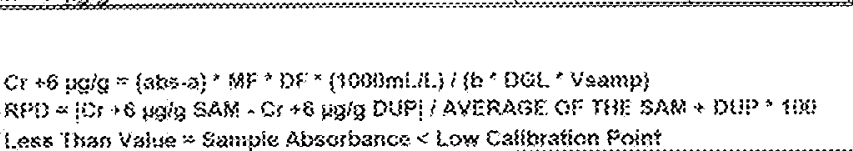 }} \\
\hline Whos & & & \\
\hline 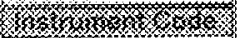 & & & \\
\hline on & & & \\
\hline Wras & UPESUT Y & sample & OUFLLCAYS \\
\hline \% & \multirow[b]{2}{*}{ Crts yojig } & \multirow[b]{2}{*}{ रA\}.313 } & \multirow[b]{2}{*}{$\times 40.328$} \\
\hline $3,10,86$ & & & \\
\hline
\end{tabular}

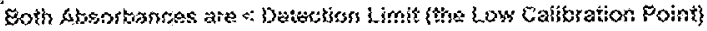

\begin{tabular}{|c|c|c|c|}
\hline Res\{3\}; $>\{$ & Gosavery $\%$ Wifseremoe & t & SHA \\
\hline
\end{tabular}

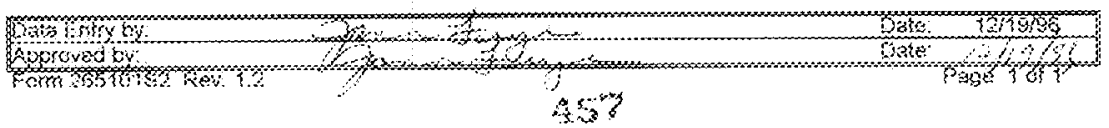




\begin{tabular}{|c|c|c|c|c|}
\hline 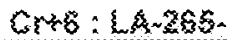 & LSOSBOS ST & & mbins & SBSPSBCATE \\
\hline \% $\%$ z & 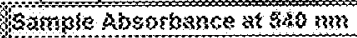 & (abs) & 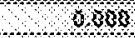 & \\
\hline $3 \mathrm{mor}$ & sample volume in ml & Ysamma & 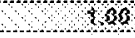 & \\
\hline 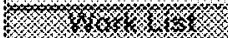 & 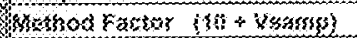 & $\{k s\}$ & 11 & 10 \\
\hline $1 / 8 \leqslant s$ & ossutron Fretory & (n) & 3 & \\
\hline 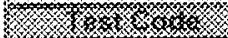 & ousess butson taststs & $\{$ ans & 3 & \\
\hline $03 \times 1131$ & Low valiurstion point & & 2.335 & 0.925 \\
\hline 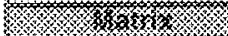 & Gigh Calibration Point & & 0.379 & 0.739 \\
\hline Lous\}彳 & S shlentape & (2) & $0.005 \mathrm{37}$ & 0.00537 \\
\hline 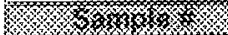 & Gloge & $\{w\}$ & 0.78283 & 0.78283 \\
\hline 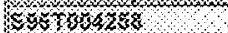 & $\alpha+5+6$ wsism & & $\angle 0,30$ & $\mathrm{ERX}$ \\
\hline
\end{tabular}

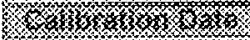

321,38

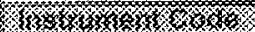
sons \% (1)

stm 緅 12\%48 \%

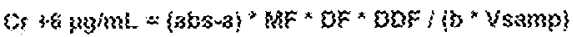

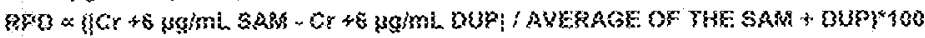

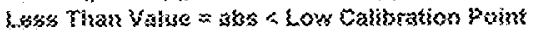

\begin{tabular}{|c|c|c|}
\hline 4 RESULT Y & bnst & DUPLIOAYE \\
\hline cr+s zuspmi. $\approx$ & $<0.220$ & $E x$ \\
\hline
\end{tabular}

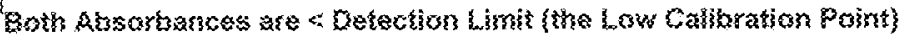

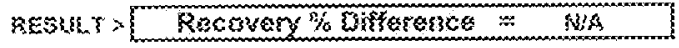

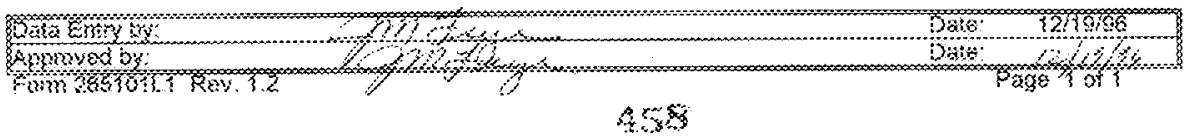




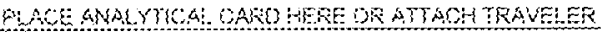

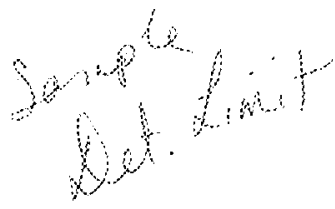

\begin{tabular}{|c|c|c|c|}
\hline Cr+G:1.4265-301 & $\cong O L D D \triangle A O P L$ & sampie & EUPLSASTE \\
\hline $4 \%$ r 8 r & Soniple Absorbance at SAE nm & 10000 & mosos3 \\
\hline 63000 & Sampse vatume in ms & 1302 & 306 \\
\hline$\alpha x$ x & sossodfactor $(10+$ vsampi & 31 & 31 \\
\hline 3306,000 & BGoton fsckar & +1 & 1 \\
\hline x $x$ x & brosst ant. & 403 & 48s \\
\hline $6401 \mathrm{Q}$ & Cow saikration shs poont & 0.321 & $0.82 i$ \\
\hline $4 \%$ & Wighs satibration acs nom & 9778 & 0.773 \\
\hline 80,0 & Simereent & 00033 & 0.00833 \\
\hline 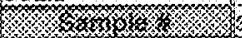 & logs & 0,72385 & 8,78288 \\
\hline$\times 400 \% 30$ & $5 s+6$ sogsy & 48883 & $<48031$ \\
\hline 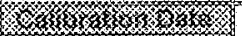 & \multirow{5}{*}{\multicolumn{3}{|c|}{ 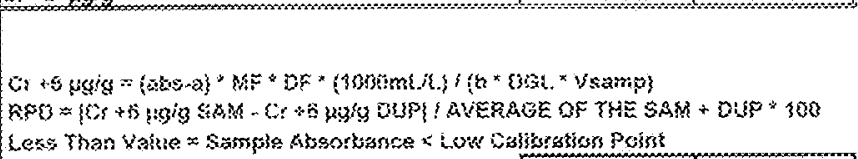 }} \\
\hline $4 \mathrm{~s} \% \mathrm{n}$ & & & \\
\hline 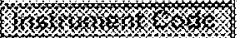 & & & \\
\hline & & & \\
\hline 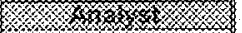 & & & \\
\hline 46 & VECSULY $\}$ & sample & BUFLESATE \\
\hline \% & \multirow[b]{2}{*}{$\mathrm{Cr}+5$ pyss $=$} & \multirow[b]{2}{*}{ Сष.698 } & \multirow[b]{2}{*}{$<A 5,041$} \\
\hline 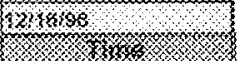 & & & \\
\hline
\end{tabular}

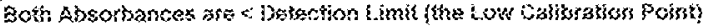

RESULT Pocouery \% Diferenex $=$ NA

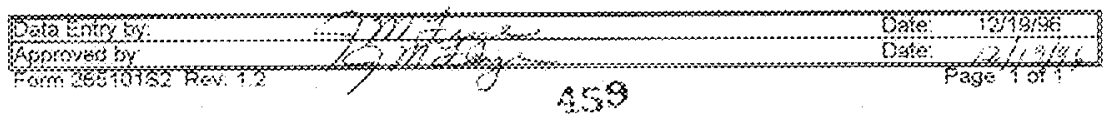




\begin{tabular}{|c|c|c|}
\hline$C r+5: 14-265-1$ & 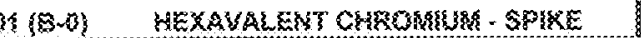 & SPIXE \\
\hline $8<x_{0 / 2}$ & SSAWL A & (10,3 \\
\hline $6916 \mathrm{~s}$ & 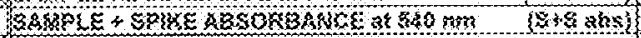 & 4,38 \\
\hline 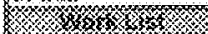 & 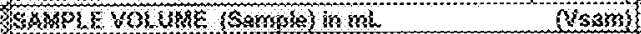 & 2003 \\
\hline Kusor & 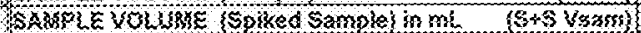 & 100 \\
\hline s/ & 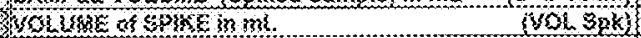 & 300 \\
\hline 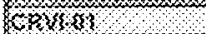 & GPKS OLOL HON \& ACTOR & +1 \\
\hline$\gamma / x / 4$ & SPMOESHLYE & 8 \\
\hline 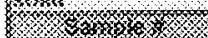 & KOW OALSBRBTHSN DOMT & 3.021 \\
\hline 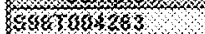 & WGK OAUZRRATGN FONKT & 0.779 \\
\hline 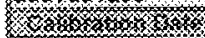 & HWRQST & 0,00833 \\
\hline 300 & $8 \mathrm{son}$ & 0,528 \\
\hline
\end{tabular}

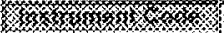

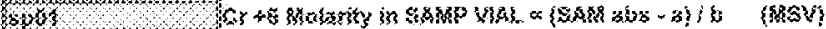

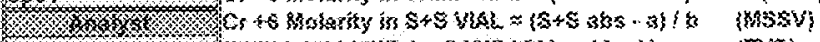

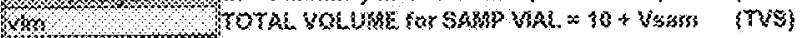

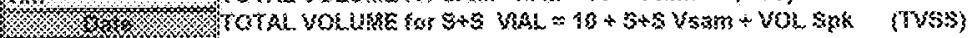

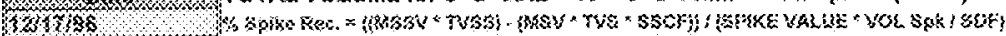

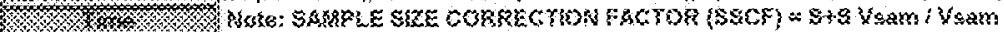

is

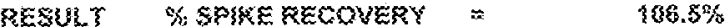

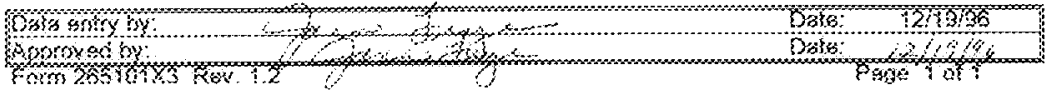




\begin{tabular}{|c|c|c|c|}
\hline$C r+6: 2,4-265-16$ & 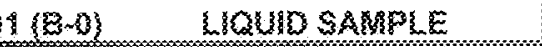 & sta & DUPLICATE \\
\hline W & Sissugle Ahsosbaniza at S40 smi & 0,52 & \\
\hline 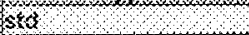 & Sample Yshome in mb & 3,00 & \\
\hline 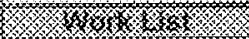 & 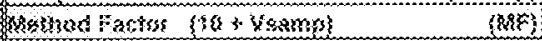 & 31 & 30 \\
\hline 13860 & OWlstion E Es:ser & 1 & \\
\hline 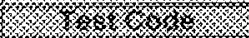 & Dogest g3instion froctors & 1 & \\
\hline $6 \% 861$ & Low Cosibsstion Psim & a.a? & 0.023 \\
\hline 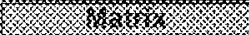 & 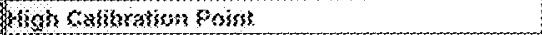 & 0.778 & 0.779 \\
\hline LIOSSO & smercept & 0.0859 & B.,BDSM? \\
\hline \% & $\operatorname{sig} \%$ & $9.732 \pi 3$ & B.7528S \\
\hline $45879862583 n<028 \%$ & $c r+6 y 3 m L$ & $8,28 \mathrm{E}+60$ & $\Leftrightarrow 8 R$ \\
\hline 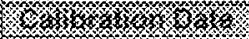 & \multirow{5}{*}{\multicolumn{3}{|c|}{ 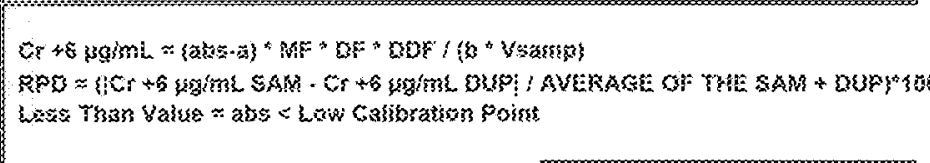 }} \\
\hline $3 / 5$ & & & \\
\hline m & & & \\
\hline 60010 & & & \\
\hline 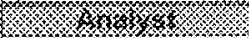 & & & \\
\hline$\omega_{n}$ & Q BESUMT V & 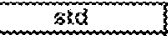 & DUPLICKE \\
\hline 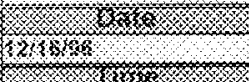 & 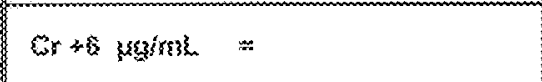 & $5.28 \div 300$ & ERR \\
\hline
\end{tabular}

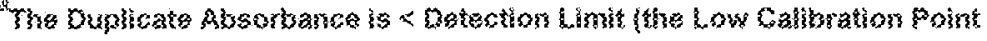

\begin{tabular}{|c|c|c|}
\hline 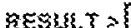 & Ragouery of OFferares & wa \\
\hline
\end{tabular}

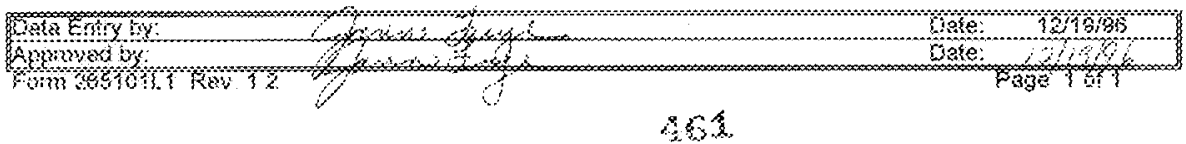




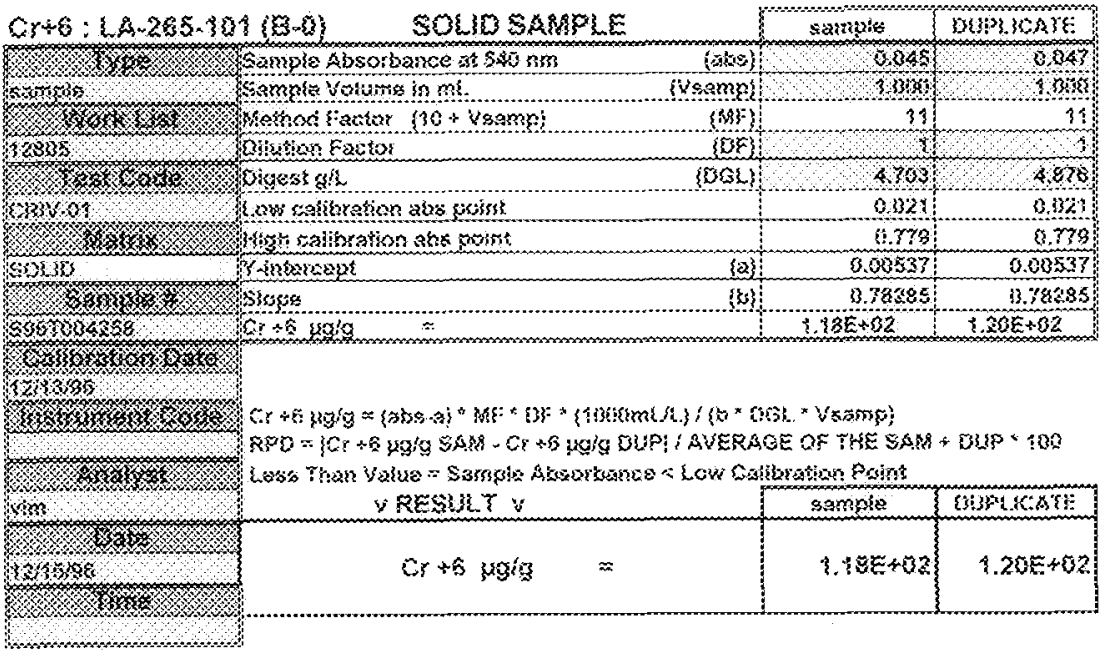

\begin{tabular}{|c|}
\hline Opcovam so \\
\hline
\end{tabular}

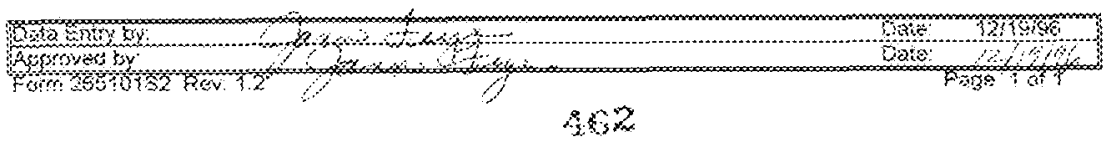




\begin{tabular}{|c|c|c|}
\hline$\angle r+6: 3,253-10$ & HEXAVALEST SWFOMASHA - SRIKE & SYKE \\
\hline 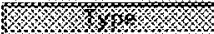 & 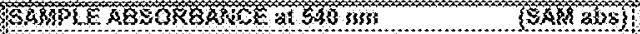 & 0,345 \\
\hline SMllo & SAMPLE S SMISE ABSORSANOE AA SAB RM & 335 \\
\hline \% & 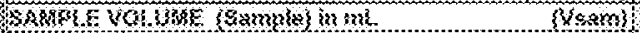 & 1) \\
\hline $1<838+8$ & 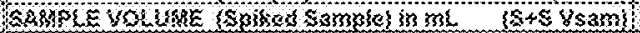 & 0,03 \\
\hline \% & 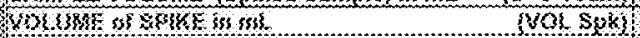 & 13330 \\
\hline s. & SPME OU US RON EX KroR & \\
\hline xk & SPUE VAUUE & 3 \\
\hline$\$$ & & \\
\hline \%x & OW OASBOATOS $80 \%$ & Q32! \\
\hline $3010345 \%$ & 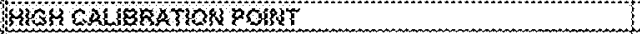 & 3.779 \\
\hline x & YUWTERSER & Q.3ass? \\
\hline 3,3386 & Sose & 3.7828 \\
\hline 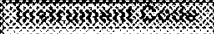 & & \\
\hline 001 & 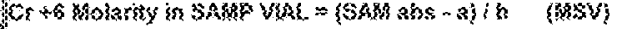 & \\
\hline 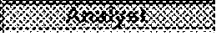 & 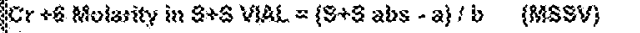 & \\
\hline 33, & 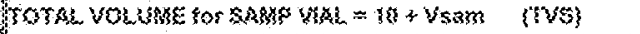 & \\
\hline & 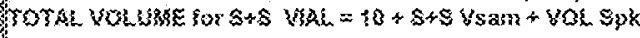 & k \{ryas \\
\hline $3 / 195$ & 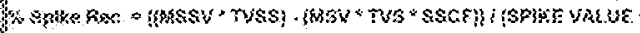 & 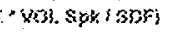 \\
\hline 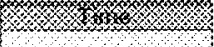 & 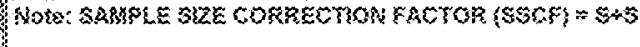 & S Vsam / Ysam \\
\hline
\end{tabular}

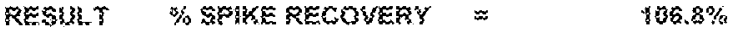

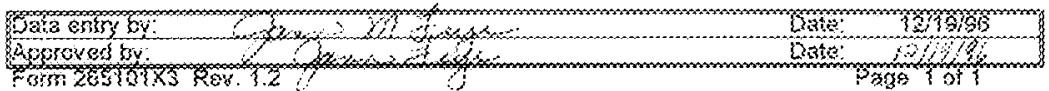




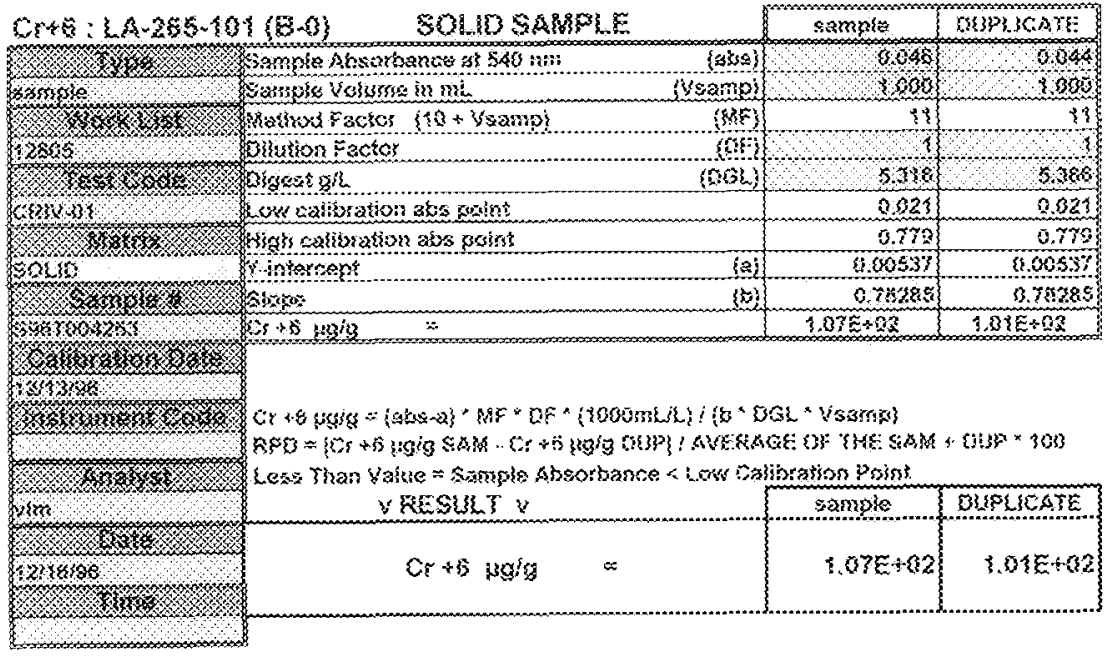

\begin{tabular}{|c|c|c|}
\hline orpas $x=$ & Monovery is oltsarenas & 5.38 \\
\hline
\end{tabular}

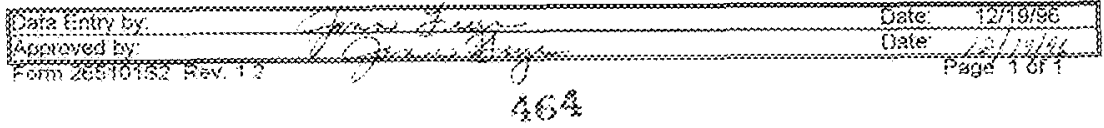




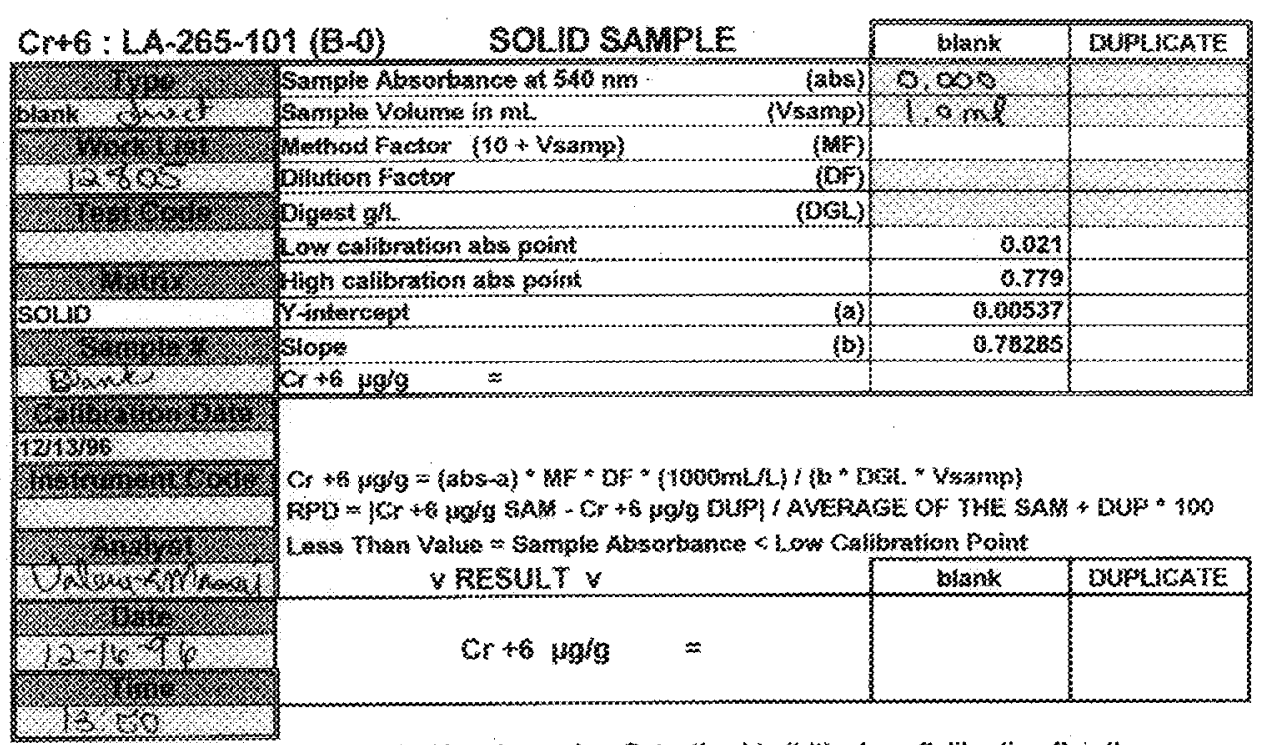

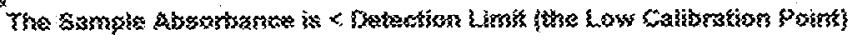

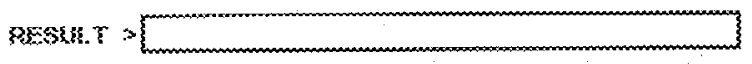

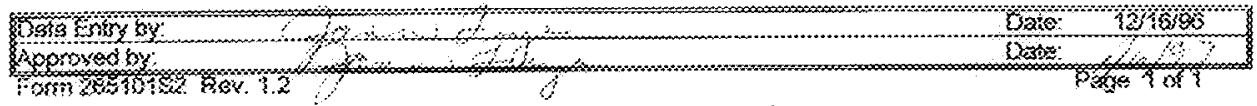




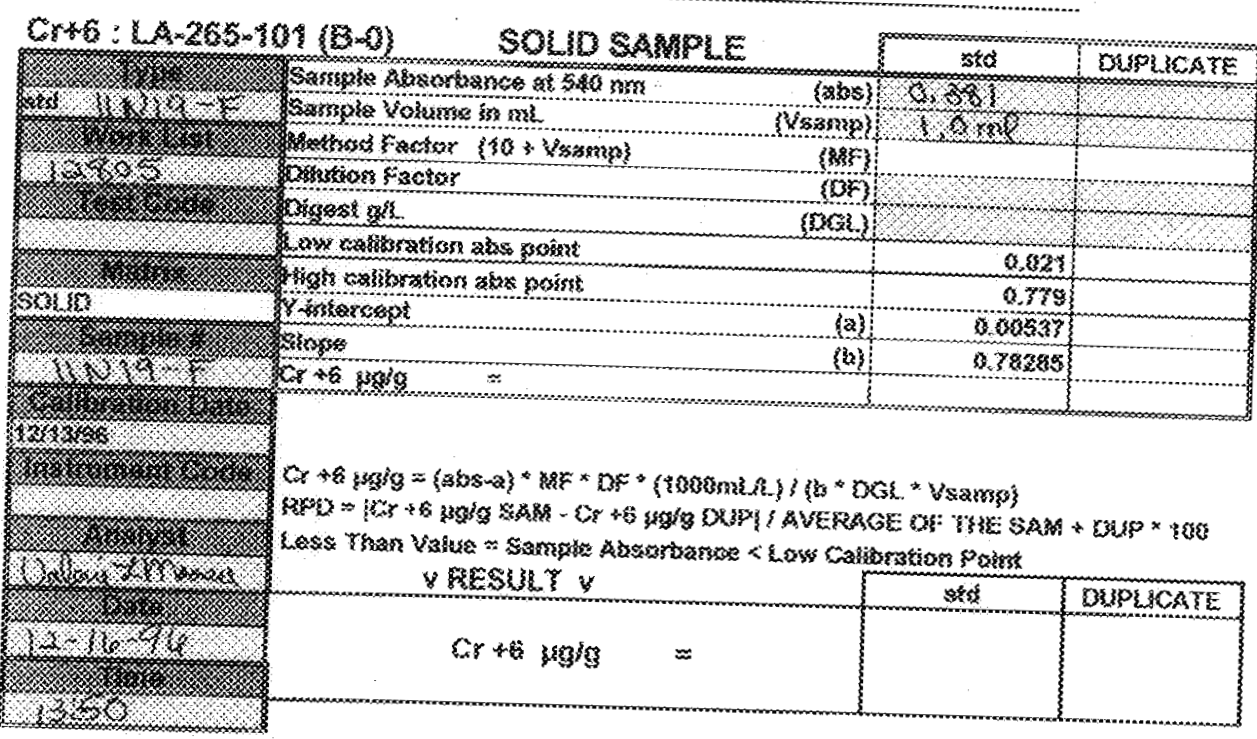

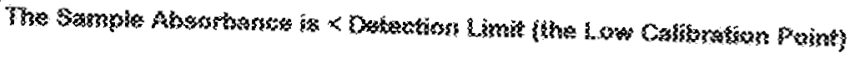

REOXYT:

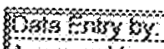

4oposed of

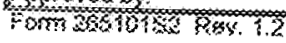

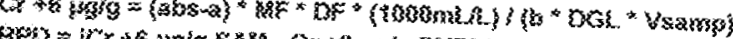

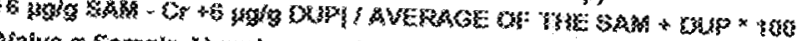
- RESLY

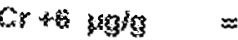




\begin{tabular}{|c|c|c|c|c|}
\hline \multicolumn{2}{|c|}{$C 5+5:\{A-265-1\}$} & SOLW & \multirow{2}{*}{ samplos } & \multirow{2}{*}{ BUPMSATE } \\
\hline & 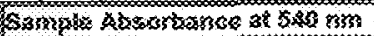 & gabsil & & \\
\hline 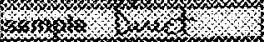 & 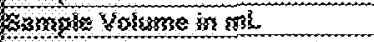 & Fasarga & 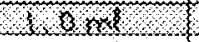 & $3 \%$ n \\
\hline 淁 & 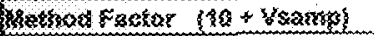 & (BgFu & & \\
\hline x $x$ x & Silutiogs Fastar & (10) & ? & \\
\hline 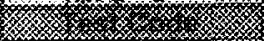 & Woost $\beta h$ & (x) & 212 & \%1k \\
\hline & ow osfisrosson ass point & & 0.827 & \\
\hline Ky & 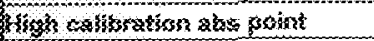 & & 0.773 & \\
\hline 000 & $Y$ hndxycent & $\{\otimes\}$ & 3,00057 & \\
\hline ry & 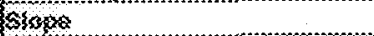 & 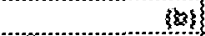 & 3.78283 & \\
\hline 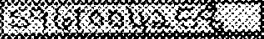 & Sols & & & \\
\hline$\gamma$ & & & & \\
\hline 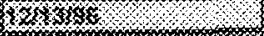 & & & & \\
\hline 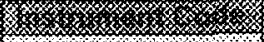 & 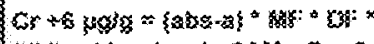 & 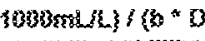 & 6L * Ys:mo) & \\
\hline & 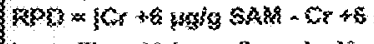 & 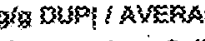 & उE OF THE & $+201 p * 100$ \\
\hline 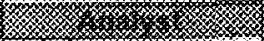 & 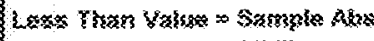 & 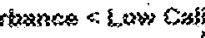 & uraksion Prim & \\
\hline 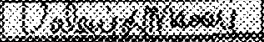 & $y$ Kร & & sargpie & DUSPLSCATE \\
\hline 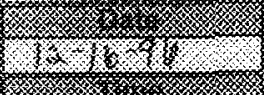 & 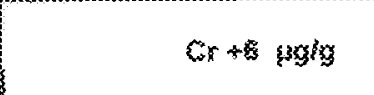 & $=$ & & \\
\hline 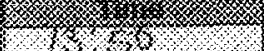 & 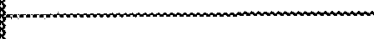 & & & \\
\hline
\end{tabular}

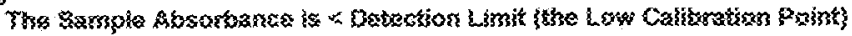

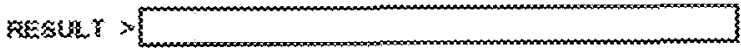

Watrox

4onons by: fom 2 (h)
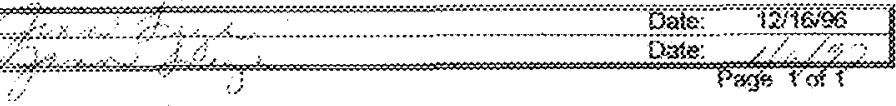


\begin{tabular}{|c|c|c|c|}
\hline 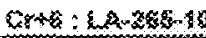 & 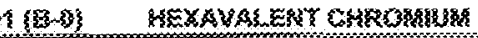 & SPlke & SFYKE \\
\hline 聯慈 & 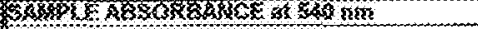 & SSAXS ahs\} & $\$ 0 \mathrm{~s}$ \\
\hline 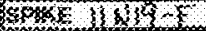 & 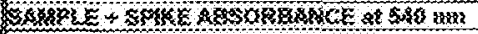 & $1848,2 b 3$ & 3,31 \\
\hline 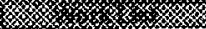 & 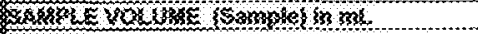 & (Yaon? & Ho 20 \\
\hline w & 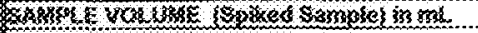 & 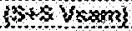 & \\
\hline 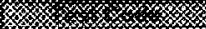 & 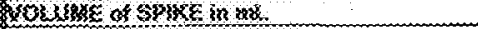 & Nol 3as & 1010 \\
\hline & 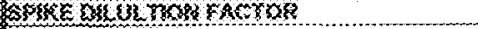 & -30 & \\
\hline & So B E KaL UL & kogers & \\
\hline & & & \\
\hline & 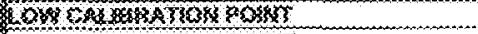 & & \$nas \\
\hline \% & 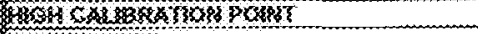 & & 3.378 \\
\hline & HSOCKe & (a) & 0.3033 \\
\hline 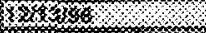 & $\% \propto$ & (b) & 073282 \\
\hline
\end{tabular}

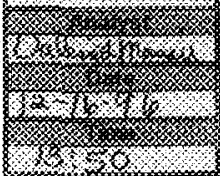

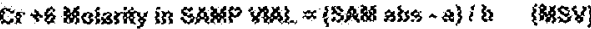

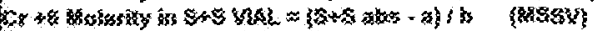

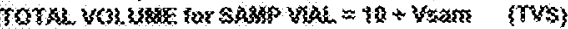

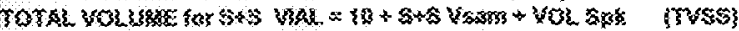

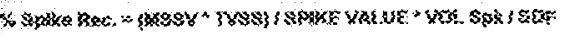

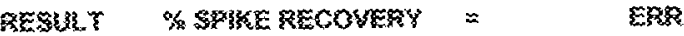

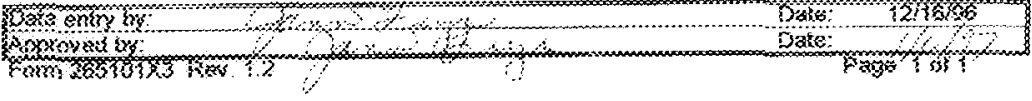




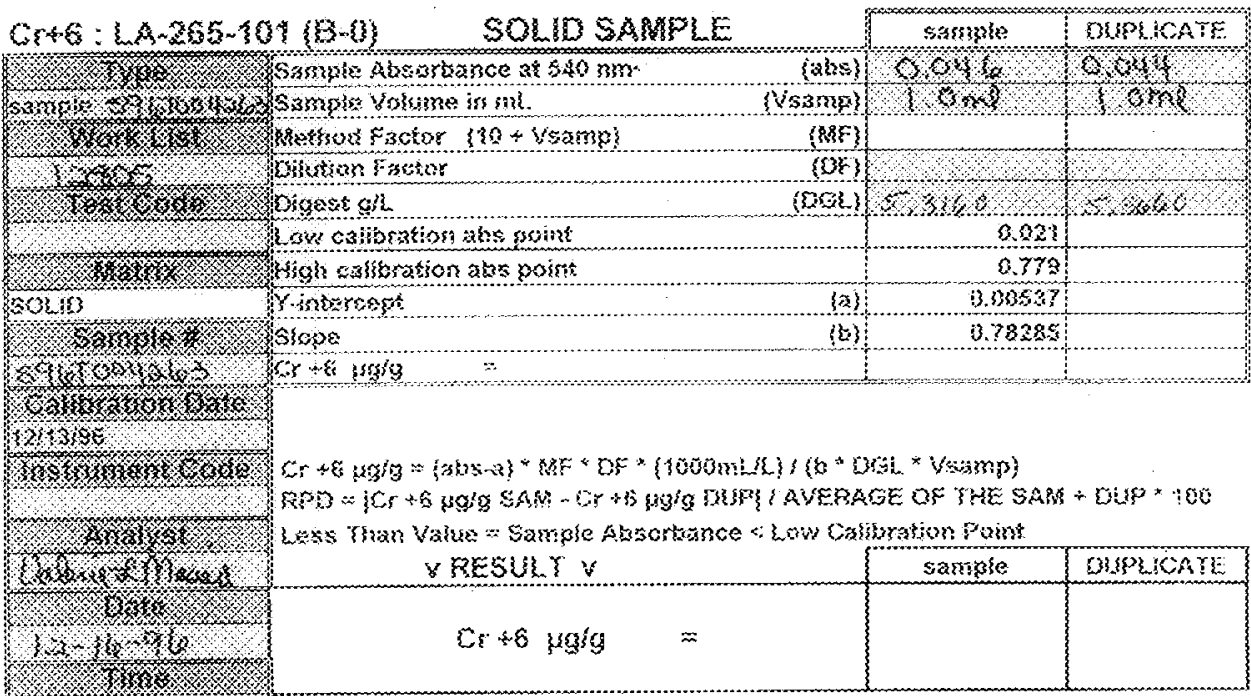

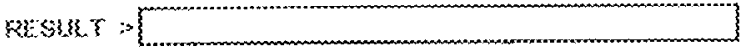

ata thy 5

Arocesediv:

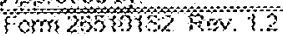

ote: Oate: itos Posjex is 1 


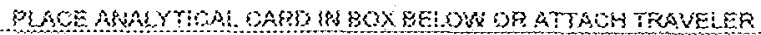

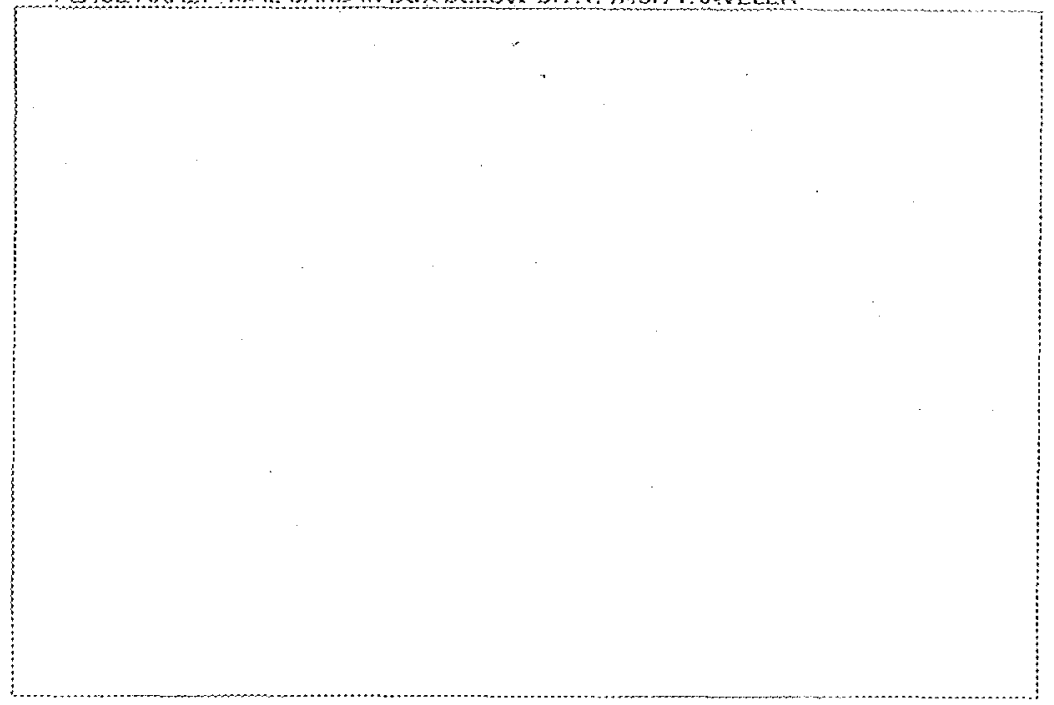

\begin{tabular}{|c|c|c|}
\hline $\sin 8:-43-255-1$ & 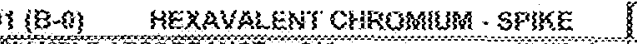 & Sßs \\
\hline$\alpha z$ & 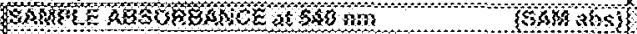 & 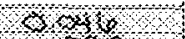 \\
\hline mot in $15-4$ & 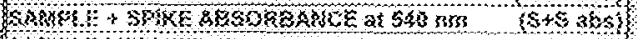 & \% \\
\hline s & 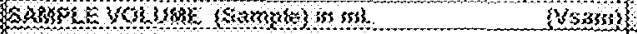 & +1, \\
\hline 30 & 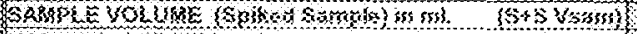 & \\
\hline $3 x$ & 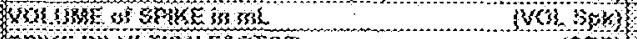 & 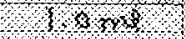 \\
\hline & 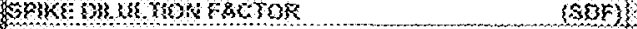 & \\
\hline 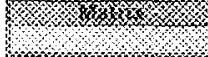 & ofus 89001 & \\
\hline $608 \%$ & 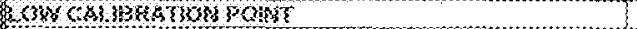 & a.02? \\
\hline $3 \%<>3$ & 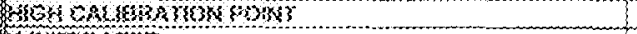 & 0.753 \\
\hline$\%<$ & YSMTEKCEPS & Mos33 \\
\hline 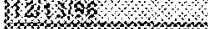 & 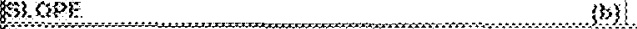 & 0,7003 \\
\hline$\psi_{4}$ & 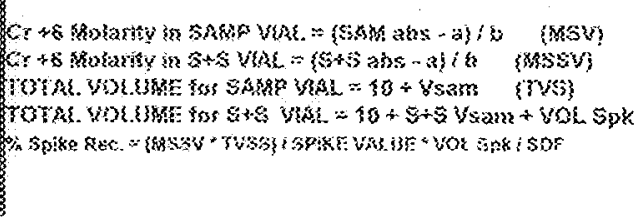 & $\{r y s\}$ \\
\hline
\end{tabular}

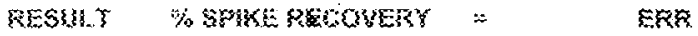

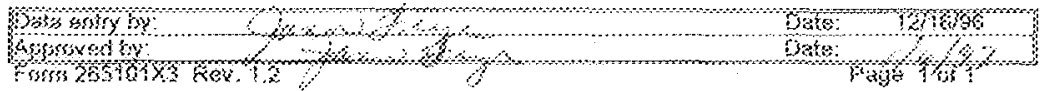




\section{LACOBg}

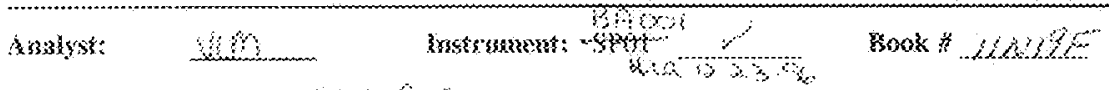

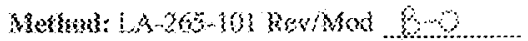

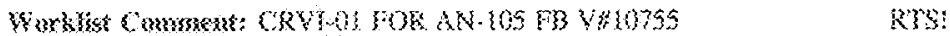

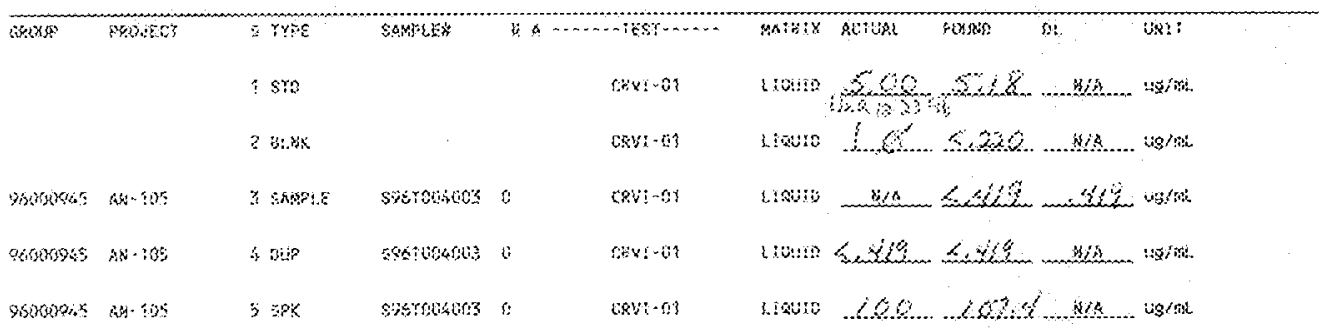

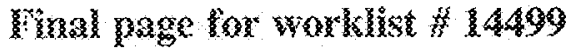

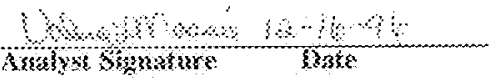

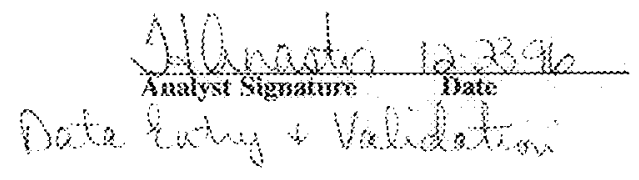

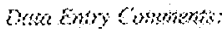

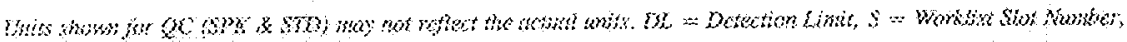




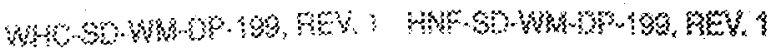

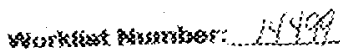

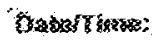

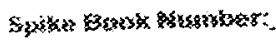

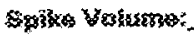

\begin{tabular}{|c|c|c|c|c|c|c|c|}
\hline 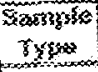 & 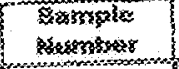 & 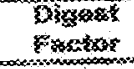 & 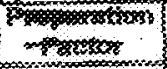 & 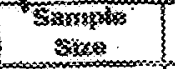 & 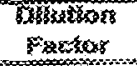 & \multicolumn{2}{|l|}{ Gormmsomsts } \\
\hline 580 & $\{N\}\}-F^{2}$ & & $x_{1000}$ & $\xi .0$ & & & \\
\hline 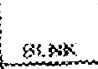 & & & 0.000 & 1.8 & & 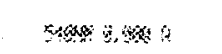 & \\
\hline 5 & $\times x<s 04003$ & $N / d$ & $=0,000$ & $6,60-10-1,19$ & & 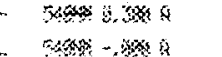 & - \\
\hline 8 & sereatress & X & 0.800 & $100,30-10 m$ & & $\%$ & 7 \\
\hline 0 & 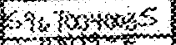 & )$^{3} s$ & 0,248 & $m b-j 0-1, n m$ & & उस\% \& 3 & $m$ \\
\hline & F 302,5 & & & & & & \\
\hline & & & inim & 8 & & & \\
\hline s. & $13 \times 19-5$ & 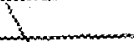 & $3 \mathrm{~s} \alpha$ & $1.0 \times 1$ & & & \\
\hline & (G) & & $\$ \infty$ & $1.8 \mathrm{~ms}$ & & 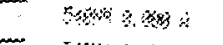 & \\
\hline$D$ & sforas:as & & $\mathrm{ses}$ & $100-40-5 \times 3$ & & 54 에 25 & \\
\hline 3 & 896 rosussios & & 2.00 & $x_{3}-10,803$ & & 4âे & \\
\hline 3 & $44600 \times x=$ & Q & 9.31 & $30-5<0$ & & 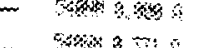 & \\
\hline & mor $\%$ & 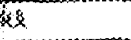 & & & & on 3, & \\
\hline & & & & & & & \\
\hline & & & & & (3) & 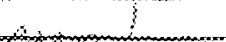 & \\
\hline & & & & & & ? & \\
\hline & & & & & & 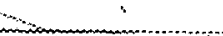 & \\
\hline & & & & & & & \\
\hline & & & & & & & \\
\hline & & & & & & & \\
\hline & & & & & & & \\
\hline & & & & & & & \\
\hline
\end{tabular}




\begin{tabular}{|c|c|c|c|c|}
\hline$\xi r+5:\{4-z \xi\}$ & LOSUSB & & $\sin x$ & DUPLSCATE \\
\hline 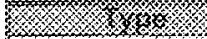 & Soropse Abssssmance as SAG num & $\{a s\}$ & ossus & \\
\hline 330 & Sample yolume in $\mathrm{ml}$. & (Ssangs) & 303 & \\
\hline zron & 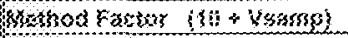 & $\{B r\}$ & 11 & 10 \\
\hline 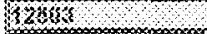 & ossiftor Fasear & $(\mathrm{D})$ & 3 & \\
\hline 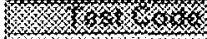 & sigess Qubution kastsa & $\{P D P\}$ & 2 & \\
\hline$\left(x_{3} / 3\right) 3$ & ow enibrasion puins & & 0,021 & 0,021 \\
\hline 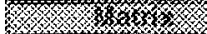 & Stzon Caibrstion Psibls & & 0.378 & 0.778 \\
\hline $\mathrm{SO}_{3} 33 \mathrm{S3}$ & Sungerst & $\{a\}$ & 5.05857 & 3.60337 \\
\hline 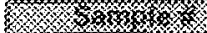 & 6 sope & (b) & 5.78288 & 3.78285 \\
\hline 8,3303018 & or \& ugimh & & $\therefore 0.220$ & ERS \\
\hline
\end{tabular}

$8 \times 3,3,3,3<$

2230

$\%$ \% 10 \% 803

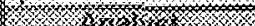

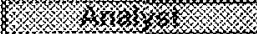

Sing

\%ow os

(12)

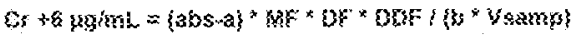

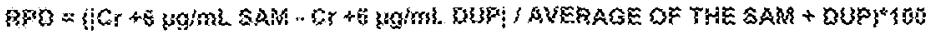

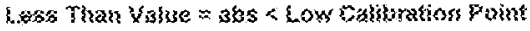

\begin{tabular}{|c|c|c|}
\hline$\checkmark$ RESUR $v$ & bensm & DUPYMASE \\
\hline or + agimi $=$ & 0.220 & ERR \\
\hline
\end{tabular}

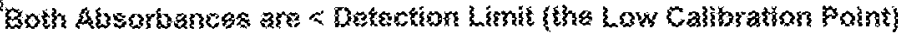

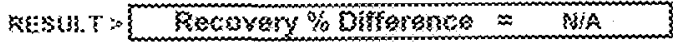

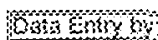

Argervel by: tom

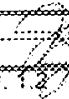

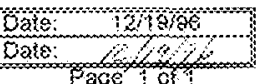

page of 
HQAOE ANALTTCAS CARD MERE OH RTSACH TPAVELEQ

\begin{tabular}{|c|c|c|c|c|}
\hline$C r+3:\left\{x_{2} 265-\right\}$ & KUUBD SA & & stros & OUELLATE \\
\hline 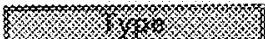 & 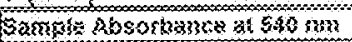 & $\{305$ & B.YYT & \\
\hline 35 & S smpe Yolume in m! & Usang & 303 & \\
\hline 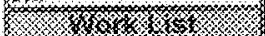 & 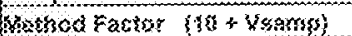 & $\{x \in\}$ & 11 & 30 \\
\hline Kusk & Oobsion raver & $(0)$ & 1 & \\
\hline 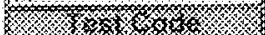 & dorgens billstion Factsss & 1005) & 1 & \\
\hline WWh & Low Calbrotion 9 ont & & .0 .323 & 0.021 \\
\hline 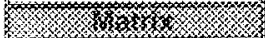 & Mign osibratuan posint & & 2.379 & 0.379 \\
\hline $4 \Omega O S$ & ronkersept & (3) & 0.00837 & 0.00837 \\
\hline 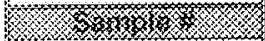 & sogse & (6) & 0.78285 & $0.72 z R s$ \\
\hline & $\mathrm{Cr}+\mathrm{s}$ yshm & & $\$ .32 \approx 400$ & Ep \\
\hline
\end{tabular}

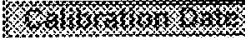

\section{8}

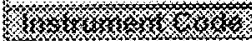

\% 03

\%

00003 s.j.

stm

楼

$12 \% 68$

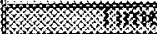

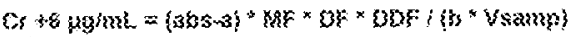

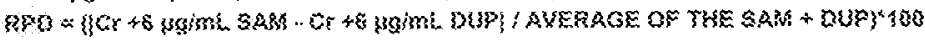

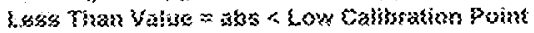

$$
4 \text { RESULT } 8
$$

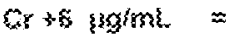

SADS DUPLKATS

5. 38506

Exh

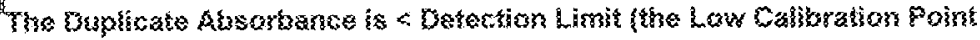

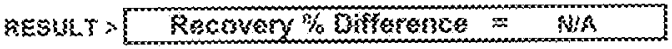

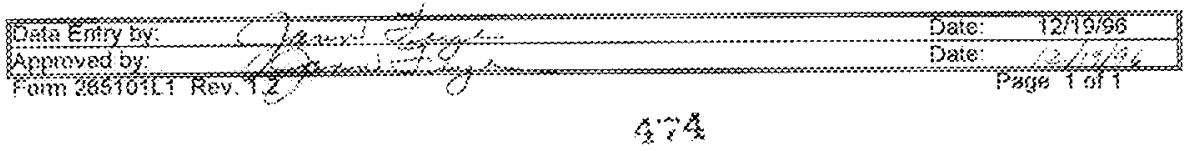




\begin{tabular}{|c|c|c|c|c|}
\hline Cr+5: $: 3-26$ & $\{\{\}\{\}\}$ & & sampla & DUPSLEATE \\
\hline 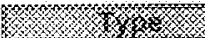 & 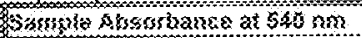 & Sahs\} & 10100 & 0,0630 \\
\hline$\$ 330 \mathrm{le}$ & sample Volunza in rat & (Xsasssy) & 0,13 & os \\
\hline 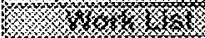 & kolinod snstor (10 + vasmp! & 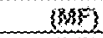 & 10.5 & 10.5 \\
\hline 3333 & Dikifoss Pactor & (5K) & + & 3 \\
\hline$\%$ k $k$ z & Sbuzes Dhution F Fstor & $\{0$ DE $\}$ & $\lcm{1}$ & से \\
\hline $6 \times 103$ & son cobismations going & & 0.021 & 0,021 \\
\hline $10 \%$ \% & Stigns Calitssition Point & & 0.723 & 0.729 \\
\hline souno & Y Enforsegst & \{a\} & 90093 & 0.00637 \\
\hline $3 / x_{x}$ & stope & (b) & 4.732ks & 0.78235 \\
\hline Whosus & $\mathrm{Cr}+\mathrm{s}$ jgiml & & 00.439 & $\angle 3,4.34$ \\
\hline
\end{tabular}

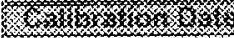

\section{$1238 \%$}

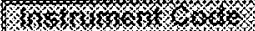

8015

W 3 or

SSss.

\%os

1218 sis

\%

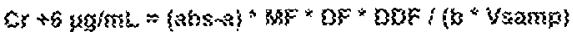

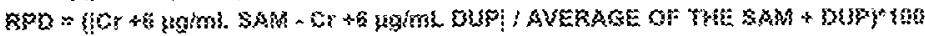

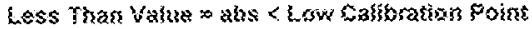

$$
\text { V RESUET * }
$$

Or +8 sigim

\begin{tabular}{c|c|}
\hline Sampls & DUPIUASE \\
$<0.419$ & $<0.419$ \\
\hline
\end{tabular}

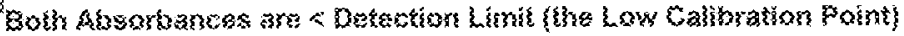

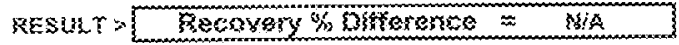

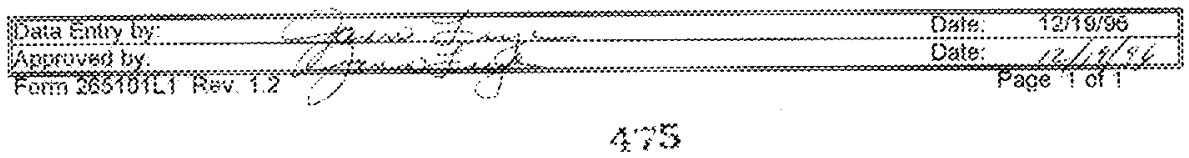




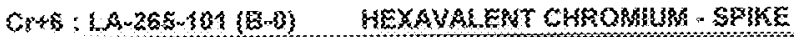

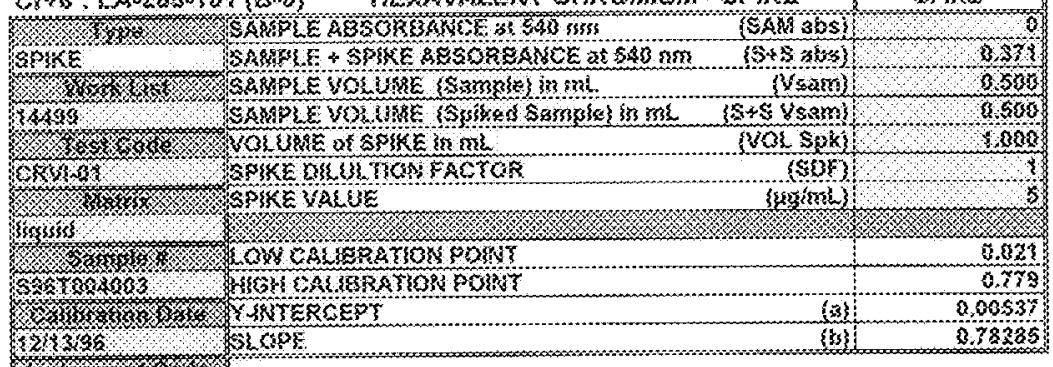

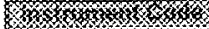

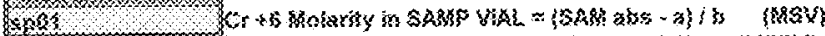

\%

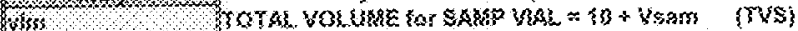

W.

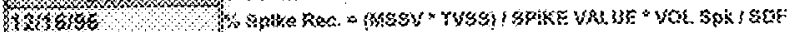

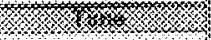

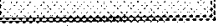

ZRESULT "5PIKE REKOYERY = $\quad 307.4 \%$

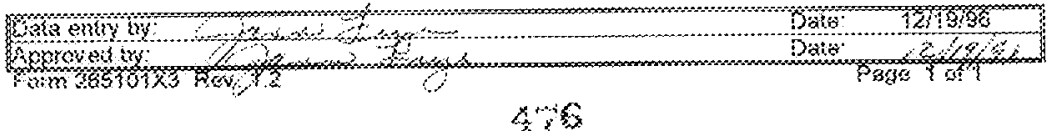




\begin{tabular}{|c|c|c|c|c|}
\hline$\{S+3: \xi A-3,55-3$ & $\{\{3\}] \$$ & & Wiank & DUPLICATE \\
\hline 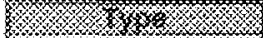 & Sasmule 4 bsortsasse at $340 \mathrm{~nm}$ & $(a b s)$ & 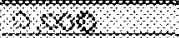 & \\
\hline 63035 & 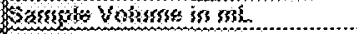 & (Xsump) & +1, & \\
\hline$\alpha x / \%$ K & 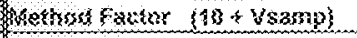 & $(8 \times F)$ & & \\
\hline ४ & Oustion Fanios & (OF) & & \\
\hline 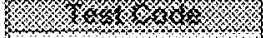 & orgest gisugross fichor & Mok: & & \\
\hline & Loss saliseratons soim & & 0.021 & \\
\hline $8 \%$ z & Hhos Exalbratioss quint & & 0.779 & \\
\hline LNOW & Yontarseps & (a) & 0.30537 & \\
\hline 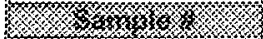 & siops & (b) & 3.76268 & \\
\hline । म ४े & $6+t$ ygiml & & & \\
\hline
\end{tabular}

\%

$3,3,3$

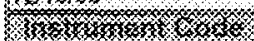

. 000000000000000 $\%$ \% $3 \%$

+ $1,1,1$ \%

33 \%

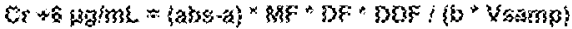

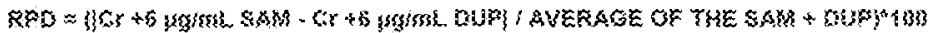

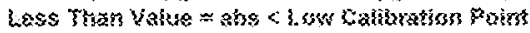

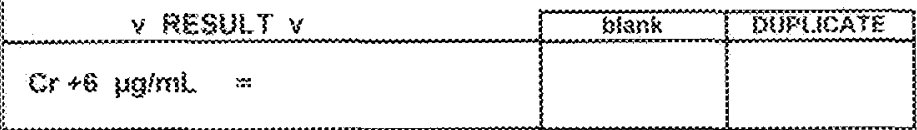

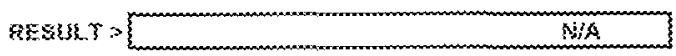

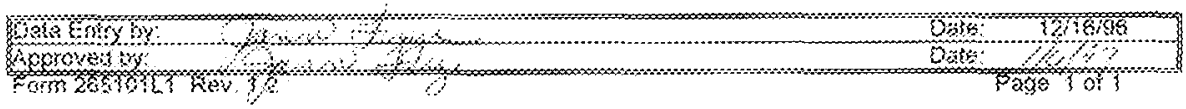




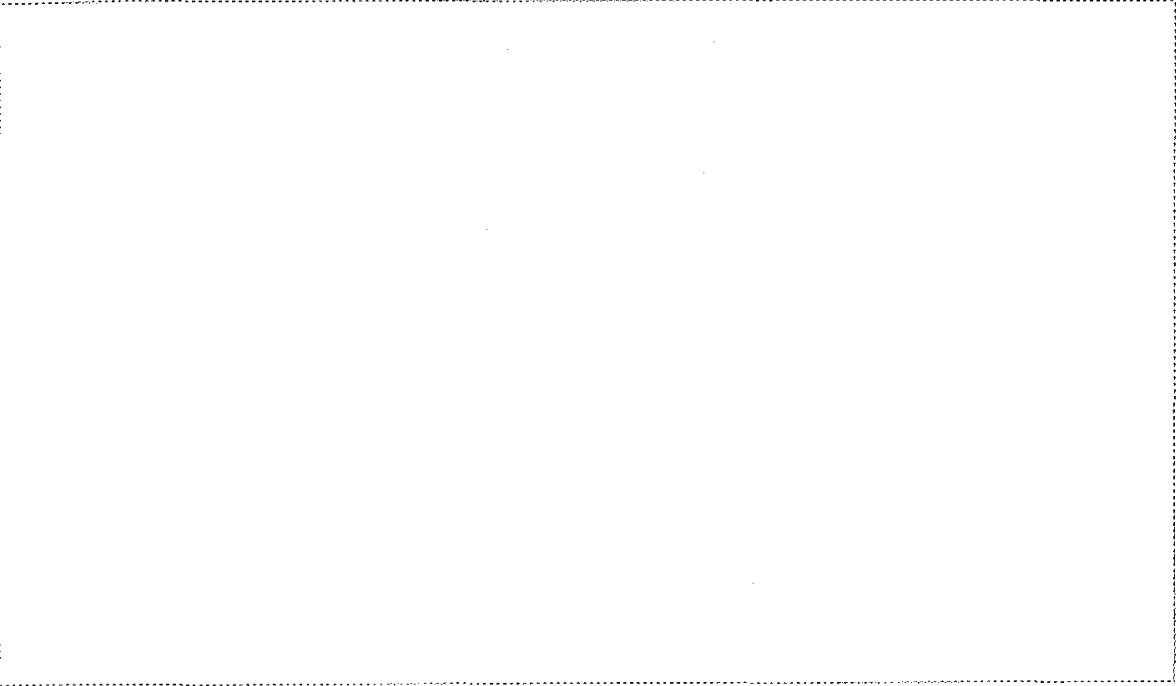

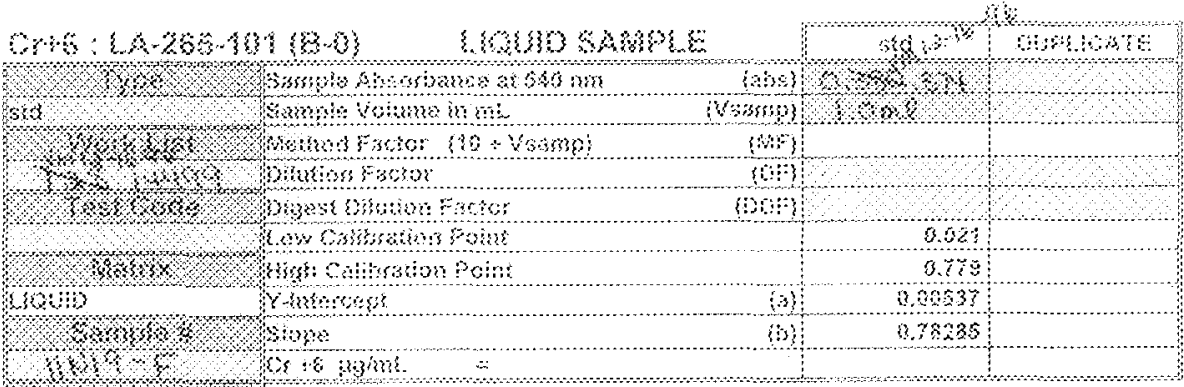

\section{$1 \% \times \%$}

ins \% $1 \%, \%$ (1) से,$+\infty$ स 1,13, s. सेखे का

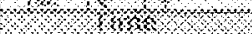

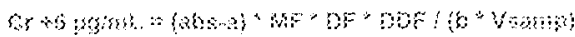

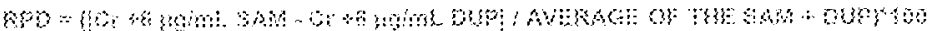

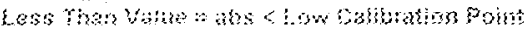

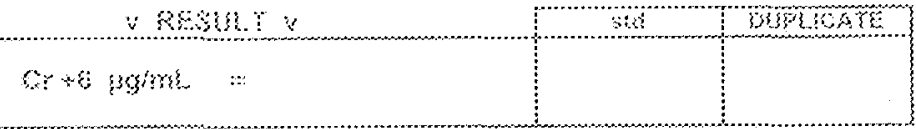

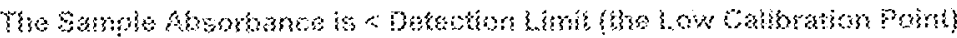

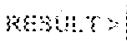
WiA

How

apmona: molी क्षि,

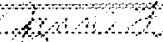

ath

$\alpha \mathrm{AOH}$

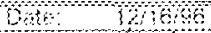

me $\quad 1$ as 


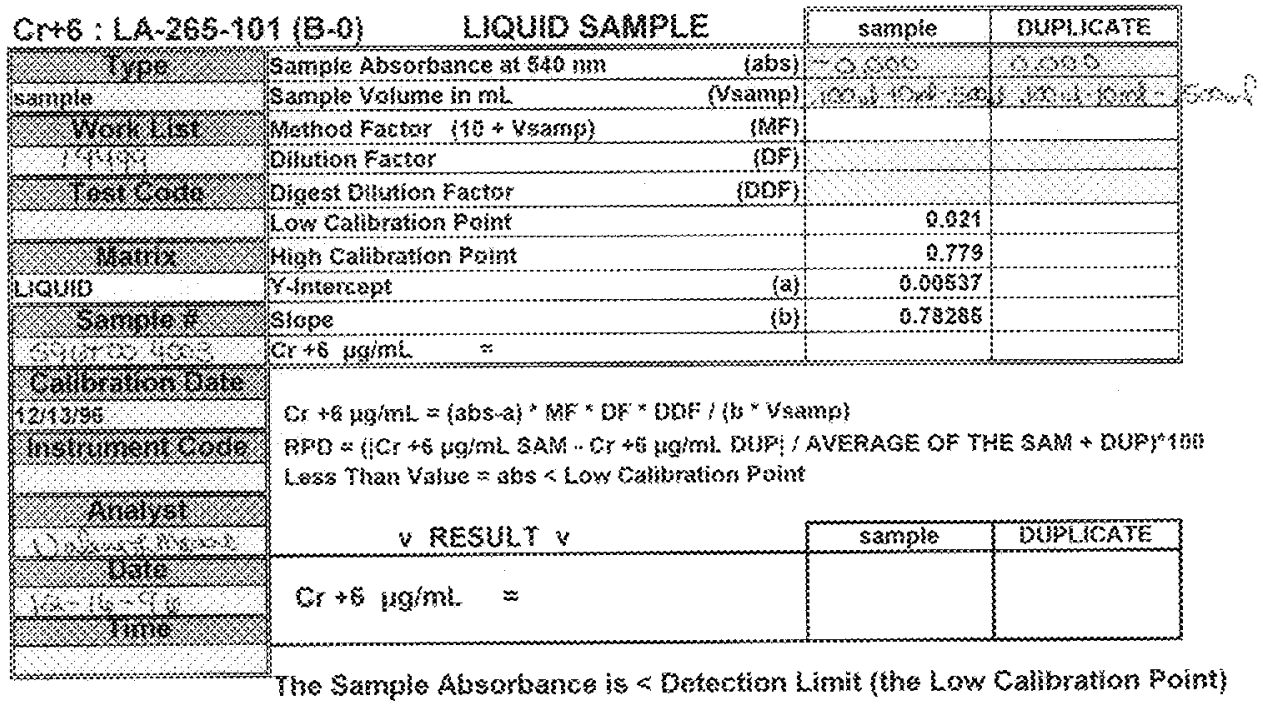

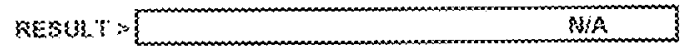

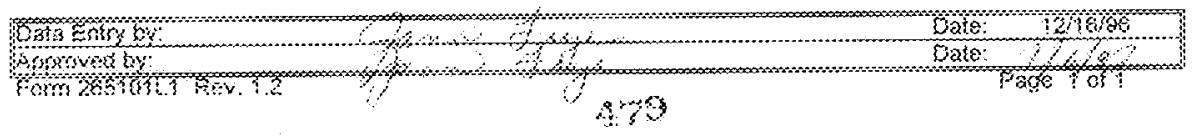




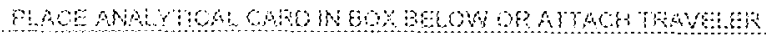

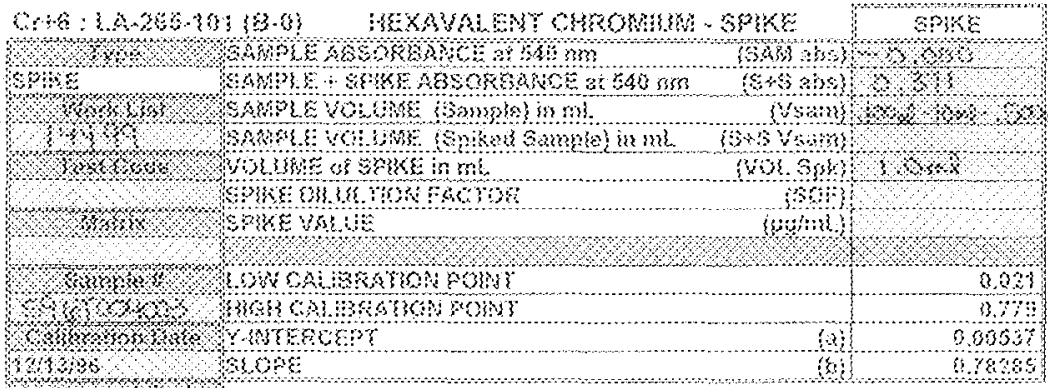

\% $\$$ \% $\$$ \%

-

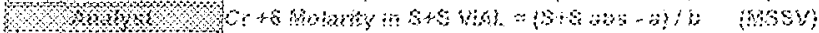

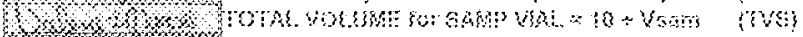

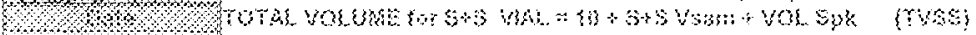

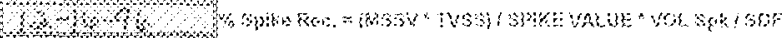

अ,

?

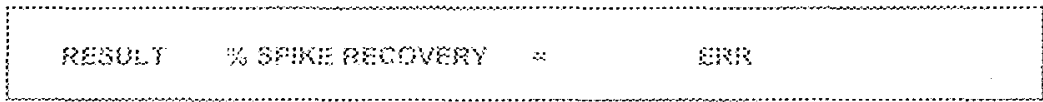

कीph 


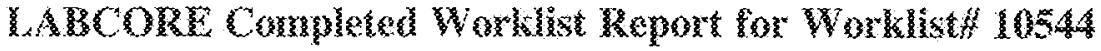

Assust: sal

Instranmert; अ?

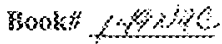

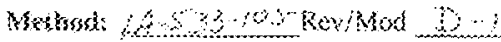

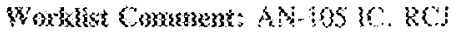

\begin{tabular}{|c|c|c|c|c|c|c|c|c|c|}
\hline meq $y^{2}$ & Sorkplot \& & $A$ & & ES & Mutsix & Axtras? & Foumd & Wis. sse bioka? & Init \\
\hline $\operatorname{sen}$ & & 6 & $35 \pi-b$ & $s$ & so & $t$ & $-\alpha \cos$ & & Whot \\
\hline$\therefore \infty$ & & $\therefore$ & $x_{2}-1 x:$ & $\infty$ & $x$ & $x$ & $2,200-6$ & $\therefore 032$ & spitst. \\
\hline$+\infty$ & & 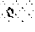 & worose & no & $x$ & $j$ & atolos & & now \\
\hline$\pm \infty$ & & $:$ & atc.go & $3 R$ & $x$ & 2 & 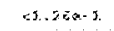 & & verst \\
\hline 200 & & 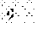 & $\alpha s<\mathrm{s}$ & $n$ & BC & 2 & $x+x_{0}$ & mes & $n \times y=$ \\
\hline $2 \infty$ & & $\therefore$ & $\theta \cos -\theta$ & 905 & 40 & ? & $=2.350-7$ & & as. \\
\hline $1-8$ & & 6 & $60+\infty 2$ & $s 0 s$ & 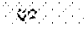 & 3 & $\operatorname{loson}$ & & $49 \times 00$ \\
\hline$\therefore: 08$ & & 8 & $2 x-2 x$ & 4thokes & 20 & 1 & Choset. & & saips. \\
\hline 26 & & 6 & $x>-0$ & 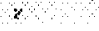 & $\alpha$ & ssokos. & C.Ssopos & 10272 & oonovens \\
\hline$=\mathrm{cos}$ & & $\therefore$ & sxe.60 & $a$ & $\theta$ & $\because$ Nosos & $3(40+0)$ & 505.938 & * 8onovory \\
\hline $3 \%$ & & $\theta$ & Qस< & $\$ 2$ & 86 & 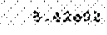 & sison & $20 \times-34$ & s xomolot \\
\hline $2 \cos$ & & $s$ & $\Delta \pi+\alpha^{\circ}$ & $\therefore$ & 80 & $20004:$ & $5.512 \pi<42$ & $202.83 \%$ & $\Rightarrow 2 x \operatorname{ses} y$ \\
\hline 20 & & 8 & $n+\infty n$ & sox & $x$ & $\$, \$ \$ \times 02$ & s Nhork & $x+2 x$ & ponotss \\
\hline$\because \forall \mathrm{s}$ & & $\therefore$ & सrato & 30 & $2 \%$ & $5, a \in x+2$ & $5,20+32$ & 29,927 & solovexpr \\
\hline$=0$ & & 9 & 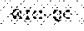 & 80 & 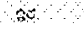 & B shool & arsingt & sox $d y$ & 1 $\$ 000 \mathrm{vo} \times$ \\
\hline $2 a \%$ & & 3 & $\forall \ddot{x}$ & $2 \times 01.0002$ & $\infty$ & 3.25002 & $5 \leqslant 5 \times 0+2$ & $20 \% 305$ & s kesorety \\
\hline Ioksess & 200000 & $\because$ & $6 t-2$ & $\operatorname{ses} \quad$ & M+ons & $2 x+2$ & +onom & $14 \infty<1$ & स \\
\hline$\therefore \sin 5 x$ & vorects & 3 & $x<-02$ & $x-02$ & vaxprs & ... $x$ & 1. $28 \% 0$ art & 20.590 & spios. \\
\hline Aowons & nonss & . & 6002 & $x+x$ & $x_{2}+\infty$ & $\mathrm{W} / \mathrm{x}<$ & 101490.42 & 20200 & $\mathrm{Non}_{2}$ \\
\hline$=\sin 00 t x$ & stoxoses & 3 & atent: & axpsc & $\sin \theta \pi$ & $N A$ & 2.270000 & ton.200 & 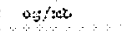 \\
\hline 20400 & $80 \times 60<5$ & 0 & and & 90,02 & Shoo & $n \alpha$ & +soseco & Ts sos & $\checkmark / \mathrm{ar}$ \\
\hline $22 x \cos 25$ & moroses: & $?$ & $0 \pi \cdot 0 t$ & $504-02$ & $2 \times 400$ & ota $=$ & $\therefore 32 x \times 20$ & 32.200 & soins \\
\hline ankms & 2 rowes & 3 & 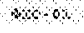 & $80 x-1$ & $\mathrm{x} \times \mathrm{x} \times \mathrm{s}$ & $\mathrm{N} / 2 \ldots$ & मnoph & 1क $6 \times 6$ & no/ow \\
\hline 8 sparas & toconess & $\therefore$ & $n<c-02$ & $\therefore 2 \times 5 \times 2 x$ & $\operatorname{scos} 2 x$ & $x^{\prime}-x^{4}$ & $\therefore+1810+52$ & 250,780 & rosou \\
\hline ons & $\$<000,0<$ & 0 & $\mathrm{sen}_{\mathrm{s}}$ & $6 \phi$ & sxotio & $21,4<0$ & $<44$ & & $2 x$ \\
\hline$x$ xpy & xpstoviszz & $\therefore$ & mon & 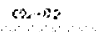 & $2 x+2$ & $\angle A, 4502$ & $\therefore 2,000$ & & 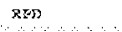 \\
\hline sty & shoorox & $n$ & $\alpha+a$ & $100<-3$ & subre & os & $d x s \mathrm{~s}$ & $\because \backsim$ & $\mathrm{sos}$ \\
\hline 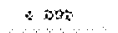 & syonosest & 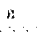 & $0.65-6.2$ & ex. $x_{2}$ & texerss? & 3.52astise & $2.23 \cos \theta$ & 0.825 & Nov \\
\hline $48 \mathrm{so}$ & $\theta 06+00<<<1$ & 6 & ascos & woth & reorsts & aseoroz & $3 n n n+12$ & $35-5$ & sos \\
\hline 2035 & poconsest & 6 & otc-os & $p(x-1 z$ & $=50022$ & $25,=202$ & 27,5262 & & $m+$ \\
\hline तts & sorotest & 8 & on $\mathrm{c}$ & sodet & $1+0020$ & 2,10 & $45 \pi$ & & $\theta$ \\
\hline$A n 0 r$ & S25ME02621 & a & $2 x+2+2$ & STREATS2 & xspmon & $=3,2103$ & $.2,270$ & & RW \\
\hline
\end{tabular}

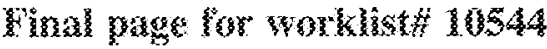

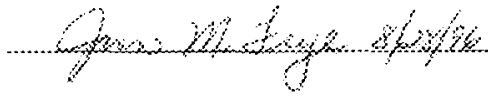




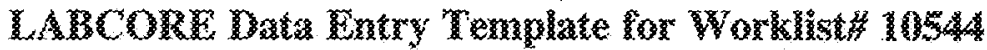

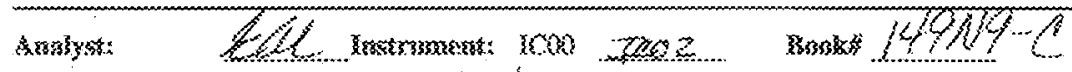

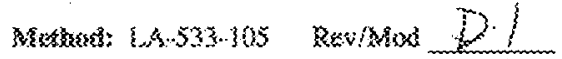

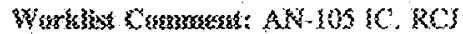

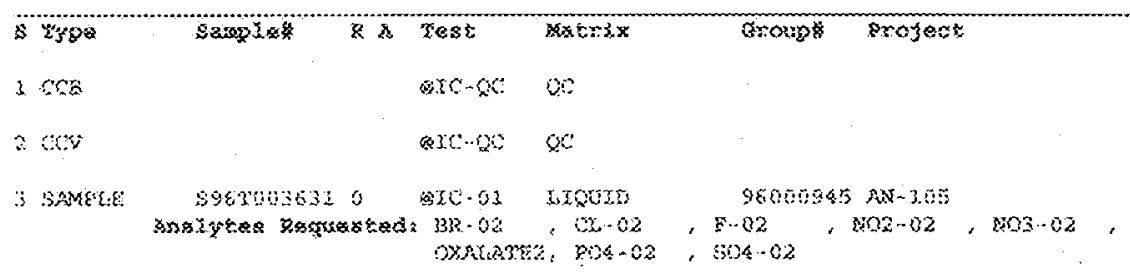

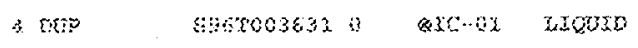

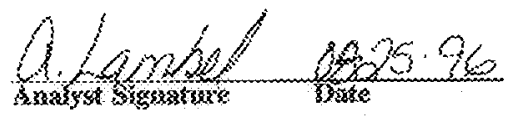

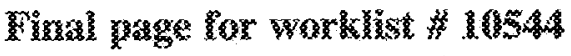

$1052 / 4 / 6, C .5$

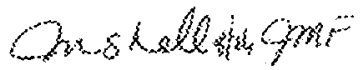

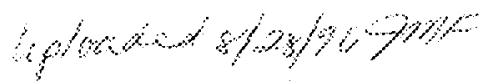

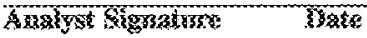

\section{Dats Sorro Comnests:}




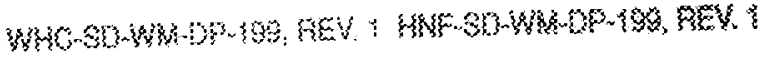

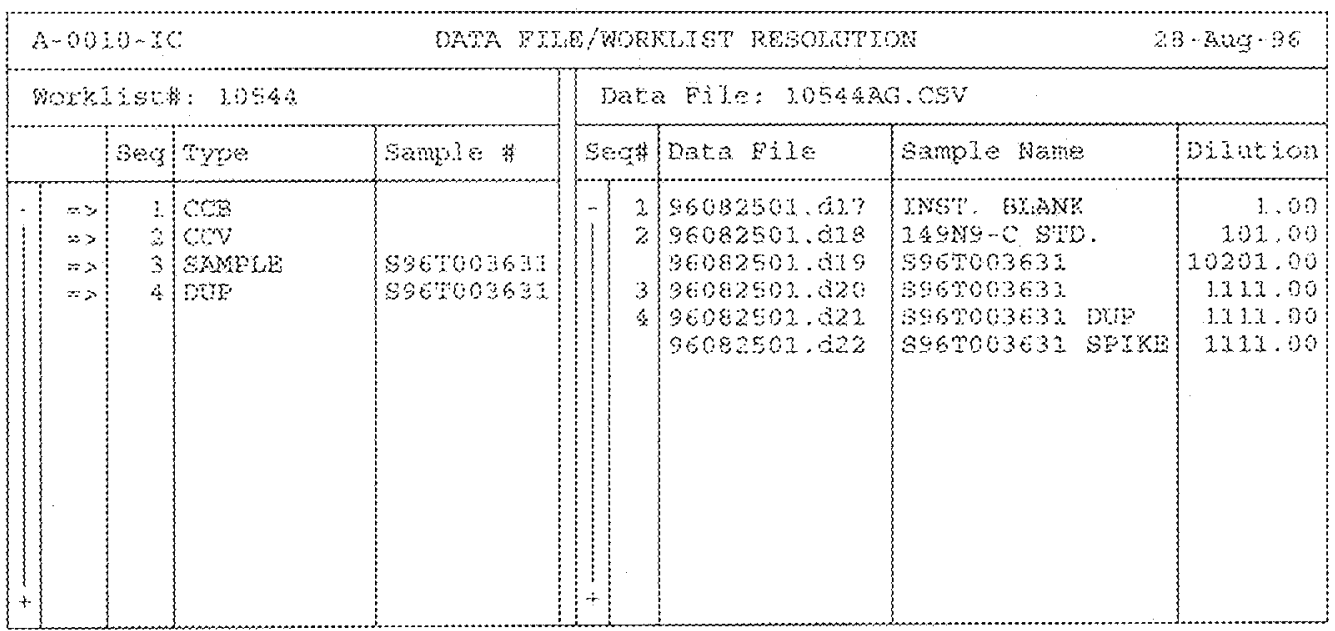

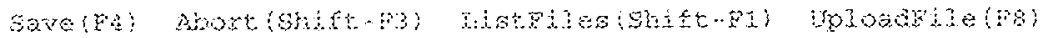




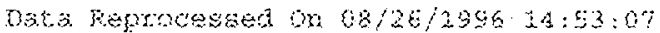

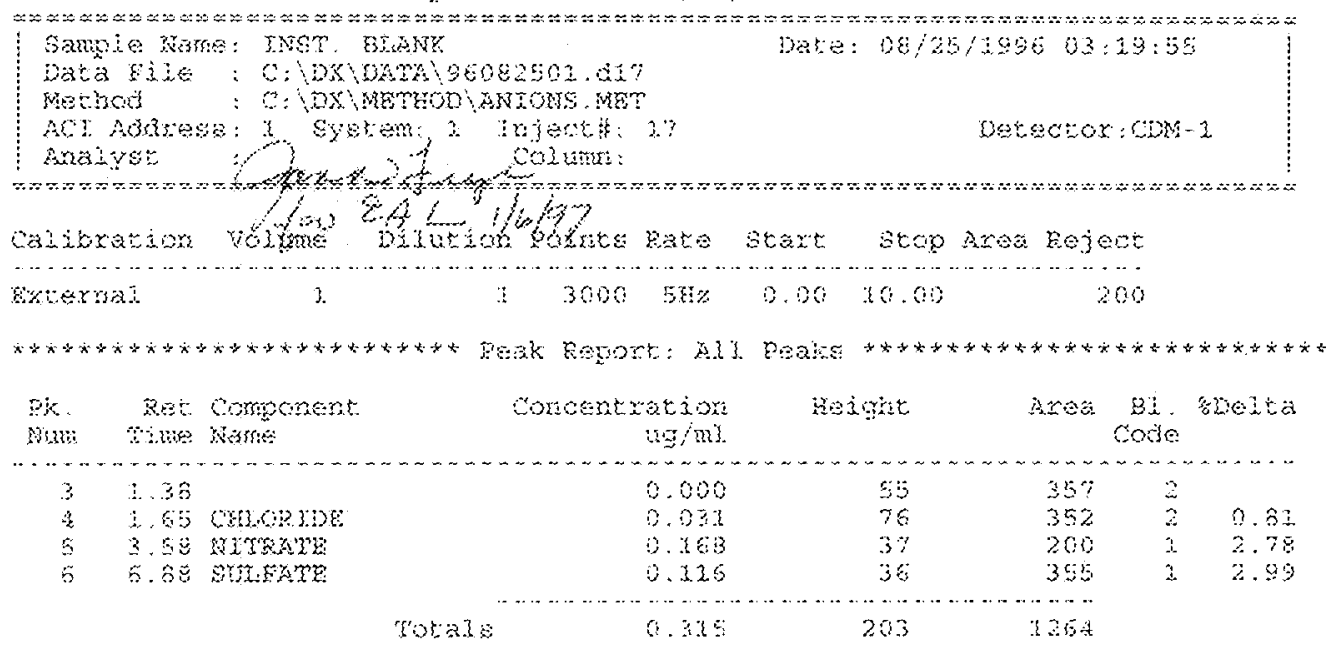

Fib: 96082601.4\%7 Sample: INST, BLAKK

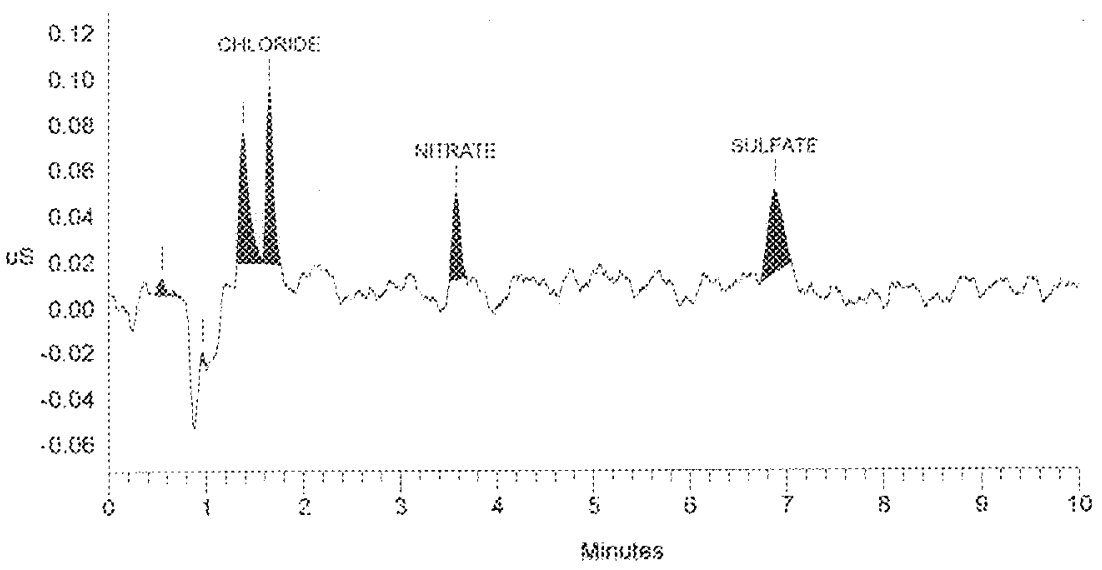

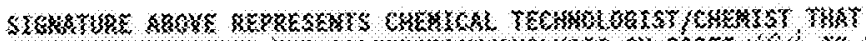

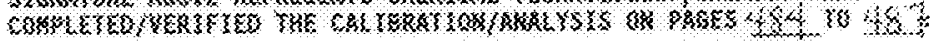




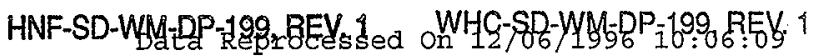

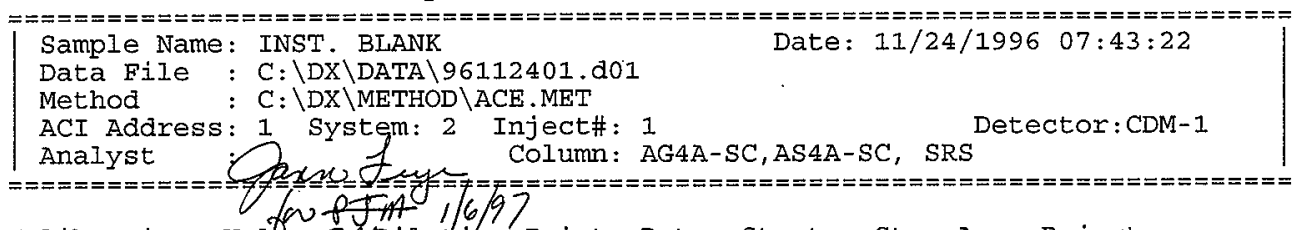

Calibration VolbumezAfilution Points Rate start stop Area Reject

\begin{tabular}{|c|}
\hline External \\
\hline
\end{tabular}

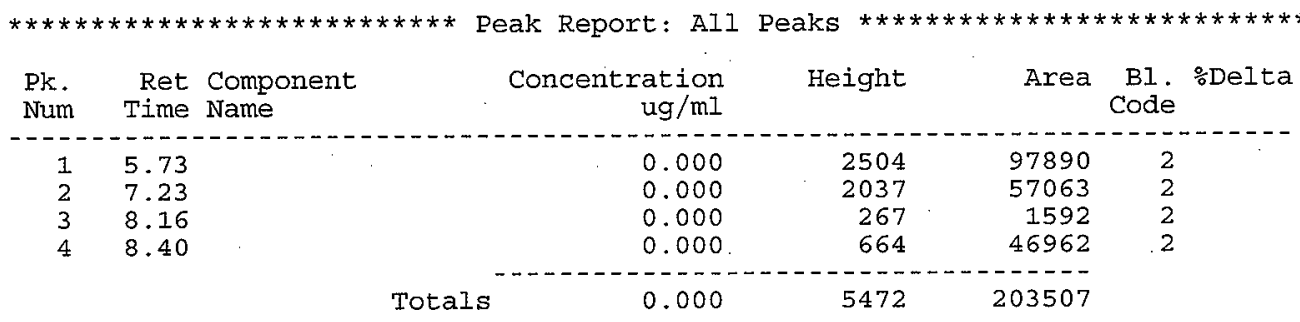

\section{File: 96112401.d01 Sample: INST. BLANK}

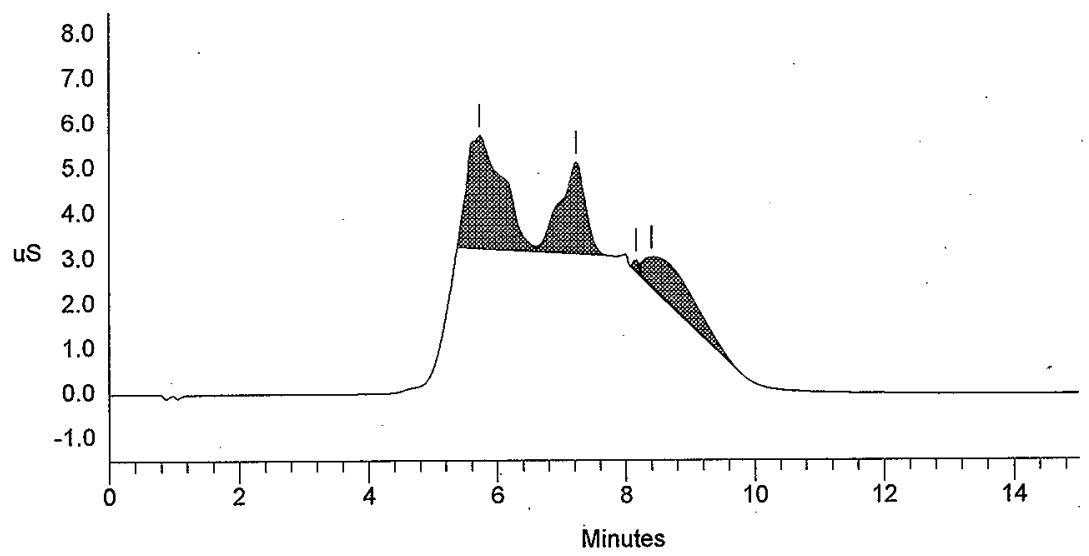

SIONATURE ABOVE REPRESENTS CHEMICAL TECHNOLOGIST/CHEMIST THAT

COMPLETEDIVERIFIED THE CALIBRATIONIANALYSIS ON PAGES 986 TO 995 


\section{DISTRIBUTION SHEET}

\begin{tabular}{|c|c|c|c|c|c|}
\hline \multirow{2}{*}{$\begin{array}{l}\text { To } \\
\text { Distribution }\end{array}$} & \multirow{2}{*}{\multicolumn{3}{|c|}{ From Production Planning \& Control }} & \multicolumn{2}{|c|}{ Page 1 of 1} \\
\hline & & & & \multicolumn{2}{|c|}{ Date:01/23/97 } \\
\hline \multirow{2}{*}{\multicolumn{4}{|c|}{$\begin{array}{l}\text { Project Tit]e/Work Order } \\
\text { HNF-SD-WM-DP-199, Rev. 1, "Tank 241-AN-105, Cores } 152 \text { and } 153 \\
\text { Analytical Results for the Final Report" }\end{array}$}} & \multicolumn{2}{|c|}{ EDT N0.: N/A } \\
\hline & & & & \multicolumn{2}{|c|}{ ECN N0.: ECN-633339 } \\
\hline & Name & MSIN & $\begin{array}{l}\text { Text With } \\
\text { a11 Attach }\end{array}$ & $\begin{array}{c}\text { EDT/ECN } \\
\text { ONLY }\end{array}$ & \\
\hline
\end{tabular}

DE\&S Hanford, Inc.

J. E. Meacham

S7-14 X

U.S. Department of Energy, RL

C. A. Babe1

S7-54 X

Lockheed Martin Hanford Corp.

J. N. Appe1

J. Jo

T. J. Kelley

N. W. Kirch

M. J. Kupfer

J. B. Schaffer

L. A. Stauffer

J. A. Voogd

A. E. Young

Lockheed Martin Services. Inc.

Central Files

Los Alamos Technical Associates

M. T. Ellsworth

Pacific Northwest National Laboratory

J. R. Gormsen

S. J. Harris

K. L. Silvers

Rust Federal Services of Hanford, Inc.

M. W. Barnes

D. C. Hetzer

K. L. Powe17

C. M. Seidel

F. H. Steen

LTIC

SGN Eurisys Services Corp.

B. A. Higley

Defense Nuclear Federal Safety Board

Rich Tontodonato

625 Indiana Ave. N. W.

Washington D.C. 20004
G3-21

R2-12

S7-21

R2-11

H5- 49

R2-12

$\mathrm{R} 2-11$

$\mathrm{H} 5-03$

R2-12

A3-88

T6-06

$\begin{array}{cc}x & x \\ x & X \\ x & X \\ x & X \\ x & \end{array}$

$\mathrm{K} 7-28$

K $7-22$

K9-08

T6-16

H5-27

T6-04

T6-04

T6-06

T6-03

H5-27
2

$x$

X

$x$

$x$

$X$

$X^{*}$

$x$

$X$

$x$

$X$
$X$

$X$
$X$

$x$

$x$

* Needs only releasing paperwork, not a copy of the released document. 
WHC-SD-WM-DP-199, REV. $\mid$

HNF-SD-WM-DP-199, REV. 1

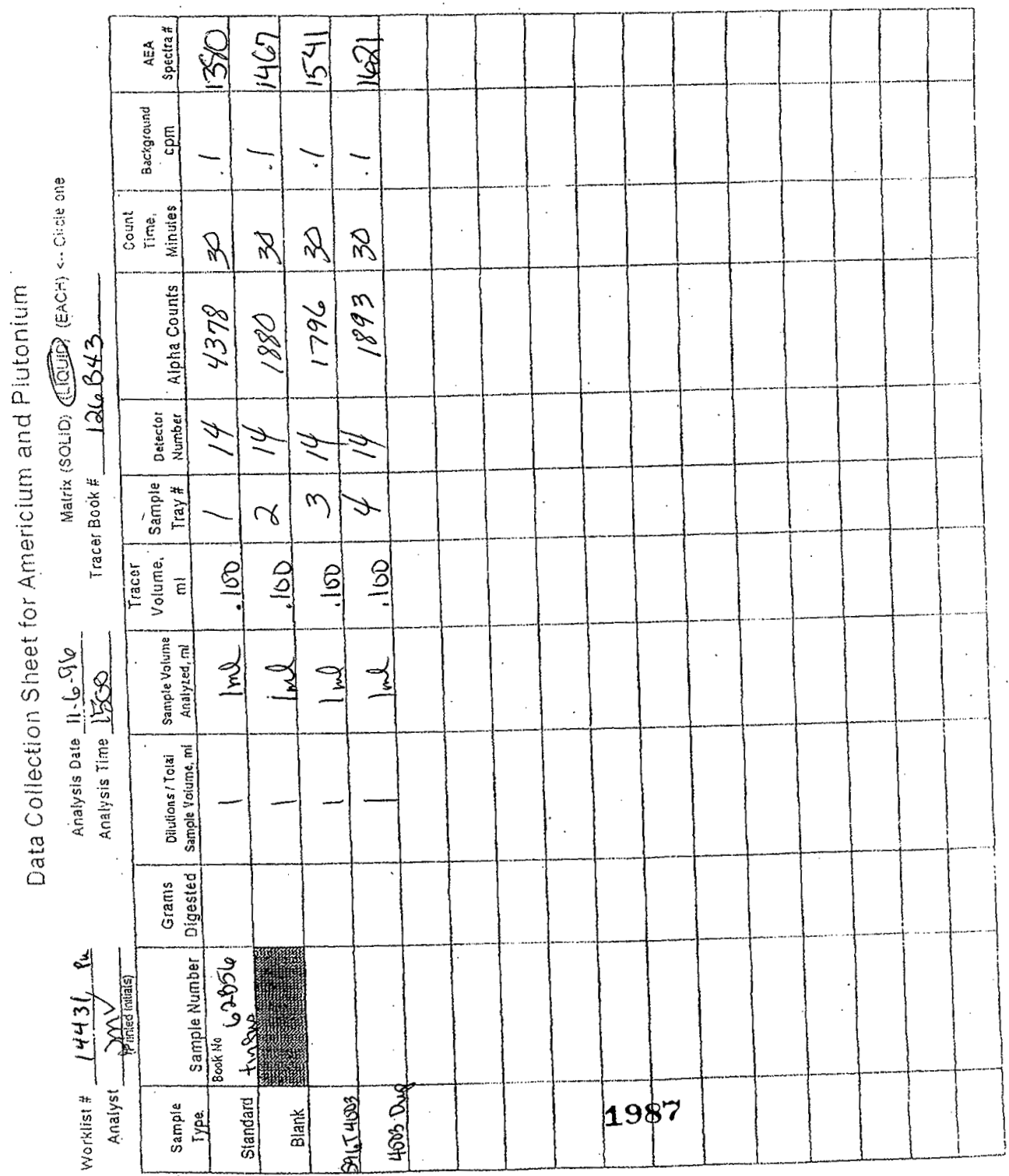


WHC-SD-WM-DP-199, REV. 1

HNF-SD-WAR-DP-199, REV. 1

AT : LA-508-101 (D-2)

LIQUIDS

\begin{tabular}{|c|c|c|}
\hline Fing & DETECTOR NUMBER & 16 \\
\hline SAMPLE & DISH SIZE $(1,2$, or 5$)$ & \\
\hline 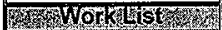 & GROSS COUNTS & \\
\hline $1 . \quad 11612$ & COUNT TIME in MINUTES & 3 \\
\hline 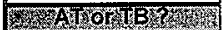 & BACKGROUND in cpm & 0.1 \\
\hline AT & SAMPLE SIZE in $\mathrm{mL}$ & 0.250 \\
\hline W & DILUTION FACTOR & 1020 \\
\hline @ALPHA01 & DIGEST DILUTION FACTOR & \\
\hline 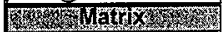 & $\begin{array}{ll}\text { EFFICIENCY FACTOR } & \text { (EFF) } \\
\end{array}$ & 0.210 \\
\hline LIQUID & Lc, Rmax, or Rs,(SAMPLE RATE) as APPROPRIATE & 0.2 \\
\hline \multicolumn{3}{|c|}{ 3atchungor } \\
\hline 96007946 & Blank Concentration in $\mu \mathrm{CI} / \mathrm{L}$ & $2.58 E+$ \\
\hline 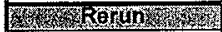 & Replicate Concentration in $\mu \mathrm{Ci} / \mathrm{L}$ & $2.94 \mathrm{E}+0$ \\
\hline & Maximum Concentration in $\mu \mathrm{Ci} / \mathrm{L}$ & $2.9398 \mathrm{E}+$ \\
\hline
\end{tabular}

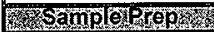

N/A $\quad$ Rs (Sample Count Rate) $=$ (TC /CT) - BKG

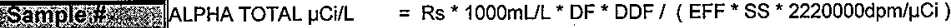

$\$ 96 T 003975$ ALPHA TOTAL $\mu \mathrm{Ci} / \mathrm{mL}=$ ALPHA TOTAL $\mu \mathrm{Ci} / \mathrm{L} / 1000 \mathrm{~mL} / \mathrm{L}$

minstimeng

WB27806

Detection Levels and Less Than Values are determined from Procedure LA-508-002.

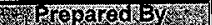

SLH2

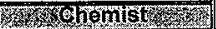

\begin{tabular}{|c|c|c|c|}
\hline SLF & ALPHA TOTAL in $\mu \mathrm{Ci} / \mathrm{mL}$ & $<2.94 \mathrm{E}-02$ & \multirow{2}{*}{$\begin{array}{c}\text { DETECTION } \\
\text { LEVEL }\end{array}$} \\
\hline X & \multirow{3}{*}{\multicolumn{2}{|c|}{ LESS THAN Value was Determined from Rmax. }} & \\
\hline TAM & & & 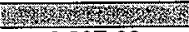 \\
\hline Daterompleter & & & 3.86 E-02 \\
\hline $08 / 12 / 96$ & RELATIVE COUNTING ERROR & $244.6 \%$ & $\mu \mathrm{Ci} / \mathrm{mL}$ \\
\hline
\end{tabular}

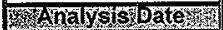

08/08/96

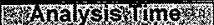
03:00 AM

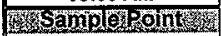
AN-105

Analyst:
Signature of Chemist:
SAMPLE.WB1 Rev. 1.0




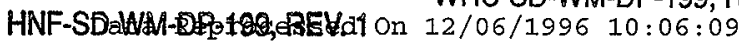

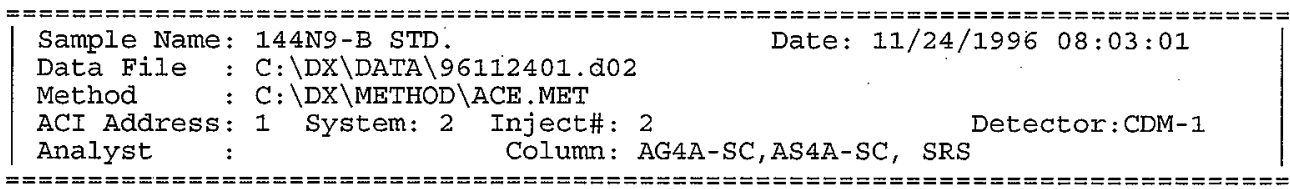

Calibration Volume Dilution Points Rate start stop Area Reject

$\begin{array}{lllllll} & & & \end{array}$

$* * * * * * * * * * * * * * * * * * * * * * * * * * *$ Peak Report: AlI Peaks $* * * * * * * * * * * * * * * * * * * * * * * * * * * *$

$\begin{array}{lrrr}\text { Pk. Ret Component } & \text { Concentration } & \text { Height } & \text { Area Bl. } \\ \text { Num } & \text { Time Name } & \text { Code }\end{array}$

2.10 fluoride

2.37 acetate

3.11 formate

5.87

7.23

8.16

8.41
66.909

122.877

121.776

0.000

0.000

0.000

0.000
1956

707

1361

4308

1751

273

678

Totals

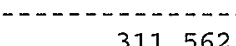

11035

13931

5002

9537

243930

68108

1619

46693

$\begin{array}{ll}2 & 0.00 \\ 2 & 0.99 \\ 1 & 2.19 \\ 2 & \\ 2 & \\ 2 & \\ 2 & \end{array}$

311.562

388819

File: 96112401.d02 Sample: 144N9-B STD.

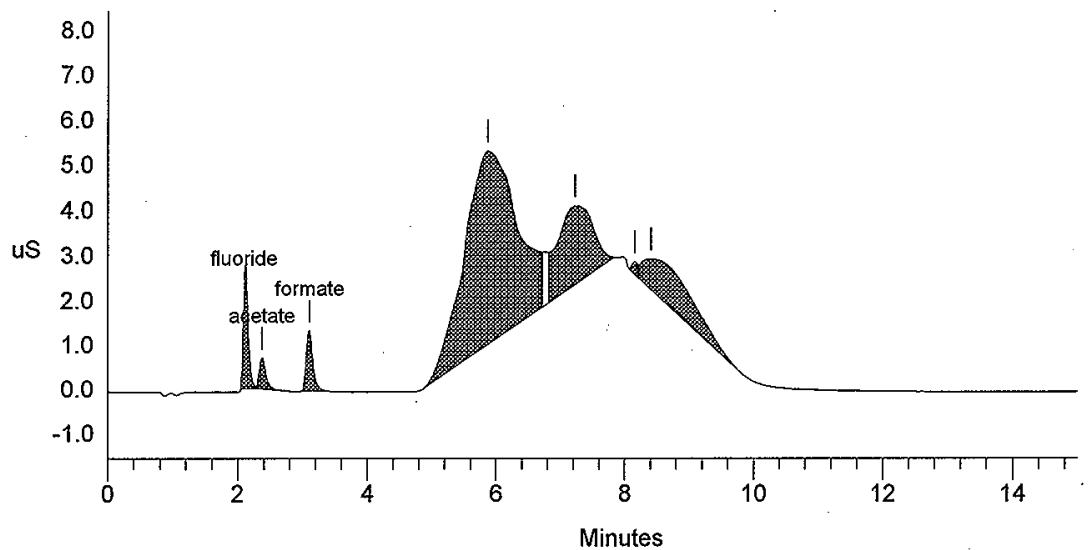


Data Reprocessed On 08/30/1996 14:17:18

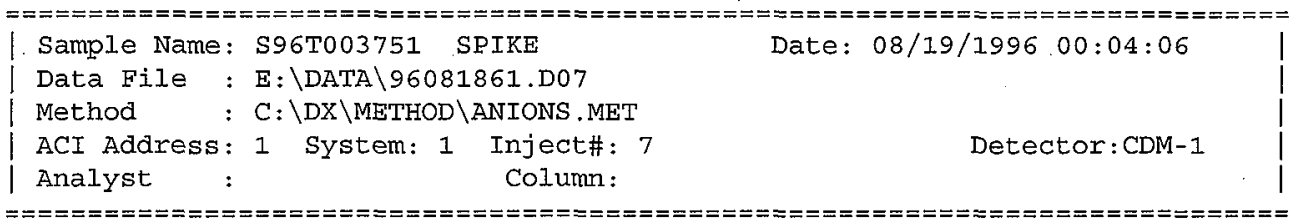

Calibration Volume Dilution Points Rate start stop Area Reject

External $\quad 1 \quad 3141 \quad 3000 \quad 5 \mathrm{~Hz} \quad 0.00 \quad 10.00 \quad 200$

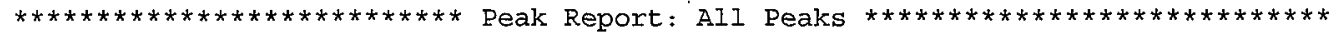

$\begin{array}{lrrr}\text { Pk. Ret Component } & \text { Concentration } & \text { Height } & \text { Area Bl. } \\ \text { Num } & \text { Time Name } / \mathrm{ml} & \text { Code }\end{array}$

10.88

21.06 FLUORIDE

31.54 CHLORIDE

41.87 NITRITE

52.76 BROMTDE

63.02 NITRATE

74.55 PHOSPHATE

85.89 SULFATE

97.70 OXALATE

.

0.000

988.406

12436.302

139482.456

23922.446

180858.007

23750.655

25309.131

21156.856

Totals

427904.259
661

988

6910

53748

6014

43137

1326

4866

2208

\section{$3410 \quad 1$}

$\begin{array}{lll}4609 & 1 & 1.60\end{array}$

39869

332860

35413

413448

16799

62949

38067

$-1.28$

$-1.06$

20.98

20.11

10.89

10.91

11.58

File: 96081861.D07 Sample: S96T003751 SPIKE

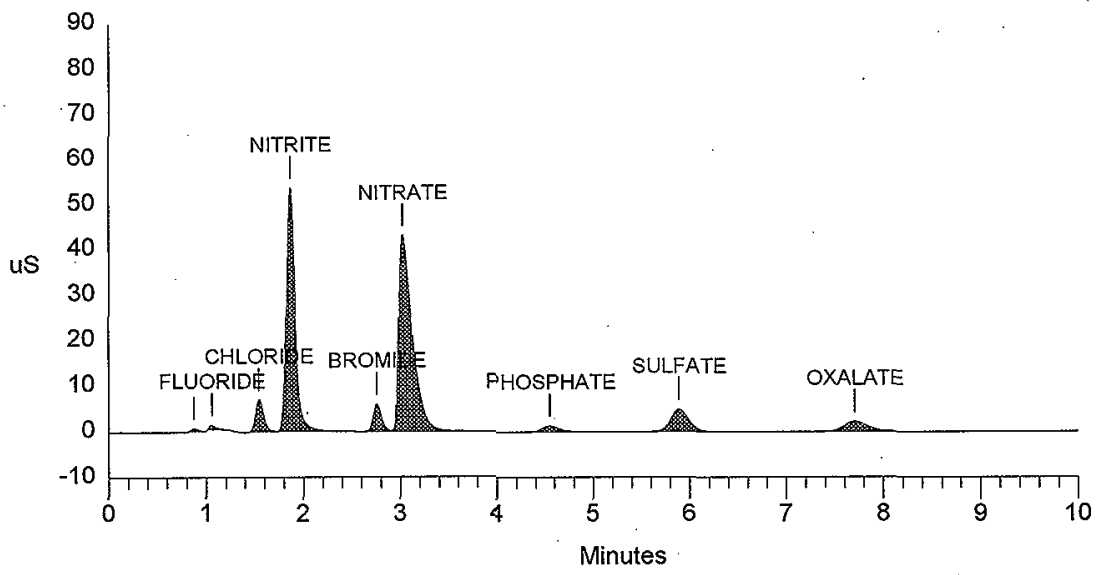


Sample Name: S96T004448 DUP

Data File : C:\DX\DATA \96100801.D05

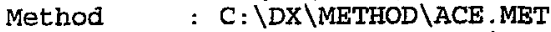

ACI Address: 1 System: 2 Inject\#: 5

Analyst : Ed Colvin

Column: AG4A-SC,AS4A-SC, SRS

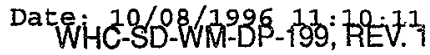

Detector : CDM-1

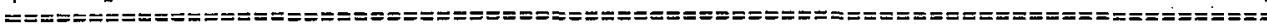

Dalibration Volume Dilution Points Rate start stop Area Reject

\section{External}

1

$11 \quad 4320 \quad 5 \mathrm{~Hz}$

0.00

14.40

0

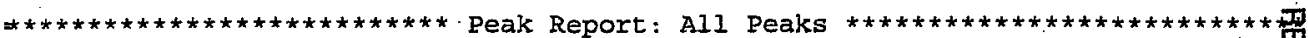

Pk. Ret Component
Num Time Name

Concentration

ug/ml

Height

Area.

Bl. Delta $\rightarrow$

2.13 fluoride

2.40 acetate

2.55

3.11 formate

3.95

5.40

5.76

7.41

8.15

8.35
7.368

4.233

0.000

$5: 931$

0.000

0.000

0.000

0.000

0.000

0.000

\section{4}

229

200

547

275

13881

42593

36446

1260

2203

Code

Totals

17.532

100159

13191

1338

1199

3911

2183

110784

661785

721753

9073

175220

$\begin{array}{rr}2 & 0.16 \\ 2 & -1.23 \\ 2 & \\ 1 & -0.75 \\ 1 & \\ 2 & \\ 2 & \\ 3 & \\ 4 & \\ 4 & \end{array}$

\footnotetext{
.
}

\section{File: 96100801:D05 Sample: S96T004448 DUP}

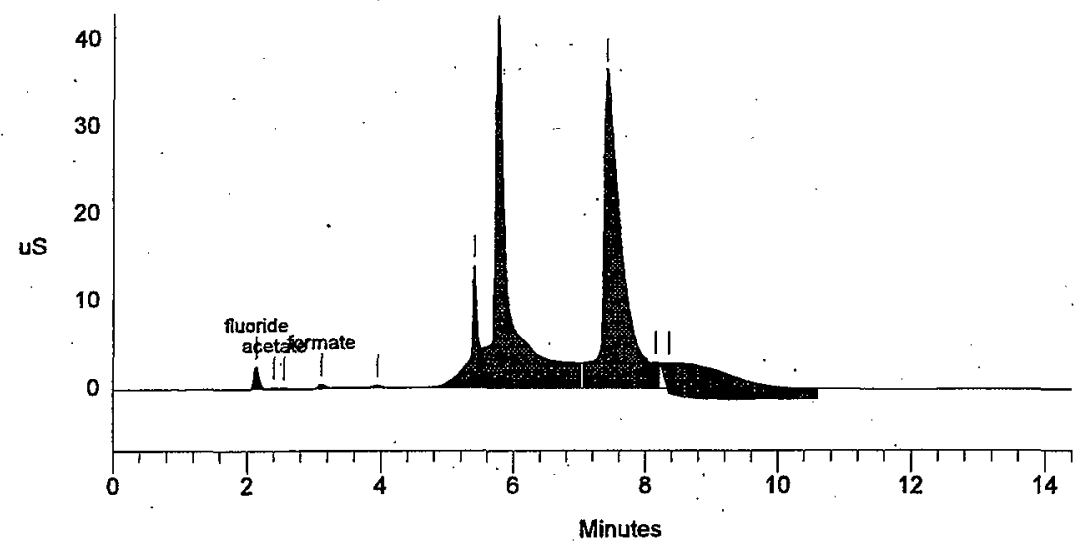




\section{LABCORE Data Entry Template for Worklist\# 12463}

Analyst:

$D C D$ Instrument: $\mathrm{AB} 00$ IS

Book\# 47856

Method: LA-508-101 Rev/Mod D-2

Worklist Comment: AN-105.Determine sample size using ludlum.Spike:0.1ml. new

\begin{tabular}{|c|c|c|c|c|c|c|c|c|}
\hline $\mathrm{s}$ & Type & Sample\# & $\mathbf{R}$ & A & Test & Matrix & Group\# & Project \\
\hline 1 & STD & & & & @ATPHA01 & SOLID & & \\
\hline 2 & BLNK-PREP & & & & $@$ ALPHA01 & SOLID & & \\
\hline 3 & BLNK/BKG & & & & $@$ @ALPHA01 & SOLID & & \\
\hline 4 & SAMPLE & $\begin{array}{l}\text { S96T004317 } \\
\text { Analytes Reque }\end{array}$ & $\begin{array}{l}0 \\
\text { est }\end{array}$ & $\begin{array}{l}\mathrm{F} \\
\text { ted: }\end{array}$ & $\begin{array}{r}\text { @ALPHA01 } \\
: \text { ALPHA0I }\end{array}$ & $\begin{array}{l}\text { SOLID } \\
\text {, ALPHAOIE }\end{array}$ & $\begin{array}{c}: \\
96000945\end{array}$ & AN-105 \\
\hline 5 & DUP & S96T004317 & 0 & F & @ALPHA01 & SOLID & & \\
\hline 6 & SPK & S96T004317 & 0 & F & $@ A L P H A 01$ & SOLID & & \\
\hline 7 & SAMPLE & $\begin{array}{l}\text { S96T004337 } \\
\text { Analytes Reque }\end{array}$ & $\begin{array}{c}0 \\
\text { est }\end{array}$ & $\begin{array}{l}\text { F } \\
\text { ted: }\end{array}$ & $\begin{array}{r}\text { @ALPHA01 } \\
: \text { ALPHA01 }\end{array}$ & $\begin{array}{l}\text { SOLID } \\
\text { ALPHAOIE }\end{array}$ & 96000945 & $A N-105$ \\
\hline 8 & DUP & $S 96 T 004337$ & 0 & $\mathrm{~F}$ & @ALPHA01 & SOLID & & : \\
\hline 9 & SPK & $s 96 \mathrm{~T} 004337$ & 0 & $\mathrm{~F}$ & @ALPHA01 & SOLID & & \\
\hline
\end{tabular}

Final page for worklist \# 12463
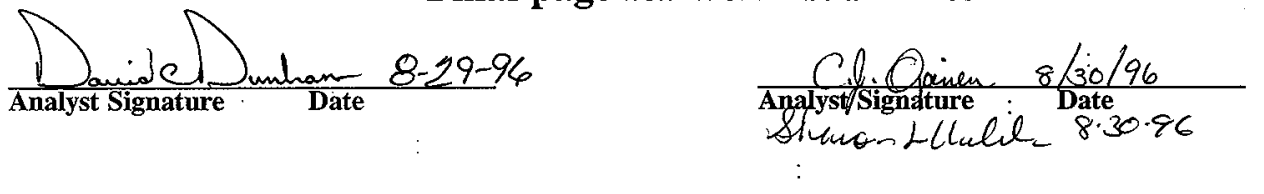

Data Entry Comments:

$S=$ Worklist Slot Number, $R=$ Replicate Number, $A=$ Aliquot Code. 


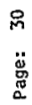

WHC-SD-WM-DP-199, REV. 0

HNF-SD-WM-DP-199, REV. 1

WHC-SD-WM-DP-199, REV. 1
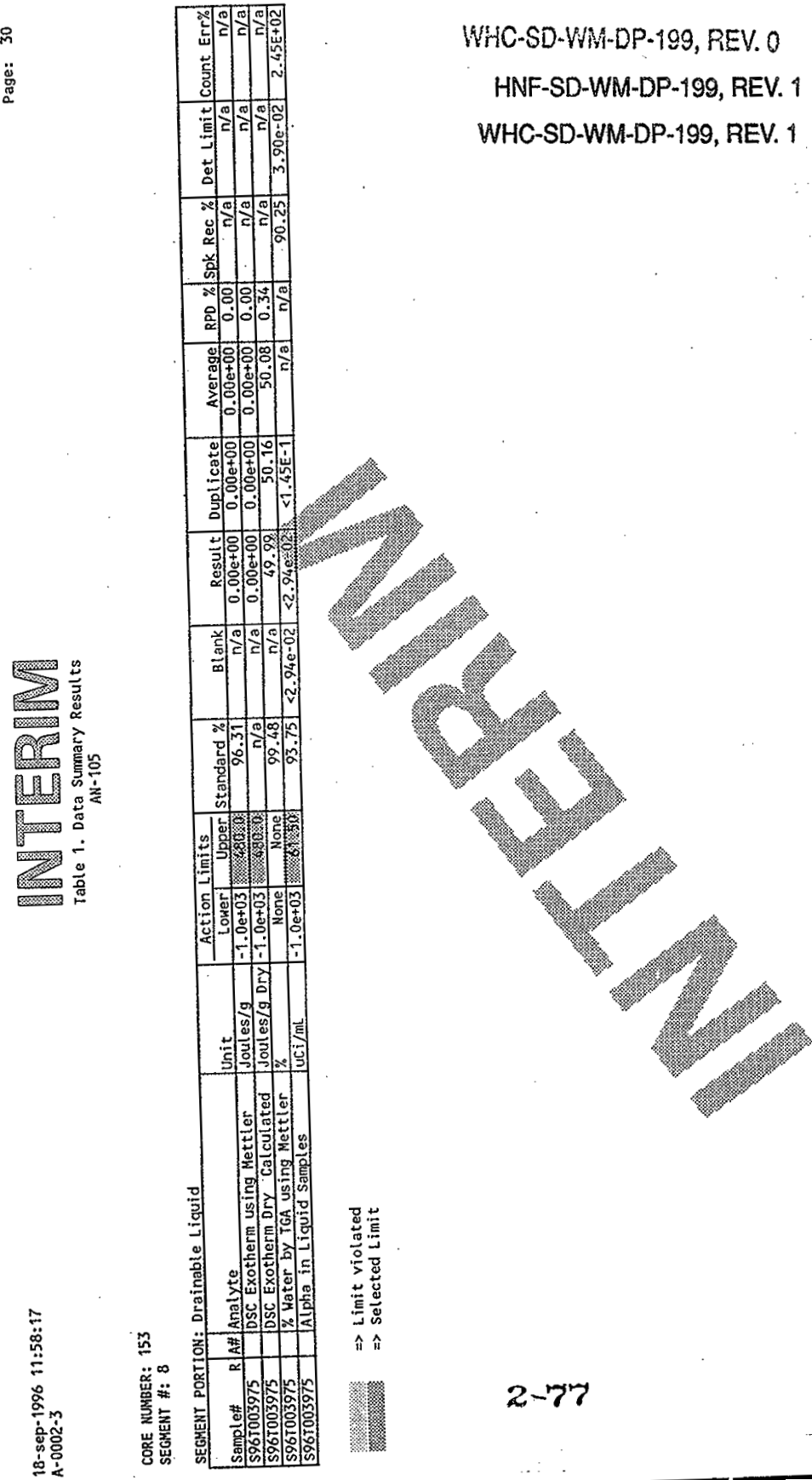

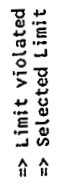

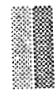

